\title{
Lecture Notes on Inverse Theory
}

\section{Andreas Fichtner}

Department of Earth Sciences, ETH Zurich, Switzerland

Summer 2021

License: These Lecture Notes are published on the Cambridge Open Engage Platform under the Creative Commons Attribution-NonCommercial-NoDerivatives 4.0 International license (CC BY-NC-ND 4.0). You are therefore free to share this material and use it for non-commercial purposes. When using these Lecture Notes for your work, you must give appropriate credit, mentioning author, title and doi. 



\section{Contents}

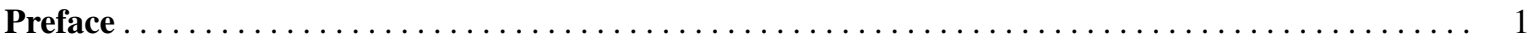

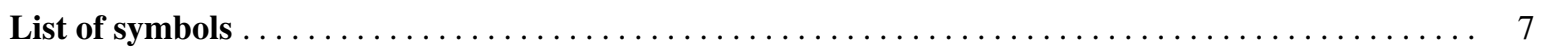

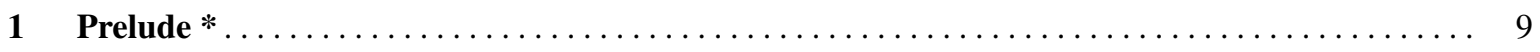

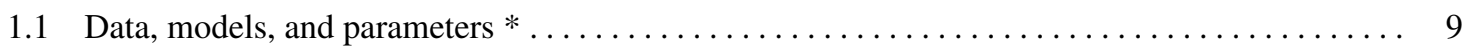

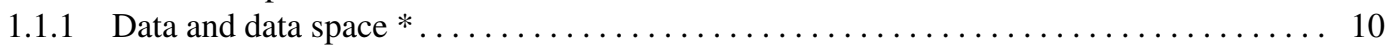

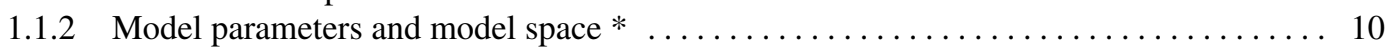

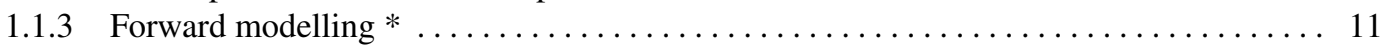

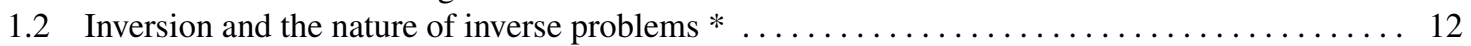

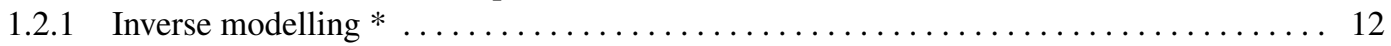

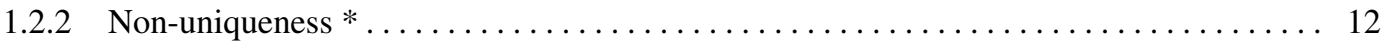

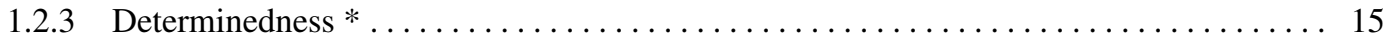

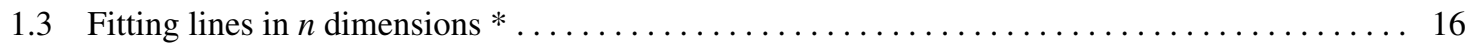

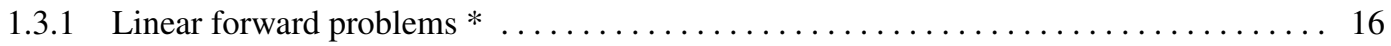

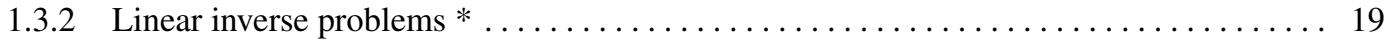

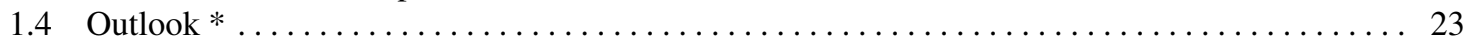

Part I Bayesian Inference and Monte Carlo Methods

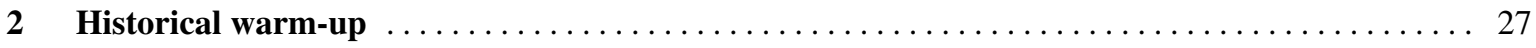

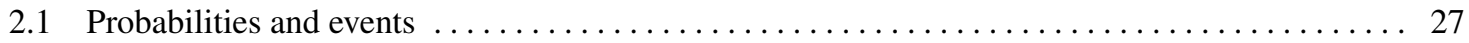

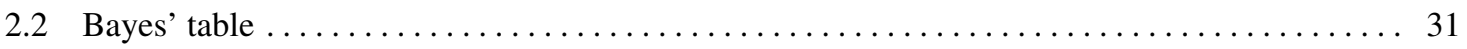

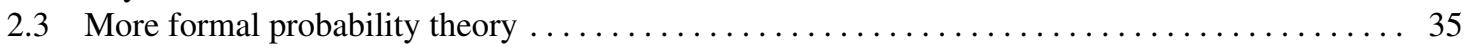

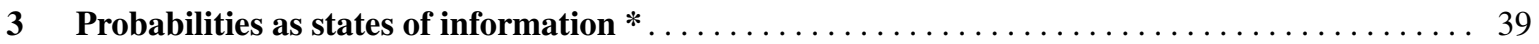

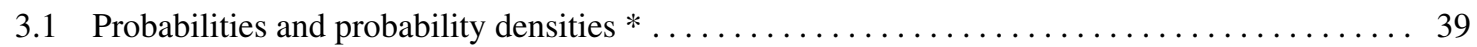

3.2 Frequentist and Bayesian interpretation of probabilities * $\ldots \ldots \ldots \ldots \ldots \ldots \ldots \ldots \ldots \ldots \ldots$

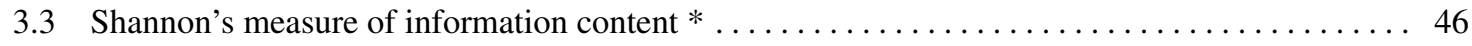

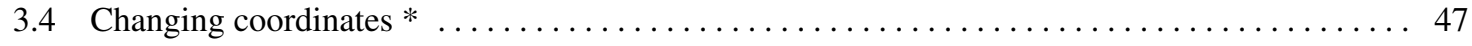

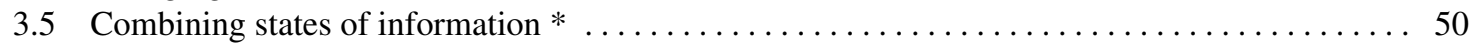

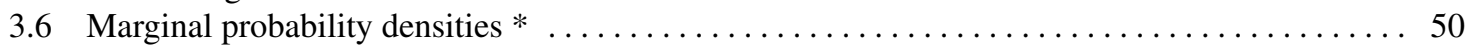

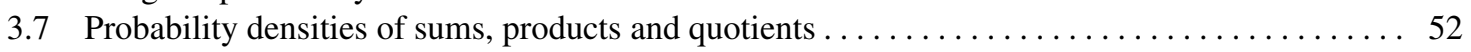

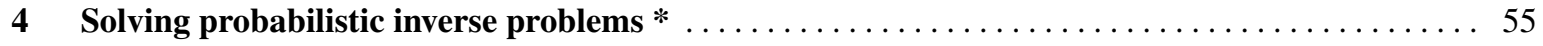

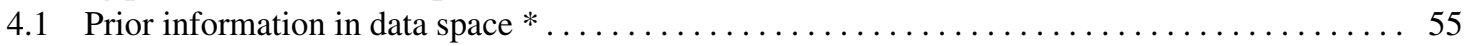

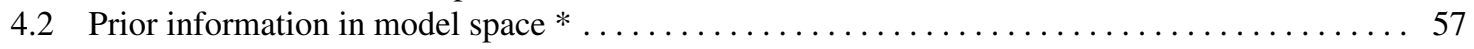

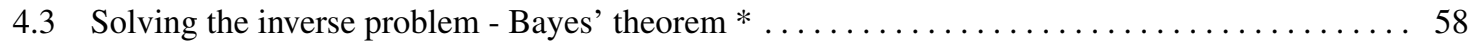

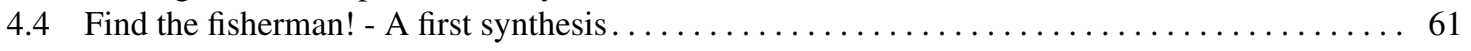




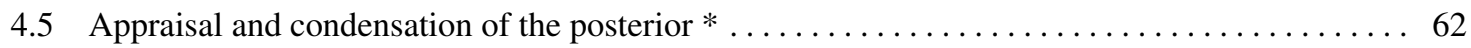

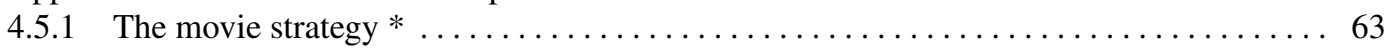

4.5.2 Computing probabilities $* \ldots \ldots \ldots \ldots \ldots \ldots \ldots \ldots \ldots \ldots \ldots \ldots \ldots \ldots \ldots \ldots \ldots$

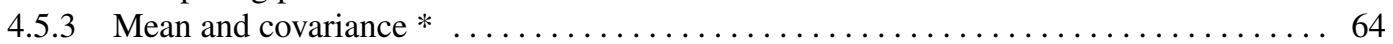

4.5.4 Maximum-likelihood model $* \ldots \ldots \ldots \ldots \ldots \ldots \ldots \ldots \ldots \ldots \ldots \ldots \ldots \ldots \ldots \ldots$

4.6 The relation between probabilistic and deterministic inversion $* \ldots \ldots \ldots \ldots \ldots$

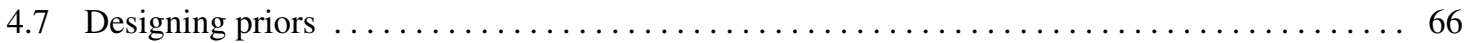

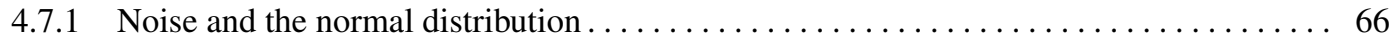

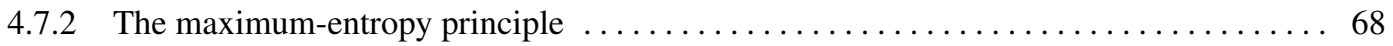

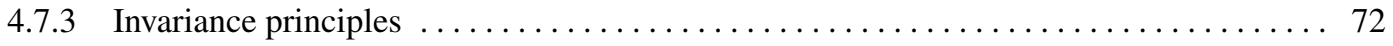

4.7.4 Physical intuition, common sense and subjective relativity $\ldots \ldots \ldots \ldots \ldots \ldots \ldots$

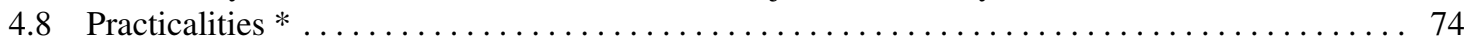

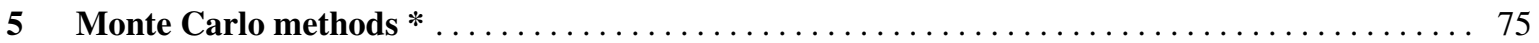

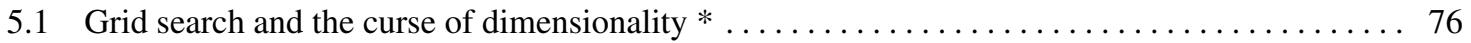

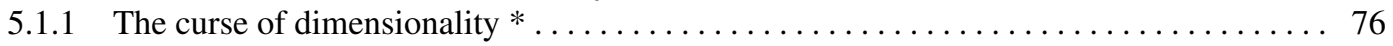

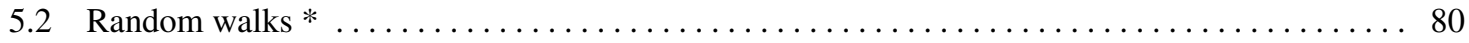

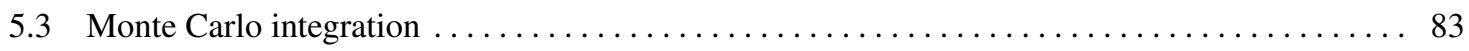

5.3.1 Approximating integrals with random samples $\ldots \ldots \ldots \ldots \ldots \ldots \ldots \ldots \ldots \ldots$

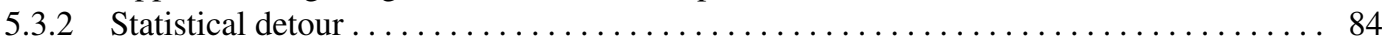

5.3 .3 Convergence of Monte Carlo integration $\ldots \ldots \ldots \ldots \ldots \ldots \ldots \ldots \ldots \ldots \ldots \ldots$

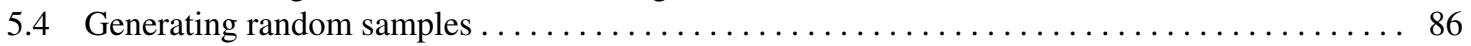

5.5 The Metropolis-Hastings algorithm and its variants $* \ldots \ldots \ldots \ldots \ldots \ldots \ldots \ldots \ldots \ldots$

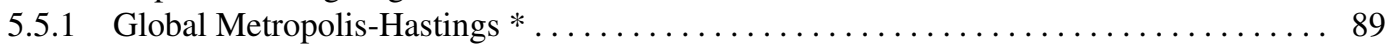

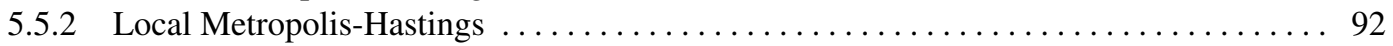

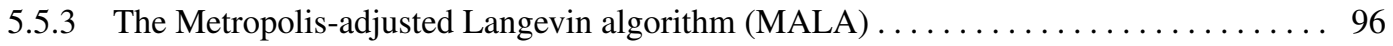

5.6 Tempering and Simulated Annealing $* \ldots \ldots \ldots \ldots \ldots \ldots \ldots \ldots \ldots \ldots \ldots \ldots \ldots \ldots$

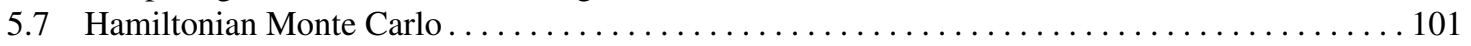

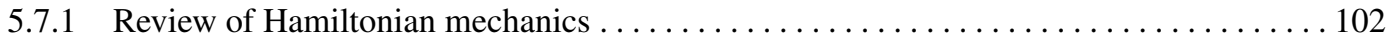

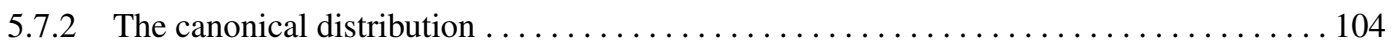

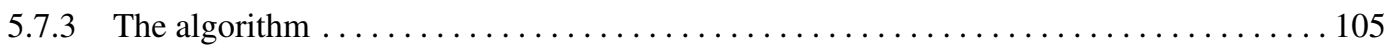

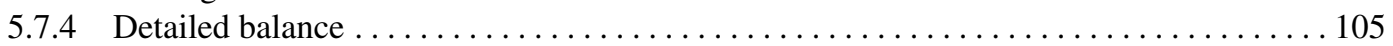

5.7.5 Numerical integration of Hamilton's equations . . . . . . . . . . . . . . . . . . 106

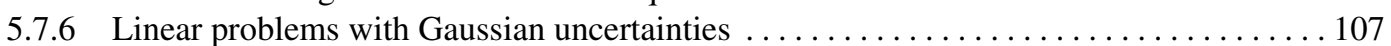

5.7 .7 Tuning Hamiltonian Monte Carlo . . . . . . . . . . . . . . . . . . . . . . . . . . . . . . . 109

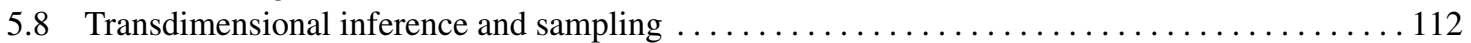

5.8 .1 Bayes' theorem for transdimensional problems $\ldots \ldots \ldots \ldots \ldots \ldots \ldots \ldots \ldots \ldots \ldots \ldots \ldots$

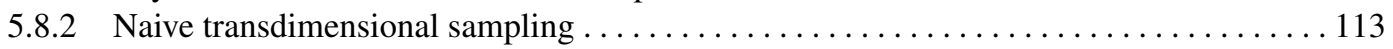

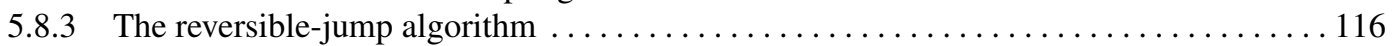

\section{Part II Linear And Weakly Nonlinear Problems}

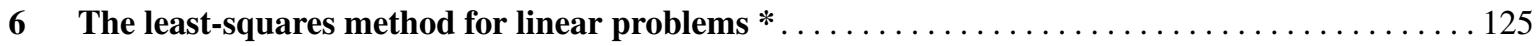

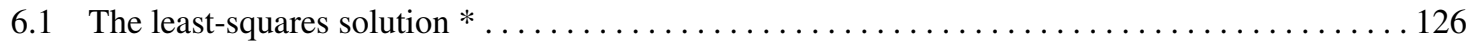

6.2 Model problem: Linear traveltime tomography $* \ldots \ldots \ldots \ldots \ldots \ldots \ldots \ldots \ldots \ldots \ldots \ldots$

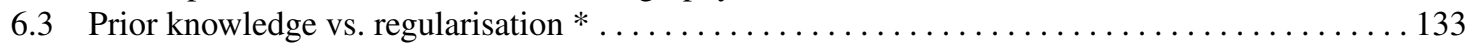

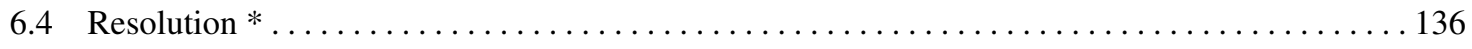

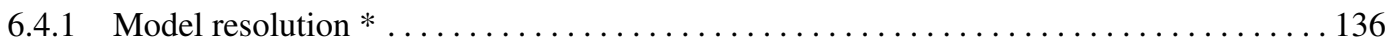

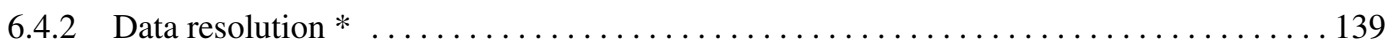

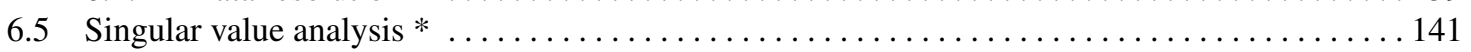

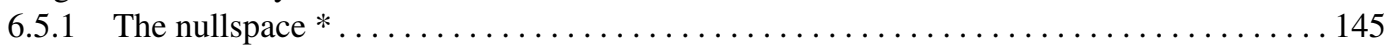

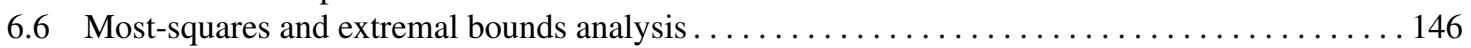




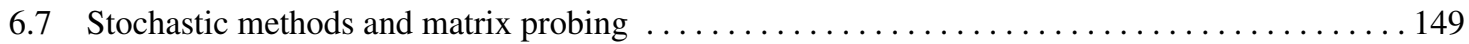

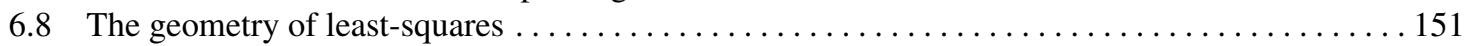

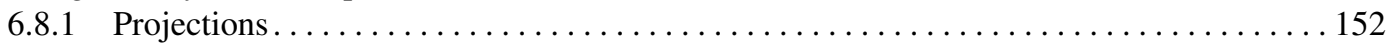

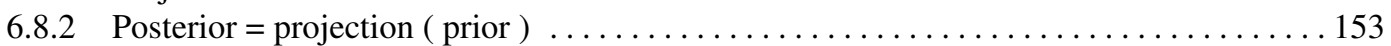

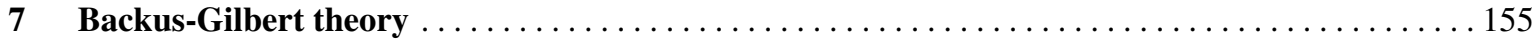

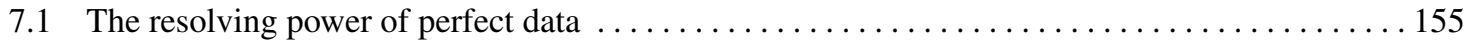

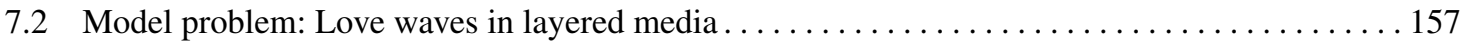

7.3 Inferences from inaccurate data . . . . . . . . . . . . . . . . . . . . . . . . . . . 159

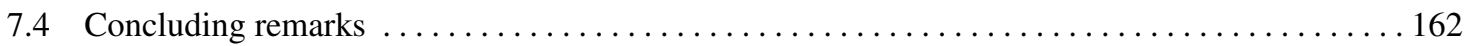

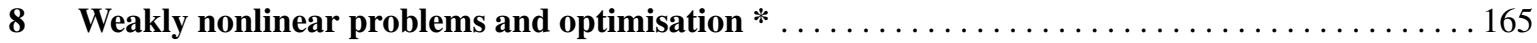

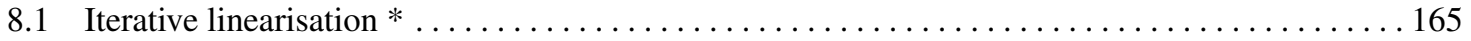

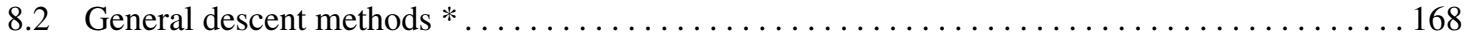

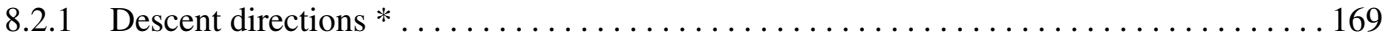

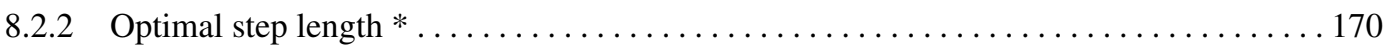

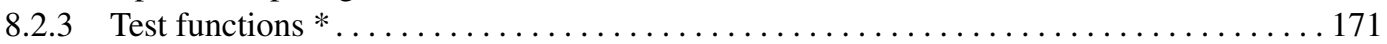

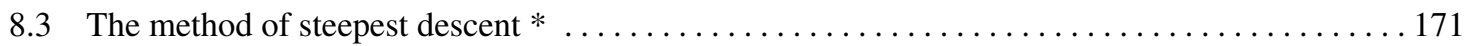

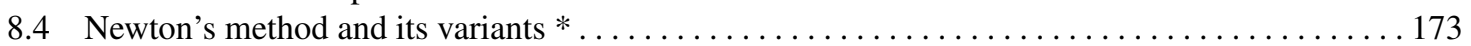

8.4.1 Damped and regularised Newton methods .......................... 174

8.4.2 Gauss-Newton, Levenberg and Levenberg-Marquardt methods . . . . . . . . . . . 176

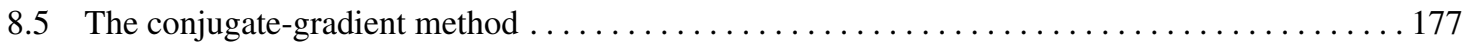

8.5.1 Derivation of the method for quadratic misfit functions . . . . . . . . . . . . . . 177

8.5.2 The benefit of using conjugate descent directions ........................... 181

8.5.3 The conjugate-gradient method for non-quadratic misfit functions $\ldots \ldots \ldots \ldots \ldots \ldots 18$

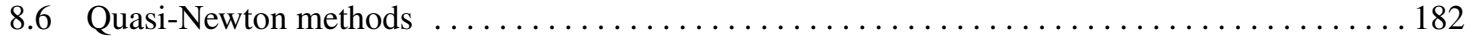

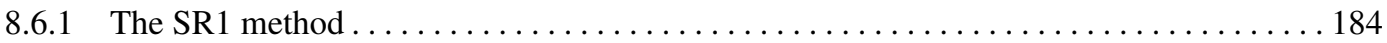

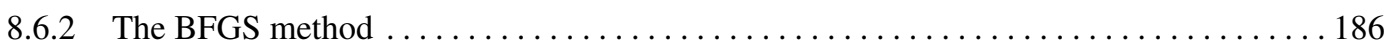

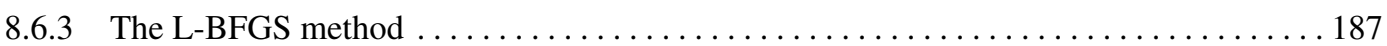

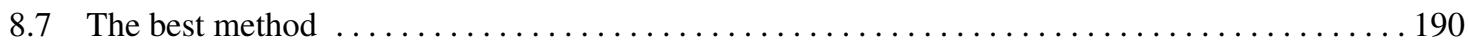

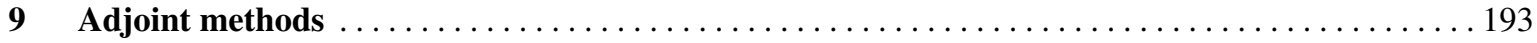

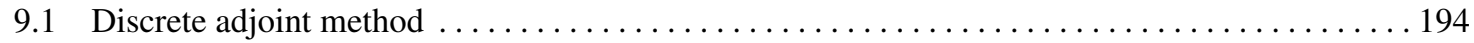

9.1.1 Derivation of the adjoint equations ................................... 194

9.1.2 Model problem: The frequency-domain wave equation with attenuation in 1-D . . . . 195

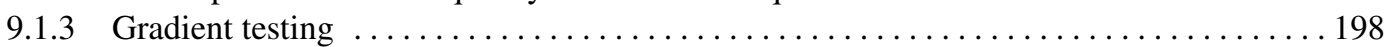

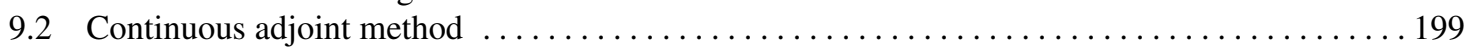

9.2.1 Fréchet derivatives and Fréchet kernels . . . . . . . . . . . . . . . . . . . . . . . . . 200

9.2.2 Derivation of the continuous adjoint equations . . . . . . . . . . . . . . . . . . 202

9.2.3 Kernels and gradients - Linking to the discrete case . . . . . . . . . . . . . . . 203

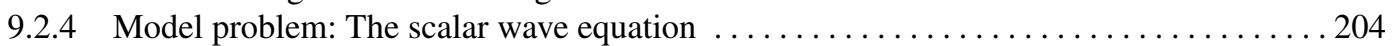

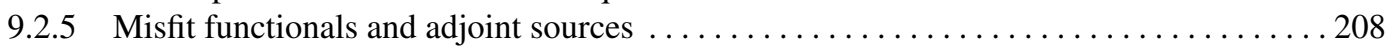

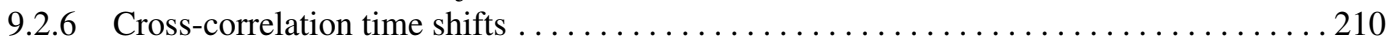

9.2.7 Model problem: The visco-elastic wave equation $\ldots \ldots \ldots \ldots \ldots \ldots \ldots \ldots \ldots \ldots \ldots \ldots \ldots \ldots$

\section{Part III Analytically Solvable Inverse Problems}

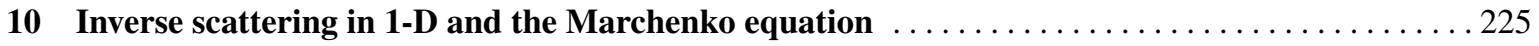

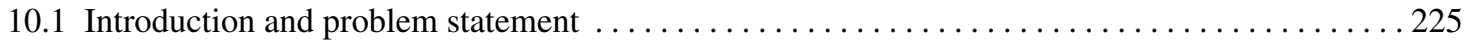

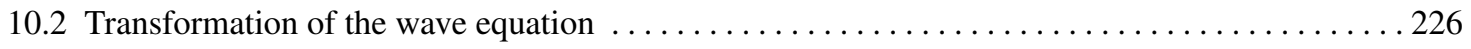

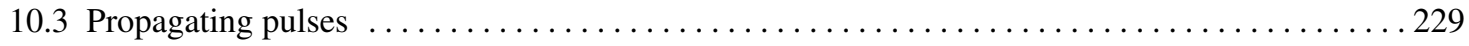

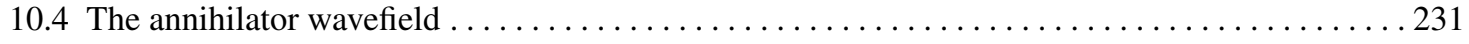

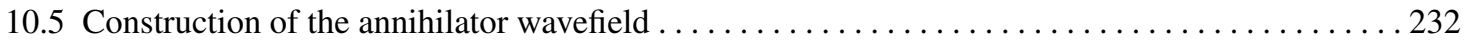




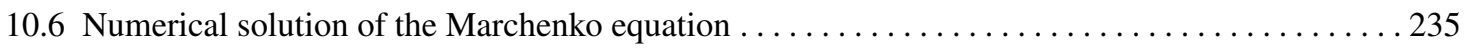

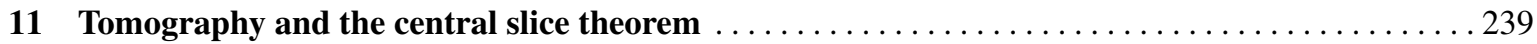

\section{Part IV Advanced and Integrated Topics}

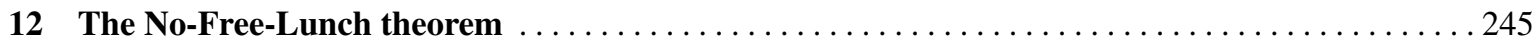

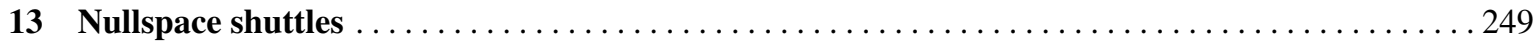

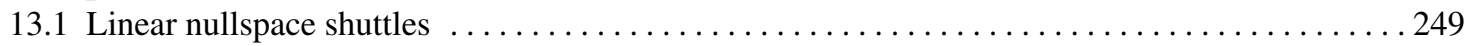

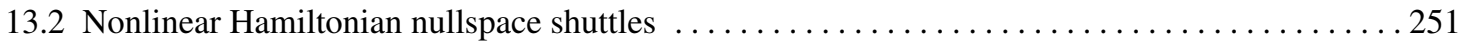

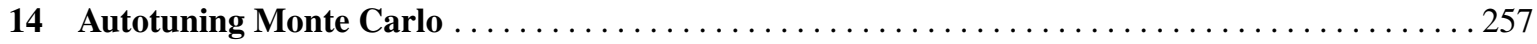

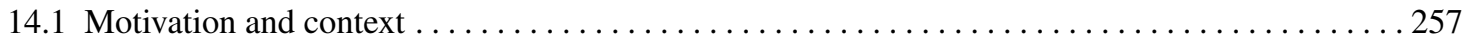

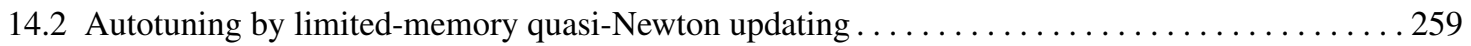

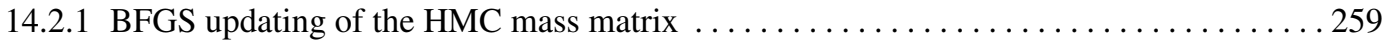

14.2.2 Factorised BFGS method .................................... 260

14.2.3 Limited-memory factorised BFGS method (LF-BFGS) $\ldots \ldots \ldots \ldots \ldots \ldots \ldots \ldots \ldots$

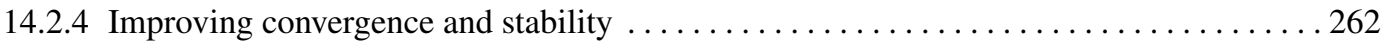

14.2.5 Loss of the Markov property and variations of the theme $\ldots \ldots \ldots \ldots \ldots \ldots \ldots 263$

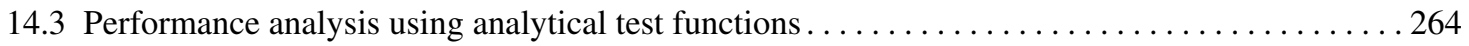

14.3.1 Return to the high-dimensional Gaussian .............................. 264

14.3.2 Modified Styblinski-Tang function ............................... 265

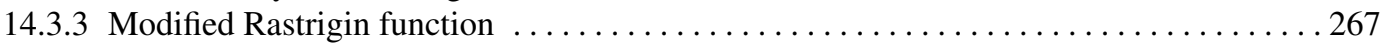

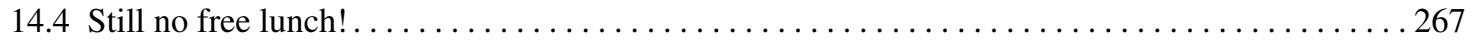

\section{Part V Appendix}

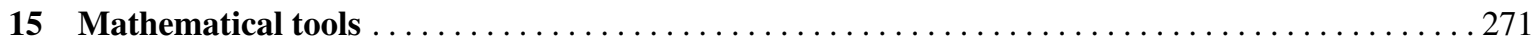

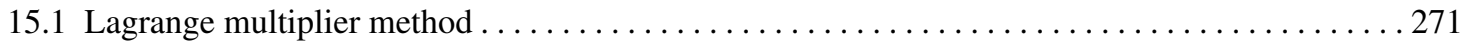

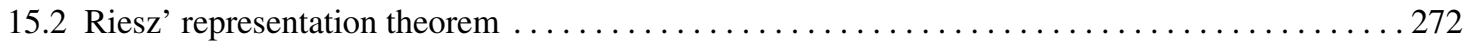

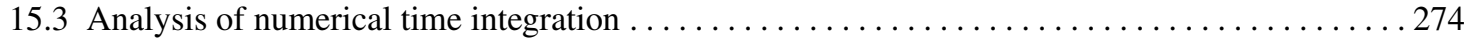

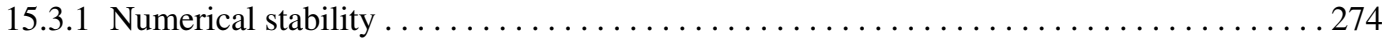

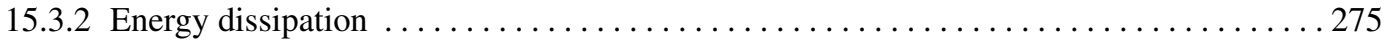

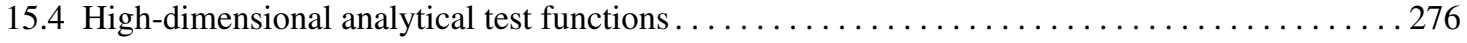

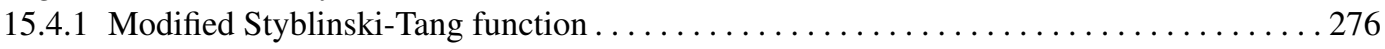

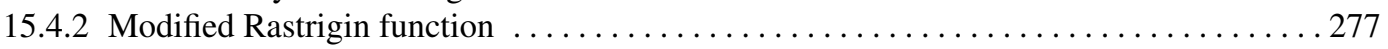

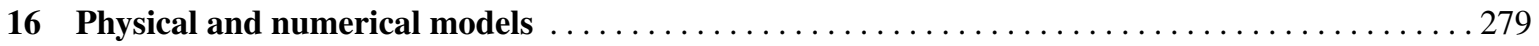

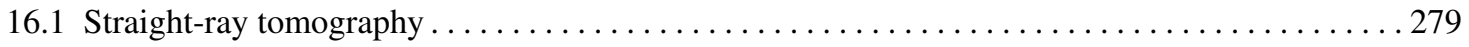

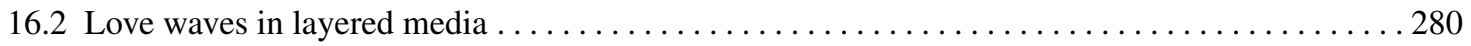

16.2.1 Displacement and stress functions of Love waves $\ldots \ldots \ldots \ldots \ldots \ldots \ldots \ldots \ldots \ldots \ldots 281$

16.2.2 Energy equation and sensitivity kernels ............................ 283

16.3 Finite-difference solution of the 2-D scalar wave equation ....................... 286

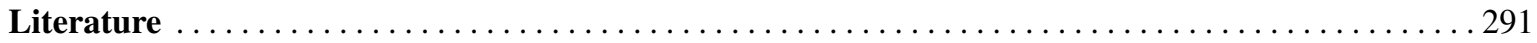

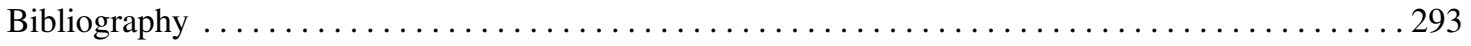

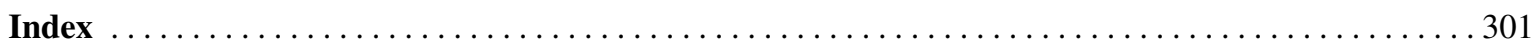




\section{Preface}

Inverse theory is the art of inferring properties of a physical system from observations. It is used to transform recordings of wavefields into estimates of medium properties in seismic tomography, medical imaging and nondestructive testing. It serves to constrain the distribution of density inside the Earth from gravity measurements, and to obtain probabilities of life on exoplanets based on telescope observations of electromagnetic spectra. The list of examples is endless.

On a grander scale, inverse theory may be considered a mathematisation of scientific progress. It formalises the Popperian process of formulating an educated and falsifiable hypothesis, and testing it with newly acquired observations (Popper, 1935). Hence, inverse theory is at the heart of all natural sciences.

What makes inverse theory an art is the nature of our observations, which are typically noisy, sparse and to some extent inconsistent. As a consequence, the properties that we would like to infer can usually not be constrained uniquely. Inverse theory offers a broad collection of concepts and methods to address this nonuniqueness. Some of these explicitly require the injection of prior knowledge, which is often not available in a strict mathematical form, but as subjective physical intuition or experience. Moreover, the choice of a specific concept or method is in itself an act of subjectivity, which is unavoidably influenced by practical considerations, including the finiteness of our resources. The explicit presence of subjective choices, with all their consequences for the inferences that we make, distinguishes inverse theory from pure mathematics, and it adds an artistic, and sometimes even philosophical component to this exciting field.

The primary goal of these lecture notes is to equip the reader with a solid theoretical background and with an extensive toolbox that can be used to solve real-world problems. A central theme is the recognition that the best theory or the best method do not exist. A good solution strategy is application- and data-specific, and so these notes are intended to help with making a reasonable choice.

\section{Structure and organisation}

Under construction and open to feedback: These lecture notes are a preliminary and incomplete version of a more comprehensive book on inverse theory. They were born out of my students' wish to reduce the amount of material that needs to be copied from the blackboard, and the list of included topics keeps growing. Still being under development, these lecture notes are naturally imperfect. The reporting of errors and typos will be very much appreciated, and rewarded with Swiss chocolate! $!^{1}$

Within this context, the purpose of this preprint is also to receive honest and constructive feedback, not only on all the smaller and bigger mistakes, but also on topics that may still be missing (and there are many). In the end, the finished book should be useful for many students and their teachers.

\footnotetext{
${ }^{1}$ You need to be the first person to discover a specific error. Otherwise it does not count. ;-)
} 
Suggestion for a course schedule: Despite being called 'lecture notes', the content of this document goes far beyond the topics that can be covered in a single class. Hence, one needs to make a rather difficult choice. The chapters and sections marked with an asterisk $(*)$ in the table of contents, contain the absolute basics, including an introduction to inverse problems, elementary probability theory and Bayesian inference, Monte Carlo methods, linear inverse theory, and a beginner's guide to nonlinear optimisation. These topics are suitable for a one-semester class with an intensity of two lecture hours per week, plus one hour for exercises. All other chapters and sections are written to be as independent as possible, and therefore can be selected in a more or less random order.

Interactive figures and codes: Many of the figures in these lecture notes are quasi-interactive in the sense that a Python Jupyter Notebook is available to play with. All codes can be downloaded from the Gitlab pages of the Seismology and Wave Physics Group at ETH Zurich, which should look similar to figure 0.1.

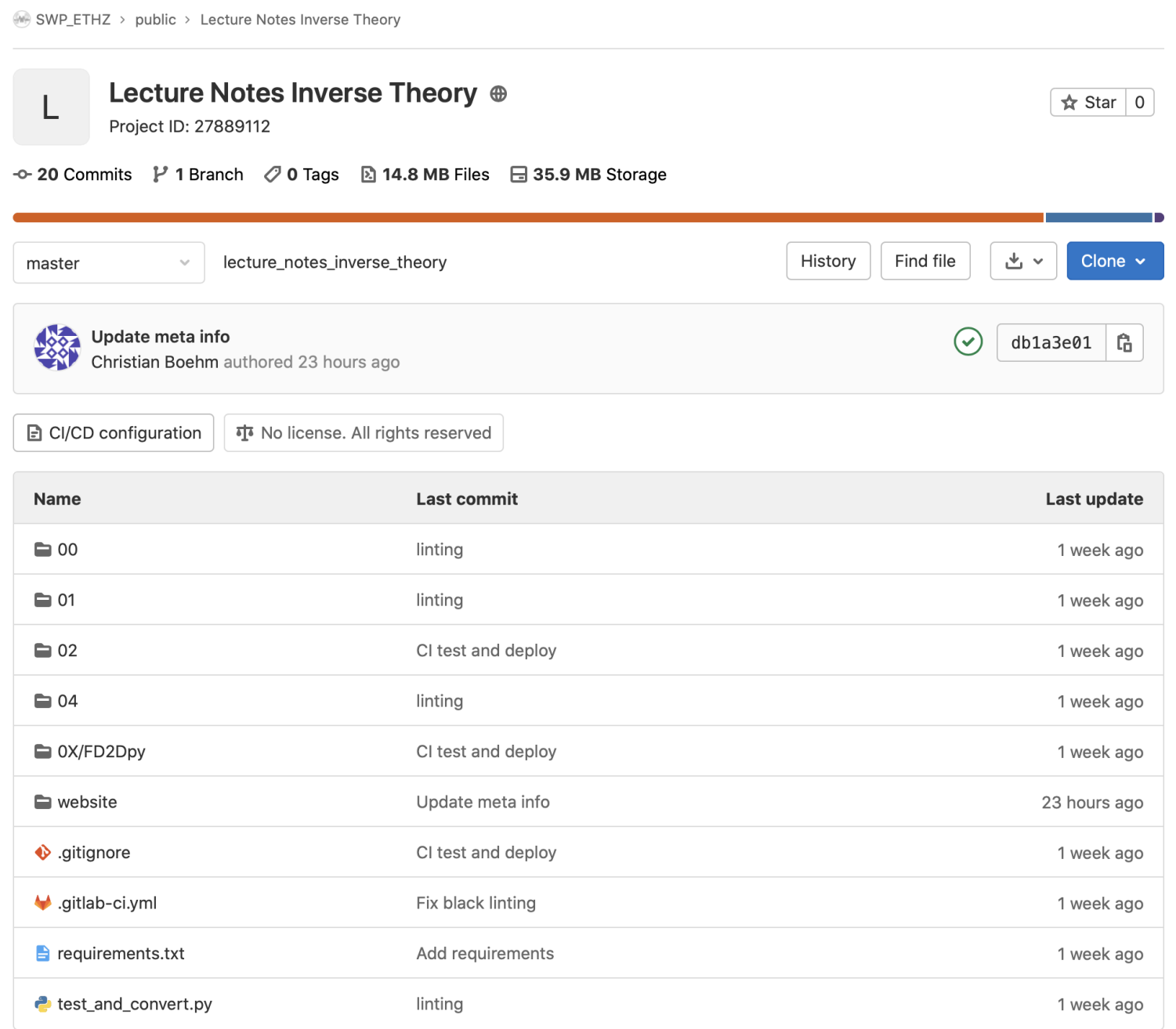

Fig. 0.1: Gitlab website where all Python codes for interactive figures can be downloaded.

Via the Lecture Notes on Inverse Theory Website, the Jupyter Notebooks can also be accessed and run directly in a web browser. This feature makes use of binder, which may take a little while to initialise. Furthermore, the captions of the interactive figures contain a hyperlink that leads to the relevant code, so that readers may start 
experimenting directly. The first interactive figure appears in section 1.3.2, and it treats a simple line-fitting problem. The hyperlink Line-fitting in 1-D should automatically open a web browser and display a markdown version of the notebook, as shown in figure 0.2 .

\section{Lecture Notes on Inverse Theory}

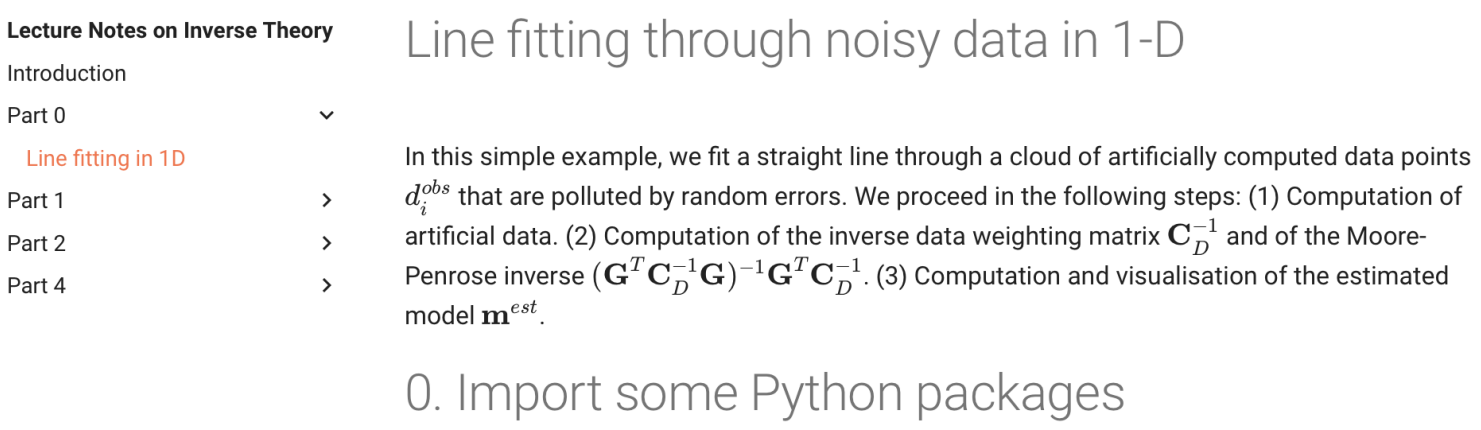

Before running any computations, we import a couple of Python packages, mostly for plotting and for matrix inversion.

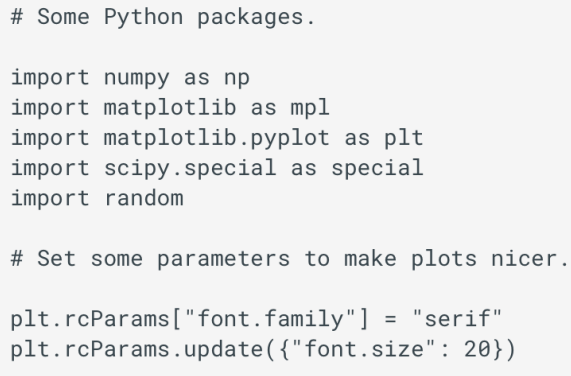

\section{Setup and artificial data}

In the absence of any real observed data, we compute artificial data $\mathbf{d}^{\text {obs }}$ using a random number generator. The artificial data will therefore look different each time you run the code.

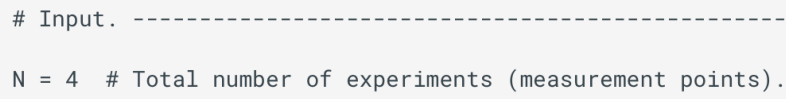

Fig. 0.2: Beginning of the markdown version of the Python Jupyter Notebook for the 1-D line-fitting problem in section 1.3.2.

Grey boxes: contain additional pieces of information, little interesting details, side notes or just some curiosity. They are, of course, recommended, but strictly speaking not required to follow the developments in the main text. 
Model problems: In addition to the purely theoretical and methodological developments, the book contains a series of model problems that are intended to serve as illustrations and means to train intuition. Though most of them are deliberately simplistic, they can often be translated to some real-world problems.

These lecture notes are organised into four different parts:

Part I, following a short introduction on basic concepts, is concerned with Bayesian inference, which can be regarded as the foundation of inverse theory. Bayesian inference elegantly provides solutions to inverse problems in the form of probability densities. Useful information can be extracted from these probability densities with the help of Monte Carlo methods, which are the second big focus of Part I.

Part II covers inverse problems that are characterised by a linear or only weakly nonlinear dependence of the observations on the parameters that we wish to infer. Despite being a special case of general Bayesian inference, this kind of inverse problems is of outstanding practical relevance. In addition to numerical methods to find (approximate) solutions, Part II will also provide a range of tools to analyse solution quality.

Part III is dedicated to a small but very interesting class of inverse problems that can be solved analytically, that is, by direct inversion, as opposed to indirect inversion. Analytical solutions exist mostly under very idealised circumstances. Those idealisations are often removed from real-world situations, which tends to limit their practical applicability. However, what we do gain is a deeper understanding about the nature of the physical system that we are interested in.

Part IV is an appendix containing a collection of useful mathematical tools, as well as detailed descriptions of various physical and numerical models that appear in many of the examples and illustrations.

\section{Notation and mathematical precision}

Finding a useful mathematical notation that is correct without being clumsy, is amost an impossible task. Since the purpose of these lecture notes is mostly educational, we frequently use notation that is simplified in order to emphasise the structure of equations and the logic behind derivations. This sometimes comes with a loss of precision that pure mathematicians may complain about. Similarly, to avoid the heavy machinery of functional analysis, some proofs are simplified.

Adhering to rather common practice, scalar quantities will be represented in regular font. A good example is the time variable $t$. Vector-valued quantities, such as velocity with its components $v_{i}$, are displayed in lower-case bold symbols, $\mathbf{v}$. With few exceptions, that are mostly historical in nature, upper-case bold symbols are used for matrices. An example is the Hessian or second-derivative matrix $\mathbf{H}$.

For the scalar product of two vectors $\mathbf{u}$ and $\mathbf{v}$, we use different notations, depending on which one is most useful and transparent in a particular equation. The most frequently employed ones are

$$
\mathbf{u}^{T} \mathbf{v}=\mathbf{u} \cdot \mathbf{v}=\sum_{i} u_{i} v_{i},
$$

where the sum runs from $i=1$ to the number of dimensions of $\mathbf{u}$ and $\mathbf{v}$. To avoid confusion, we generally do not use the Einstein summation convention, where repeated indices imply notation. The number of cases where it fails is simply too large. 


\section{Audience and prerequisites}

These lecture notes are intended to be accessible for readers with a basic education in some natural science. Specific prior knowledge that will be useful includes undergraduate-level physics, linear algebra (vectors, matrices and their inverses, determinants, index notation, ...), statistics (means and variances, probabilities and probability distributions), and calculus (differentiation and integration of scalar- and vector-valued functions, finding minima and maxima of functions). Knowledge of the programming language Python is beneficial for the interactive figures and related Jupyter Notebooks, but not essential to understand the lecture notes themselves.

\section{Thanks and acknowledgements}

First and foremost, I would like to thank my students who pushed me to start writing, and who continue to provide inspiration for further additions and improvements. Many friends and colleagues contributed to these lecture notes in the course of many years. Large parts of this book were written in the lovely village of Mönkeberg near Kiel, in the middle of the Corona pandemic. The quiet time of concetrated work in Mönkeberg would not have been possible without the help of Tom Kwasnitschka and Heidrun Kopp. The sections on linear inverse problems were largely inspired by an inverse theory manuscript of Hansruedi Maurer. The section on Hamiltonian Monte Carlo is the result of a collaboration with Andrea Zunino and Lars Gebraad. Klaus Mosegaard kindly introduced me to the No-Free-Lunch Theorem and mentioned its relation to Lewis Carrol and Alice in Wonderland. Seeing connections and the broader context, and looking at problems of different angles, would not have been possible without the help of many colleagues who I had the pleasure to work and discuss with: Christian Boehm, Ebru Bozdağ, Joeri Brackenhoff, Hans-Peter Bunge, Michel Campillo, Yann Capdeville, Andrew Curtis, Alexandre Fournier, Bernhard Forkmann, Heiner Igel, Andy Jackson, Brian L. N. Kennett, Amir Khan, Barbara Romanowicz, Malcolm Sambridge, Frederik Simons, Roel Snieder, Hrvoje Tkalčić , Jeroen Tromp, Martin van Driel, Peter Jan van Leeuwen, Tristan van Leeuwen, Ivan Vasconcelos, Kees Wapenaar, and many others. Christian Boehm kindly implemented the direct linking between the interactive figures and the Jupyter Notebooks, which is one of the unique features of these Lecture Notes. Finally, I would like to acknowledge Jeannot Trampert for his patience and long brainstorming sessions about inverse theory. 



\section{List of symbols}

The following is a non-exhaustive list of symbols. It is unavoidable that some symbols are used more than once. In such cases, their meaning should be obvious from the context.

\begin{tabular}{l|l}
$A^{i \leftarrow j}$ & acceptance probability \\
$b_{i}$ & basis function \\
$c$ & phase velocity of a wave \\
$\mathbf{c}$ & elastic tensor \\
$\mathbf{C}$ & generic covariance matrix \\
$\mathbf{C}_{D}$ & data covariance matrix \\
$\mathbf{C}_{M}$ & model covariance matrix \\
$\mathbf{d}$ & generic data vector \\
$\mathbf{d}^{\text {est }}$ & data vector computed from the estimated model $\mathbf{m}^{\text {est }}$ \\
$\mathbf{d}^{\text {obs }}$ & vector of observed data \\
$\mathbb{D}$ & data space \\
$\mathbf{e}_{i}$ & unit vector in $i$-direction \\
$\mathbf{f}$ & external source, force term \\
$\mathbf{f}^{\dagger}$ & adjoint source \\
$\mathbf{G}$ & forward modelling operator or data kernel \\
$\mathbf{h}$ & descent direction in iterative optimisation \\
$I$ & information content \\
$\mathbf{J}$ & Jacobian (of the forward modelling operator) \\
$K$ & Fréchet or sensitivity kernel \\
$\mathbf{L}$ & forward modelling operator \\
$\mathbf{L}^{\dagger}$ & adjoint operator \\
$\mathscr{L}$ & differential operator \\
$\mathbf{m}$ & generic model vector \\
$\mathbf{m}^{\text {est }}$ & estimated model vector \\
$\mathbf{m}^{\text {prior }}$ & prior model vector \\
$\mathbf{m}_{0}$ & mean model of a Gaussian distribution \\
$\tilde{\mathbf{m}}$ & posterior mean model \\
$\hat{\mathbf{m}}$ & posterior maximum-likelihood model \\
$\mathbf{m}_{i}$ & model vector in the $i$ th iteration of an optimisation algorithm \\
$\mathbf{m}^{i}$ & $i^{\text {th }}$ \\
$\mathbf{m}^{i}$ & vector of continuously distributed model parameters \\
$\mathbb{M}$ & model space \\
& some subvolume of model space \\
\hline &
\end{tabular}




\begin{tabular}{|c|c|}
\hline $\begin{array}{l}n \\
\mathbf{n} \\
N \\
N_{s} \\
N_{r} \\
N_{\mathrm{eff}} \\
\mathscr{N}\left(\mathbf{m}_{0}, \mathbf{C}\right) \\
P \\
\mathbf{R}_{M} \\
\mathbf{R}_{D} \\
T^{i \leftarrow j} \\
\mathscr{T} \\
\mathbf{u}^{\mathrm{obs}} \\
\mathbf{u} \\
\mathbf{v} \\
V \\
\partial V \\
\mathbf{x}^{r} \\
X\end{array}$ & $\begin{array}{l}\text { model space dimension } \\
\text { outward surface normal vector } \\
\text { data space dimension } \\
\text { number of samples } \\
\text { number of receivers } \\
\text { effective sample size } \\
\text { normal (Gaussian) distribution with mean } \mathbf{m}_{0} \text { and covariance } \mathbf{C} \\
\text { probability } \\
\text { model resolution matrix } \\
\text { data resolution matrix } \\
\text { transition probability } \\
\text { cross-correlation time shift } \\
\text { observed physical field } \\
\text { calculated physical field } \\
\text { adjoint field } \\
\text { volume, spatial domain of interest } \\
\text { boundary of } V \\
\text { receiver position } \\
\text { statistical event }\end{array}$ \\
\hline $\begin{array}{l}\delta \chi \\
\Gamma \\
\Lambda \\
\mu \\
\rho \\
\rho_{h} \\
\rho_{\text {ref }} \\
\sigma, \sigma_{i} \\
\sigma \\
\chi \\
\chi^{\mathrm{est}} \\
\chi^{\mathrm{rms}}\end{array}$ & $\begin{array}{l}\text { Fréchet (functional) derivative (of a misfit functional) } \\
\text { Gamma function } \\
\text { bulk modulus } \\
\text { singular value matrix } \\
\text { shear modulus } \\
\text { probability density (or mass density) } \\
\text { homogeneous probability density } \\
\text { reference probability density (for the computation of relative information) } \\
\text { standard deviation or singular value or search radius in Metropolis-Hastings algorithm } \\
\text { stress tensor } \\
\text { misfit function, misfit functional } \\
\text { misfit value computed from the estimated model } \mathbf{m}^{\text {est }} \\
\text { root-mean-square misfit functional }\end{array}$ \\
\hline $\begin{array}{l}\nabla \\
\Delta \\
\langle., .\rangle \\
(., .)\end{array}$ & $\begin{array}{l}\text { gradient operator } \\
\text { Laplace operator, Laplacian } \\
\text { averaging integral (Backus-Gilbert theory) } \\
\text { scalar product }\end{array}$ \\
\hline
\end{tabular}




\section{Chapter 1 \\ Prelude *}

Since the number of observations is finite, it is intuitively reasonable that

the collection of acceptable [Earth] models will be 'very' infinite.

George E. Backus and J. Freeman Gilbert, The resolving power of gross Earth data, 1968.

A major task of many physical sciences is the study of properties and processes within media that are not accessible to direct observations. Concrete examples include the detection of density anomalies inside the Earth, the characterisation of tumors inside the human body, or the identification of corrosion in building materials.

Therefore, indirect methods are used to infer properties and processes from signals that carry information about the internal structure of the medium of interest. Inverse theory provides the mathematical background and the practical methods needed to solve such inference problems.

\subsection{Data, models, and parameters *}

Inverse theory has three main ingredients, schematically summarised in figure 1.1: (i) Observed data, such as acoustic waves received by an ultrasound transducer, or a gravity signal recorded by a satellite. (ii) Unknown material properties or processes that we wish to infer, for instance, the density of human tissue, or the elastic properties inside the Earth. (iii) A physical relationship or a theory that allows us to predict observations, given some material properties or processes. In the following, we will consider these three ingredients in more detail.

\begin{tabular}{|c|c|c|}
\hline $\begin{array}{l}\text { Observed Data } \\
\text { acoustic / seismic wave } \\
\text { recordings, gravity } \\
\text { measurements, GPS } \\
\text { displacements, ... }\end{array}$ & $\begin{array}{c}\text { Physics } \\
\text { wave equation, Maxwell's } \\
\text { equations, Newton's law of } \\
\text { gravitation, Navier-Stokes' } \\
\text { equation, ... }\end{array}$ & $\begin{array}{l}\text { Unknown Properties } \\
\text { seismic velocities, density, } \\
\text { viscosity, conductivity, ..., in } \\
\text { the Earth or some other } \\
\text { inaccessible medium }\end{array}$ \\
\hline
\end{tabular}

Fig. 1.1: The ingredients of inverse theory. Observed data and unknown properties are linked by physics. 


\subsubsection{Data and data space *}

Any inference rests on observations or data. For all practical purposes, data are discrete in both time and space. This is because our measurement devices record signals only with a finite sampling rate, and because the number of measurements is limited in space, for instance by the available number of seismometers and the finite number of pixels of a camera. We may thus collect our observed data into an $N$-dimensional vector $\mathbf{d}^{\text {obs }}$. The ensemble of all conceivable measurements spans the data space $\mathbb{D}$.

Observed data are a function of control parameters $\mathbf{c}$, that can, to some extent, be chosen by the experimenter. Typical examples include the positions and the sampling rate of seismometers or ultrasound transducers used to record a wavefield that has propagated through some unknown medium. Typically, the control parameters are themselves controlled by financial, logistic or physical constraints. For instance, the size of an ultrasound transducer naturally limits the number of transducers per area. Similarly, the sheer cost of a broadband seismometer limits the density of seismic arrays. Clearly, the control parameters can have a significant effect on the quality of our inferences, for example, the resolution of a tomographic image. Choosing the control parameters as good as possible, taking additional constraints into account, is known as optimal design.

Unsurprisingly, data have errors. They fall into three categories that may not always be clearly separated:

Random errors may result from ambient noise, the limited accuracy of any recording device, thermal noise, or data analysis errors, including, for instance, inaccuracies in the picking of arrival times of a wave. Provided that the random nature of the errors, that is, their statistical distribution, is known, inverse theory provides tools to take this class of errors into account and to minimise its effect.

Systematic errors are deterministic (not random) inaccuracies in the experimental setup. Classic examples include incorrect positions or orientations of instruments. While systematic errors are in principle easy to repair, the difficulty lies in their detection. Often, systematic errors are only found a posteriori, when the results look unreasonable.

Outliers or blunders are data errors that are typically much larger than random errors, and they tend to be outright (human) mistakes. Examples include the recording of an incorrect value or misreading a scale. If only a small percentage of the data are contaminated by outliers, it is usually quite easy to detect and remove them from the data set. There exist also specialised inversion techniques that minimise the influence of outliers.

\subsubsection{Model parameters and model space *}

On the basis of observed data, we wish to infer physical model parameters, symbolically written as a finitedimensional vector $\mathbf{m}$. The set of all possible $\mathbf{m}$ spans the model space $\mathbb{M}$. At this point, the terminology used in different branches of the physical sciences is a bit ambiguous. For instance, in the atmospheric and ocean sciences, the term model refers to the equations relating parameters, such as the viscosity of water, to observations. In contrast, geophysicists reserve the term model for the model parameters. Having to make some choice, we will henceforth use the geophysical terminology.

For some inverse problems, the definition of the model space is quite trivial. For example, the earthquake location problem includes four unknowns contained in $\mathbf{m}$, namely the three hypocentral coordinates that determine position in space, and the origin time. Another trivial example is fitting a polynomial. Let us assume that we made temperature measurements in a borehole, and we would like to approximate the temperature-depth profile with a polynomial of degree $n$. This can be cast in the form of an inverse problem, and the corresponding model vector $\mathbf{m}$ includes the $n+1$ polynomial coefficients.

The above examples include a naturally limited number of discrete model parameters. The situation becomes more complicated when we try, for example, to estimate properties that are continuous functions of a space variable $\mathbf{x}$, for instance, wave speeds or electrical conductivity inside the Earth or other inaccessible bodies. While theories for continuous inverse problems do exist, the vast majority of practically relevant problems is 
unavoidably discrete because they are solved using a digital computer. It follows that space must be discretised. This can be done by representing a continuous model parameter $\mathfrak{m}(\mathbf{x})$ in terms of a finite number of basis functions $b_{i}(\mathbf{x})$, that is,

$$
\mathfrak{m}(\mathbf{x})=\sum_{i=1}^{n} m_{i} b_{i}(\mathbf{x}) .
$$

The $n$ basis functions may, for instance, be small blocks within which material properties are constant, or spline functions, wavelets, spherical harmonics, etc. Collecting the coefficients $m_{i}$ into an $n$-dimensional vector, then yields the finite-dimensional model

$$
\mathbf{m}=\left(m_{1}, m_{2}, \ldots, m_{n}\right)^{T} .
$$

Discretisation of a continuous model space always adds some subjectivity because it is not obvious how to subdivide the region of interest. Figure 1.2 illustrates the problem. It shows a simple seismic or medical tomography experiment where the region of interest is delimited by the black square. Within the region of interest, we wish to constrain, for instance, the distribution of wave speed, which is a continuous quantity. For this, a collection of sources (e.g., earthquakes, explosions, or ultrasound transducers) emit waves that propagate towards some receivers. For simplicity, we assume that wave propagation takes place along straight lines, also called rays, to make the analogy with a ray of sunlight, which is an electromagnetic wave. The 2-D discretisation of the domain is shown in the form of square cells. The experiment in figure 1.2a employs large cells. Therefore, many of the cells are traversed by a large number of crossing ray paths. As we will learn later, the number of crossing ray paths in a particular cell is indicative of how well the physical parameter, in this case the wave speed, of this cell can be resolved. We call our ability to resolve a particular model parameter the formal resolution. The formal resolution in figure 1.2a is comparatively good, but the large cells will prevent us from resolving features that may be smaller than the cells. Therefore, the spatial resolution of the experiment in figure $1.2 \mathrm{a}$ is rather poor.

In contrast, the experiment shown in figure $1.2 \mathrm{~b}$ includes a large number of small cells. This comes at the expense of a low formal resolution. In fact, some of the cells are traversed by any ray at all, meaning that their physical properties are entirely unconstrained. However, in some parts of the region of interest, the spatial resolution is potentially quite good. Ideally, we would like to have both, good formal and spatial resolution, but this is often not possible. In the experiment in figure 1.2, one could simply increase the number of sources and receivers, but in many other experimental setups this may not be an option. Finding a good compromise between formal and spatial resolution is a non-trivial task and requires a good physical understanding of the problem. How large are the smallest expected anomalies? What is the theoretical resolution power of the method employed? We will revisit this problem later when we discuss transdimensional inference methods that determine suitable parameterisations automatically (section 5.8), and when we introduce the concept of regularisation (section 6.3).

\subsubsection{Forward modelling *}

Forward modelling on the basis of a physical theory provides the link between model space $\mathbb{M}$ and data space $\mathbb{D}$. Specifically, assuming some model parameters $\mathbf{m}$ and some control parameters $\mathbf{c}$, the forward modelling operator $\mathbf{G}$ produces synthetic or predicted data $\mathbf{d}$,

$$
\mathbf{d}=\mathbf{G}(\mathbf{m}, \mathbf{c}) .
$$

For convenience, we will mostly omit $\mathbf{c}$ in the notation, unless it is explicitly needed. The forward modelling operator $\mathbf{G}$ may, for instance, be Newton's law of gravitation that links the masses of two bodies (model parameters) to a gravitational force that can be measured (data). More often, however, $\mathbf{G}$ is not given explicitly, but in the form of a differential equation that needs to be solved numerically. Common examples include the wave equation, Maxwell's equations, or the Navier-Stokes equation. 
a) High formal resolution, low spatial resolution

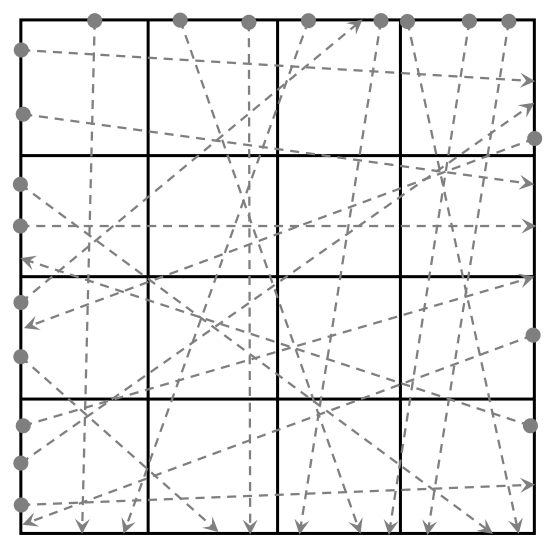

b) High spatial resolution, low formal resolution
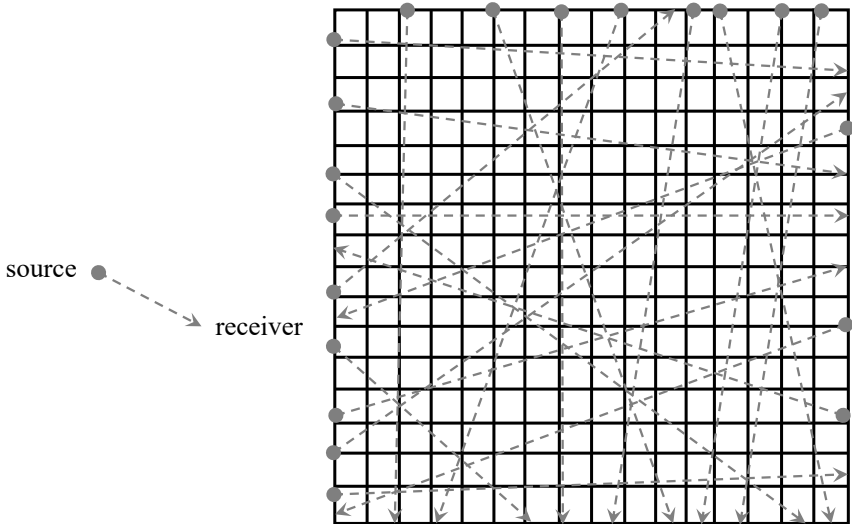

Fig. 1.2: Trade-off between spatial and formal resolution. Grey arrows indicate straight ray paths from sources to receivers, black lines show the model parameterisation in 2-D cells. The model parameterisation shown in (a) offers a good formal and a poor spatial resolution, whereas the model parameterisation in (b) offers a poor formal and a good spatial resolution. (Figure modified after Menke (2012).)

Ideally, it seems, we would like to have a model $\mathbf{m}$ that exactly reproduces the observations $\mathbf{d}^{\text {obs }}$. This model could then be considered an optimal representation of the actual medium ${ }^{1}$. However, the data have errors that we would not like to fit, and the model that we found may actually not be the only possible one!

\subsection{Inversion and the nature of inverse problems *}

\subsubsection{Inverse modelling *}

Inverse modelling means to estimate a model $\mathbf{m}^{\text {est }}$ using observations. At least symbolically, this requires an inversion of equation (1.3),

$$
\mathbf{m}^{\mathrm{est}}=\mathbf{G}^{-1}\left(\mathbf{d}^{\mathrm{obs}}\right) .
$$

The outstanding difficulty of inverse modelling is that the inverse $\mathbf{G}^{-1}$ of the forward modelling operator $\mathbf{G}$ does usually not exist. Already in the simple case where the forward model is a matrix-vector multiplication, $\mathbf{d}=\mathbf{G}(\mathbf{m})=\mathbf{G m}$, the matrix $\mathbf{G}$ may not be square, or it may have vanishing eigenvalues. This illustrates that inverse modelling is a significantly more challenging task than forward modelling.

\subsubsection{Non-uniqueness *}

The large majority of inverse problems is non-unique, meaning that more than one model explains the data to within the observational errors. Non-uniqueness has several origins:

Physical or inherent non-uniqueness is a property of physics that cannot be avoided, regardless of data volume and quality. A well-known example from gravity is illustrated in figure 1.3. It shows three different distributions of density within a spherically shaped body that all produce exactly the same external gravity field. The

${ }^{1}$ In this context, the term true model is often used. We note, however, that there is no such thing as a true model. Building a physical model involves simplifications and an abstraction of reality. Therefore, a model is, by construction, never true. 
spherically symmetric part of the gravity field can be generated by any arbitrary, spherically symmetric density distribution with the same total mass. It follows that the inverse problem is inherently ambiguous. As a consequence, the inverse operator $\mathbf{G}^{-1}$ - even if it existed explicitly - could not provide a unique answer concerning the distribution of density inside the body. Hypothetically acquiring an infinite number of data points with prefect accuracy would not change this situation.

Inherent non-uniqueness of the gravity potential: Certainly the most prominent example of physical or inherent non-uniqueness are measurements of the gravitational potential or gravitational acceleration. The gravitational potential $\phi$ at any point in space $\mathbf{x}$ is determined by the spatial distribution of mass density $\rho(\mathbf{x})$ via Gauss' law of gravitation,

$$
\Delta \phi(\mathbf{x})=4 \pi G \rho(\mathbf{x}),
$$

where $G=6.674 \cdot 10^{-11} \mathrm{~m}^{3} \mathrm{~kg}^{-1} \mathrm{~s}^{-2}$ is the gravitational constant, and $\Delta=\nabla \cdot \nabla$ is the Laplace operator. Equation (1.5) is a Poisson equation.

As illustrated in figure 1.3a, we may decompose the density distribution $\rho(\mathbf{x})$ into a spherically symmetric component $\rho_{0}(\mathbf{x})$, and a component $\delta \rho(\mathbf{x})=\rho(\mathbf{x})-\rho_{0}(\mathbf{x})$, which contains the remaining nonspherically symmetric density variations. Since the Poisson equation (1.5) is linear, we obtain separate equations for the potential $\phi_{0}(\mathbf{x})$ caused by $\rho_{0}(\mathbf{x})$ and the potential $\delta \phi(\mathbf{x})$ caused by $\delta \rho(\mathbf{x})$,

$$
\Delta \phi_{0}(\mathbf{x})=4 \pi G \rho_{0}(\mathbf{x}), \quad \Delta \delta \phi(\mathbf{x})=4 \pi G \delta \rho(\mathbf{x}), \quad \phi(\mathbf{x})=\phi_{0}(\mathbf{x})+\delta \phi(\mathbf{x}) .
$$

To demonstrate non-uniqueness, we consider a spherical volume $V$ that completely surrounds the spherically symmetric part of the density distribution, $\rho_{0}(\mathbf{x})$. Integrating the first of equations (1.6) over $V$, we obtain

$$
\int_{V} \Delta \phi_{0}(\mathbf{x}) d^{3} \mathbf{x}=\int_{\partial V} \nabla \phi_{0}(\mathbf{x}) \cdot \mathbf{n}(\mathbf{x}) d^{2} \mathbf{x}=4 \pi G \int_{V} \rho_{0}(\mathbf{x}) d^{3} \mathbf{x}=4 \pi G M_{0},
$$

where the second identity follows from Gauss' divergence theorem, which transforms the volume integral into an integral over the surface $\partial V$ of the volume $V$. The vector $\mathbf{n}(\mathbf{x})$ denotes the outward surface normal on $\partial V$, and $M_{0}$ is the total mass of the spherically symmetric density distribution. Since $\rho_{0}(\mathbf{x})$ is spherically symmetric, the equipotential surfaces, that is, the surfaces of constant potential, are also spherically symmetric. Therefore, the integrand $\nabla \phi_{0}(\mathbf{x}) \cdot \mathbf{n}(\mathbf{x})$ is constant across $\partial V$. Denoting the distance of $\partial V$ from the coordinate origin by $r$, and recalling that $-\nabla \phi_{0}(\mathbf{x}) \cdot \mathbf{n}(\mathbf{x})=g_{0}$ is the radial component of the gravitational acceleration $\mathbf{g}_{0}$, we obtain

$$
-4 \pi r^{2} g_{0}(r)=4 \pi G M_{0}
$$

or

$$
g_{0}(r)=-G \frac{M_{0}}{r^{2}}
$$

Equation (1.9) implies that the spherically symmetric component of the gravitational acceleration $g_{0}$ only depends on the total mass of the spherically symmetric density component $\rho_{0}$, and not on how this density is actually distributed in space. Hence, any other spherically symmetric density distribution $\rho_{0}^{\prime}(\mathbf{x})$ that has the same mass, $M_{0}=\int_{V} \rho_{0}^{\prime}(\mathbf{x}) d^{3} \mathbf{x}$, produces exactly the same potential $\phi_{0}$. In other words, the spherically symmetric component of the density distribution $\rho(\mathbf{x})$ cannot be constrained by gravity observations, no matter how dense and perfect they are. This fact of gravity non-uniqueness is illustrated in figure $1.3 \mathrm{~b}$.

Similar arguments hold for other physical quantities that are governed by Poisson equations. These in- 
clude static electric and magnetic fields, and the steady-state temperature field cause by a time-invariant heat source. Inherent non-uniqueness is indeed widespread in the physical sciences.

In addition to inherent physics, non-uniqueness may result from an insufficient number of data. Again using the example of a linear problem, $\mathbf{d}=\mathbf{G}(\mathbf{m})=\mathbf{G m}$, the number of model parameters may be larger than the number of data points. Consequently, there may not be enough equations to fully constrain $\mathbf{m}$.

a) Decomposition of $\rho(\mathbf{x})$ into spherically symmetric and non-spherically symmetric part

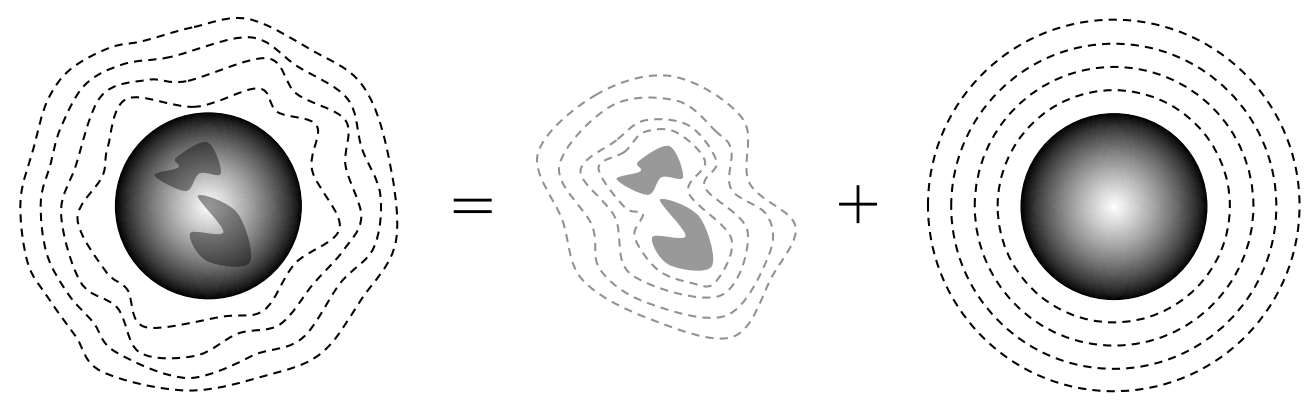

b) Equivalent density distributions
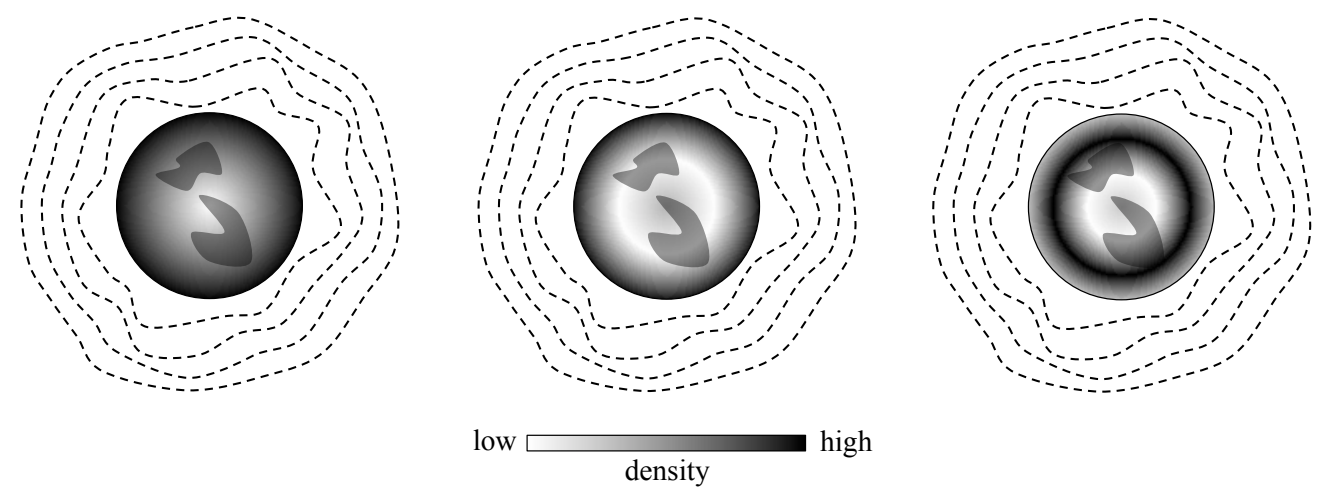

Fig. 1.3: Illustration of physical non-uniqueness related to gravity measurements. (a) The density distribution to the left produces a gravity field, the equipotential surfaces of which are schematically shown in the form of dashed curves. It can be decomposed into a spherically symmetric part (right) and a remainder that is not spherically symmetric (centre). The equipotential surfaces of the spherically symmetric part are perfect spheres. (b) Schematic collection of density distributions that produce exactly the same gravity field as in the upper left. Though the spherically symmetric density distributions differ, they are distinguished by all having the same total mass, and therefore the same potential field outside the body.

A further source of non-uniqueness, closely related to an insufficient number of data, is lack of coverage. Considering, for example, figure $1.2 \mathrm{~b}$, there are cells that are not traversed by any ray. No information on velocity in these cells reaches any of the receivers. As a consequence, velocity in these uncovered cells may take any arbitrary value without having an effect on the observed data.

Finally, as noted before, data have errors. Generally, there will be many models that explain the data to within 
these errors, which makes all of these models plausible.

An immediate consequence of non-uniqueness is that inversion cannot consist in the construction of a single model $\mathbf{m}$ that explains the data acceptably well. Solving an inverse problem must involve an appraisal of the whole set of plausible models. Finding only one model without any assessment of non-uniqueness is just data fitting, not inversion.

\subsubsection{Determinedness *}

Non-uniqueness has many aspects, one of them being the determinedness of the inverse problem. The concept is most easily explained by the example in figure 1.4, which displays a very simple tomographic experiment involving only two cells with wave speeds $v_{1}$ and $v_{2}$, respectively. The grey dots indicate source or receiver positions, and the connecting grey lines show the source-receiver configurations (rays). In the upper-left panel, there are four rays. The two vertical ones allow the two wave speeds to be determined. (The dimension of the cell is assumed to be known, and the traveltimes from the sources to the receivers are the observed data.) The two horizontal rays add some further redundancy to the system, because they allow the average wave speed of the two cells to be determined. Such a scenario is referred to as an over-determined problem.

In the upper-right panel of figure 1.4, we have only two vertical rays, which allow both wave speeds to be determined. With two data points (traveltimes) and two unknowns (velocities) we have an even-determined problem. In the lower-left panel, there is only one ray crossing the cell with wave speed $v_{2}$. This allows $v_{2}$ to be determined, but there is no information on $v_{1}$. With one data point and two unknowns we have an underdetermined problem.

Finally, we consider the scenario shown in the lower-right panel of figure 1.4. Here, we have four parallel rays crossing both cells. This allows the average wave speed of the two cells to be determined, but the individual wave speeds remain unresolved. Although we have four data points and two unknowns, there is an underdetermined component in this problem. Since the traveltimes observed offer only partial information on the wave speeds, we call such problems mixed-determined.

over-determined

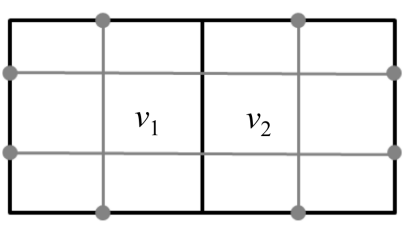

under-determined

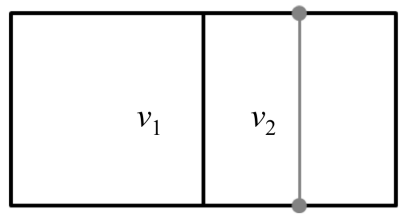

even-determined

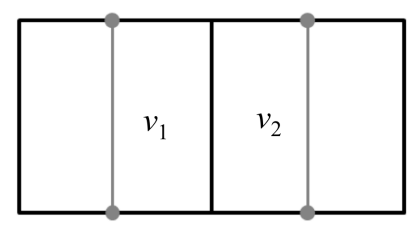

mixed-determined

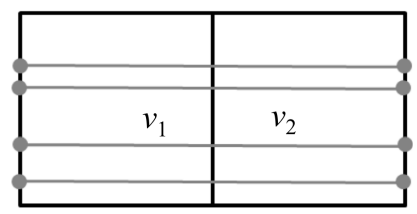

Fig. 1.4: Simple tomographic experiment including only two model cells with wave speeds $v_{1}$ and $v_{2}$. The grey dots indicate source or receiver positions, and the connecting grey lines are the straight ray paths connecting the actual source-receiver pairs. Depending on the source-receiver geometry, the inverse problem is over-, even-, under-, or mixed-determined. 
In a more realistic scenario, there are many more cells of unknown wave speeds and rays. Some of the cells are well resolvable, but others may be only partially resolved or may remain unresolved. Therefore, such problems are usually over-, under-, and mixed-determined at the same time.

\subsection{Fitting lines in $n$ dimensions *}

We continue our brief introduction to basic concepts of inverse theory by considering a very simple, but yet important, class of problems where the forward modelling equations are linear.

\subsubsection{Linear forward problems *}

Generally, a function $G(\mathbf{m})$ is said to be linear when, for two arbitrary models $\mathbf{m}^{1}$ and $\mathbf{m}^{2}$,

$$
G\left(a_{1} \mathbf{m}^{1}+a_{2} \mathbf{m}^{2}\right)=a_{1} G\left(\mathbf{m}^{1}\right)+a_{2} G\left(\mathbf{m}^{2}\right),
$$

for all possible choices of the scalars $a_{1}$ and $a_{2}$. Probably the simplest of all linear inverse problems is the fitting of a straight line through a collection of points. In this case, the predicted data $d_{i}$, that is, the elements of the data vector $\mathbf{d}$, are related to the model parameters $\mathbf{m}=\left[m_{1}, m_{2}\right]^{T}$ via the equation

$$
d_{i}=m_{1} c_{i}+m_{2},
$$

where $m_{1}$ is the slope of the curve, and $m_{2}$ is the intersection with the vertical axis. The control parameters $c_{i}$ may, for instance, be temperature, location, or any other parameter of the experimental setup. In this linear scenario, $m_{1}$ and $m_{2}$ are two physical model parameters that we wish to infer on the basis of $N$ observed data $d_{i}^{\text {obs }}$. Solving this inference problem means to fit a line through the observations.

As shown in figure 1.5, our line fitting problem is not as simple as it may seem. First of all, we have more measurements (four) than unknowns (two), meaning that the problem is over-determined. Furthermore, our measurements $d_{i}$ clearly do not fall onto a straight line. This may be due to measurement errors, deficiencies of our forward modelling theory which may in reality not be as nicely linear, or both.

The problem of fitting a line may be extended to higher dimensions, for instance, to the fitting of a plane through a point cloud in three dimensions. In this case, we have two control parameters, $c^{1}$ and $c^{2}$, and three model parameters, $m_{1}, m_{2}$ and $m_{3}$, that enter the linear forward problem

$$
d_{i}=m_{1} c_{i}^{1}+m_{2} c_{i}^{2}+m_{3} .
$$

A further generalisation to the fitting of an $n$-dimensional hyperplane is straightforward,

$$
d_{i}=m_{1} c_{i}^{1}+\ldots+m_{j} c_{i}^{j}+\ldots+m_{n-1} c_{i}^{n-1}+m_{n},
$$

where $n$ is the dimension of the model space (number of unknown model parameters), and $i=1, \ldots, N$ is again the index of the measurement. For notational convenience, we write the forward problems in equations (1.10) to (1.13) in matrix-vector notation, that is, as $\mathbf{d}=\mathbf{G m}$. For the line fitting problem in (1.11) we have

$$
\mathbf{G}=\left[\begin{array}{cc}
c_{1} & 1 \\
c_{2} & 1 \\
\vdots & \vdots \\
c_{N-1} & 1 \\
c_{N} & 1
\end{array}\right], \quad \mathbf{m}=\left[\begin{array}{l}
m_{1} \\
m_{2}
\end{array}\right]
$$




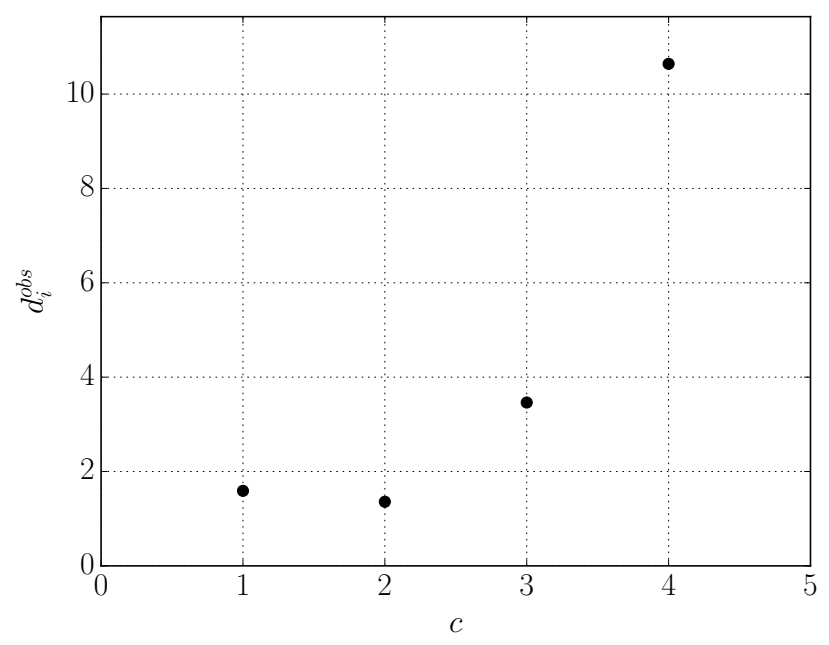

Fig. 1.5: Illustration of the line fitting problem. Measurements $d_{i}$ are taken for four control parameters $c_{i}$, representing, for instance, temperature, location, ..., of the experiment. Measurement values are shown as black dots.

Similarly, for the plane fitting problem (1.12)

$$
\mathbf{G}=\left[\begin{array}{ccc}
c_{1}^{1} & c_{1}^{2} & 1 \\
c_{2}^{1} & c_{2}^{2} & 1 \\
\vdots & \vdots & \vdots \\
c_{N-1}^{1} & c_{N-1}^{2} & 1 \\
c_{N}^{1} & c_{N}^{2} & 1
\end{array}\right], \quad \mathbf{m}=\left[\begin{array}{c}
m_{1} \\
m_{2} \\
m_{3}
\end{array}\right]
$$

and for the general $N$-dimensional problem

$$
\mathbf{G}=\left[\begin{array}{ccccc}
c_{1}^{1} & c_{1}^{2} & \ldots & c_{1}^{n-1} & 1 \\
c_{2}^{1} & c_{2}^{2} & \ldots & c_{2}^{n-1} & 1 \\
\vdots & \vdots & \vdots & \vdots & \vdots \\
c_{N-1}^{1} & c_{N-1}^{2} & \ldots & c_{N-1}^{n-1} & 1 \\
c_{N}^{1} & c_{N}^{2} & \ldots & c_{N}^{n-1} & 1
\end{array}\right], \quad \mathbf{m}=\left[\begin{array}{c}
m_{1} \\
m_{2} \\
\vdots \\
m_{n-1} \\
m_{n}
\end{array}\right]
$$

Admittedly, fitting a line through a point cloud may not seem to be the most relevant inverse problem. Yet, there are real-world problems taking exactly that form. One of these is vertical seismic profiling (VSP), illustrated in figure 1.6. The goal is to estimate the velocities of layers in the subsurface using measurements of seismic waves inside a borehole. The source, located at the surface, emits seismic waves, generated, for instance, by an explosion. Subsequently, the waves are recorded by receivers at known depths $z_{i}^{r}$ with $i=1, \ldots, n$. The traveltime $t_{i}$ of a wave from the source to a receiver at $z_{i}^{r}$ is given by the integral

$$
t_{i}=\int_{z=z_{1}}^{z_{i}^{r}} \frac{1}{v(z)} d z,
$$

where $v(z)$ is the depth-dependent velocity, that is, our unknown. Introducing slowness $s(z)$ as the inverse of velocity 


$$
s(z)=\frac{1}{v(z)}
$$

we can write the forward problem as

$$
t_{i}=d_{i}=\sum_{j=1}^{i-1}\left(z_{j+1}-z_{j}\right) m_{j}+\left(z_{i}^{r}-z_{i}\right) m_{i}
$$

In equation (1.19), the unknown model parameters are $m_{i}=s_{i}$, which is slowness within the layer between $z_{i}$ and $z_{i+1}$. Assuming the positions of the layer interfaces, $z_{i}$, known, equation (1.19) constitutes a linear forward modelling equation, similar to (1.13). As such, it may also be written in the generic matrix-vector notation as $\mathbf{d}=\mathbf{G m}$.

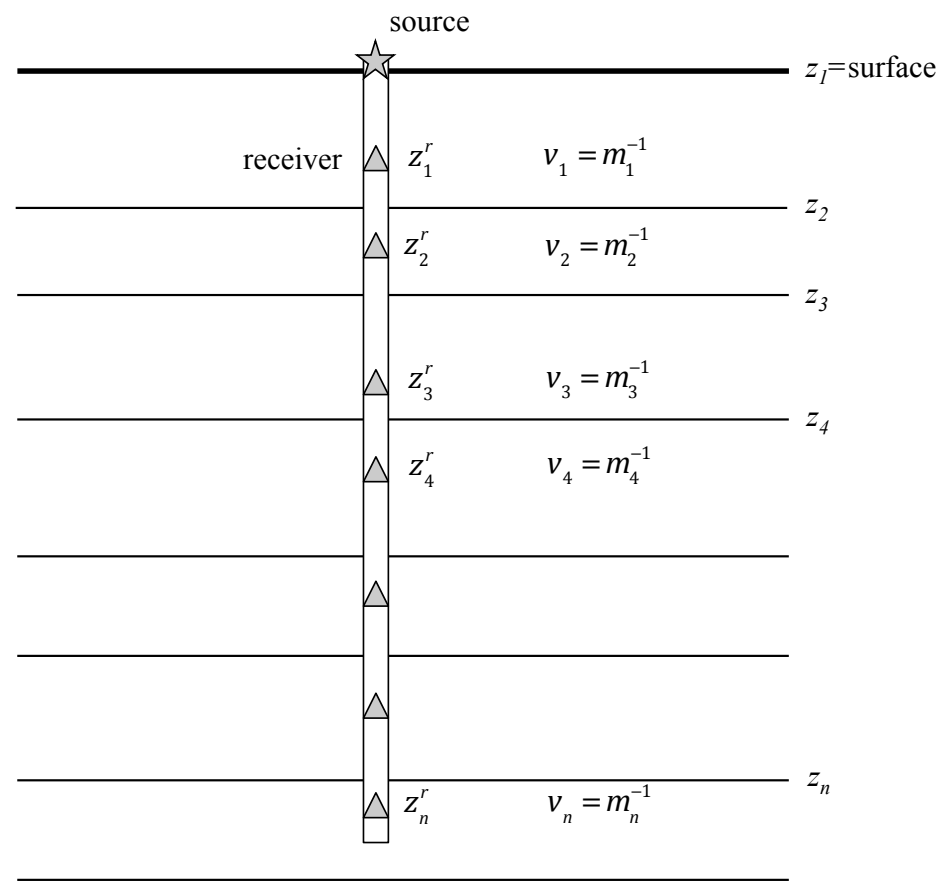

Fig. 1.6: Schematic illustration of vertical seismic profiling (VSP). The source (star) is located at the surface. The seismic waves it emits are recorded by receivers in a borehole (triangles). Assuming the locations of the layer interfaces, $z_{i}$, known, the model parameters are the layer slownesses (inverse velocities), $m_{i}=s_{i}=v_{i}^{-1}$.

An interesting aspect of the VSP problem, and in fact of many tomographic problems, is the transformation of the model parameters from velocity $v$ to its inverse, slowness $s$. Of course, we may easily rewrite the forward modelling equation (1.19) in terms of the layer velocities $v_{j}$,

$$
t_{i}=d_{i}=\sum_{j=1}^{i-1}\left(z_{j+1}-z_{j}\right) \frac{1}{v_{j}}+\left(z_{i}^{r}-z_{i}\right) \frac{1}{v_{i}}
$$


We then note, however, that the forward modelling equation is not linear anymore. As a consequence, we may not apply the powerful machinery for the solution of linear inverse problems, that we will start to develop in the following section.

\subsubsection{Linear inverse problems *}

Based on the forward modelling equations that produce synthetic data d, we wish to find a set of model parameters $\mathbf{m}$ that explains the observations $\mathbf{d}^{\text {obs }}$ to within their errors. The most straightforward, though somewhat ad hoc, approach is to search for a model $\mathbf{m}^{\text {est }}$ that minimises the least-squares misfit functional

$$
\chi(\mathbf{m})=\frac{1}{2}\left(\mathbf{d}^{\mathrm{obs}}-\mathbf{G m}\right)^{T}\left(\mathbf{d}^{\mathrm{obs}}-\mathbf{G m}\right)=\frac{1}{2} \sum_{i=1}^{N}\left(d_{i}^{\mathrm{obs}}-\sum_{j=1}^{n} G_{i j} m_{j}\right)^{2} .
$$

In real-data applications, the misfit $\chi$ will never be exactly zero because the observations $\mathbf{d}^{\text {obs }}$ are contaminated by errors, and because the forward modelling theory is unavoidably inaccurate. All physical models are just simplified abstractions of nature. To find an optimal model, $\mathbf{m}^{\text {est }}$, we differentiate equation (1.21) with respect to the model vector component $m_{q}$,

$$
\frac{\partial \chi}{\partial m_{q}}=-\sum_{i=1}^{N} G_{i q}\left(d_{i}^{\mathrm{obs}}-\sum_{k=1}^{n} G_{i k} m_{k}\right)=-\mathbf{G}^{T}\left(\mathbf{d}^{\mathrm{obs}}-\mathbf{G m}\right) .
$$

Forcing equation (1.22) to zero, gives the estimated model, in the least-squares sense, as

$$
\mathbf{m}^{\mathrm{est}}=\left(\mathbf{G}^{T} \mathbf{G}\right)^{-1} \mathbf{G}^{T} \mathbf{d}^{\mathrm{obs}} .
$$

The matrix $\left(\mathbf{G}^{T} \mathbf{G}\right)^{-1} \mathbf{G}^{T}$ is known as pseudoinverse or Moore-Penrose inverse $e^{2}$. Equation (1.23) deserves a little bit of discussion. First, we note that the computation of $\mathbf{m}^{\text {est }}$ requires the inversion of the $n \times n$ matrix $\mathbf{G}^{T} \mathbf{G}$. When the number of model parameters $n$ is large, this may become a formidable computational task. Furthermore, there is no guarantee that $\mathbf{G}^{T} \mathbf{G}$ is actually invertible.

Figure 1.7a shows the application of equation (1.23) to the 2-D line fitting problem shown in figure 1.5. The line follows the overall trend of the point cloud (a very small cloud with only four members), without precisely matching the observed data.

Fooling yourself with least-squares - The consistency problem: The least-squares solution (1.23) seems nice and simple. However, it can lead to confusing results, as pointed out in a small example by Snieder \& Trampert (1999). Consider an experiment where we are interested in the masses of two objects, $m_{1}$ and $m_{2}$. We start by putting object one on a scale, and we read $d_{1}^{\text {obs }}=1 \mathrm{~kg}$. The second object gives the measurement $d_{2}^{\text {obs }}=2 \mathrm{~kg}$. Finally, we put both objects together on the scale. Since the scale is a bit old and rusty, we obtain a measurement that is obviously inconsistent with the previous two, $d_{3}^{\text {obs }}=2 \mathrm{~kg}$. In equations, we now have the following:

$$
m_{1}=d_{1}^{\mathrm{obs}}=1 \mathrm{~kg}, \quad m_{2}=d_{2}^{\mathrm{obs}}=2 \mathrm{~kg}, \quad m_{1}+m_{2}=d_{3}^{\mathrm{obs}}=2 \mathrm{~kg} .
$$

The corresponding forward modelling matrix is

2 The Moore-Penrose inverse is named after the American mathematician Eliakim Hastings Moore (1862-1932), who probably described it first, and the English physicist and science philosopher Sir Roger Penrose (born in 1931). 
a)

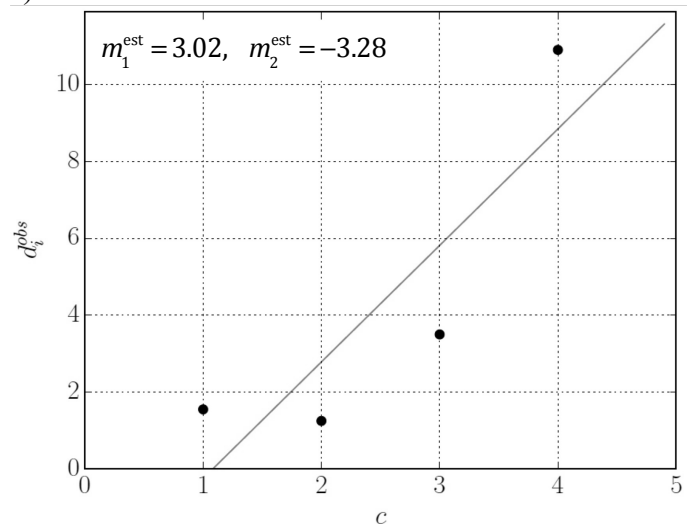

b)

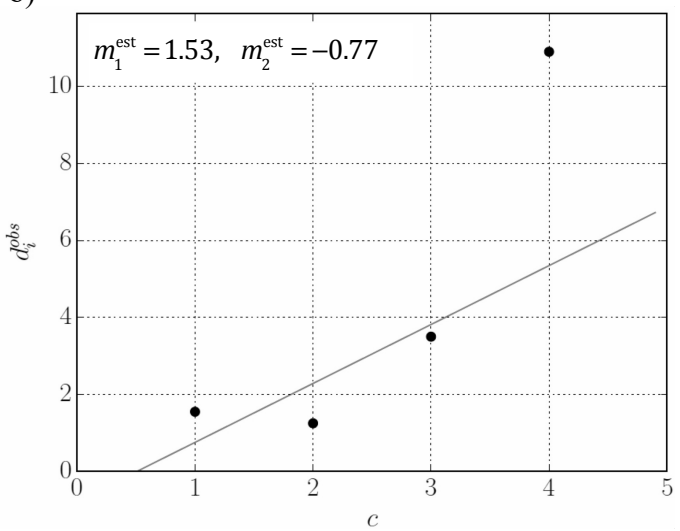

Fig. 1.7: Least-squares solutions of the line fitting problem from figure 1.5. (a) Best fitting line (grey) in the least-squares sense, ignoring observational errors. (b) Best fitting line after assigning a large error to the assumed outlier at $c=4$. [Line fitting in 1-D]

$$
\mathbf{G}=\left[\begin{array}{ll}
1 & 0 \\
0 & 1 \\
1 & 1
\end{array}\right]
$$

Inserting $\mathbf{G}$ and $\mathbf{d}^{\text {obs }}$ into equation (1.23) we obtain a solution that consititutes a compromise between our apparently conflicting pieces of information,

$$
m_{1}^{\text {est }}=2 / 3 \mathrm{~kg}, \quad m_{2}^{\text {est }}=5 / 3 \mathrm{~kg} .
$$

From a purely mathematical perspective, nothing prevents us from multiplying the third equation in (1.24) by a factor of 2 , yielding

$$
m_{1}=d_{1}^{\mathrm{obs}}=1 \mathrm{~kg}, \quad m_{2}=d_{2}^{\mathrm{obs}}=2 \mathrm{~kg}, \quad 2 m_{1}+2 m_{2}=d_{3}^{\mathrm{obs}}=4 \mathrm{~kg} .
$$

Each of these equations is fully equivalent to the corresponding equations in (1.24). Again solving the least-squares problem with the new forward modelling matrix

$$
\mathbf{G}=\left[\begin{array}{ll}
1 & 0 \\
0 & 1 \\
2 & 2
\end{array}\right],
$$

gives the new result

$$
m_{1}^{\text {est }}=5 / 9 \mathrm{~kg}, \quad m_{2}^{\text {est }}=14 / 4 \mathrm{~kg} .
$$

This is obviously different from $m_{1}^{\text {est }}=2 / 3 \mathrm{~kg}$ and $m_{2}^{\text {est }}=5 / 3 \mathrm{~kg}$, as found before. What has happened? We will return to this apparent paradox a little later.

Up to this point, we ignored the fact that our measurements have errors, and that these errors may be different for each measurement. As a consequence, measurements that may be outliers - such as the one taken at $c=4$ in figure 1.5 - receive more weight than they deserve. This problem may be addressed with a modification of the original misfit functional in equation (1.21). In fact, inserting weights $\varepsilon_{i}$ that are proportional to the error of measurement $d_{i}^{\text {obs }}$, we obtain a version of equation (1.21) that balances data according to their uncertainties, 


$$
\chi(\mathbf{m})=\frac{1}{2} \sum_{i=1}^{N} \frac{1}{\varepsilon_{i}^{2}}\left(d_{i}^{\mathrm{obs}}-\sum_{j=1}^{n} G_{i j} m_{j}\right)^{2}
$$

Introducing the diagonal matrix

$$
\mathbf{C}_{D}=\left[\begin{array}{ccccc}
\varepsilon_{1}^{2} & 0 & \ldots & 0 & 0 \\
0 & \varepsilon_{2}^{2} & \ldots & 0 & 0 \\
\vdots & \vdots & \vdots & \vdots & \vdots \\
0 & 0 & \ldots & \varepsilon_{N-1}^{2} & 0 \\
0 & 0 & \ldots & 0 & \varepsilon_{N}^{2}
\end{array}\right]
$$

we can rewrite equation (1.30) in the more condensed matrix-vector notation,

$$
\chi(\mathbf{m})=\frac{1}{2}\left(\mathbf{d}^{\mathrm{obs}}-\mathbf{G m}\right)^{T} \mathbf{C}_{D}^{-1}\left(\mathbf{d}^{\mathrm{obs}}-\mathbf{G m}\right) .
$$

The matrix $\mathbf{C}_{D}$ is commonly referred to as the data covariance matrix. Repeating the previous steps of differentiating and forcing the derivative to vanish, results in a modified version of equation (1.23) for the estimated model,

$$
\mathbf{m}^{\text {est }}=\left(\mathbf{G}^{T} \mathbf{C}_{D}^{-1} \mathbf{G}\right)^{-1} \mathbf{G}^{T} \mathbf{C}_{D}^{-1} \mathbf{d}^{\text {obs }}
$$

The effect of accounting for observational errors on the line fitting problem from figure 1.5 is illustrated in figure $1.7 \mathrm{~b}$. Assigning a large error $\varepsilon_{i}$ to the outlier measurement at $c_{4}$ leads to significantly different model parameter estimates, and to a completely reoriented best-fitting line.

Revisiting the consistency problem: The concept of the data covariance allows us to elegantly resolve the consistency problem, condensed in equations (1.27) and (1.29). In fact, the consistency problem arises from ignoring that measurements have errors. Assuming that the values we read from the scale are nearly identical for all measurements, a suitable data covariance matrix would be

$$
\mathbf{C}_{D}=\left[\begin{array}{lll}
1 & 0 & 0 \\
0 & 1 & 0 \\
0 & 0 & 1
\end{array}\right]
$$

When the third equation in (1.24) is multiplied by a factor of 2 , the measurement error of the new datum, $d_{3}^{\text {obs' }}=2 d_{3}^{\text {obs }}$, is twice as large as the error of the original datum, $d_{3}^{\text {obs }}$. Hence, the appropriately modified data covariance matrix is

$$
\mathbf{C}_{D}^{\prime}=\left[\begin{array}{lll}
1 & 0 & 0 \\
0 & 1 & 0 \\
0 & 0 & 2^{2}
\end{array}\right]
$$

Indeed, now using equation (1.33) for the solution of the least-squares problem, we find

$$
m_{1}^{\text {est }}=2 / 3 \mathrm{~kg}, \quad m_{2}^{\text {est }}=5 / 3 \mathrm{~kg} \text {. }
$$

In addition to making the least-squares solution invariant under arbitrary scalings, the data covariance also ensures that the equations have proper units, and specifically that the unit of the misfit $\chi$ is generally 1. This is particularly important when working with data that have different physical units.

Based on the modified misfit definition (1.32), we may produce a first simple assessment of the solution quality. For this, we define the estimated data $\mathbf{d}^{\text {est }}$ as the synthetic data computed with the estimated model, that is,

$$
\mathbf{d}^{\text {est }}=\mathbf{G m}^{\text {est }} \text {. }
$$


Inserting $\mathbf{d}^{\text {est }}$ for $\mathbf{G m}$ in equation (1.30), gives the estimated misfit,

$$
\chi^{\mathrm{est}}=\frac{1}{2} \sum_{i=1}^{N} \frac{1}{\varepsilon_{i}^{2}}\left(d_{i}^{\mathrm{obs}}-d_{i}^{\mathrm{est}}\right)^{2} .
$$

Ideally, when observations are fit roughly to within their observational errors, we should have $d_{i}^{\text {obs }}-d_{i}^{\text {est }} \approx \varepsilon_{i}$, for all $i=1, \ldots, N$. Thus, summed over all observations, we expect

$$
\chi^{\text {est }} \approx \frac{N}{2}
$$

When $\chi^{\text {est }}$ is significantly larger than $N / 2$, the fit is worse than we would expect, given the observational errors. This situation is referred to as under-fitting the data. Underfitting may result, for instance, from overly optimistic (too small) observational errors, or from an insufficient forward model that may just not be able to fit the data. In our case, the model may not be linear. The opposite scenario, where $\chi^{\text {est }}$ is significantly smaller than $N / 2$, is called over-fitting. Typically, over-fitting means that we fit noise instead of credible data. A quantity that is closely related to the estimated misfit, and which frequently appears in the literature, is the root-mean-square misfit,

$$
\chi^{\mathrm{rms}}=\sqrt{\frac{1}{N} \sum_{i=1}^{N} \frac{1}{\varepsilon_{i}^{2}}\left(d_{i}^{\mathrm{obs}}-d_{i}^{\mathrm{est}}\right)^{2}} .
$$

In terms of the root-mean-square misfit, over-fitting occurs for $\chi^{\text {rms }}$ significantly smaller than 1 , and underfitting for $\chi^{\text {rms }}$ significantly larger than 1 .

Having incorporated measurement uncertainties, we add another level of complexity, related to prior knowledge that we may have about the model parameters. Without any analysis of data, we usually have prior knowledge, for instance, from physical constraints. Velocities, temperatures, conductivities, and other quantities are always known to be positive. In addition, velocities are known to be lower than the speed of light in vacuum. In the interest of simplicity, we start with prior knowledge on the proximity of model parameters $\mathbf{m}$ to some prior model parameters $\mathbf{~ m}^{\text {prior }}$. Mathematically, this proximity may be captured by adding yet another component to our misfit definition,

$$
\chi(\mathbf{m})=\frac{1}{2} \sum_{i=1}^{N} \frac{1}{\varepsilon_{i}^{2}}\left(d_{i}^{\mathrm{obs}}-\sum_{j=1}^{n} G_{i j} m_{j}\right)^{2}+\frac{1}{2} \sum_{i=1}^{n} \frac{1}{\gamma_{i}^{2}}\left(m_{i}-m_{i}^{\mathrm{prior}}\right)^{2} .
$$

For small values of $\gamma_{i}$, the misfit is dominated by differences between $\mathbf{m}$ and our prior model $\mathbf{m}^{\text {prior }}$, meaning that solutions close to $\mathbf{m}^{\text {prior }}$ are favoured. Otherwise, when the $\gamma_{i}$ are large, our prior knowledge only has a small influence on the solution. Again defining a diagonal matrix,

$$
\mathbf{C}_{M}=\left[\begin{array}{ccccc}
\gamma_{1}^{2} & 0 & \ldots & 0 & 0 \\
0 & \gamma_{2}^{2} & \ldots & 0 & 0 \\
\vdots & \vdots & \vdots & \vdots & \vdots \\
0 & 0 & \ldots & \gamma_{n-1}^{2} & 0 \\
0 & 0 & \ldots & 0 & \gamma_{n}^{2}
\end{array}\right]
$$

the new misfit condenses to

$$
\chi(\mathbf{m})=\frac{1}{2}\left(\mathbf{d}^{\mathrm{obs}}-\mathbf{G m}\right)^{T} \mathbf{C}_{D}^{-1}\left(\mathbf{d}^{\mathrm{obs}}-\mathbf{G m}\right)+\frac{1}{2}\left(\mathbf{m}-\mathbf{m}^{\text {prior }}\right)^{T} \mathbf{C}_{M}^{-1}\left(\mathbf{m}-\mathbf{m}^{\text {prior }}\right) .
$$

Following the previously employed procedure, the estimated model is now given by

$$
\mathbf{m}^{\mathrm{est}}=\left(\mathbf{G}^{T} \mathbf{C}_{D}^{-1} \mathbf{G}+\mathbf{C}_{M}^{-1}\right)^{-1}\left(\mathbf{G}^{T} \mathbf{C}_{D}^{-1} \mathbf{d}^{\text {obs }}+\mathbf{C}_{M}^{-1} \mathbf{m}^{\text {prior }}\right) .
$$


As expected, we obtain $\mathbf{m}^{\text {est }}=\mathbf{m}^{\text {prior }}$ as $\gamma_{i} \rightarrow 0$, that is, when we introduce a strong bias towards the prior model.

Before closing this section on linear inverse problems, we briefly reflect on what we just did and observed. Several issues clearly deserve more attention:

1. Despite being intuitive and mathematically easy to handle, our misfit definitions are rather arbitrary. Why did we use the least-squares misfit and not some other misfit measure, for instance, a sum of absolute values?

2. Related to this, we observe that the least-squares misfit is apparently very impressed by the outlier at $c=4$ in figure 1.7. This property, known as non-robustness, is due to the fact that individual measurements with their potentially large errors enter the least-squares misfit quadratically. Therefore, the impact of outliers is disproportionately amplified, unless they are clearly identified as outliers (in which case they would have been removed from the dataset anyway).

3. We only found one 'solution'. This is in conflict with the fact that more than one model is able to explain the observations to within the uncertainties. How could we construct alternative plausible solutions? How different will they be from each other? So, have we actually solved an inverse problem?

4. What happens when $\mathbf{G}^{T} \mathbf{G}$ or the modified variants of it are not invertible?

5. Finding an optimal solution via least squares works beautifully for linear forward problems. Is there any chance to tackle non-linear problems as well?

These problems remain to be addressed in the following chapters.

\subsection{Outlook*}

The immediately following chapters in Part I are dedicated to Bayesian inference, which forms the conceptual backbone of inverse theory. Bayesian inference provides probabilistic solutions to inverse problems, meaning that the solution consists of an ensemble of plausible models, with each model being ranked according to its probability. This very general flavour of inverse theory is also referred to as probabilistic inversion. In addition to providing a whole ensemble of models, probabilistic inversion is able to handle non-linear problems, and to deal with any kind of prior knowledge that we may wish to use. Elevated computational costs are the price to pay for this generality and elegance of probabilistic inversion.

The least-squares approach introduced in the previous section is clearly not probabilistic. It provides a single 'solution', and is therefore referred to as deterministic inversion. Despite several drawbacks, deterministic inversion is widely used for pragmatic reasons. The mathematics are easily understood, and the computational requirements are manageable for many practically relevant problems. Part II is dedicated to various forms of deterministic inversion.

Part III contains a collection of special cases where inverse problems can be solved exactly, sometimes even in closed form, assuming that data are perfect. While these special cases may initially seem to have little relevance, they often provide invaluable physical insight and intuition that is crucial for more realistic scenarios where data insufficient and polluted by measurement errors. 

Part I Bayesian Inference and Monte Carlo Methods 



\section{Chapter 2 \\ Historical warm-up}

I send you an essay which I have found among the papers of our deceased friend Mr. Bayes, and which, in my opinion, has great merit, and well deserves to be preserved. [...] Every judicious person will be sensible that the problem now mentioned is by no means merely a curious speculation in the doctrine of chances, but necessary to be solved in order to a sure foundation for all our reasonings concerning past facts, and what is likely to be hereafter.

Richard Price, 1763.

Richard Price wrote these words to John Canton, his fellow member of the Royal Society in London, in December 1763, more than two and a half years after his friend Thomas Bayes ${ }^{1}$ had passed away (Bayes \& Price, 1763). While Price was obviously aware of the importance of Bayes' work, he could certainly not foresee that Bayes' essay would one day become The foundation of scientific inference.

Today, more than 250 years after the publication of the essay, it is difficult to penetrate, and without the proper historical context its significance may be difficult to discover. Price' language sounds unusual to us. Many mathematical facts that we would describe elegantly using well-established symbolisms are described tediously in words. Nevertheless, a loose translation of Bayes' work into modern mathematical language will serve us as a useful introduction to some of the basic concepts needed to understand Bayesian inference, the heart of inverse theory.

\subsection{Probabilities and events}

The first section of Bayes' work is concerned with the probabilities of events, where the term event is abstract and not further specified. It could be the event of a sunny day in London ${ }^{2}$ (where Bayes was born in 1701), the event of having an apple for breakfast, or the specific outcome of an experiment. Interestingly, Bayes is unspecific to the extent that he does not seem to require that the event be repeatable, such as throwing darts. Instead, it is just some event $X$ to which we shall attach a probability of occurrence $P(X)$. The probability that event $X$ does not happen, or is considered false, is denoted by $P(\bar{X})$.

\footnotetext{
${ }^{1}$ The reverend Thomas Bayes (1701 - 1761) was an English Presbyterian minister who studied theology and logic in Edinburgh. His theorem, contained in unpublished manuscripts on probability theory, was passed to his friend Richard Price after his death. In 1763, Price read Bayes' work to the Royal Society. The theorem was rediscovered in 1812 by Pierre-Simon Laplace, and it now forms the basis of Bayesian inference.

${ }^{2}$ Note that the probability of that event is not exactly zero. In fact, London receives around 1400 hours of sunshine per year, which is 100 more than Reykjavik!
} 
Prior to any mathematics, Bayes classifies different types of events. For instance, events that exclude each other mutually are called inconsistent. Two events are contrary when one of them must happen, but both cannot happen together. Most importantly, events are said to be independent when the happening of one does not affect the probability of any of the other events.

Though this is strictly speaking not required, we will consider a specific event, simply for easier imagination: An experimenter takes a coloured building block out of a large box, notes the colour, and puts it back into the box. In total, there are $N$ blocks; $b$ of them are black, $g$ of them are grey, and $w$ of them are white. The probabilities of observing a black $(B)$, a grey $(G)$ or a white $(W)$ block are

$$
P(X=B)=\frac{b}{N}, \quad P(X=G)=\frac{g}{N}, \quad P(X=W)=\frac{w}{N},
$$

respectively. Since each block can only have one colour, the events $X=B, X=G$ and $X=W$ are inconsistent in Bayes' sense. In the interest of a lighter notation, we will simplify $P(X=B)$ to $P(B)$, etc. Note that there may of course also be blocks of other colours inside the box. Now the experimenter performs the experiment $n<N$ times, not having any preference for a particular colour, and lets a second person, the observer, guess the number of black, grey and white blocks he has noted. Bayes then defines probability such that the observer expects $n P(B)$ black, $n P(G)$ grey, and $n P(w)$ white blocks. Interestingly, Bayes does not provide a rigorous definition of expectation, very much leaving it to the intuition of the reader to fill this term with meaning. While this may seem lax, it reflects very well that expectation and probability are to some extent subjective, unless, of course, they are defined statistically in terms of an experiment that can be repeated in identical form infinitely many times.

Without using the modern mathematical terminology, Bayes continues to define the logical or, also called the disjunction of events. Denoted by $X \vee Y$, the disjunction of events $X$ and $Y$ is true if either $X$ or $Y$, or both, are true. (In the literature, the disjunction is sometimes alternatively referred to as the logical sum and written as $X+Y$.) Since the events of getting blocks with a certain colour are inconsistent, the new event of getting a black or a grey or a white one is given by the sum of individual probabilities,

$$
P(B \vee G \vee W)=P(B)+P(G)+P(W)=\frac{b+g+w}{N} .
$$

If the events were not inconsistent, equation (2.2) would not hold. As a corollary we find that

$$
r+g+b=N,
$$

for the special case where only black, grey, and white blocks are inside the box. This is because one of the individual events $-B, G$ or $W$-then necessarily has to happen, so that the disjunction in equation (2.2) becomes

$$
P(B \vee G \vee W)=1 .
$$

Implicitly, equation (2.4) introduces a normalisation of probabilities in the sense that the disjunction of all possible events must sum to 1 .

To account for more complex scenarios, we now abandon the requirement that the events be mutually inconsistent by allowing for blocks that have two colours. This leads to the definition of the logical and, or the conjunction of events $X$ and $Y$, denoted by $X \wedge Y$. It is true if both $X$ and $Y$ are true. (Alternatively, the conjunction is sometimes referred to as the logical product and denoted by $X Y$.) For instance, assuming that $b^{\prime}$ of the $N$ blocks have some black and white, the probability of the event $B \wedge W$ is given by

$$
P(B \wedge W)=\frac{b^{\prime}}{N} .
$$

In total, we let $b$ of the $N$ blocks have some black. As before, the experimenter takes a random block out of the box, reports to the observer that it contains some black, and asks him about the probability that the block also 


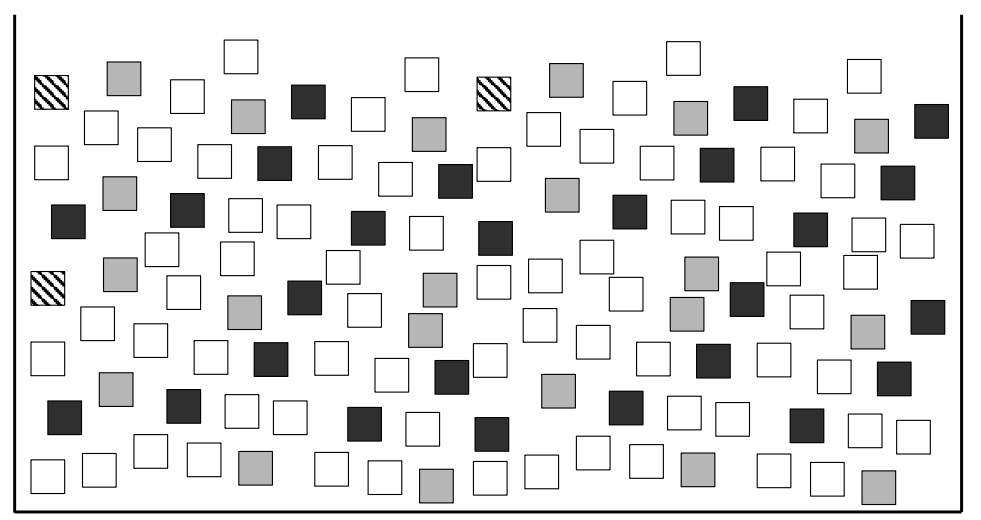

Fig. 2.1: Though Bayes \& Price (1763) never used a specific example to introduce basic probability concepts, it is still useful to have one in mind in order to gain some intuition, for instance a box containing $N$ coloured building blocks. An event consists of taking a block out of the box. More generally, an event could be anything, including the outcomes of repeatable experiments, or less repeatable events such as meteorite impacts in your backyard. What changes is the (subjective) interpretation of what probability is supposed to mean.

contains some patches of white. To determine this conditional probability $P(W \mid B)$, we note that it equals the number of blocks that are both black and white, divided by the total number of blocks that have some black, that is,

$$
P(W \mid B)=\frac{b^{\prime}}{b}=\frac{\frac{b^{\prime}}{N}}{\frac{b}{N}}=\frac{P(B \wedge W)}{P(B)} .
$$

In more general scenarios, equation (2.5) is taken as the definition of a conditional probability. As a trivial corollary we find that the joint probability (conjunction) $P(B \wedge W)$ is the product of the conditional probability $P(W \mid B)$ and the unconditional probability $P(B)$,

$$
P(B \wedge W)=P(W \mid B) P(B) .
$$

The seemingly harmless definition (2.6) has important consequences that will become clear later. Obviously, the conjunction $P(B \wedge W)$ is symmetric. The number of blocks that are black and white is the same as the number of blocks that are white and black, that is, $P(W \wedge B)$. It then follows from (2.7) that

$$
P(W \mid B) P(B)=P(B \mid W) P(W) .
$$

Rearranging equation (2.8) gives the conditional probability of receiving a black block, given that a white one has already been taken out of the box,

$$
P(B \mid W)=\frac{P(W \mid B) P(B)}{P(W)} .
$$

Equation (2.9), referred to as Bayes' theorem, is arguably the most important equation for the solution of any inference or inverse problem. The philosophical depth of the equation very much depends on the definition of what an event is supposed to mean. It can range from trivial, as in our toy example, all the way to attempts to calculate the probability of God (Unwin, 2004).

Equation (2.7) also provides a rule to compute the joint probability of independent events. By definition, events $B$ and $W$ are independent when the probability of $W$ is unaffected by the occurrence of $B$. In other words, the conditional probability of $W$ given $B$, that is $P(W \mid B)$, is the same as the probability of $W$ itself, 


$$
P(W \mid B)=P(W)
$$

It follows that the joint probability of $W \wedge B$ is given by

$$
P(W \wedge B)=P(W \mid B) P(B)=P(W) P(B) .
$$

Equation (2.11) may be iterated to obtain the joint probability of $n$ independent events $X_{i}$,

$$
P\left(\wedge_{i=1}^{n} X_{i}\right)=\prod_{i=1}^{n} P\left(X_{i}\right)
$$

To conclude this section, and to prepare for the following one, we consider an event that occurs with probability $\alpha$, and does not occur with probability $\beta$. For instance, $P(B)=\frac{b}{N}=\alpha$ and $P(\sim B)=1-\frac{b}{N}=\beta$. Now assume that the experimenter takes a block out of the box, looks at it, puts it back, and repeats this procedure $n$ times. Of the $n$ blocks, $p$ were indeed black, and $q$ happened to be of some other colour. What is the probability of receiving this set of blocks, assuming that their ordering does not matter?

First, we note that the events $B$ and $\sim B$ are independent because each block was carefully put back into the box before getting the next one. Consequently, by equation (2.12), the probability of one specific realisation of this joint event (receiving this sequence of blocks) is $\alpha^{p} b^{q}$. The total number of such sequences, possibly with a different ordering of the blocks, is

$$
\left(\begin{array}{l}
n \\
p
\end{array}\right)=\frac{n !}{(n-p) ! p !}
$$

where $\left(\begin{array}{l}n \\ p\end{array}\right)$ is the binomial coefficient. Thus, we find that the probability of our joint event is given by

$$
P[(p \text { black }) \wedge(q \text { not black })]=P(p)=\left(\begin{array}{l}
n \\
p
\end{array}\right) \alpha^{p} \beta^{q} .
$$

Equation (2.14) defines a binomial distribution in the integer $p$, ranging between 0 and $n$. The binomial distribution is our first example of a discrete probability distribution in one parameter, $\alpha$. Examples for $n=10$ and different choices of $\alpha$ are shown in figure 2.2.
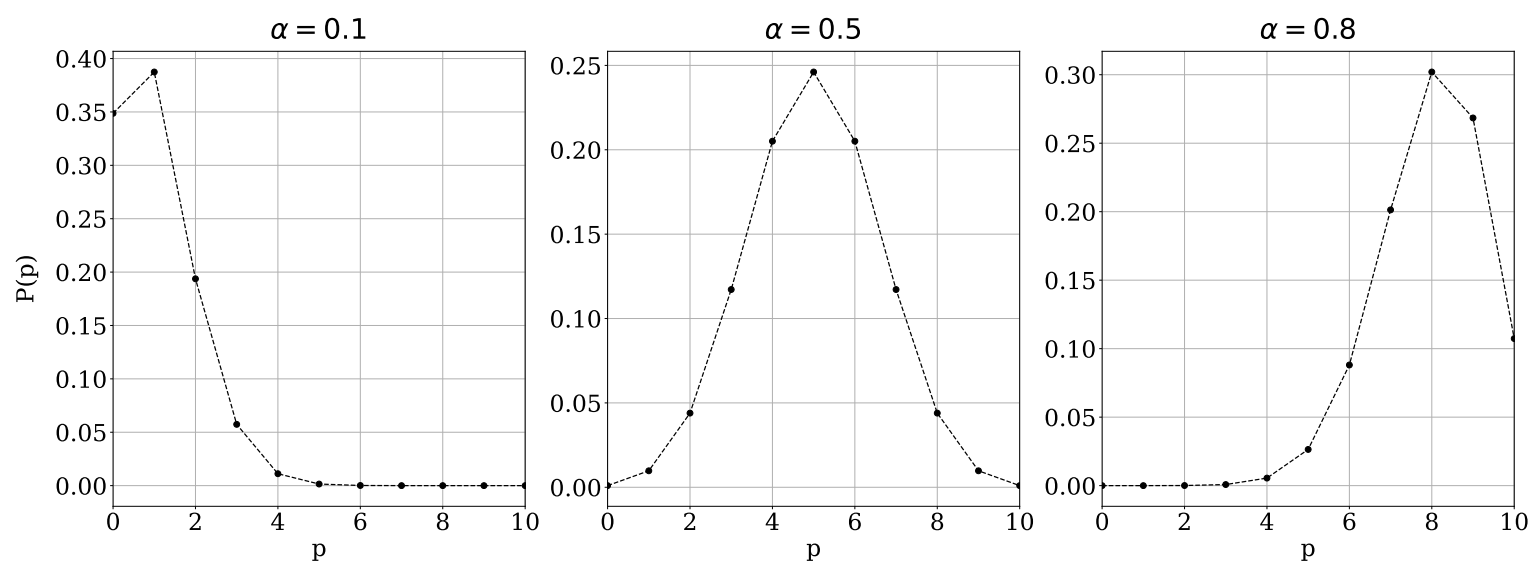

Fig. 2.2: Examples of binomial distributions, $P(p)$ for $n=10$ and different values of $\alpha$. In the context of our toy example, it is most likely to obtain sequences with a low number of black blocks $(p)$ when the probability of receiving an individual black block $(\alpha)$ is low, and vice versa. 
The de Moivre-Laplace approximation: The binomial distributions in figure 2.2 appear roughly bellshaped, and indeed it can be shown that they approach a Gaussian distribution

$$
P_{g}(p)=\frac{1}{\sqrt{2 \pi} \sigma} e^{-\frac{(p-\mu)^{2}}{2 \sigma^{2}}},
$$

with mean $\mu$ and standard deviation $\sigma$ for $N \rightarrow \infty$. This result is known as the de Moivre-Laplace approximation ${ }^{a}$. To show that the binomial distribution $P(p)$ in (2.14) indeed approaches the Gaussian, we loosely consider $p$ as a continuous variable. We then note that the Gaussian in (2.15) is the solution of the differential equation

$$
\frac{1}{P_{g}(p)} \frac{d P_{g}(p)}{d p}=-\frac{p-\mu}{\sigma^{2}}
$$

with the initial condition $P_{g}(\mu)=\frac{1}{\sqrt{2 \pi} \sigma}$, which determines the normalisation constant. For the binomial distribution, we may approximate the derivative with respect to $p$ by the difference $P(p+1)-P(p)$. Making use of the relation

$$
\left(\begin{array}{c}
n \\
p+1
\end{array}\right)=\left(\begin{array}{c}
n \\
p
\end{array}\right) \frac{n-p}{p+1}
$$

we have

$$
P(p+1)-P(p)=\left(\begin{array}{l}
n \\
p
\end{array}\right) \frac{n-p}{p+1} \frac{\alpha}{\beta} \alpha^{p} \beta^{n-p}-P(p)=-\frac{p-n \alpha+\beta}{b(\beta+1)} P(p) .
$$

For large $n$ and $p$, the contribution of $\beta$ (a number $\leq 1$ ) in the numerator and the contribution of 1 in the denominator can be neglected, and we find

$$
\frac{1}{P(p)} \frac{d P(p)}{d p} \approx \frac{P(p+1)-P(p)}{P(p)} \approx=-\frac{p-n \alpha}{\beta p} .
$$

Relation (2.19) shows that the maximum of the binomial distribution occurs around $p=n \alpha$, in accord with what we see in figure 2.2. Thus restricting our attention to values of $p$ in the vicinity of $n \alpha$, we may further approximate (2.19) by

$$
\frac{1}{P(p)} \frac{d P(p)}{d p} \approx-\frac{p-n \alpha}{n \alpha \beta}
$$

Comparing (2.20) to the differential equation (2.16), we conclude that the binomial distribution indeed approaches the Gaussian, with mean

$$
\mu=n \alpha
$$

and standard deviation

$$
\sigma=\sqrt{n \alpha \beta}
$$

${ }^{a}$ Abraham de Moivre (1667-1754) was a French mathematician best known for his work on probability theory and for de Moivre's formula, $(\cos x+i \sin x)^{n}=\cos (n x)+i \sin (n x)$.

\subsection{Bayes' table}

In the previous section, we introduced by example several basic concepts such as the conjunction and disjunction of probabilities, conditional probabilities, and Bayes' theorem. In section II of their essay, Bayes \& Price (1763) complement these developments by what we call today a probability density. The concept of probability 
densities is then used to make inferences concerning future events on the basis of past observations. It is this notion of looking into the future in a quantitative fashion that seems to have motivated Richard Price to present the work of his deceased friend to the illustrious Royal Society.

While section I of the essay is rather abstract, section II works with the concrete example of a perfectly planar table in order to illustrate the potential of the previous formal developments. The table, illustrated in figure 2.3, extends from $x=0$ to $x=l_{x}$ in $x$-direction, and from $y=0$ to $y=l_{y}$ in $y$-direction. The experiment starts by throwing a ball $A$ onto the table. It comes to rest at some random point $\left(x_{A}, y_{A}\right)$. This point divides the table into two domains, $D_{l}$ to the left and $D_{r}$ to the right. Then, the experimenter throws a second ball, $B$, which lands on another point $\left(x_{B}, y_{B}\right)$. Subsequently, the second ball is thrown again, and again, $\ldots, n$ times in total. Next to the experimenter stands an observer who cannot see the table. The experimenter merely tells the observer if the second ball $B$ has landed inside the left domain $D_{l}$, an event that we shall call $L$.

Initially, the observer has no information on where $A$ has landed, nor can he make any prediction where the next $B$ is likely to land. But could he make any quantitative predictions based on the information conveyed to him by the experimenter?

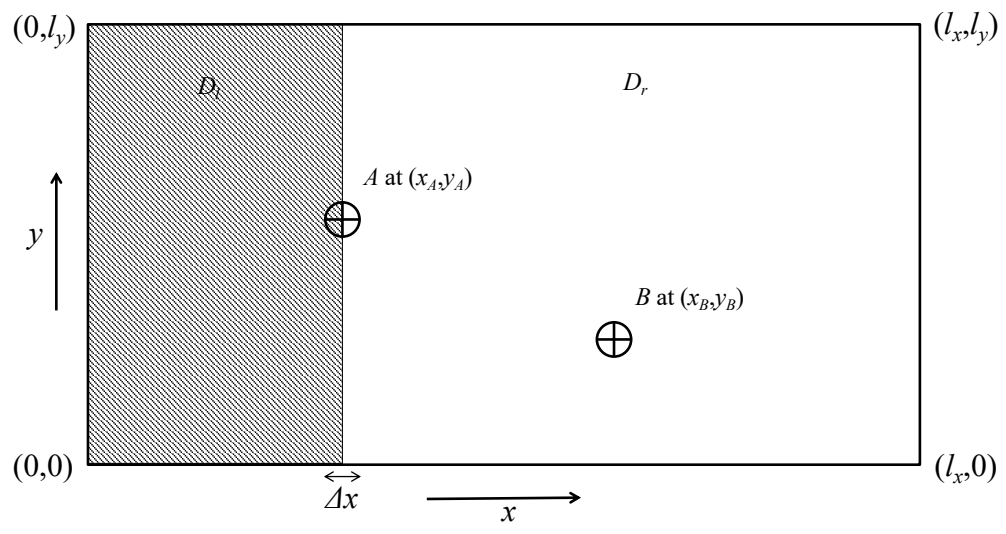

Fig. 2.3: Bayes' table with corners $(0,0),\left(l_{x}, 0\right),\left(0, l_{y}\right)$, and $\left(l_{x}, l_{y}\right)$. Ball $A$ landing at $\left(x_{A}, y_{A}\right)$ divides the table into a left domain, $D_{l}$, and a right domain, $D_{r}$. The landing of ball $B$ inside $D_{l}$ is defined as event $L$.

First, we note that the probability of event $L$ is a conditional one, conditioned on the $x$-position where $A$ has landed. Looking at figure 2.3 , we find

$$
P\left(L \mid x_{A}\right)=\frac{x_{A}}{l_{x}} .
$$

Second, referring to equation (2.14), we know that the conditional probability that $L$ has occurred $p$ times and failed $q=n-p$ times is given by

$$
P\left(p \mid x_{A}\right)=\left(\begin{array}{c}
n \\
p
\end{array}\right)\left[P\left(L \mid x_{A}\right)\right]^{p}\left[1-P\left(L \mid x_{A}\right)\right]^{q}=\left(\begin{array}{c}
n \\
p
\end{array}\right)\left(\frac{x_{A}}{l_{x}}\right)^{p}\left(1-\frac{x_{A}}{l_{x}}\right)^{n-p} .
$$

Prior to being communicated any experimental results, the observer has no information on the $x$-coordinate $x_{A}$ of ball $A$, meaning that the prior probability $P\left(x_{A}\right)$ is identical for all possible $x_{A}$. At this point, we face a conceptual difficulty. Obviously, $x_{A}$ is a continuous variable, unlike $p$, which can only take a finite number of integer values ranging from 0 to $N$. Being continuous, $x_{A}$ can take infinitely many values, namely any real number between 0 and $l_{x}$. Since the probabilities for all the inifinitely many possible values of $x_{A}$ must sum to 1 , it follows that the probability of each individual $x_{A}$ must be 0 . In other words, the probability of landing at 
exactly one of infinitely many points vanishes.

For the moment, we circumvent this problem by dividing the $x$-axis between 0 and $l_{x}$ into many small segments of equal length $\Delta x$. Then, instead of a specific point $x_{A}$, we consider $A$ landing within the interval $\Delta x$ around $x_{A}$, the probability of which is

$$
P\left(x_{A}\right)=\frac{\Delta x}{l_{x}}
$$

Then, using Bayes' theorem (2.9), we find

$$
P\left(x_{A} \mid p\right)=k P\left(p \mid x_{A}\right) P\left(x_{A}\right)=k\left(\begin{array}{l}
n \\
p
\end{array}\right)\left(\frac{x_{A}}{l_{x}}\right)^{p}\left(1-\frac{x_{A}}{l_{x}}\right)^{n-p} \frac{\Delta x}{l_{x}} .
$$

Equation (2.26) deserves a more detailed discussion. The number $k=1 / P(p)$ acts as a normalisation constant, chosen such that $P\left(x_{A} \mid p\right)$ summed over all possible $x_{A}$ is equal to 1 , that is

$$
\sum_{x_{A}} P\left(x_{A} \mid p\right)=1
$$

The right-hand side of equation (2.26), that is, $P\left(p \mid x_{A}\right) P\left(x_{A}\right)$, contains all prior knowledge. This includes the knowledge about $x_{A}$ prior to receiving any experimental results, $P\left(x_{A}\right)$, and the knowledge about the probability $P\left(p \mid x_{A}\right)$ of a certain $p$ given some $x_{A}$, which we derived from simple geometric arguments. Bayes' theorem combines this prior knowledge to produce the posterior probability $P\left(x_{A} \mid p\right)$. The posterior provides a quantitative probability for the occurrence of a specific $x_{A}$ given the observations $p$ provided by the experimenter.

Directly from equation (2.26) we can see various special cases. For instance, when event $L$ has been observed in every experiment $(p=n)$, we have $P\left(x_{A} \mid p\right) \propto\left(x_{A} / l_{x}\right)^{n}$. This implies that the posterior probability is generally largest for $x_{A}=l_{x}$, and as $n$ grows, all alternative values, $x_{A} \neq l_{x}$, become increasingly unlikely. In the other extreme case where $L$ has never been observed $(p=0)$, we similarly find $P\left(x_{A} \mid p\right) \propto\left(1-x_{A} / l_{x}\right)^{n}$, meaning that $x_{A}=0$ is the most likely $x$-coordinate of ball $A$.

A range of other scenarios is shown in figure 2.4. They illustrate, for example, that the posterior distribution $P\left(x_{A} \mid p\right)$ becomes increasingly peaked as the number of observations, $n$, grows. This means that some events become increasingly probable at the expense of other events becoming increasingly improbable. In other words, we gain information, and our knowledge becomes stronger, as the number of observations grows. In chapter 3 , we will formalise this notion of information.

As a side note we remark that we have just solved an inverse problem. A priori, we had no information on $x_{A}$ in the sense that all positions were equally likely. Through the incorporation of observations, $p$, however, we accumulated probabilistic constraints on $x_{A}$ that honour the random nature of the experiment.

A particularly attractive component of Bayesian inference lies in the possibility to iterate. Through Bayes' theorem, prior knowledge is transformed into posterior knowledge, which then becomes the new prior knowledge to use for the incorporation of new data, ... . Furthermore, having solved an inference or inverse problem, we can make predictions, for example in the form of probabilistic statements on the future occurrence of event $L$. Using equation (2.7) for the conjunction of events, we obtain the joint probability of observing event $L$ and position $x_{A}$ as

$$
P\left(L \wedge x_{A}\right)=P\left(L \mid x_{A}\right) P\left(x_{A}\right) .
$$

Inserting for $P\left(x_{A}\right)$ the posterior (conditional) probabilty of $x_{A}$ after observing $p$, yields the conditional joint probability

$$
P\left(L \wedge x_{A} \mid p\right)=P\left(L \mid x_{A}\right) P\left(x_{A} \mid p\right) .
$$

Substituting equations (2.23) and (2.26), we then find

$$
P\left(L \wedge x_{A} \mid p\right)=k\left(\begin{array}{l}
n \\
p
\end{array}\right)\left(\frac{x_{A}}{l_{x}}\right)^{p+1}\left(1-\frac{x_{A}}{l_{x}}\right)^{n-p} \frac{\Delta x}{l_{x}} .
$$



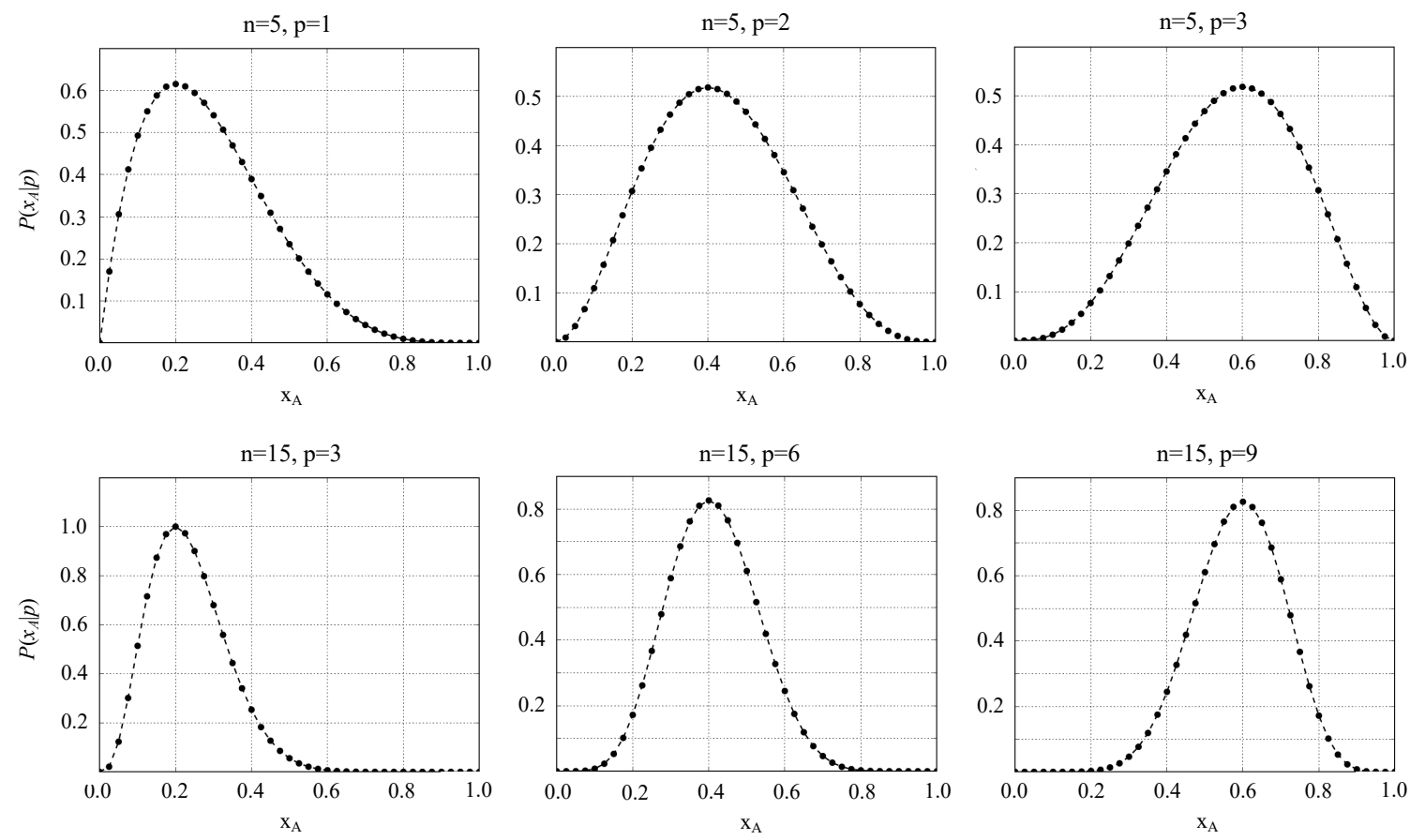

Fig. 2.4: Posterior probability $P\left(x_{A} \mid p\right)$ for the position of ball $A$ given the number of occurrences $p$ of event $L$ as observation. The interval length is set to $\Delta x=0.025$. Top: The number of experiments is $N=n$, and the number of times that event $L$ has been observed takes the values $p=1,2,3$. For $p=1$, the probability is largest for $x_{A}=0.2$. However, the distribution is rather broad, meaning that information on the location $x_{A}$ is weak. The situation is similar for the other values of $p$. Bottom: As the number of experiments increases from $n=5$ to $n=15$, the probability distributions become more peaked. As a consequence, certain values of $x_{A}$ can be ruled out with greater certainty. The posterior distributions contain more precise information on $x_{A}$. [Bayes' table]

Since the individual $x_{A}$ represent inconsistent events - ball $A$ cannot land in two intervals at the same time we can use equation (2.2) to compute the probability of observing event $L$ regardless of whether $A$ has landed in the first, or the second, or in any other interval,

$$
P(L \mid p)=\sum_{x_{A}} P\left(L \wedge x_{A} \mid p\right)
$$

A visualisation of $P(L \mid p)$ is shown in figure 2.5. Note that the probability of observing $L$ is never exactly 0 even when $L$ has never been observed before $(p=0)$. However, the probability at $p=0$ approaches zero as the number of observations, $n$, grows. Similarly, the probability of observing $L$ steadily increases when it continues to be observed in all experiments $(p=n)$.

In modern terminology, the action of summing over all possible values of one variable of a joint probability, as in equation (2.31), is referred to as marginalising. The resulting probability distribution is called the marginal probability.

Before closing this chapter, we briefly return to our small dilemma of $x_{A}$ actually being a continuous and not a discrete variable with a finite number of values. Our crude solution was to subdivide the $x$-axis into small intervals of equal length $\Delta x$, that we chose rather arbitrarily. A more elegant approach is to work with probability densities. To illustrate the concept, we rewrite equation (2.25) in a more correct form, 

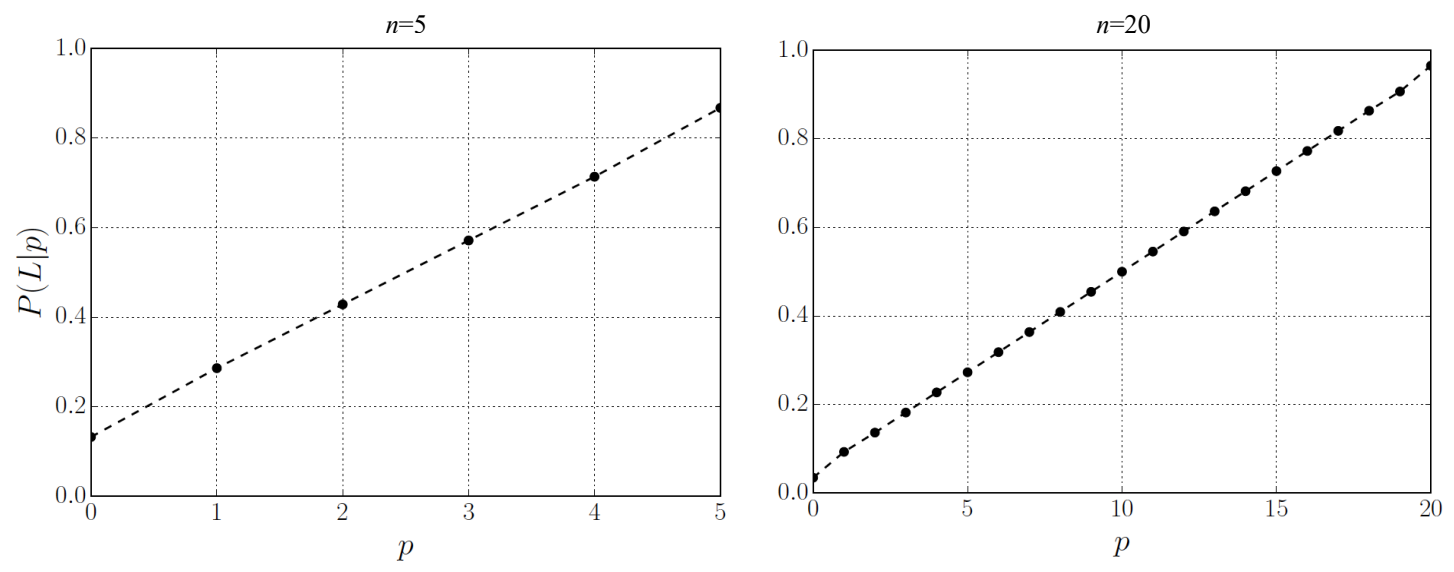

Fig. 2.5: Occurrence probability $P(L \mid p)$ of event $L$ given observations $p$. The number of observations is $n=5$ in the left panel, and $n=20$ in the right panel. [Bayes' table]

$$
P\left(x_{A}\right)=P\left(x \in\left[x_{A}, x_{A}+\Delta x\right]\right)=\frac{\Delta x}{l_{x}} .
$$

Though it may seem to be an unnecessary complication at this point, we write the right-hand side of equation (2.32) in terms of an integral,

$$
P\left(x \in\left[x_{A}, x_{A}+\Delta x\right]\right)=\int_{x_{A}}^{x_{A}+\Delta x} \frac{1}{l_{x}} d x=\int_{x_{A}}^{x_{A}+\Delta x} \rho(x) d x .
$$

The quantity $\rho(x)=l_{x}^{-1}$ is referred to as the probability density of $P$. In our case, $\rho(x)$ is constant within our domain of interest, $\left[0, l_{x}\right]$, reflecting or prior knowledge that $A$ may land in any interval with equal probability ${ }^{3}$. All the developments in the previous paragraph may be reformulated in terms of probability densities, prior and posterior ones. A more detailed treatment of probability densities and their role in Bayesian inference will be the subject of section 3.1 .

\subsection{More formal probability theory}

All our previous developments, including the important equations (2.6) and (2.9), derive from counting a finite number of coloured blocks. These equations appear reasonable to us because they conform with our intuitive expectations when this specific experiment is repeated many times. However, two problems arise immediately: (1) Already in section 2.2, we somewhat blindly translated equations from the block counting experiment to an entirely different scenario. How can we be sure that these equations are still meaningful within the new context? (2) In inverse theory, the notion of probability is often not used in a statistical sense where an experiment with a finite number of outcomes can in principle be repeated infinitely often. Instead, as we will see in chapter 3, it quantifies the probability that a model parameter, typically inferred from error-contaminated and incomplete data, has certain properties. Examples would be the probability that the average density of the Earth's inner core is above $13,000 \mathrm{~kg} / \mathrm{m}^{3}$, or that the temperature in a certain volume of the atmosphere is above the freezing

\footnotetext{
${ }^{3}$ One may argue that this is not really prior knowledge because we do not actually know a priori where $A$ is located. However, being able to state that $\rho(x)$ is constant, and not any other function, is also a form of knowledge.
} 
point ${ }^{4}$. In both cases, there is not even a repeatable experiment that would allow us to establish rules on the basis of statistical arguments. How do we handle probabilities in such applications?

Obviously, it is desirable to have a more formal derivation of equations for conjunctions, disjunctions, conditional probabilities, and relations between them. Historically, there are numerous approaches to formal probability theory, including the celebrated Kolmogorov axioms ${ }^{5}$ (Kolmogorov, 1950). One of the most appealing and intuitive ones is due to Jaynes (2003), which we shall summarise here. It starts with three desiderata that may initially seem to be somewhat vague and non-mathematical:

1) Degrees of probability are represented by real numbers. Furthermore, these numbers shall increase continuously with increasing probability.

\section{2) Probabilities shall have a qualitative correspondence with common sense.}

3) Probabilities shall be consistent. This includes that conclusions are identical if they can be reached in more than one way, that all evidence is taken into account without ideological bias, and that the same numbers are assigned when two states of knowledge are identical.

In a remarkable sequence of arguments, Jaynes continues to demonstrate that these desiderata lead to concrete mathematical statements concerning some arbitrary events $A, B$ and $C$. These are the product rule

$$
P(A \wedge B \mid C)=P(A \mid C) P(B \mid A \wedge C)=P(B \mid C) P(A \mid B \wedge C),
$$

the sum rule

$$
P(A \mid B)+P(\bar{A} \mid B)=1,
$$

and the requirements that impossibility of $A$ corresponds to $P(A)=0$, and certainty of $A$ corresponds to $P(A)=$ 1. The product and the sum rule may be used, for example, to derive a useful equation for the conditional probability of the disjunction $A \vee B$. For this, we first note that

$$
A \vee B=\overline{\bar{A} \wedge \bar{B}},
$$

which can easily be verified using the truth table below,

\begin{tabular}{c|c|c|c}
$A$ & $B$ & $A \vee B$ & $\bar{A} \wedge \bar{B}$ \\
\hline false & false & false & false \\
\hline true & false & true & true \\
\hline false & true & true & true \\
\hline true & true & true & true
\end{tabular}

Repeated application of the sum and product rules then yields

$$
\begin{aligned}
P(A \vee B \mid C) & =1-P(\overline{A \vee B} \mid C)=1-P(\bar{A} \wedge \bar{B} \mid C)=1-P(\bar{A} \mid C) P(\bar{B} \mid \bar{A} \wedge C) \\
& =1-P(\bar{A} \mid C)[1-P(B \mid \bar{A} \wedge C)]=P(A \mid C)+P(\bar{A} \wedge B \mid C)=P(A \mid C)+P(B \mid C) P(\bar{A} \mid B \wedge C) \\
& =P(A \mid C)+P(B \mid C)[1-P(A \mid B \wedge C)]=P(A \mid C)+P(B \mid C)-P(B \mid C) P(A \mid B \wedge C),
\end{aligned}
$$

and finally

$$
P(A \vee B \mid C)=P(A \mid C)+P(B \mid C)-P(A \wedge B \mid C) .
$$

\footnotetext{
${ }^{4}$ The density of the Earth's inner core, mostly composed of an iron-nickel alloy under high pressure and temperture, is believed to be mostly below $13,000 \mathrm{~kg} / \mathrm{m}^{3}$.

${ }^{5}$ Andrey Nikolaevich Kolmogorov (1903 - 1987) was a Soviet mathematician, famous not only for his contributions in probability theory, mechanics and complexity theory, but also for his life-long dedication to childhood education.
} 
In the special case where $A$ and $B$ are mutually exclusive, or inconsistent in Bayes' words, $A \wedge B$ is generally false, irrespective of $C$, and so (2.38) simplifies to

$$
P(A \vee B \mid C)=P(A \mid C)+P(B \mid C) .
$$

Furthermore, if both $A$ and $B$ are independent of $C$, we obtain

$$
P(A \vee B)=P(A)+P(B) .
$$

which is equivalent to equation (2.2), that we used before in our box counting experiment. Also the product rule (2.34) simplifies if $A$ and $B$ do not depend on $C$ :

$$
P(A \wedge B)=P(A) P(B \mid A)=P(B) P(A \mid B) .
$$

This is the same as our intuitive definition of conditional probability in equation (2.7), from which we derived Bayes' theorem. When $B$ is independent of $A$, that is, when the probability of $B$ is unaffected by the truth or occurence of $A$, equation (2.41) further condenses to

$$
P(A \wedge B)=P(A) P(B) .
$$

We have seen this before in (2.11).

Having established the foundations of probability theory, largely by example, we will continue to use them for the very general solution of inverse problems. As we will see in the following chapter, this will not require much more than a specific interpretation of the generic events $A$ and $B$. 



\section{Chapter 3 \\ Probabilities as states of information *}

Information demands respect!

Klaus Mosegaard, 2019.

My greatest concern was what to call it. I thought of calling it 'information,' but the word was overly used, so I decided to call it 'uncertainty.' When I discussed it with John von Neumann, he had a better idea. Von Neumann told me, 'You should call it entropy, for two reasons. In the 1st place your uncertainty function has been used in statistical mechanics under that name, so it already has a name. In the second place, and more important, no one knows what entropy really is, so in a debate you will always have the advantage.

Claude E. Shannon to Myron Tribus, 1971.

This chapter provides the basic mathematical machinery needed to define and solve probabilistic inverse problems. The concepts introduced are deliberately kept on a quite general level. They will then be applied to concrete inverse problems in the following chapters.

\subsection{Probabilities and probability densities *}

The fundamental idea of probabilistic inverse theory is to express all knowledge concerning model parameters, data and the physical theory in terms of probabilities. Probability theory, as we have seen in chapter 2 , is concerned with abstract events that are not further specified. Hence, the question arises what a more specific definition of events might be, that is useful for our purposes.

A seemingly natural definition of an event $A$ could be that a one-dimensional model parameter $m$ takes exactly the value $m_{0}$, and so we would be interested in the probability $P\left(m=m_{0}\right)=P_{0}$. However, in all practically relevant scenarios, our knowledge about $m$ will not be perfect, for instance, because our data are not perfect either. Therefore, we can generally find an interval $\left[m_{0}-\varepsilon, m_{0}+\varepsilon\right]$ that contains alternative parameter values $m_{1}, m_{2}, \ldots$, that are essentially as probable as $m_{0}$, that is, $P\left(m=m_{i}\right)=P_{0}$ for any $i=1,2, \ldots$. Despite being potentially very small, the interval $\left[m_{0}-\varepsilon, m_{0}+\varepsilon\right]$ contains infinitely many of these alternative parameter values $m_{i}$, and to be meaningful, their associated probability must sum to a value that is smaller or equal to 1 , 


$$
\sum_{i=0}^{\infty} P\left(m=m_{i}\right)=\sum_{i=0}^{\infty} P_{0} \leq 1
$$

It immediately follows from (3.1) that the probability of $m$ taking exactly one specific value must be 0 . We learn from this argument that choosing the event $A$ to be $m=m_{0}$ is in principle possible. However, we know the answer immediately in any case, and so this choice is not particularly interesting.

Looking at the example above, it may be more useful to consider the event that $m$ falls into a certain interval. Assuming, for the sake of illustration, that a one-dimensional model space $\mathbb{M}$ consists of the interval $[0,1]$, we may define $A$ as the event that $m \in\left[0, m_{1}\right]$, with $m_{1} \leq 1$. Letting $m_{1}$ be variable, the probability $P(A)=P(m \in$ $\left.\left[0, m_{1}\right]\right)$ becomes a function of $m_{1}$. The derivative of this function,

$$
\frac{d}{d m_{1}} P\left(m \in\left[0, m_{1}\right]\right)=\rho\left(m_{1}\right),
$$

defines the probability density $\rho$ of $P$. As we will see later, much of our (intuitive) understanding of inverse problems rests on the analysis of probability densities. From the probability density $\rho$ we can uniquely reconstruct the probability $P$. In fact, upon integrating (3.2), we find

$$
\int_{0}^{m_{1}} \rho(m) d m=P\left(m \in\left[0, m_{1}\right]\right)+c,
$$

where $c$ is an integration constant. Setting $m_{1}=0$, the integral on the left-hand side of (3.3) vanishes, and we obtain $c=-P(m \in[0,0])$, which is the negative probability of $m_{1}$ being exactly equal to 0 . As we have seen above, the probability of $m$ taking any specific value vanishes, and so we infer that the integration constant is given by $c=0$.

To gain more flexibility, we now also allow the lower interval boundary to be chosen freely, and consider the event $A: m \in\left[m_{1}, m_{2}\right]$. This is identical to the conjunction of two other events, namely $A_{1}: m \in\left[0, m_{2}\right]$ and $A_{2}: \overline{m \in\left[0, m_{1}\right]}$, and so we have

$$
\begin{aligned}
P\left(m \in\left[m_{1}, m_{2}\right]\right) & =P\left(A_{1} \wedge A_{2}\right)=P\left(\overline{\bar{A}_{1} \vee \bar{A}_{2}}\right)=1-P\left(\bar{A}_{1} \vee \bar{A}_{2}\right)=1-P\left(\bar{A}_{1}\right)-P\left(\bar{A}_{2}\right) \\
& =P\left(A_{1}\right)-P\left(\bar{A}_{2}\right)=P\left(m \in\left[0, m_{2}\right]\right)-P\left(m \in\left[0, m_{1}\right]\right) .
\end{aligned}
$$

Making use of the integral expression of probabilities introduced in (3.3), we can express (3.4) as

$$
P\left(m \in\left[m_{1}, m_{2}\right]\right)=\int_{0}^{m_{2}} \rho(m) d m-\int_{0}^{m_{1}} \rho(m) d m=\int_{m_{1}}^{m_{2}} \rho(m) d m .
$$

Equation (3.5) states that the probability of $m$ falling into the interval $\left[m_{1}, m_{2}\right]$ is equal to the area between $m_{1}$ and $m_{2}$ under the probability density curve $\rho(m)$. This is illustrated in figure 3.1.

While the above line of arguments uses a specific one-dimensional interval, it can in fact be generalised to any arbitrary model space dimension $n \geq 1$, where the probability of $\mathbf{m}$ falling into some subvolume $M$ of model space can be written as an integral,

$$
P(\mathbf{m} \in M)=\int_{M \in \mathbb{M}} \rho(\mathbf{m}) d \mathbf{m} .
$$

Admittedly, some of the arguments given above are somewhat handwavy, for example, when we considered an infinite number of points in (3.1) with 'essentially' equal probability. A rigorous derivation of equations 3.5 and (3.6) requires more mathematical machinery from functional analysis. It results in the celebrated Radon- 
Nikodym theorem, stating that a probability can be expressed in terms of an integral over a probability density under very general assumptions.

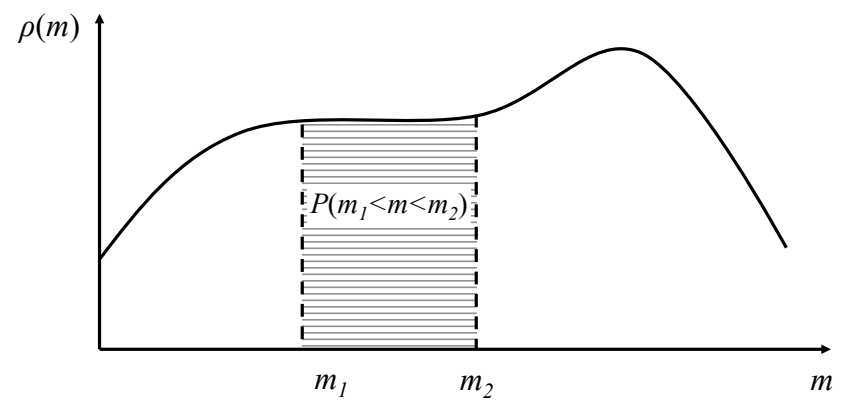

Fig. 3.1: Illustration of a one-dimensional probability density $\rho(m)$. The probability of $m$ being between $m_{1}$ and $m_{2}$ is equal to the striped area under the curve, that is $P\left(m \in\left(m_{1}, m_{2}\right)\right)=\int_{m_{1}}^{m_{2}} \rho(m) d m$.

To be interpretable in terms of a proper probability, we require that the integral of $\rho(\mathbf{m})$ over the complete model space $\mathbb{M}$ is equal to 1 ,

$$
P(\mathbf{m} \in \mathbb{M})=\int_{\mathbb{M}} \rho(\mathbf{m}) d \mathbf{m}=1,
$$

meaning that the model vector $\mathbf{m}$ lies with $100 \%$ probability anywhere in the model space. Probability densities $\rho$ that satisfy equation (3.7) are called normalisable.

The one-dimensional Gaussian distribution: The best known example of a normalisable probability density is the Gaussian distribution or normal distribution, given in one dimension by

$$
\rho(m)=\frac{1}{\sqrt{2 \pi} \sigma} e^{-\frac{\left(m-m_{0}\right)^{2}}{2 \sigma^{2}}},
$$

with mean $m_{0}$ and standard deviation $\sigma$. A small $\sigma$ indicates that the model $m$ is located near the mean $m_{0}$ with relatively high probability. In contrast, a large $\sigma$ describes the less precise knowledge that $m$ may be located in a relatively wide range around $m_{0}$. An example of a Gaussian is shown in figure 3.2a. 
a) Gaussian

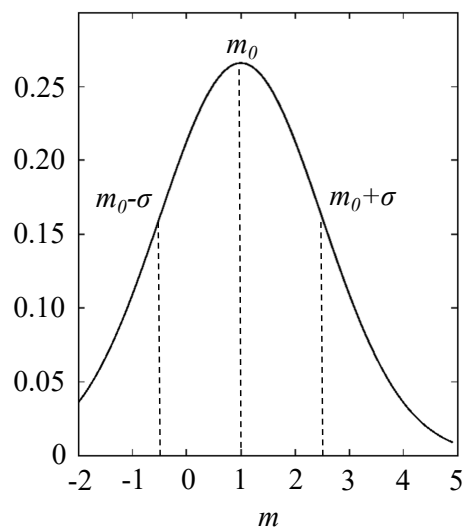

b) delta

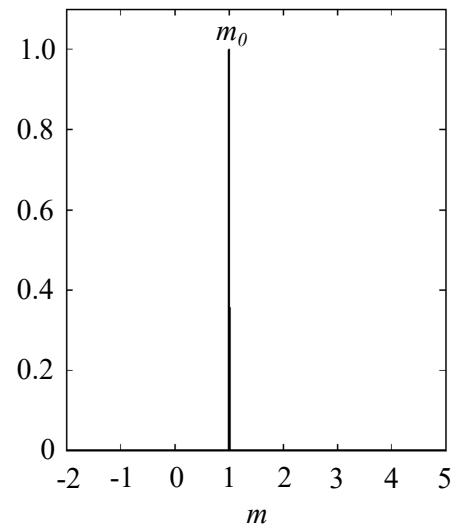

c) uniform

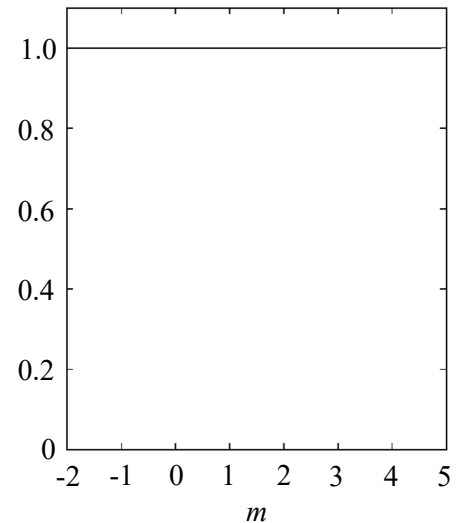

Fig. 3.2: Examples of probability densities. (a) Gaussian distribution with mean $m_{0}=1$ and standard deviation $\sigma=1.5$. (b) $\delta$-distribution, $\delta\left(m-m_{0}\right)$, with $m_{0}=1$. c) Uniform distribution, $\rho(m)=1$.

Perfect knowledge - the $\delta$-distribution: As $\sigma$ tends to 0 , the Gaussian (3.8) converges towards the $\delta$-function

$$
\rho(m)=\delta\left(m-m_{0}\right)
$$

which is the normalisable probability density describing absolute certainty that $m=m_{0}$. Figure $3.2 \mathrm{~b}$ shows an example. The $\delta$-distribution is pathological in the sense of being interesting as an idealised limiting case. However, it is of little practical relevance because the imperfections of real data will never permit absolute certainty.

Representing ignorance - the uniform distribution: Not all probability densities are normalisable. Consider, for instance, the probability density

$$
\rho(m)=\text { constant }, \quad m \in \mathbb{R} .
$$

This probability density, illustrated in figure 3.2c, describes a state of knowledge where $m$ is located with equal probability within any interval $\left[m_{0}, m_{0}+\Delta m\right]$ of length $\Delta m$. The constant or uniform probability density is useful for encoding a relative state of ignorance, meaning that we do not know where in model space $m$ is located. However, the constant probability density cannot be normalised.

While non-normalisable probability densities can be useful, they should still be employed with some caution. Jaynes (2003), for example, notes that the product rule (2.1) and the sum rule (2.2), which are fundamental for probability theory, need to be modified when absolute certainty of some event $X$ is not represented by $P(X)=1$.

Normalising Gaussians: The normalisation constant $c$ of the one-dimensional Gaussian

$$
\rho(x)=c e^{-\frac{x^{2}}{2 \sigma^{2}}}
$$

can be found with a simple integration trick. Integrating the product of two Gaussians, we have

$$
\frac{1}{c^{2}}=\int_{-\infty}^{\infty} \int_{-\infty}^{\infty} e^{-\frac{x^{2}}{2 \sigma^{2}}} e^{-\frac{y^{2}}{2 \sigma^{2}}} d x d y=\int_{-\infty}^{\infty} \int_{-\infty}^{\infty} e^{-\frac{\left(x^{2}+y^{2}\right)}{2 \sigma^{2}}} d x d y
$$

Transforming this two-dimensional integral into polar coordinates, we obtain 


$$
\frac{1}{c^{2}}=\int_{\phi=0}^{2 \pi} \int_{0}^{\infty} e^{-\frac{r^{2}}{2 \sigma^{2}}} r d \phi d r
$$

Realising that

$$
-\sigma^{2} \frac{d}{d r} e^{-\frac{r^{2}}{2 \sigma^{2}}}=r e^{-\frac{r^{2}}{2 \sigma^{2}}}
$$

and computing the integral over $\phi$, equation (3.13) simplifies to

$$
\frac{1}{c^{2}}=-2 \pi \sigma^{2} \int_{0}^{\infty} \frac{d}{d r} e^{-\frac{r^{2}}{2 \sigma^{2}}} d r .
$$

The trivial solution of (3.15) yields the normalisation constant

$$
c=\frac{1}{\sqrt{2 \pi} \sigma} .
$$

In higher dimensions, we use the fact that an $n$-dimensional Gaussian is a product of $n$ one-dimensional Gaussians, and so we find for

$$
\frac{1}{c}=\int_{-\infty}^{\infty} \ldots \int_{-\infty}^{\infty} e^{-\frac{1}{2}\left(\frac{x_{1}^{2}}{\sigma_{1}^{2}}+\frac{x_{2}^{2}}{\sigma_{2}^{2}}+\ldots+\frac{x_{n}^{2}}{\sigma_{n}^{2}}\right)} d x_{1} d x_{2} \ldots d x_{n}
$$

the solution

$$
c=\frac{1}{(2 \pi)^{\frac{n}{2}} \sigma_{1} \sigma_{2} \ldots \sigma_{n}} .
$$

The multi-dimensional Gaussian distribution: The one-dimensional Gaussian in equation (3.8) can be generalised to an arbitrary $n$-dimensional model vector $\mathbf{m}$,

$$
\rho(\mathbf{m})=\frac{1}{\sqrt{(2 \pi)^{n} \operatorname{det} \mathbf{C}}} e^{-\frac{1}{2}\left(\mathbf{m}-\mathbf{m}_{0}\right)^{T} \mathbf{C}^{-1}\left(\mathbf{m}-\mathbf{m}_{0}\right)} .
$$

The symmetric and positive definite matrix $\mathbf{C}$ is called the covariance matrix. When the covariance matrix is diagonal, with diagonal entries $\sigma_{i}^{2}$, the joint probability density of all model parameters can be written as a product of one-dimensional Gaussians,

$$
\rho(\mathbf{m})=c e^{-\frac{1}{2}\left[\frac{\left(m_{1}-m_{0,1}\right)^{2}}{\sigma_{1}^{2}}+\frac{\left(m_{2}-m_{0,2}\right)^{2}}{\sigma_{2}^{2}}+\ldots+\frac{\left(m_{n}-m_{0, n}\right)^{2}}{\sigma_{n}^{2}}\right]}=c e^{-\frac{1}{2} \frac{\left(m_{1}-m_{0,1}\right)^{2}}{\sigma_{1}^{2}}} e^{-\frac{1}{2} \frac{\left(m_{2}-m_{0,2}\right)^{2}}{\sigma_{2}^{2}}} \ldots e^{-\frac{1}{2} \frac{\left(m_{n}-m_{0, n}\right)^{2}}{\sigma_{n}^{2}}}
$$

with the normalisation constant $c$ from equation (3.18). In this case, the model parameters are independent. Examples are shown in figure 3.3. In the interest of a more condensed notation, a variable $\mathbf{m}$ that is distributed according to a Gaussian with mean $\mathbf{m}_{0}$ and covariance $\mathbf{C}$ is often written as

$$
\mathbf{m} \sim \mathscr{N}\left(\mathbf{m}_{0}, \mathbf{C}\right) .
$$

More generally, and in accord with equation (2.42) for the probability of the conjunction of independent events, model parameters (or any variables) are independent when their joint probability density can be written as a product of one-dimensional probability densities, that is 
a) independent

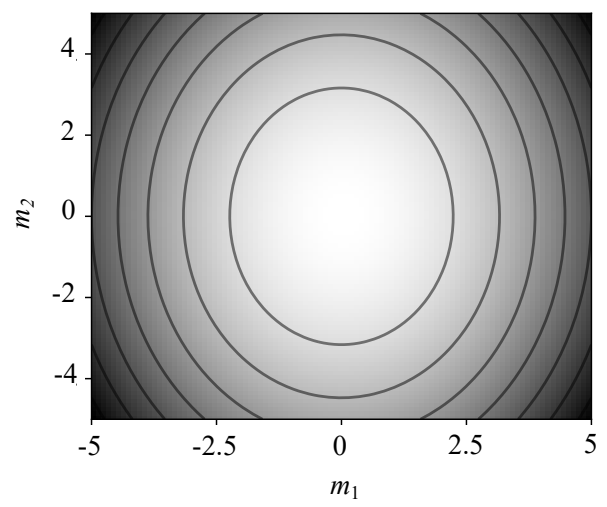

b) dependent

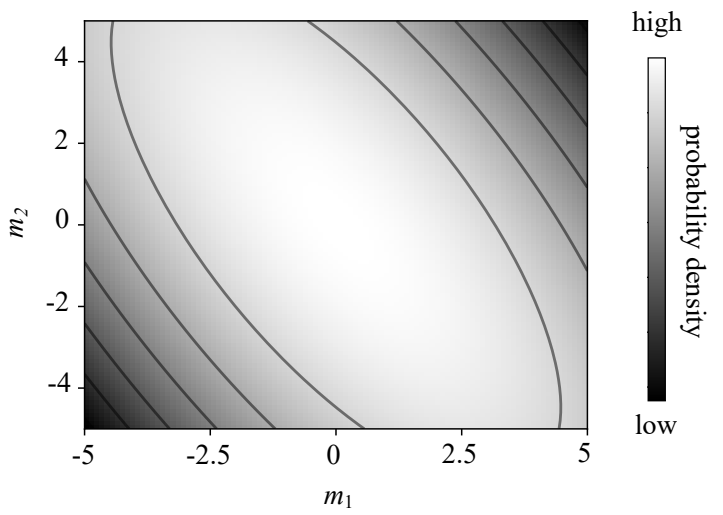

Fig. 3.3: Examples of two-dimensional Gaussians with (a) independent parameters and (b) dependent parameters.

$$
\rho(\mathbf{m})=\rho_{1}\left(m_{1}\right) \rho_{2}\left(m_{2}\right) \ldots \rho_{n}\left(m_{n}\right)
$$

Otherwise, they are dependent.

The uniform probability density is a special case of a homogeneous probability density $\rho_{h}$, frequently used to encode ignorance. The homogeneous probability density is defined as being proportional to the $n$-dimensional volume element, which may, in fact, be a surface element when $n=2$. This means that a homogeneous probability density ascribes to any volume $V$ of a certain size the same probability, no matter where in model space that volume is located. In other words, probability is proportional to volume.

Example 3.1. The homogeneous probability density in 2-D Cartesian coordinates: Note that the homogeneous probability density depends on the coordinate system. For instance, when $\mathbf{m}$ consists of the 2-D Cartesian coordinates $x$ and $y$, that is $\mathbf{m}=(x, y)$, in $\mathbb{R}^{2}$, the homogeneous probability density is

$$
\rho_{h}(x, y)=\text { constant }
$$

because the probability within any volume of size $V=\Delta x \Delta y$ is always equal to a constant times $\Delta x \Delta y$, no matter where in model space that volume is actually located. The homogeneous distribution (3.23) is visualised in figure $3.4 \mathrm{a}$.

Example 3.2. The homogeneous probability density in 2-D polar coordinates: In contrast, the homogeneous probability density in 2-D polar coordinates, $r$ and $\phi$ is

$$
\rho_{h}(r, \phi)=r \times \text { constant }
$$

that is, proportional to the 2-D volume element $r d r d \phi$, which is just a surface element in this case. The distribution (3.24) is shown in figure 3.4b. Homogeneous probability densities for different coordinate systems or model space parameterisations can formally be transformed into each other, as shown in section 3.4.

The dependence of the homogeneous (or in fact any) probability density on the coordinate system may initially be somewhat counter-intuitive. However, it can be illustrated by straightforward examples. Imagine, for instance, a blind dart player. In Cartesian coordinates, his chances of hitting some square of dimension $\Delta x \Delta y$ on the dart board are the same, no matter where the square is located. In polar coordinates, however, we do not consider squares but polar subsections with angular increment $d \phi$ and radial increment $d r$. These spherical subsections are much smaller close to the coordinate origin where $r$ is small. Therefore, the dart player's 


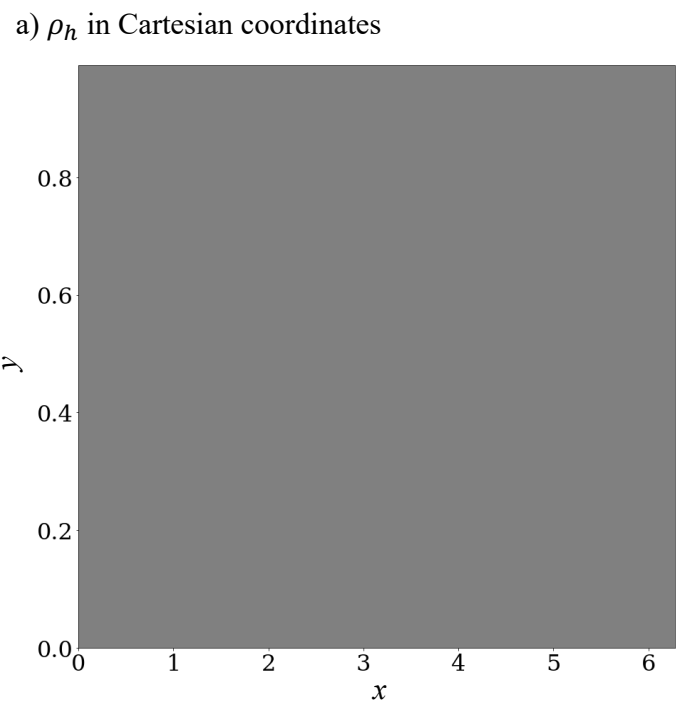

b) $\rho_{h}$ in polar coordinates

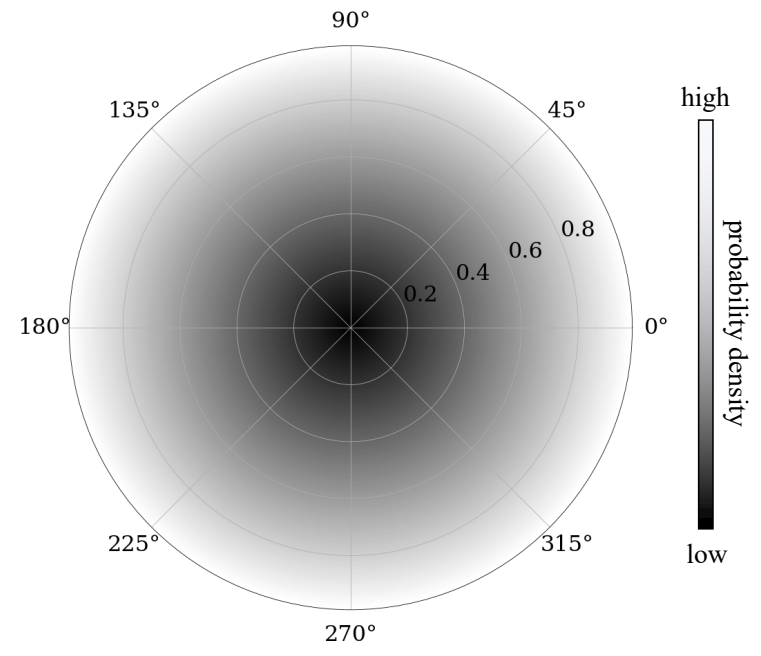

Fig. 3.4: Examples two-dimensional homogeneous probability densities. (a): The homogeneous probability density $\rho_{h}$ in Cartesian coordinates is constant. (b): In polar coordinates, the homogeneous probability density is proportional to radius $r$, meaning that it increases linearly with distance from the coordinate origin.

chances of hitting a small subsection near $r=0$ are much smaller. In other words, the probability density is lower.

While the homogeneous probability density may be used to describe a lack of knowledge, it is important to note that an uninformative probability density does strictly speaking not exist. Any probability density contains some sort of information, as we will see later.

\subsection{Frequentist and Bayesian interpretation of probabilities *}

We are most familiar with the frequentist interpretation of probabilities. In this interpretation, we consider a large number of repeatable experiments, for instance throwing darts or flipping a coin. A probability then describes the relative frequency, or number of occurrences of a specific experimental result. Our block-counting experiment in section 2.1 was clearly related to probabilities in the frequentist sense.

Often, however, we do not consider repeatable experiments, but specific objects or events, like the interior of the Earth or of a human body, or the probability of a meteorite impact in our backyard. Clearly, there is only one Earth, and there is no meaning in repeating the Earth many times. Also, meteorite impacts hopefully are not too frequent, at least in our backyard. Thus, in probabilistic inversion, we adopt the Bayesian interpretation of probabilities, where they represent a (subjective) state of information. As in the examples above, a probability density may describe a state of more or less certain information on the values of a model parameter $m$. While the Bayesian interpretation obviously differs from the frequentist interpretation, they both use the same mathematical machinery, as we will see in the following sections. 


\subsection{Shannon's measure of information content *}

The concept of information in probability densities can be formalised by a rigorous definition of the information content, introduced by Claude Shannon ${ }^{1}$ : For this, we consider the probability density $\rho(\mathbf{m})$ and the reference probability density $\rho_{\text {ref }}(\mathbf{m})$. The relative information content of $\rho(\mathbf{m})$ relative to $\rho_{\text {ref }}(\mathbf{m})$ is then given by an integral over model space $\mathbb{M}$,

$$
I\left(\rho, \rho_{\mathrm{ref}}\right)=\int_{\mathbb{M}} \rho(\mathbf{m}) \log _{b} \frac{\rho(\mathbf{m})}{\rho_{\mathrm{ref}}(\mathbf{m})} d \mathbf{m} .
$$

In this definition, the base of the logarithm, $b$, is undetermined. It can be chosen freely; and depending on this choice, the information content has a different unit. For $b=2$, the unit is the $b i t^{2}$, for $b=e \approx 2.71828$ the $n e p^{3}$, and for $b=10$ the digit.

Using the homogeneous probability density $\rho_{h}$ as reference, we may define an absolute information content as

$$
I(\rho)=\int_{\mathbb{M}} \rho(\mathbf{m}) \log _{b} \frac{\rho(\mathbf{m})}{\rho_{h}(\mathbf{m})} d \mathbf{m} .
$$

Clearly, the absolute information content is 0 when $\rho$ is equal to the homogeneous (or uninformative) probability density $\rho_{h}$. Alluding to concepts in statistical thermodynamics, the negative information context, $-I$, is often referred to as entropy.

Relative information content of two Gaussians: As an example, we consider the relative information content of the two Gaussians

$$
\rho(m)=\frac{1}{\sqrt{2 \pi} \sigma} e^{-\frac{m^{2}}{2 \sigma^{2}}}, \quad \rho_{\mathrm{ref}}(m)=\frac{1}{\sqrt{2 \pi} \sigma_{\mathrm{ref}}} e^{-\frac{m^{2}}{2 \sigma_{\mathrm{ref}}^{2}}} .
$$

After a series of integrations by part, we find

$$
I\left(\rho, \rho_{\mathrm{ref}}\right)=\ln \frac{\sigma_{\mathrm{ref}}}{\sigma}+\frac{1}{2}\left(\frac{\sigma^{2}}{\sigma_{\mathrm{ref}}^{2}}-1\right) .
$$

Two special cases deserve particular attention: For $\sigma=\sigma_{\text {ref }}$, we have $I=0$, meaning that $\rho$ contains no new in-

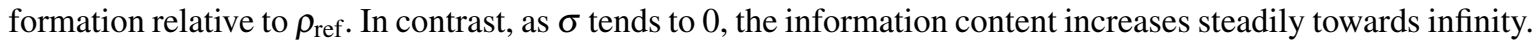

Measuring differences between probability densities: Shannon's measure of information content, also referred to as the Kullback-Leibler divergence, is only one of many possible measures for the difference between probability densities. Generalisations of the information content are $f$-divergences, defined as

$$
D\left(\rho, \rho_{\mathrm{ref}}\right)=\int_{\mathbb{M}} f\left(\frac{\rho}{\rho_{\mathrm{ref}}}\right) \rho_{\mathrm{ref}} d \mathbf{m},
$$

for any convex function $f$ with $f(1)=0$. The Kullback-Leibler divergence is the special case where $f(x)=x \log x$.

\footnotetext{
${ }^{1}$ Claude Elwood Shannon (1916 - 2001) was a US-American mathematician and electrical engineer. He is considered the founder of information theory.

2 The bit, introduced in the 1940's by John Tuckey, stands for binary digit.

3 The unit nep honours John Napier (1550 - 1617), considered the inventor of logarithms.
} 


\subsection{Changing coordinates *}

Very often, there is considerable freedom in the choice of parameterisation. For instance, one may use velocity or its inverse, slowness, to forward model the time needed by a wave or a particle to travel a certain distance. This naturally poses the question of how a probability density transforms as we go from one parameterisation $m$ to another one $m^{*}$.

To find a transformation rule, we start with the definition of probability in terms of probability density, already introduced in equation (3.6):

$$
P(m \in M)=\int_{M \in \mathbb{M}} \rho(m) d m .
$$

Transforming $m$ to the new parameter $m^{*}(m)$ also transforms the subvolume $M$ to a new subvolume $M^{*}$, and the corresponding integral becomes

$$
P\left(m^{*} \in M^{*}\right)=\int_{M^{*} \in \mathbb{M}^{*}} \rho^{*}\left(m^{*}\right) d m^{*},
$$

with the transformed probability density $\rho^{*}$. Since the two integrals (3.30) and (3.31) must return the same number - probability cannot change just because we reparameterise - we have

$$
\int_{M \in \mathbb{M}} \rho(m) d m=\int_{M^{*} \in \mathbb{M}^{*}} \rho^{*}\left(m^{*}\right) d m^{*}=\int_{M \in \mathbb{M}} \rho^{*}\left[m^{*}(m)\right] \frac{d m^{*}}{d m} d m,
$$

where the last identity follows from the transformation rule of integrals. Comparing the first and the last integrands in (3.32), we find the transformation rule for probability densities

$$
\rho^{*}\left(m^{*}\right)=\rho(m)\left|\frac{d m}{d m^{*}}\right|
$$

The absolute value in equation (3.33) is introduced by convention, to ensure that the transformed probability density is always positive. For non-scalar parameters, equation (3.33) generalises to

$$
\rho^{*}\left(\mathbf{m}^{*}\right)=\rho(\mathbf{m})\left|\operatorname{det} \frac{d \mathbf{m}}{d \mathbf{m}^{*}}\right|
$$

where $\operatorname{det} d \mathbf{m} / d \mathbf{m}^{*}$ denotes the determinant of the Jacobian of the transformation. Specific examples for the application of (3.34) will follow in section 3.7, where it will be used to derive the probability densities of sums, products and quotients of model parameters.

Bulk modulus and compressibility: As an example, we consider the bulk modulus $\kappa$ and its inverse, the compressibility $\kappa^{*}=\frac{1}{\kappa}$. For $\kappa$ we define a constant probability density, expressing a state of relative ignorance,

$$
\rho(\kappa)=\text { constant }
$$

Then, the distribution for $\kappa^{*}$ is

$$
\rho^{*}\left(\kappa^{*}\right)=\rho(\kappa)\left|\frac{d \kappa}{d \kappa^{*}}\right|=\text { constant }\left|\frac{d}{d \kappa^{*}} \frac{1}{\kappa^{*}}\right|=\operatorname{constant} \frac{1}{\kappa^{* 2}} .
$$

Clearly, the functional form of the probability density changes drastically as we replace the bulk modulus by its inverse, which is an entirely legitimate subjective choice. This result underlines that the description of 
information - or lack thereof - generally has a subjective component, not only in probabilistic inversion. The probability densities $\rho$ and $\rho^{*}$ are shown in figure 3.5.
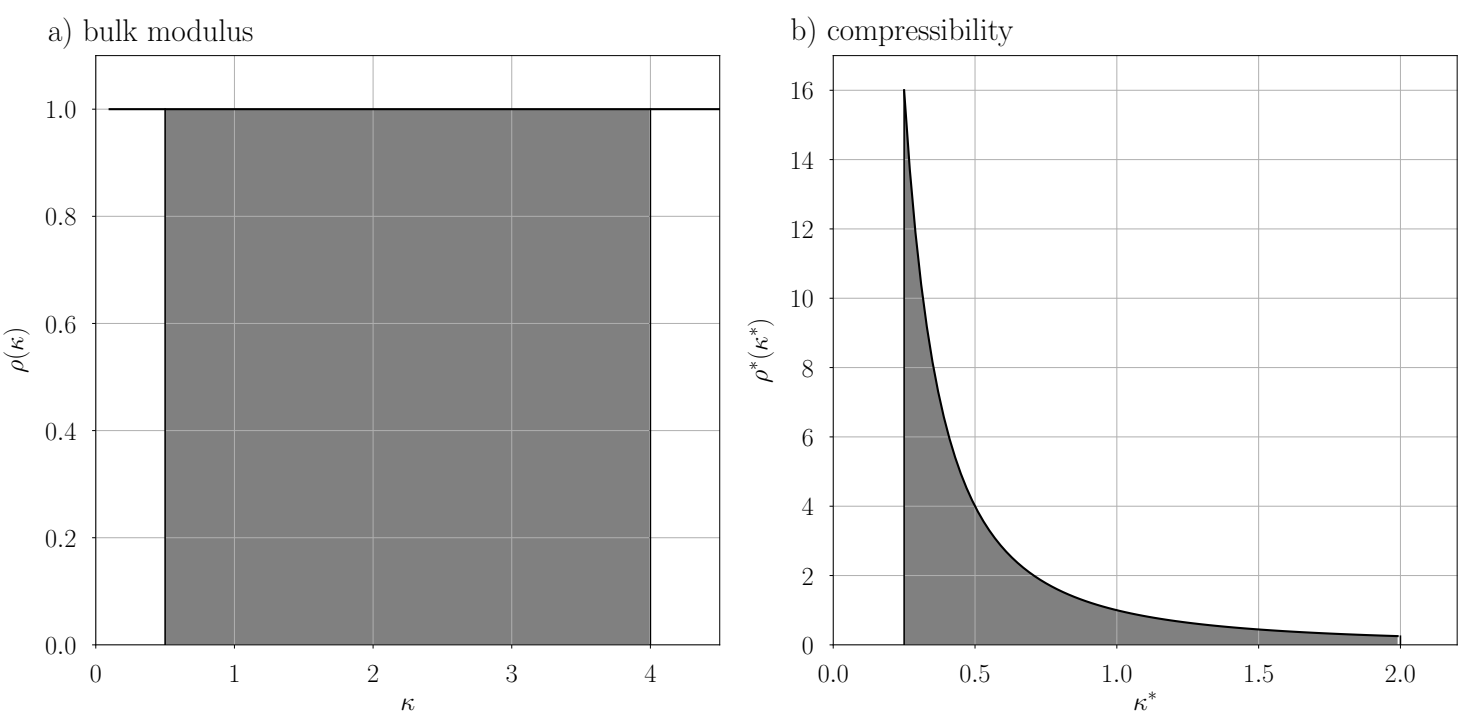

Fig. 3.5: Illustration of the coordinate transformation from bulk modulus $\kappa$ and compressibility $\kappa^{*}$. (a): Uniform probability density for $\kappa$. (b): The corresponding, transformed, probability density for $\kappa^{*}$. Note that the shaded areas between $\kappa=0.5$ and $\kappa=4$, and $\kappa^{*}=1 / 4$ and $\kappa^{*}=1 / 0.5$ are, by design, equal. Thus, probabilities are properly preserved under coordinate transformation, as desired.

Removing parameter dependence in a multi-dimensional Gaussian: A coordinate transformation may be used to transform a Gaussian distribution of dependent model parameters $\mathbf{m}$ into a Gaussian of independent model parameters $\mathbf{m}^{*}$. To see this, we consider the Gaussian

$$
\rho(\mathbf{m})=\frac{1}{\sqrt{(2 \pi)^{n} \operatorname{det} \mathbf{C}}} e^{-\frac{1}{2} \mathbf{m}^{T} \mathbf{C}^{-1} \mathbf{m}} .
$$

Being a covariance matrix, $\mathbf{C}$ is by definition positive definite. Therefore, $\mathbf{C}^{-1}$ has the Cholesky ${ }^{4}$ decomposition $\mathbf{C}^{-1}=\mathbf{L}^{T} \mathbf{L}$, with a lower triangular matrix $\mathbf{L}$. (See the following box.) Using the coordinate transformation

$$
\mathbf{m}^{*}=\mathbf{L}^{T} \mathbf{m},
$$

we find another Gaussian distribution for $\mathbf{m}^{*}$,

$$
\rho^{*}\left(\mathbf{m}^{*}\right)=\frac{1}{\sqrt{(2 \pi)^{n} \operatorname{det} \mathbf{C}}} \frac{1}{\operatorname{det} \mathbf{L}} e^{-\frac{1}{2}\left(\mathbf{m}^{*}\right)^{T} \mathbf{m}^{*}}=\frac{1}{(2 \pi)^{n / 2}} e^{-\frac{1}{2}\left(\mathbf{m}^{*}\right)^{T} \mathbf{m}^{*}} .
$$

The last equality follows because the determinants of two matrices are related by $\operatorname{det}(\mathbf{A B})=\operatorname{det} \mathbf{A} \operatorname{det} \mathbf{B}$. By construction, the covariance of $\rho^{*}\left(\mathbf{m}^{*}\right)$ is the unit matrix, meaning that all model parameters are independent.

\footnotetext{
${ }^{4}$ André-Louis Cholesky (1875-1918) was a French geodesist, mathematician and military officer. He developed the decomposition of a symmetric, positive definite matrix into a lower triangular matrix and its transpose in the context of the least-squares method, applied to geodetic problems. He was killed in combat just two months before the end of World War I. The decomposition named in his honour was published posthumously.
} 
Why do probability densities change under coordinate transformations?: It is important to see that the need to transform probability densities under a change of coordinates arises from the definition of probability in terms of probability density, introduced in equation (3.6). The integral is strictly over $d \mathbf{m}=d m_{1} d m_{2} \ldots d m_{n}$, which is not generally equal to the volume element in the $n$-dimensional model space.

Cholesky decomposition: The Cholesky decomposition factorises a symmetric and positive-definite matrix $\mathbf{A}$ into a lower triangular matrix $\mathbf{L}$ and its transpose $\mathbf{L}^{T}$, which is an upper triangular matrix,

$$
\mathbf{A}=\mathbf{L} \mathbf{L}^{T} .
$$

The concept of the Cholesky decomposition is most easily shown by example. Consider the $3 \times 3$ matrix

$$
\mathbf{A}=\left[\begin{array}{ccc}
4 & 12 & -16 \\
12 & 37 & -43 \\
16 & -43 & 98
\end{array}\right]
$$

Gauss elimination applied to $\mathbf{A}$ is equivalent to the application of a lower triangular matrix $\mathbf{L}_{1}$ to $\mathbf{A}$, which yields an upper triangular matrix $\mathbf{U}_{1}$,

$$
\mathbf{L}_{1} \mathbf{A}=\left[\begin{array}{ccc}
1 & 0 & 0 \\
-3 & 1 & 0 \\
19 & -5 & 1
\end{array}\right]\left[\begin{array}{ccc}
4 & 12 & -16 \\
12 & 37 & -43 \\
16 & -43 & 98
\end{array}\right]=\left[\begin{array}{ccc}
4 & 12 & -16 \\
0 & 1 & 5 \\
0 & 0 & 9
\end{array}\right]=\mathbf{U}_{1}
$$

Being triangular, the matrix $\mathbf{L}_{1}$ is easy to invert, and so we can represent $\mathbf{A}$ as

$$
\mathbf{A}=\mathbf{L}_{1}^{-1} \mathbf{U}_{1}=\left[\begin{array}{ccc}
1 & 0 & 0 \\
3 & 1 & 0 \\
-4 & 5 & 1
\end{array}\right]\left[\begin{array}{ccc}
4 & 12 & -16 \\
0 & 1 & 5 \\
0 & 0 & 9
\end{array}\right]
$$

Since $\mathbf{L}_{1}$ is a lower triangular matrix, its inverse is a lower triangular matrix, too. Furthermore, the diagonal elements of $\mathbf{L}_{1}^{-1}$ are all equal to 1 , just as the diagonal elements of $\mathbf{L}_{1}$. Introducing a diagonal matrix $\Lambda$, wwe can normalise the diagonal elements of the upper triangular matrix $\mathbf{U}_{1}$ to 1 as well,

$$
\mathbf{A}=\mathbf{L}_{1}^{-1} \Lambda \mathbf{U}_{1}^{\prime}=\left[\begin{array}{ccc}
1 & 0 & 0 \\
3 & 1 & 0 \\
-4 & 5 & 1
\end{array}\right]\left[\begin{array}{lll}
4 & 0 & 0 \\
0 & 1 & 0 \\
0 & 0 & 9
\end{array}\right]\left[\begin{array}{ccc}
1 & 3 & -4 \\
0 & 1 & 5 \\
0 & 0 & 1
\end{array}\right]
$$

The triangular matrices in (3.44) are transposes of each other, and since $\mathbf{A}$ is positive definite, the diagonal matrix $\Lambda$ only contains values that are strictly greater than 0 . Thus, we may take the square root of $\Lambda$ and write

$$
\mathbf{A}=\mathbf{L}_{1}^{-1} \Lambda \mathbf{L}_{1}^{-T}=\mathbf{L}_{1}^{-1} \sqrt{\Lambda}\left(\mathbf{L}_{1}^{-1} \sqrt{\Lambda}\right)^{T}
$$

where ${ }^{-T}$ denotes the transpose of an inverse matrix. Defining $\mathbf{L}=\mathbf{L}_{1}^{-1} \sqrt{\Lambda}$, we find the Cholesky decomposition of $\mathbf{A}$,

$$
\mathbf{A}=\mathbf{L L}^{T}=\left[\begin{array}{ccc}
2 & 0 & 0 \\
6 & 1 & 0 \\
-8 & 5 & 3
\end{array}\right]\left[\begin{array}{ccc}
2 & 6 & -8 \\
0 & 1 & 5 \\
0 & 0 & 3
\end{array}\right]
$$




\subsection{Combining states of information*}

Different experts or different datasets often provide different pieces of information that may be encoded by the probability densities $\rho_{1}(\mathbf{m})$ and $\rho_{2}(\mathbf{m})$, respectively. Alluding to the conjunction of events, defined in section 2.1, we can combine these states of information through the conjunction of probability densities

$$
\left(\rho_{1} \wedge \rho_{2}\right)(\mathbf{m})=k \frac{\rho_{1}(\mathbf{m}) \rho_{2}(\mathbf{m})}{\rho_{h}(\mathbf{m})}
$$

where $k$ is a normalisation constant. A schematic illustration of the conjunction is shown in figure 3.6. The homogeneous (uninformative) probability density in equation (3.47) ensures that the conjunction of information $\rho_{1}$ with the no-information $\rho_{h}$ does not yield any new information, that is

$$
\rho_{1} \wedge \rho_{h}=\rho_{1}
$$

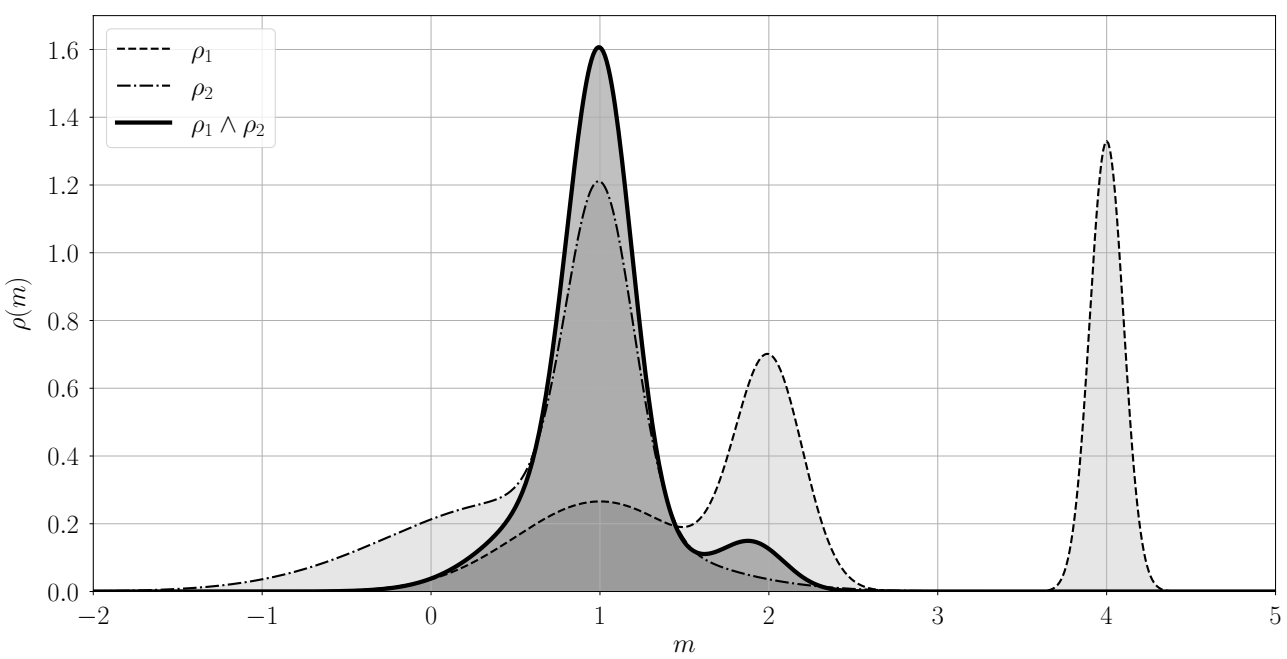

Fig. 3.6: Illustration of the conjunction of two probability densities, $\rho_{1}$ and $\rho_{2}$, shown with dashed and dashdotted curves, respectively. The conjunction, shown as solid curve, is the normalised product of the two. It represents the information that is compatible with both $\rho_{1}$ and $\rho_{2}$. Therefore, it does not feature the isolated spike in $\rho_{1}$ around $m=4$, where $\rho_{2}$ is zero.

\subsection{Marginal probability densities *}

Consider a two-dimensional model space with $\mathbf{m}=\left(m_{1}, m_{2}\right)$. While the probability density $\rho(\mathbf{m})=\rho\left(m_{1}, m_{2}\right)$ describes the complete joint information about $m_{1}$ and $m_{2}$, we are only interested in one of the parameters, say $m_{1}$. The probability that $m_{1}$ falls into the interval $\left[\tilde{m}_{1}, \tilde{m}_{1}+\Delta m_{1}\right]$, regardless of where $m_{2}$ is located, is given by the integral 


$$
P\left(m_{1} \in\left[\tilde{m}_{1}, \tilde{m}_{1}+\Delta m_{1}\right], m_{2} \text { anywhere }\right)=\int_{m_{1}=\tilde{m}_{1}}^{\tilde{m}_{1}+\Delta m_{1}} \int_{m_{2}} \rho\left(m_{1}, m_{2}\right) d m_{1} d m_{2}=\int_{m_{1}=\tilde{m}_{1}}^{\tilde{m}_{1}+\Delta m_{1}} \rho\left(m_{1}\right) d m_{1} .
$$

The marginal probability density $\rho\left(m_{1}\right)$, defined as

$$
\rho\left(m_{1}\right)=\int_{m_{2}} \rho\left(m_{1}, m_{2}\right) d m_{2}
$$

contains all information on $m_{1}$, independent of the information on $m_{2}$. Equation (3.50), that is the process of marginalisation, can be generalised to higher dimensions by simply integrating over all the model parameters that we are not interested in. An illustration for two dimensions is shown in figure 3.7.
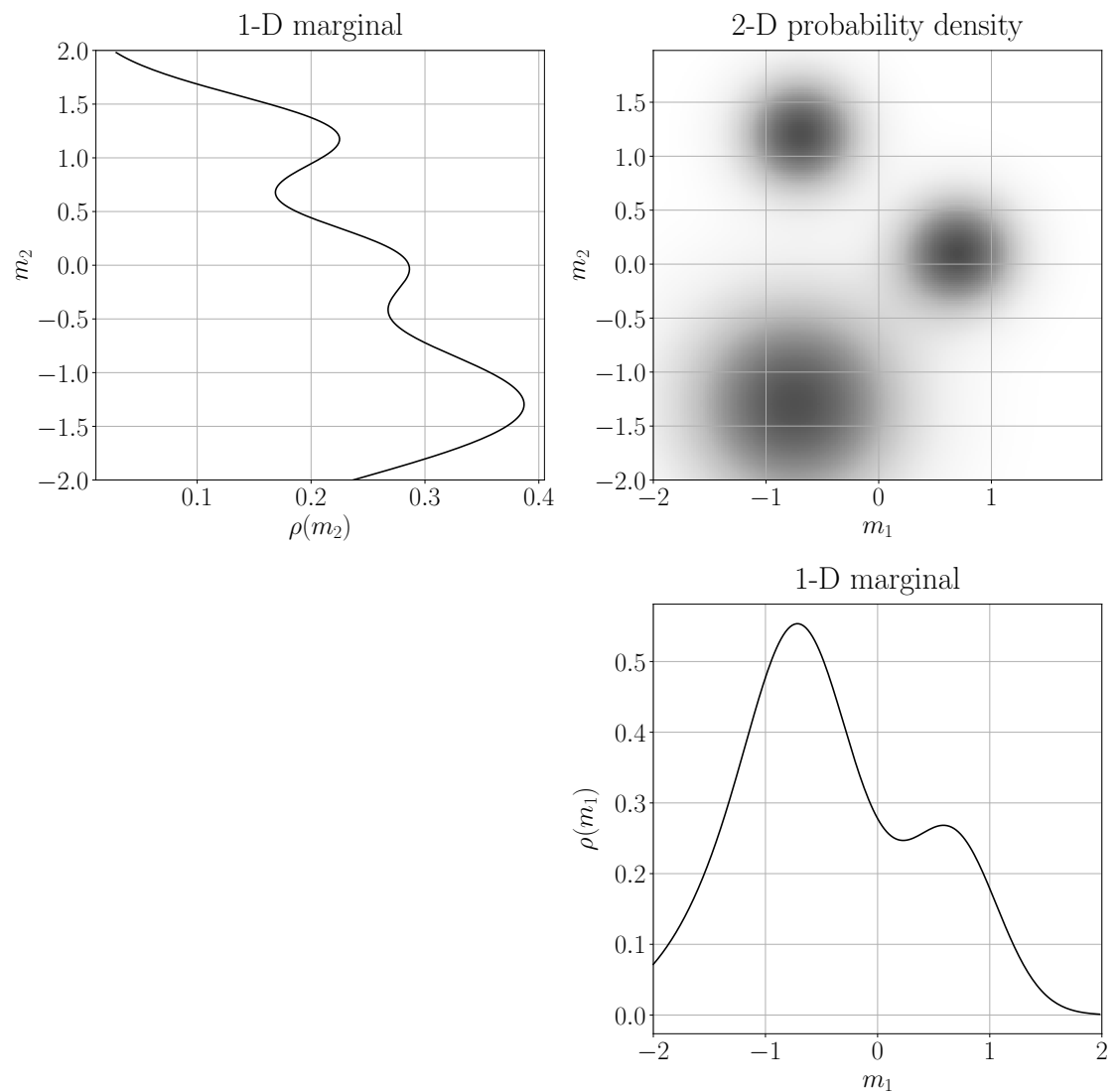

Fig. 3.7: Illustration of marginalising a two-dimensional probability density, $\rho\left(m_{1}, m_{2}\right)$, shown in the upperright panel. The $m_{1}$ and $m_{2}$ marginals are shown below and to the left, respectively. 


\subsection{Probability densities of sums, products and quotients}

In many cases, we are interested not only in the probability densities of model parameters themselves, but also in the probability densities of their sum, product or quotient. For example, the propagation speed of an acoustic wave in a gas or fluid is proportional to the ratio of bulk modulus and density. Under the assumption that two model parameters $m_{1}$ and $m_{2}$ are independent, with distributions $\rho_{1}\left(m_{1}\right)$ and $\rho_{2}\left(m_{2}\right)$, we can derive the distribution of their sum, product and quotient. The transformation rule introduced in section 3.4, combined with the concept of marginalisation from section 3.6, will be particularly useful for this.

We begin with the sum $m_{1}+m_{2}$. Since $m_{1}$ and $m_{2}$ are independent by assumption, equation (2.42) for the conjunction of independent events implies that their joint distribution $\rho\left(m_{1}, m_{2}\right)$ is given by

$$
\rho\left(m_{1}, m_{2}\right)=\rho_{1}\left(m_{1}\right) \rho_{2}\left(m_{2}\right) \text {. }
$$

To obtain an expression for the sum $m_{1}+m_{2}$, we introduce the coordinate transform

$$
m_{1}^{*}=m_{1}+m_{2}, \quad m_{2}^{*}=m_{2} .
$$

For the determinant of the Jacobian of this transformation we find

$$
\operatorname{det} \frac{d \mathbf{m}}{d \mathbf{m}^{*}}=\left|\begin{array}{ll}
\frac{d m_{1}}{d m_{1}^{*}} & \frac{d m_{1}}{d m_{2}^{*}} \\
\frac{d m_{2}}{d m_{1}^{*}} & \frac{d m_{2}}{d m_{2}^{*}}
\end{array}\right|=\left|\begin{array}{ll}
1 & 0 \\
0 & 1
\end{array}\right|=1
$$

Inserting (3.52) and (3.53) into the transformation rule (3.34), we obtain the probability density of $m_{1}^{*}$ and $m_{2}^{*}$,

$$
\rho^{*}\left(m_{1}^{*}, m_{2}^{*}\right)=\rho_{1}\left(m_{1}^{*}-m_{2}^{*}\right) \rho_{2}\left(m_{2}^{*}\right)
$$

Since our interest is only in the sum $m_{1}^{*}=m_{1}+m_{2}$ and not in $m_{2}^{*}=m_{2}$, we marginalise over $m_{2}^{*}$ and obtain

$$
\rho_{1+2}\left(m_{1}^{*}\right)=\int_{m_{2}^{*}} \rho_{1}\left(m_{1}^{*}-m_{2}^{*}\right) \rho_{2}\left(m_{2}^{*}\right) d m_{2}^{*} .
$$

Equation (3.55) reveals that the probability density of the sum of two independent variables is the convolution integral or their respective probability densities.

To fill equation (3.55) with life, we consider the simple case where $m_{1}$ and $m_{2}$ are both distributed according to a standard Gaussian,

$$
\rho_{1,2}\left(m_{1,2}\right)=\frac{1}{\sqrt{2 \pi}} e^{-\frac{1}{2} m_{1,2}^{2}} .
$$

Substituting (3.56) into (3.55), and realising that

$$
\left(m_{1}^{*}-m_{2}^{*}\right)^{2}+m_{2}^{* 2}=2\left(\frac{1}{2} m_{1}^{*}-m_{2}^{*}\right)^{2}+\frac{1}{2} m_{1}^{* 2},
$$

we obtain

$$
\rho_{1+2}\left(m_{1}^{*}\right)=\frac{1}{2 \sqrt{\pi}} e^{-\frac{1}{4} m_{1}^{* 2}} \frac{1}{\sqrt{\pi}} \int_{m_{2}^{*}} e^{-\left(m_{2}^{*}-m_{1}^{*} / 2\right)^{2}} d m_{2}^{*} .
$$

Comparing to (3.8), we note that the integrand with its pre-multiplier $1 / \sqrt{\pi}$ is a properly normalised Gaussian with standard deviation $1 / \sqrt{2}$ and mean $m_{1}^{*} / 2$. Hence, it integrates to 1 , and we are left with

$$
\rho_{1+2}\left(m_{1}^{*}\right)=\frac{1}{2 \sqrt{\pi}} e^{-\frac{1}{4} m_{1}^{* 2}} .
$$


The distribution of a sum of independent model parameters with standard Gaussian distribution is again a Gaussian. However, as illustrated in figure 3.8a, the standard deviation has increased from 1 to $\sqrt{2}$.

The recipe that led to the sum distribution (3.55) can equally be applied to obtain the distribution of the product $m_{1} m_{2}$. For this, we merely define a new transformation,

$$
m_{1}^{*}=m_{1} m_{2}, \quad m_{2}^{*}=m_{2},
$$

which has the Jacobian

$$
\operatorname{det} \frac{d \mathbf{m}}{d \mathbf{m}^{*}}=\left|\begin{array}{ll}
\frac{d m_{1}}{d m_{1}^{*}} & \frac{d m_{1}}{d m_{2}^{*}} \\
\frac{d m_{2}}{d m_{1}^{*}} & \frac{d m_{2}}{d m_{2}^{*}}
\end{array}\right|=\left|\begin{array}{cc}
\frac{1}{m_{2}^{*}} & -\frac{m_{1}^{*}}{m_{2}^{* 2}} \\
0 & 1
\end{array}\right|=\frac{1}{m_{2}^{*}} .
$$

It follows that the transformed version of the joint distribution $\rho\left(m_{1}, m_{2}\right)$ is

$$
\rho^{*}\left(m_{1}^{*}, m_{2}^{*}\right)=\rho_{1}\left(\frac{m_{1}^{*}}{m_{2}^{*}}\right) \rho_{2}\left(m_{2}^{*}\right)\left|\frac{1}{m_{2}^{*}}\right| .
$$

Again marginalising over $m_{2}^{*}$, we obtain the product distribution

$$
\rho_{1 \cdot 2}\left(m_{1}^{*}\right)=\int_{m_{2}^{*}} \rho_{1}\left(\frac{m_{1}^{*}}{m_{2}^{*}}\right) \rho_{2}\left(m_{2}^{*}\right)\left|\frac{1}{m_{2}^{*}}\right| d m_{2}^{*} .
$$

Making use of (3.63) to compute the product distribution of the two standard Gaussians (3.56), we find

$$
\rho_{1 \cdot 2}\left(m_{1}^{*}\right)=\frac{1}{\pi} \int_{m_{2}^{*}=0}^{\infty} e^{-\frac{1}{2}\left(\frac{m_{1}^{*}}{m_{2}^{*}}\right)^{2}} e^{-\frac{1}{2} m_{2}^{* 2}} \frac{1}{m_{2}^{*}} d m_{2}^{*} .
$$

To the best of our knowledge, the intregal in (3.64) has no closed-form solution. However, an approximation can be found numerically. It is shown in figure $3.8 \mathrm{~b}$.

It remains to consider the distribution of the quotient of two independent distributions. Following the previous procedure, we define a suitable coordinate transformation,

$$
m_{1}^{*}=\frac{m_{1}}{m_{2}}, \quad m_{2}^{*}=m_{2} .
$$

The determinant of the Jacobian of (3.65) is equal to $m_{2}^{*}$, and so the marginalisation over the transformed joint distribution yields the quotient distribution

$$
\rho_{1 / 2}\left(m_{1}^{*}\right)=\int_{m_{2}^{*}} \rho_{1}\left(m_{1}^{*} m_{2}^{*}\right) \rho_{2}\left(m_{2}^{*}\right)\left|m_{2}^{*}\right| d m_{2}^{*} .
$$

Again applied to the example standard Gaussian (3.56) we find their quotient distribution to be

$$
\rho_{1 / 2}\left(m_{1}^{*}\right)=\frac{1}{\pi\left(1+m_{1}^{* 2}\right)} .
$$

Equation (3.67) represents a standard Cauchy distribution, visualised in figure 3.8c.

Equations (3.55), (3.63) and (3.66) for the sum, product and quotient distributions are strictly valid only for independent model parameters, the joint distribution of which can be written as a product. As we know, for example from equation (2.41), the joint distribution of dependent model parameters is more complicated and involves conditional probabilities. Nevertheless, when the joint distribution $\rho\left(m_{1}, m_{2}\right)$ of $m_{1}$ and $m_{2}$ is known, 

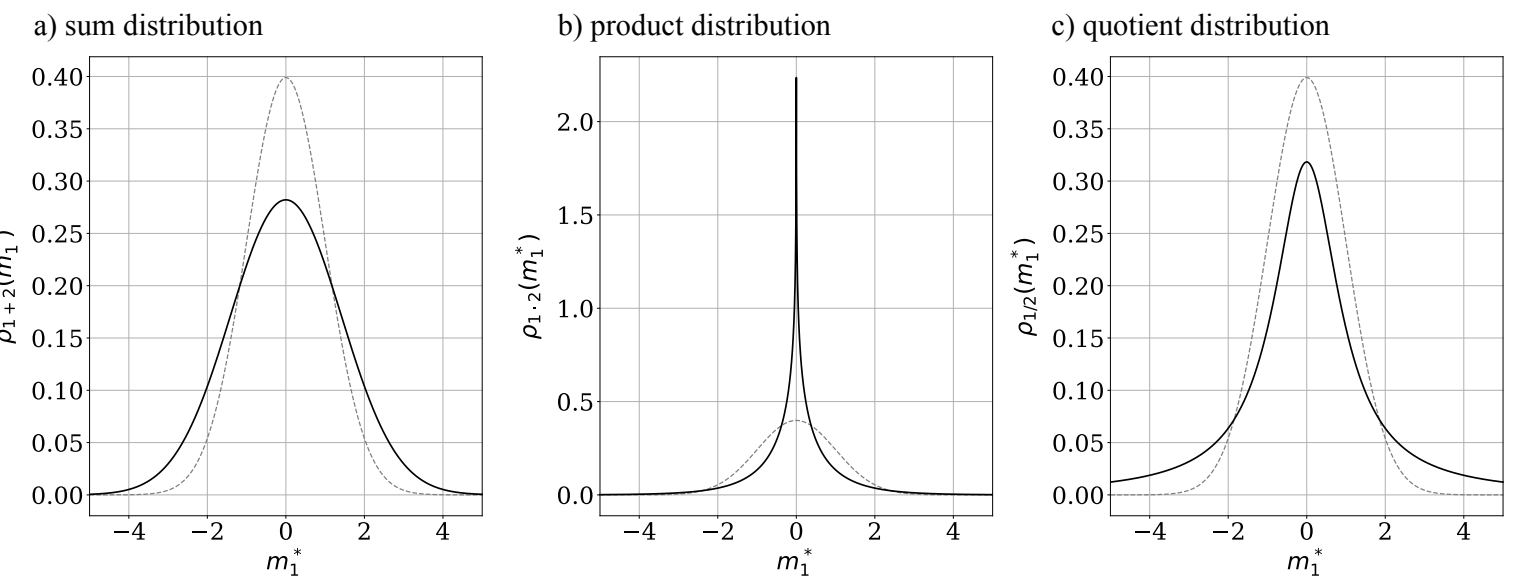

Fig. 3.8: Probability densities of the sum (a), the product (b) and the quotient (c) of independent model parameters $m_{1}$ and $m_{2}$ that are both distributed according to the standard Gaussian 3.56, shown as light grey dashed curve. The sum distribution in (a) is another Gaussian, and the quotient distribution in (c) is the standard Cauchy distribution. The product distribution in (b) can only be approximated numerically.

we can still borrow some of the above ideas to derive, for instance, the sum distribution. In fact, taking the transformation (3.52), we find the transformed joint distribution

$$
\rho^{*}\left(m_{1}^{*}, m_{2}^{*}\right)=\rho\left(m_{1}^{*}-m_{2}^{*}, m_{2}^{*}\right)
$$

Marginalising over $m_{2}^{*}$ yields a more general expression for the sum distribution,

$$
\rho_{1+2}\left(m_{1}^{*}\right)=\int_{m_{2}^{*}} \rho\left(m_{1}^{*}-m_{2}^{*}, m_{2}^{*}\right) d m_{2}^{*} .
$$

As a consequence of parameter dependence, the sum distribution does not take the form of a convolution integral as in (3.55). Similar generalisations of product and quotient distributions for dependent parameters can be derived based on the same recipes as above. Doing so will be left to the reader as an exercise. 


\section{Chapter 4 \\ Solving probabilistic inverse problems *}

In general we look for a new law by the following process. First we guess it. Then we compute the consequences of the guess to see what would be implied if this law that we guessed is right. Then we compare the result of the computation to nature, with experiment or experience, compare it directly with observation, to see if it works. If it disagrees with experiment it is wrong. In that simple statement is the key to science. It does not make any difference how beautiful your guess is. It does not make any difference how smart you are, who made the guess, or what his name is - if it disagrees with experiment it is wrong. That is all there is to it.

Richard Feynman, The character of physical law, 1964.

Solving a probabilistic inverse problem is equivalent to finding a posterior probability density in model space that describes all available information on the model parameters. This posterior information in model space is the result of combining prior information on model parameters, observed data, and the forward modelling theory. In the following sections we will introduce these different types of prior information and learn how to combine them.

\subsection{Prior information in data space *}

On 9 October 1971 a nuclear bomb detonated at Murzhik ${ }^{1}$ in today's north-eastern Kazakhstan. The underground explosion liberated energy equivalent to a magnitude 5.4 earthquake. Just like an earthquake, it excited waves that travelled through the interior of the Earth and could be recorded by seismometers worldwide. At a distance of nearly $4000 \mathrm{~km}$, near the Indian city of Gauribidanur, the nuclear test was recorded in the form of a slight ground displacement with a maximum amplitude of around $100 \mathrm{~nm}$, as shown in figure 4.1a.

More nuclear tests of similar size and within a few hundred metres distance took place throughout the 1970 s. The corresponding ground displacements at Gauribidanur, displayed for two other examples in figures 4.1a and $4.1 \mathrm{~b}$, are nearly identical. However, small differences can be observed. Prior to the actual wave from the nuclear tests, the recordings are dominated by ambient vibrations, or ambient seismic noise, shown in the zoom of figure 4.1c. Ambient seismic noise is primarily excited by human activity, ocean waves, and atmospheric turbulence; and it is superimposed on the actual nuclear test recording. Also following the largest-amplitude wave, the recordings differ slightly because the test sites were not exactly identical. In fact, the explosions

${ }^{1}$ Murzhik is part of the Semipalatinsk Test Site, where the former Soviet Union conducted 456 nuclear tests between 1949 and 1989. Operations at Murzhik stopped already in 1980 after 26 tests. 
a) Ground motion at Gauribidanur from Soviet nuclear explosions

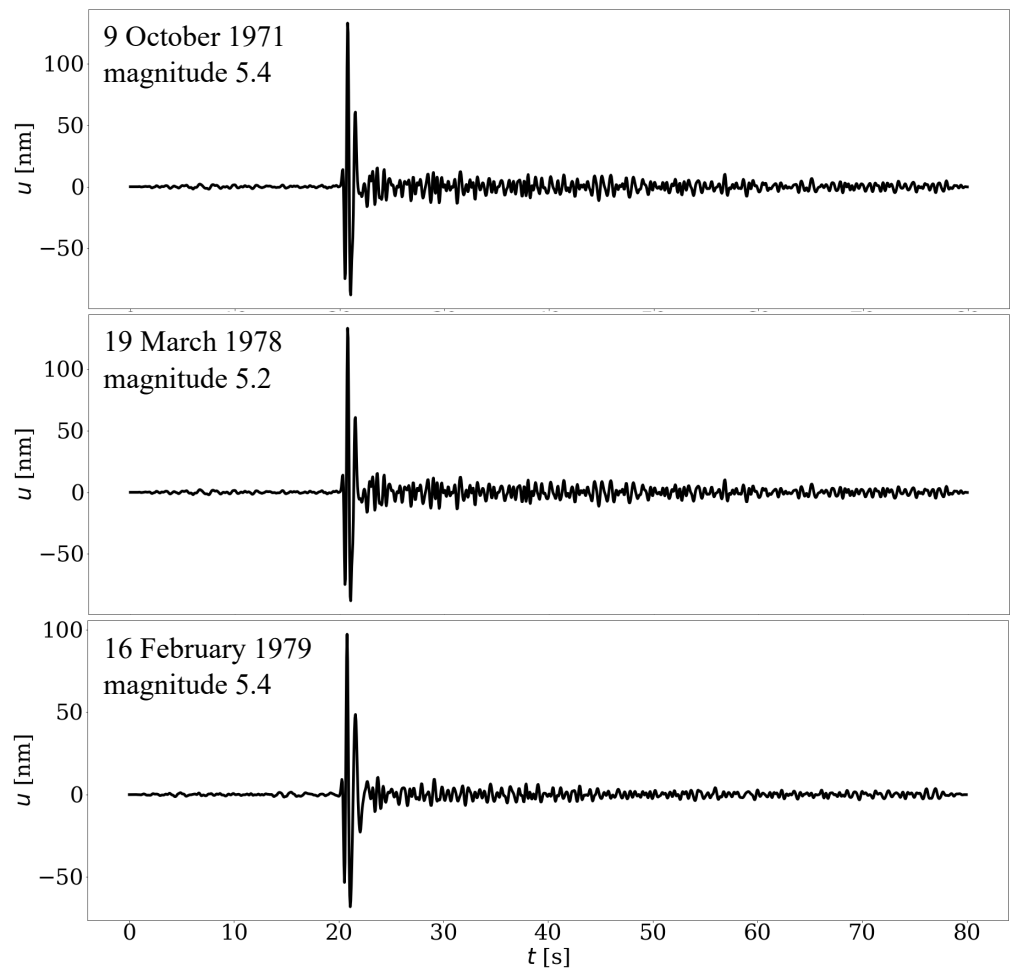

b) Overlay of normalised waveforms

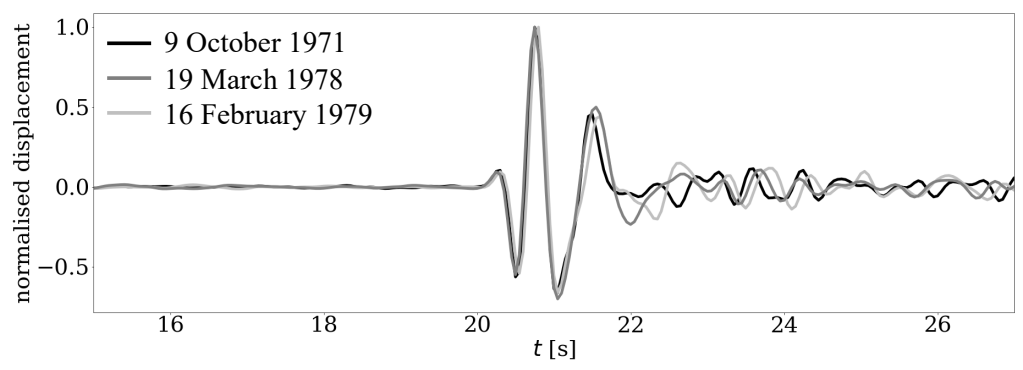

c) Ambient seismic noise prior to the first arrival

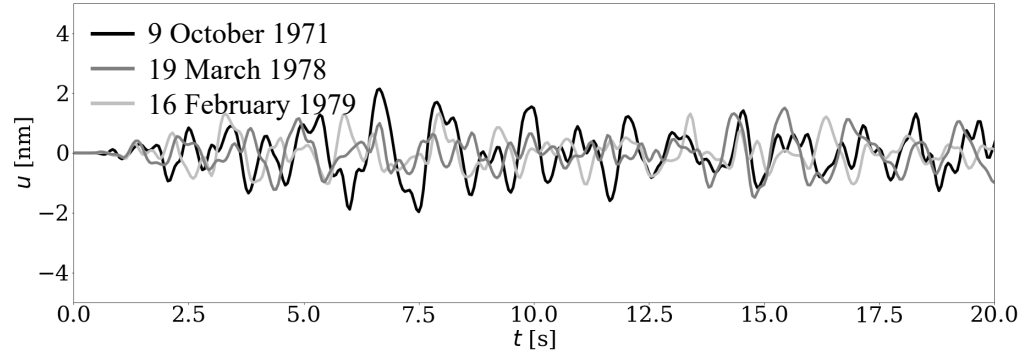

Fig. 4.1: Vertical-component ground motion recorded at Gauribidanur, India, originating from underground nuclear tests at Murzhik in today's Kazakhstan. (a) Vertical ground displacement in nm from three nuclear tests in the 1970s that were separated only by a few hundred metres. The energy liberated by the explosions corresponded to earthquakes of magnitude 5.2 - 5.4. (b) Overlay of the first-arriving compressional waves. (c) Overlay of the ambient seismic noise, recorded prior to the first arrival. Data kindly provided by AWE Blacknest with the help of Marta Pienkowska. 
themselves modify the structure of the underground, thereby causing waveforms to differ in their details.

Figure 4.1 illustrates that observed data, $\mathbf{d}^{\text {obs }}$, generated by repeated experiments of any kind are generally not identical. Differences can originate from the presence of noise and from our inability to exactly reproduce experimental conditions. Therefore, $\mathbf{d}^{\text {obs }}$ is effectively a realisation of a random variable, even though we may only work with one or few specific observations.

The character of $\mathbf{d}^{\text {obs }}$ as random variable has consequences for the comparison of observations with computed data $\mathbf{d}=\mathbf{G}(\mathbf{m})$. For a fixed model $\mathbf{m}$, the difference $\mathbf{d}^{\text {obs }}-\mathbf{G}(\mathbf{m})$ behaves like a random variable as well. The statistics of this random variable can be described by a conditional probability density, conventionally denoted by

$$
\rho\left(\mathbf{d}^{\text {obs }} \mid \mathbf{m}\right),
$$

and named the prior in data space. The probability density $\rho\left(\mathbf{d}^{\text {obs }} \mid \mathbf{m}\right)$ allows us to encode our prior knowledge about the observed data, as we will see in the following examples.

Perfect data and perfect theory: In the unrealistic, though instructive, case where the data have no errors (e.g., no noise and exact repeatability of experiments), the prior in data space takes the form of a $\delta$-function,

$$
\rho\left(\mathbf{d}^{\mathrm{obs}} \mid \mathbf{m}\right)=\delta\left[\mathbf{G}(\mathbf{m})-\mathbf{d}^{\mathrm{obs}}\right]=\delta\left(\mathbf{d}-\mathbf{d}^{\mathrm{obs}}\right) .
$$

Equation (4.2) can be interpreted as follows: Given a model $\mathbf{m}$, we compute synthetic data $\mathbf{d}=\mathbf{G}(\mathbf{m})$ using a forward modelling theory. Unless the synthetic data are exactly equal to the observed data $\mathbf{d}^{\text {obs }}$, the likelihood is zero, meaning that these cannot be the right synthetic data. While the data prior $\rho\left(\mathbf{d}^{\text {obs }} \mid \mathbf{m}\right)$ is a probability density in data space, describing the likelihood of the synthetic data, it clearly establishes a link to the model parameters $\mathbf{m}$ because a model $\mathbf{m}$ that does not reproduce the observations $\mathbf{d}^{\text {obs }}$ exactly, also seems unlikely. We will later formalise this relation.

Non-zero measurement errors: Vanishing measurement errors are certainly not very realistic, and in a realworld application we want to encode them in the data prior. Assuming, for simplicity, that the measurement errors are normally distributed, the data prior takes the form

$$
\rho\left(\mathbf{d}^{\mathrm{obs}} \mid \mathbf{m}\right)=\text { const. } e^{-\frac{1}{2}\left(\mathbf{d}-\mathbf{d}^{\mathrm{obs}}\right)^{T} \mathbf{C}_{D}^{-1}\left(\mathbf{d}-\mathbf{d}^{\mathrm{obs}}\right)},
$$

with a suitably chosen normalisation constant. The data covariance matrix $\mathbf{C}_{D}$ describes the observational uncertainties that may arise, for instance, from noise or imperfectly calibrated instruments. In contrast to the $\delta$-distribution in the previous example, the Gaussian does not require the forward model to perfectly reproduce the observed data in order to result in a non-zero likelihood. Even when a model $\mathbf{m}$ produces synthetic data d that are not exactly equal to $\mathbf{d}^{\text {obs }}$, the probability density $\rho\left(\mathbf{d}^{\mathrm{obs}} \mid \mathbf{m}\right)$ may not vanish because the presence of measurement errors does not preclude that the actual perfect data (that unfortunately we cannot observe in reality) are equal to $\mathbf{d}$.

While probabilistic inversion offers a natural framework for the description and incorporation of observational errors, it must be noted that these are often difficult to quantify.

\subsection{Prior information in model space *}

In the previous section we considered the likelihood of observed data $\mathbf{d}^{\text {obs }}$ that are related to a model $\mathbf{m}$ via a forward modelling theory and the data prior $\rho\left(\mathbf{d}^{\text {obs }} \mid \mathbf{m}\right)$. While a model may in principle be chosen arbitrarily to produce some synthetic data, we often have prior information that tells us if a certain model is actually meaningful. Such prior information in model space exists often independently of any observed data, and it may result from fundamental laws or constraints of physics. Examples are quantities such as velocities, temperatures, energies, etc., that cannot be negative. Similarly, the speed of light in some medium is bounded above by the 
speed of light in a vacuum. More complex prior information in model space could include correlations between different model parameters. We describe prior knowledge in model space with a probability density,

$$
\rho(\mathbf{m}),
$$

referred to as the prior in model space.

Positive velocities: The prior information that a velocity $m$ must be positive can be described with the model space prior

$$
\rho(m)= \begin{cases}0, & m<0, \\ 1, & m \geq 0 .\end{cases}
$$

Gaussian prior: Prior knowledge in model space is also frequently described with a Gaussian,

$$
\rho(\mathbf{m})=\text { const. } e^{-\frac{1}{2}\left(\mathbf{m}-\mathbf{m}^{\text {prior }}\right)^{T} \mathbf{C}_{M}^{-1}\left(\mathbf{m}-\mathbf{m}^{\text {prior }}\right)},
$$

with a mean model $\mathbf{m}^{\text {prior }}$ that is the a priori most likely model, and the model covariance $\mathbf{C}_{M}$ that captures a priori correlations between model parameters and our a priori certainty that $\mathbf{m}$ is actually close to $\mathbf{m}^{\text {prior }}$. While the Gaussian is convenient, it should be noted that it cannot be used, for instance, to describe prior information on model parameters that must be strictly positive because the likelihood is always non-zero on the negative axis.

The nature of prior knowledge in model space: While one may often think that one does not know anything about the model parameters $\mathbf{m}$, this is almost certainly never true. Most frequently, prior knowledge results from constraints of the forward modelling theory or from plain physical intuition. The latter is, of course, often subjective. However, subjectivity is unavoidable in any realistic inverse problem with insufficient and erroneous data, and it does not pose problems as long as the results are interpreted accordingly.

While a homogeneous distribution may be used to encode a state of relative ignorance on model parameters, it is again important to note that a truly uninformative probability density does not exist. Any probability density contains some sort of information, and information that appears weak in one coordinate system may appear stronger in another one, as we have seen in section 3.4. A selection of approaches to and arguments for the design of suitable priors will be presented in section 4.7 .

\subsection{Solving the inverse problem - Bayes' theorem *}

In sections 4.1 and 4.2 we introduced prior information in data and in model space. It remains to combine this information to obtain the posterior in model space. This combination can be achieved using Bayes' theorem that we will derive in the following paragraphs:

We consider two sets, $M$ and $D$. The probability of an event being in both $M$ and $D$ at the same time is given by the conjunction

$$
P(M \wedge D)=P(M \mid D) P(D),
$$

where $P(D)$ is the probability for the event being in $D$, and $P(M \mid D)$ is the probability for the event being in $M$ given that it is already in $D$. Equation (4.7) can be considered the definition of the conditional probability $P(M \mid D)$. Since $P(M \wedge D)=P(D \wedge M)$, we also have

$$
P(M \wedge D)=P(D \mid M) P(M) .
$$

Combining equations (4.7) and (4.8), we obtain

$$
P(M \mid D) P(D)=P(D \mid M) P(M) .
$$


Slightly rearranging equation (4.9), leads to Bayes' theorem for probabilities:

$$
P(M \mid D)=\frac{P(D \mid M) P(M)}{P(D)} .
$$

A simple visual illustration of this derivation is provided in figure 4.2.

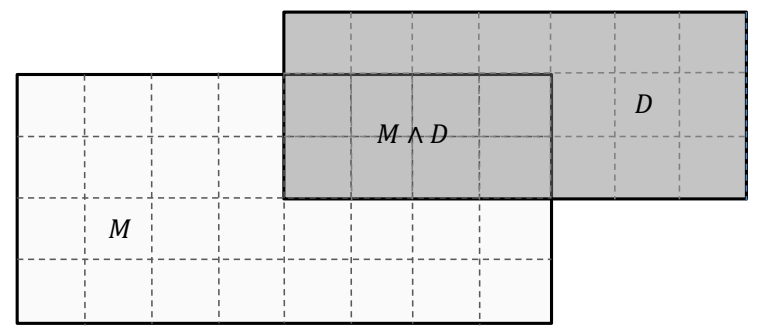

Fig. 4.2: Illustration of the derivation of Bayes' theorem using simple Venn diagrams. In total, there are 45 equally sized squares. Of those, 32 are in the model space domain $M, 21$ are in the data space domain $D$, and 8 are in both. The probability of being in $M$ is therefore $P(M)=32 / 45$, and the probability of being in $D$ is $P(D)=21 / 45$. Given that we are already in $M$, the probability of also being in $D$ is given by the conditional $P(D \mid M)=8 / 32$, that is, the number of those squares in $M$ that are also in $D$. Using Bayes' theorem, we infer that the probability of being in $M$ while already being in $D$ is $P(M \mid D)=(8 / 32)(32 / 45) /(21 / 45)=8 / 21$. The same result is obtained by directly counting the number of squares in $M$ that are already contained in $D$.

Since we are mostly concerned with probability densities, we derive a variant of Bayes' theorem that is more useful for our purposes. For this, we consider some arbitrary small subvolumes of model and data space, $d \mathbb{M}$ and $d \mathbb{D}$, with increments $d \mathbf{m}$ and $d \mathbf{d}^{\text {obs }}$, respectively. Repeating the derivation of equation (4.9) for $d \mathbb{M}$ and $d \mathbb{D}$ instead of $\mathbb{M}$ and $\mathbb{D}$, yields

$$
\rho\left(\mathbf{m} \mid \mathbf{d}^{\mathrm{obs}}\right) \rho\left(\mathbf{d}^{\mathrm{obs}}\right) d \mathbf{m} d \mathbf{d}^{\mathrm{obs}}=\rho\left(\mathbf{d}^{\mathrm{obs}} \mid \mathbf{m}\right) \rho(\mathbf{m}) d \mathbf{m} d \mathbf{d}^{\mathrm{obs}} .
$$

Since equation (4.11) must hold for any small $d \mathbb{M}$ and $d \mathbb{D}$, we generally have

$$
\rho\left(\mathbf{m} \mid \mathbf{d}^{\mathrm{obs}}\right)=\frac{\rho\left(\mathbf{d}^{\mathrm{obs}} \mid \mathbf{m}\right) \rho(\mathbf{m})}{\rho\left(\mathbf{d}^{\mathrm{obs}}\right)}
$$

which is Bayes' theorem for probability densities. It gives the posterior probability density of the model parameters $\mathbf{m}$ given some observed data $\mathbf{d}^{\text {obs }}$, in terms of

1) the prior in data space, $\rho\left(\mathbf{d}^{\mathrm{obs}} \mid \mathbf{m}\right)$, also named the likelihood function, and introduced in section 4.1,

2) the prior in model space, $\rho(\mathbf{m})$, introduced in section 4.2 ,

3) and a normalisation factor $\rho\left(\mathbf{d}^{\mathrm{obs}}\right)=\int_{\mathbb{M}} \rho\left(\mathbf{d}^{\mathrm{obs}} \mid \mathbf{m}\right) \rho(\mathbf{m}) d \mathbf{m}$, named the evidence. The evidence scales the posterior, and its numerical value does not affect relative likelihoods of different models $\mathbf{m}$.

The posterior in model space, $\rho\left(\mathbf{m} \mid \mathbf{d}^{\text {obs }}\right)$ is the solution of the probabilistic inverse problem. It contains all information that we can possibly obtain by combining our prior information on the data, the forward modelling theory, and the model parameters. Unlike in deterministic inversion, such as the line fitting problems in section 1.3 , the computation of $\rho\left(\mathbf{m} \mid \mathbf{d}^{\text {obs }}\right)$ does not require the inversion of any matrix. Thus, we do not need to worry 
if a certain matrix is actually invertible. Furthermore, there are no constraints on the nature of the forward problem. In particular, it is allowed to be highly non-linear, non-differentiable, etc.

It is important to recognise that the variables on the left- and the right-hand sides of Bayes's theorem (4.12) have different interpretations. On the right-hand side, the observations $\mathbf{d}^{\text {obs }}$ are considered a random variable, and the data-space prior $\rho\left(\mathbf{d}^{\mathrm{obs}} \mid \mathbf{m}\right)$ describes the likelihood of an observation under the assumption of a fixed a priori model $\mathbf{m}$. On the left-hand side, the posterior $\rho\left(\mathbf{m} \mid \mathbf{d}^{\text {obs }}\right)$ gives the likelihood of variable model realisations $\mathbf{m}$, given that a specific observation $\mathbf{d}^{\text {obs }}$ has actually been made.

The evidence: The evidence $\rho\left(\mathbf{d}^{\mathrm{obs}}\right)$ is often ignored in the solution of inverse problems because it 'only' scales the posterior, and because it is usually difficult to compute. (It requires the solution of an integral over the complete model space $\mathbb{M}$, which may have a high dimension.) However, the evidence is far from being a useless quantity.

To see the meaning of the evidence, we first note that any inverse, and in fact also forward, problem comes with a set of hypotheses $\mathscr{H}$. These hypotheses include, but are not limited to, the parameterisation of the model space, the number of model parameters, and the simplifications applied to the forward modelling physics. Reflecting the dependence on these inevitable choices, Bayes' theorem may be more appropriately written as

$$
\rho\left(\mathbf{m} \mid \mathbf{d}^{\text {obs }}, \mathscr{H}\right)=\frac{\rho\left(\mathbf{d}^{\text {obs }} \mid \mathbf{m}, \mathscr{H}\right) \rho(\mathbf{m} \mid \mathscr{H})}{\rho\left(\mathbf{d}^{\text {obs }} \mid \mathscr{H}\right)} .
$$

Now, we consider two different sets of hypotheses, $\mathscr{H}_{1}$ and $\mathscr{H}_{2}$, and we would like to know which one of these is more likely, i.e., more strongly supported by the data. To solve this problem, we first apply Bayes' theorem to compute the likelihood of $\mathscr{H}_{1}$ given some observed data $\mathbf{d}^{\text {obs }}$,

$$
\rho\left(\mathscr{H}_{1} \mid \mathbf{d}^{\mathrm{obs}}\right)=\frac{\rho\left(\mathbf{d}^{\mathrm{obs}} \mid \mathscr{H}_{1}\right) \rho\left(\mathscr{H}_{1}\right)}{\rho\left(\mathbf{d}^{\mathrm{obs}}\right)} .
$$

In equation (4.14), $\rho\left(\mathscr{H}_{1}\right)$ describes the extent of our prior preference for hypothesis $\mathscr{H}_{1}$. Similarly, the likelihood of the alternative hypothesis, $\mathscr{H}_{2}$, given $\mathbf{d}$, can be computed as

$$
\rho\left(\mathscr{H}_{2} \mid \mathbf{d}^{\mathrm{obs}}\right)=\frac{\rho\left(\mathbf{d}^{\mathrm{obs}} \mid \mathscr{H}_{2}\right) \rho\left(\mathscr{H}_{2}\right)}{\rho\left(\mathbf{d}^{\mathrm{obs}}\right)} .
$$

Dividing equation (4.14) by equation (4.15), we obtain

$$
\frac{\rho\left(\mathscr{H}_{1} \mid \mathbf{d}^{\mathrm{obs}}\right)}{\rho\left(\mathscr{H}_{2} \mid \mathbf{d}^{\mathrm{obs}}\right)}=\frac{\rho\left(\mathbf{d}^{\mathrm{obs}} \mid \mathscr{H}_{1}\right) \rho\left(\mathscr{H}_{1}\right)}{\rho\left(\mathbf{d}^{\mathrm{obs}} \mid \mathscr{H}_{2}\right) \rho\left(\mathscr{H}_{2}\right)} .
$$

Assuming that we have no more prior preference for $\mathscr{H}_{1}$ than for $\mathscr{H}_{2}$, we have $\rho\left(\mathscr{H}_{1}\right)=\rho\left(\mathscr{H}_{2}\right)$, and equation (4.16) simplifies to

$$
\frac{\rho\left(\mathscr{H}_{1} \mid \mathbf{d}^{\mathrm{obs}}\right)}{\rho\left(\mathscr{H}_{2} \mid \mathbf{d}^{\mathrm{obs}}\right)}=\frac{\rho\left(\mathbf{d}^{\mathrm{obs}} \mid \mathscr{H}_{1}\right)}{\rho\left(\mathbf{d}^{\mathrm{obs}} \mid \mathscr{H}_{2}\right)} .
$$

Equation (4.17) finally reveals the meaning of the evidence. It gives the posterior likelihood ratio of the competing hypotheses, $\rho\left(\mathscr{H}_{1} \mid \mathbf{d}^{\mathrm{obs}}\right) / \rho\left(\mathscr{H}_{2} \mid \mathbf{d}^{\text {obs }}\right)$, in terms of the evidence ratio, $\rho\left(\mathbf{d}^{\text {obs }} \mid \mathscr{H}_{1}\right) / \rho\left(\mathbf{d}^{\text {obs }} \mid \mathscr{H}_{2}\right)$. Hence, the evidence measures how well a hypothesis explains the data, taking all possible combinations of its parameters into account. The evidence ratio indicates which hypothesis is a posteriori more likely, that is, more probable after bringing actual data into the problem.

In recent years, the evidence has received more of the recognition that it deserves, especially in the context of transdimensional inverse problems where the dimension of the model space is among the 
unknowns (e.g. Sambridge et al., 2006). In the transdimensional case, different model space dimensions $N_{m}$ represent the different hypotheses $\mathscr{H}$. A detailed introduction to transdimensional inversion is given in section 5.8.

\subsection{Find the fisherman! - A first synthesis}

So far, our developments have been deliberately general and abstract. To make them more intuitively understandable, and to illustrate a Bayesian inference workflow, we now consider a more concrete example. It is a slightly more complicated version of the Lighthouse Problem studied by Gull (1988).

The scenery, depicted in figure 4.3a, is as follows: On a cold and foggy morning, the coastguard of a lovely fishing village receives an emergency call from one of the local fishermen. After his boat was hit by a gigantic wave, both the engine and the navigation system failed. All he is left with is an old compass and a flashlight that emits highly collimated light pulses. The coastguard instructs the fisherman to point his compass in the direction of the coast and to send as many flashes as he can. As he arrives on the shore, he can indeed see the light flashes through the fog, and he records them at positions $x_{i}^{\text {obs }}=d_{i}^{\text {obs }}$, for $i=1,2, \ldots, N$. Can the coastguard infer the position of the fisherman based on these observations?

a) experimental setup

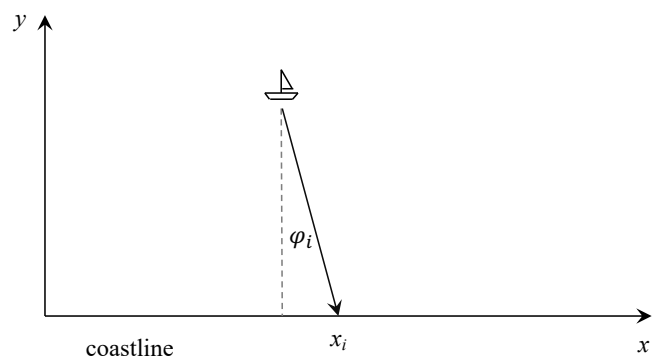

b) von Mises distributions

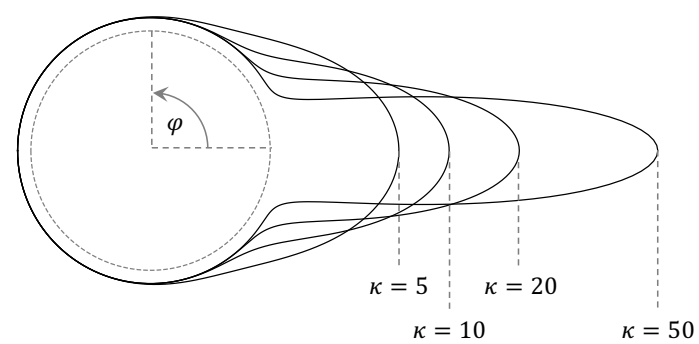

Fig. 4.3: Finding the fisherman. (a) Geometric setup of the experiment. (b) von Mises distributions (4.20) for different choices of the parameter $\kappa$.

Using the terminology introduced in the previous sections, the model parameters of interest are the $x$ - and $y$-coordinates of the boat, that is, $\mathbf{m}=(x, y)$. The forward problem relates $\mathbf{m}$ to the error-free synthetic observations,

$$
d_{i}=G(\mathbf{m})=x, \quad \text { for all } i=1, \ldots, N,
$$

meaning that each flash is recorded exactly at the $x$-position of the boat. However, the compass is not perfect. Hence, instead of pointing his flashlight precisely towards the coast, the light beam deviates from the normal to the coast by some angle $\varphi_{i}$. From figure 4.3 a we infer that $\varphi_{i}$ is related to the observations $d_{i}^{\text {obs }}=x_{i}^{\text {obs }}$ via

$$
d_{i}^{\text {obs }}=x+y \tan \varphi_{i}
$$


Having studied the statistics of compass misorientations in great detail, the coastguard knows that the random variations of $\varphi$ can be modelled by a von Mises distribution ${ }^{2}$, also known as the circular normal distribution,

$$
\rho_{\mathrm{VM}}(\varphi)=k e^{\kappa \cos \varphi},
$$

where $k$ is the normalisation constant, and the parameter $\kappa$ is a measure of concentration. A selection of von Mises distributions for different choices of $\kappa$ is shown in figure 4.3b. (The von Mises distribution will reappear in section 4.7.2, where we show that it is a maximum-entropy distribution, that is, a distribution with minimum information content.) The distribution of $\varphi$ relates to the distribution of observational errors. However, the coastguard observes realisations $d_{i}^{\text {obs }}$ of the random variable $d^{\text {obs }}$ and not of $\varphi$. To obtain the distribution of $d^{\text {obs }}$, we apply a coordinate transformation using (3.33) together with (4.19),

$$
\rho\left(d^{\mathrm{obs}} \mid \mathbf{m}\right)=\rho_{\mathrm{vM}}[\varphi(\mathbf{m})]\left|\frac{d \varphi}{d d^{\mathrm{obs}}}\right|=\rho_{\mathrm{vM}}\left[\arctan \left(\frac{d^{\mathrm{obs}}-x}{y}\right)\right] \frac{y}{y^{2}+\left(x-d^{\mathrm{obs}}\right)^{2}} .
$$

Equation (4.21) is the prior probability density in data space for a single observation. In the limiting case where $\kappa$ tends to 0 , the von Mises distribution becomes a constant, and (4.21) simplifies to a Cauchy distribution, that we found to be the quotient distribution of two Gaussians in section 3.7. Since the individual realisations of the observations $d_{i}^{\text {obs }}$ are independent and identically distributed ${ }^{3}$, the distribution of the observed data vector $\mathbf{d}^{\mathrm{obs}}=\left(d_{1}^{\mathrm{obs}}, \ldots, d_{N}^{\mathrm{obs}}\right)$ equals the product of the individual distributions, that is,

$$
\rho\left(\mathbf{d}^{\mathrm{obs}} \mid \mathbf{m}\right)=\prod_{i=1}^{N} \rho\left(d_{i}^{\mathrm{obs}} \mid \mathbf{m}\right) .
$$

Invoking Bayes' theorem (4.12), the posterior distribution is now given by

$$
\rho\left(\mathbf{m} \mid \mathbf{d}^{\mathrm{obs}}\right)=\rho\left(\mathbf{d}^{\mathrm{obs}} \mid \mathbf{m}\right) \rho(\mathbf{m})=\rho(\mathbf{m}) \prod_{i=1}^{N} \rho_{\mathrm{vM}}\left[\arctan \left(\frac{d_{i}^{\mathrm{obs}}-x}{y}\right)\right] \frac{y}{y^{2}+\left(x-d_{i}^{\mathrm{obs}}\right)^{2}} .
$$

To implement (4.23) in the absence of observations from an actual fisherman lost at sea, we compute artificial data by drawing random angles $\varphi_{i}$ from a von Mises distribution and inserting them in (4.19) to obtain $x_{i}^{\text {obs }}$. For this, we use a fixed value of $\kappa=10$ and a location $(x, y)=(5,2)$, which we would like to estimate. In the interest of simplicity, we choose a model space prior $\rho(\mathbf{m})$ that is constant within the intervals $x \in[3,7]$ and $y \in[0.5,6]$, and zero outside.

The resulting posterior probability densities for $N=5$ and $N=100$ observations are displayed in figure 4.4. In accord with our intuition, the posterior distribution based on five observations only is rather broad, and so the coastguard needs to search within a radius of several kilometres in order to find the fisherman. Increasing the number of observations to 100 , condenses the posterior into a much smaller region with a radius on the order of 100 metres. In both cases, the input location of the boat does not coincide with the maximum of the posterior, due to the randomness in the dataset. However, it generally falls into the region of relatively high probability density.

\subsection{Appraisal and condensation of the posterior *}

In the previous section, we defined the posterior $\rho\left(\mathbf{m} \mid \mathbf{d}^{\mathrm{obs}}\right)$ as the solution of the probabilistic inverse, or Bayesian inference, problem. In contrast to a deterministic inversion, such as the simplistic line fitting example

\footnotetext{
${ }^{2}$ Richard Edler von Mises (1883 - 1953) was an Austrian mathematician. After serving as a test pilot in World War I, he made important contributions to probability theory and mechanics. In addition to the von Mises distribution, also the von Mises yield criterion in plasticity theory is named after him.

${ }^{3}$ In the literature, the expression 'independent and identically distributed' is very often abbreviated as iid.
} 
a) posterior distributions, $\kappa=10, N=5$

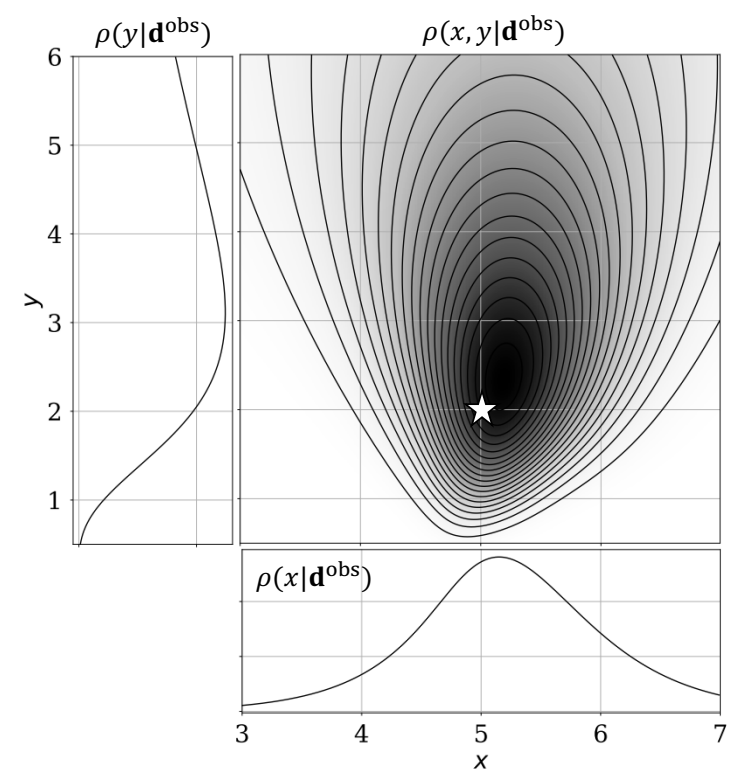

b) posterior distributions, $\kappa=10, N=100$

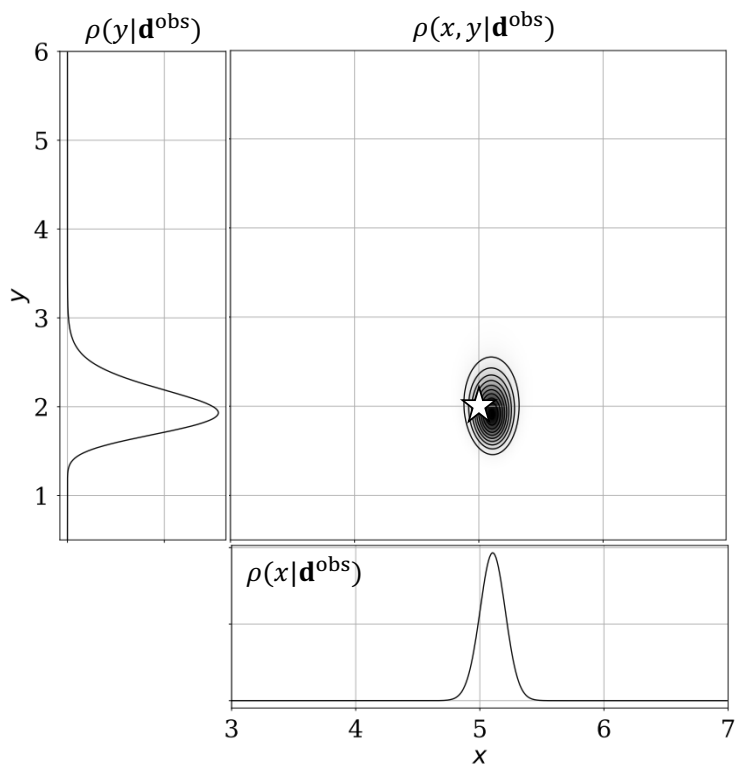

Fig. 4.4: Posterior probability densities $\rho\left(x, y \mid \mathbf{d}^{\text {obs }}\right)$ and their 1-D marginals $\rho\left(x \mid \mathbf{d}^{\text {obs }}\right)$ and $\rho\left(y \mid \mathbf{d}^{\text {obs }}\right)$ for $N=5$ observations (a) and $N=100$ observations (b). The location of the boat, used to compute artificial observations, is marked by the white star. [Find the fisherman]

in section 1.3, the solution is not given as a single model but in the form of a probability density that attaches a measure of plausibility, namely the likelihood $\rho\left(\mathbf{m} \mid \mathbf{d}^{\text {obs }}\right)$, to potentially infinitely many models. How do we exploit this wealth of information? How can we extract meaningful and digestible information from this highdimensional function?

Since the high-dimensional posterior may be difficult to interpret, it is common practice to condense $\rho\left(\mathbf{m} \mid \mathbf{d}^{\text {obs }}\right)$ into fewer numbers, including probabilities, means and covariances, the maximum-likelihood model, or lowerdimensional marginals that can be visualised. The concrete way of reducing a potentially complex and highdimensional posterior to more interpretable quantities, upon which important decisions may be based, will in the end depend on the requirements of a specific application. Hence, the examples provided below are merely a small collection of options.

\subsubsection{The movie strategy *}

Arguably the simplest way of appraising the posterior is to display large numbers of plausible models, that is, models for which the posterior attains values above a certain threshold. Since this may be done in the form of a movie, this is commonly known as the movie strategy. Our ability to visually discern features that many of the displayed models share, may indeed help us to quickly gain qualitative insight into the properties of the posterior.

Simple as it is, the movie strategy is obviously not very scalable. Depending on the dimension of the model space, our typical human attention span, or even our lives, may be a bit short compared to the sheer number of plausible models. Thus, more condensed, and also more quantitative, appraisal methods are certainly desirable. 


\subsubsection{Computing probabilities *}

The most straightforward use of $\rho\left(\mathbf{m} \mid \mathbf{d}^{\text {obs }}\right)$ is to compute actual probabilities for $\mathbf{m}$ falling into a specific subvolume $M$ of the model space $\mathbb{M}$ :

$$
P(M \in \mathbb{M})=\int_{M} \rho\left(\mathbf{m} \mid \mathbf{d}^{\mathrm{obs}}\right) d \mathbf{m} .
$$

The number $P(M \in \mathbb{M})$ is a measure of plausibility. While the absolute value of $P(M \in \mathbb{M})$ may be difficult to interpret, it can be compared to the plausibility of another subvolume $M^{\prime}$, that is $P\left(M^{\prime} \in \mathbb{M}\right)$. As noted already in section 3.1 , the probability of $\mathbf{m}$ being exactly equal to some $\mathbf{m}_{0}$ is zero because the subvolume $M=\left\{\mathbf{m} \in \mathbb{M} \mid \mathbf{m}=\mathbf{m}_{0}\right\}$ occupies zero volume. The only exception occurs in the pathological case where $\rho\left(\mathbf{m} \mid \mathbf{d}^{\text {obs }}\right)=\delta\left(\mathbf{m}-\mathbf{m}_{0}\right)$. This means, in other words, that the probability of a specific model - found for instance through deterministic inversion - is zero, unless the posterior happens to be a $\delta$-function. However, this will never happen in practice because there are always non-zero uncertainties introduced by measurement errors.

\subsubsection{Mean and covariance *}

Based on the posterior $\rho\left(\mathbf{m} \mid \mathbf{d}^{\text {obs }}\right)$, we can compute the mean model through an integral over the whole model space $\mathbb{M}$ :

$$
\tilde{\mathbf{m}}=\int_{\mathbb{M}} \mathbf{m} \rho\left(\mathbf{m} \mid \mathbf{d}^{\mathrm{obs}}\right) d \mathbf{m} .
$$

Loosely interpreting the integral in (4.25) as a sum, the mean can be considered as an average over all models $\mathbf{m}$, weighted by their posterior likelihood $\rho\left(\mathbf{m} \mid \mathbf{d}^{\text {obs }}\right)$. As a measure of uncertainty or spread of the posterior distribution, we may compute the average of the squared distance of models from the mean, that is, the posterior covariance

$$
\tilde{\mathbf{C}}_{M}=\int_{\mathbb{M}}(\mathbf{m}-\tilde{\mathbf{m}})(\mathbf{m}-\tilde{\mathbf{m}})^{T} \rho\left(\mathbf{m} \mid \mathbf{d}^{\mathrm{obs}}\right) d \mathbf{m}
$$

In the special case where the posterior $\rho\left(\mathbf{m} \mid \mathbf{d}^{\text {obs }}\right)$ is an $n$-dimensional Gaussian

$$
\rho(\mathbf{m} \mid \mathbf{d})=\frac{1}{(2 \pi)^{\frac{n}{2}} \operatorname{det} \mathbf{C}_{M}} e^{-\frac{1}{2}\left(\mathbf{m}-\mathbf{m}_{0}\right)^{T} \mathbf{C}_{M}^{-1}\left(\mathbf{m}-\mathbf{m}_{0}\right)},
$$

the mean and the posterior covariance can be computed analytically:

$$
\tilde{\mathbf{m}}=\mathbf{m}_{0}, \quad \tilde{\mathbf{C}}_{M}=\mathbf{C}_{M}
$$

While the mean and posterior covariance are convenient quantities with intuitive interpretations, they may not always be meaningful. For instance, the mean of a probability density that is bimodal may actually have a very low likelihood. Also, the covariance of a probability density that is not at least approximately Gaussian, may be hard to interpret if not meaningless.

The mean and covariance are also frequently referred to as the first and second moments of the distribution $\rho\left(\mathbf{m} \mid \mathbf{d}^{\text {obs }}\right)$. Using index notation, moments of arbitrary order $k$ can be defined as

$$
\tilde{M}_{i_{1}, \ldots, i_{k}}=\int_{\mathbb{M}}\left(m_{i_{1}}-\tilde{m}_{i_{1}}\right) \cdot \ldots \cdot\left(m_{i_{k}}-\tilde{m}_{i_{k}}\right) \rho\left(\mathbf{m} \mid \mathbf{d}^{\mathrm{obs}}\right) d \mathbf{m}
$$


where the indices $i_{k}$ take values between 1 and $n$. The third moment, called the skewness is a measure of lopsidedness of a distribution. It is zero for distributions that are symmetric with respect to their mean, positive when tilted to the right, and negative when tilted to the left. The fourth moment, referred to as kurtosis, is a generally non-negative quantity that measures the heaviness of the tail of a distribution. Even higher moments may in principle be computed, however, their interpretation becomes increasingly difficult, and so they are hardly used in practice.

\subsubsection{Maximum-likelihood model *}

To find the posterior maximum-likelihood model, also known as the maximum a posteriori (MAP) model, we consider some small volume element of fixed size, $d V(\mathbf{m})$. Remembering that the volume element is proportional to the homogeneous probability density, we may write $d V(\mathbf{m})$ as

$$
d V(\mathbf{m})=\text { constant } \rho_{h}(\mathbf{m}) d m_{1} \ldots d m_{n} .
$$

It follows that the probability of $\mathbf{m}$ falling into that volume element is

$$
P(\mathbf{m} \in d V)=\rho\left(\mathbf{m} \mid \mathbf{d}^{\mathrm{obs}}\right) d m_{1} \ldots d m_{n} \propto \frac{\rho\left(\mathbf{m} \mid \mathbf{d}^{\mathrm{obs}}\right)}{\rho_{h}(\mathbf{m})} d V(\mathbf{m}) .
$$

Since we fixed the size of $d V(\mathbf{m})$ regardless of its position, we infer from (4.31) that the posterior maximumlikelihood model must be given by

$$
\hat{\mathbf{m}}=\arg \max \frac{\rho\left(\mathbf{m} \mid \mathbf{d}^{\mathrm{obs}}\right)}{\rho_{h}(\mathbf{m})} .
$$

Sometimes one finds the maximum-likelihood model somewhat incorrectly defined as the model that maximises the posterior $\rho\left(\mathbf{m} \mid \mathbf{d}^{\text {obs }}\right)$. However, this would imply that the maximum-likelihood model may change under a change of coordinates.

In the case of a Gaussian, the mean and the maximum-likelihood models are identical. For other distributions, however, this need not be the case. Finding the maximum-likelihood model is an optimisation problem that may be solved, for instance, with the help of Simulated Annealing, introduced later in chapter 5.6.

Frequently, the posterior maximum-likelihood model $\hat{\mathbf{m}}$ is subjectively declared to be the estimated model $\mathbf{m}^{\text {est }}$. In fact, we somewhat naïvely did so in section 1.3. While being intuitive, this common practice ignores that the model does not exists as a consequence of non-uniqueness, and that other models, for instance, the mean, may be considered as well.

\subsection{The relation between probabilistic and deterministic inversion *}

While the use of Gaussians in some of our previous examples may appear somewhat artificial, it allows us to make a simple connection between probabilistic inversion and the deterministic solution of least-squares problems. Assuming that both the data and the model priors are Gaussian, and that the forward problem is linear with $\mathbf{G}(\mathbf{m})=\mathbf{G m}$, we have

$$
\rho(\mathbf{m})=\text { const. } e^{-\frac{1}{2}\left(\mathbf{m}-\mathbf{m}^{\text {prior }}\right)^{T} \mathbf{C}_{M}^{-1}\left(\mathbf{m}-\mathbf{m}^{\text {prior }}\right)},
$$

and

$$
\rho\left(\mathbf{d}^{\mathrm{obs}} \mid \mathbf{m}\right)=\text { const. } e^{-\frac{1}{2}\left(\mathbf{G m}-\mathbf{d}^{\mathrm{obs}}\right)^{T} \mathbf{C}_{D}^{-1}\left(\mathbf{G m}-\mathbf{d}^{\mathrm{obs}}\right)} .
$$


It follows that the posterior is given by

$$
\rho\left(\mathbf{m} \mid \mathbf{d}^{\text {obs }}\right)=\text { const. } e^{-\frac{1}{2}\left(\mathbf{m}-\mathbf{m}^{\text {prior }}\right)^{T} \mathbf{C}_{M}^{-1}\left(\mathbf{m}-\mathbf{m}^{\text {prior }}\right)-\frac{1}{2}\left(\mathbf{G m}-\mathbf{d}^{\text {obs }}\right)^{T} \mathbf{C}_{D}^{-1}\left(\mathbf{G m}-\mathbf{d}^{\mathrm{obs}}\right)} .
$$

Finding the maximum-likelihood model $\tilde{\mathbf{m}}=\hat{\mathbf{m}}$ of the posterior corresponds to the solution of a least-squares problem where the misfit function $\chi$ is the negative exponent in equation (4.35), that is

$$
\tilde{\mathbf{m}}=\arg \min \chi(\mathbf{m}),
$$

with

$$
\chi(\mathbf{m})=\frac{1}{2}\left(\mathbf{m}-\mathbf{m}^{\text {prior }}\right)^{T} \mathbf{C}_{M}^{-1}\left(\mathbf{m}-\mathbf{m}^{\text {prior }}\right)+\frac{1}{2}\left(\mathbf{G m}-\mathbf{d}^{\text {obs }}\right)^{T} \mathbf{C}_{D}^{-1}\left(\mathbf{G m}-\mathbf{d}^{\mathrm{obs}}\right) .
$$

In summary, finding the maximum-likelihood $\hat{\mathbf{m}}$ model of a purely Gaussian distribution with linear forward problem corresponds to the solution of a deterministic least-squares problem. When the priors are not Gaussian or the forward problem is not linear, the least-squares solution may have limited meaning. In section 6, we will analyse the least-squares problem in more detail, giving explicit expressions for the posterior mean and the posterior covariance.

\subsection{Designing priors}

Bayes' theorem explicitly contains prior information in data and model space, thereby naturally raising the question where these may come from. Under favourable conditions, the data prior may be derived from careful statistical analyses that allow us to cast the random occurrence of observational errors in the form of the probability density $\rho\left(\mathbf{d}^{\text {obs }} \mid \mathbf{m}\right)$. The model space prior $\rho(\mathbf{m})$ may be constrained by laws of physics, or it may be the result of previous experiments. In this sense, the current prior may actually be the previous posterior.

Often, however, information is not sufficiently abundant to define prior probability densities easily. For example, the amount of data may be too small for a robust analysis of their errors, or there may simply not be previous experiments that produces a posterior probability density. In such cases, we require additional arguments and tools for the design of useful priors. Some, though by far not all, of these will be presented in the following paragraphs.

\subsubsection{Noise and the normal distribution}

The central, normal or Gaussian distribution,

$$
\rho(d)=\frac{1}{\sqrt{2 \pi} \sigma} e^{-\frac{d^{2}}{2 \sigma^{2}}},
$$

is arguably the most widely used and most frequently contested probability density. The young Carl Friedrich Gauss ${ }^{4}$ discovered the distribution named after him in the years between 1801 and 1809 (or possibly even earlier) during his studies of celestial mechanics, largely motivated by the discovery of the dwarf planet Ceres. His research culminated in his monumental work Theoria motus corporum coelestium in sectionibus conicis solem ambientum (Theory of motion of the celestial bodies moving in conic sections around the Sun) (Gauss, 1809). In this context, he considered some error-contaminated observations $d_{1}, \ldots, d_{N}$, of which he wanted to know the

${ }^{4}$ Carl Friedrich Gauss (1777 - 1866) was a German universal genius who made profound contributions to mathematics already in his teenager years. The perfectionist Gauss used to publish his results only when they were complete beyond criticism, which often was years after an actual discovery. In addition to mathematics, he was active in numerous branches of science, including astronomy, geodesy, and magnetism. 
maximum-likelihood datum $\hat{d}$. Gauss demonstrated that $\hat{d}$ equals the arithmetic mean of the observations if and only if they are distributed according to (4.38).

We already encountered the normal distribution several times in earlier sections as a limiting case of other distributions. For instance, in section 2.1, we found that the binomial distribution approaches a Gaussian as the number of observations increases. The von Mises distribution, considered in section 4.4, is also closely approximated by a Gaussian when the concentration parameter $\kappa$ is large. Numerous other examples of this gravitating phenomenon exist (Jaynes, 2003), suggesting that there is more to it.

Throughout the centuries, the normal distribution has been derived multiple times, based on remarkably diverse arguments. In the context of inverse problems using data of natural, and therefore generally noise-contaminated data, the Landon derivation is of particular interest (Jaynes, 2003). To illustrate the idea, we briefly return to figure 4.1c, showing the recording of seismic ambient noise, recorded prior to three nuclear explosions in the 1970s. This kind of noise is being excited continuously on literally every little patch of the ocean floor and the Earth's surface by tiny pressure variations, which in turn may be due to ocean waves, atmospheric turbulence, or anthropogenic activity (Nakata et al., 2019).

Computing a histogram from noise amplitudes of 400 time samples prior to the explosion on 9 October 1971, reveals an approximately normal distribution, as shown in figure 4.5. Repeating the computation for noise recorded prior to the events on 19 March 1978 and 16 February 1979, yields similar results, that is, normal distributions with zero mean but slightly different variance.

The similarity of the histograms in figure 4.5 is remarkable in that the sources of noise producing them, acted years apart and were certainly very different. This suggests that the addition or the removal of noise sources leaves the distribution invariant, up to a change in variance.
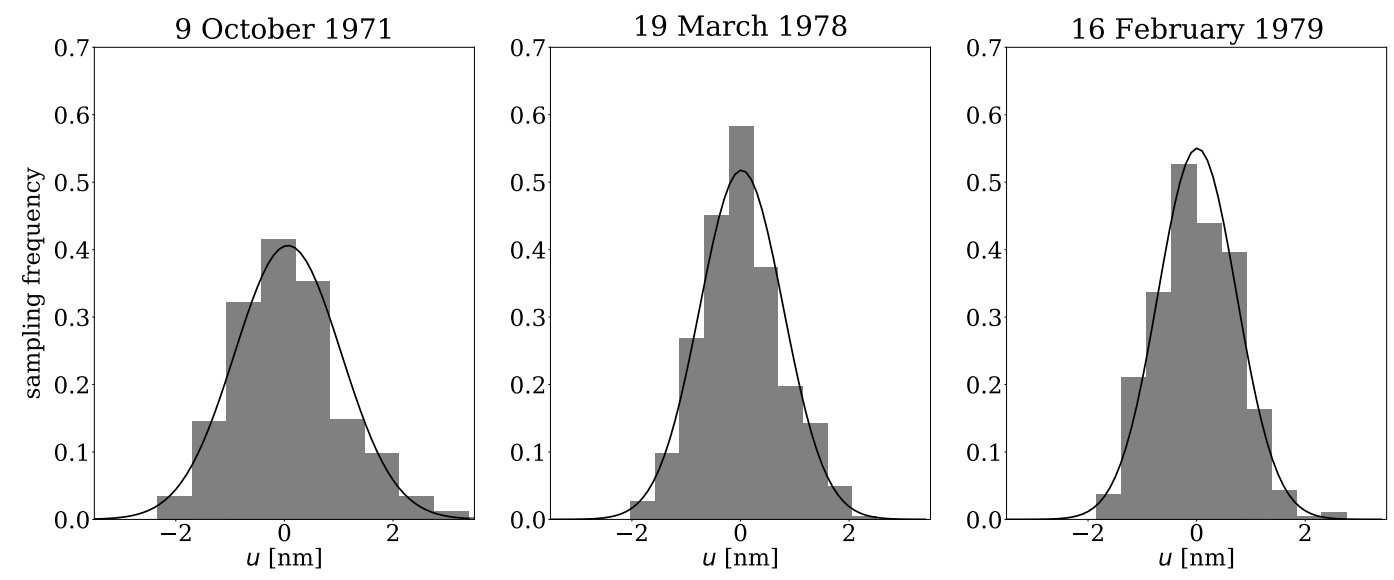

Fig. 4.5: Histograms and best-fitting Gaussians (in a least-squares sense) of the seismic ambient noise $u(t)$ from figure 4.1, recorded prior to three nuclear explosions in the 1970s. The histograms are based on 400 time samples of noise amplitude.

To understand our observation quantitatively, we let $\rho\left(d \mid \sigma_{d}^{2}\right)$ be the distribution of our data, where we made the dependence of $\rho$ on the variance $\sigma_{d}^{2}$ explicit. At this point, we do not further specify the functional form of $\rho$, meaning that it could be any distribution for which a variance $\sigma^{2}$ can be computed. In our concrete case, the data $d$ are given by the vertical displacement $u(t)$ of the Earth's surface at some time $t$.

The addition of a small noise source will lead to a slight modification of the data from $d$ to $d^{*}=d+\varepsilon$, where the random perturbations $\varepsilon$ follow an equally unspecified distribution $\rho^{\prime}(\varepsilon)$. Assuming that $\rho^{\prime}$ is independent from the original distribution $\rho$, the probability density of the sum $d^{*}=d+\varepsilon$ is given by the convolution 


$$
\rho^{*}\left(d^{*}\right)=\int \rho\left(d^{*}-\varepsilon \mid \sigma_{d}^{2}\right) \rho^{\prime}(\varepsilon) d \varepsilon
$$

as we have seen in section 3.7. Expanding $\rho\left(d^{*}-\varepsilon \mid \sigma_{d}^{2}\right)$ in a Taylor series with respect to $\varepsilon$, and dropping terms above second order, we obtain

$$
\rho^{*}\left(d^{*}\right)=\rho\left(d^{*} \mid \sigma_{d}^{2}\right)-\tilde{\varepsilon} \frac{d}{d d} \rho\left(d^{*} \mid \sigma_{d}^{2}\right)+\frac{1}{2} \sigma_{\varepsilon}^{2} \frac{d^{2}}{d d^{2}} \rho\left(d^{*} \mid \sigma_{d}^{2}\right),
$$

with the mean $\tilde{\varepsilon}=\int \varepsilon \rho^{\prime}(\varepsilon) d \varepsilon$ and the variance $\sigma_{\varepsilon}^{2}=\int \varepsilon^{2} \rho^{\prime}(\varepsilon) d \varepsilon$. In the interest of simplicity, we assume that the perturbations $\varepsilon$ are positive or negative with equal probability, so that the mean vanishes, that is, $\tilde{\varepsilon}=0$. Based on our observation, we furthermore request that the sum distribution $\rho^{*}$ has the same functional form as the original distribution $\rho$, but with variance increased from $\sigma_{d}^{2}$ to $\sigma_{d}^{2}+\sigma_{\varepsilon}^{2}$,

$$
\rho^{*}\left(d^{*}\right)=\rho\left(d^{*} \mid \sigma_{d}^{2}+\sigma_{\varepsilon}^{2}\right)
$$

Expanding (4.41) to first order in $\sigma_{\varepsilon}^{2}$ and combining the result with (4.42), yields the diffusion equation

$$
\frac{d \rho}{d \sigma^{2}}=\frac{1}{2} \frac{d^{2} \rho}{d d^{2}}
$$

Invoking the initial condition $\rho\left(d \mid \sigma_{d}^{2}=0\right)$, the solution of (4.42) is just the normal distribution (4.38).

Our derivation shows that the normal distribution has a remarkable stability property. Once some collection of noise sources has produced a normal distribution, the addition of any other noise sources will not change this. The resulting distribution remains Gaussian for all times, regardless of the distribution $\rho_{\varepsilon}$ of an individual noise source. Conversely, this implies that no individual noise source can be distinguished from the distribution of the noise source ensemble.

Not strictly mathematically but in spirit, the above line of arguments corresponds to the Central Limit Theorem, which establishes that the sum of an increasing number of independent random variables with finite means and variances tends towards a Gaussian, even when the individual variables follow some other distributions.

The practical relevance of the Landon derivation becomes obvious through the fact that the electrical engineer Vernon D. Landon was not interested in seismic noise but in the electrical noise of communication channels. In fact, the derivation is free of any assumptions on a particular application, and so it is useful in a wide variety of scenarios where observational errors are due to noise with a large number of diverse sources. Ideally, of course, we would like to have detailed statistics of observational errors, cast into the form of a probability density. More often than not, however, such detailed statistics are unavailable or difficult to obtain. In such cases, the Landon argument can help to define a reasonable data space prior $\rho\left(\mathbf{d}^{\text {obs }} \mid \mathbf{m}\right)$.

\subsubsection{The maximum-entropy principle}

The maximum-entropy principle can be used to design a prior probability density in model space, $\rho(\mathbf{m})$, given that only the a priori expected mean of the model parameters, $\tilde{\mathbf{m}}$ is actually known before the experiment. The principle states that the prior $\rho(\mathbf{m})$ should be such that its information content relative to a homogeneous probability density $\rho_{h}(\mathbf{m})$,

$$
I(\rho)=\int_{\mathbb{M}} \rho(\mathbf{m}) \log _{b} \frac{\rho(\mathbf{m})}{\rho_{h}(\mathbf{m})} d \mathbf{m},
$$

is as small as possible. (See equation 3.27 for the definition of absolute information.) Minimising the information content corresponds to maximising entropy, that is, the negative information. Finding $\rho(\mathbf{m})$ constitutes a constrained optimisation problem. On one hand, we need to minimise (4.43). On the other hand we need to 
satisfy the constraints

$$
\tilde{\mathbf{m}}=\int_{\mathbb{M}} \mathbf{m} \rho(\mathbf{m}) d \mathbf{m}
$$

and

$$
1=\int_{\mathbb{M}} \rho(\mathbf{m}) d \mathbf{m} .
$$

This can be solved conveniently using the method of Lagrange multipliers (see appendix 15.1), where the Lagrange function

$$
L(\rho)=\int_{\mathbb{M}} \rho(\mathbf{m}) \log _{b} \frac{\rho(\mathbf{m})}{\rho_{h}(\mathbf{m})} d \mathbf{m}+\mathbf{l}_{1}^{T}\left[\tilde{\mathbf{m}}-\int_{\mathbb{M}} \mathbf{m} \rho(\mathbf{m}) d \mathbf{m}\right]+l_{2}\left[1-\int_{\mathbb{M}} \rho(\mathbf{m}) d \mathbf{m}\right],
$$

is required to vanish for infinitesimally small variations $\delta \rho$ of $\rho$. The vector $\mathbf{l}_{1}$ and the scalar $l_{2}$ are the Lagrange multipliers. Indeed, we see that forcing the derivative of (4.46) with respect to $\mathbf{l}_{1}$ and $l_{2}$ to zero, automatically implies the constraints (4.44) and (4.45). Explicitly computing the variation of the Lagrange function with respect to $\rho$, that is,

$$
\delta L(\rho)=L(\rho+\delta \rho)-L(\rho)=0,
$$

we find

$$
\delta L(\rho)=\int_{\mathbb{M}}\left[\frac{1}{\ln b}+\log _{b} \frac{\rho(\mathbf{m})}{\rho_{h}(\mathbf{m})}-\mathbf{l}_{1}^{T} \mathbf{m}-l_{2}\right] \delta \rho(\mathbf{m}) d \mathbf{m},
$$

where we used the first-order approximation

$$
\log _{b} \frac{\rho(\mathbf{m})+\delta \rho(\mathbf{m})}{\rho_{h}(\mathbf{m})} \approx \log _{b} \frac{\rho(\mathbf{m})}{\rho_{h}(\mathbf{m})}+\frac{1}{\ln b} \frac{\delta \rho(\mathbf{m})}{\rho(\mathbf{m})} .
$$

Since $\delta L$ in equation (4.47) must vanish for arbitrary variations $\delta \rho$, we force the integrand in (4.48) to zero, which yields, after renaming constants,

$$
\rho(\mathbf{m})=c_{1} \rho_{h}(\mathbf{m}) b^{\mathbf{c}_{2}^{T} \mathbf{m}} .
$$

The constants $c_{1}$ and $\mathbf{c}_{2}$ in (4.50) can be computed from the constraints (4.44) and (4.45). Thus, the maximumentropy principle states that the most uninformative prior is equal to the homogeneous distribution $\rho_{h}$, multiplied by an exponential, $b^{\mathbf{c}_{2}^{T} \mathbf{m}}$; assuming that only the prior mean $\tilde{\mathbf{m}}$ is known.

The maximum-entropy principle makes intuitive sense. It allows us to construct a prior in model space from only one vector, namely the prior mean $\tilde{\mathbf{m}}$. In doing so, we ensure that the information content of the prior is as small as possible. Nevertheless, it is important to keep in mind that the maximum-entropy principle does not remove subjectivity from the design of the prior, because the acceptance of the principle is an act of subjectivity in itself. The maximum-entropy principle is not a law of physics, and we are therefore free to ignore it, if we like. A small detail is that the choice of the base $b$ is still arbitrary.

The maximum-entropy principle may be modified or expanded by using different constraints. For instance, instead of having prior knowledge of the mean, one may have prior knowledge of the variance. This would lead to a Gaussian distribution.

As presented above, the maximum-entropy principle is a very general concept that leads to equation (4.50). The numerical values and interpretations of the constants $c_{1}$ and $\mathbf{c}_{2}$ largely depend on the definition of the model space $\mathbb{M}$. Below, we will consider some special cases that are of broader interest.

The unit interval: A particularly interesting case occurs when the model space $\mathbb{M}$ is the 1-D unit interval $[-1,1]$, and when the prior mean is zero. Inserting (4.50) into the definition of the mean, gives 


$$
\int_{-1}^{1} m e^{c_{2} m} d m=0
$$

where we already divided by $c_{1}$. Solving the integral, yields

$$
-\left.\frac{1-c_{2} m}{c_{2}^{2}} e^{c_{2} m}\right|_{-1} ^{1}=\frac{\left(1+c_{2}\right)}{c_{2}^{2}} e^{-c_{2}}-\frac{\left(1-c_{2}\right)}{c_{2}^{2}} e^{c_{2}}=0 .
$$

The unique solution of (4.52) is $c_{2}=0$, meaning that the general exponential in equation (4.50) takes the special form of a constant function. Thus, for $\mathbb{M}=[-1,1]$ and $\tilde{m}=0$, the least informative prior is indeed $\rho(m)=\frac{1}{2}$.

The unit circle: In many applications where rotating objects or the directionality of some phenomenon are of interest, $\mathbf{m}$ is defined in the unit circle $S_{2}$, where it can be written in polar coordinates as

$$
\left[\begin{array}{l}
m_{1} \\
m_{2}
\end{array}\right]=\left[\begin{array}{l}
\cos \varphi \\
\sin \varphi
\end{array}\right]
$$

Starting in Cartesian coordinates, the maximum-entropy distribution is given by

$$
\rho(\mathbf{m})=c_{1} e^{\mathbf{c}_{2}^{T} \mathbf{m}} .
$$

In (4.54) we chose the basis $b=e$ and used the fact that the homogeneous distribution in 2-D Cartesian coordinates is constant. For simplicity, and without a significant loss of generality, we let the vector $\mathbf{c}_{2}$ point in $m_{1}$-direction, so that (4.54) simplifies to

$$
\rho(\mathbf{m})=c_{1} e^{\kappa \cos \varphi},
$$

with $\kappa$ being the $m_{1}$-component of $\mathbf{c}_{2}$. Before using the constraints (4.44) and (4.45) to determined the constants, we define the Bessel function

$$
I_{n}(\kappa)=\frac{1}{\pi} \int_{0}^{\pi} \cos (n \varphi) e^{\kappa \cos \varphi} d \varphi
$$

Inserting (4.55) into the normalisation constraint (4.45), we find

$$
1=c_{1} \int_{S_{2}} e^{\kappa \cos \varphi} d m_{1} d m_{2}=c_{1} \int_{-\pi}^{\pi} e^{\kappa \cos \varphi} d \varphi=2 \pi c_{1} I_{0}(\kappa)
$$

It follows that the normalised maximum-entropy distribution on the unit circle is given by

$$
\rho(\mathbf{m})=\frac{1}{2 \pi I_{0}(\kappa)} e^{\kappa \cos \varphi}
$$

Equation (4.58) is the von Mises distribution, which we previously encountered in section 4.4, where we tried to find the fisherman lost at sea. A selection of von Mises distributions for different values of $\kappa$ are shown in figure $4.3 \mathrm{~b}$. Considering the $m_{1}$-component of the averaging constraint (4.44), we obtain

$$
\tilde{m}_{1}=\int_{-\pi}^{\pi} \cos \varphi \rho[\mathbf{m}(\varphi)] d \varphi=\frac{1}{2 \pi I_{0}(\kappa)} \int_{-\pi}^{\pi} \cos \varphi e^{\kappa \cos \varphi} d \varphi=\frac{I_{1}(\kappa)}{I_{0}(\kappa)}
$$

Equation (4.59) relates the mean $\tilde{m}_{1}$ to the parameter $\kappa$, which is a measure of concentration or peakedness. Another interesting aspect of (4.59) is that it does not involve the average over $\phi$, which would be an integral 
over $\phi \rho[\mathbf{m}(\varphi)]$, but the average over $\cos \phi$, often referred to as the circular mean. Expanding the cosine in (4.58) into a Taylor series up to second order, we see that the von Mises distribution approximates a Gaussian in $\varphi$ for sufficiently small values of $\kappa$. This explains why the von Mises distribution is also known as circular normal distribution.

The unit sphere: Probability densities defined on the unit sphere $S_{3}$ play a major role in the Earth sciences, astronomy, and many other domains. A model vector on the unit sphere can be expressed in spherical coordinates,

$$
\left[\begin{array}{l}
m_{1} \\
m_{2} \\
m_{3}
\end{array}\right]=\left[\begin{array}{c}
\cos \varphi \sin \theta \\
\sin \varphi \sin \theta \\
\cos \theta
\end{array}\right]
$$

where $\varphi$ is the azimuthal angle (between the $m_{1}$ - and $m_{2}$-axes) and $\theta$ the polar angle (between the $m_{3}$-axis and the $m_{1}-m_{2}$-plane. Again starting in Cartesian coordinates where the homogeneous probability density is constant, the maximum-entropy distribution is

$$
\rho(\mathbf{m})=c_{1} e^{\kappa \mu^{T} \mathbf{m}},
$$

with a constant $\kappa$ and a unit vector $\boldsymbol{\mu}$. To determine the normalisation constant $c_{1}$ we let $\boldsymbol{\mu}$ point in $m_{3}$-direction. Other choices are equally possible, but they require significantly more effort to reach the same result. Invoking the normalisation constraint (4.45), we have

$$
1=c_{1} \int_{S_{3}} e^{\kappa \cos \theta} d m_{1} d m_{2} d m_{3}=c_{1} \int_{\varphi=-\pi}^{\pi} \int_{\theta=0}^{\pi} e^{\kappa \cos \theta} \sin \theta d \varphi d \theta .
$$

Realising that

$$
-\frac{1}{\kappa} \frac{d}{d \theta} e^{\kappa \cos \theta}=\sin \theta e^{\kappa \cos \theta},
$$

the integral (4.62) can be solved analytically,

$$
1=\frac{4 \pi c_{1}}{\kappa} \sinh \kappa
$$

It follows that the normalised maximum-entropy distribution on the unit sphere is given by

$$
\rho(\mathbf{m})=\frac{\kappa}{4 \pi \sinh \kappa} e^{\kappa \boldsymbol{\mu}^{T} \mathbf{m}}
$$

The probability density (4.65) is commonly referred to as the Fisher distribution ${ }^{5}$.

Since $\mathbf{m}$ is defined on the unit sphere, it is often more convenient to express $\rho(\mathbf{m})$ not in Cartesian coordinates but in terms of the spherical coordinate angles $\varphi$ and $\theta$. Unfortunately, the simple coordinate transformation recipe introduced in section 3.4 is not applicable in this case, because the Jacobian of (4.60) is not square, meaning that it does not have a determinant. Nevertheless, we can apply the principle that probabilities must not change upon a coordinate transform of the probability density. For this, we consider an arbitrary part $S_{3}^{\prime}$ of the unit sphere $S_{3}$. The probability of $\mathbf{m}$ being in $S_{3}^{\prime}$ is

$$
P\left(\mathbf{m} \in S_{3}^{\prime}\right)=\int_{S_{3}^{\prime}\left(m_{1}, m_{2}, m_{3}\right)} \rho(\mathbf{m}) d m_{1} d m_{2} d m_{3}=\int_{S_{3}^{\prime}(\varphi, \theta)} \rho[\mathbf{m}(\varphi, \theta)] \sin \theta d \varphi d \theta .
$$

From (4.66) we infer that the Fisher distribution in spherical coordinates is given by

${ }^{5}$ Sir Ronald Aylmer Fisher (1890 - 1962) was a British mathematician. He is best known for his work in statistics, often with applications in biology. His mathematical contributions to natural selection led to a revival of Darwinism in the early 20 th century. 


$$
\rho(\mathbf{m})=\frac{\kappa \sin \theta}{4 \pi \sinh \kappa} e^{\kappa \boldsymbol{\mu}^{T} \mathbf{m}} .
$$

A collection of Fisher distributions in Cartesian coordinates for different values of $\kappa$ is shown in figure 4.6.

$$
\kappa=1
$$

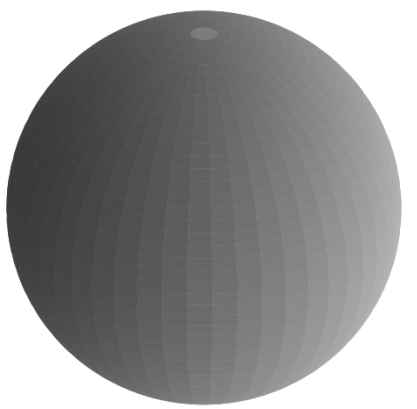

$$
\kappa=5
$$

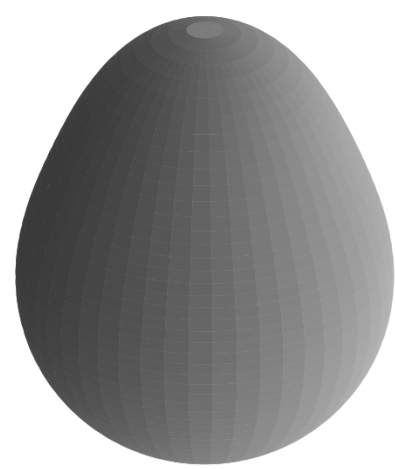

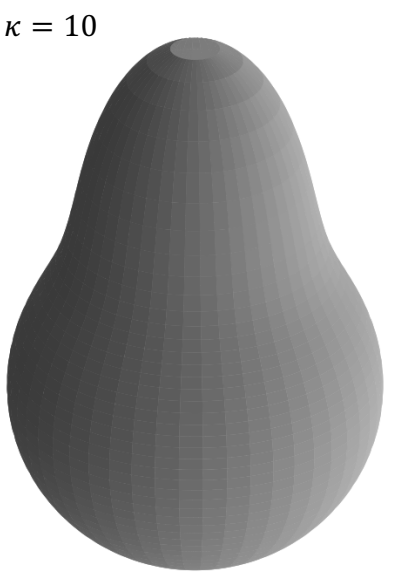

Fig. 4.6: Fisher distributions in Cartesian coordinates for different values of $\kappa$, and $\boldsymbol{\mu}$ oriented in the vertical direction. The radius of the surfaces is equal to $1+\rho(\mathbf{m})$.

\subsubsection{Invariance principles}

In numerous applications, especially in physics, we encounter model parameters $m$ that are as meaningful as their inverses $1 / \mathrm{m}$. Examples include period and frequency, resistivity and conductivity, mass density and lightness, and velocity and slowness. Hence, in the absence of other information, it seems reasonable to request that a prior probability density $\rho(m)$ must not change upon a coordinate transform from $m$ to $m^{*}=1 / m$. Using the transformation rule (3.33) we find that the pair of probability densities

$$
\rho(m)=\frac{1}{m}, \quad \rho^{*}\left(m^{*}\right)=\frac{1}{m^{*}},
$$

fulfils this requirement. In honour of Sir Harold Jeffreys ${ }^{6}$, who first studied probability densities of the form (4.68) (Jeffreys, 1939), parameters that are as popular as their inverses are referred to as Jeffreys parameters.

While the probability densities in (4.68) are invariant under a change of coordinates, their interpretation is non-trivial and potentially misleading. In fact, the maximum-likelihood model of $\rho(m)=1 / m$ is $\hat{m}=0$, even though we did not inject any prior knowledge that would favour one model over another. An elegant solution to this problem is the definition of the logarithmic parameters

$$
\mu=\log \frac{m}{m_{\mathrm{ref}}}, \quad \mu^{*}=\log \frac{m^{*}}{m_{\mathrm{ref}}^{*}},
$$

\footnotetext{
${ }^{6}$ Sir Harold Jeffreys (1891 - 1989) was a British mathematician, geophysicist and astronomer. His 1939 book Theory of Probability is widely considered as one of major works in Bayesian inference. He is credited with the idea that the Earth's core is liquid. From 1939 - 1952, he served as Director of the International Seismic Summary, which later became the International Seismological Centre (ISC). He was knighted in 1953.
} 
with some suitably chosen reference parameters $m_{\mathrm{ref}}=1 / m_{\mathrm{ref}}^{*}$. Defining a constant prior that attributes equal likelihood to all logarithmic models,

$$
\rho(\mu)=\text { constant }
$$

and applying the transformation rule (3.33), we indeed find

$$
\rho^{*}\left(\mu^{*}\right)=\text { constant }
$$

An additional benefit of using logarithmic parameters is the preservation of distance between models upon a coordinate change. Letting $\mu_{1}$ and $\mu_{2}$ be two models, we can compute their mutual distance by taking the absolute value of their difference, that is, $d=\left|\mu_{1}-\mu_{2}\right|$. For the distance of the transformed models, we have

$$
d^{*}=\left|\mu_{1}^{*}-\mu_{2}^{*}\right|=\left|\mu_{2}-\mu_{1}\right|=d .
$$

Hence, distance remains invariant as well. Yet, just as the maximum-entropy principle in section 4.7.2, the invariance principle is not an irrevocable law of nature. Instead, it is ultimately founded on our subjective and widely shared notions of elegance and symmetry, which implicitly seem to suggest some kind of correctness. However, an alien arriving on Earth from a distant planet may find invariance under the transformation $m^{*}=$ $\arctan m$ even more beautiful, and since beauty is in the eye of the beholder, we would not have a strong argument against this choice.

In the interest of simplicity, we merely considered invariance for a scalar parameter. Idea on how to generalise this concept to vectorial and tensorial quantities may be found in Tarantola (2005).

\subsubsection{Physical intuition, common sense and subjective relativity}

In the previous paragraphs we considered methods for the construction of plausible prior probability densities. These were based on an assumed mechanism of noise generation (section 4.7.1), the notion of maximum entropy or minimum information (section 4.7.2), or invariance under certain symmetry operations (section 4.7.3). Many more arguments could be added to this list.

All of these arguments are to some degree subjective. Maximum entropy and symmetry in the prior, for instance, are certainly good to have, but by no means enforced by laws of nature. Nevertheless, they are in accord with what many of us would agree to be common sense. Hence, it is fair to ask why common sense, often based on physical intuition and experience, should not be used more directly, instead of hiding it behind mathematical procedures.

In fact, the more direct use of common sense and intuition is entirely legitimate if one accepts that the solution of an inverse problem often has an inevitable subjective component. The beauty of Bayesian inference is that this is made explicit through the appearance of the data and model space priors in Bayes' theorem. There is nothing fundamentally wrong with subjectivity when it is used transparently, and when results are interpreted not as being the ultimate truth but as being relative to the prior.

Unless a probability density carries a clear frequentist meaning, for instance, via the error statistics of identical experiments that can be repeated many times, its interpretation is to some extent subjective as well. What $P\left(m \in\left[m_{1}, m_{2}\right]\right)$ really means to us depends on the decisions that we decided to make a priori in case this event would actually happen a posteriori. In this regard, Bayes' theorem links our personal definition of a prior to our personal interpretation and understanding of the posterior.

The reader may object that the acceptance of subjectivity compromises the comparability of results, obtained, for example, with different data $\mathbf{d}_{1}^{\text {obs }}$ and $\mathbf{d}_{2}^{\text {obs }}$. Indeed, it may seem that the posterior $\rho_{1}\left(\mathbf{m} \mid \mathbf{d}_{1}^{\text {obs }}\right)$ obtained by person 1 using the prior $\rho_{1}(\mathbf{m})$ differs from $\rho_{2}\left(\mathbf{m} \mid \mathbf{d}_{2}^{\text {obs }}\right)$ obtained by person 2 on the basis of the prior $\rho_{2}(\mathbf{m})$. Bayesian inference, however, naturally allows us to resolve this problem. In case person 1 and person 2 agree that their priors are both meaningful, they may simply combine their posteriors by computing their conjunc- 
tion. Otherwise, person 1 may bring the posterior $\rho_{2}\left(\mathbf{m} \mid \mathbf{d}_{2}^{\text {obs }}\right)$ in accord with their subjective understanding of Bayesian probability by eliminating the influence of $\rho_{2}(\mathbf{m})$ and inserting $\rho_{1}(\mathbf{m})$ instead,

$$
\frac{\rho_{2}\left(\mathbf{m} \mid \mathbf{d}_{2}^{\mathrm{obs}}\right)}{\rho_{2}(\mathbf{m})} \rho_{1}(\mathbf{m}) \propto \rho\left(\mathbf{d}_{2}^{\mathrm{obs}} \mid \mathbf{m}\right) \rho_{1}(\mathbf{m}) \propto \rho_{1}\left(\mathbf{m} \mid \mathbf{d}_{2}^{\mathrm{obs}}\right) .
$$

How to perform this change in subjective perspective in practice will be the topic of the following chapter.

\subsection{Practicalities*}

Despite its simplicity and elegance, Bayes' theorem comes with a significant drawback, namely that the posterior $\rho\left(\mathbf{m} \mid \mathbf{d}^{\text {obs }}\right)$ can usually not be computed analytically, that is, we cannot write down a simple explicit equation that takes some $\mathbf{m}$ and returns $\rho\left(\mathbf{m} \mid \mathbf{d}^{\text {obs }}\right)$ without significant computation. Instead, for each candidate $\mathbf{m}$, we must solve the forward problem, evaluate the likelihood function $\rho\left(\mathbf{d}^{\mathrm{obs}} \mid \mathbf{m}\right)$, and then multiply with the prior in model space. This process, that needs to be repeated for all candidate models $\mathbf{m}$, is referred to as sampling. Sampling can be a computationally expensive exercise, especially when the forward problem solution is already computationally expensive, and when the model space has a large number of dimensions.

Not only the plain computation of $\rho\left(\mathbf{m} \mid \mathbf{d}^{\text {obs }}\right)$ itself can be difficult in practice. Also the condensation of a potentially high-dimensional posterior into lower-dimensional quantities, discussed in section 4.5 , often cannot be performed analytically. This is because the posterior may not be given explicitly, or because there are no analytical solutions to the high-dimensional integrals that define means, covariances, probabilities or marginals. The discrete sampling of the posterior can help in such cases, too, as it allows us to replace a high-dimensional integral by a finite sum.

During the past decades, a whole branch of mathematics has evolved around the sampling of probability densities that cannot be handled analytically, because they are too complicated or too high-dimensional. How shall we distribute discrete samples in model space in order to learn what we wish to learn about the posterior? How can we ensure that the number of required samples is as small as possible? These questions motivate the following chapter on Monte Carlo methods. 


\section{Chapter 5 Monte Carlo methods *}

Den Zufall gibt die Vorsehung. Zum Zwecke muss der Mensch ihn gestalten.

Friedrich Schiller, Don Carlos, 1787.

Since all models are wrong the scientist must be alert to what is importantly wrong. It is inappropriate to be concerned about mice when there are tigers abroad.

George Box, Science and statistics, 1976.

The main purpose of science is simplicity, and as we understand more things, everything is becoming simpler.

Edward Teller, Conversations on the Dark Secrets of Physics, 1991.

It was around 1941 when the young physicist John Mauchly ${ }^{1}$ realised that the electronic circuits in Geiger counters may as well be used to perform simple arithmetic, and hence to solve difference equations. Supported by the United States Army, mostly interested in the laborious calculation of firing tables for new guns used in World War II, Mauchly initiated the construction of the Electronic Numerical Integrator and Computer $(E N I A C)$. Being orders of magnitude faster than all other technologies available at that time, ENIAC was able to add 5000 numbers per second, thus allowing its users to solve problems that were previously unsolvable.

The ENIAC soon attracted the attention of John von Neumann ${ }^{2}$, consultant to Mauchly's research laboratory. Affectionately called "Johnny" by his fellow scientists at Los Alamos, von Neumann was seriously interested in thermonuclear reactions. In the spring of 1946, von Neumann visited Mauchly to discuss the first results of thermonuclear simulations performed on ENIAC. Together with von Neumann, another East-European exile, Stanisław "Stan" Ulam ${ }^{3}$, attended the meeting. Having an extensive mathematical background, Ulam realised the potential of using ENIAC for a class of methods that had so far been deemed entirely out of reach: statistical sampling, that is, the simulation of physical processes, such as the diffusion of neutrons during thermonuclear

\footnotetext{
${ }^{1}$ John William Mauchly (1907-1980) was a U.S. American electrical engineer and physicist. Together with his colleague J. Presper Eckert, he constructed ENIAC, the first general-purpose electronic computer. They introduced numerous fundamental concepts of digital computing, including programming languages, subroutines and stored programs.

2 John von Neumann (1903-1957), born as Neumann János Lajos in Budapest, was a Hungarian-U.S. American mathematician and physicist. In his short life, he made groundbreaking contributions in mathematics, the foundations of computing and programming, quantum physics and statistics. His colleague at Los Alamos and later Nobel Laureate Hans Bethe considered Neumann to belong to a new species, superior to that of ordinary human beings.

${ }^{3}$ Stanisław Marcin Ulam (1909-1984) was a Polish-U.S. American mathematician and nuclear physicist. He is best known for his work on thermonuclear weapons and for the recognition that newly emerging digital computers would enable statistical sampling techniques.
} 
reactions, using random numbers. Alluding to the fact that Ulam's uncle, a gambler, would borrow money from relatives because he "had just gone to Monte Carlo", another Los Alamos scientist, Nicholas Metropolis ${ }^{4}$, jokingly called the revived class of algorithms the Monte Carlo method. Obviously, the name stuck.

In a 1987 special issue of Los Alamos Science, Metropolis recalls these times (Metropolis, 1987): "The year was 1945. Two earthshaking events took place: the successful test at Alamogordo ${ }^{5}$ and the building of the first electronic computer. Their combined impact was to modify qualitatively the nature of global interactions between Russia and the West. No less perturbative were the changes wrought in all of academic research and in applied science. On a less grand scale these events brought about a renascence of a mathematical technique known to the old guard as statistical sampling." Metropolis foresaw the impact and potential of Monte Carlo methods, that are becoming ever more attractive as available computational power continues to increase, far beyond what Mauchly, von Neumann and Ulam might have imagined. Today, Monte Carlo methods allow us, for instance, to solve large Bayesian inverse problems and to obtain detailed, quantitative information on the properties of physical systems.

In the following sections, we will highlight the challenge of sampling high-dimensional model spaces, and explain how this challenge may be addressed using random sampling techniques. This will lead us to the concept of random walks and to a diverse family of Monte Carlo techniques that may be used to solve Bayesian inverse problems.

\subsection{Grid search and the curse of dimensionality *}

The benefit of using Monte Carlo methods becomes most obvious when not using them. Thus, before delving into statistical sampling, we briefly analyse the properties of a systematic, deterministic exploration of model space, known as grid search.

\subsubsection{The curse of dimensionality *}

Let us assume, for the purpose of illustration, that our posterior is the $n$-dimensional Gaussian

$$
\rho\left(\mathbf{m} \mid \mathbf{d}^{\mathrm{obs}}\right)=\frac{1}{(2 \pi)^{\frac{n}{2}}} e^{-\frac{1}{2} \mathbf{m}^{T} \mathbf{m}} .
$$

Loosely speaking, all relevant models are located within the $n$-dimensional hypersphere with radius 1 because the covariance of the posterior is the $n$-dimensional unit matrix $\mathbf{I}_{n}$. Models that are located outside this $n$ dimensional unit-hypersphere are more irrelevant, meaning that their likelihood is comparatively small.

Assume that we wish to explore $\rho\left(\mathbf{m} \mid \mathbf{d}^{\text {obs }}\right)$ with a grid search. For this, we discretise the $n$-dimensional hypercube $[-1,1]^{n}$ with a regular grid, and we then evaluate the posterior at those grid points. How many of these models that we draw from the grid are relevant, compared to the total number of models (grid points)?

For a two-dimensional model space with $n=2$ this question is easy to answer. The volume of the unit 2-sphere, that is, the area of the unit circle, is $\pi$. The volume of the unit 2-cube, that is, the area of the unit square, is 4. It follows that $100 \times \pi / 4 \approx 79 \%$ of all models are relevant, and $100 \times(1-\pi / 4) \approx 21 \%$ of all models are irrelevant. In three dimensions, where $n=3$, we find that $100 \times \frac{4}{3} \pi / 2^{3} \approx 52 \%$ of all models on the grid are

\footnotetext{
${ }^{4}$ Nicholas Constantine Metropolis (1915-1999) was a Greek-U.S. American physicist. He was one of the first 50 scientists who arrived in the newly founded Los Alamos Laboratory in 1943 to work on the construction of the first nuclear bomb. After World War II, he focused on the development of digital computers (the successors of ENIAC) and on Monte Carlo computation.

5 Alamogordo is a city in the desert of New Mexico, U.S.A., known for its proximity to the site where the first nuclear bomb, Trinity, was tested on 16 July 1945.
} 
relevant, whereas $100 \times\left(1-\frac{4}{3} \pi / 2^{3}\right) \approx 48 \%$ of them are irrelevant.

How does this evolve as we go to higher dimensions? While the volume of the $n$-dimensional unit hypercube, $V_{n, \text { cube }}$ is easily computed as

$$
V_{n, \text { cube }}=2^{n},
$$

the computation of the volume of the $n$-dimensional unit hypersphere, $V_{n \text {,sphere }}$ is more involved:

$$
V_{n, \text { sphere }}=\frac{\pi^{\frac{n}{2}}}{\Gamma\left(\frac{n}{2}+1\right)},
$$

where $\Gamma(n)$ is the gamma function. (See the box below for a derivation of this formula and the definition of the gamma function.) It follows that the fraction of relevant models is given by

$$
\frac{V_{n, \text { sphere }}}{V_{n, \text { cube }}}=\frac{\pi^{\frac{n}{2}}}{2^{n} \Gamma\left(\frac{n}{2}+1\right)} .
$$

Using equation (5.4) we can compute the fraction of relevant models for any dimension. For $n=4$, we obtain $\approx 0.31$, for $n=6$ we have $\approx 0.081$, and for $n=10$, the fraction of relevant models drops to $\approx 0.0024$. For $n=20$, only $\approx 2.46 \cdot 10^{-8}$ of all models on the grid are actually relevant.

Obviously, the fraction of relevant models decreases dramatically with increasing dimension. This is because the gamma function in equation (5.4) is proportional to the factorial of the dimension $n$, which increases much more rapidly than the $n^{\text {th }}$ power of any number. This phenomenon is referred to as the curse of dimensionality ${ }^{6}$.

The curse of dimensionality effectively means that the very large majority of models that we evaluate in a grid search is useless, already when the dimension of the model space is not very large. Another interpretation is that the very large majority of models in a high-dimensional space are located along the boundaries of that space. In other words: "High-dimensional model spaces are terribly empty." (A. Tarantola).

While we have here considered the curse of dimensionality for the specific example of a Gaussian distribution, it of course extends to any other distribution. In a more general framework, the volume of relevant models that contribute, for instance, to the computation of probabilities or expectations, is called the typical set.

The gamma function: The gamma function can be considered as an interpolation of the factorial of an integer $z$, denoted by $z$ !, to positive non-integer numbers. It is defined by the integral

$$
\Gamma(z)=\int_{0}^{\infty} x^{z-1} e^{-x} d x .
$$

Integration by parts yields a recursion relation for the gamma function:

$$
\Gamma(z)=(z-1) \int_{0}^{\infty} x^{z-2} e^{-x} d x=(z-1) \Gamma(z-1) .
$$

With $\Gamma(1)=1$, we therefore have $\Gamma(2)=1=1$ !, $\Gamma(3)=2=2$ !, $\Gamma(4)=6=3$ !, or more generally

$$
\Gamma(z)=(z-1) !, \quad \text { for } z \in \mathbb{N} .
$$

${ }^{6}$ In fact, the curse of dimensionality is a very diffuse expression, and what we see here is just one of its many facets. More generally, the curse of dimensionality refers to any problem that becomes dramatic very quickly as the dimension of the problem increases just slightly. Another example would be the computational cost for numerically solving a partial differential equation in one, two or three dimensions. 


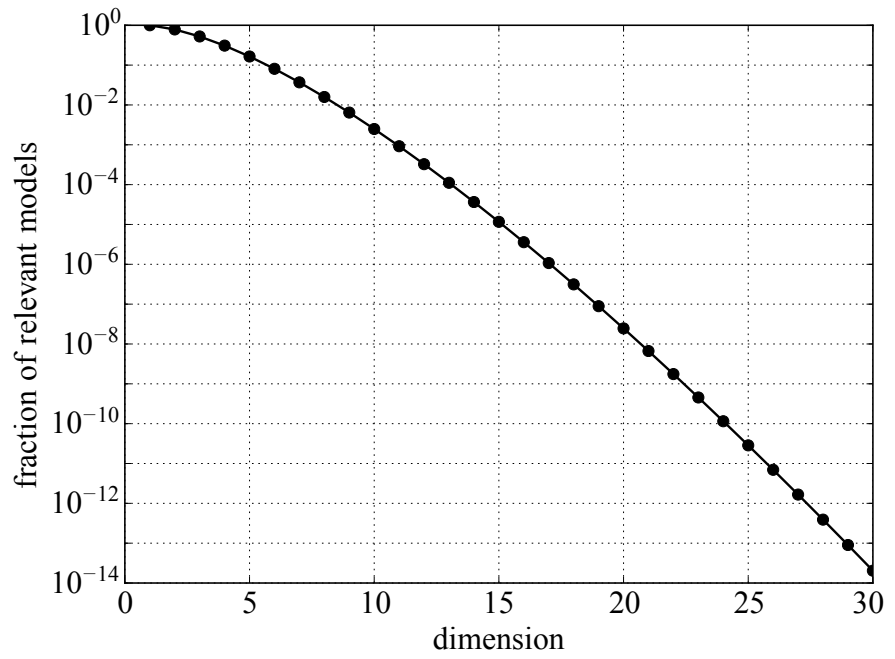

Fig. 5.1: Illustration of the curse of dimensionality. Shown is the fraction of relevant models (within the $n$ dimensional unit sphere) compared to all models in the $n$-dimensional unit cube. Note the logarithmic axis.

For arbitrary positive non-integers, $\Gamma(z)$ is not an integer. Some special values are $\Gamma\left(\frac{1}{2}\right)=\sqrt{\pi}, \Gamma\left(\frac{3}{2}\right)=$ $\frac{1}{2} \sqrt{\pi}$ and $\Gamma\left(\frac{5}{2}\right)=\frac{3}{4} \sqrt{\pi}$.

The volume of the $n$-sphere: Computing the volume $V_{n}$ of the $n$-dimensional hypersphere can either be done brute force by directly evaluating an integral in $n$-dimensional spherical coordinates, or again by some insightful tricks, as in the following.

First, we note that $V_{2}$, the volume of the unit 2-sphere (the surface of the unit circle) can be computed by summing (integrating) $S_{2}$, the surfaces of the unit 2-sphere (the circumference of the unit circle) scaled by the radius $r$ :

$$
V_{2}=\int_{0}^{1} S_{2} r d r=S_{2} \int_{0}^{1} r d r=\frac{S_{2}}{2}=\pi .
$$

Similarly, the volume of the unit 3 -sphere (volume of the unit sphere) $V_{3}$ can be computed by summing (integrating) the surfaces of unit 3-spheres $S_{3}$ scaled by $r^{2}$ :

$$
V_{3}=\int_{0}^{1} S_{3} r^{2} d r=\frac{S_{3}}{3}=\frac{4}{3} \pi .
$$

Analogously, we find a general relation between the volume and the surface of an $n$-sphere:

$$
V_{n}=\int_{0}^{1} S_{n} r^{n-1} d r=\frac{S_{n}}{n} .
$$

The surface of the $n$-sphere, $S_{n}$, can be computed with the help of the Gaussian integral, 


$$
(2 \pi)^{\frac{n}{2}}=\int_{\mathbb{R}^{n}} e^{-\frac{1}{2} \mathbf{x}^{T} \mathbf{x}} d \mathbf{x}
$$

previously considered in equation (3.18). Replacing, as in equations (5.8) to (5.10) the volume integral by an integral over radius, we find

$$
(2 \pi)^{\frac{n}{2}}=\int_{0}^{\infty} S_{n} r^{n-1} e^{-\frac{1}{2} r^{2}} d r .
$$

Substituting $x=\frac{1}{2} r^{2}$, transforms this integral to

$$
(2 \pi)^{\frac{n}{2}}=\frac{1}{2} S_{n} 2^{\frac{n}{2}} \int_{0}^{\infty} x^{\frac{n}{2}-1} e^{-x} d x .
$$

Using the definition of the gamma function, we see that the integral in equation (5.13) is equal to $\Gamma\left(\frac{n}{2}\right)$, and we finally obtain the surface of the $n$-sphere as

$$
S_{n}=\frac{2 \pi^{\frac{n}{2}}}{\Gamma\left(\frac{n}{2}\right)} .
$$

The volume of the $n$-sphere then follows from the combination of equations (5.14) and (5.10):

$$
V_{n}=\frac{2 \pi^{\frac{n}{2}}}{n \Gamma\left(\frac{n}{2}\right)}=\frac{\pi^{\frac{n}{2}}}{\Gamma\left(\frac{n}{2}+1\right)} .
$$




\subsection{Random walks *}

As we have seen in the previous section, grid search is not an option for model spaces with slightly elevated dimension. To improve the inefficient grid search, we introduce, in the following paragraphs, a new class of approaches known as Monte Carlo methods. Instead of systematically exploring the model space, Monte Carlo methods test models randomly, trying to avoid those regions of the model space that are less relevant by taking prior knowledge into account.

To reduce the notational burden, we first introduce a terminology that is somewhat lighter than the one we have used so far. For this, we partition the model space $\mathbb{M}$ into equal-sized subvolumes $\mathbb{M}^{i}$ around some model $\mathbf{m}^{i}$. Assuming the $\mathbb{M}^{i}$ are sufficiently small, their prior probability is $P^{i}=M \rho\left(\mathbf{m}^{i}\right)$, with $M$ being the volume of the subvolumes $\mathbb{M}^{i}$. Similarly, we define $L^{i}=M \rho\left(\mathbf{d}^{\mathrm{obs}} \mid \mathbf{m}^{i}\right)$ for the discretised likelihood function (prior in data space $)$, and $\hat{P}^{i}=M \rho\left(\mathbf{m}^{i} \mid \mathbf{d}^{\text {obs }}\right)$ for the discretised posterior in model space. Furthermore, we denote by $Q^{i}=M q\left(\mathbf{m}^{i}\right)$ an arbitrary generic probability with its distribution $q$.

Instead of a systematic grid search, we now consider a random walk, where a sequence of models $\mathbf{m}^{i}$ is visited randomly according to some rules that remain to be specified. When the rules are designed such that the number of visits at model $\mathbf{m}^{i}$ is proportional to $Q^{i}$, the random walker is said to sample the distribution $q$. The ensemble of models with a frequency distribution proportional to $Q^{i}$ are called samples of the distribution $q$. Furthermore, the walker's preference for models with high $Q^{i}$ is referred to as importance sampling of $q$.

Assume that the random walker is currently located at $\mathbf{m}^{j}$. The conditional probability of going from $\mathbf{m}^{j}$ to $\mathbf{m}^{i}$ in the next step of the walk, conditioned by the probability that the walker is actually at $\mathbf{m}^{j}$, is called the transition probability, $T^{i \leftarrow j}=T\left(\mathbb{M}^{i} \mid \mathbb{M}^{j}\right)$. Thus, if the probability of currently being at $\mathbf{m}^{j}$ is $Q^{j}$, the unconditional probability of walking to $\mathbf{m}^{i}$ is given by $T^{i \leftarrow j} Q^{j}$. This situation is schematically illustrated in figure 5.2. Since the walker must go somewhere, including the option of staying at $\mathbf{m}^{j}$, the transition probabilities satisfy

$$
\sum_{i} T^{i \leftarrow j}=1
$$

Our particular interest is in random walks that equilibrate asymptotically as the number of steps tends to infinity. Since the statistics of infinity are a bit difficult to grasp intuitively, we simplistically imagine the average number of visits ${ }^{7}$ of the walker at model parameter $\mathbf{m}^{j}$ in terms of particles, as shown in figure 5.3. Considering just one model parameter, or block in figure 5.3, with index $i$, the walk is equilibrated when the number of particles moving to the block is equal to the number of particles leaving the block, such that the total number of particles inside the block is always equal to is relative probability. This includes the option of staying in block $i$. In probabilistic terms, equilibrium means that the combined probability to arrive at $\mathbf{m}^{i}$ from any $\mathbf{m}^{j}$ is equal to the probability of actually being at $\mathbf{m}^{i}$, that is

$$
\sum_{j} T^{i \leftarrow j} Q^{j}=Q^{i}
$$

In other words, the random walk has an equilibrium when $Q^{j}$ is an eigenvector of the transition probability matrix $T^{i \leftarrow j}$ with eigenvalue 1 .

Random walks that satisfy the equilibrium condition (5.17) are difficult to design. Therefore, we are interested in a simpler one. In fact, a sufficient condition of equilibration at the distribution $Q^{i}$ is detailed balance of the flow, meaning that as many particles flow from a specific $\mathbf{m}^{j}$ to a specific $\mathbf{m}^{i}$ as from $\mathbf{m}^{i}$ to $\mathbf{m}^{j}$, that is,

$$
T^{i \leftarrow j} Q^{j}=T^{j \leftarrow i} Q^{i}
$$

Summing equation (5.18) over $j$, and using equation (5.16), we indeed retrieve the equilibration condition (5.17). We note that the flow illustrated in figure 5.3 does not satisfy detailed balance. For instance, 2 of 10

${ }^{7}$ This is to be understood as the average over a number of moves that is large compared to the number of model parameters. 


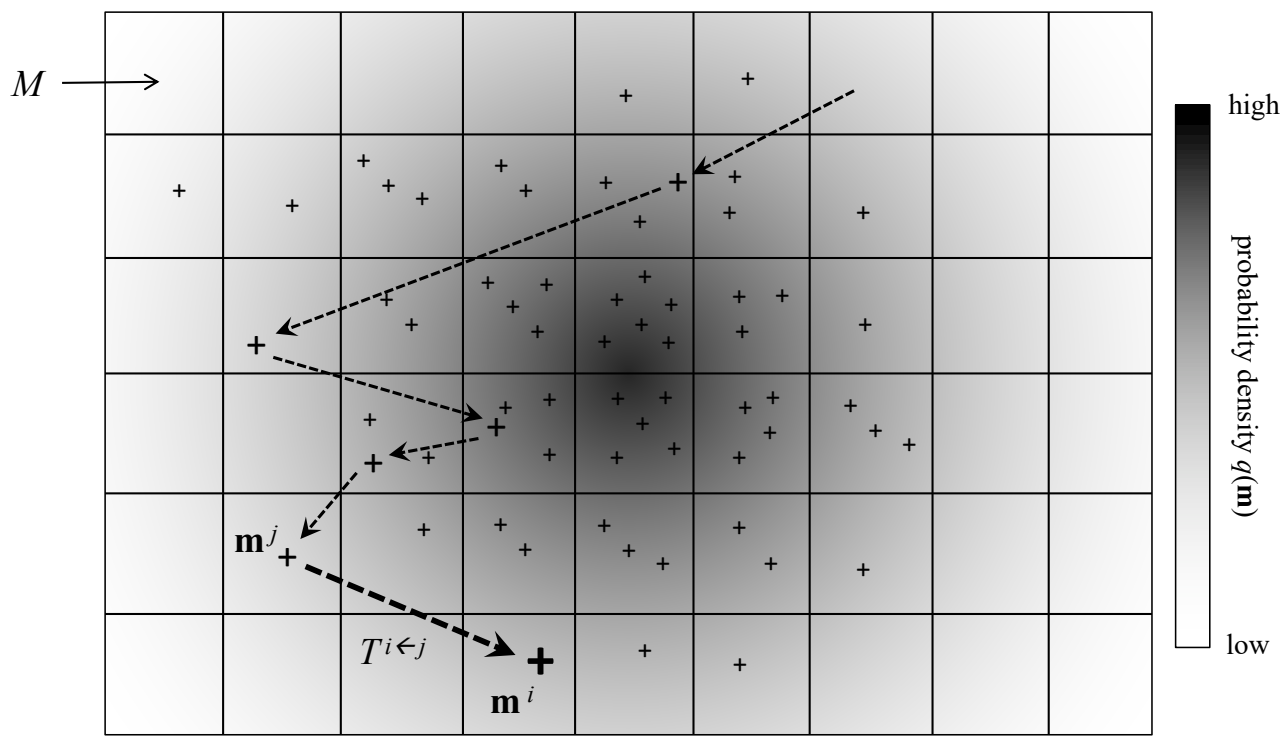

Fig. 5.2: Schematic illustration of a random walker sampling the generic probability density $q(\mathbf{m})$, shown in grey shading. The domain is partitioned into equal-size squares of area $M$. The density of samples (black + signs), that is, the locations visited by the walker, is asymptotically proportional to the value of $q(\mathbf{m})$. The probability of walking from the current $\mathbf{m}^{j}$ to some new location $\mathbf{m}^{i}$ is given by the transition probability $T^{i \leftarrow j}$. Previous moves are indicated by thinner arrows.

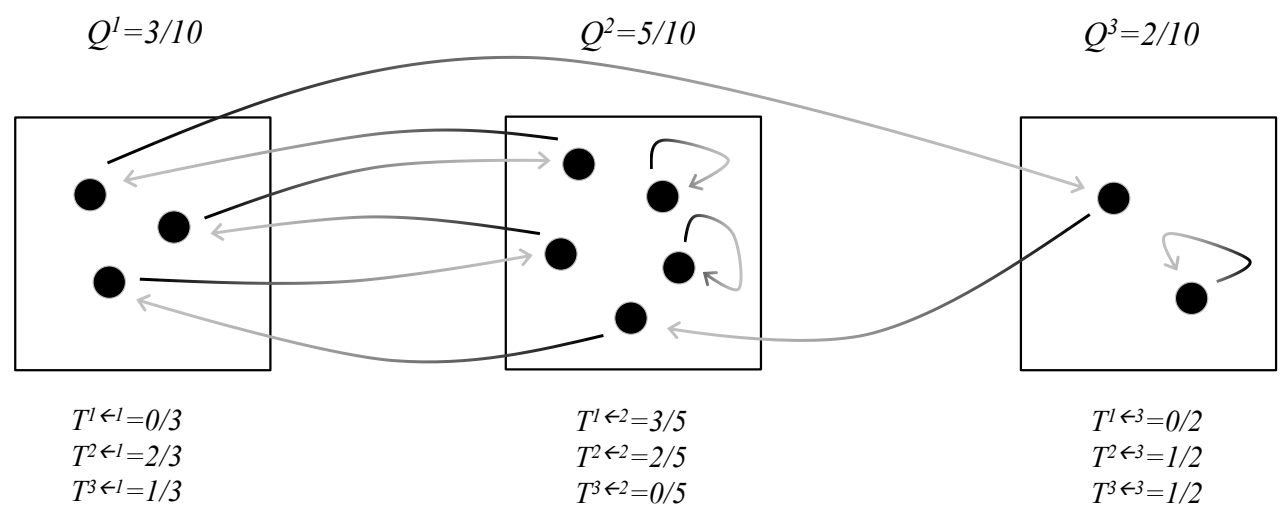

Fig. 5.3: Schematic illustration of an equilibrated random walk en miniature. For simplicity, we imagine only three possible model parameters, symbolised by the black squares, with probabilities $Q^{1}=3 / 10, Q^{2}=5 / 10$ and $Q^{3}=2 / 10$. These probabilities are visualised by black particles, of which there are 10 in total. The transition probabilities $T^{i \leftarrow j}$ correspond to the particles moving between or within model parameter blocks. For instance, 3 of 5 particles from block 2 move to block 1, 2 of 5 particles stay within block 2, and none moves from block 2 to block 3 . Therefore, $T^{1 \leftarrow 2}=3 / 5, T^{2 \leftarrow 2}=2 / 5$ and $T^{3 \leftarrow 2}=0 / 5$. We may easily check that the set of transitions is equilibrated. Taking, as an example, block 3, we find $T^{3 \leftarrow 1} Q^{1}+T^{3 \leftarrow 2} Q^{2}+T^{3 \leftarrow 3} Q^{3}=2 / 10=Q^{3}$. 
particles move from 1 to $2\left(T^{2 \leftarrow 1} Q^{1}=2 / 10\right)$, but 3 of 10 particles move from 2 to $1\left(T^{1 \leftarrow 2} Q^{2}=3 / 10\right)$. A version that does satisfy detailed balance is shown in figure 5.4.

$Q^{l}=3 / 10 \quad Q^{2}=5 / 10 \quad Q^{3}=2 / 10$

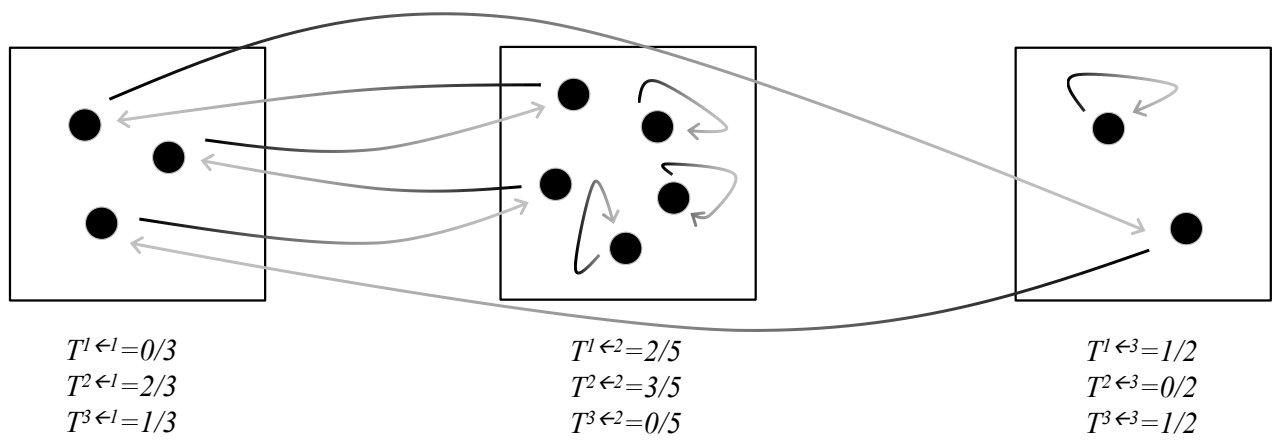

Fig. 5.4: A version of the scenario from figure 5.3 that does satisfy detailed balance.

A typical random walk, of which we will see examples in the following sections, explores a distribution in three phases that are usually not sharpy separated. In the first phase, referred to as burn-in, the walker approaches the typical set, starting from its initial position. The second phase consists of the rough and usually very efficient exploration of the distribution. This is followed by the third phase during which details are found in a much less efficient manner. While the convergence of a random walk towards the target distribution depends very much

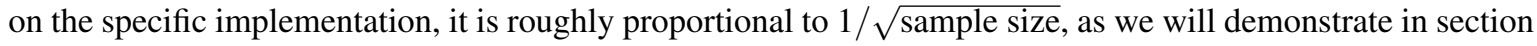
5.3.3. Thus, in accord with the description of the three phases, few samples are needed for the broad structure, and disproportionately many samples are needed for the details.

Continuous detailed balance: In the previous paragraphs, we discretised the model space, mostly in the interest of notational simplicity, into a discrete number of models $\mathbf{m}^{i}$ surrounded by equal-sized volumes $\mathbb{M}^{i}$. In the following, we show how the derivation of detailed balance may be generalised to the continuous case and to partitionings of model space into differently-sized subvolumes. This will be particularly useful in the derivation of transdimensional sampling strategies in section 5.8.3.

We again start with the assumption that the walker is currently located at model $\mathbf{m}^{j}$, and we denote the conditional transition probability of moving from $\mathbf{m}^{j}$ into subvolume $\mathbb{M}^{i}$ by $T\left(\mathbb{M}^{i} \mid \mathbf{m}^{j}\right)$. Note that $T\left(\mathbb{M}^{i} \mid \mathbf{m}^{j}\right)$ is a probability with respect to the subvolume $\mathbb{M}^{i}$ but a probability density with respect to the model $\mathbf{m}^{j}$. The transition probability may be expressed in terms of a transition probability density, $\tau\left(\mathbf{m}^{i} \mid \mathbf{m}^{j}\right)$,

$$
T\left(\mathbb{M}^{i} \mid \mathbf{m}^{j}\right)=\int_{\mathbf{m}^{i} \in \mathbb{M}^{i}} \tau\left(\mathbf{m}^{i} \mid \mathbf{m}^{j}\right) d \mathbf{m}^{i} .
$$

The probability of landing anywhere in model space, obtained by summing (5.19) over all subvolumes, must be equal to 1 , that is,

$$
\sum_{\mathbb{M}^{i} \mathbf{m}^{i} \in \mathbb{M}^{i}} \tau\left(\mathbf{m}^{i} \mid \mathbf{m}^{j}\right) d \mathbf{m}^{i}=\int_{\mathbf{m}^{i} \in \mathbb{M}} \tau\left(\mathbf{m}^{i} \mid \mathbf{m}^{j}\right) d \mathbf{m}^{i}=1 .
$$


Equation (5.20) is the continuous equivalent of equation (5.16). The unconditional probability of landing inside $\mathbb{M}^{i}$, coming from somewhere in $\mathbb{M}^{j}$, follows from the conditional probability (5.19),

$$
\int_{\mathbf{m}^{j} \in \mathbb{M}^{j}} T\left(\mathbb{M}^{i} \mid \mathbf{m}^{j}\right) q\left(\mathbf{m}^{j}\right) d \mathbf{m}^{j}=\int_{\mathbf{m}^{j} \in \mathbb{M}^{j}} \int_{\mathbf{m}^{i} \in \mathbb{M}^{i}} \tau\left(\mathbf{m}^{i} \mid \mathbf{m}^{j}\right) q\left(\mathbf{m}^{j}\right) d \mathbf{m}^{i} d \mathbf{m}^{j} .
$$

To ensure that the walk equilibrates at the distribution $q$, we again require that the unconditional probability of landing in $\mathbb{M}^{i}$, coming from anywhere in model space, is equal to the probability of being in $\mathbb{M}^{i}$. Thus, summing (5.21) over all subvolumes $\mathbb{M}^{j}$, the equilibrium condition is

$$
\int_{\mathbf{m}^{j} \in \mathbb{M}} \int_{\mathbf{m}^{i} \in \mathbb{M}^{i}} \tau\left(\mathbf{m}^{i} \mid \mathbf{m}^{j}\right) q\left(\mathbf{m}^{j}\right) d \mathbf{m}^{i} d \mathbf{m}^{j}=\int_{\mathbf{m}^{i} \in \mathbb{M}^{i}} q\left(\mathbf{m}^{i}\right) d \mathbf{m}^{i} .
$$

A sufficient requirement that ensures (5.22) is the continuous detailed balance

$$
\int_{\mathbf{m}^{j} \in \mathbb{M}^{j}} \int_{\mathbf{m}^{i} \in \mathbb{M}^{i}} \tau\left(\mathbf{m}^{i} \mid \mathbf{m}^{j}\right) q\left(\mathbf{m}^{j}\right) d \mathbf{m}^{i} d \mathbf{m}^{j}=\int_{\mathbf{m}^{j} \in \mathbb{M}^{j}} \int_{\mathbf{m}^{i} \in \mathbb{M}^{i}} \tau\left(\mathbf{m}^{j} \mid \mathbf{m}^{i}\right) q\left(\mathbf{m}^{i}\right) d \mathbf{m}^{i} d \mathbf{m}^{j} .
$$

Equation (5.23) states that the unconditional probability of transitioning from $\mathbb{M}^{j}$ to $\mathbb{M}^{i}$ (left-hand side) is equal to the unconditional probability of transitioning from $\mathbb{M}^{i}$ to $\mathbb{M}^{j}$ (right-hand side). It is the continuous equivalent of the discrete detailed balance equation (5.18). Summing equation (5.23) over all subvolumes $\mathbb{M}^{j}$, and using the normalisation condition (5.20), we indeed retrieve the equilibrium condition (5.22).

\subsection{Monte Carlo integration}

\subsubsection{Approximating integrals with random samples}

Having samples of a distribution, $q(\mathbf{m})$, not only allows us to approximate the distribution by simply counting the number of samples within a small model space subvolume. It also helps us to approximate integrals over the distribution. This is needed, for instance, to compute lower-dimensional condensations of the potentially high-dimensional posterior, as discussed in section 4.5. These include the mean model (equation 4.25), the covariance (equation 4.26), marginals, or probabilities. All of these quantities can be generically written as integrals over $q(\mathbf{m})$ times some function $f(\mathbf{m})$, that is,

$$
F=\int_{\mathbb{M}} f(\mathbf{m}) q(\mathbf{m}) d \mathbf{m} .
$$

To approximate the integral, we again partition the model space $\mathbb{M}$ into small subvolumes $\mathbb{M}^{i}$ with equal volume $M$. Based on this discretisation, we obtain

$$
\left.F \approx \sum_{i=1}^{N_{M}} f(\mathbf{m}) q(\mathbf{m}) M\right|_{\mathbf{m} \in \mathbb{M}^{i}}
$$

where $N_{M}$ is the number of subvolumes. By construction, the samples of $q$ are distributed such that the number $N^{i}$ of samples within $\mathbb{M}^{i}$ divided by the total number of samples $N_{s}$ is equal to the probability of landing inside 
$\mathbb{M}^{i}$, that is,

$$
\left.q(\mathbf{m}) M\right|_{\mathbf{m} \in \mathbb{M}^{i}}=\frac{N^{i}}{N_{s}} .
$$

Of course, this assumes that sufficiently many samples have been drawn to approximate the distribution. Substituting (5.26) into (5.25), we obtain

$$
\left.F \approx \frac{1}{N_{s}} \sum_{i=1}^{N_{M}} N^{i} f(\mathbf{m})\right|_{\mathbf{m} \in \mathbb{M}^{i}}
$$

Using the samples $\mathbf{m}^{j}\left(j=1, \ldots, N_{s}\right)$ of $q$, and assuming that $f$ is nearly constant within $\mathbb{M}^{i}$, we can replace $N^{i} f(\mathbf{m})$ by $\sum_{\mathbf{m}^{j} \in \mathbb{M}^{i}} f\left(\mathbf{m}^{j}\right)$, that is, by the sum over $f$ evaluated a the samples within the subvolume $\mathbb{M}^{i}$. Thus, finally, we obtain an approximation $F_{N_{s}}$ of the integral $F$,

$$
F \approx F_{N_{s}}=\frac{1}{N_{s}} \sum_{j=1}^{N_{s}} f\left(\mathbf{m}^{j}\right)
$$

Applying (5.28) specifically to the approximation of the posterior mean and covariance, we find

$$
\tilde{\mathbf{m}}=\int_{\mathbb{M}} \mathbf{m} \rho\left(\mathbf{m} \mid \mathbf{d}^{\mathrm{obs}}\right) d \mathbf{m} \approx \tilde{\mathbf{m}}_{N_{s}}=\frac{1}{N_{s}} \sum_{j=1}^{N_{s}} \mathbf{m}^{j},
$$

and

$$
\tilde{\mathbf{C}}_{M}=\int_{\mathbb{M}}(\mathbf{m}-\tilde{\mathbf{m}})(\mathbf{m}-\tilde{\mathbf{m}})^{T} \rho\left(\mathbf{m} \mid \mathbf{d}^{\mathrm{obs}}\right) d \mathbf{m} \approx \tilde{\mathbf{C}}_{M, N_{s}}=\frac{1}{N_{s}} \sum_{j=1}^{N_{s}}\left(\mathbf{m}^{j}-\tilde{\mathbf{m}}\right)\left(\mathbf{m}^{j}-\tilde{\mathbf{m}}\right)^{T},
$$

where $\tilde{\mathbf{m}}_{N_{s}}$ and $\tilde{\mathbf{C}}_{M, N_{s}}$ are referred to as the posterior sample mean and the sample covariance, respectively. Furthermore, we find that the normalisation condition is trivially satisfied,

$$
\int_{\mathbb{M}} \rho\left(\mathbf{m} \mid \mathbf{d}^{\mathrm{obs}}\right) d \mathbf{m} \approx \frac{1}{N_{s}} \sum_{j=1}^{N_{s}} 1=1 .
$$

Equations (5.28) to (5.31) naturally raise important questions concerning the quality of the approximations. How many samples do we actually need in order to approximate the integrals reasonably well? How exactly should the samples be generated? Before trying to answer these questions, we remind ourselves of some basic statistical concepts.

\subsubsection{Statistical detour}

In Monte Carlo methods, models $\mathbf{m}$ play the role of random variables. Hypothetically drawing infinitely many models $\mathbf{m}^{j}$, allows us to compute the average model or expectation,

$$
\mu[\mathbf{m}]=\lim _{N_{s} \rightarrow \infty} \frac{1}{N_{s}} \sum_{j=1}^{N_{s}} \mathbf{m}^{j}
$$

The deviation of individual samples $\mathbf{m}^{j}$ from their expectation $\mu[\mathbf{m}]$ can be quantified in terms of the variance,

$$
\sigma^{2}[\mathbf{m}]=\lim _{N_{s} \rightarrow \infty} \frac{1}{N_{s}} \sum_{j=1}^{N_{s}}\left(\mathbf{m}^{j}-\mu[\mathbf{m}]\right)\left(\mathbf{m}^{j}-\mu[\mathbf{m}]\right)^{T} .
$$


The square root of the variance, $\sigma[\mathbf{m}]$, is the standard deviation. Expanding the right-hand side of equation (5.33), we can express the variance in terms of the expectation

$$
\sigma^{2}[\mathbf{m}]=\mu\left[\mathbf{m m}^{T}\right]-\mu[\mathbf{m}] \mu[\mathbf{m}]^{T} .
$$

Equation (5.34) is often regarded as the definition of the variance. Now consider another chain of random samples $\mathbf{n}^{j}$, generated by the same or possibly another random walk algorithm. The two chains are said to be independent when the expectation of their outer product, $\mu\left[\mathbf{m n}^{T}\right]$, is equal to the product of their individual expectations, that is,

$$
\mu\left[\mathbf{m n}^{T}\right]=\mu[\mathbf{m}] \mu[\mathbf{n}]^{T} .
$$

Equipped with this definition, we consider the variance of a sum of random variables, that is, $\sigma^{2}[\mathbf{m}+\mathbf{n}]$. Making use of equation (5.34), we find

$$
\begin{aligned}
\sigma^{2}[\mathbf{m}+\mathbf{n}] & =\mu\left[(\mathbf{m}+\mathbf{n})(\mathbf{m}+\mathbf{n})^{T}\right]-\mu[\mathbf{m}+\mathbf{n}] \mu[\mathbf{m}+\mathbf{n}]^{T} \\
& =\mu\left[\mathbf{m m}^{T}+\mathbf{m n}^{T}+\mathbf{n m}^{T}+\mathbf{n n} \mathbf{n}^{T}\right]-\mu[\mathbf{m}] \mu[\mathbf{m}]^{T}-\mu[\mathbf{m}] \mu[\mathbf{n}]^{T}-\mu[\mathbf{n}] \mu[\mathbf{m}]^{T}-\mu[\mathbf{n}] \mu[\mathbf{n}]^{T} \\
& =\sigma^{2}[\mathbf{m}]+\sigma^{2}[\mathbf{n}]+\mu\left[\mathbf{m} \mathbf{n}^{T}\right]-\mu[\mathbf{m}] \mu[\mathbf{n}]^{T}+\mu\left[\mathbf{n} \mathbf{m}^{T}\right]-\mu[\mathbf{n}] \mu[\mathbf{m}]^{T} .
\end{aligned}
$$

Equation (5.36) is complicated, but an interesting special case arises when $\mathbf{m}$ and $\mathbf{n}$ are independent in the sense of (5.35). Then we find

$$
\sigma^{2}[\mathbf{m}+\mathbf{n}]=\sigma^{2}[\mathbf{m}]+\sigma^{2}[\mathbf{n}] .
$$

Thus, when two random variables are independent, the variance of their sum is equal to the sum of their variances.

\subsubsection{Convergence of Monte Carlo integration}

Returning to our questions concerning the approximation quality, we focus on the simplest case: the estimation of the mean, according to equation (5.29). Assume that we repeat the estimation of $\tilde{\mathbf{m}}_{N_{s}}$ from random samples $\mathbf{m}^{j}$ many times. Each time, we draw a new set of $N_{s}$ random samples, and so we will obtain somewhat different estimates $\tilde{\mathbf{m}}_{N_{s}}$ of the true but unknown mean $\tilde{\mathbf{m}}$. On average, we will obtain the estimate $\mu\left[\tilde{\mathbf{m}}_{N_{s}}\right]$. The individual estimates $\tilde{\mathbf{m}}_{N_{s}}$ obtained from the different sets of random samples will scatter around $\mu\left[\tilde{\mathbf{m}}_{N_{s}}\right]$, and this scatter can be expressed in terms of the standard deviation $\sigma\left[\tilde{\mathbf{m}}_{N_{s}}\right]$.

For a very precise estimate of $\tilde{\mathbf{m}}$ we would expect the scatter to be small. Conversely, when the scatter is large, the individual estimates $\tilde{\mathbf{m}}_{N_{s}}$ are probably not very good approximations of $\tilde{\mathbf{m}}$. Thus, we will use the standard deviation $\sigma\left[\tilde{\mathbf{m}}_{N_{s}}\right]$ to measure the quality of our estimate. To compute $\sigma\left[\tilde{\mathbf{m}}_{N_{s}}\right]$, we first consider the variance, that is, the squared standard deviation,

$$
\sigma^{2}\left[\tilde{\mathbf{m}}_{N_{s}}\right]=\sigma^{2}\left[\frac{1}{N_{s}} \sum_{j=1}^{N_{s}} \mathbf{m}^{j}\right]=\frac{1}{N_{s}^{2}} \sigma^{2}\left[\sum_{j=1}^{N_{s}} \mathbf{m}^{j}\right] .
$$

When the samples $\mathbf{m}^{j}$ are strictly independent, the sum can be taken out of the variance, as shown in the derivation of equation (5.37). This leads to

$$
\sigma^{2}\left[\tilde{m}_{N_{s}}\right]=\frac{1}{N_{s}^{2}} \sum_{j=1}^{N_{s}} \sigma^{2}\left[\mathbf{m}^{j}\right]=\frac{1}{N_{s}} \sigma^{2}\left[\mathbf{m}^{j}\right]
$$

where we assume that the variances of all samples are equal to the variance of one specific sample $\mathbf{m}^{j}$. Taking the square root of (5.39), we obtain the desired estimate of the estimation error, 


$$
\sigma\left[\tilde{\mathbf{m}}_{N_{s}}\right]=\frac{1}{\sqrt{N_{s}}} \sigma\left[\mathbf{m}^{j}\right]
$$

Equation (5.40) has interesting and profound consequences. As expected, the quality of the approximation increases with increasing number of samples, $N_{s}$. However, the improvement is rather slow. Decreasing the error by a factor of 10, requires 100 times more samples. This disadvantage of Monte Carlo integration is balanced by the surprising result that the error is independent of the model space dimension $n$. This is in sharp contrast to brute force grid search introduced in section 5.1, where the required number of samples is proportional to the power of $n$.

Another important aspect of (5.40) is that it only holds when successive samples are statistically independent. In fact, this can be understood intuitively. When successive samples are not independent, they are statistically similar to each other. This situation corresponds to a chain of samples that are rather close to each other, without really exploring model space. In the extreme case, the chain consists of identical models. We may have as many identical models as we can possibly generate. However, this will not at all improve the estimate of the mean, which requires model space exploration.

Though we have derived equation (5.40) for the simple case of estimating the mean, it can in fact be generalised to other integrals. The main features, such as the proportionality of $\sigma\left[\tilde{\mathbf{m}}_{N_{s}}\right]$ to $\frac{1}{\sqrt{N_{s}}}$, and the independence of model space dimension, are universal.

In sections 5.5 and the following, we will see that the independence of the samples is one of the main challenges in Monte Carlo methods. If the random walker makes steps through model space that are too small, subsequent samples are actually dependent, and so convergence of a Monte Carlo integral may slow down considerably. Conversely, if the steps are too large, the walker risks to leave the typical set of plausible models, especially when the model space dimension is high. Hence, the application of Monte Carlo methods will require us to find some reasonable balance.

\subsection{Generating random samples}

Random walks are based on the random proposition of new models. Therefore, they rely on the generation of random numbers from a suitable probability distribution, where the precise notion of suitable remains to be addressed. Thus, before we continue with the description of specific random walk methods, we briefly discuss some basic methods to generate samples from non-uniform probability densities. For this, we assume that some method is available to draw random numbers from the 1-D uniform distribution in the interval $[0,1]$. This functionality is available in any modern programming language.

At least in one dimension, we can transform the samples of the uniform distribution in the interval $[0,1]$ into the samples of any arbitrary distribution by using the transformation rule for probability densities (3.33) in reverse sense. Denoting by $m^{*}$ the samples of the uniform distribution $\rho^{*}\left(m^{*}\right)=1$, we wish to find transformed samples $m=m\left(m^{*}\right)$ that sample the prior $\rho(m)$. Applying the transformation rule without taking the conventional absolute value, we find

$$
\rho^{*}\left(m^{*}\right)=\rho(m) \frac{d m}{d m^{*}} .
$$

Rearranging equation (5.41) and integrating, yields

$$
m^{*}(m)=\int_{-\infty}^{m} \rho(x) d x=\mathscr{P}(m),
$$

where $\mathscr{P}$ is the cumulative density function of the prior. Applying the inverse of $\mathscr{P}$ to both sides of equation (5.42), gives the desired transformation of the uniformly distributed samples $m^{*}$, 


$$
m=\mathscr{P}^{-1}\left(m^{*}\right)
$$

Of course, integrating $\rho$ analytically and finding the inverse of the integral, may still be a difficult task. Due to the use of the inverse cumulative density function, this method of generating samples of $\rho$ is often referred to as inverse transform sampling or inverse probability integral transform.

For many frequently used probability densities or in more than one dimension, inverse transform sampling is difficult because the inverse of the cumulative density function may not be found analytically. This includes the normal distribution. Interestingly, however, the normal distribution with zero mean and unit variance in one dimension can be sampled analytically via a detour through the two-dimensional plane. Assuming that $m_{1}^{*}$ and $m_{2}^{*}$ are both distributed according to the uniform (or homogeneous) distribution $\rho_{h}$ in $[0,1]$, we define the Box-Muller transform, named after mathematicians George Edward Pelham Box and Mervin Edgar Muller, as

$$
\begin{aligned}
& m_{1}=\sqrt{-2 \ln m_{1}^{*}} \cos \left(2 \pi m_{2}^{*}\right), \\
& m_{2}=\sqrt{-2 \ln m_{1}^{*}} \sin \left(2 \pi m_{2}^{*}\right) .
\end{aligned}
$$

The inverse transformation is

$$
\begin{aligned}
& m_{2}^{*}=\frac{1}{2 \pi} \arctan \frac{m_{2}}{m_{1}}, \\
& m_{1}^{*}=e^{-\frac{1}{2}\left(m_{1}^{2}+m_{2}^{2}\right)} .
\end{aligned}
$$

The distribution of the transformed variables is then given by

$$
\rho\left(m_{1}, m_{2}\right)=\left|\operatorname{det} \frac{d \mathbf{m}^{*}}{d \mathbf{m}}\right|=\frac{1}{\sqrt{2 \pi}} e^{-\frac{1}{2} m_{1}^{2}} \frac{1}{\sqrt{2 \pi}} e^{-\frac{1}{2} m_{2}^{2}} .
$$

It follows that the transformed variables are independently and normally distributed in $2 \mathrm{D}$ with zero mean and unit variance.

The Box-Muller transform is easily generalised to higher dimensions, where it allows us to generate samples from the multidimensional Gaussian

$$
\rho(\mathbf{m})=\frac{1}{\sqrt{(2 \pi)^{n} \operatorname{det} \mathbf{C}}} e^{-\frac{1}{2} \mathbf{m}^{T} \mathbf{C}^{-1} \mathbf{m}},
$$

with diagonal covariance matrix $\mathbf{C}$. In the more general case where $\mathbf{C}$ is not diagonal, we can use the approach from section 3.4, based on the Cholesky decomposition of the covariance matrix, $\mathbf{C}^{-1}=\mathbf{L} \mathbf{L}^{T}$. The transformed model

$$
\mathbf{m}^{*}=\mathbf{L}^{T} \mathbf{m}
$$

corresponds to the transformed distribution

$$
\rho^{*}\left(\mathbf{m}^{*}\right)=\frac{1}{(2 \pi)^{n / 2}} e^{-\frac{1}{2}\left(\mathbf{m}^{*}\right)^{T} \mathbf{m}^{*}}
$$

which is again a Gaussian with unit covariance. Thus, conversely, random samples $\mathbf{m}^{*}$ of the distribution with unit covariance (3.34) may be transformed into samples $\mathbf{m}$ of the general multi-dimensional Gaussian via the linear transformation

$$
\mathbf{m}=\mathbf{L}^{-T} \mathbf{m}^{*}
$$

where ${ }^{-T}$ denotes the inverse of the transpose. An illustration of samples drawn from a Gaussian with nondiagonal covariance using equation (5.52) is shown in figure 5.5.

In addition to the analytical approaches presented above, Monte Carlo methods themselves, such as the Metropolis-Hastings algorithm covered in section 5.5, may be used for the purpose of drawing samples not from the posterior but also from some arbitrary prior distribution. However, this sampling approach will usu- 

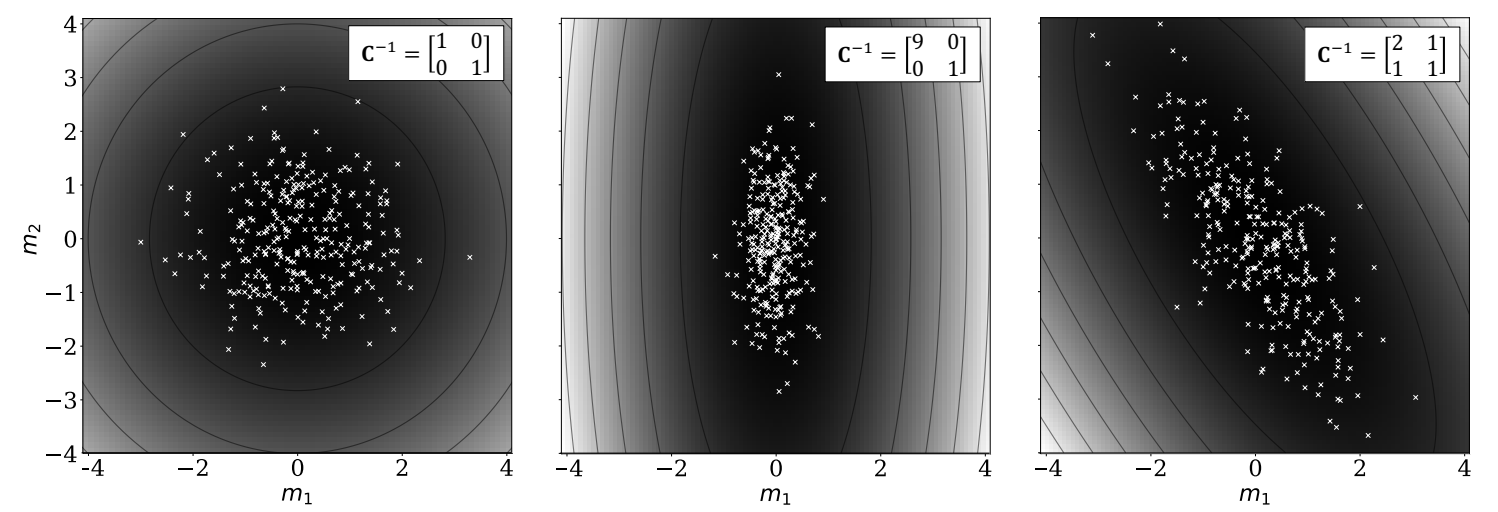

Fig. 5.5: Examples of 2-D Gaussians with different covariance matrices and 300 samples (white crosses) drawn with the help of the coordinate transform approach. [Gaussian samples]

ally require a burn-in phase before it produces samples from the typical set. Hence, despite always being a possible option, it tends to be less efficient than analytical approaches. 


\subsection{The Metropolis-Hastings algorithm and its variants *}

Our interest is of course not to sample an arbitrary distribution $q(\mathbf{m})$ but to sample the posterior $\rho\left(\mathbf{m} \mid \mathbf{d}^{\text {obs }}\right)$. For this, we assume that we already have an algorithm that allows us to propose a move from the current model $\mathbf{m}^{j}$ to some other model $\mathbf{m}^{i}$ with a transition probability $T^{i \leftarrow j}$. Unless we are extremely lucky, $T^{i \leftarrow j}$ will not satisfy the detailed balance relation of the posterior. This means that we cannot just blindly accept any of these proposals. Instead, the proposal must be subjected to some acceptance criterion. Whether the proposal is actually accepted, thus depends on the acceptance probability $A^{i \leftarrow j}$ that modifies the original transition probability $T^{i \leftarrow j}$ to yield a new transition probability $\hat{T}^{i \leftarrow j}$,

$$
\hat{T}^{i \leftarrow j}=A^{i \leftarrow j} T^{i \leftarrow j}
$$

Our goal is to design $A^{i \leftarrow j}$ such that the random walker ends up sampling the posterior $\rho\left(\mathbf{m} \mid \mathbf{d}^{\text {obs }}\right)$. For this, we choose $A^{i \leftarrow j}$ such that the new transition probability $\hat{T}^{i \leftarrow j}$ produces an equilibrium distribution that is equal to the posterior distribution $\rho\left(\mathbf{m} \mid \mathbf{d}^{\text {obs }}\right)$, meaning that we require the detailed balance relation

$$
\hat{T}^{i \leftarrow j} \hat{P}^{j}=\hat{T}^{j \leftarrow i} \hat{P}^{i}
$$

Inserting equation (5.53) into (5.54), we obtain

$$
\frac{A^{i \leftarrow j}}{A^{j \leftarrow i}}=\frac{T^{j \leftarrow i} \hat{P}^{i}}{T^{i \leftarrow j} \hat{P}^{j}} .
$$

Clearly, there are infinitely many acceptance probabilities $A^{i \leftarrow j}$ that satisfy equation (5.55). One possible solution is the logistic rule

$$
A^{i \leftarrow j}=\frac{T^{j \leftarrow i} \hat{P}^{i}}{T^{j \leftarrow i} \hat{P}^{i}+T^{i \leftarrow j \hat{P}^{j}}} .
$$

An alternative to the logistic rule is the Metropolis rule ${ }^{8}$, where the acceptance probability is given by

$$
A^{i \leftarrow j}=\min \left(1, \frac{T^{j \leftarrow i} \hat{P}^{i}}{T^{i \leftarrow j} \hat{P}^{j}}\right) .
$$

The choice of the transition proposals $T^{i \leftarrow j}$ and of the acceptance criterion control critical properties of the random walk, including the number of steps needed to achieve reasonable convergence. We will investigate this issue in the following paragraphs.

\subsubsection{Global Metropolis-Hastings *}

A particularly interesting special case of the Metropolis rule arises when the prior distribution in model space, $\rho(\mathbf{m})$ is used to make transition proposals. This means that the probability of moving from the current $\mathbf{m}^{j}$ to some new $\mathbf{m}^{i}$ only depends on the prior probability of $\mathbf{m}^{i}$, that is,

$$
T^{i \leftarrow j}=P^{i}
$$

\footnotetext{
${ }^{8}$ The history of the Metropolis rule does not seem to be very clear, and various people are credited with its (re)invention. It is named after Nicholas Metropolis (1915 - 1999), co-author of a 1953 paper entitled Equation of state calculations by fast computing machines. In that work, the Metropolis rule was used to sample the Boltzmann distribution $p(x) \propto e^{-x}$. However, according to his co-authors Arianna W. Rosenbluth (who programmed the computer), Marshall N. Rosenbluth (Arianna's husband), Augusta H. Teller, and Edward Teller (1908 - 2003); one of the main inventors of the hydrogen bomb), Metropolis' contribution to the work was rather minor.
} 
By construction, the transition probabilites $T^{i \leftarrow j}$ then satisfy the detailed balance relation of the prior,

$$
T^{i \leftarrow j} P^{j}=T^{j \leftarrow i} P^{i}
$$

Further taking into account that the posterior equals prior times likelihood, that is $M \hat{P}^{i}=L^{i} P^{i}$, we obtain a simplified version of equation (5.55),

$$
\frac{A^{i \leftarrow j}}{A^{j \leftarrow i}}=\frac{L^{i}}{L^{j}} .
$$

The corresponding version of the logistic rule is

$$
A^{i \leftarrow j}=\frac{L^{i}}{L^{i}+L^{j}},
$$

and for the Metropolis rule we find

$$
A^{i \leftarrow j}=\min \left(1, \frac{L^{i}}{L^{j}}\right)
$$

According to the Metropolis rule, the transition from $\mathbf{m}^{j}$ to $\mathbf{m}^{i}$ is always accepted when the likelihood of the new model, $L^{i}$, is higher than the likelihood of the current model, $L^{j}$, because then we have $A^{i \leftarrow j}=1$. When $L^{i}<L^{j}$, however, there is still a chance to make the transition, namely with probability $A^{i \leftarrow j}=\frac{L^{i}}{L^{j}}$. Thus, the proposed transition becomes less probable when the new model is very unlikely.

It is easy to see that the Metropolis rule is more efficient than the logistic rule in the sense that it accepts a proposed transition with generally higher probability. Therefore, the Metropolis rule is more exploratory, whereas the logistic rule has higher tendency to spend more time in a corner of the model space that it already knows, unless it accidentally discovers a new region of the model space that is radically better.

In fact, the Metropolis rule can be shown to be optimal in the sense of being as exploratory as possible: Requesting that a transition from $\mathbf{m}^{i}$ to $\mathbf{m}^{j}$ is always accepted when the likelihood increases, that is $A^{j \leftarrow i}=1$ for $L^{j}>L^{i}$, equation (5.60) transforms to

$$
A^{i \leftarrow j}=\frac{L^{i}}{L^{j}}<1,
$$

which is equivalent to the Metropolis rule. The Metropolis rule as stated in equation (5.62), is the basis of the (global) Metropolis-Hastings algorithm ${ }^{9}$. It implements a random walk where the prior in model space, $\rho(\mathbf{m})$, is used as proposal density, and where transitions are accepted based on the Metropolis rule. Using the prior as proposal density ensures that models that are a priori more likely are visited more frequently, so that less computational resources are wasted on models that are a priori unlikely.

The posterior sampling strategy of the global Metropolis-Hastings algorithm can be formulated in the form of a computational recipe:

${ }^{9}$ Wilfred Keith Hastings (1930 - 2016) generalised the Metropolis rule to enable sampling of arbitrary probability densities, not just the Boltzmann distribution. 


\section{Global Metropolis-Hastings algorithm}

1) Starting from a model $\mathbf{m}^{j}$, propose a transition to $\mathbf{m}^{i}$ according to the prior distribution.

2) Solve the forward problem for the candidate model $\mathbf{m}^{i}$ and evaluate the likelihood function $L^{i}$.

3) Apply the Metropolis rule (5.62) to check if the transition is accepted. Most commonly, the Metropolis rule is implemented numerically using a random number generator that produces uniformly distributed random numbers in the interval $[0,1]$. The transition is accepted if the random number is smaller than $\frac{L^{i}}{L^{j}}$.

4) If the move is accepted, add 1 to the number of times that the walker visited the subvolume around $\mathbf{m}^{i}$. Then go back to 1) and repeat.

5) If the move is not accepted, add 1 to the number of times that the walker visited the subvolume around the original model $\mathbf{m}^{j}$. Not moving is counted as moving from $\mathbf{m}^{j}$ to itself. Then also go back to 1) and try another move.

This procedure is repeated until the relative number of visits to the models has stabilised. Since the number of visits is, by design, proportional to $\hat{P}^{i}$, we can simply visualise the posterior by plotting a histogram that shows the normalised frequency of visits.

The Metropolis-Hastings algorithm constitutes a special case of a Markov chain Monte Carlo (MCMC) method. In the context of random walks through model space, a Markov ${ }^{10}$ chain is a sequence of models where the successor of a model depends at most on itself, but not on any of the predecessors. This means that the development of a Markov chain from a certain model onwards is completely determined by that current model. There is no memory of previous models. Clearly, the Metropolis-Hastings algorithm constructs a Markov chain because the proposal is independent even of the current model, and the acceptance of a proposed step only depends on the likelihood ratio of the current and the proposed model.

Metropolis-Hastings sampling of a ten-dimensional Gaussian: For illustration, we consider a Gaussian prior with a diagonal covariance taking the value 4 :

$$
\rho(\mathbf{m})=\text { constant } e^{-\frac{1}{8} \mathbf{m}^{T} \mathbf{m}} .
$$

The model vector $\mathbf{m}$ is ten-dimensional, that is $\mathbf{m} \in \mathbb{R}^{10}$. In the interest of simplicity, we let the forward problem be linear, $\mathbf{d}=\mathbf{G m}$, and choose a diagonal matrix $\mathbf{G}$ with entries $G_{i i}=i / 10$. Setting the observed data vector components to $d_{i}^{\text {obs }}=i / 5$ suggests $m_{i}=2$ for all $i=1, \ldots, 10$, in the hypothetical case of vanishing measurement errors. Assuming, however, that the measurement errors are independent and normally distributed with unit covariance, we obtain the likelihood function

$$
\rho\left(\mathbf{d}^{\mathrm{obs}} \mid \mathbf{m}\right)=\text { const. } e^{-\frac{1}{2}\left(\mathbf{d}^{\mathrm{obs}}-\mathbf{G m}\right)^{T}\left(\mathbf{d}^{\mathrm{obs}}-\mathbf{G m}\right)} .
$$

Running the Metropolis-Hasting algorithm with $N_{s}=500^{\prime} 000$ samples produces the histograms of the posterior shown in figure 5.6. We observe that $m_{1}$ is less constrained than $m_{10}$ because the posterior is significantly wider. This is to be expected because the small $G_{11}$ allows for large variations in $m_{1}$ that still fall within the measurement error.

\footnotetext{
${ }^{10}$ Andrey Andreyevich Markov (1856 - 1922) was a Russian mathematician who made important contributions to probability theory and differential calculus. Performing rather poorly in school in most subjects, it was realised early that he had enormous talent for mathematics. He was allowed to study at St. Petersburg University where he later became a lecturer, succeeding his own teacher Pafnuty Chebyshev (1821 - 1894). When he refused to monitor his students during the 1908 student riots, he was removed from the university. Following the 1917 February revolution, however, he became an honorary member of St. Petersburg University.
} 

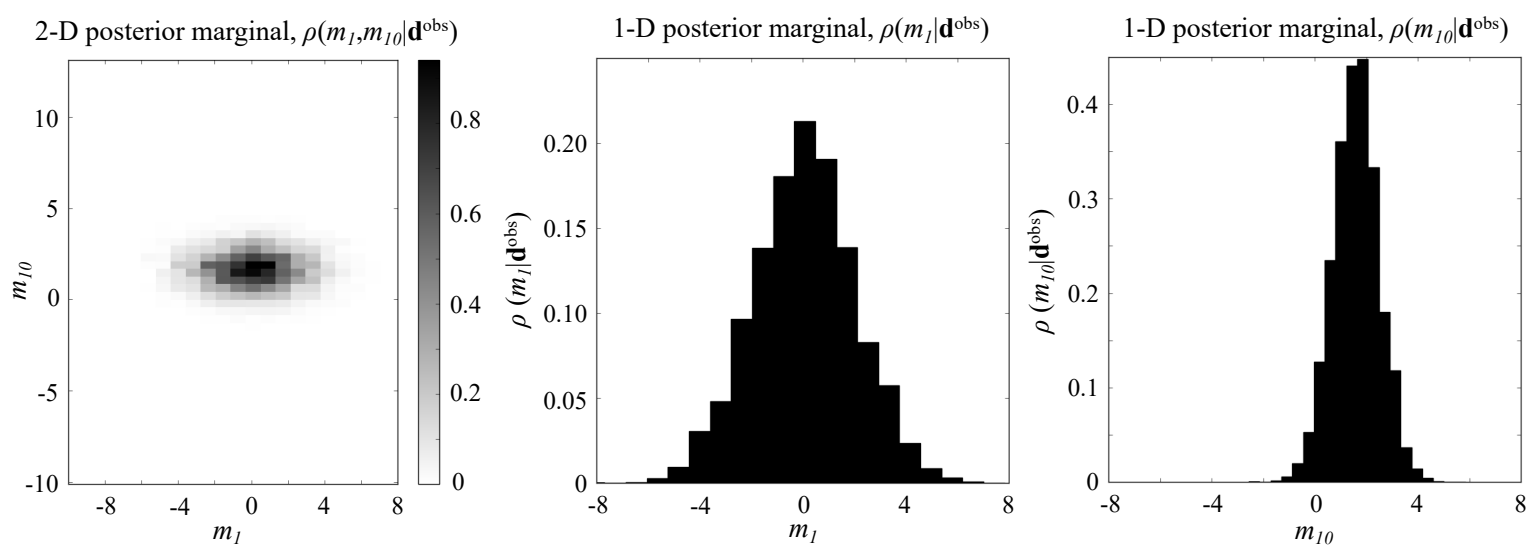

Fig. 5.6: Two- and one-dimensional marginals of the posterior defined through the priors in equations (5.64) and (5.65). Since $d_{1}$ depends only weakly on $m_{1}$ through the small value of $G_{11}$, we find that $m_{1}$ is poorly constrained. Its marginal posterior is nearly identical to the prior. In contrast, $m_{10}$ is well constrained to be approximately 2. Better approximations of the posterior may be obtained using more random samples. [MetropolisHastings]

\subsubsection{Local Metropolis-Hastings}

In the global Metropolis-Hastings algorithm, described in the previous section, proposals to move from the current $\mathbf{m}^{j}$ to a new $\mathbf{m}^{i}$ are based on the prior probability density in model space, $\rho(\mathbf{m})$. Let us, for illustration, consider the very unlikely scenario that our prior $\rho(\mathbf{m})$ happens to equal the posterior $\rho\left(\mathbf{m} \mid \mathbf{d}^{\text {obs }}\right)$. Using this special prior to make proposals in the global Metropolis-Hastings algorithm, means that the transition probability is given by

$$
T^{i \leftarrow j}=P^{i}=\hat{P}^{i} .
$$

The Metropolis rule (5.57) now simplifies to

$$
A^{i \leftarrow j}=\min \left(1, \frac{T^{j \leftarrow i} \hat{P}^{i}}{T^{i \leftarrow j} \hat{P}^{j}}\right)=\min (1,1)=1,
$$

meaning that all proposals are accepted. Usually, our prior knowledge is not that perfect. Otherwise we would not need to solve inverse problems in the first place. Consequently, not all of the proposed models are accepted. The ratio of the total number of proposed samples $N_{s}$ to the number of accepted moves $N_{\text {acc }}$ is called the acceptance rate. A low acceptance ratio means that many proposals are tested without actually making the random walker move to a new position. Therefore, it takes very long to explore the model space.

Low acceptance rates in the global Metropolis-Hastings algorithm are very closely linked to the curse of dimensionality, described in section 5.1.1. As the dimension of the model space increases, the probability of actually finding plausible models decreases very rapidly, and the acceptance rate may drop to uselessly low values. This does not mean that the Metropolis-Hastings algorithm does not converge in theory. However, in practice it may take more samples than we can possibly generate in any reasonable amount of time.

The need to make proposals very carefully, was recognised early on in the pioneering work of Metropolis $e t$ al. (1953). Instead of making proposals globally, independent of $\mathbf{m}^{j}$, they suggested to only go in little steps, not too far from the current position of the walker. For instance, one may choose a Gaussian transition probability,

$$
T^{i \leftarrow j} \propto e^{-\frac{1}{2} \frac{\left(\mathbf{m}^{i}-\mathbf{m}^{j}\right)^{2}}{\sigma^{2}}} .
$$


Equation (5.68) means that moves are preferentially proposed within a distance or search radius $\sigma$ from the current model $\mathbf{m}^{j}$. Long-distance moves, beyond $\sigma$, are rather unlikely. Since $T^{i \leftarrow j}$ is symmetric, $T^{i \leftarrow j}=T^{j \leftarrow i}$, the Metropolis rule (5.57) specialises to

$$
A^{i \leftarrow j}=\min \left(1, \frac{\hat{P}^{i}}{\hat{P}^{j}}\right) .
$$

Thus, instead of the likelihood ratio as in (5.62), the posterior ratio needs to be evaluated. Finding the optimal search radius $\sigma$ is a delicate balancing act that has attracted much interest in applied mathematics research (e.g., Rosenthal, 2011, for a review). It is illustrated in figure 5.7 for the exemplary case of a 2-D bimodal distribution that we wish to sample. When $\sigma$ is too small, the walker is only allowed to go in little steps away from the current position. This makes it unlikely that an implausible model is proposed, and therefore, the acceptance rate is high. The price to pay is a slow exploration of model space. The walker simply does not make much progress, and it takes many samples before high-probability regions are actually discovered. It is said that the mixing of the Markov chain is poor. The opposite behaviour occurs for large search radii. The likelihood of proposing a low-probability model, far from the current position, is rather high. Consequently, the acceptance rate drops, and many proposals are wasted. The benefit, however, is that the walker makes fast progress in exploring model space.

Though algorithms for finding an optimal search radius exist, the tuning of $\sigma$ is mostly performed manually in practice. While not being quantitative, the Goldilocks principle ${ }^{11}$ of just not too much and not too little often yields reasonable results. In terms of the acceptance rate, the Goldilocks principle suggests that $N_{\text {acc }} / N_{s}$ should range roughly between 0.5 and 0.9 . Lower values indicate that the search radius may be too large, whereas higher values are likely to result from a search radius that is too small.

A useful visual diagnostic for the performance of a Markov chain are trace plots, which are simply plots of selected components of the model vector as a function of sample index. Examples are shown in the top row of figure 5.8. For a Markov chain that is well mixed, a trace plot should roughly look like uncorrelated noise or a hairy caterpillar. In the case of poor mixing, the trace plots indicate that the walker remained in similar positions for a relatively long time, thereby causing the sampling to be rather inefficient.

A complementary tuning approach is based on the concept of the effective sample size $N_{\text {eff }}$. It related back to the convergence analysis of Monte Carlo integration in section 5.3.3, which was based on the assumption of perfectly independent samples. The Metropolis-Hastings algorithm, however, cannot produce samples that are perfectly independent because the choice of moving to the next position depends on the current position of the walker via the Metropolis rule. Only after a certain number of steps will the samples effectively become independent. Hence, we would like to know what that number of steps is. The ratio $N_{s} / N_{\text {eff }}$ answers precisely this question.

The Monte Carlo literature is replete with various estimates of $N_{\text {eff }}$. A widely used one is based on the autocorrelation of the sample chain

$$
c(k)=\frac{\sum_{i=1}^{N_{s}} m^{i} m^{i+k}}{\sum_{i}\left(m^{i}\right)^{2}},
$$

where $m^{i}$ is one component of the $N$-dimensional model vector $\mathbf{m}^{i}$. The autocorrelation is a measure of sample dependence. An autocorrelation that drops to 0 only slowly, indicates relatively strong dependence, and vice versa. Examples for the sample chains from figure 5.7 are shown in figure 5.8.

Based on the sample autocorrelation, an estimate of the effective sample size can be computed as (e.g. Ripley, 1987)

$$
N_{\text {eff }}=\frac{1}{1+2 \sum_{k=1}^{\infty} c(k)} .
$$

\footnotetext{
${ }^{11}$ In the 19th-century fairy-tale The Three Bears, a little girl named Goldilocks tastes three different bowls of porridge. She finds that she prefers porridge that is neither too hot nor too cold, but has just the right temperature.
} 
a) $\sigma=1.5$ (useful)
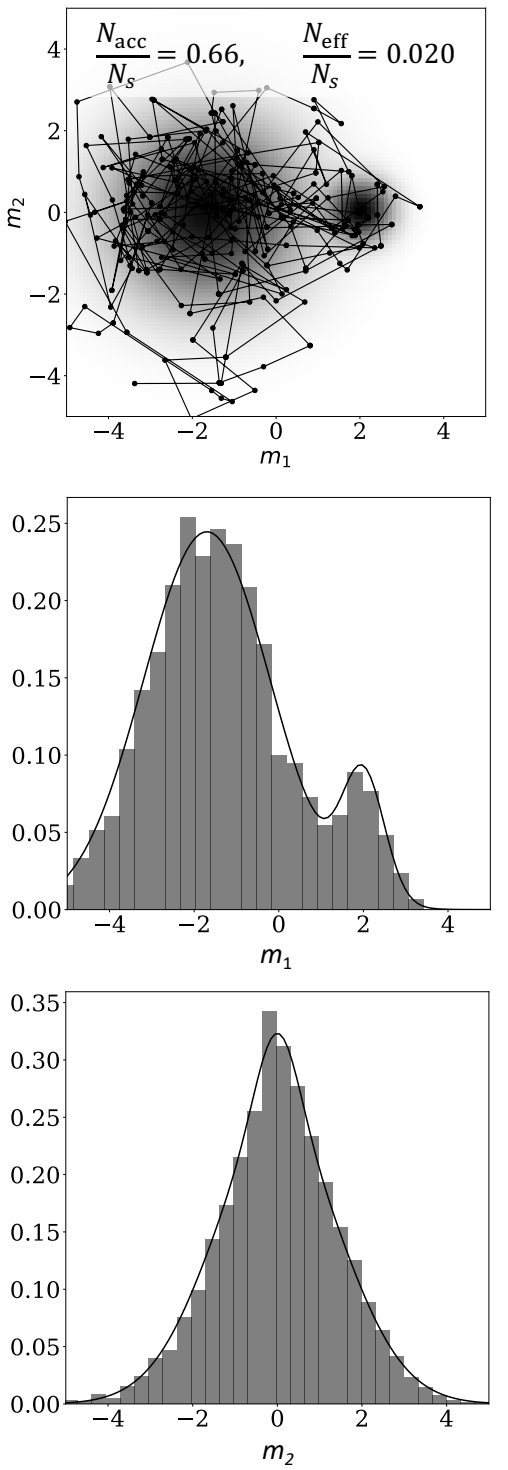

b) $\sigma=0.15$ (too small)
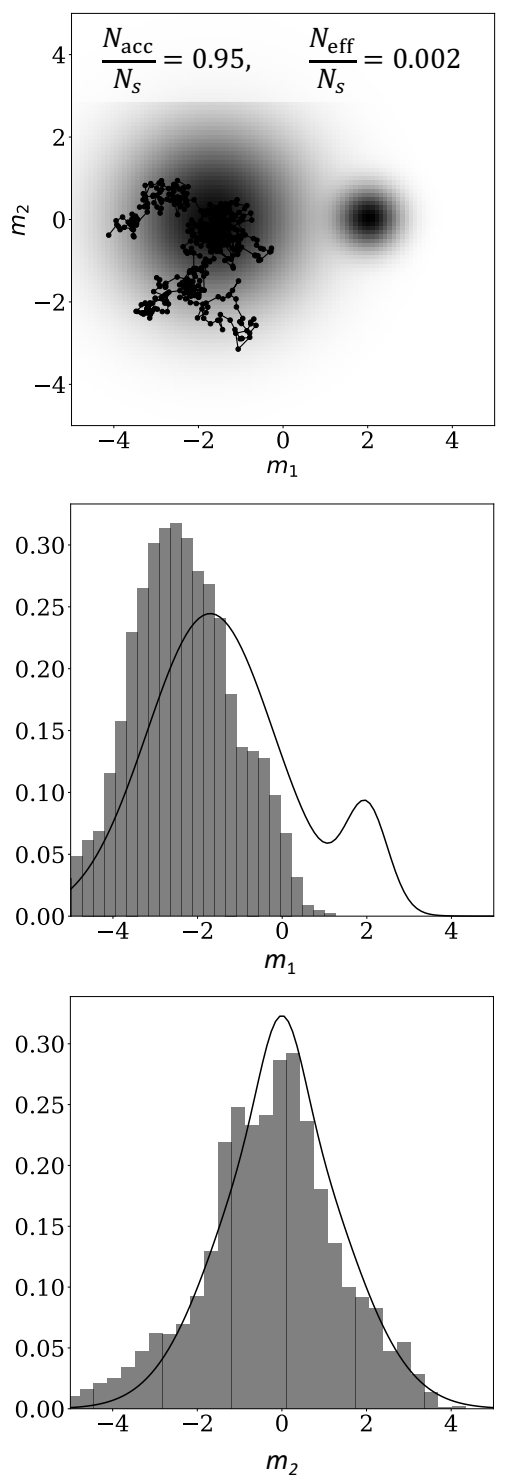

c) $\sigma=15.0$ (too large)
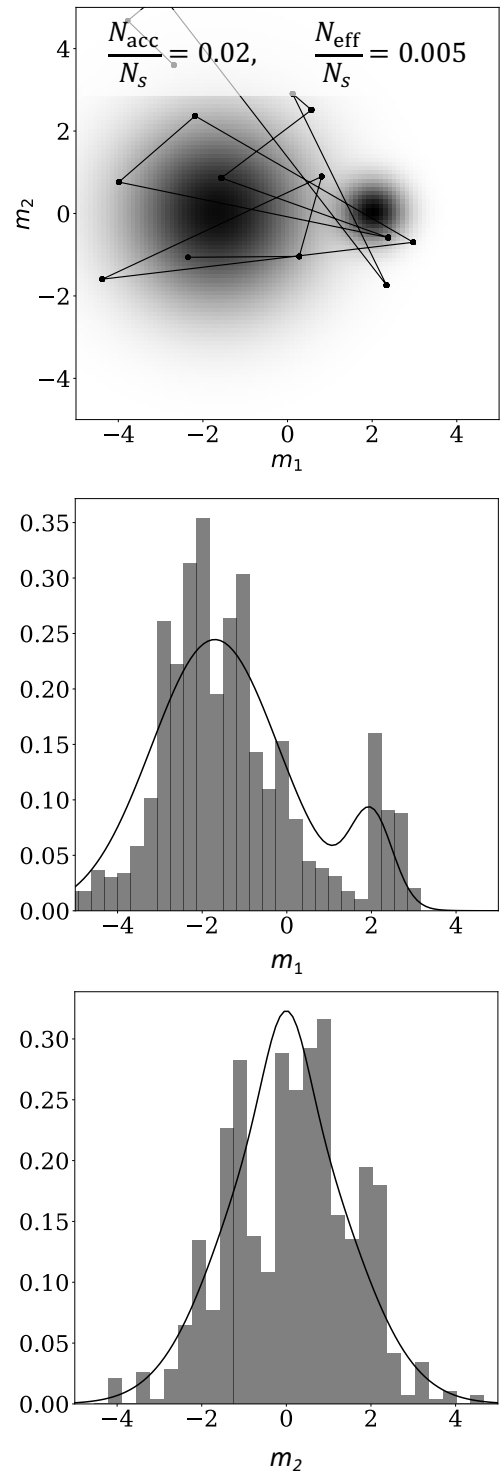

Fig. 5.7: Illustration of the local Metropolis-Hastings algorithm for different choices of the search radius $\sigma$ in (5.69) and a total of $N_{s}=10^{\prime} 000$ samples. The top panels show the first 500 samples superimposed on the target probability density, where darker colours mean larger values. The middle and lower panels contain the two 1-D marginals in their exact form as black curve and the sample-based histogram approximation. While $\sigma=1.5$ (a) is a useful value that leads to a good approximation of the 1-D marginals by the sample histograms, $\sigma=0.15$ (b) and $\sigma=15.0$ (c) are too small and too large, respectively. Excessively large or small search radii cause subsequent samples to be highly correlated, either because a single step is too small or because a large step is unlikely to be accepted. In the extreme cases, the marginals are poorly approximated by the sample histogram. [Metropolis-Hastings] 
In practice, the number of available samples is of course not infinite, and so the sum in (5.71) is typically truncated, for instance, at the index $k$ where the sum of subsequent autocorrelation values is below 0 for the first time (Gelman et al., 2013). The effective sample sizes for different choices of the search radius are indicated in figure 5.7. They confirm that both excessively small or large search radii produce very small effective sample sizes.

a) $\sigma=1.5$ (useful)
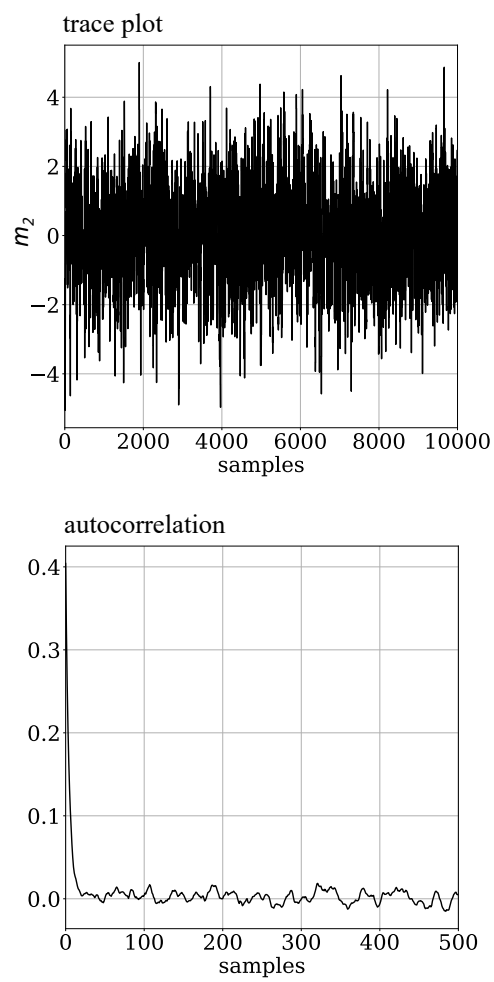

b) $\sigma=0.15$ (too small)
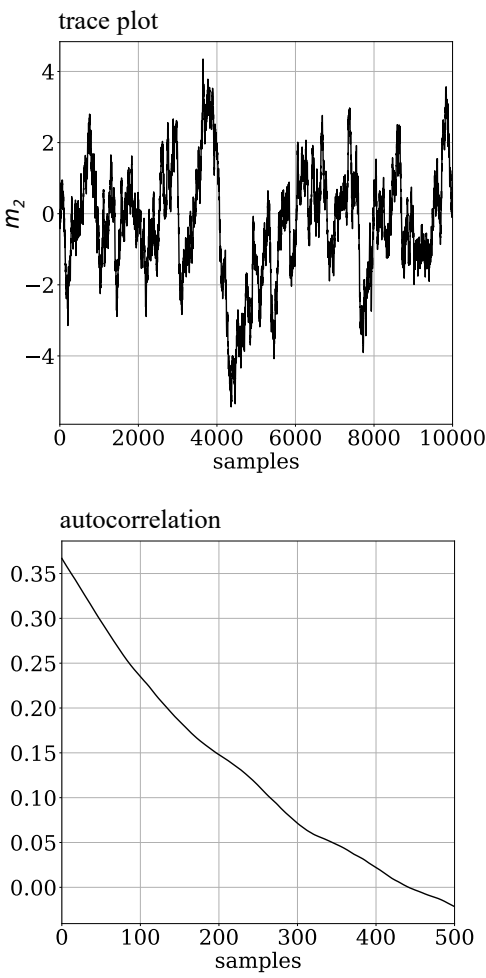

c) $\sigma=15.0$ (too large)
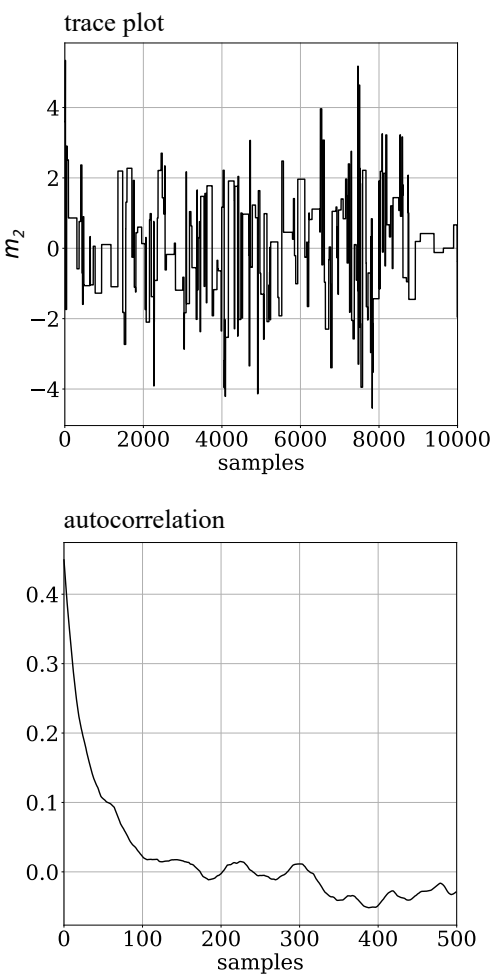

Fig. 5.8: Trace plots (top) and autocorrelations (bottom) for the $m_{2}$-component of scenarios from figure 5.7. For search radii that are either too large or too small, the trace plots show structure that indicates that the random walker remained in similar positions for a relatively long time. This is reflected in the autocorrelations, which decay to 0 only slowly. In contrast, for $\sigma=1.5$, the trace plot visually appears like nearly uncorrelated noise or a hairy caterpillar, which translates to an autocorrelation that drops to 0 after only few samples. [MetropolisHastings]

The search radius $\sigma$ that maximises the effective sample size depends on many factors, including the distribution that we wish to sample. Trying to find a reasonable $\sigma$ by running some tests with smaller sample sizes, is a good thing to do. However, there is no point in over-doing it in practice. 


\subsubsection{The Metropolis-adjusted Langevin algorithm (MALA)}

The two basic variants of the Metropolis-Hastings algorithm presented in sections 5.5.1 and 5.5.2 gather information about the target density only through the evaluation of the Metropolis rule, whereas the proposal itself is purely random. As a consequence, they can be slow in approaching and sampling the typical set. To produce more informed proposals, we first write the local Metropolis-Hastings algorithm, so far described by the acceptance probability (5.69), as an update of the current model,

$$
\mathbf{m}^{i}=\mathbf{m}^{j}+\sigma \Delta \mathbf{m}^{j}
$$

with an update $\Delta \mathbf{m}^{j}$ drawn from a Gaussian with unit covariance matrix. Our goal is to modify (5.72) such that the update becomes biased towards regions of model space where the posterior $\rho\left(\mathbf{m} \mid \mathbf{d}^{\text {obs }}\right)$ is large. To achieve this, we may add a new term to (5.72) that involves the relative gradient of $\rho\left(\mathbf{m} \mid \mathbf{d}^{\text {obs }}\right)$,

$$
\frac{1}{\rho\left(\mathbf{m} \mid \mathbf{d}^{\mathrm{obs}}\right)} \nabla \rho\left(\mathbf{m} \mid \mathbf{d}^{\mathrm{obs}}\right)=\nabla \ln \rho\left(\mathbf{m} \mid \mathbf{d}^{\mathrm{obs}}\right) \text {. }
$$

The gradient $\nabla \rho\left(\mathbf{m} \mid \mathbf{d}^{\text {obs }}\right)$ points into the direction of steepest ascent, that is, the direction in which $\rho\left(\mathbf{m} \mid \mathbf{d}^{\text {obs }}\right)$ increases most rapidly. (See also section 8.3 on the steepest descent method.) The division by $\rho\left(\mathbf{m} \mid \mathbf{d}^{\text {obs }}\right)$ promotes large moves towards towards more probable regions of model space especially when the current probability density is low. Adding a scaled version of (5.73) to (5.72), we obtain

$$
\mathbf{m}^{i}=\mathbf{m}^{j}+\frac{\sigma^{2}}{2} \nabla \ln \rho\left(\mathbf{m}^{j} \mid \mathbf{d}^{\mathrm{obs}}\right)+\sigma \Delta \mathbf{m}^{j} .
$$

For $\sigma \ll 1$, the update (5.74) resembles the local Metropolis-Hastings algorithm with small search radius. In contrast, for larger values of $\sigma$, the update becomes increasingly deterministic and driven towards higherprobability regions. Still drawing the increment $\Delta \mathbf{m}^{j}$ from a Gaussian with unit covariance matrix, the transition probability is given by

$$
T^{i \leftarrow j} \propto e^{-\frac{1}{2 \sigma^{2}}\left(\Delta \mathbf{m}^{j}\right)^{T}\left(\Delta \mathbf{m}^{j}\right)} .
$$

Using (5.75) in the general Metropolis rule (5.57), gives rise to a variant of the Metropolis-Hastings algorithm known as the Metropolis-adjusted Langevin ${ }^{12}$ algorithm (MALA).

In contrast to the pure random-walk Metropolis-Hastings (RWMH) variants in sections 5.5.1 and 5.5.2, MALA has a deterministic component. By design, this component biases the walker towards regions of higher probability, meaning that the typical set may be approached and explored faster. This behaviour is illustrated in figure 5.9a,b, showing the first ten steps of MALA compared to a local Metropolis-Hastings sampler for the same example distribution used in figure 5.7.

Methodologically, MALA is a hybrid between the local Metropolis-Hastings algorithm and the method of steepest descent (section 8.3), which can be used to approximate the minimum of a function by iteratively walking opposite to the gradient direction. As we will see later in section 5.7, MALA can also be interpreted as a variant of the , which makes proposals not on the basis of one but multiple gradient evaluations.

MALA can be shown to converge faster towards the target distribution than RWMH, and the benefit increases for increasing model space dimension (Rosenthal, 2011). However, this comes at the price of having to compute the derivative (5.73), which can be challenging, especially when the forward problem involves the solution of a differential equation. A possible solution are adjoint methods, covered in chapter 9.

\footnotetext{
12 Paul Langevin (1872 - 1946) was a French physicist known for his contributions to magnetism, statistical mechanics, and ultrasonic submarine detection. Being a vocal opponent to Nazism, he co-founded the Watchfulness Committee of Antifascist Intellectuals, and was president of the Human Rights League.
} 
a) $\operatorname{MALA}(\sigma=1.0)$

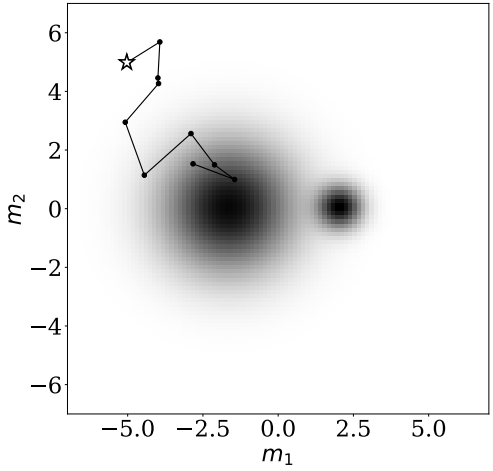

b) local Metropolis-Hastings $(\sigma=1.0)$

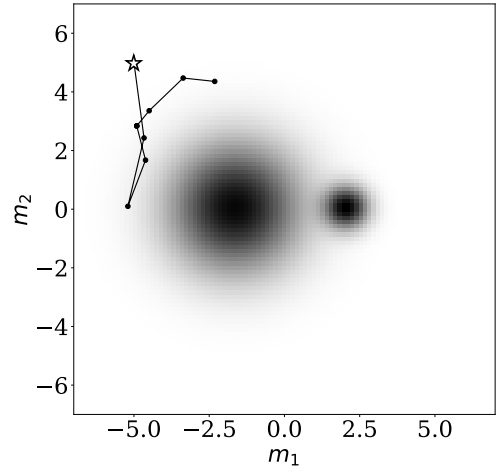

c) Brownian motion $(\sigma=0.25)$

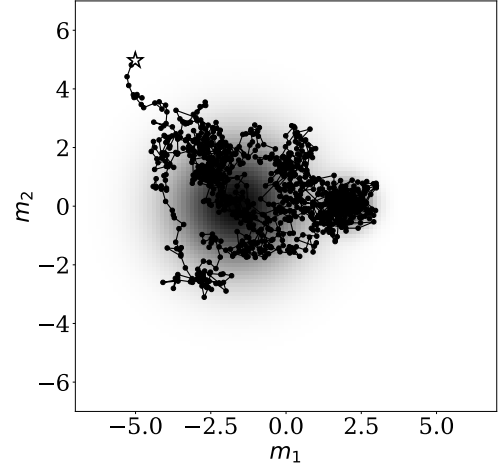

Fig. 5.9: Comparison of MALA and the local Metropolis-Hastings algorithm for a 2-D probability density. Panels (a) and (b) show a typical example of the first ten steps using the two algorithms, superimposed in the target probability density. (Darker colours mean higher probability density.) The initial model is indicated by a star. While MALA approaches the typical set within a few steps, the local Metropolis-Hastings algorithm requires more samples to discover the regions of higher probability density. Not all ten moves are visible because some proposed transitions have been rejected. For smaller values of $\sigma$, nearly $100 \%$ of the moves are accepted, and MALA effectively becomes a simulation of Brownian motion, as illustrated in panel (c) and explained in the box on Langevin diffusion. [MALA]

Langevin diffusion and Brownian motion: MALA inherits its name from the Langevin equation, which appears in the study of Brownian motion ${ }^{a}$,

$$
\delta \mathbf{v}=-\frac{1}{2} \delta t \lambda \mathbf{v}+\delta \mathbf{f}
$$

Equation (5.76) describes the infinitesimal velocity change $\delta \mathbf{v}$ of a small particle moving through some fluid medium during an infinitesimal time interval $\delta t$. The driving force $\delta \mathbf{f}$ of this velocity change, or acceleration, results from collisions of the particle with the molecules of the fluid. At the same time, the acceleration is counteracted by viscous forces within the fluid, which are proportional to the current velocity $\mathbf{v}$, the time increment $\delta t$, and the material-specific damping coefficient $\lambda$.

Owing to the randomness of the collisions between the particle and the surrounding molecules, the force increment $\delta \mathbf{f}$ is a random variable, commonly thought to have a normal distribution with variance $\delta t$, that is, $\delta \mathbf{f} \sim \mathscr{N}(\mathbf{0}, \delta t \mathbf{I})$. Hence, longer time intervals $\delta t$ lead to force increments $\delta \mathbf{f}$ that are on average larger. We may rewrite the force increment as $\delta \mathbf{f}=\sqrt{\delta t} \mathbf{f}$, where $\mathbf{f}$ follows a standard normal distribution, $\mathbf{f} \sim \mathscr{N}(\mathbf{0}, \mathbf{I})$. Furthermore, denoting the current time $t$ with superscript $j$ and time $t+\delta t$ with superscript $i$, and writing the velocity change as $\delta \mathbf{v}=\mathbf{v}^{i}-\mathbf{v}^{j}$, we obtain a version of the Langevin equation (5.76) that closely resembles the MALA update equation (5.74),

$$
\mathbf{v}^{i}=\mathbf{v}^{j}-\frac{1}{2} \delta t \lambda \mathbf{v}+\sqrt{\delta t} \mathbf{f} .
$$

We obtain an exact copy of (5.74) by renaming the time increment, $\delta t=\sigma^{2}$, and writing the viscous deceleration $-\lambda \mathbf{v}$ as the derivative of the logarithm of a standard Gaussian distribution,

$$
-\lambda \mathbf{v}=\nabla \ln c e^{-\lambda \mathbf{v}^{T} \mathbf{v}}
$$


with some normalisation constant $c$. This analogy reveals that MALA can be interpreted as a simulation of the Brownian motion of a small particle, when the search radius $\sigma$ is so small that practically all proposals are accepted. Seen from the opposite direction, the fact that MALA produces a random walk with equilibrium distribution $\rho\left(\mathbf{m} \mid \mathbf{d}^{\text {obs }}\right)$, allows us to infer that the velocity distribution of Brownian motion must, in accord with (5.78), be a Gaussian with zero mean and variance $\lambda^{-1}$, that is, $\mathbf{v} \sim$ $\mathscr{N}\left(\mathbf{0}, \lambda^{-1} \mathbf{I}\right)$. Strong viscous damping, expressed in terms of large values of $\lambda$, is therefore expected to produce small velocities, and vice versa.

${ }^{a}$ Brownian motion is named after the Scottish botanist Robert Brown (1773 - 1858), who made significant contributions to his field thanks to his pioneering use of microscopes. Observing plant pollen suspended in water, he observed a continuous, random motion of small particles, which he incorrectly interpreted as an expression of microscopic life. 


\subsection{Tempering and Simulated Annealing *}

The Metropolis-Hastings algorithm solves the very general problem of sampling an arbitrary probability density. Its efficiency, however, depends critically on the proximity of the prior to the posterior that we wish to sample, and on the complexity of the posterior. Using, for instance, a uniform prior, the global MetropolisHastings algorithm may have difficulties finding the isolated peak shown in figure 5.10b, simply because it occupies a very small volume of the model space. Due to the curse of dimensionality, this detectability problem becomes more severe in higher dimensions. Though the Metropolis-Hastings algorithm will ultimately converge, the number of samples that is required before one of them accidentally hits the isolated peak may be excessively large.

Since the development of the Metropolis-Hastings algorithm, many solutions to this problem have been proposed. The most straightforward one is certainly tempering. The simple idea is to raise the target probability density that we wish to sample, for instance, $\rho\left(\mathbf{m} \mid \mathbf{d}^{\text {obs }}\right)$, to the power of $T_{0} / T$, where $T_{0}$ is interpreted as a reference temperature, and $T$ as the current temperature:

$$
\rho_{T}\left(\mathbf{m} \mid \mathbf{d}^{\mathrm{obs}}\right)=\left[\rho\left(\mathbf{m} \mid \mathbf{d}^{\mathrm{obs}}\right)\right]^{T_{0} / T} .
$$

For temperatures that are high compared to the reference temperature, $T>T_{0}$, the modified distribution $\rho_{T}$ is much more smooth. In fact, the peaked distribution in figure $5.10 \mathrm{~b}$ is transformed into the more slowly varying distribution shown in figure 5.10c, as temperature is increased from $T=T_{0}=1$ to $T=20$. The isolated peak in the original distribution has become broader, and even merged with the other peaks. As a consequence, it is easier to detect by the Metropolis-Hastings algorithm. Using, as another example, the simple Gaussian with variance $\sigma$,

$$
\rho\left(\mathbf{m} \mid \mathbf{d}^{\mathrm{obs}}\right)=\text { const. } e^{-\frac{1}{2 \sigma^{2}} \mathbf{m}^{T} \mathbf{m}},
$$

tempering produces the modified Gaussian

$$
\rho_{T}\left(\mathbf{m} \mid \mathbf{d}^{\text {obs }}\right)=\text { const. } e^{-\frac{T_{0}}{2 T \sigma^{2}} \mathbf{m}^{T} \mathbf{m}} .
$$

Thus, tempering has changed the original standard deviation from $\sigma$ to $\sigma \sqrt{\frac{T}{T_{0}}}>\sigma$. Using the MetropolisHastings algorithm in any of its variants to sample the heated distribution $\rho_{T}$ will produce histograms that approximate $\rho_{T}$ increasingly well, as the number of samples increases. Since we are ultimately not interested in $\rho_{T}$ but in $\rho$, it then suffices to rescale the histogram by raising it to the power of $T / T_{0}$ in order to obtain the desired histogram of $\rho$.

If we change our objectives from sampling the posterior to finding the posterior maximum-likelihood model, we can reverse the concept of tempering, which leads to annealing. In fact, decreasing temperature to $T<T_{0}$ has the opposite effect of tempering. The variance of the Gaussian in (5.80) decreases, and the isolated peak in figure 5.10b becomes even more isolated, as shown in figure 5.10a. Ultimately, when cooling sufficiently, the highest peak of the orignal distribution $\rho$ ends up being the only peak of the cooled distribution $\rho_{T}$.

This simple observation is the basis of Simulated Annealing ${ }^{13}$, first proposed by Kirkpatrick et al. (1983). Starting at a very high temperature, $T \gg T_{0}$, the Metropolis-Hastings algorithm is used to sample the heated $\rho_{T}$. Then, temperature is successively lowered. Ultimately, the Metropolis-Hastings algorithm will sample a very cool distribution $\rho_{T \ll T_{0}}$, consisting of a single narrow peak centred on the maximum-likelihood model.

The strength of Simulated Annealing is the guaranteed convergence towards the global maximum-likelihood model, without getting trapped in local maxima. This property results from the ability to transition from models

${ }^{13}$ Simulated Annealing can be interpreted as a mathematical model of a cooling melt where molecules try to find the minimumenergy position while temperature decreases. During the annealing process, the molecules randomly test a large number of configurations until the optimum is found, and the melt has turned into a crystal. If cooling is too fast, only a meta-stable local optimum is found, and we obtain a glass instead of a well-ordered crystal. 
a) annealed ('cooled') probability density

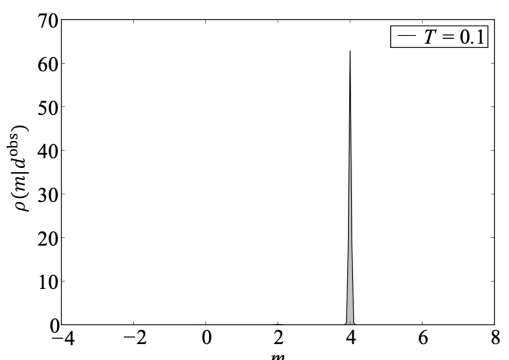

b) original probability density

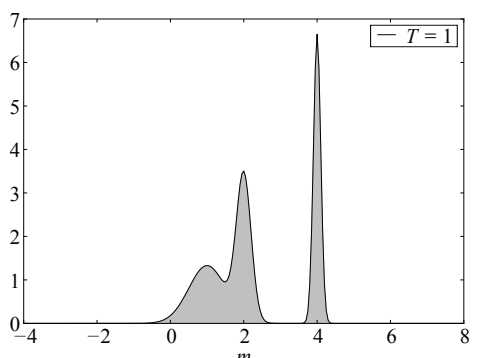

c) tempered ('heated') probability density

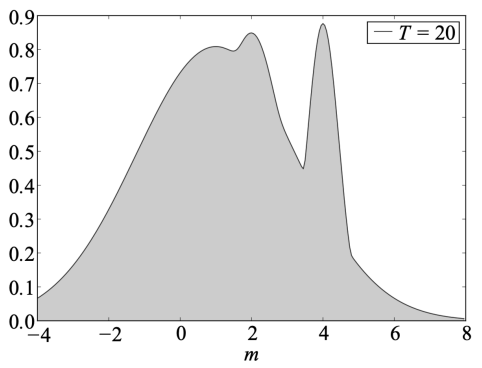

decreasing temperature

'annealing'

'tempering'

Fig. 5.10: Illustration of tempering and annealing. Increasing the original distribution $\rho$ in the centre from temperature $T=T_{0}=1$ to $T>T_{0}$ is called tempering. It produces a smoother variant of $\rho$ that can be sampled more easily. Decreasing temperature, referred to as annealing, emphasises the peak centred around the maximum-likelihood model.

with higher likelihood to models with lower likelihood, which is a consequence of the Metropolis rule. Thus, even when the algorithm samples the vicinity of a local maximum, it can still escape. It is, in this sense, a global optimiser.

The weakness of Simulated Annealing is that guaranteed convergence only holds for infinitely slow cooling, which is not possible in practice. In real-world applications, we require a cooling schedule that reduces the temperature to practically zero in a finite number of steps. If cooling is too fast, the algorithm may lock into a local maximum without any chance to ever escape again. Though efficient cooling schedules can be derived rigorously, some trial and error usually yields satisfactory results.

Global optimisation using Simulated Annealing: In addition to finding the maximum-likelihood model of a probability distribution, one may use Simulated Annealing in order to find the global minimum of some cost or misfit function $\chi(\mathbf{m})$, not necessarily related to the solution of an inverse problem. For instance, $\chi$ may literally be the cost of travelling by train from A to B, as a function of the trajectory m. To apply Simulated Annealing in this context, we define an auxiliary probability density

$$
\rho_{a}(\mathbf{m})=\text { const. } e^{-\chi(\mathbf{m})} \text {. }
$$

Running Simulated Annealing on $\rho_{a}$ will then give the model (trajectory) $\mathbf{m}$ that minimises the cost function $\chi$.

A disadvantage of Simulated Annealing is that most of the proposed models are rejected when the temperature is low, meaning that computational resources tend to be wasted. Without details, we mention here the Fast Simulated Annealing and the Very Fast Simulated Reannealing variants by Szu \& Hartley (1987) and Ingber (1989), respectively. These and other modifications of the original algorithm are summarised in Sen \& Stoffa (2013). 


\subsection{Hamiltonian Monte Carlo}

The advantage of the global Metropolis-Hasting algorithm (section 5.5.1) over a brute-force grid search lies in its ability to take prior knowledge into account. Only samples that are a priori relevant are proposed for testing via the Metropolis rule. Unsurprisingly, the efficiency of the global Metropolis-Hastings algorithm depends critically on the quality of the prior knowledge. When the prior is already a good approximation of the posterior, the sampling will be very efficient, and a good approximation of the posterior may be obtained with a reasonably small number of samples. However, when the prior is far from the posterior, many test models will be wasted in regions of the model space that are irrelevant to the posterior.

The magnitude of this effect is illustrated in figure 5.11, which uses the ten-dimensional Gaussian toy problem from figure 5.6. The left panel shows the trajectory of the random walker projected onto the $\left(m_{1}, m_{10}\right)$ plane when the standard deviation of the prior, from which proposals are drawn, is $\sigma=1$. Each grey dot represents an accepted test model. There are $60^{\prime} 176$ of these dots, meaning that around $12 \%$ of the $500^{\prime} 000$ proposed samples have been accepted. Increasing the standard deviation of the prior from $\sigma=1$ to $\sigma=3$, corresponds to a weakening of our prior knowledge. A priori, we know less precisely where a posteriori relevant models may be located. Therefore, the proposed samples scatter more widely, and more of them will not be accepted by the Metropolis rule. As a consequence, only $7^{\prime} 121$ of the $500^{\prime} 000$ proposed samples are accepted; roughly $1.4 \%$. Further weakening our prior knowledge by setting the prior standard deviation to $\sigma=7$, reduces the number of accepted models to 142, that is around $0.028 \%$. For $\sigma=10$, only 7 models are accepted (on average over many runs), that is around $0.0014 \%$. In other words, the random walker remains at a certain position for a very long time before a proposed model actually passes the Metropolis test.

This undesirable phenomenon can be explained with the curse of dimensionality, analysed in section 5.1.1. In a high-dimensional model space, the volume of relevant models defined by the posterior becomes excessively small compared to the volume of the whole model space. If the proposal density, that is the prior in the case of global Metropolis-Hastings, does not limit the search space very significantly, the probability to find a new model that is better than an already relatively good one becomes negligibly small. Therefore, the random walker will mostly stay at its current position without really exploring the model space. To some extent, the low acceptance rate can be overcome by the local Metropolis-Hastings sampler (section 5.5.2), however, at the price of very slow model space exploration.

Hamiltonian Monte Carlo (HMC) is designed to overcome the problem of inefficient sampling when the prior knowledge is not sufficiently close to the posterior. In contrast to being drawn randomly, a new proposal is already influenced by the data. Starting from the current model, the random walker follows a trajectory towards a new model, just as if the walker were a particle in $n$-dimensional space moving on a surface under the influence of gravity.

Hamiltonian Monte Carlo was developed for problems where the derivative of the target probability density with respect to the model parameters can be computed quickly (e.g. Betancourt, 2017; Neal, 2011). Using derivatives, in contrast, for instance, to the Metropolis-Hastings algorithm, helps to focus on those regions of model space that are more plausible, thus wasting less samples. Since its first formulation for applications in lattice quantum chromodynamics (Duane et al., 1987), HMC has found widespread use in neural networks and machine learning (Bishop, 2006; Gutmann \& Hyvärinen, 2012; Neal, 1996), molecular simulations (e.g. Dubbledam et al., 2016), nuclear physics (e.g. Elhatisari et al., 2015), genomics (e.g. Honkela et al., 2015), signal processing (e.g. Wei et al., 2015), and quantum mechanics (e.g. Seah et al., 2015). Applications to geophysical problems may be found, for instance, in Muir \& Tkalčić (2015), Biswas \& Sen (2017), Sen \& Biswas (2017), Fichtner \& Simute (2018), Fichtner et al. (2019), Muir \& Tkalčić (2020), and Gebraad et al. (2020).

The basic idea of Hamiltonian Monte Carlo is to treat a model $\mathbf{m}$ of dimension $n$ as an $n$-dimensional particle. This particle is under the influence of a force, which exerts an attraction towards model space regions with higher posterior probability. The trajectory of the particle, that is, the model, is controlled by Hamilton's equa- 

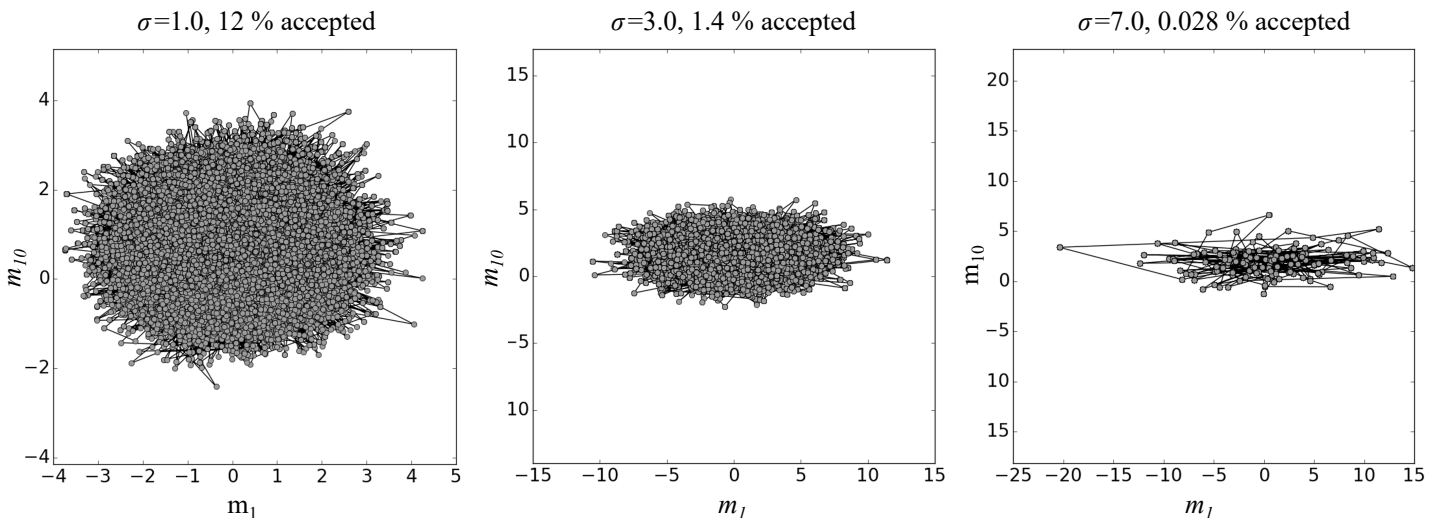

Fig. 5.11: Trajectory of the random walker from the ten-dimensional Gaussian example from figure 5.6 for three different standard deviations of the Gaussian prior, $\sigma=1, \sigma=3$, and $\sigma=7$, used to make proposals in the global Metropolis-Hastings algorithm. Each grey dot represents an accepted model projected onto the $\left(m_{1}, m_{10}\right)$ plane in the ten-dimensional model space. The total number of proposed samples is $500^{\prime} 000$. As the prior standard deviation increases, the number of accepted samples decreases dramatically, leading to a very poor exploration of the model space and an enormous waste of computing time.

tions from classical mechanics. Thus, before delving into the details of the Hamiltonian Monte Carlo algorithm, we provide a brief recapitulation of Hamiltonian mechanics.

\subsubsection{Review of Hamiltonian mechanics}

The trajectory of a particle as a function of time $t$ in an $n$-dimensional space is determined by Hamilton's ${ }^{14}$ equations

$$
\frac{d m_{i}}{d t}=\frac{\partial H}{\partial p_{i}}
$$

and

$$
\frac{d p_{i}}{d t}=-\frac{\partial H}{\partial m_{i}}
$$

where $i$ ranges from 1 to $n$. In the context of Hamiltonian mechanics, $m_{i}$ plays the role of a generalised position, and $p_{i}$ is a generalised momentum defined through

$$
p_{i}=\mu_{i} \frac{d m_{i}}{d t}
$$

with positive $\mu_{i}$ interpreted as masses. The function $H(\mathbf{m}, \mathbf{p})$ is the total energy of the particle, that is $H=K+U$ with the kinetic energy $K(\mathbf{m}, \mathbf{p})$ and the potential energy $U(\mathbf{m}, \mathbf{p})$. It is referred to as the Hamiltonian. The dependence of the model parameters on time is of course artificial, and only a means of translating one model

\footnotetext{
${ }^{14}$ William Rowan Hamilton (1805-1865) was an Irish physicist, astronomer and mathematician. Among his many contributions, the reformulation of Newtonian mechanics (now known as Hamiltonian mechanics) and the invention of quaternions are probably the best known. Hamilton is said to have shown an extraordinary talent for languages at a very early age. However, when he lost in an arithmetic contest against the American prodigy Zerha Colburn, he decided to spend more time with mathematics. Colburn was 9 , Hamilton 8 years old.
} 
into another one, as we will see later. The momenta $\mathbf{p}$ are elements of the momentum space $\mathbb{P}$. Together, $\mathbf{m}$ and p are said to be in phase space $\mathbb{X}=\mathbb{M} \times \mathbb{P}$.

A key property of Hamiltonian systems with a time-independent Hamiltonian $H$ is time reversability. Indeed, replacing $t$ by $-t$ transforms the generalised momenta according to

$$
p_{i} \rightarrow \mu_{i} \frac{d m_{i}}{d(-t)}=-p_{i}
$$

For Hamilton's equations (5.83) and (5.84) we then find

$$
\frac{d m_{i}}{d t}=\frac{\partial H}{\partial p_{i}} \rightarrow \frac{d m_{i}}{d(-t)}=\frac{\partial H}{\partial\left(-p_{i}\right)} \quad \Rightarrow \quad \frac{d m_{i}}{d t}=\frac{\partial H}{\partial p_{i}},
$$

and

$$
\frac{d p_{i}}{d t}=-\frac{\partial H}{\partial m_{i}} \rightarrow \frac{d\left(-p_{i}\right)}{d(-t)}=-\frac{\partial H}{\partial m_{i}} \Rightarrow \frac{d p_{i}}{d t}=-\frac{\partial H}{\partial m_{i}} .
$$

Thus, Hamilton's equations are invariant under time reversal, provided that $H$ has no explicit time dependence. Time reversability means that we cannot infer the direction of the time arrow from observing the trajectory of a particle. Note that changing the sign of time and momentum goes hand in hand, according to equation (5.86). If a particle takes time $\tau$ to move from phase space position $(\mathbf{m}, \mathbf{p})$ to the new position $\left(\mathbf{m}_{\tau}, \mathbf{p}_{\tau}\right)$, we can take $\left(\mathbf{m}_{\tau},-\mathbf{p}_{\tau}\right)$ as new initial condition, reverse time, and at $t=0$ the particle will be back at $(\mathbf{m}, \mathbf{p})$. In this sense, a trajectory is reversible under Hamiltonian mechanics.

An important consequence of time reversability is the preserved partitioning of the phase space under movement along a Hamiltonian trajectory: When the $\mathbb{X}^{i}$ partition phase space $\mathbb{X}$, meaning that they completely fill $\mathbb{X}$ without overlapping, then the images $\mathbb{X}_{\tau}^{i}$ partition the phase space as well. To see this, we first assume that the image space has holes, so that there is an element $\left(\mathbf{m}_{\tau}, \mathbf{p}_{\tau}\right)$ that is not an image. Using time reversal, $\left(\mathbf{m}_{\tau}, \mathbf{p}_{\tau}\right)$ translates to some $(\mathbf{m}, \mathbf{p})$ of the phase space. Then, again by time reversal, $(\mathbf{m}, \mathbf{p})$ maps to $\left(\mathbf{m}_{\tau}, \mathbf{p}_{\tau}\right)$, thus contradicting the assumption that it is not an image. Conversely, assume that images $\mathbb{X}_{\tau}^{i}$ and $\mathbb{X}_{\tau}^{j}$ overlap, meaning that there is an $\left(\mathbf{m}_{\tau}, \mathbf{p}_{\tau}\right)$ located within both $\mathbb{X}_{\tau}^{i}$ and $\mathbb{X}_{\tau}^{j}$. It follows that there must have been distinct $\left(\mathbf{m}^{i}, \mathbf{p}^{i}\right) \in \mathbb{X}^{i}$ and $\left(\mathbf{m}^{j}, \mathbf{p}^{j}\right) \in \mathbb{X}^{j}$ mapping to the same point. This, however, contradicts time reversability which maps $\left(\mathbf{m}_{\tau}, \mathbf{p}_{\tau}\right)$ back to one unique $(\mathbf{m}, \mathbf{p})$.

A particular facet of time reversability is energy conservation. If the total energy $H$ of a particle were not preserved along its trajectory, we could infer the direction of the time arrow by observing a decrease or increase in total energy. Conservation of energy can also be derived directly from Hamilton's equations as follows:

$$
\frac{d H}{d t}=\sum_{i=1}^{n}\left(\frac{\partial H}{\partial p_{i}} \frac{d p_{i}}{d t}+\frac{\partial H}{\partial m_{i}} \frac{d m_{i}}{d t}\right)=\sum_{i=1}^{n}\left(\frac{d m_{i}}{d t} \frac{d p_{i}}{d t}-\frac{d p_{i}}{d t} \frac{d m_{i}}{d t}\right)=0 .
$$

In addition to being time-reversible, motion under Hamiltonian mechanics preserves volume in phase space. To see this, we consider a group of particles with position vectors $\mathbf{x}=(\mathbf{m}, \mathbf{p})$ within a volume $X$ in phase space $\mathbb{X}$. As illustrated in figure 5.12, a small movement of the particles induces a small volume change given by

$$
\mathbf{n} \cdot d \mathbf{x} d A=\mathbf{n} \cdot \frac{d \mathbf{x}}{d t} d t d A
$$

where $d A$ is a small surface element with normal $\mathbf{n}$. The total volume change per time, integrated over the surface $\partial X$ of the volume $X$ is then

$$
\frac{d X}{d t}=\int_{\partial X} \mathbf{n} \cdot \frac{d \mathbf{x}}{d t} d A=\int_{X} \nabla \cdot \frac{d \mathbf{x}}{d t} d \mathbf{x},
$$


where we applied Gauss' theorem to transform the surface into a volume integral. Using Hamilton's equations, we now find

$$
\frac{d X}{d t}=\int_{X} \nabla \cdot \frac{d \mathbf{x}}{d t} d \mathbf{x}=\int_{X} \sum_{i=1}^{n}\left(\frac{\partial}{\partial m_{i}} \frac{d m_{i}}{d t}+\frac{\partial}{\partial p_{i}} \frac{d p_{i}}{d t}\right) d \mathbf{x}=\int_{X} \sum_{i=1}^{n}\left(\frac{\partial}{\partial m_{i}} \frac{\partial H}{\partial p_{i}}-\frac{\partial}{\partial p_{i}} \frac{\partial H}{\partial m_{i}}\right) d \mathbf{x}=0 .
$$

This proves that the phase space volume is preserved under Hamiltonian motion. In other words, the volume occupied by the group of particles remains constant. Equipped with these prerequisites, we now continue with the description of the actual Hamiltonian Monte Carlo algorithm.

a)

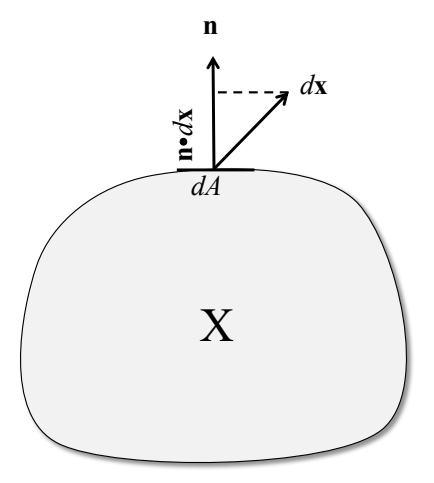

b)

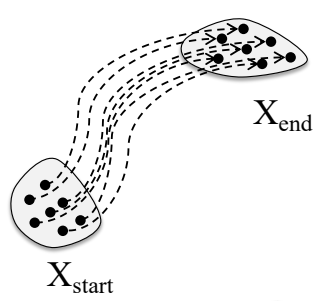

c)

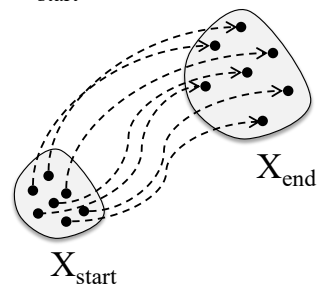

Fig. 5.12: Volume preservation in phase space under Hamiltonian mechanics. (a) A volume $X$ in phase space. On the boundary, a particle is displaced by $d \mathbf{x}$, which induces a small volume change $\mathbf{n} \cdot \mathbf{d x} d A$, where $\mathbf{n}$ is the surface normal, and $d A$ is a small surface element. (b) Hamiltonian trajectory in phase space. When a group of particles moves according to Hamiltonian mechanics, the region occupied by the particles may be deformed, but its volume remains constant, that is $X_{\text {end }}=X_{\text {start }}$. (c) In non-Hamiltonian mechanics the phase space volume may change along the trajectory.

\subsubsection{The canonical distribution}

The trick behind Hamiltonian Monte Carlo is to sample an auxiliary distribution in the $2 n$-dimensional phase space, the canonical distribution, from which samples of the posterior can be obtained by ignoring the momentum space component. Defining kinetic and potential energies as

$$
K(\mathbf{p})=\frac{1}{2} \sum_{i=1}^{n} \frac{p_{i}^{2}}{\mu_{i}}
$$

and

$$
U(\mathbf{m})=-\log \rho\left(\mathbf{m} \mid \mathbf{d}^{\mathrm{obs}}\right),
$$

the canonical distribution is defined as

$$
\rho_{c}(\mathbf{m}, \mathbf{p})=e^{-H(\mathbf{m}, \mathbf{p})}=e^{-U(\mathbf{m})-K(\mathbf{p})}=\rho\left(\mathbf{m} \mid \mathbf{d}^{\mathrm{obs}}\right) e^{-\frac{1}{2} \sum_{i=1}^{n} \frac{p_{i}^{2}}{\mu_{i}}} .
$$


In other words, the canonical distribution is the product of the posterior $\rho\left(\mathbf{m} \mid \mathbf{d}^{\text {obs }}\right)$ and a Gaussian distribution of the momenta. If we manage to sample $\rho_{c}(\mathbf{m}, \mathbf{p})$, we may simply take the model space component of the samples to obtain samples of the posterior.

\subsubsection{The algorithm}

The step of a random walker in Hamiltonian Monte Carlo consists of two phases: In the first phase, momentum variables $p_{i}$ are drawn from their Gaussian distribution

$$
e^{-\frac{1}{2} \sum_{i=1}^{n} \frac{p_{i}^{2}}{\mu_{i}}},
$$

as previously defined in equation (5.95). In the second phase, the current state $(\mathbf{m}, \mathbf{p})$ is propagated for some time $\tau$ along a Hamiltonian trajectory by numerically solving Hamilton's equations (5.83) and (5.84). The new state $\left(\mathbf{m}_{\tau}, \mathbf{p}_{\tau}\right)$ is then accepted with the probability

$$
\min \left(1, \frac{\rho_{c}\left(\mathbf{m}_{\tau}, \mathbf{p}_{\tau}\right)}{\rho_{c}(\mathbf{m}, \mathbf{p})}\right)=\min \left(1, \frac{\rho\left(\mathbf{m}_{\tau} \mid \mathbf{d}^{\mathrm{obs}}\right)}{\rho\left(\mathbf{m} \mid \mathbf{d}^{\mathrm{obs}}\right)} e^{-K\left(\mathbf{p}_{\tau}\right)+K(\mathbf{p})}\right),
$$

which can be seen as a modified version of the Metropolis rule (5.57). If the transition is accepted, the new model $\left(\mathbf{m}_{\tau}, \mathbf{p}_{\tau}\right)$ is counted. Otherwise, the original model $(\mathbf{m}, \mathbf{p})$. Then, the walker's next step starts again with the first phase, that is, the random generation of momenta. This procedure is then repeated until the sample density has converged sufficiently well. To obtain samples of the posterior, the momentum component of the phase space samples of the canonical distribution are simply ignored.

In the light of the conservation of total energy, shown in equation (5.88), the Hamiltonian Monte Carlo algorithm can be interpreted as a random exploration of energy level sets. During each Hamiltonian trajectory, the total energy $H$ is preserved, up to numerical errors. Randomly choosing a new momentum at the beginning of the next iteration changes the energy level at which the walker will develop its new trajectory.

\subsubsection{Detailed balance}

To demonstrate that the Hamiltonian Monte Carlo algorithm indeed has the canonical distribution $\rho_{c}$ as equilibrium distribution, we need to assert reversibility of the transition in phase space in the sense of the detailed balance equation (5.18). Thus, for two phase space points $\left(\mathbf{m}^{i}, \mathbf{v}^{i}\right)$ and $\left(\mathbf{m}^{j}, \mathbf{v}^{j}\right)$, we must show

$$
T\left(\mathbb{X}^{j} \mid \mathbb{X}^{i}\right) P_{c}\left(\mathbb{X}^{i}\right)=T\left(\mathbb{X}^{i} \mid \mathbb{X}^{j}\right) P_{c}\left(\mathbb{X}^{j}\right) .
$$

Since the phase space is partitioned into small subvolumes, $\left(\mathbf{m}^{i}, \mathbf{p}^{i}\right)$ is located inside some $\mathbb{X}^{i}$. As we have seen in section 5.7.1, all the images under movement along a Hamiltonian trajectory partition the phase space as well. This implies that $\left(\mathbf{m}^{j}, \mathbf{p}^{j}\right)$ must be inside some $\mathbb{X}_{\tau}^{j}$, and is therefore equal to some particular image, that is $\left(\mathbf{m}^{j}, \mathbf{p}^{j}\right)=\left(\mathbf{m}_{\tau}^{j}, \mathbf{p}_{\tau}^{j}\right)$. Since Hamiltonian mechanics uniquely translates $\mathbb{X}^{i}$ into its image $\mathbb{X}_{\tau}^{i}$, the transition probability $T\left(\mathbb{X}_{\tau}^{j} \mid \mathbb{X}^{i}\right)$ must be zero unless $i=j$. Furthermore, since Hamiltonian mechanics is reversible, $T\left(\mathbb{X}^{i} \mid \mathbb{X}_{\tau}^{j}\right)$ is also zero unless $i=j$. In summary,

$$
T\left(\mathbb{X}_{\tau}^{j} \mid \mathbb{X}^{i}\right)=T\left(\mathbb{X}^{i} \mid \mathbb{X}_{\tau}^{j}\right)=0, \quad \text { if } i \neq j .
$$

Thus, equation (5.98) is trivially satisfied for $i \neq j$, and it remains to consider the more special case

$$
T\left(\mathbb{X}_{\tau}^{i} \mid \mathbb{X}^{i}\right) P_{c}\left(\mathbb{X}^{i}\right)=T\left(\mathbb{X}^{i} \mid \mathbb{X}_{\tau}^{i}\right) P_{c}\left(\mathbb{X}_{\tau}^{i}\right)
$$


Under the assumption that our partitioning is so fine that we can consider the canonical distribution constant within each $\mathbb{X}^{i}$, we have

$$
P_{c}\left(\mathbb{X}^{i}\right)=X \rho_{c}\left(\mathbf{m}^{i}, \mathbf{p}^{i}\right), \quad \text { and } \quad P_{c}\left(\mathbb{X}_{\tau}^{i}\right)=X \rho_{c}\left(\mathbf{m}_{\tau}^{i}, \mathbf{p}_{\tau}^{i}\right)
$$

Note that equation (5.101) only holds because Hamiltonian mechanics preserves the volume $X$ along the trajectory. With the help of equation (5.97), which determines the acceptance probability, equation (5.100) now transforms to

$$
\min \left(1, \frac{\rho_{c}\left(\mathbf{m}_{\tau}^{i}, \mathbf{p}_{\tau}^{i}\right)}{\rho_{c}\left(\mathbf{m}^{i}, \mathbf{p}^{i}\right)}\right) \rho_{c}\left(\mathbf{m}^{i}, \mathbf{p}^{i}\right)=\min \left(1, \frac{\rho_{c}\left(\mathbf{m}^{i}, \mathbf{p}^{i}\right)}{\rho_{c}\left(\mathbf{m}_{\tau}^{i}, \mathbf{p}_{\tau}^{i}\right)}\right) \rho_{c}\left(\mathbf{m}_{\tau}^{i}, \mathbf{p}_{\tau}^{i}\right) .
$$

That equation (5.102), and therefore detailed balance, indeed holds, is now easily shown by considering the two cases $\rho_{c}\left(\mathbf{m}_{\tau}^{i}, \mathbf{p}_{\tau}^{i}\right)>\rho_{c}\left(\mathbf{m}^{i}, \mathbf{p}^{i}\right)$ and $\rho_{c}\left(\mathbf{m}_{\tau}^{i}, \mathbf{p}_{\tau}^{i}\right) \leq \rho_{c}\left(\mathbf{m}^{i}, \mathbf{p}^{i}\right)$.

\subsubsection{Numerical integration of Hamilton's equations}

As we will see in the following section 5.7.6, Hamilton's equations may be solved analytically in some special cases, for instance when the forward problem is linear and all uncertainties are Gaussian. More generally, however, Hamilton's equations need to be solved numerically, which raises the question for suitable numerical integrators.

Among the wealth of available integration schemes we would like to choose one with properties that were critical in our proof of detailed balance in section 5.7.4, namely time reversability and volume preservation. Such schemes, often specifically designed for the solution of Hamilton's equations, are called symplectic integrators ${ }^{15}$. Probably the best known example is the leap-frog scheme, which proceeds in the following three steps

$$
\begin{gathered}
p_{i}(t+h / 2)=p_{i}(t)-\frac{h}{2} \frac{\partial U}{\partial m_{i}}[\mathbf{m}(t)], \\
m_{i}(t+h)=m_{i}(t)+\frac{h}{\mu_{i}} p_{i}(t+h / 2), \\
p_{i}(t+h)=p_{i}(t+h / 2)-\frac{h}{2} \frac{\partial U}{\partial m_{i}}[\mathbf{m}(t+h)],
\end{gathered}
$$

where $h$ is the time increment. To show time reversability, we use equations (5.103) to (5.105) in order to propagate from $\mathbf{m}(t+h)$ to $\mathbf{m}(t+2 h)$ starting with the initial momentum $-\mathbf{p}(t+h)$, that is the inverse of the momentum after the step from $t$ to $t+h$. The corresponding equations are

$$
\begin{gathered}
p_{i}(t+3 h / 2)=-p_{i}(t+h)-\frac{h}{2} \frac{\partial U}{\partial m_{i}}[\mathbf{m}(t+h)], \\
m_{i}(t+2 h)=m_{i}(t+h)+\frac{h}{\mu_{i}} p_{i}(t+3 h / 2), \\
p_{i}(t+2 h)=p_{i}(t+3 h / 2)-\frac{h}{2} \frac{\partial U}{\partial m_{i}}[\mathbf{m}(t+2 h)] .
\end{gathered}
$$

Combining (5.103) to (5.105) with (5.106) to (5.108), we find after some tedious but straightforward manipulations

$$
m_{i}(t+2 h)=m_{i}(t), \quad p_{i}(t+2 h)=-p_{i}(t)
$$

\footnotetext{
15 The term symplectic derives from the Ancient Greek sym-plektos, meaning weaved or braided together. In mathematics it has various meanings, depending on the context, for instance in symplectic group, symplectic matrix, or symplectic geometry. In the framework of numerical integration, symplectic is often equated with volume-preserving.
} 
meaning that the trajectory returns exactly to its initial position at time $t$, just with reversed momentum. For the demonstration of volume preservation, we interpret equations (5.103) to (5.105) as three distinct transformations. The first transformation maps $p_{i}(t)$ into $p_{i}(t)-\frac{h}{2} \frac{\partial U}{\partial m_{i}}[\mathbf{m}(t)]$ and $m_{i}(t)$ into itself. The Jacobian of this transformation is

$$
J=\operatorname{det}\left(\begin{array}{cc}
1 & -\frac{h}{2} \frac{\partial U}{\partial m_{i}}[\mathbf{m}(t)] \\
0 & 1
\end{array}\right)=1,
$$

meaning that the volume is preserved during the first step. Repeating exactly the same analysis for the remaining two steps, shows that their respective Jacobians are also equal to 1, meaning that the volume during the complete leap-frog iteration is preserved.

It follows that the key properties needed to achieve detailed balance are satisfied exactly, even though the numerical scheme is of course not completely free of errors. Alternative symplectic integrators are well-documented in the literature (e.g. Blanes et al., 2014; de Vogelaere, 1956; Leimkuhler \& Reich, 1994; Ruth, 1983; Sanz-Serna $\&$ Calvo, 1994). In our numerical examples we consistently use the leapfrog scheme, mostly for simplicity and ease of implementation.

While the leap-frog scheme has the necessary properties of time reversability and volume preservation, it does not generally preserve the total energy $H$. This kind or numerical error does not break the Hamiltonian Monte Carlo algorithm, but it has consequences for the acceptance probability (5.97). In fact, slightly rewriting equation (5.97) in terms of the total energy $H=U+K$, we find

$$
\min \left(1, \frac{\rho_{c}\left(\mathbf{m}_{\tau}, \mathbf{p}_{\tau}\right)}{\rho_{c}(\mathbf{m}, \mathbf{p})}\right)=\min \left(1, e^{-H\left(\mathbf{m}_{\tau}, \mathbf{p}_{\tau}\right)+H(\mathbf{m}, \mathbf{p})}\right),
$$

where we used the definition of potential energy from equation (5.94). If $H$ were correctly preserved along the trajectory, we would have $H\left(\mathbf{m}_{\tau}, \mathbf{p}_{\tau}\right)=H(\mathbf{m}, \mathbf{p})$, and all proposals would be accepted. Failure to preserved energy leads to acceptance ratios below 1 , and also to a dependence of the acceptance ratio on the numerical time step $h$. In practice, the time step is therefore often chosen such that the acceptance ratio remains acceptably high.

Of course, the leap-frog scheme is not the only available numerical integrator. However, all other explicit numerical integrators do not preserve energy either. For linear problems, the implicit Crank-Nicolson scheme (Crank \& Nicolson, 1947) preserves energy exactly. For the more interesting nonlinear case, energy-preserving schemes have been developed more recently (e.g. Quispel \& McLaren, 2008; Simo et al., 1992). These schemes are also generally implicit and therefore prohibitively expensive for modern large-scale inverse problems.

\subsubsection{Linear problems with Gaussian uncertainties}

Though the solution of linear problems is not the prime target of Hamiltonian Monte Carlo, we illustrate some more practical basics of the method by continuing the example from figure 5.11, where we assumed Gaussian uncertainties and a linear forward model,

$$
\rho\left(\mathbf{d}^{\mathrm{obs}} \mid \mathbf{m}\right)=\text { const. } e^{-\frac{1}{2}\left(\mathbf{d}^{\mathrm{obs}}-\mathbf{G m}\right)^{T}\left(\mathbf{d}^{\mathrm{obs}}-\mathbf{G m}\right)} .
$$

In order to have a particularly weak prior, we furthermore choose

$$
\rho(\mathbf{m})=1 .
$$

Hamilton's equations (5.83) and (5.84) now take the specific form

$$
\frac{d m_{i}}{d t}=\frac{\partial H}{\partial p_{i}}=\frac{\partial K}{\partial p_{i}}=\frac{p_{i}}{\mu_{i}}
$$




$$
\frac{d p_{i}}{d t}=-\frac{\partial H}{\partial m_{i}}=-\frac{\partial U}{\partial m_{i}}=\sum_{k=1}^{n} d_{k} G_{k i}-\sum_{j, k=1}^{n} G_{k i} G_{k j} m_{j}
$$

Equations (5.114) and (5.115) constitute a coupled system of two linear, first-order, ordinary differential equations. Differentiating equation (5.114) with respect to time, and substituting $\frac{d p_{i}}{d t}$ from equation (5.115), we obtain an independent, second-order differential equation for the model parameters $m_{i}$,

$$
\frac{d^{2} m_{i}}{d t^{2}}+\frac{1}{\mu_{i}} \sum_{j, k} G_{k i} G_{k j} m_{j}=\frac{1}{\mu_{i}} \sum_{k} d_{k} G_{k i}
$$

Denoting by $A_{i j}$ the square root of the positive-semidefinite symmetric matrix $\frac{1}{\mu_{i}} \sum_{k} G_{k i} G_{k j}$, and renaming $\frac{1}{\mu_{i}} \sum_{k} d_{k} G_{k i}=f_{i}$, we can write equation (5.116) in a more condensed vector-matrix notation,

$$
\frac{d^{2} \mathbf{m}}{d t^{2}}+\mathbf{A} \mathbf{A} \mathbf{m}=\mathbf{f}
$$

Using the matrix sine and cosine, the general solution of (5.117) is given by

$$
\mathbf{m}=\sin (\mathbf{A} t) \mathbf{a}+\cos (\mathbf{A} t) \mathbf{b}+(\mathbf{A} \mathbf{A})^{-1} \mathbf{f} .
$$

The initial values $\mathbf{a}$ and $\mathbf{b}$ can be expressed in terms of the initial model $\mathbf{m}_{0}$ and the initial momentum $\mathbf{p}_{0}$ of the Hamiltonian trajectory,

$$
\mathbf{a}=\mathbf{A}^{-1} \mathbf{M}^{-1} \mathbf{p}_{0}, \quad \mathbf{b}=\mathbf{m}_{0}-\mathbf{f},
$$

where $\mathbf{M}$ is a diagonal matrix with entries $M_{i i}=\mu_{i}^{-1}$. An analogous equation can be obtained for the momentum vector p. Equation (5.118) already indicates that the Hamiltonian trajectories for a linear problem with Gaussian uncertainties are harmonic oscillations. This becomes even more apparent when the forward problem matrix $G_{i j}$ is assumed to be square and diagonal. In this special case, $\mathbf{A}$ is diagonal, with entries

$$
A_{i i}=\frac{1}{\sqrt{\mu_{i}}} G_{i i}
$$

and the model space trajectories can be written in terms of conventional sines and cosines,

$$
m_{i}=a_{i} \sin \left(\frac{1}{\sqrt{\mu_{i}}} G_{i i} t\right)+b_{i} \cos \left(\frac{1}{\sqrt{\mu_{i}}} G_{i i} t\right)+f_{i},
$$

where $a_{i}$ and $b_{i}$ are again the initial values specified in equation (5.119). Equation (5.121) demonstrates that the trajectory of the random walker from the current model to a new proposal model oscillates harmonically through phase space - provided of course that the forward problem is linear, and uncertainties are Gaussian. The oscillation period is controlled by the coefficients of the forward problem $G_{i i}$ and the masses of the particles $\mu_{i}$. Larger forward problem derivatives $G_{i i}$ result in faster oscillations. Conversely, small derivatives lead to slow oscillations and a slow traversal of the model space. In the extreme case where $G_{i i}=0$, the model component $m_{i}$ does not move at all. The particle masses $\mu_{i}$ can serve as tuning parameters to adjust the speed with which the phase space is traversed.

The particle masses also seemingly enter the oscillation amplitude through the initial value a from equation (5.119). Since the initial momenta of the trajectory $\mathbf{p}_{0}$ are drawn randomly from the canonical distribution, they have a standard deviation of $\sqrt{\mu_{i}}$. This is multiplied by a factor $\sqrt{\mu_{i}}$ from the inverse of $\mathbf{A}$ defined in (5.120), and devided by $\mu_{i}$ contained in $\mathbf{M}^{-1}$. It follows that the oscillation amplitude is on average independent of the particle masses. It depends, however, on $G_{i i}^{-1}$. Model vector components with large $G_{i i}$ that oscillate rapidly have a small oscillation amplitude, reflecting the fact that they are less constrained by the data.

To make this example more concrete, we reuse the specific numbers already employed for figure 5.6. The model vector $\mathbf{m}$ is ten-dimensional, and we choose a diagonal matrix $\mathbf{G}$ with entries $G_{i i}=i / 10$. Furthermore, we set 
the observed data vector components to $d_{i}^{\text {obs }}=i / 5$. For illustration, we choose $\mu_{i}=1$ for all $i=1, \ldots, 10$.

Figure 5.13 shows a random realisation of a Hamiltonian trajectory through the ten-dimensional model space projected onto various two-dimensional subspaces. It is clearly visible that model parameters with small derivatives $G_{i i}$ traverse the model space comparatively slowly, and have a large oscillation amplitude.

A concrete Hamiltonian Monte Carlo run with 10'000 test models is summarised in figure 5.14. Using a Hamiltonian trajectory length of $20 \mathrm{~s}, 2681$ of the proposed models, around $27 \%$, are accepted. Their distribution is so favourable that the posterior can be approximated very well, despite the small number of samples.

To put this result into perspective, we compare to the numbers from the introductory example where a Gaussian prior with standard deviation $\sigma=10$, which is far from being uninformative, led to an acceptance rate of around $0.0014 \%$. If we wanted to also have about 2700 accepted models with the Metropolis-Hastings algorithm, we would need to propose more than 19 million test models.
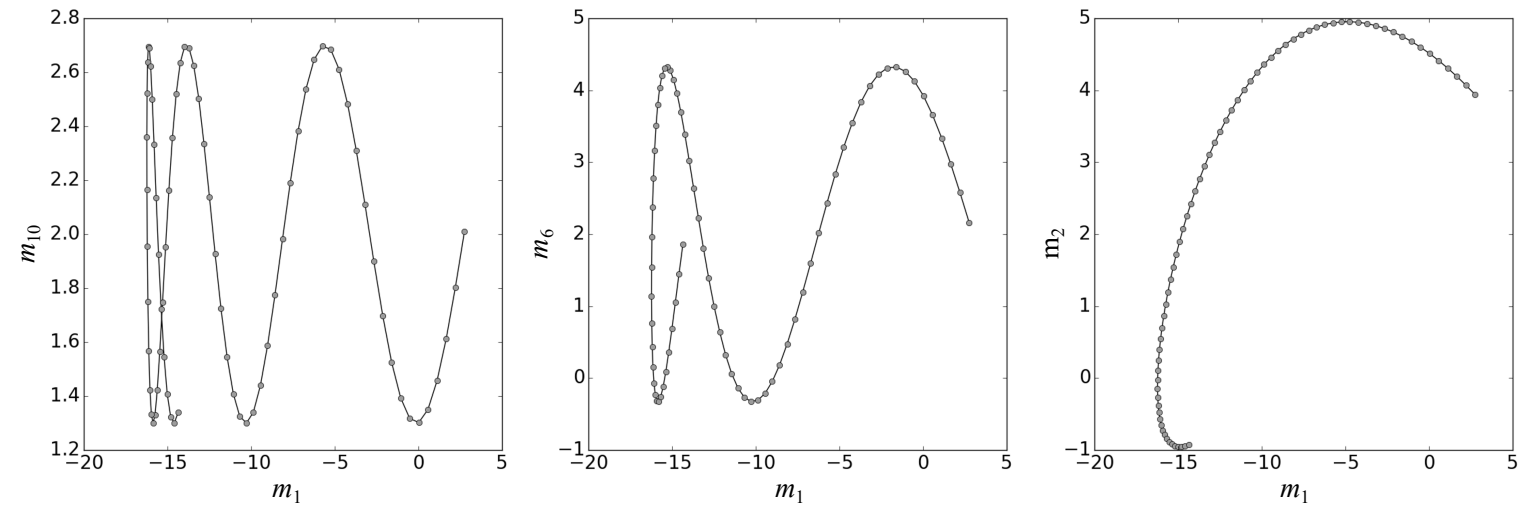

Fig. 5.13: Realisation of a ten-dimensional Hamiltonian trajectory projected onto the two-dimensional planes $\left(m_{1}, m_{10}\right),\left(m_{1}, m_{6}\right)$ and $\left(m_{1}, m_{2}\right)$. Model parameters with small forward problem derivative $G_{i i}$, such as $m_{1}$, oscillate slowly with large amplitude, and vice versa.

\subsubsection{Tuning Hamiltonian Monte Carlo}

In contrast to the global Metropolis-Hastings algorithm, Hamiltonian Monte Carlo has a large number of parameters that are not a priori determined, and the choice of which strongly affects the performance of the algorithm. They include the masses $\mu_{i}$ of the particles, the integration time step, and the length of the individual trajectories. Thus, we are presented with a delicate tuning problem.

An obvious potential improvement by tuning is most apparent in figure 5.13. While $m_{1}$ traverses the energy level set roughly once during the Hamiltonian trajectory, $m_{10}$ traverses it more than 6 times. From the perspective of $m_{1}$, the trajectory has just about the right length, but it is much too long for $m_{10}$, which keeps visiting the same positions again and again without seeing anything new. This deficiency can be repaired easily by choosing the particle masses as $\mu_{i}=G_{i i}^{2}$. According to equation (5.121), the circular frequency of the oscillations through phase space is now equal to $\frac{1}{\sqrt{\mu_{i}}} G_{i i}=1$. All particles oscillate equally fast. The result of this tuning is shown in figure 5.15, which is based on the example from figure 5.13. This simple tuning allows us to reduce the temporal duration of a trajectory significantly because it is not controlled anymore by a potentially very slow model space component. 

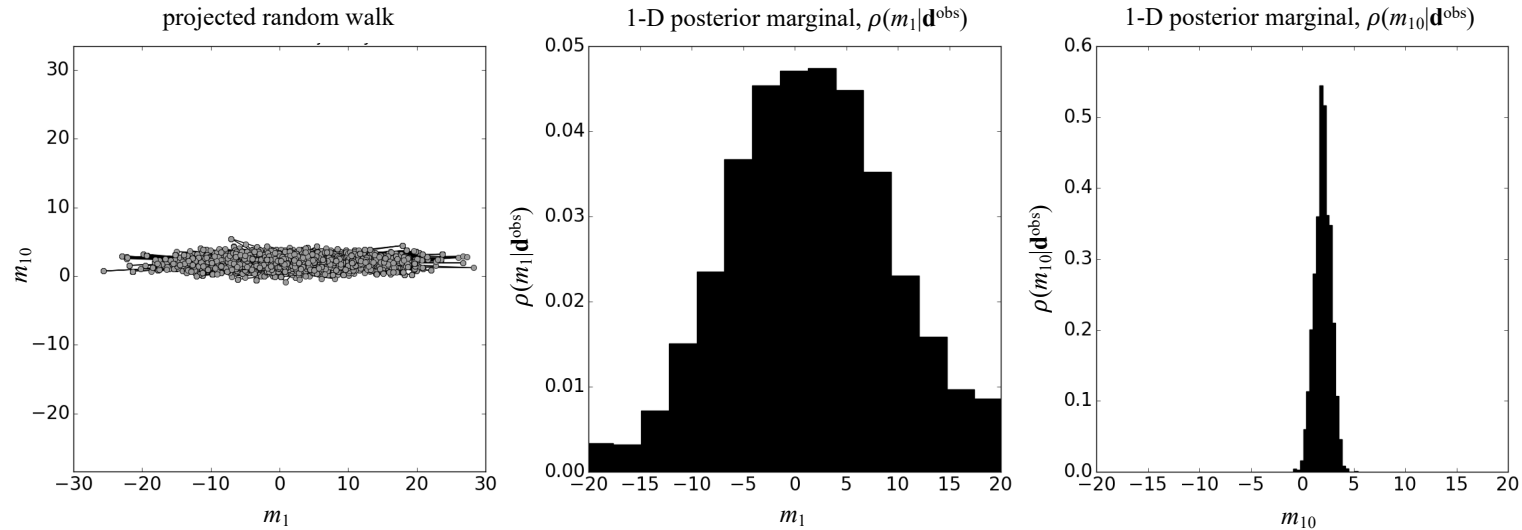

Fig. 5.14: Hamiltonian Monte Carlo applied to the example problem. The random walker's trajectory is shown in the left panel. Out of $10^{\prime} 000$ proposed models, $2^{\prime} 681$ were accepted, that is around $27 \%$. The samples are distributed very favourably so that a good approximation of the posterior, shown as 1D marginals, can be obtained despite their small number.

Hamiltonian Monte Carlo and the associated tuning can be generalised by replacing the original definition of the momentum variables $p_{i}$ from equation (5.85) with

$$
p_{i}=\sum_{j=1}^{n} M_{i j} \frac{d m_{j}}{d t},
$$

where the $n^{2}$ numbers $M_{i j}$ are the components of the mass matrix. The mass matrix may be non-diagonal, but is required to be positive definite in order to ensure that kinetic energy $K$ is strictly positive. Introducing $n^{2}$ mass matrix components instead of only $n$ masses $\mu_{i}$ provides more opportunities for tuning, as described, for instance, in Fichtner et al. (2019).
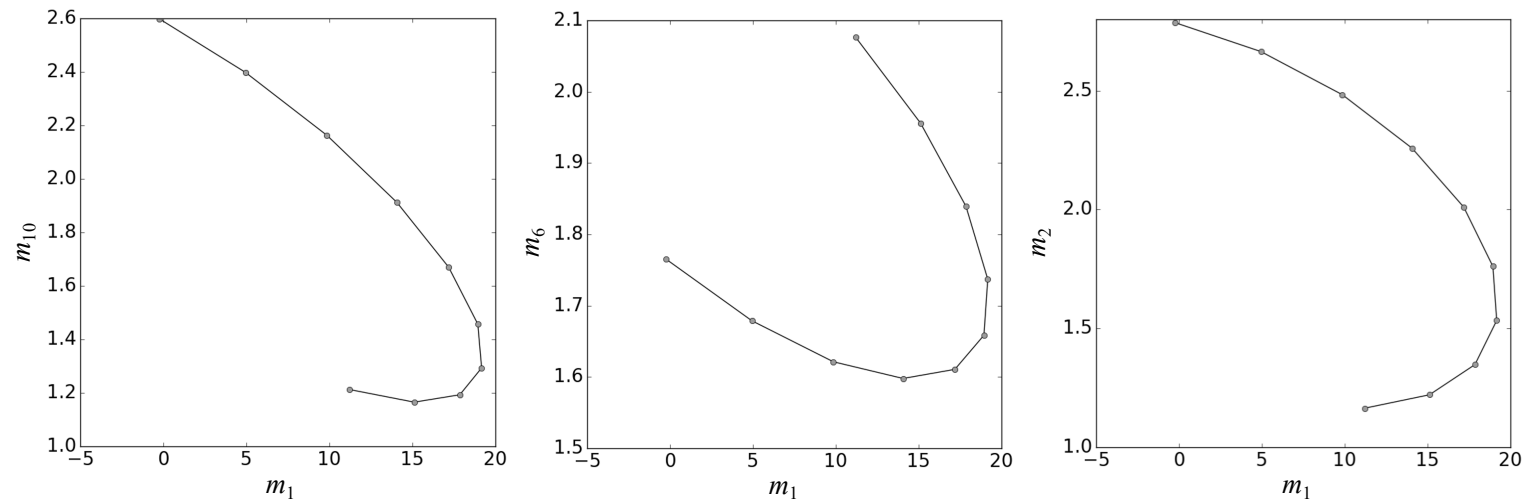

Fig. 5.15: The same as figure 5.13, but with the particle masses set to $\mu_{i}=G_{i i}^{2}$. All components of the model vector traverse the phase space with equal oscillation frequency. 
The main computational requirements of Hamiltonian Monte Carlo, compared to derivative-free random walks, come from the need to evaluate the derivative of the Hamiltonian along the trajectory, that is, in each time step of the numerical integration. Thus, the computational cost of Hamiltonian Monte Carlo is strongly controlled by the average trajectory length.

Fig. 5.13 indicates that trajectories can be too short or too long. If too short, the next proposal is very close to the current position, leading to slow exploration of model space. In contrast, a trajectory that is too long revisits points in model space that it has seen before, thereby wasting valuable computing time. Furthermore, the time needed to traverse a specific energy level set depends on the energy itself. Low-energy level sets require less, and high-energy level sets require more time for a traversal. An additional complication arises in the presence of periodic trajectories, such as those shown for the linear problem in Fig. 5.13. Accidentally fixing the trajectory length to an integer multiple of the oscillation frequency will cause the random walker to always revisit the same positions, thus preventing convergence towards the posterior distribution.

At least for (nearly) linear problems with Gaussian priors, we can estimate suitable trajectory lengths. In the case of the optimal mass matrix, we have $\mathbf{A}=\mathbf{I}$, and a trajectory length of $\pi$ will achieve the maximum distance from the starting point of the trajectory. When the forward problem is weakly nonlinear or when a diagonal approximation of $\mathbf{A}$ is used, a trajectory length of approximately $\pi$ may still serve as a useful guiding value. To avoid locking in periodic trajectories, the trajectory length may be chosen randomly, as suggested by Mackenzie (1989) and Neal (2011). Alternatively, the length of the trajectory may be chosen adaptively, e.g., using the NoU-Turn criterion that terminates the integration when the trajectory begins to return towards its starting point (Betancourt, 2017; Gelman et al., 2013; Hoffmann \& Gelman, 2014).

Often, 5 - 10 time steps per trajectory are optimal. Using fewer time steps, limits model space exploration and results in slow convergence. A larger number of time steps further decreases the accurace of the numerical integrator, thereby leading to low acceptance rates.

The tuning of Hamiltonian Monte Carlo can be a tedious exercise, especially for higher-dimensional problems that require substantial computational resources, and thereby do not permit a large number of trial-and-error runs. In chapter 14, we present an autotuning approach that finds a nearly optimal mass matrix and a reasonable time step on the fly, that is, while sampling the target distribution. 


\subsection{Transdimensional inference and sampling}

In a famous speech, former U.S. Secretary of Defence Donald Rumsfeld explained in 2002: "There are known knowns; there are things we know we know. We also know there are known unknowns; that is to say we know there are some things we do not know. But there are also unknown unknowns - there are things we do not know we don't know.' Rumsfeld's attempt to justify military intervention in Iraq despite his government's failure to find "weapons of mass destruction", may have seemed ridiculous; but he had a point in saying that some things we (consciously or unconsciously) assume known, may actually be unknown, thereby leading to potentially incorrect inferences.

Many inverse problems suffer from the unknown unknowns, either for physical or for practical reasons. Our physical models are models, that is, abstractions of reality. In their construction, typically based on a finite number of experiments, we made simplifications by omitting physical phenomena that we may actually not be aware of. The consequences of these simplifications may or may not play a role in a new experiment that was not used to build the physical model in the first place.

For practical reasons, we often pretend that specific aspects of the problem are known, even though they are not. (In Donald Rumsfeld's words, some of the known knowns are actually unknowns.) The classic example is the dimension of the model space. In problems that require discretisation, the number of unknown model parameters is often guided by a combination of physical intuition and technical limitations. However, we rarely try to determine the number of model parameters, or model complexity, that is really warranted by our data.

It is precisely at this point where transdimensional inference becomes relevant. Instead of imposing a fixed number of model parameters $n$, we treat $n$ itself as an unknown. Our more general goal is then to obtain a joint posterior for the model parameters $\mathbf{m}$ and for their dimension.

The outstanding advantage of transdimensional inversion is natural parsimony. This means that Occam's razor is applied automatically, thereby alowing us to find the optimal model complexity requested by the data. This benefit comes at the price of requiring prior knowledge on the model space dimension. In some cases the dimension of the model space may have actual physical meaning. It may, for instance, be the number of substances in a mixture that we wish to indentify. In other cases, however, model parameters are the result of an arbitrary or convenience-driven discretisation process. The lack of physics-based prior knowledge in such applications is one of the most frequent criticisms of transdimensional inversion. It can, nevertheless, be a useful tool to infer a suitable level of model complexity.

To introduce transdimensional inversion, we will start in section 5.8.1 with an extension of Bayes' theorem from the fixed-dimensional case to the case where the model space dimension is among the unknowns that we wish to infer. This will be followed, in section 5.8.2, by the description of a simple algorithm that enables transdimensional inference by actually running a series of fixed-dimensional samplers. Though inefficient in practice, this naive transdimensional sampler will be useful to train our intuition. Finally, section 5.8.3, introduces the reversible-jump algorithm, which serves as a template for a large family of transdimensional samplers that can be adapted to the specifics of a particular application.

Though the reversible-jump algorithm was originally introduced by Green (1995), we more closely follow the unpublished chapter of Green \& Hastie (2009), and the work of Sambridge et al. (2006) that includes numerous illustrative examples.

\subsubsection{Bayes' theorem for transdimensional problems}

We start with a generalisation of Bayes' theorem, introduced in section 4.3 for fixed model space dimension $n$, to the case of model spaces with variable dimension. First, based on the definition of a conditional probability density, we have

$$
\rho\left(\mathbf{m}, n \mid \mathbf{d}^{\text {obs }}\right)=\rho\left(\mathbf{m} \mid \mathbf{d}^{\text {obs }}, n\right) \rho\left(n \mid \mathbf{d}^{\text {obs }}\right),
$$


where $\rho\left(\mathbf{m}, n \mid \mathbf{d}^{\text {obs }}\right)$ is the joint posterior of $\mathbf{m}$ and $n$, and $\rho\left(n \mid \mathbf{d}^{\text {obs }}\right)$ is the posterior of $n$ given synthetic data $\mathbf{d}^{\text {obs }}$. We may substitute $\rho\left(\mathbf{m} / \mathbf{d}^{\text {obs }}, n\right)$ by explicitly including the conditional dependence on $n$ into the fixeddimensional Bayes' theorem (4.12), that is,

$$
\rho\left(\mathbf{m} \mid \mathbf{d}^{\mathrm{obs}}, n\right)=\frac{\rho\left(\mathbf{d}^{\mathrm{obs}} \mid \mathbf{m}, n\right) \rho(\mathbf{m} \mid n)}{\rho\left(\mathbf{d}^{\mathrm{obs}} \mid n\right)} .
$$

Furthermore, we may express $\rho\left(n \mid \mathbf{d}^{\text {obs }}\right)$ in equation (5.123) using Bayes' theorem applied to $n$ and $\mathbf{d}^{\text {obs }}$, that is,

$$
\rho\left(n \mid \mathbf{d}^{\mathrm{obs}}\right)=\frac{\rho\left(\mathbf{d}^{\mathrm{obs}} \mid n\right) \rho(n)}{\rho\left(\mathbf{d}^{\mathrm{obs}}\right)},
$$

with $\rho(n)$ being the prior on the number of model parameters. Finally, substituting (5.124) and (5.125) into (5.123), we find

$$
\rho\left(\mathbf{m}, n \mid \mathbf{d}^{\mathrm{obs}}\right)=\frac{\rho\left(\mathbf{d}^{\mathrm{obs}} \mid \mathbf{m}, n\right) \rho(\mathbf{m} \mid n) \rho(n)}{\rho\left(\mathbf{d}^{\mathrm{obs}}\right)} .
$$

Equation (5.126) is Bayes' theorem for a transdimensional inference problem. We note that the conditional evidence $\rho\left(\mathbf{d}^{\mathrm{obs}} \mid n\right)$ has cancelled, and only the unconditional or total evidence $\rho\left(\mathbf{d}^{\mathrm{obs}}\right)$ enters the equation.

Beyond variable dimensions: The concept of transdimensionality introduced above may be further generalised without much additional notational burden. In fact, we may not just vary the number of model parameters, we may even switch between models with entirely different parameterisations. For instance, a 3-D distribution of some quantity (e.g., velocity, conductivity, ...) may be represented in terms of pixels, polynomials, wavelets, or a combination of them. Instead of labelling models by their number of model parameters, $n$, they may then be labelled by some index $k$. Following the same line of arguments as above, we arrive at a generalised form of Bayes' theorem,

$$
\rho\left(\mathbf{m}, k \mid \mathbf{d}^{\mathrm{obs}}\right)=\frac{\rho\left(\mathbf{d}^{\mathrm{obs}} \mid \mathbf{m}, k\right) \rho(\mathbf{m} \mid k) \rho(k)}{\rho\left(\mathbf{d}^{\mathrm{obs}}\right)} .
$$

In the following, we will continue to work only with variable dimensions, though keeping in mind that the concepts can easily be modified to capture more complex scenarios.

\subsubsection{Naive transdimensional sampling}

Using Bayes' theorem (5.126), we can formally express the joint posterior for $\mathbf{m}$ and $n$ in terms of the fixed-dimensional model prior $\rho(\mathbf{m} \mid n)$, the prior on model dimension, $\rho(n)$, the fixed-dimensional likelihood $\rho\left(\mathbf{d}^{\text {obs }} \mid \mathbf{m}, n\right)$, and the total evidence $\rho\left(\mathbf{d}^{\text {obs }}\right)$. However, we do not yet have a sampling algorithm that is capable of switching between models of variable dimension while maintaining detailed balance. Before we derive such an algorithm in section 5.8.3, we first consider a naive sampler that generates the transdimensional posterior $\rho\left(\mathbf{m}, n \mid \mathbf{d}^{\text {obs }}\right)$ from fixed-dimensional posteriors $\rho\left(\mathbf{m} \mid \mathbf{d}^{\text {obs }}, n\right)$.

We start with the assumption that samples of the fixed-dimensional posteriors $\rho\left(\mathbf{m} \mid \mathbf{d}^{\text {obs }}, n\right)$ for some $n$ are already available. They may have been obtained, for instance, using a Metropolis-Hasting or Hamiltonian Monte Carlo algorithm where the model space dimension was not allowed to vary. Using equation (5.123), we can express the transdimensional posterior $\rho\left(\mathbf{m}, n \mid \mathbf{d}^{\mathrm{obs}}\right)$ in terms of fixed-dimensional posteriors $\rho\left(\mathbf{m} \mid \mathbf{d}^{\text {obs }}, n\right)$ and $\rho\left(n \mid \mathbf{d}^{\mathrm{obs}}\right)$, where $\rho\left(n \mid \mathbf{d}^{\mathrm{obs}}\right)$ is the posterior distribution for the model space dimension $n$. For the moment, we 
assume that $\rho\left(n \mid \mathbf{d}^{\text {obs }}\right)$ has somehow been given to us. (We will soon discover that it is actually not needed.) Equation (5.123) then suggests a simple algorithm to obtain samples of $\rho\left(\mathbf{m}, n \mid \mathbf{d}^{\text {obs }}\right)$ :

1) Choose $n$ randomly with probability $\rho\left(n \mid \mathbf{d}^{\text {obs }}\right)$.

2) With $n$ chosen, consider the samples of $\rho\left(\mathbf{m} \mid \mathbf{d}^{\text {obs }}, n\right)$ and pick one of them randomly, with uniform probability. Then go back to (1), and repeat until the distribution has converged.

The beauty of this simple algorithm is that $\rho\left(n \mid \mathbf{d}^{\mathrm{obs}}\right)$ is actually not explicitly needed. In fact, using Bayes' theorem for $n$ and $\mathbf{d}^{\text {obs }}$ from equation (5.125), the probability of picking some $n$ in step 1 of the algorithm, is given by

$$
P_{n}=\frac{\rho\left(n \mid \mathbf{d}^{\mathrm{obs}}\right)}{\sum_{n^{\prime}} \rho\left(n^{\prime} \mid \mathbf{d}^{\mathrm{obs}}\right)}=\frac{\rho\left(\mathbf{d}^{\mathrm{obs}} \mid n\right) \rho(n)}{\sum_{n^{\prime}} \rho\left(\mathbf{d}^{\mathrm{obs}} \mid n^{\prime}\right) \rho\left(n^{\prime}\right)} .
$$

The total evidence $\rho\left(\mathbf{d}^{\text {obs }}\right)$ has again cancelled, and only the conditional evidences $\rho\left(\mathbf{d}^{\text {obs }} \mid n\right)$ are needed. As we will see in the following example, $\rho\left(\mathbf{d}^{\mathrm{obs}} \mid n\right)$ may be approximated explicitly through the normalisation of the posterior samples, at least for problems of sufficiently low dimension. (Note that this approach requires the storage of all samples. This quickly becomes unfeasible when either the number of samples or the model space dimension is somewhat elevated.)

An interesting aspect of equation (5.128) is the appearance of the evidence ratios $\rho\left(\mathbf{d}^{\mathrm{obs}} \mid n^{\prime}\right) / \rho\left(\mathbf{d}^{\mathrm{obs}} \mid n\right)$, that we encountered before in equation (4.17) where we considered the relative probability of two hypotheses, $\mathscr{H}_{1}$ and $\mathscr{H}_{2}$, given some data $\mathbf{d}^{\text {obs }}$. In fact, these different hypotheses represent the different model space dimensions that we consider in a transdimensional inverse problem.

Transdimensional data fitting: To illustrate the naive transdimensional sampling, we consider a classical data fitting problem, closely following a similar example given by Sambridge et al. (2006). For this, we compute artificial data $d_{i}$ using the linear function

$$
d_{i}=m_{1}+m_{2} x_{i}+\varepsilon_{i},
$$

where $\mathbf{m}=\left(m_{1}, m_{2}\right)=(1.0,1.0)$ are the model parameters that we pretend to be unknown, $x_{i}$ are the measurement locations, and $\varepsilon_{i}$ are random Gaussian errors with standard deviation $\sigma=2.0$. The artificial data are shown in figure 5.16. Not knowing the linear function behind the artificial data, one may fit the data points with polynomials of variable order, ranging from constant to cubic, or even higher. Clearly, increasing the order improves the fit, but which order (model complexity) is still justified by the data?

For simplicity, we choose a uniform prior for the model space dimension, $\rho(n)$, meaning that all model space dimensions have equal probability. To estimate the conditional evidence, $\rho\left(\mathbf{d}^{\text {obs }} \mid n\right)$, needed to evaluate the probability $P_{n}$ in equation (5.128), we note that the fixed-dimensional Bayes' theorem implies

$$
\rho\left(\mathbf{d}^{\mathrm{obs}} \mid n\right)=\int_{\mathbb{M}} \rho\left(\mathbf{d}^{\mathrm{obs}} \mid \mathbf{m}, n\right) \rho(\mathbf{m}, n) d \mathbf{m},
$$

because the fixed-dimensional posterior $\rho\left(\mathbf{m} \mid \mathbf{d}^{\text {obs }}, n\right)$ must integrate to 1 . Using the concepts introduced in section 5.3, we can approximate the integral on the right-hand side of equation (5.130) using samples $\mathbf{~ m}^{j}$ $\left(j=1, \ldots, N_{s}\right)$ from the fixed-dimensional prior $\rho(\mathbf{m} \mid n)$. Using equation (5.28), we then obtain

$$
\rho\left(\mathbf{d}^{\mathrm{obs}} \mid n\right) \approx \frac{1}{N_{s}} \sum_{j=1}^{N_{s}} \rho\left(\mathbf{d}^{\mathrm{obs}} \mid \mathbf{m}^{j}, n\right) .
$$

Equation (5.131) suggests that the conditional evidence $\rho\left(\mathbf{d}^{\mathrm{obs}} \mid n\right)$ can be approximated by evaluating the fixeddimensional likelihood function $\rho\left(\mathbf{d}^{\mathrm{obs}} \mid \mathbf{m}^{j}, n\right)$ at samples $\mathbf{m}^{j}$ of the prior. Thus, $\rho\left(\mathbf{d}^{\mathrm{obs}} \mid n\right)$ is essentially a byproduct of the global Metropolis-Hastings algorithm introduced in section 5.5.1. At this stage, we have all 


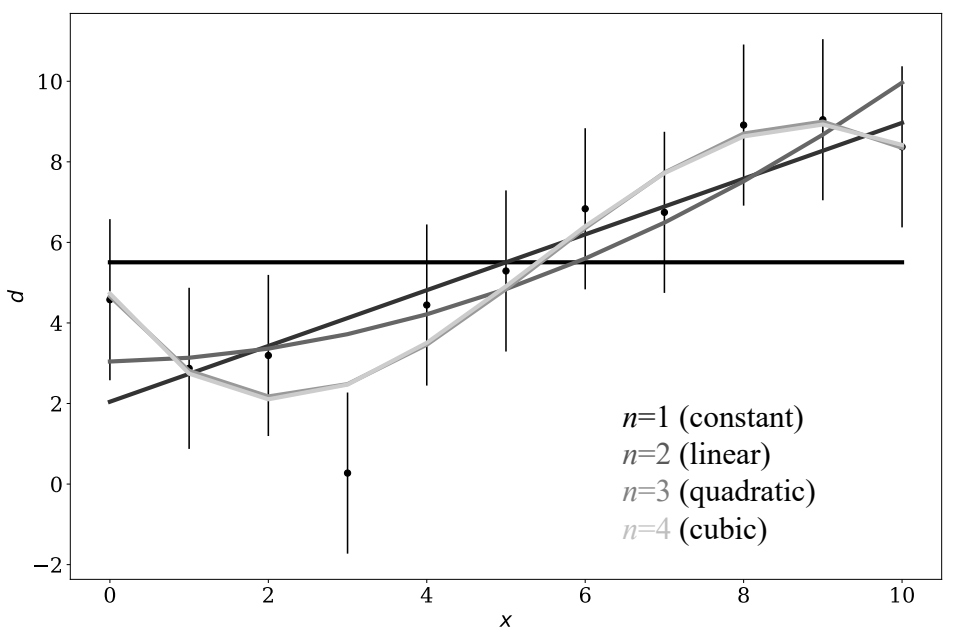

Fig. 5.16: Artificial data (black) and best-fitting polynomials of various orders (coloured) used for the naive transdimensional sampling. The observation points, $x_{i}$, are equally spaced. The error bars represent the standard deviation of the Gaussian errors, \pm 2.0 . [Transdimensional - Naive]

ingredients for the naive transdimensional sampling. The probabilities $P_{n}$ from equation (5.128) provide the posterior distribution of the model space dimension, displayed in figure 5.17. It clearly indicates that the preferred dimension is $n=2$, that is, the linear regression of the data points shown in figure 5.16.

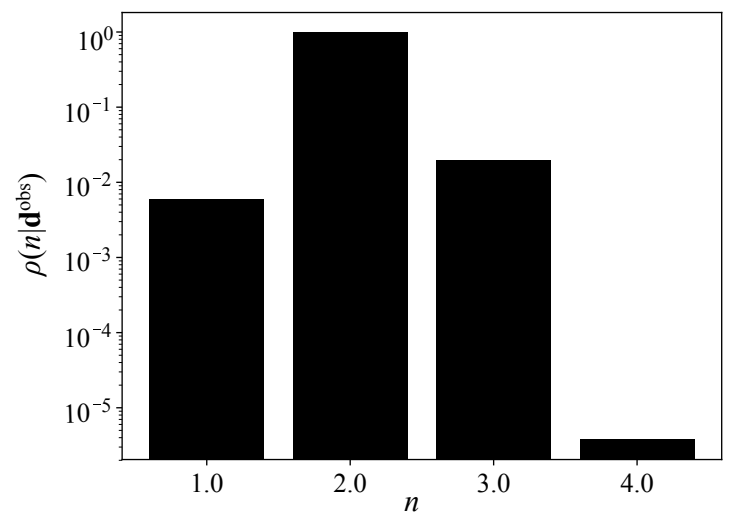

Fig. 5.17: Posterior $\rho\left(n \mid \mathbf{d}^{\text {obs }}\right)$ for the model space dimension $n$ based on the naive transdimensional sampling method. Note the logarithmic $y$-axis. [Transdimensional - Naive]

Despite being conceptually simple, the naive transdimensional sampling has the potential to be hugely inefficient because all fixed-dimensional posteriors need to be sampled beforehand, despite being potentially unlikely from the outset. The reversible-jump algorithm, introduced by Green (1995) and described in the following section, circumvents this problem through the design of a random walk that is able to transition between different dimensions while maintaining detailed balance. 


\subsubsection{The reversible-jump algorithm}

The concept of the reversible-jump algorithm can be explained most easily by comparison to fixed-dimensional samplers, such as the Metropolis-Hastings algorithm. As in section 5.2, we consider a generic probability density $q(\mathbf{m}, n)$ that we wish to sample. As so often, a detour via a higher-dimensional space will turn out to be useful.

In global Metropolis-Hastings sampling (section 5.5.1), transition proposals from the current model $\mathbf{m}^{j}$ to a new model $\mathbf{m}^{i}$ (both of the same dimension) are made by drawing random samples from the prior, $\rho(\mathbf{m})$. The reversible-jump algorithm, in contrast, achieves a random transition from $\mathbf{m}^{j}$ of dimension $n^{j}$ to $\mathbf{m}^{i}$ of dimension $n^{i}$ by first appending $\mathbf{m}^{j}$ by the dimension variable $n^{j}$, and by a random vector $\mathbf{u}^{j}$, drawn from a proposal distribution $g^{j}\left(\mathbf{u}^{j}\right)$. This produces the extended, semi-random vector

$$
\mathbf{x}^{j}=\left(n^{j}, \mathbf{m}^{j}, \mathbf{u}^{j}\right) \in \mathbb{X}
$$

Subsequently, $\mathbf{x}^{j}$ is mapped via a deterministic and invertible function $\mathbf{h}$ onto a new vector, $\mathbf{x}^{i}=\left(n^{i}, \mathbf{m}^{i}, \mathbf{u}^{i}\right)$, that is,

$$
\mathbf{x}^{i}=\mathbf{h}\left(\mathbf{x}^{j}\right)
$$

The dimension $n^{i}$ of the proposed model vector $\mathbf{m}^{i}$ is allowed to differ from the current model vector dimension $n^{j}$, but the combined dimension of $\mathbf{x}^{i}=\left(n^{i}, \mathbf{m}^{i}, \mathbf{u}^{i}\right)$ is still the same as the dimension of $\mathbf{x}^{j}=\left(n^{j}, \mathbf{m}^{j}, \mathbf{u}^{j}\right)$. A visual description of the fixed- and transdimensional proposal procedures is shown in figure 5.18, which also introduces an indexing of the extended vectors $\mathbf{x}^{j}$ and $\mathbf{x}^{i}$ that will turn out to be convenient later.

fixed-dimensional random walk

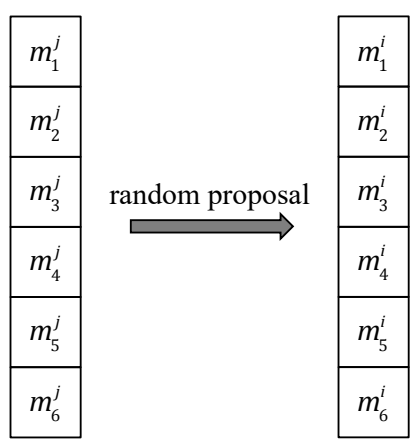

transdimensional random walk

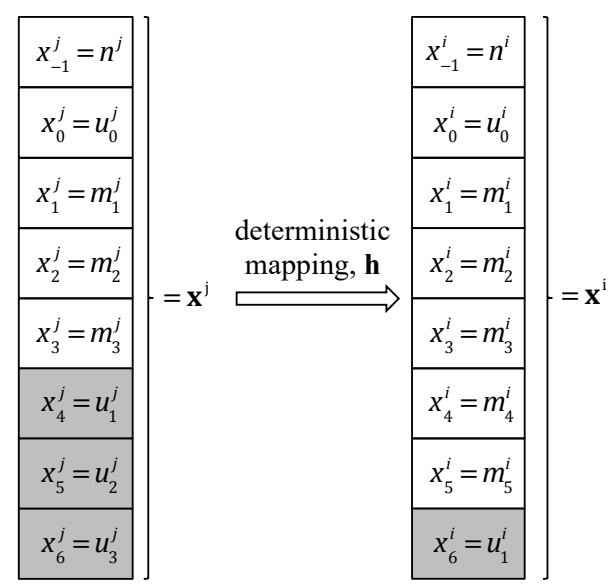

Fig. 5.18: Comparison of fixed-dimensional (left) and transdimensional (right) random-walk proposals. The random components of the two procedures are shown in grey. In fixed-dimensional random walks, the transition from a given $\mathbf{m}^{j}$ to some new $\mathbf{m}^{i}$ is proposed in a random fashion, for instance, by drawing random samples from the posterior, as in the global Metropolis-Hastings algorithm (section 5.5.1). Transdimensional random walks, in contrast, append the current model $\mathbf{m}^{j}$ by the model space dimension $n^{j}$ and by a set of randomly chosen numbers $\mathbf{u}^{j}$. The extended vector $\mathbf{x}^{j}$ is then mapped via a deterministic function $\mathbf{h}$ to a new extended vector $\mathbf{x}^{i}$ of the same dimension. For later convenience, we start the indexing of $\mathbf{x}^{i, j}$ at $x_{-1}^{i, j}$, which contains the model space dimension $n^{i, j}$. The next element, $x_{0}^{i, j}$, takes the first component of the random number vectors, $u_{0}^{i, j}$. The remaining components are filled by the actual model vector and the remaining random numbers. 
An important element of the reversible-jump algorithm, that is different from Metropolis-Hastings sampling, is that the proposition of the move $\mathbf{x}^{j} \rightarrow \mathbf{x}^{i}$ is deterministic in the sense that once $\mathbf{x}^{j}$ is known, the proposition of $\mathbf{x}^{i}$ is absolutely certain. Furthermore, since $\mathbf{h}$ is required to be invertible, it maps disjoint subvolumes $\mathbb{X}^{j}$ that partition $\mathbb{X}$ into another set of subvolumes $\mathbb{X}^{i}$ that partition $\mathbb{X}$ as well. This is illustrated in figure 5.19.

mapping to extended model space $\mathbb{X}$ deterministic proposal

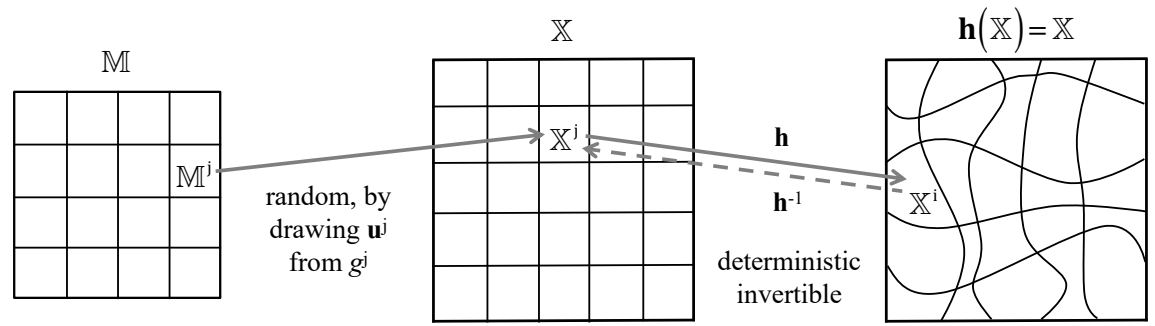

Fig. 5.19: Illustration of the proposal procedure of the reversible-jump algorithm. Coming from $\mathbf{x}^{j} \in \mathbb{M}^{j}$, an element $\mathbf{x}^{j}$ within subvolume $\mathbb{X}^{j}$ of the extended model space is found by drawing a random vector $\mathbf{u}^{j}$ from the distribution $g^{j}$, that we are free to choose. From that point onwards, the proposal is deterministic, meaning that the invertible function $\mathbf{h}$ maps $\mathbf{x}^{j}$ onto a proposal $\mathbf{x}^{i} \in \mathbb{X}^{i}$.

It follows that the proposal density of $\mathbf{x}^{i}$ is equal to the extended probability density

$$
q^{j}\left(\mathbf{x}^{j}\right)=q\left(\mathbf{m}^{j}, n^{j}\right) g^{j}\left(\mathbf{u}^{j}\right),
$$

for actually being at $\mathbf{x}^{j}$. Denoting by $\alpha\left(\mathbf{x}^{i}, \mathbf{x}^{j}\right)$ the acceptance probability density for the proposed move, we infer that the unconditional probability of landing within the subvolume $\mathbb{X}^{i}$ of the extended space $\mathbb{X}$ is given by

$$
T^{i \leftarrow j} Q^{j}=\int_{\mathbb{X}^{j}} \alpha\left(\mathbf{x}^{i}, \mathbf{x}^{j}\right) q^{j}\left(\mathbf{x}^{j}\right) d \mathbf{x}^{j},
$$

where we used the notation introduced in section 5.2 on general random walks ${ }^{16}$. Requesting detailed balance, that is $T^{i \leftarrow j} Q^{j}=T^{j \leftarrow i} Q^{i}$ (see equation (5.18)), we obtain

$$
\int_{\mathbb{X}^{j}} \alpha\left(\mathbf{x}^{i}, \mathbf{x}^{j}\right) q^{j}\left(\mathbf{x}^{j}\right) d \mathbf{x}^{j}=\int_{\mathbb{X}^{i}} \alpha\left(\mathbf{x}^{j}, \mathbf{x}^{i}\right) q^{i}\left(\mathbf{x}^{i}\right) d \mathbf{x}^{i}
$$

Requesting that the transition probability density $\alpha$ only depends on $\mathbf{m}^{j}$ and $\mathbf{m}^{i}$, and noting that $d \mathbf{x}^{j}=d \mathbf{m}^{j} d \mathbf{u}^{j}$, equation (5.136) may equivalently be written as

$$
\int_{\mathbb{M}^{j}} \int_{\mathbb{U}^{j}} \alpha\left(\mathbf{m}^{i}, \mathbf{m}^{j}\right) q\left(\mathbf{m}^{j}, n^{j}\right) g^{j}\left(\mathbf{u}^{j}\right) d \mathbf{m}^{j} d \mathbf{u}^{j}=\int_{\mathbb{M}^{i} \mathbb{U}^{i}} \alpha\left(\mathbf{m}^{j}, \mathbf{m}^{i}\right) q\left(\mathbf{m}^{i}, n^{i}\right) g^{i}\left(\mathbf{u}^{i}\right) d \mathbf{m}^{i} d \mathbf{u}^{i},
$$

where we also made use of equation (5.134). The integrals on both sides of equation (5.137) are over different subvolumes of the extended model space. With the help of a coordinate transformation, we may convert the integral on the right-hand side into an integral over $\mathbb{M}^{j} \times \mathbb{U}^{j}$,

\footnotetext{
${ }^{16}$ Strictly speaking, $T^{i \leftarrow j} Q^{j}$ is the uncoditional probability of moving from $\left(\mathbf{m}^{j}, n^{j}\right)$ to $\left(\mathbf{m}^{i}, n^{i}\right)$, and not for a move from $\mathbf{x}^{j}$ to $\mathbf{x}^{i}$. The latter is deterministic, and only $\mathbf{x}^{i}$ is accessible from $\mathbf{x}^{j}$ anyway.
} 


$$
\int_{\mathbb{M}^{j}} \int_{\mathbb{U}^{j}} \alpha\left(\mathbf{m}^{i}, \mathbf{m}^{j}\right) q\left(\mathbf{m}^{j}, n^{j}\right) g^{j}\left(\mathbf{u}^{j}\right) d \mathbf{m}^{j} d \mathbf{u}^{j}=\int_{\mathbb{M}^{j} \mathbb{U}^{j}} \alpha\left(\mathbf{m}^{j}, \mathbf{m}^{i}\right) q\left(\mathbf{m}^{i}, n^{i}\right) g^{i}\left(\mathbf{u}^{i}\right) J d \mathbf{m}^{j} d \mathbf{u}^{j},
$$

with $J$ being the Jacobian of the coordinate transformation $\mathbf{h}$, that is,

$$
J=\left|\operatorname{det} \frac{\partial \mathbf{h}\left(n^{j}, \mathbf{m}^{j}, \mathbf{u}^{j}\right)}{\partial\left(n^{j}, \mathbf{m}^{j}, \mathbf{u}^{j}\right)}\right|=\left|\operatorname{det} \frac{\partial \mathbf{x}^{i}}{\partial \mathbf{x}^{j}}\right| .
$$

For $J$ to exist and to be generally non-zero, we require that $\mathbf{h}$ be a diffeomorphism, that is, not only invertible but also differentiable. With equation (5.138) holding for any (infinitesimally small) subvolume $\mathbb{X}^{j}=\mathbb{M}^{j} \times \mathbb{U}^{j}$, the integrands on both sides must be equal, meaning that

$$
\frac{\alpha\left(\mathbf{m}^{i}, \mathbf{m}^{j}\right)}{\alpha\left(\mathbf{m}^{j}, \mathbf{m}^{i}\right)}=\frac{q\left(\mathbf{m}^{i}, n^{i}\right) g^{i}\left(\mathbf{u}^{i}\right)}{q\left(\mathbf{m}^{j}, n^{j}\right) g^{j}\left(\mathbf{u}^{j}\right)} J\left(n^{i}, \mathbf{m}^{i}, \mathbf{u}^{i}\right) .
$$

As described before in section 5.5, many possible choices of acceptance probability densities satisfy equation (5.140), which is the varible-dimension equivalent of equation (5.55). The most efficient choice is the Metropolis rule

$$
\alpha\left(\mathbf{m}^{i}, \mathbf{m}^{j}\right)=\min \left(1, \frac{q\left(\mathbf{m}^{i}, n^{i}\right) g^{i}\left(\mathbf{u}^{i}\right)}{q\left(\mathbf{m}^{j}, n^{j}\right) g^{j}\left(\mathbf{u}^{j}\right)} J\left(n^{i}, \mathbf{m}^{i}, \mathbf{u}^{i}\right)\right) .
$$

So far, we considered the transdimensional sampling of a generic probability density $q$. Specifying the Metropolis rule to the sampling of the transdimensional posterior $\rho\left(\mathbf{m}, n \mid \mathbf{d}^{\text {obs }}\right)$ from the generalised Bayes' theorem (5.126), gives

$$
\alpha\left(\mathbf{m}^{i}, \mathbf{m}^{j}\right)=\min \left(1, \frac{\rho\left(\mathbf{d}^{\mathrm{obs}} \mid \mathbf{m}^{i}, n^{i}\right) \rho\left(\mathbf{m}^{i} \mid n^{i}\right) \rho\left(n^{i}\right) g^{i}\left(\mathbf{u}^{i}\right)}{\rho\left(\mathbf{d}^{\mathrm{obs}} \mid \mathbf{m}^{j}, n^{j}\right) \rho\left(\mathbf{m}^{j} \mid n^{j}\right) \rho\left(n^{j}\right) g^{j}\left(\mathbf{u}^{j}\right)} J\left(n^{i}, \mathbf{m}^{i}, \mathbf{u}^{i}\right)\right) .
$$

Again, we note that the total evidence $\rho\left(\mathbf{d}^{\text {obs }}\right)$ has canceled. In fact, as in the fixed-dimensional case, the Metropolis rule can be evaluated purely on the basis of the priors in model and data space.

Addition or removal of dimensions: We consider cases where a transition corresponds to a simple increase or decrease of dimension. Most of the work will be in designing the transformation $\mathbf{h}$ and the corresponding probability densities for random numbers, $g^{j}$ and $g^{i}$. Denoting by $N$ the maximum allowable model space dimension, we start by drawing the random numbers, $\mathbf{u}^{j}=\left(u_{0}^{j}, u_{1}^{j}, \ldots, u_{N-n^{j}}\right)$ from the probability distribution $g^{j}$ that we partition into two components,

$$
g^{j}\left(\mathbf{u}^{j}\right)=g_{d}^{j}\left(u_{0}^{j}\right) g_{m}^{j}\left(u_{1}^{j}, \ldots, u_{n^{i}-n^{j}}\right) .
$$

The component $g_{d}^{j}$ of the distribution will be used to determine the dimension of the newly proposed model. Here, we choose a uniform, discrete distribution, producing the values $(1, \ldots, N)$ with equal probability. Thus, $g_{d}^{j}=N^{-1}$. The component $g_{m}^{j}$ will serve to generate random model vector perturbations. For convenience, we choose an $\left(N-n^{j}\right)$-dimensional uniform distribution in $[0,1]^{N-n^{j}}$, that is, $g_{m}^{j}=1$. We first consider the scenario where the newly proposed model space dimension $n^{i}=u_{j}^{0}$ is greater or equal than the current dimension $n^{j}$. In this case, we define $\mathbf{h}$ as follows,

$$
\mathbf{x}^{i}=\mathbf{h}\left(\mathbf{x}^{j}\right)=\left\{\begin{array}{ll}
n^{i}=u_{0}^{j}, \\
u_{0}^{i}=n^{j}, \\
m_{k}^{i}=m_{k}^{j}, & \text { for } k=1, \ldots, n^{j} \\
m_{k}^{i}=\Delta m u_{k-n^{j}}^{j}, & \text { for } k=n^{j}+1, \ldots, n^{i} \\
u_{k-n^{i}}^{i}=u_{k-n^{j}}^{j}, & \text { for } k=n^{i}+1, \ldots, N
\end{array}= \begin{cases}x_{-1}^{i}=x_{0}^{j}, & \\
x_{0}^{i}=x_{-1}^{j}, & \\
x_{k}^{i}=x_{k}^{j}, & \text { for } k=1, \ldots, n^{j} \\
x_{k}^{i}=\Delta m x_{k}^{j}, & \text { for } k=n^{j}+1, \ldots, n^{i} \\
x_{k}^{i}=x_{k}^{j} & \text { for } k=n^{i}+1, \ldots, N .\end{cases}\right.
$$


In the first two lines of equation (5.144), we simply swap the first two components of the extended vectors $\mathbf{x}^{j}$ and $\mathbf{x}^{i}$. Specifically, the dimension of the proposed model $n^{i}$ is set equal to the randomly drawn number $u_{0}^{j}$. In return, we assign the current model vector dimension $n^{j}$ to the extended vector component $x_{0}^{i}$. This is needed only to make the transformation invertible.

The third line of equation (5.144) copies the $n^{j}$ components of the current model vector $\mathbf{m}^{j}$ into the first $n^{j}$ components of the proposed model vector $\mathbf{m}^{j}$. Thus, the proposed and the current model vectors differ only in the newly added dimensions. For these, we use $n^{i}-n^{j}$ components of the randomly generated vector $\mathbf{u}^{j}$, as we can see in the fourth line of equation (5.144). The added model vector components $m_{k}^{i}$ are set equal to the uniformly distributed numbers $u_{k-n^{j}}^{j}$ times some scaling $\Delta m$, which controls the spread of the model parameter values. Finally, in the fifth line of equation (5.144), we copy the remaining, so far unused, components of the extended model vectors into each other.

The transformation (5.144) may also be written in a more readable vector-matrix notation,

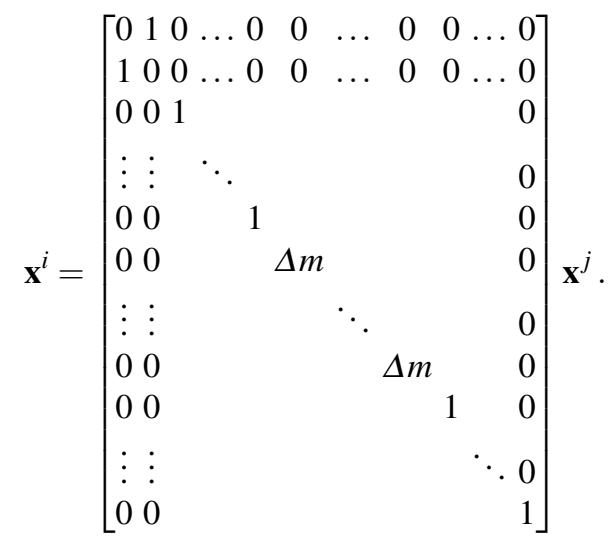

As requested, the transformation $\mathbf{h}$ represented by the matrix in equation (5.145) is invertible and differentiable. The Jacobian of $\mathbf{h}$ is

$$
J=|\Delta m|^{n^{i}-n^{j}} .
$$

The inverse of equations (5.144) and (5.145) corresponds to a reduction in model space dimension, from $n^{i}$ to $n^{j} \leq n^{i}$. It is given by

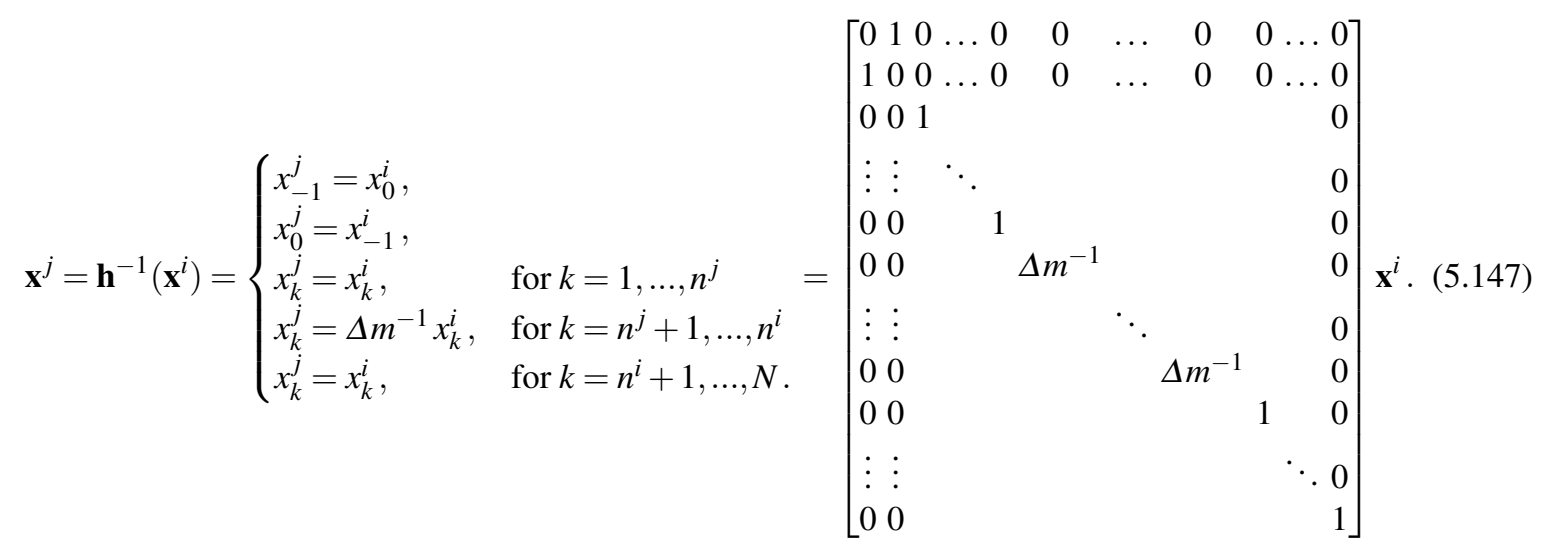

The inverse transformation (5.147) provides information on how to design the probability distributions $g_{d}^{i}$ and $g_{m}^{i}$ for the random numbers $u_{0}^{i}$ and $u_{k}^{i}$, with $k=1, \ldots, N-n^{i}$, respectively. In fact, the fifth line in the middle part of equation (5.147), which corresponds to $u_{k-n^{j}}^{j}=u_{k-n^{i}}^{i}$ for $k=n^{i}+1, \ldots, N$, determines that the distribution of the $N-n^{i}$ random numbers in $\mathbf{u}^{i}$ must be the same as the distribution of the last $N-n^{i}$ components of $\mathbf{u}^{j}$. 
It follows that $g_{m}^{i}=1$, just as $g_{m}^{j}=1$. Equation (5.147) does not impose a similar constraint on $g_{d}^{i}$, and so we conveniently choose it to be the same as $g_{d}^{j}$, that is, uniform between 1 and $N$.

It remains to choose the fixed-dimensional model priors. For simplicity, we define them to be uniform as well, that is,

$$
\rho(\mathbf{m} \mid n)=\frac{1}{|\Delta m|^{n}} .
$$

The Metropolis rule (5.142) then simplifies to

$$
\alpha\left(\mathbf{m}^{i}, \mathbf{m}^{j}\right)=\min \left(1, \frac{\rho\left(\mathbf{d}^{\mathrm{obs}} \mid \mathbf{m}^{i}, n^{i}\right) \rho\left(n^{i}\right)}{\rho\left(\mathbf{d}^{\mathrm{obs}} \mid \mathbf{m}^{j}, n^{j}\right) \rho\left(n^{j}\right)}\right) .
$$

Equation (5.161) holds for the case of increasing dimension. For decreasing dimension, the transformation defined in (5.144) must be used, with $i$ and $j$ interchanged. Repeating the analysis, then again leads to the same Metropolis rule as in equation (5.161). This shows that transitions between dimensions are not affected by the size of the respective model spaces. Instead, they are controlled by the ratio of the data priors and the prior knowledge (preference) for specific model space dimensions, $\rho(n)$. In the special case of equal dimensions, $n^{i}=n^{j}$, equation (5.161) further reduces to the Metropolis rule for the fixed-dimensional case (5.57).

Alternative transformations: The transformation proposed in equation (5.144) is by no means the only option. For instance, instead of drawing random numbers $u_{k>1}^{j}$ uniformly between 0 and 1 and then scaling them by $\Delta m$, we could have drawn them uniformly between 0 and $\Delta m$, without the need of any scaling. The corresponding probability density is

$$
g_{m}^{j}=\Delta m^{n^{j}-N},
$$

and the transformation for an increase of dimension from $n^{j}$ to $n^{i} \geq n^{j}$ is

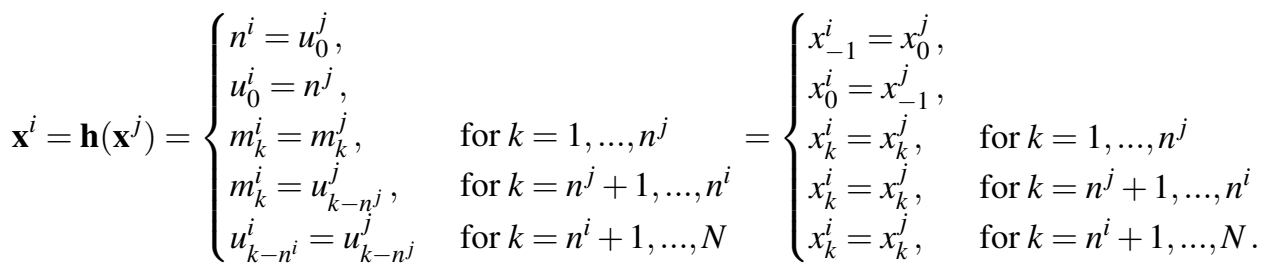

The Jacobian of this transformation is $J=1$. For the inverse transformation that reduces the model space dimension, we find

$$
\mathbf{x}^{j}=\mathbf{h}^{-1}\left(\mathbf{x}^{i}\right)= \begin{cases}x_{-1}^{j}=x_{0}^{i}, & \\ x_{0}^{j}=x_{-1}^{i}, & \\ x_{k}^{j}=x_{k}^{i}, & \text { for } k=1, \ldots, n^{j} \\ x_{k}^{j}=x_{k}^{i}, & \text { for } k=n^{j}+1, \ldots, n^{i} \\ x_{k}^{j}=x_{k}^{i}, & \text { for } k=n^{i}+1, \ldots, N .\end{cases}
$$

As in the previous example, the fifth line of equation (5.152) determines the probability distribution $g_{m}^{i}$. In fact, since the $x_{k>n^{j}+1}^{j}$ vary uniformly between 0 and $\Delta m$, the $x_{k>n^{i}+1}^{i}$ must vary uniformly between 0 and $\Delta m$ as well. Therefore,

$$
g_{m}^{i}=\Delta m^{n^{i}-N} .
$$

Choosing the same uniform fixed-dimension model priors $\rho(\mathbf{m} \mid n)$ as in equation (5.160), finally leads to the acceptance probability

$$
\alpha\left(\mathbf{m}^{i}, \mathbf{m}^{j}\right)=\min \left(1, \frac{\rho\left(\mathbf{d}^{\mathrm{obs}} \mid \mathbf{m}^{i}, n^{i}\right) \rho\left(n^{i}\right)}{\rho\left(\mathbf{d}^{\mathrm{obs}} \mid \mathbf{m}^{j}, n^{j}\right) \rho\left(n^{j}\right)}\right),
$$

which is the same as in the previous example. Thus, different transformations can effectively lead to the same algorithm. 
The birth-death algorithm: The algorithms designed in the previous two examples allow us to access any model space dimension $n^{i}$ from the current model space dimension $n^{j}$. Large jumps in dimension can lead to rather low acceptance ratios. When dimension strongly decreases, the new and much simpler model is unlikely to explain the observations. Conversely, when dimension strongly increases, the curse of dimensionality (section SS:curse) makes it unlikely to randomly find a plausible model. To avoid that model space exploration becomes uselessly slow, we may limit the dimension of the proposed model to be either one more or one less than the current model. This is referred to as the birth-death variant of the reversible-jump algorithm. For its implementation, we modify the tranformation $\mathbf{h}$ from equation (5.144) to

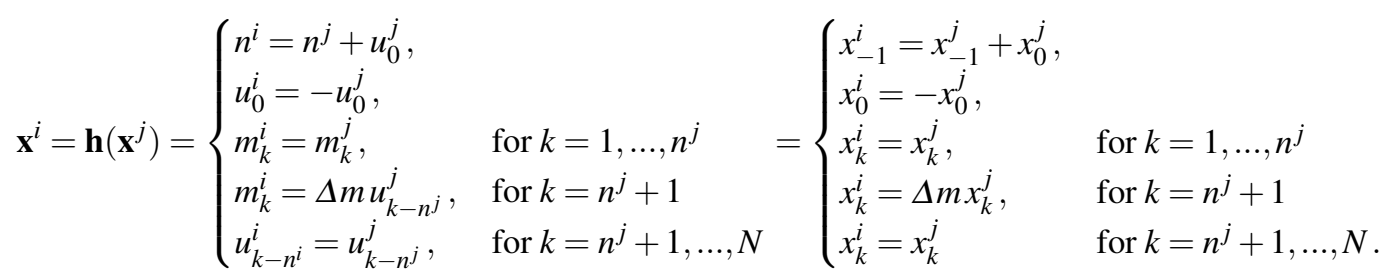

The random number $u_{0}^{j}$ is now purely binary. It takes the value +1 with probability $b_{j}$ (adding a dimension (birth)), and the value -1 with probability $d_{j}=b_{j}-1$ (removing a dimension (death)). Thus, in symbols,

$$
g_{0}^{j}(1)=b_{j}, \quad g_{0}^{j}(-1)=d_{j} .
$$

The Jacobian of the transformation (5.155) is $J=\Delta m$. When dimension increases, $u_{0}^{j}=1$, the second line of (5.155) implies $u_{0}^{j}=-1$. Therefore

$$
g_{0}^{i}\left(u_{0}^{i}\right)=g_{0}^{i}(-1)=d_{i},
$$

which is the probability of decreasing model space dimension from the proposed dimension $n^{i}$ back to $n^{j}=$ $n^{i}-1$. We then deduce the acceptance probability

$$
\alpha\left(\mathbf{m}^{i}, \mathbf{m}^{j}\right)=\min \left(1, \frac{\rho\left(\mathbf{d}^{\mathrm{obs}} \mid \mathbf{m}^{i}, n^{i}\right) \rho\left(n^{i}\right)}{\rho\left(\mathbf{d}^{\mathrm{obs}} \mid \mathbf{m}^{j}, n^{j}\right) \rho\left(n^{j}\right)} \frac{d_{i}}{b_{j}}\right) .
$$

Similarly, for a reduction in dimension, we have $u_{0}^{j}=-1$ and $u_{0}^{i}=1$, and therefore $g_{0}^{j}\left(u_{0}^{j}\right)=d_{j}$ and $g_{0}^{i}\left(u_{0}^{i}\right)=b_{i}$. The resulting acceptance probability is

$$
\alpha\left(\mathbf{m}^{i}, \mathbf{m}^{j}\right)=\min \left(1, \frac{\rho\left(\mathbf{d}^{\mathrm{obs}} \mid \mathbf{m}^{i}, n^{i}\right) \rho\left(n^{i}\right)}{\rho\left(\mathbf{d}^{\mathrm{obs}} \mid \mathbf{m}^{j}, n^{j}\right) \rho\left(n^{j}\right)} \frac{b_{i}}{d_{j}}\right) .
$$

Typical choices for the probabilities $b_{j}$ and $d_{j}$ are $b_{j}=d_{j}=0.5$ for $j=2, \ldots, N, b_{N}=d_{1}=0$, and $b_{1}=d_{N}=1$.

Transdimensional data fitting revisited: For illustration, we revisit the polynomial data fitting problem that we solved by the naive transdimensional sampler in section 5.8.2. Keeping the same setup, the birth-death variant of the reversible-jump algorithm, described above, produces the posterior marginals shown in figure 5.20. The 1D posterior marginal for the model space dimension is practically identical to the one shown in figure 5.17 for the fixed-dimensional sampler, as it should be. The 2D posterior marginals indicate that the posterior distributions for model parameters $m_{1}$ and $m_{2}$ are strongly dependent on the model space dimension (polynomial degree).

Reduction to the fixed-dimensional Metropolis-Hastings algorithm: In the case of a fixed-dimensional problem with $n^{i}=n^{j}=n$, we may indeed show that the Metropolis-Hastings algorithm follows from the reversiblejump algorithm. For this, we draw $n$ random numbers $\mathbf{u}^{j}$ from the model prior $\rho(\mathbf{m} \mid n)$, and we define the transformation $h$ as 
a) $1 \mathrm{D}$ posterior marginal for model space dimension

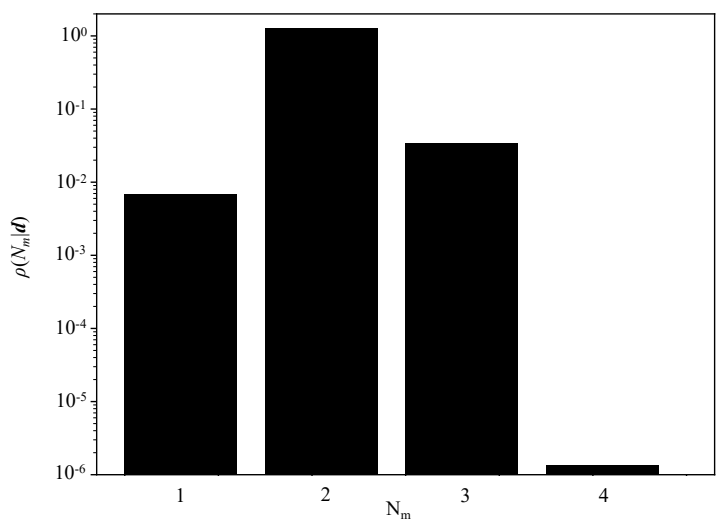

b) 2D posterior marginals

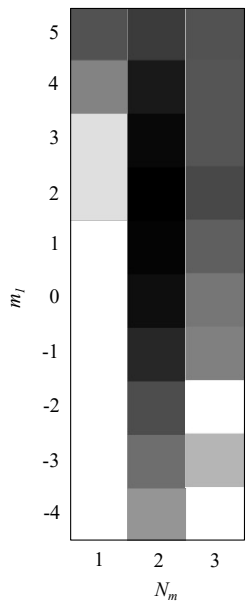

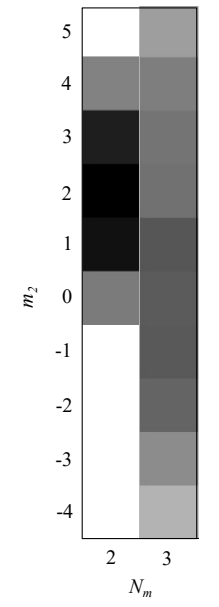

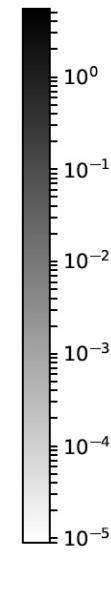

Fig. 5.20: Posterior marginals for the polynomial data fitting example from section 5.8.2, obtained with the birth-death variant of the reversible-jump algorithm. (a) 1D posterior marginal for the model space dimension $n$. The result is practically identical with the posterior computed with the naive transdimensional sampler, shown in figure 5.17. (b) 2D posterior marginals for the model space dimension $n$ and the model parameters $m_{1}$ (left) and $m_{2}$ (right). The maximum-likelihood values of $m_{1}$ and $m_{2}$ strongly depend on the degree of the polynomial. Note the logarithmic scales in all plots. [Transdimensional - Reversible Jump]

$$
m_{n}^{i}=u_{n}^{j},
$$

for $n=1, \ldots, n$. Equation (5.160) means that model parameter proposals are drawn from the model prior, as in Metropolis-Hastings sampling. It follows that $J=1$ and $\rho\left(\mathbf{m}^{i}\left(\mathbf{u}^{j}\right) \mid n\right)=g^{j}\left(\mathbf{u}^{j}\right)$. Similarly, for the reverse move, we have $\rho\left(\mathbf{m}^{j}\left(\mathbf{u}^{i}\right) \mid n\right)=g^{i}\left(\mathbf{u}^{i}\right)$. From this we deduce that the transdimensional Metropolis rule (5.142) again condenses to the fixed-dimensional Metropolis rule (5.57), that is

$$
\alpha\left(\mathbf{m}^{i}, \mathbf{m}^{j}\right)=\min \left(1, \frac{\rho\left(\mathbf{d}^{\mathrm{obs}} \mid \mathbf{m}^{i}, n^{i}\right)}{\rho\left(\mathbf{d}^{\mathrm{obs}} \mid \mathbf{m}^{j}, n^{j}\right)}\right) .
$$


Part II

Linear And Weakly Nonlinear Problems 



\section{Chapter 6 \\ The least-squares method for linear problems *}

Dear Lord, please make the world linear and normally distributed.

Statistician's prayer.

Already in section 4.6 we briefly examined the special case where all prior information is Gaussian and the forward modelling equations are linear. Invoking Bayes' theorem (4.12), we found the posterior to be given by

$$
\rho\left(\mathbf{m} \mid \mathbf{d}^{\text {obs }}\right)=\text { const. } e^{-\frac{1}{2}\left(\mathbf{m}-\mathbf{m}^{\text {prior }}\right)^{T} \mathbf{C}_{M}^{-1}\left(\mathbf{m}-\mathbf{m}^{\text {prior }}\right)-\frac{1}{2}\left(\mathbf{G m}-\mathbf{d}^{\text {obs }}\right)^{T} \mathbf{C}_{D}^{-1}\left(\mathbf{G m}-\mathbf{d}^{\text {obs }}\right)} .
$$

Equation (6.1) is remarkable for various reasons. First of all, we note that the maximum-likelihood model $\tilde{\mathbf{m}}$ may be found by minimising the misfit function

$$
\chi(\mathbf{m})=\frac{1}{2}\left(\mathbf{m}-\mathbf{m}^{\text {prior }}\right)^{T} \mathbf{C}_{M}^{-1}\left(\mathbf{m}-\mathbf{m}^{\text {prior }}\right)+\frac{1}{2}\left(\mathbf{G m}-\mathbf{d}^{\mathrm{obs}}\right)^{T} \mathbf{C}_{D}^{-1}\left(\mathbf{G m}-\mathbf{d}^{\mathrm{obs}}\right) .
$$

This corresponds to finding the minimum of the weighted squared differences between observed and synthetically computed data, $\left(\mathbf{G m}-\mathbf{d}^{\text {obs }}\right)^{T} \mathbf{C}_{D}^{-1}\left(\mathbf{G m}-\mathbf{d}^{\text {obs }}\right)$, plus an additional quadratic term that captures our preference for the prior model, $\left(\mathbf{m}-\mathbf{m}^{\text {prior }}\right) \mathbf{C}_{M}^{-1}\left(\mathbf{m}-\mathbf{m}^{\text {prior }}\right)$. In other words, finding the maximum-likelihood model corresponds to the solution of a least-squares problem, first encountered in section 1.3.2.

Second, we note that equation (6.2) is quadratic in $\mathbf{m}$ and may therefore be rewritten as

$$
\chi(\mathbf{m})=\frac{1}{2}(\mathbf{m}-\tilde{\mathbf{m}})^{T} \tilde{\mathbf{C}}_{M}^{-1}(\mathbf{m}-\tilde{\mathbf{m}})+c,
$$

where the constant $c$ absorbs all terms that do not depend on the model parameter vector $\mathbf{m}$. It follows that the posterior $\rho\left(\mathbf{m} \mid \mathbf{d}^{\text {obs }}\right)=$ const. $e^{-\chi(\mathbf{m})}$ is again a pure Gaussian in the model parameters $\mathbf{m}$, with the posterior model mean $\tilde{\mathbf{m}}$ and the posterior model covariance $\tilde{\mathbf{C}}_{M}$.

Being able to compute $\tilde{\mathbf{m}}$ and $\tilde{\mathbf{C}}_{M}$ explicitly, is particularly convenient, because these two quantities fully describe the posterior. It follows that the posterior can be expressed in closed form, analytically. This eliminates the need to approximate the posterior, for instance, by sampling with the help of Monte Carlo methods. In the following paragraphs we will provide explicit formulas for $\tilde{\mathbf{m}}$ and $\tilde{\mathbf{C}}_{M}$.

Interpreting prior knowledge as additional data: The incorporation of prior knowledge in the form of the contribution $\left(\mathbf{m}-\mathbf{m}^{\text {prior }}\right)^{T} \mathbf{C}_{M}^{-1}\left(\mathbf{m}-\mathbf{m}^{\text {prior }}\right)$ has an interesting interpretation in terms of artificial data added to the inverse problem. To see this, we write the positive-definite inverse covariance matrices $\mathbf{C}_{M}^{-1}$ and $\mathbf{C}_{D}^{-1}$ as the square of their respective matrix square roots (that are not uniquely determined), 


$$
\mathbf{C}_{M}^{-1}=\mathbf{W}_{M}^{T} \mathbf{W}_{M}, \quad \mathbf{C}_{D}^{-1}=\mathbf{W}_{D}^{T} \mathbf{W}_{D} .
$$

Using the weight matrices $\mathbf{W}_{M}$ and $\mathbf{W}_{D}$, we can rewrite the least-squares misfit function from equation $(6.2)$ as

$$
\chi(\mathbf{m})=\frac{1}{2}\left|\left[\begin{array}{c}
\mathbf{W}_{M} \mathbf{m} \\
\mathbf{W}_{D} \mathbf{G m}
\end{array}\right]-\left[\begin{array}{c}
\mathbf{W}_{M} \mathbf{m}^{\text {prior }} \\
\mathbf{W}_{D} \mathbf{d}^{\text {obs }}
\end{array}\right]\right|^{2} .
$$

Defining the extended forward modelling operator $\hat{\mathbf{G}}$ and the extended data $\hat{\mathbf{d}}^{\mathrm{obs}}$ as

$$
\hat{\mathbf{G}}=\left[\begin{array}{c}
\mathbf{W}_{M} \\
\mathbf{W}_{D} \mathbf{G}
\end{array}\right], \quad \hat{\mathbf{d}}^{\mathrm{obs}}=\left[\begin{array}{c}
\mathbf{W}_{M} \mathbf{m}^{\text {prior }} \\
\mathbf{W}_{D} \mathbf{d}^{\text {obs }}
\end{array}\right],
$$

the least-squares misfit finally takes the form

$$
\chi(\mathbf{m})=\frac{1}{2}\left|\hat{\mathbf{G}} \mathbf{m}-\hat{\mathbf{d}}^{\mathrm{obs}}\right|^{2} .
$$

Equation (6.7) suggests that prior knowledge can indeed be re-interpreted as additional data, at least in the simple case of a purely Gaussian prior. The weight matrices control the relative contributions of the actual data and the artificial data to the overall least-squares misfit.

\subsection{The least-squares solution *}

Explicitly, the posterior mean $\tilde{\mathbf{m}}$ and the posterior covariance $\tilde{\mathbf{C}}_{M}$ are given by

$$
\tilde{\mathbf{m}}=\left(\mathbf{G}^{T} \mathbf{C}_{D}^{-1} \mathbf{G}+\mathbf{C}_{M}^{-1}\right)^{-1}\left(\mathbf{G}^{T} \mathbf{C}_{D}^{-1} \mathbf{d}^{\text {obs }}+\mathbf{C}_{M}^{-1} \mathbf{m}^{\text {prior }}\right),
$$

and

$$
\tilde{\mathbf{C}}_{M}=\left(\mathbf{G}^{T} \mathbf{C}_{D}^{-1} \mathbf{G}+\mathbf{C}_{M}^{-1}\right)^{-1}
$$

Proving equations (6.8) and (6.9) directly from (6.2) and (6.3) is a rather tedious exercise. Instead, we go the other direction, showing (6.8) and (6.9) by substitution into (6.3). First expanding (6.3), we obtain

$$
\chi(\mathbf{m})=\frac{1}{2} \mathbf{m}^{T} \tilde{\mathbf{C}}_{M}^{-1} \mathbf{m}-\mathbf{m}^{T} \tilde{\mathbf{C}}_{M}^{-1} \tilde{\mathbf{m}}+c .
$$

Substituting (6.8) and (6.9) into (6.10), yields

$$
\chi(\mathbf{m})=\frac{1}{2} \mathbf{m}^{T}\left(\mathbf{G}^{T} \mathbf{C}_{D}^{-1} \mathbf{G}+\mathbf{C}_{M}^{-1}\right) \mathbf{m}-\mathbf{m}^{T}\left(\mathbf{G}^{T} \mathbf{C}_{D}^{-1} \mathbf{d}^{\text {obs }}+\mathbf{C}_{M}^{-1} \mathbf{m}^{\text {prior }}\right)+c^{\prime},
$$

where $c^{\prime}$ is a new constant containing all terms that are independent of $\mathbf{m}$. Finally, we note that expanding equation (6.2) gives exactly the same expression, which demonstrates the correctness of (6.8) and (6.9). Though formally correct, there are various more useful and interpretable versions of equations (6.8) and (6.9). For instance, we may rewrite $\tilde{\mathbf{m}}$ as follows: 


$$
\begin{aligned}
\tilde{\mathbf{m}} & =\tilde{\mathbf{C}}_{M}\left(\mathbf{G}^{T} \mathbf{C}_{D}^{-1} \mathbf{d}^{\text {obs }}+\mathbf{C}_{M}^{-1} \mathbf{m}^{\text {prior }}\right)=\tilde{\mathbf{C}}_{M} \mathbf{G}^{T} \mathbf{C}_{D}^{-1} \mathbf{d}^{\text {obs }}+\tilde{\mathbf{C}}_{M} \mathbf{C}_{M}^{-1} \mathbf{m}^{\text {prior }} \\
& =\tilde{\mathbf{C}}_{M} \mathbf{G}^{T} \mathbf{C}_{D}^{-1} \mathbf{d}^{\text {obs }}+\tilde{\mathbf{C}}_{M}\left(\mathbf{G}^{T} \mathbf{C}_{D}^{-1} \mathbf{G}+\mathbf{C}_{M}^{-1}-\mathbf{G}^{T} \mathbf{C}_{D}^{-1} \mathbf{G}\right) \mathbf{m}^{\text {prior }} \\
& =\tilde{\mathbf{C}}_{M} \mathbf{G}^{T} \mathbf{C}_{D}^{-1} \mathbf{d}^{\text {obs }}+\tilde{\mathbf{C}}_{M}\left(\tilde{\mathbf{C}}_{M}^{-1}-\mathbf{G}^{T} \mathbf{C}_{D}^{-1} \mathbf{G}\right) \mathbf{m}^{\text {prior }} \\
& =\mathbf{m}^{\text {prior }}+\tilde{\mathbf{C}}_{M} \mathbf{G}^{T} \mathbf{C}_{D}^{-1}\left(\mathbf{d}^{\text {obs }}-\mathbf{G} \mathbf{m}^{\text {prior }}\right) .
\end{aligned}
$$

Equation (6.12) expresses the posterior mean $\tilde{\mathbf{m}}$ in terms of an update to the prior mean $\mathbf{m}^{\text {prior }}$. The update is controlled by the differences between observed and calculated data, $\mathbf{d}^{\text {obs }}-\mathbf{G m}^{\text {prior }}$. When the prior measurement errors are large, that is when the entries of $\mathbf{C}_{D}^{-1}$ are small, the update will be small, and vice versa. Using the matrix identities (6.17) and (6.18), shown below, we arrive at a set of equivalent expressions for the posterior mean

$$
\begin{aligned}
\tilde{\mathbf{m}} & =\left(\mathbf{G}^{T} \mathbf{C}_{D}^{-1} \mathbf{G}+\mathbf{C}_{M}^{-1}\right)^{-1}\left(\mathbf{G}^{T} \mathbf{C}_{D}^{-1} \mathbf{d}^{\text {obs }}+\mathbf{C}_{M}^{-1} \mathbf{m}^{\text {prior }}\right) \\
& =\mathbf{m}^{\text {prior }}+\tilde{\mathbf{C}}_{M} \mathbf{G}^{T} \mathbf{C}_{D}^{-1}\left(\mathbf{d}^{\text {obs }}-\mathbf{G m}^{\text {prior }}\right) \\
& =\mathbf{m}^{\text {prior }}+\mathbf{C}_{M} \mathbf{G}^{T}\left(\mathbf{C}_{D}+\mathbf{G C}_{M} \mathbf{G}^{T}\right)^{-1}\left(\mathbf{d}^{\text {obs }}-\mathbf{G m}^{\text {prior }}\right)
\end{aligned}
$$

and the posterior covariance,

$$
\begin{aligned}
\tilde{\mathbf{C}}_{M} & =\left(\mathbf{G}^{T} \mathbf{C}_{D}^{-1} \mathbf{G}+\mathbf{C}_{M}^{-1}\right)^{-1} \\
& =\mathbf{C}_{M}-\mathbf{C}_{M} \mathbf{G}^{T}\left(\mathbf{C}_{D}+\mathbf{G} \mathbf{C}_{M} \mathbf{G}^{T}\right)^{-1} \mathbf{G C}_{M} .
\end{aligned}
$$

Similar to equation (6.12), also equation (6.14) expresses the posterior quantity (the covariance) as a modification of the prior quantity. Interestingly, for any unit vector e, we have

$$
\mathbf{e}^{T} \tilde{\mathbf{C}}_{M} \mathbf{e}=\mathbf{e}^{T} \mathbf{C}_{M} \mathbf{e}-\left(\mathbf{G C}_{M} \mathbf{e}\right)^{T}\left(\mathbf{C}_{D}+\mathbf{G C}_{M} \mathbf{G}^{T}\right)^{-1}\left(\mathbf{G C}_{M} \mathbf{e}\right)<\mathbf{e}^{T} \mathbf{C}_{M} \mathbf{e}
$$

because $\left(\mathbf{C}_{D}+\mathbf{G C}_{M} \mathbf{G}^{T}\right)$ is a positive definite matrix. Therefore, the posterior variances $\mathbf{e}^{T} \tilde{\mathbf{C}}_{M} \mathbf{e}$ are always smaller than the prior variances $\mathbf{e}^{T} \mathbf{C}_{M} \mathbf{e}$, which is what we expect from the addition of new information.

Providing a range of different expressions for $\tilde{\mathbf{m}}$ and $\tilde{\mathbf{C}}_{M}$ as in equations (6.13) and (6.14) may seem redundant. However, choosing one or the other may have significant computational advantages. In fact, denoting by $N$ the dimension of the data vector and by $n$ the dimension of the model vector, we note that $\left(\mathbf{G}^{T} \mathbf{C}_{D}^{-1} \mathbf{G}+\mathbf{C}_{M}^{-1}\right)$ in (6.14) is an $n \times n$ matrix that includes the inverse of the $n \times n$ prior model covariance $\mathbf{C}_{M}$. On the other hand, $\left(\mathbf{C}_{D}+\mathbf{G C}_{M} \mathbf{G}^{T}\right)$ is a matrix of dimension $N \times N$ that can be computed without any matrix inversion. Thus, depending on the relative sizes of the data and model spaces, different expression will be preferred.

Some useful matrix identities: When dealing with the posterior mean and posterior covariance in equations (6.8) and (6.9), two matrix identities turn out to be useful. First, we note that

$$
\begin{aligned}
\mathbf{G}^{T}+\mathbf{G}^{T} \mathbf{C}_{D}^{-1} \mathbf{G} \mathbf{C}_{M} \mathbf{G}^{T} & =\mathbf{G}^{T} \mathbf{C}_{D}^{-1}\left(\mathbf{C}_{D}+\mathbf{G} \mathbf{C}_{M} \mathbf{G}^{T}\right) \\
& =\left(\mathbf{G}^{T} \mathbf{C}_{D}^{-1} \mathbf{G}+\mathbf{C}_{M}^{-1}\right) \mathbf{C}_{M} \mathbf{G}^{T}
\end{aligned}
$$

Multiplying by $\left(\mathbf{G}^{T} \mathbf{C}_{D}^{-1} \mathbf{G}+\mathbf{C}_{M}^{-1}\right)^{-1}$ from the left, and by $\left(\mathbf{C}_{D}+\mathbf{G C}_{M} \mathbf{G}^{T}\right)^{-1}$ from the right, gives

$$
\left(\mathbf{G}^{T} \mathbf{C}_{D}^{-1} \mathbf{G}+\mathbf{C}_{M}^{-1}\right)^{-1} \mathbf{G}^{T} \mathbf{C}_{D}^{-1}=\mathbf{C}_{M} \mathbf{G}^{T}\left(\mathbf{C}_{D}+\mathbf{G C}_{M} \mathbf{G}^{T}\right)^{-1}
$$

Making use of equation (6.17), we furthermore find 


$$
\begin{aligned}
& \mathbf{C}_{M}-\mathbf{C}_{M} \mathbf{G}^{T}\left(\mathbf{C}_{D}+\mathbf{G} \mathbf{C}_{M} \mathbf{G}^{T}\right)^{-1} \mathbf{G} \mathbf{C}_{M} \\
= & \mathbf{C}_{M}-\left(\mathbf{G}^{T} \mathbf{C}_{D}^{-1} \mathbf{G}+\mathbf{C}_{M}^{-1}\right)^{-1} \mathbf{G}^{T} \mathbf{C}_{D}^{-1} \mathbf{G} \mathbf{C}_{M} \\
= & \left(\mathbf{G}^{T} \mathbf{C}_{D}^{-1} \mathbf{G}+\mathbf{C}_{M}^{-1}\right)^{-1}\left[\left(\mathbf{G}^{T} \mathbf{C}_{D}^{-1} \mathbf{G}+\mathbf{C}_{M}^{-1}\right) \mathbf{C}_{M}-\mathbf{G}^{T} \mathbf{C}_{D}^{-1} \mathbf{G} \mathbf{C}_{M}\right] .
\end{aligned}
$$

Noticing that the term in square brackets is equal to the unit matrix I, we finally find

$$
\left(\mathbf{G}^{T} \mathbf{C}_{D}^{-1} \mathbf{G}+\mathbf{C}_{M}^{-1}\right)^{-1}=\mathbf{C}_{M}-\mathbf{C}_{M} \mathbf{G}^{T}\left(\mathbf{C}_{D}+\mathbf{G C}_{M} \mathbf{G}^{T}\right)^{-1} \mathbf{G C}_{M}
$$

\subsection{Model problem: Linear traveltime tomography *}

To illustrate the general least-squares solution introduced in section 6.1, we consider the specific example of a linear traveltime tomography, where we try to estimate the propagation velocity of waves inside a medium based on observations along its boundary. The geometric setup, shown in figure 6.1, consists of a 2-D domain, extending $50 \mathrm{~m}$ in both $x$ - and $y$-directions. Along the left boundary, 11 sources are evenly spaced. They emit waves (rays) recorded by 21 evenly spaced receivers along the right boundary of the domain. Thus, the number of data points is $N=11 \times 21=231$. All 231 rays connecting sources and receivers are straight lines, meaning that the prior velocity distribution is assumed to be homogeneous. In the following, we will work with the inverse of velocity, slowness, as this leads to a more linear forward problem, as explained in Appendix 16.1. Similar configurations may be found in medical ultrasound tomography, where transducers are positioned on opposite sides of a patient, or in geophysical cross-hole tomography, where explosions in one borehole act as sources that excite waves recorded in another borehole.

The 2-D slowness model is discretised in terms of $20 \times 20$ blocks of constant slowness. Thus, the total number of unknown model parameters is $n=20^{2}=400$. As shown in the ray density plot 6.2, the number of rays crossing a block is highly variable, ranging from around 10 to more than 50. In Appendix 16.1 we show that the forward problem of linear traveltime tomography can be written in the form

$$
\mathbf{d}=\mathbf{G m},
$$

where $\mathbf{d}$ is the synthetic data vector containing traveltime measurements, and $\mathbf{m}$ is the vector of unknown model parameters. In our case, $\mathbf{d} \in \mathbb{R}^{231}$ and $\mathbf{m} \in \mathbb{R}^{400}$, meaning that the inverse problem is from the outset underdetermined. The 400 entries in the $i^{\text {th }}$ row of the $231 \times 400$ matrix $\mathbf{G}$ are equal to the length of the $i^{\text {th }}$ ray within the discrete slowness blocks.

Before we proceed with the analysis of our specific example, we note that the linear forward modelling equation (6.20) is merely a convenient approximation. In fact, if we require a heterogeneous slowness distribution to explain our traveltime observations to within their uncertainties, the rays connecting sources and receivers cannot be straight lines. Instead, the rays would bend according to Fermat's principle. Consequently, the matrix $\mathbf{G}$ containing the lengths of ray segments would depend on the slowness distribution, that is, $\mathbf{G}=\mathbf{G}(\mathbf{m})$, thereby introducing nonlinearity into the problem. Nevertheless, the assumption of linearity is often a reasonable one for media with sufficiently weak heterogeneities ${ }^{1}$.

Though the domain seems visually well covered by rays, the number of non-zero values in $\mathbf{G}$ is very small, as

\footnotetext{
${ }^{1}$ The term weak is unavoidably diffuse. Assuming linearity effectively introduces a forward modelling error which then translates into increased posterior errors. Whether the heterogeneities can be considered weak thus depends on our willingness to accept posterior errors of a certain level.
} 


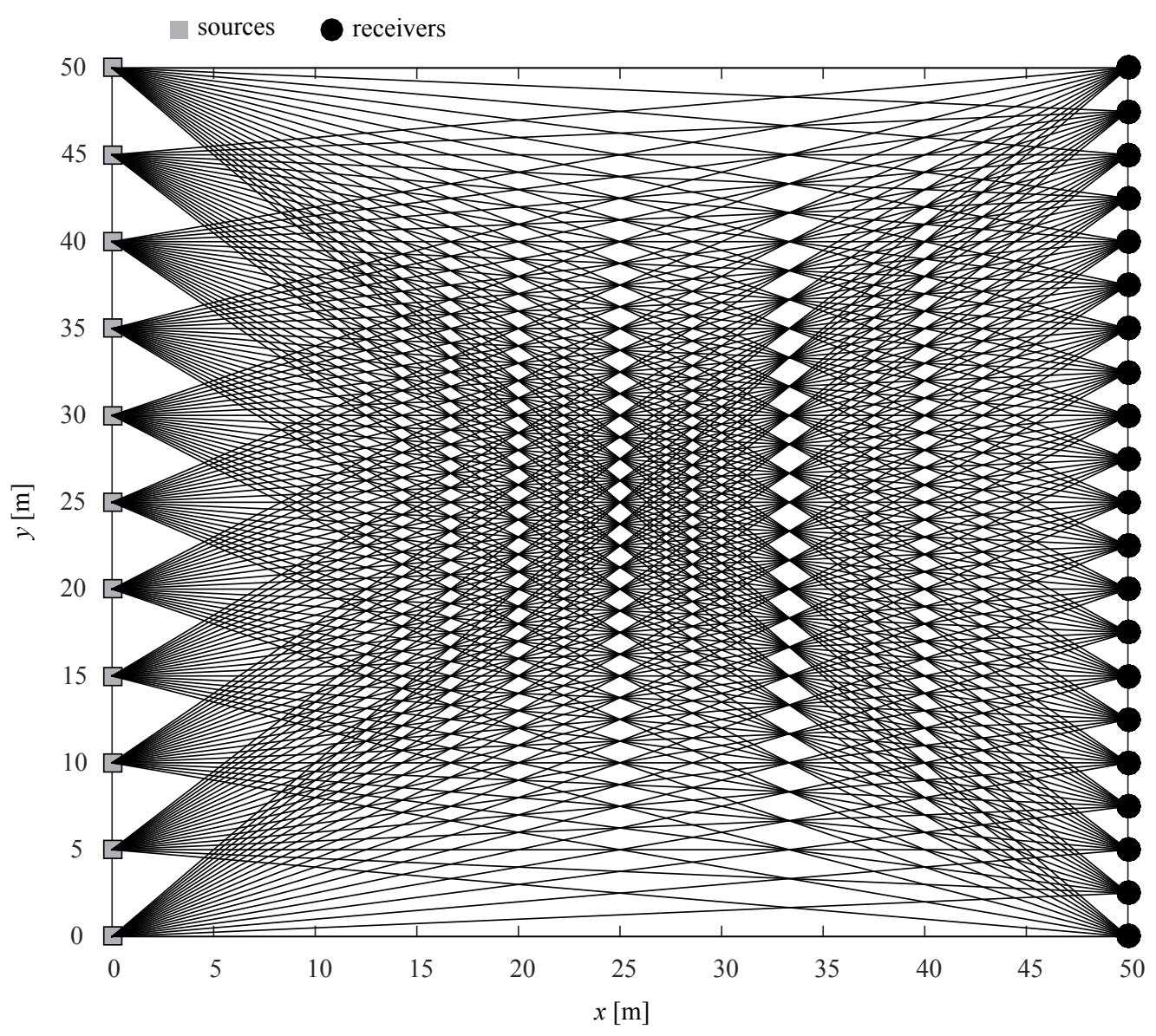

Fig. 6.1: Source-receiver setup of a linear traveltime tomography. Sources (grey squares) along the left boundary emit waves recorded by receivers (black circles) along the right boundary. Assuming a homogeneous slowness (or velocity) distribution, all rays connecting sources are straight lines. The 2-D slowness model is discretised in terms of $2.5 \mathrm{~m} \times 2.5 \mathrm{~m}$ blocks of constant slowness. [Straight-ray tomography]

shown in figure 6.3a. In fact, from a total of $231 \times 400=92^{\prime} 400$ only $6^{\prime} 349$ entries are non-zero, that is, around $7 \%$. Thus, $\mathbf{G}$ is very sparse, which may lead us to suspect already that not all details of the unknown slowness distribution can be recovered. This is confirmed by an eigenvalue analysis of $\mathbf{G}^{T} \mathbf{G}$, which enters the MoorePenrose inverse $\left(\mathbf{G}^{T} \mathbf{G}\right)^{-1} \mathbf{G}^{T}$ (see also section 1.3.2). As shown in figure 6.3b, only around 200 of the 400 eigenvalues of $\mathbf{G}^{T} \mathbf{G}$ are significantly non-zero. It follows that the least-squares problem without the injection of prior knowledge in the form of the covariance matrices $\mathbf{C}_{D}$ and $\mathbf{C}_{M}$ is not solvable because it requires the inversion of $\mathbf{G}^{T} \mathbf{G}$.

The condition number and eigenvalues of $\mathbf{G}^{T} \mathbf{G}$ : The eigenvalues of $\mathbf{G}^{T} \mathbf{G}$ or its weighted variant $\left(\mathbf{G}^{T} \mathbf{C}_{D}^{-1} \mathbf{G}+\mathbf{C}_{M}^{-1}\right)$ from equation (6.8) are particularly relevant for the stability of the least-squares problem. For notational convenience, we limit ourselves to the solution of the least-squares problem in terms of the Moore-Penrose inverse, $\tilde{\mathbf{m}}=\left(\mathbf{G}^{T} \mathbf{G}\right)^{-1} \mathbf{G}^{T} \mathbf{d}^{\text {obs }}$. Our interest is to quantify changes in the posterior mean, $\Delta \tilde{\mathbf{m}}$ that may be induced by changes in the observations, $\Delta \mathbf{d}^{\text {obs }}$. In other words: How susceptible is our solution to potential errors in the data? To answer this question, we consider the 


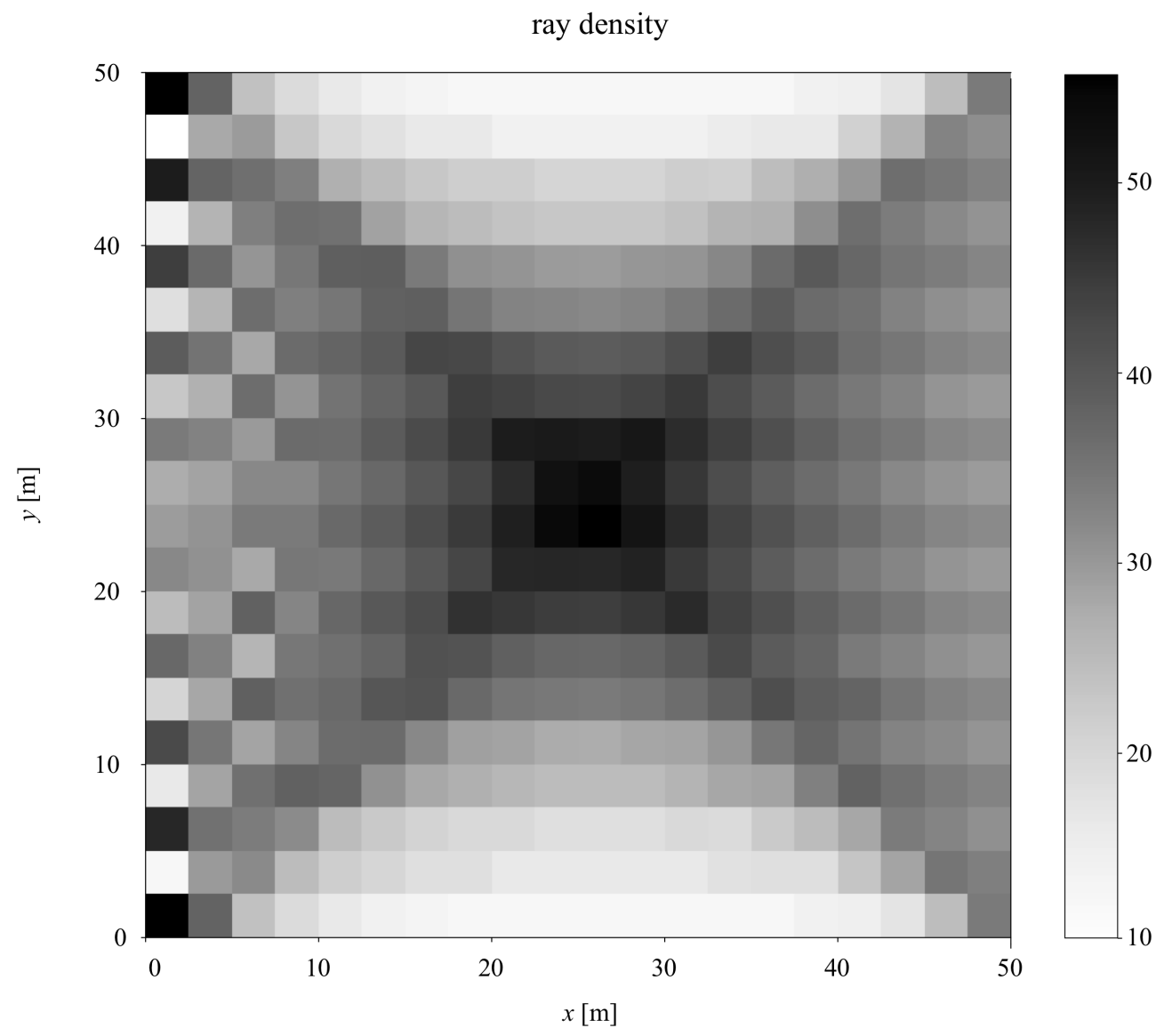

Fig. 6.2: Raydensity plot. Grey scale indicates the number of rays going through a grid cell. [Straight-ray tomography]
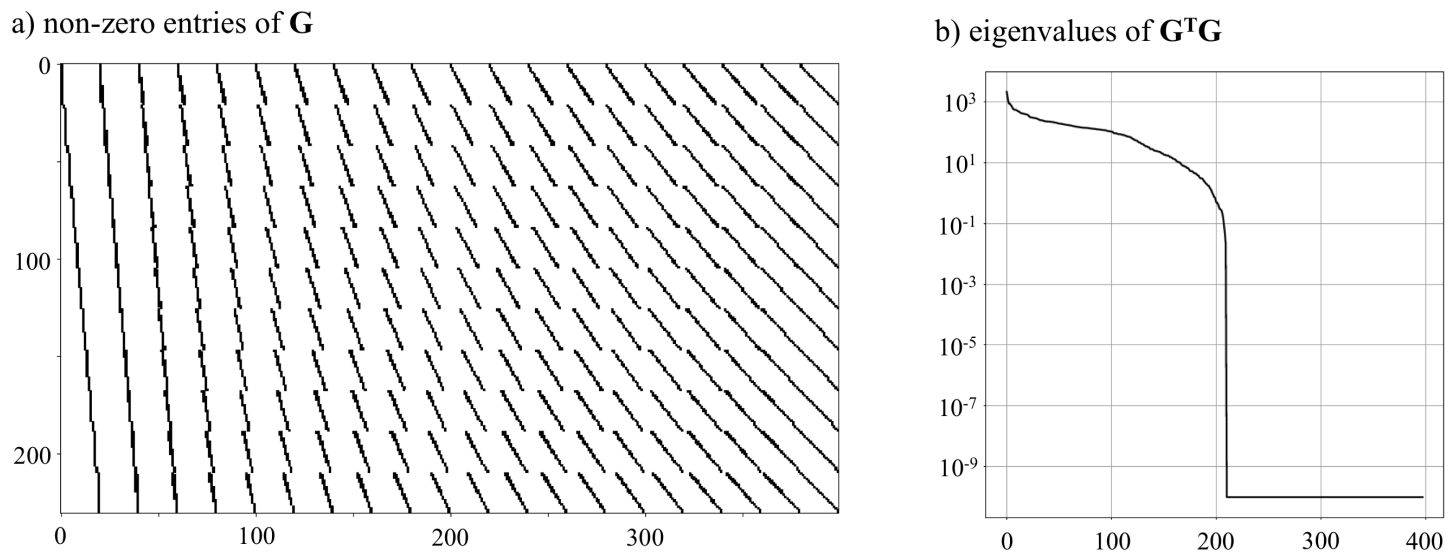

Fig. 6.3: Analysis of $\mathbf{G}$ and $\mathbf{G}^{T} \mathbf{G}$. (a) Display of the $441 \times 231$ matrix $\mathbf{G}$, with non-zero values shown in black. From a total of $231 \times 400=92^{\prime} 400$ entries, only 6'349 are non-zero, that is, around $7 \%$. In other words, $\mathbf{G}$ is very sparse. (b) Logarithmic plot of the eigenvalues of $\mathbf{G}^{T} \mathbf{G}$. Only non-zero eigenvalues are shown. The number of significantly non-zero eigenvalues $(>1)$ is only around 200. [Straight-ray tomography] 
maximum relative change in $\tilde{\mathbf{m}}$ with respect to the relative change in $\mathbf{G}^{T} \mathbf{d}^{\text {obs }}$,

$$
\xi=\max _{\Delta \mathbf{d}^{\mathrm{obs}}, \mathbf{d}^{\mathrm{obs}}} \frac{|\Delta \tilde{\mathbf{m}}| /|\tilde{\mathbf{m}}|}{\left|\mathbf{G}^{T} \Delta \mathbf{d}^{\mathrm{obs}}\right| /\left|\mathbf{G}^{T} \mathbf{d}^{\mathrm{obs}}\right|}=\max _{\Delta \mathbf{d}^{\mathrm{obs}}, \mathbf{d}^{\mathrm{obs}}} \frac{|\Delta \tilde{\mathbf{m}}|}{\left|\mathbf{G}^{T} \Delta \mathbf{d}^{\mathrm{obs}}\right|} \frac{\left|\mathbf{G}^{T} \mathbf{d}^{\mathrm{obs}}\right|}{|\tilde{\mathbf{m}}|},
$$

where $|$.$| denotes the length of a vector. The maximum relative perturbation \xi$ over all possible $\Delta \mathbf{d}^{\text {obs }}$ and $\mathbf{d}^{\text {obs }}$ is referred to as the condition number of the least-squares problem. Large condition numbers imply that even small perturbations in the data will have a large effect on the solution, and the problem is said to be ill-conditioned. In contrast, small condition numbers mean that perturbations in the data, for instance in the form of observational errors, will have little effect on the result. The problem is then well-conditioned. Equation (6.21) is not very useful, but can be brought into a more practical form. In fact, defining $\mathbf{x}=\mathbf{G}^{T} \mathbf{d}^{\text {obs }}$ and $\Delta \mathbf{x}=\mathbf{G}^{T} \Delta \mathbf{d}^{\text {obs }}$ we obtain

$$
\xi=\max _{\Delta \mathbf{x}} \frac{|\Delta \tilde{\mathbf{m}}|}{|\Delta \mathbf{x}|} \max _{\mathbf{x}} \frac{|\mathbf{x}|}{|\tilde{\mathbf{m}}|}=\max _{\Delta \mathbf{x}} \frac{\left|\left(\mathbf{G}^{T} \mathbf{G}\right)^{-1} \Delta \mathbf{x}\right|}{|\Delta \mathbf{x}|} \max _{\tilde{\mathbf{m}}} \frac{\left|\left(\mathbf{G}^{T} \mathbf{G}\right) \tilde{\mathbf{m}}\right|}{|\tilde{\mathbf{m}}|} .
$$

Equation (6.22) can be further simplified. For instance, the maximum of the term $\left|\left(\mathbf{G}^{T} \mathbf{G}\right) \tilde{\mathbf{m}}\right| /|\tilde{\mathbf{m}}|$ can be found conveniently by forcing the gradient of its square, $\left|\left(\mathbf{G}^{T} \mathbf{G}\right) \tilde{\mathbf{m}}\right|^{2} /|\tilde{\mathbf{m}}|^{2}$ to zero. From this it follows that the maximum is equal to the largest eigenvalue of $\mathbf{G}^{T} \mathbf{G}$, denoted by $\lambda_{\max }$. Similarly, we find that the maximum of $\left|\left(\mathbf{G}^{T} \mathbf{G}\right)^{-1} \Delta \mathbf{x}\right| /|\Delta \mathbf{x}|$ is the largest eigenvalue of $\left(\mathbf{G}^{T} \mathbf{G}\right)^{-1}$, which is equal to the inverse of the smallest eigenvalue of $\mathbf{G}^{T} \mathbf{G}$, that is, $\lambda_{\min }$. In summary, we find that the condition number is given by the ratio of the largest to the smallest eigenvalue of $\mathbf{G}^{T} \mathbf{G}$,

$$
\xi=\frac{\lambda_{\max }}{\lambda_{\min }} .
$$

Clearly, $\mathbf{G}^{T} \mathbf{G}$ from figure 6.3 is very ill-conditioned. The slightest error in the data would have large impact on the solution, if the pure Moore-Penrose inverse were used to solve the least-squares problem. However, adding prior knowledge and a prior data covariance may improve the conditioning of the problem.

For the illustration of basic concepts, we perform a synthetic inversion: Instead of working with actual observed data, we use artificial data computed via the solution of our forward problem. This form of cheating is frequently referred to as an inverse crime, as it does not take into account that real wave propagation through a complex medium is certainly less simple than $\mathbf{d}=\mathbf{G m}$. In this sense, a synthetic inversion leads to optimistic results, and if these are not considered satisfactory, a real-data application would have low chances of success. The benefit of a synthetic inversion is the availability of a ground truth target model to which inversion results can be compared easily. Figure 6.4 summarises our target model and the artificial data, which we perturb by random errors in order to mimic measurement uncertainties.

Before we attempt any reconstruction, the prior covariance matrices $\mathbf{C}_{D}$ and $\mathbf{C}_{M}$ must be constructed. For the data covariance matrix $\mathbf{C}_{D}$ that describes measurement uncertainties, we choose a diagonal matrix, $\mathbf{C}_{D}=\sigma_{D}^{2} \mathbf{I}$, with the standard deviation set to $\sigma_{D}=0.2 \cdot 10^{-4} \mathrm{~s}$. This choice is made under the assumption that measurement errors at different receivers and for different sources are independent, and indeed have a standard deviation of $0.2 \cdot 10^{-4} \mathrm{~s}$ with zero mean. In our synthetic example, this assumption is correct by construction. In more realistic cases where the assumption may be less precise, an incorrect $\mathbf{C}_{D}$ may lead to over- or under-fitting of the data, as we will see later in this section.

The choice of the prior model covariance $\mathbf{C}_{M}$ is more ad hoc. Realistically, we may not know a priori how slowness in one grid cell is correlated with slowness in another grid cell. Motivated more by convenience than by hard physical constraints, we adopt a functional form of $\mathbf{C}_{M}$ proposed, for instance, by Tarantola \& Valette 
a) Target slowness model $[\mathrm{ms} / \mathrm{m}]$

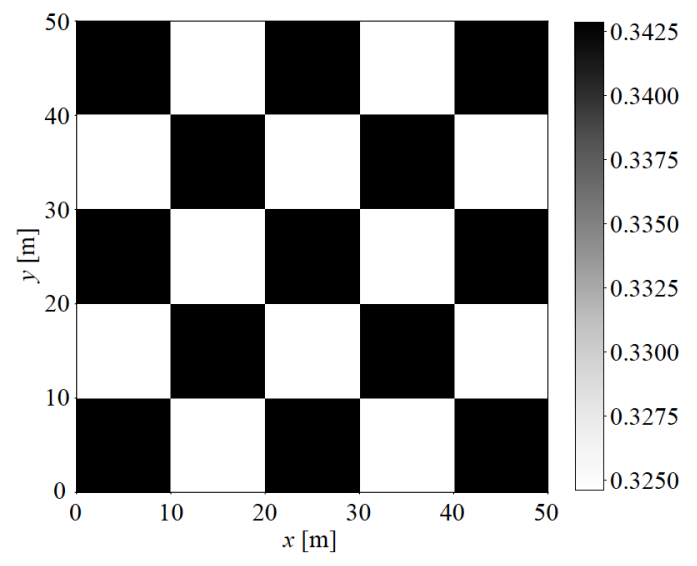

b) Traveltimes and measurement errors
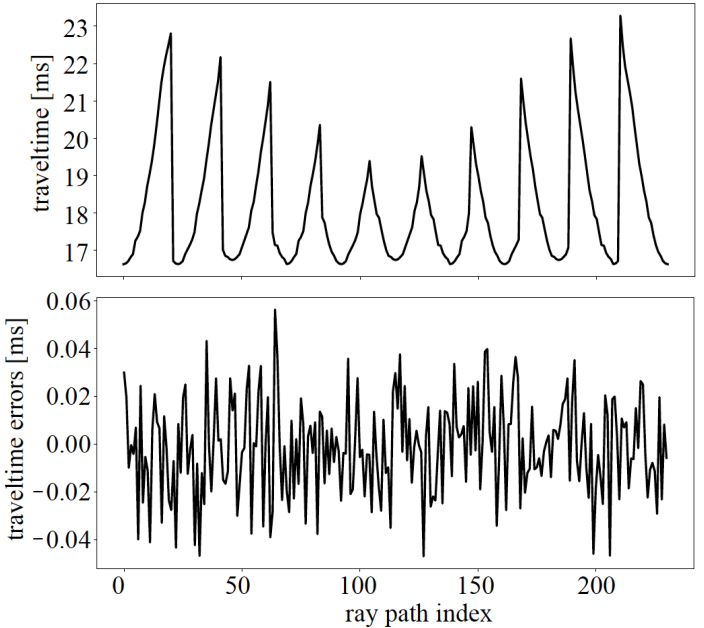

Fig. 6.4: Target model and artificial traveltime measurements. (a): The target model is a chequerboard slowness pattern. Each patch of the chequerboard is $4 \times 4$ grid cells wide. (b): Artificial traveltime data (top) are computed for the chequerboard pattern and then polluted by random Gaussian noise with zero mean and standard deviation $2.5 \mathrm{~ms}$, in order to mimic observational errors (bottom). [Straight-ray tomography]

(1982):

$$
\left(\mathbf{C}_{M}\right)_{i j}=\sigma_{M}^{2} e^{-\frac{\left|\mathbf{x}_{i}-\mathbf{x}_{j}\right|^{2}}{2 \lambda^{2}}} .
$$

In equation (6.24), $\mathbf{x}_{i}$ and $\mathbf{x}_{j}$ are the central points of grid cells $i$ and $j, \sigma_{M}$ is the prior standard deviation of individual cells, and $\lambda$ is the correlation length. Values of $\lambda$ that are large compared to the size of the grid cells imply that slowness is correlated over comparatively large distances. In contrast, values of $\lambda$ that are small compared to the size of a cell indicate that slowness is a priori allowed to vary rapidly from one cell to its neighbour. For our first example, we choose $\sigma_{M}=2 \cdot 10^{-5} \mathrm{~s} / \mathrm{m}$ and $\lambda=3 \cdot 2.5 \mathrm{~m}=7.5 \mathrm{~m}$, that is, three times the size of a cell.

Figure 6.5 summarises the results of the least-squares inversion, based on equations (6.8) and (6.9) and the parameters described in the previous paragraphs. As suspected for an under-determined problem, the reconstructed slowness distribution in figure 6.5a, that is, the posterior mean or maximum-likelihood model, is not exactly the same as the target model in figure 6.4a. Though the chequerboard pattern can be recognised, the reconstruction is rather poor along the edges of the domain where the ray coverage is less dense.

Figure $6.5 \mathrm{~b}$ displays the traveltime residuals $\mathbf{d}^{\text {obs }}-\mathbf{d}^{\text {est }}=\mathbf{d}^{\text {obs }}-\mathbf{G} \tilde{\mathbf{m}}$, that is, the differences between the (artificial) observed traveltimes and the traveltimes computed with the estimated posterior model $\tilde{\mathbf{m}}=\mathbf{m}^{\text {est }}$. While the residuals are significantly smaller than those for the homogeneous prior model, displayed in figure $6.4 \mathrm{~b}$, they are not zero, partly because of the random observational errors that we added to the observations. For our specific example, the root-mean-square misfit,

$$
\chi^{\mathrm{rms}}=\sqrt{\frac{1}{N}\left(\mathbf{d}^{\mathrm{obs}}-\mathbf{d}^{\mathrm{est}}\right)^{T} \mathbf{C}_{D}^{-1}\left(\mathbf{d}^{\mathrm{obs}}-\mathbf{d}^{\mathrm{est}}\right)}
$$

takes the value $\chi^{\mathrm{rms}}=1.2$, indicating that $\mathbf{m}^{\text {est }}$ is slightly under-fitting the observations.

Finally, figure 6.5 c compares the prior model covariance $\mathbf{C}_{M}$ to the posterior model covariance $\tilde{\mathbf{C}}_{M}$. Shown are the components with indices $(210, i)$, where index 210 corresponds to a cell in the middle of the domain. 
For $i=210$, the curves display the prior and posterior variance, which can be interpreted as the prior and posterior uncertainties of the model parameter (slowness in cell number 210). As predicted by equation (6.15), variances decrease upon the assimilation of data, meaning that uncertainties decrease when our prior knowledge is combined with actual data.

The off-diagonal elements with indices $(210, i \neq 210)$ describe prior and posterior correlations between model parameters, that is, pairs of cells at some distance from each other. The posterior indicates that the correlation length is slightly longer than assumed a priori.

a) Reconstructed slowness distribution

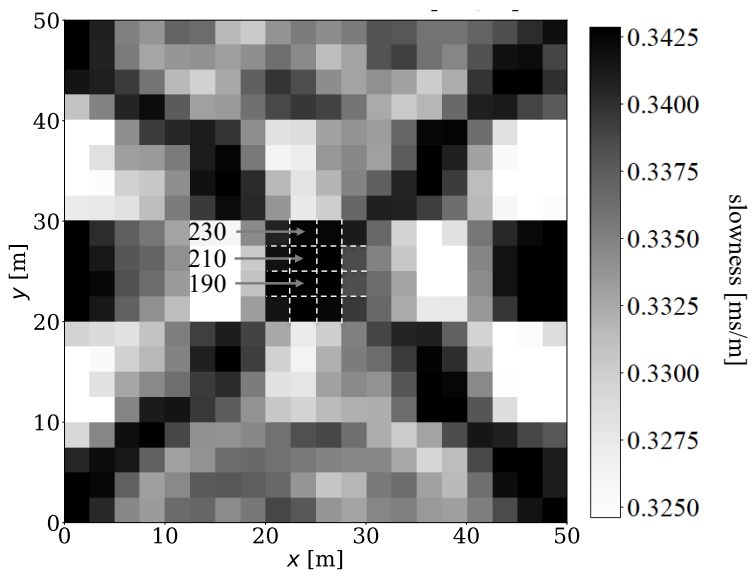

b) Traveltime residuals

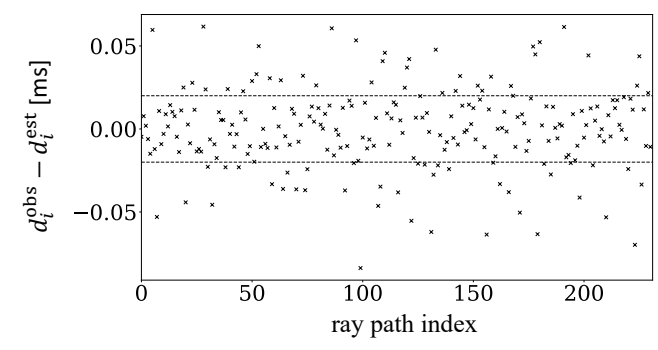

c) Prior and posterior covariance $C_{210, i}$

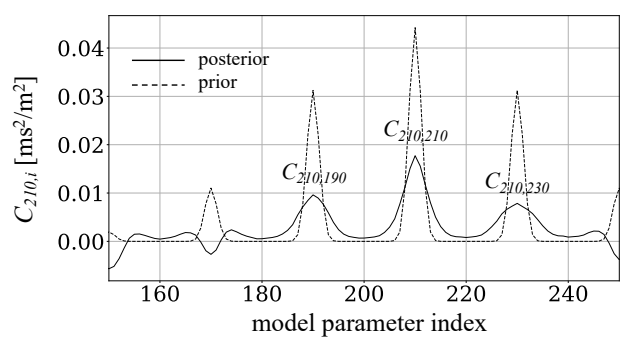

Fig. 6.5: Summary of the synthetic least-squares traveltime inversion. (a) The posterior mean, or maximumlikelihood, slowness distribution, $\tilde{\mathbf{m}}$. The grid cells with indices 190, 210 and 230 are marked. (b) Posterior traveltime residuals $\mathbf{d}^{\text {obs }}-\mathbf{d}^{\text {est }}$, where $\mathbf{d}^{\text {est }}=\mathbf{G m}^{\text {est }}$ are the traveltimes computed with the estimated model $\mathbf{m}^{\text {est }}=\tilde{\mathbf{m}}$. The dashed lines mark the locations of the prior data standard deviation, $\pm \sigma_{D}$. (c) Entries of the prior model covariance matrix $\mathbf{C}_{M}$ (solid) and the posterior model covariance matrix $\tilde{\mathbf{C}}_{M}$ (dashed) for indices $(210, i)$. Index $(210,210)$ corresponds to the prior and posterior variances of the central element in the domain. The offdiagonal elements $(210, i \neq 210)$ describe correlations between slownesses in different grid cells. [Straight-ray tomography]

\subsection{Prior knowledge vs. regularisation *}

While we may indeed obtain a good estimate of the prior data covariance $\mathbf{C}_{D}$ from careful data analysis, our formulation of prior information in model space in the form of the prior covariance in equation (6.24) was obviously ad hoc, and not at all based on physical arguments. We could have, for instance, designed a prior that bounds the admissible slowness values between some a priori plausible minimum and maximum. In seismic tomography, minimum and maximum values may be derived from petrological prior knowledge about the properties of rocks that acutally occur inside the Earth. In medical tomography, minima and maxima could be derived from known extremal slowness values of human tissue. At least, we could have imposed that any 
slowness is larger than the inverse of the speed of light. However, all these priors would not have been Gaussian, and therefore our beautifully simple theory would not have been applicable.

In addition to choosing a Gaussian ad hoc, we also imposed the functional form of the prior covariance in equation (6.24) for plain convenience. Mostly, we will just not have hard prior information on the spatial correlation of model parameters. Ideally, we would at least have chosen a prior in model space that is as uninformative as possible, simply to ensure that we do not inject information that we actually do not have. This means, in the context of the least-squares problem, that we would replace the Gaussian model space prior in equation (6.1) by a constant, uniform prior. The posterior mean would then be given by

$$
\tilde{\mathbf{m}}=\left(\mathbf{G}^{T} \mathbf{C}_{D}^{-1} \mathbf{G}\right)^{-1} \mathbf{G}^{T} \mathbf{C}_{D}^{-1} \mathbf{d}^{\mathrm{obs}} .
$$

Though formally correct, equation (6.26) suffers from the problem that $\mathbf{G}^{T} \mathbf{C}_{D}^{-1} \mathbf{G}$ may actually not be invertible, or at least very poorly conditioned. For instance, in our example 2-D traveltime tomography, $\mathbf{G}^{T} \mathbf{C}_{D}^{-1} \mathbf{G}$ is proportional to $\mathbf{G}^{T} \mathbf{G}$, which, as we have seen in figure 6.3 , has a large number of eigenvalues that are practically zero. We have thus reached an interesting limit of the least-squares method. In order to explicitly compute the posterior mean and the posterior covariance that completely describe the posterior distribution, we need to impose prior knowledge that we do not have. Otherwise, some of the matrices that we need to invert are simply not invertible.

It is important to note that this is not a failure of inverse theory or Bayesian inference per se, but a failure of the least-squares method. That $\mathbf{G}^{T} \mathbf{G}$ is not invertible does not pose a problem to Monte Carlo methods that sample the posterior without any need to invert a matrix. The posterior would just not be a Gaussian with well-defined posterior mean and posterior covariance.

Given that Monte Carlo methods may not be computationally affordable for many higher-dimensional problems, it has become common practice to handle the fundamental failure of the least-squares method in a rather pragmatic, though not very beautiful, manner. To produce some results that are compatible with the data, the problem is regularised by injecting artificial prior knowledge into the problem.

Regularisation in the form of smoothing is illustrated in figure 6.6 where we vary the prior correlation length $\lambda$ from equation (6.24), while keeping the prior standard deviation constant at $\sigma_{M}=0.2 \cdot 10^{-5} \mathrm{~s} / \mathrm{m}$. When $\lambda$ is small, slowness is allowed to vary rapidly in space in order to fit the observations. As a consequence, the root-mean-square misfit $\chi^{\mathrm{rms}}$ may drop significantly below 1 . Thus, we observe over-fitting in response to under-smoothing. The opposite scenario occurs when $\lambda$ increases, meaning that our artificial prior knowledge forces the slowness to vary more smoothly in space. For large $\lambda$, the slowness variations will be too smooth to explain the observations, and $\chi^{\mathrm{rms}}$ becomes significantly larger than 1 . In other words, over-smoothing leads to under-fitting.

The second tuning parameter in the prior covariance in equation (6.24) is the prior standard deviation $\sigma_{M}$. The effect of varying $\sigma_{M}$ while keeping the prior correlation length fixed at $\lambda=3 \mathrm{~m}$, is illustrated in figure 6.7. For decreasing values of $\sigma_{M}$, the slowness distribution is increasingly damped towards the homogeneous prior distribution. Just as in the case of smoothing, we may observe under-fitting in response to over-damping, and over-fitting in response to under-damping.

Damping, just as smoothing, affects the complexity of plausible posterior models, and of the posterior mean (or maximum-likelihood) model in particular. Stronger damping and smoothing reduce model complexity while increasing the root-mean-square misfit $\chi^{\mathrm{rms}}$. Figure 6.7a shows model complexity in terms of the difference between posterior and prior mean, $\left|\mathbf{m}^{\text {prior }}-\tilde{\mathbf{m}}\right|$, as a function of $\chi^{\mathrm{rms}}$. The curve has roughly the shape of the letter $\mathrm{L}$, and is therefore often referred to as the $L$-curve. In cases where the observational uncertainties are not well known, the L-curve may be used to guide the choice of suitable smoothing and damping (regularisation) parameters. More or less reasonable regularisation parameters should be located roughly around the kink of the L. 


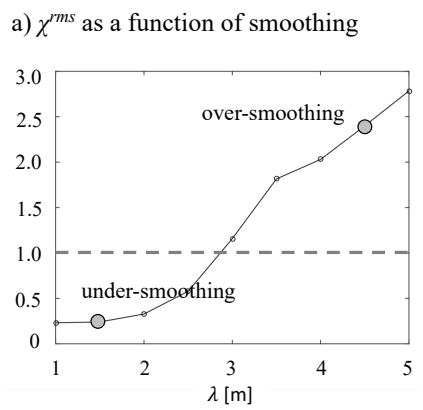

b) Under-smoothed slowness model

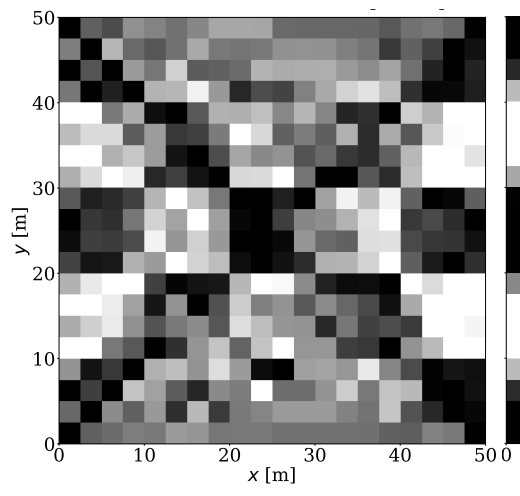

c) Over-smoothed slowness model

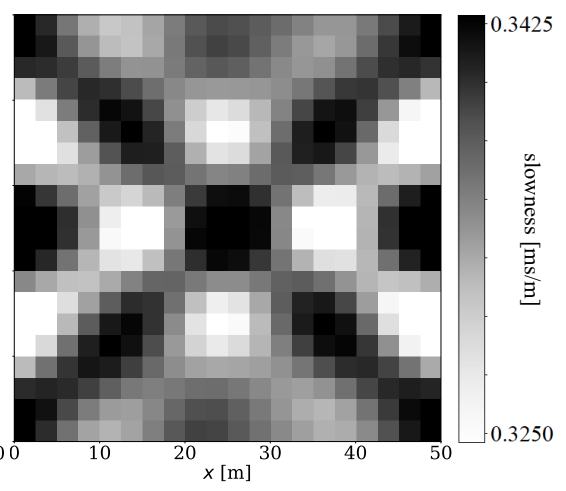

smoothing $[\lambda=1.5 \mathrm{~m} \rightarrow \lambda=4.5]$

Fig. 6.6: Effect of smoothing on the posterior mean model for a fixed prior standard deviation of $\sigma_{M}=0.2 \cdot 10^{-5}$ $\mathrm{s} / \mathrm{m}$. (a) Increasing the prior correlation length $\lambda$ from equation (6.24) causes the posterior mean models to be increasingly smooth. For small $\lambda$, the model is allowed to vary rapidly and to appear very complex. As a consequence, the observations can be fit very accurately, even below the noise level. In this sense, undersmoothing may imply over-fitting. Conversely, over-smoothing may result in under-fitting because the model cannot achieve a complexity sufficient to fit the observations to within the uncertainties. The horizontal dashed line roughly separates regions of under-smoothing (below) and over-smoothing (above). The larger grey dots mark the under- and over-smoothed models shown in (b) and (c), respectively. [Straight-ray tomography]

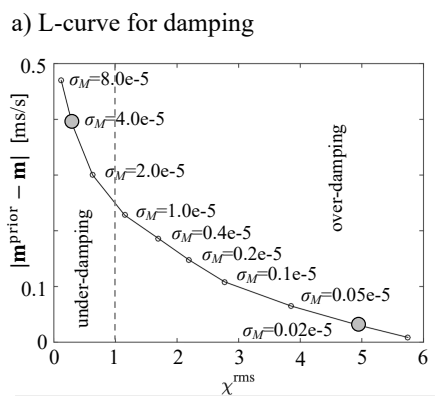

b) Over-damped slowness model

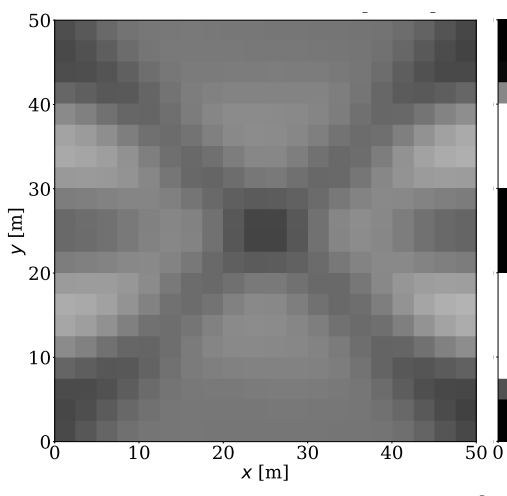

c) Under-damped slowness model

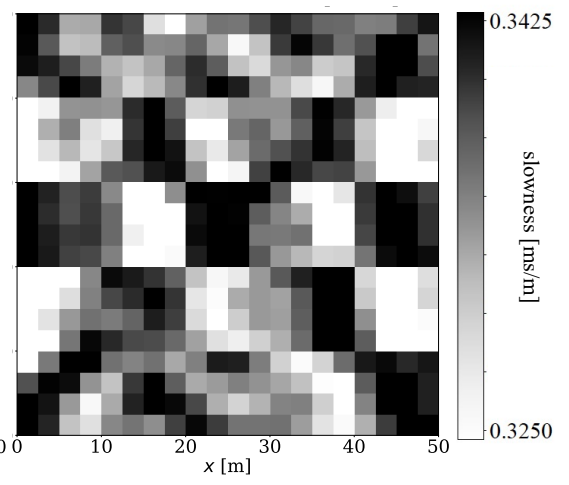

damping $\left[\sigma_{M}=0.02 \cdot 10^{-5} \mathrm{~s} / \mathrm{m} \leftarrow \sigma_{M}=4.0 \cdot 10^{-5} \mathrm{~s} / \mathrm{m}\right]$

Fig. 6.7: Effect of damping on the posterior mean model for a fixed correlation length of $\lambda=3 \mathrm{~m}$. (a) Example of an L-curve. Decreasing the prior standard deviation $\sigma_{M}$ from equation (6.24) causes the posterior mean models to be increasingly close to the prior mean model, which is homogeneous in this example. For large $\sigma_{M}$, the model is allowed to deviate strongly from the prior, meaning that $\left|\mathbf{m}^{\text {prior }}-\tilde{\mathbf{m}}\right|$ can be large. As a consequence, the observations can be fit very accurately, possibly below the noise level. Thus, under-damping may imply over-fitting. Conversely, over-damping may in result in under-fitting. The vertical dashed line roughly separates regions of under-damping (left) and over-damping (right). The larger grey dots mark the over- and under-damped models shown in (b) and (c), respectively. [Straight-ray tomography] 
To conclude this section, we remark that regularisation is an act of pragmatism. It is often unavoidable when the more holistic Bayesian approach (combined with Monte Carlo methods) is computationally too expensive. Regularisation is almost by definition ad hoc. Choosing, for instance, the functional form of the prior model covariance and the regularisation parameters, is subjective. This subjectivity leaves an imprint on the results and should be taken into account during the interpretation. One of the undesirable effects of regularisation is that the posterior uncertainties may be meaningless. Injecting artificial prior knowledge to make a matrix invertible tends to decrease the uncertainties. Specifically in the case of our traveltime tomography, slowness in a cell that is not crossed by any ray is entirely undetermined. Still, through regularisation, we will find a finite posterior variance.

Occam's razor: Concepts such as the L-curve, over-, and under-fitting are closely related to Occam's razor, also known as the Law of Parsimony (spareness) or the Principle of Simplicity. Though many formulations of Occam's razor exist, it may be summarised as the preference for simple models and theories. Given a selection of models with variable complexity that all explain the observations to within their errors, Occam's razor suggests to choose the simplest one.

Documented early versions of the Principle of Simplicity go back at least to Aristotle's (384-322 BC) Posterior Analytics. However, William of Okham (1287-1347) used it so frequently that it ended up being attributed to him. (William of Okham was an English Franciscan friar and philosopher. His biography seems to be known rather poorly. He is likely to have studied theology at Oxford University where he wrote unorthodox commentaries on religious texts that were standard in his days. To defend himself against claims of heresy, he was ordered to Avignon (France) in 1324. After leaving Avignon without permission in 1328, he was excommunicated. He spent the rest of his life in exile, under the protection of the Holy Roman Emperor Luis IV.)

Occam's razor is by no means a rigorous discriminator between different options, also because the notions of 'simplicity' and 'complexity' are often not well-defined. Instead, it serves as a heuristic guide. Scientifically, Occam's razor can be justified because simple models are usually easier to test. Furthermore, increasing complexity increases possibilities of errors. Thus, if a more complex model does not improve our ability to explain observations to within their errors, the effect is only to increase the probability that the model is wrong.

In the context of our traveltime tomography example, Occam's razor expresses a preference for slowness models that are regularised (smoothed and damped) as much as permitted by the errors in the traveltime measurements. Choosing a model that is less smoothed and less damped will of course improve the data fit, but only to within the errors. In this sense, the additional complexity is not required. As an alternative to smoothing and damping, one may regularise the inverse problem by choosing a smaller number of parameters, that is, larger grid cells in our case.

\subsection{Resolution*}

\subsubsection{Model resolution*}

To gain a deeper understanding of the least-squares solution, we take a closer look at the relation between the target model, $\mathbf{m}^{\text {target}}$, shown in figure 6.4 , and the estimated model, $\mathbf{m}^{\text {est }}=\tilde{\mathbf{m}}$. Within the framework of our synthetic inversion, we can write the artificial data as

$$
\mathbf{d}^{\mathrm{obs}}=\mathbf{G m}^{\text {target }}+\varepsilon,
$$


where $\varepsilon$ represents the random observational errors that we added. Furthermore defining $\mathbf{d}^{\text {prior }}$ as the synthetic data computed with the prior mean model, that is,

$$
\mathbf{d}^{\text {prior }}=\mathbf{G m}^{\text {prior }}
$$

we have

$$
\Delta \mathbf{d}=\mathbf{G} \Delta \mathbf{m}^{\text {target }}+\varepsilon,
$$

with the data residuals $\Delta \mathbf{d}=\mathbf{d}^{\text {obs }}-\mathbf{d}^{\text {prior }}$ and the target model difference $\Delta \mathbf{m}^{\text {target }}=\mathbf{m}^{\text {target }}-\mathbf{m}^{\text {prior }}$. Furthermore, from the third option in equation (6.13), we find that the estimated model difference $\Delta \tilde{\mathbf{m}}=\tilde{\mathbf{m}}-\mathbf{m}^{\text {prior }}$ and $\Delta \mathbf{d}$ are related by

$$
\Delta \tilde{\mathbf{m}}=\mathbf{G}^{-g} \Delta \mathbf{d}
$$

where the generalised inverse $\mathbf{G}^{-g}$ is given by

$$
\mathbf{G}^{-g}=\mathbf{C}_{M} \mathbf{G}^{T}\left(\mathbf{C}_{D}+\mathbf{G C}_{M} \mathbf{G}^{T}\right)^{-1}
$$

Substituting $\Delta \mathbf{d}$ from equation (6.29) into equation (6.31), we then find an explicit relation between the target model difference and the estimated model difference that we can actually recover,

$$
\Delta \tilde{\mathbf{m}}=\mathbf{G}^{-g}\left(\mathbf{G} \Delta \mathbf{m}^{\text {target }}+\boldsymbol{\varepsilon}\right)=\mathbf{G}^{-g} \mathbf{G} \Delta \mathbf{m}^{\text {target }}+\mathbf{G}^{-g} \boldsymbol{\varepsilon}=\mathbf{R}_{M} \Delta \mathbf{m}^{\text {target }}+\mathbf{G}^{-g} \boldsymbol{\varepsilon} .
$$

The matrix

$$
\mathbf{R}_{M}=\mathbf{G}^{-g} \mathbf{G}=\mathbf{C}_{M} \mathbf{G}^{T}\left(\mathbf{C}_{D}+\mathbf{G C}_{M} \mathbf{G}^{T}\right)^{-1} \mathbf{G}
$$

is the model resolution matrix, or simply the resolution matrix. It establishes a linear relation between the target model $\Delta \mathbf{m}^{\text {target }}$ that we wish to recover, and the usually imperfect, estimated $\Delta \tilde{\mathbf{m}}$, that we obtain as a result of limited coverage. The second term in equation (6.32) represents error propagation, that is, the direct imprint of observational errors on the estimated model.

Under (hypothetically) ideal circumstances, we have $\Delta \tilde{\mathbf{m}}=\Delta \mathbf{m}^{\text {target }}$, and the resolution matrix is equal to the identity matrix, that is $\mathbf{R}_{M}=\mathbf{I}$. In this case, the trace of the resolution matrix is equal to the number of model parameters, $\operatorname{tr} \mathbf{R}_{M}=n$. In realistic scenarios, $\mathbf{R}_{M}$ is, of course, not equal to $\mathbf{I}$, and $\operatorname{tr} \mathbf{R}_{M}$ is interpreted as the effective number of parameters that can be resolved ${ }^{2}$.

To interpret the resolution matrix in more detail, we briefly omit the error propagation term $\mathbf{G}^{-g} \boldsymbol{\varepsilon}$ and write equation (6.32) in index notation,

$$
\Delta \tilde{m}_{i}=\sum_{j=1}^{n}\left(\mathbf{R}_{M}\right)_{i j} \Delta m_{j}^{\text {target }} .
$$

Equation (6.34) reveals that the $i$-component of the estimated model difference, $\Delta \tilde{m}_{i}$, is a sum over the target model differences $\Delta m_{j}^{\text {target }}$, with the entries in the $i^{\text {th }}$ row of $\mathbf{R}_{M}$ acting as weights. In this sense, $\Delta \tilde{m}_{i}$ is a weighted average of the $\Delta m_{j}^{\text {target }}$, with the rows of the resolution matrix acting as averaging kernels.

Also the columns of $\mathbf{R}_{M}$ have concrete meaning. To see this, we assume that we wish to recover a model that differs from the prior model only in one parameter, that is, $\Delta m_{j}^{\text {target }}=m_{j}^{\text {target }}-m_{j}^{\text {prior }}=\delta_{j k}$, with a fixed index $k$. Inserting $\Delta m_{j}^{\text {target }}$ into equation (6.34), we find

$$
\Delta \tilde{m}_{i}=\left(\mathbf{R}_{M}\right)_{i k} .
$$

Equation (6.35) suggests that the recovered model, $\Delta \tilde{m}_{i}$, has non-zero components for indices $i \neq k$, unless we are in the situation of perfect resolution with $\mathbf{R}_{M}=\mathbf{I}$. In fact, the target model $\Delta m_{j}^{\text {target }}$ that is non-zero only for index $j=k$, is blurred or smeared into a broader (slowness) distribution, the values of which are contained in the $k^{\text {th }}$ column of the resolution matrix. Therefore, the columns of $\mathbf{R}_{M}$ are known as point-spread functions.

${ }^{2}$ We note that this is merely an intuitive interpretation of $\operatorname{tr} \mathbf{R}_{M}$ and not a hard definition. 
The concept of the point-spread function is used widely in astronomy and microscopy. Loosely speaking, the point-spread function is the impulse response of an imaging system, that is, the response (or image) obtained when trying to resolve a perfectly point-localised object. In astronomy, in particular, we can literally see the point-spread function when looking at a distant star (the point) through a telescope. This is illustrated for the specific example of the Hubble Space Telescope in figure 6.8. In the context of the traveltime tomography, we may imagine looking through a tomographic telescope that blurrs a hypothetically point-localised heterogeneity.

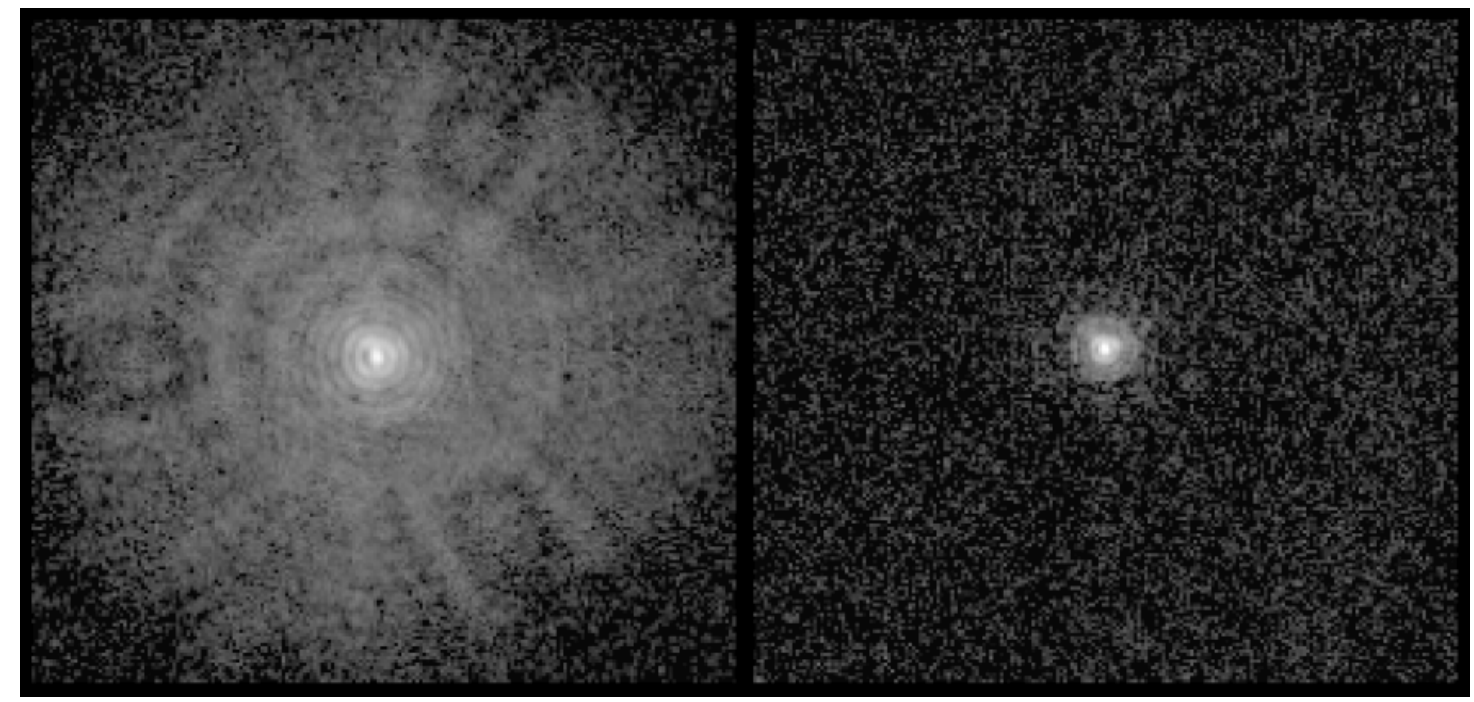

Fig. 6.8: Point-spread functions of the Faint Object Camera (FOC) of the Hubble Space Telescope. Left: FOC image of a star using Hubble's flawed mirror, which was around $2 \mu \mathrm{m}$ too flat. Right: FOC image after the COSTAR repair mission that corrected Hubble's optics by providing it with the most expensive glasses ever made. (Copyright: Patrick P Murphy, National Radio Astronomy Observatory)

The concept of model resolution deserves a little more discussion. First of all, we note the generality of equation (6.30). In our specific context, we defined the generalised inverse $\mathbf{G}^{-g}$ as in equation (6.31), which followed from the least-squares solution. However, other methods to estimate a model may lead to other expressions for $\mathbf{G}^{-g}$. In these cases, the model resolution matrix is still given generically by $\mathbf{R}_{M}=\mathbf{G}^{-g} \mathbf{G}$.

Second, equation (6.33) indicates that the computation of the resolution matrix requires the computation of a matrix inverse, $\left(\mathbf{C}_{D}+\mathbf{G C}_{M} \mathbf{G}^{T}\right)^{-1}$. However, when inserting honest prior knowledge, which may not be particularly strong, this inverse may actually not exist. Thus, as in the case of the posterior mean model, we may be required to regularise the problem by choosing artificial values for $\mathbf{C}_{D}$ and $\mathbf{C}_{M}$. It follows that the regularised resolution may be overly optimistic and not perfectly representative of the true resolution.

Finally, we remark that the concept of model resolution, as introduced above, only applies to synthetic inversions, meaning that an inverse crime is built in. This is because equation (6.27) does not strictly apply to actually observed data that can of course not be computed through the application of our unavoidably simplified forward modelling theory $\mathbf{G}$. Thus, also in this sense, resolution estimated with the help of $\mathbf{R}_{M}$ may be rather optimistic in any case. 
Model resolution and covariance: Using equation (6.14) for the posterior model covariance $\tilde{\mathbf{C}}_{M}$, we can find an alternative and more insightful expression for the model resolution $\mathbf{R}_{M}$. In fact, substituting equation (6.14) into equation (6.33), yields

$$
\mathbf{C}_{M}-\tilde{\mathbf{C}}_{M}=\mathbf{R}_{M} \mathbf{C}_{M}
$$

It follows that $\mathbf{R}_{M}$ can be expressed as

$$
\mathbf{R}_{M}=\mathbf{I}-\tilde{\mathbf{C}}_{M} \mathbf{C}_{M}^{-1}
$$

Equation (6.37) indicates that resolution and covariance are intimately linked. When the data contribute no new information, so that the posterior covariance is equal to the prior covariance $\left(\tilde{\mathbf{C}}_{M}=\mathbf{C}_{M}\right)$, model resolution vanishes completely, that is, $\mathbf{R}_{M}=\mathbf{0}$. In contrast, when the posterior covariance becomes numerically very small, we approach the case of perfect resolution, that is $\mathbf{R}_{M} \rightarrow \mathbf{I}$.

Figure 6.9 displays the model resolution matrix for the traveltime tomography problem introduced in section 6.2. Clearly, as expected, $\mathbf{R}_{M}$ is not equal to the unit matrix $\mathbf{I}$, indicating that resolution is not perfect. Though $\mathbf{R}_{M}$ is dominated by its diagonal, prominent off-diagonal elements are present, which shows that averaging and smearing do occur as a consequence of imperfect coverage. The banded structure results from trade-offs or smearing between neighbouring model parameters that are separated by around 20 parameter indices. The the total number of model parameters is 400 , the trace of $\mathbf{R}_{M}$ indicates that only 108 of them are effectively resolved.

Averaging and smearing in the form of selected averaging kernels and point-spread functions are presented in figure 6.10. The averaging kernels illustrate that an element of the posterior mean model is indeed an average over slowness values in many neighbouring grid cells. For cells located in regions of good ray coverage - in terms of both ray density and azimuthal coverage - averaging is considerably less pronounced than for cells in poorly covered regions. Complementary information is contained in the point-spread functions, which indicate how a hypothetical slowness anomaly within a single grid cell would smear into its surroundings, when seen through the tomographic telescope.

In the light of imperfect resolution, it is important to consider the extent to which the addition of data could lead to significant improvements. At least a partial answer in the context of our synthetic traveltime tomography is provided in figure 6.11. In the left panel, it shows the trace of the model resolution matrix, that is, the number of effectively resolved parameters, as function of the number of receivers positioned equidistantly along the right boundary of the domain (see figure 6.1). As the number of receivers increases from less then 10 to around 50 , the effective number of resolved parameters increases rapidly from slightly more than 50 to nearly 200 . Any further increase in the number of receivers comes, however, only with small additional benefits. A more detailed view of this saturation effect is shown in the left panel of figure 6.11, where the diagonal elements of the model resolution matrix are displayed. Clearly, some model parameters benefit more from additional data than others ${ }^{3}$.

\subsubsection{Data resolution*}

Following the solution of a least-squares problem, we may compute estimated data residuals $\Delta \mathbf{d}^{\text {est }}$ by applying the forward modelling matrix $\mathbf{G}$ to the estimated model difference $\Delta \tilde{\mathbf{m}}$, similar to equation (6.29),

\footnotetext{
${ }^{3}$ Using different source-receiver geometries does not fundamentally change the shape of the curve in figure 6.11. It always closely follows the function form $\operatorname{tr} \mathbf{R}_{M}=a\left(1-e^{-b N}\right)$, with some constants $a$ and $b$, though this is purely empirical.
} 


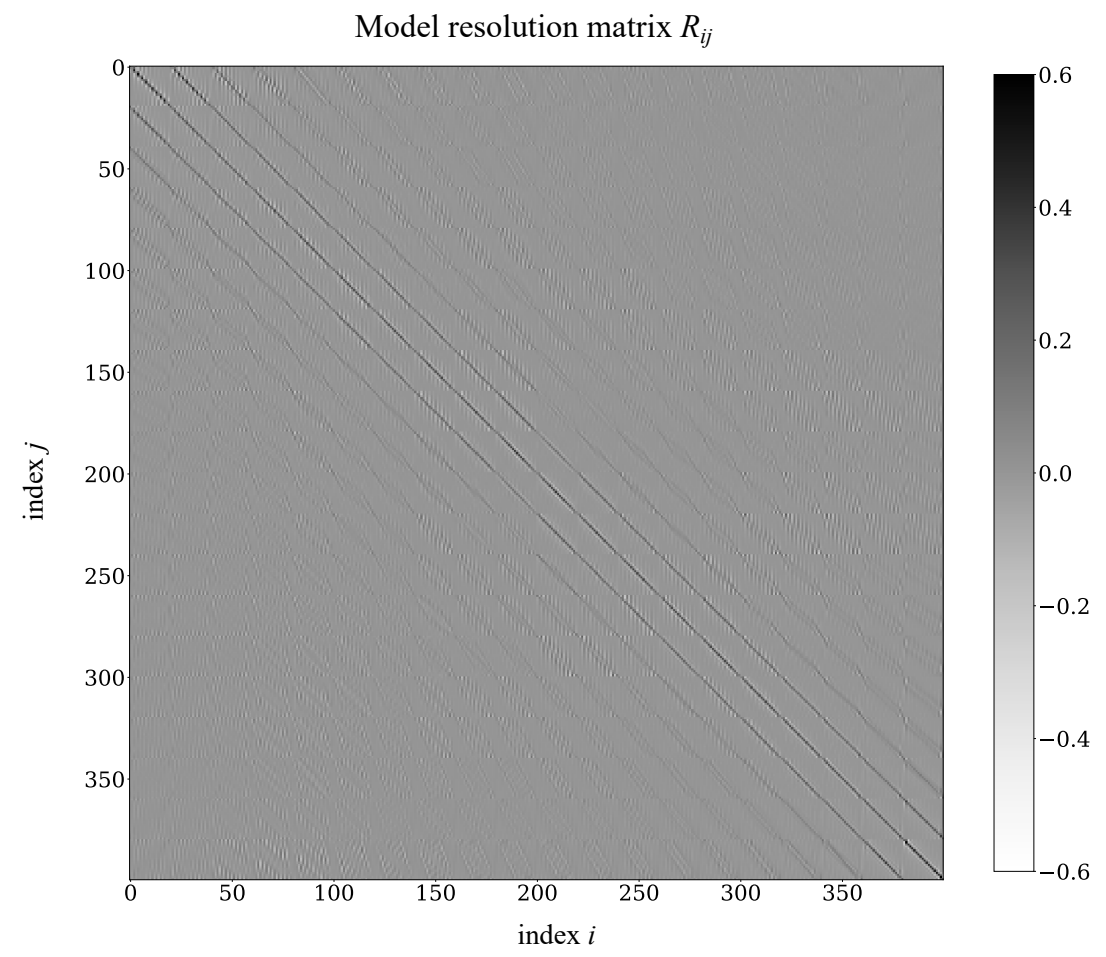

Fig. 6.9: Resolution matrix $\mathbf{R}_{M}$ for the traveltime tomography from section 6.2. The total number of resolved parameters is $\operatorname{tr} R_{M}=108$, that is, approximately $25 \%$ of the actual number of parameters, which is 400 . The conspicuous striping results from trade-offs (smearing) between neighbouring grid points that are separated by around 20 parameter indices. [Straight-ray tomography]

$$
\Delta \mathbf{d}^{\mathrm{est}}=\mathbf{G} \Delta \tilde{\mathbf{m}} .
$$

Substituting $\tilde{\mathbf{m}}$ from equation (6.30), we obtain

$$
\Delta \mathbf{d}^{\mathrm{est}}=\mathbf{G G}^{-g} \Delta \mathbf{d}^{\mathrm{obs}}=\mathbf{R}_{D} \Delta \mathbf{d}^{\mathrm{obs}},
$$

where

$$
\mathbf{R}_{D}=\mathbf{G G}^{-g}=\mathbf{G C}_{M} \mathbf{G}^{T}\left(\mathbf{C}_{D}+\mathbf{G C}_{M} \mathbf{G}^{T}\right)^{-1}
$$

is the data resolution matrix. Formally, the data resolution matrix can be interpreted similarly to the model resolution matrix. Of particular interest are the diagonal elements $\left(\mathbf{R}_{D}\right)_{i i}$ of $\mathbf{R}_{D}$, referred to as data importance. It indicates the importance of an observed datum $\Delta \mathbf{d}_{i}^{\text {obs }}$ on an estimated or predicted datum $\Delta \mathbf{d}_{i}^{\text {est }}$. Ideally, the data importances $\left(\mathbf{R}_{D}\right)_{i i}$ have approximately equal values for all $i=1, \ldots, N$, meaning that no data point is much more or much less important than the others. Experimental setups that achieve this goal are referred to as equileverage designs.

A visual summary of the data resolution matrix for the traveltime tomography introduced in section 6.2 is presented in figure 6.12. The matrix is diagonally dominant, indicating that each observed data point contributes most directly to the corresponding predicted data point. However, a closer look at the diagonal of $\mathbf{R}_{D}$ also reveals that some data points are significantly more important than others, suggesting that the experimental setup may have potential for optimisation. 

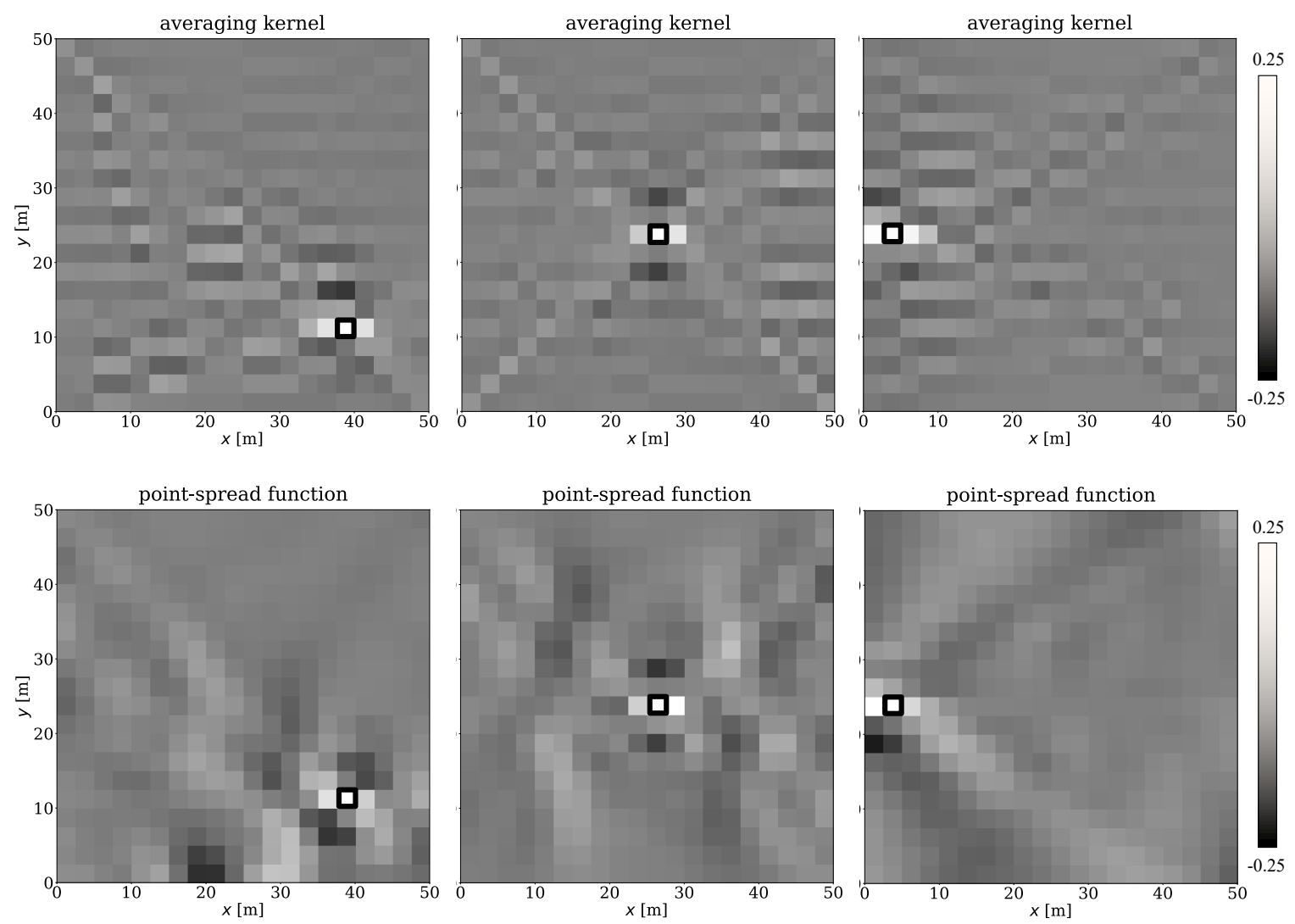

Fig. 6.10: Collection of averaging kernels (top row) and point-spread functions (bottom row) for the traveltime tomography from section 6.2. The rows and columns of the resolution matrix are reformatted to plot within the spatial domain of the slowness model. The black squares mark the grid cell for which the averaging kernels and point-spread functions have been computed. [Straight-ray tomography]

\subsection{Singular value analysis *}

In section 6.2 and figure 6.3 we already considered the eigenvalues of the matrix $\mathbf{G}^{T} \mathbf{G}$, which enters the calculation of the Moore-Penrose inverse. We will now carry this analysis a bit further, which will lead us to a more profound understanding of linear inverse problems. To set the stage, we recall some basic properties of the eigenvalues and eigenvectors of a generic, symmetric matrix $\mathbf{A} \in \mathbb{R}^{n \times n}$. The $n$ eigenvectors $\mathbf{w}$ of $\mathbf{A}$ are defined as solutions to the eigenvalue problem

$$
\mathbf{A} \mathbf{w}_{i}=\lambda_{i} \mathbf{w}_{i}
$$

where $\lambda_{i}$ is the eigenvalue corresponding to $\mathbf{w}_{i}$. Since $\mathbf{A}$ is assumed to be symmetric, the eigenvectors are orthogonal, and can be normalised such that

$$
\mathbf{w}_{i}^{T} \mathbf{w}_{j}=\delta_{i j}
$$

We may collect all $n$ eigenvectors into a matrix $\mathbf{W}$,

$$
\mathbf{W}=\left[\mathbf{w}_{1}, \ldots, \mathbf{w}_{n}\right]
$$

meaning that they play the role of column vectors in $\mathbf{W}$. This leads to a matrix version of equation (6.42), 
a) diagonal of model resolution matrix

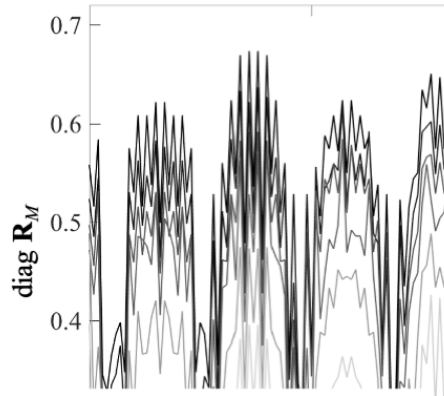

b) number of effectively resolved parameters
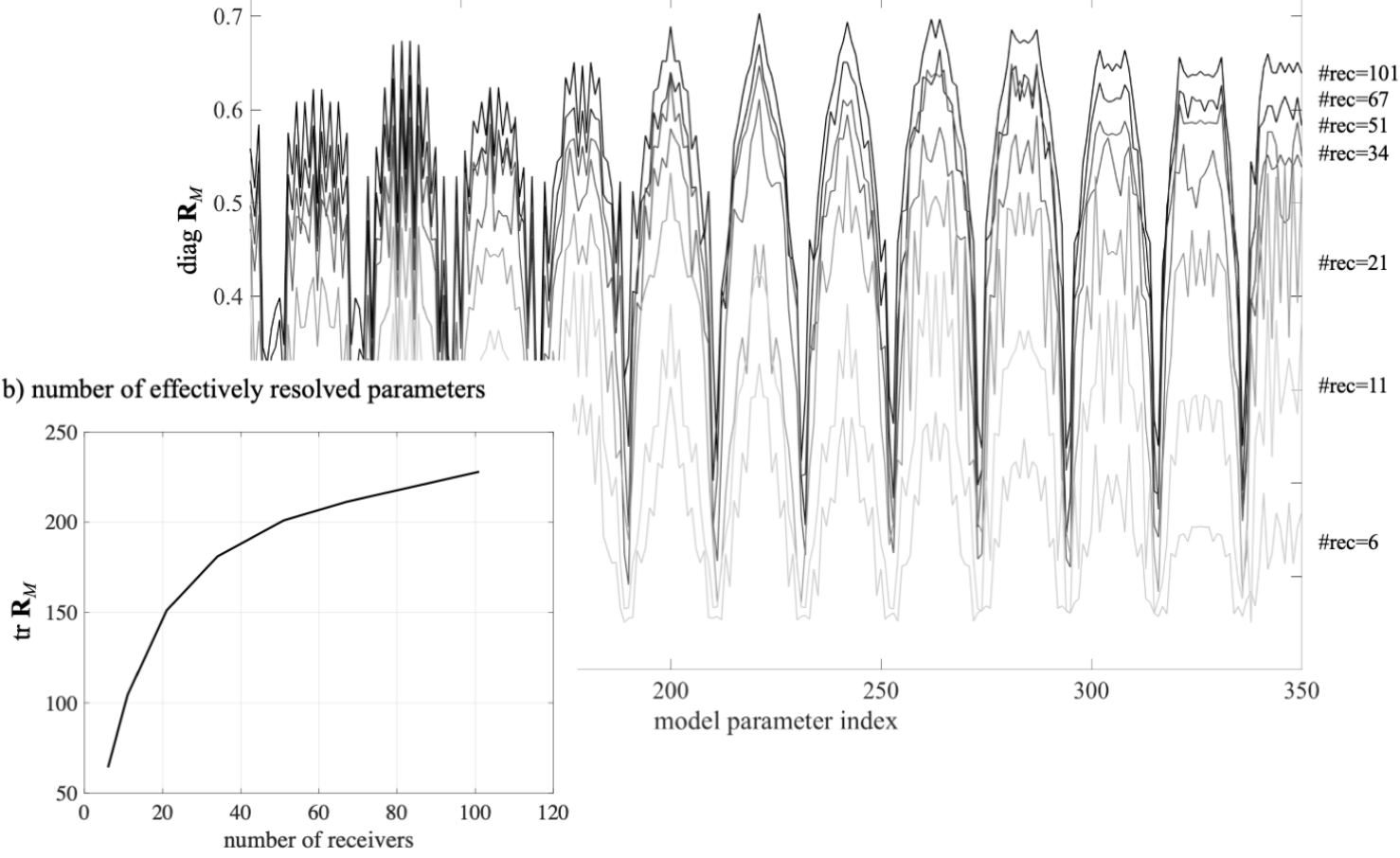

Fig. 6.11: Model resolution as a function of the number of receivers. (a) Diagonal elements of the model resolution matrix for an increasing number of receivers. (b) Development of the effective number of resolved parameters as the number of receivers increases. The clearly visible saturation effect implies that there is an optimal number of receivers around the kink of the curve beyond which the effect of additional observations is rather small.

$$
\mathbf{W}^{T} \mathbf{W}=\left[\begin{array}{c}
\mathbf{w}_{1}^{T} \\
\vdots \\
\mathbf{w}_{n}^{T}
\end{array}\right]\left[\mathbf{w}_{1}, \ldots, \mathbf{w}_{n}\right]=\mathbf{I} \in \mathbb{R}^{n \times n} .
$$

From equation (6.44) we directly derive an equation that we will find useful later:

$$
\mathbf{W} \mathbf{W}^{T}=\left(\mathbf{W}^{T} \mathbf{W}\right)^{T}=\mathbf{I}^{T}=\mathbf{I} .
$$

With equations (6.41) to (6.45) in mind, we turn to the more interesting task of analysing our linear inverse problem. Though the forward modelling matrix $\mathbf{G}$ is not generally symmetric, the matrix $\mathbf{G}^{T} \mathbf{G} \in \mathbb{R}^{n \times n}$ is symmetric by construction. It therefore has $n$ orthonormal eigenvectors $\mathbf{m}_{i} \in \mathbb{R}^{n}$, defined through the equation

$$
\mathbf{G}^{T} \mathbf{G m}_{i}=\lambda_{i} \mathbf{m}_{i} .
$$

Since $\mathbf{G}^{T} \mathbf{G}$ is positive semi-definite, the eigenvalues $\lambda_{i}$ are not negative, but they may be equal to 0 . Assuming that the eigenvalues are ordered such that the first $r \leq n$ of them are non-zero, and the remaining $n-r$ are zero, we define $\sigma_{j}=\sqrt{\lambda_{j}}$, and

$$
\mathbf{d}_{j}=\frac{1}{\sigma_{j}} \mathbf{G m}_{j} \in \mathbb{R}^{N}, \quad \text { for } j=1, \ldots, r .
$$




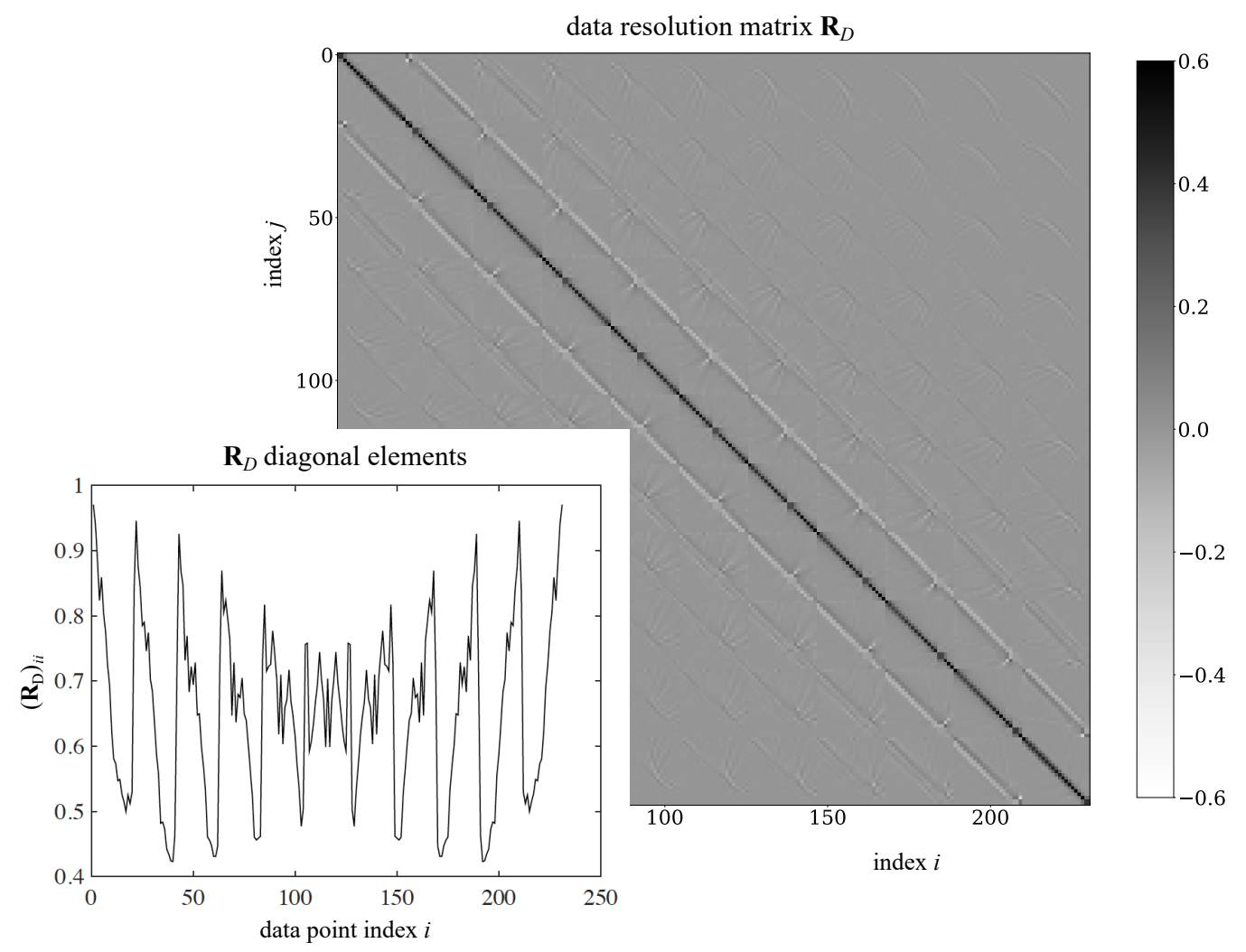

Fig. 6.12: Summary of the data resolution matrix $\mathbf{R}_{D}$ for the traveltime tomography from section 6.2. Left: Element-wise display of the data resolution matrix. Right: Diagonal elements of the data resolution matrix. [Straight-ray tomography]

The vector $\mathbf{d}_{j}$ contains the synthetic data computed for the eigenvector (eigenmodel) $\mathbf{m}_{j}$, and normalised by $\sigma_{j}$. Based on the definition in equation (6.47), we find

$$
\mathbf{d}_{j}^{T} \mathbf{d}_{i}=\frac{1}{\sigma_{i} \sigma_{j}} \mathbf{m}_{j}^{T} \mathbf{G}^{T} \mathbf{G m}_{i}=\frac{\lambda_{i}}{\sigma_{i} \sigma_{j}} \mathbf{m}_{j}^{T} \mathbf{m}_{i}=\delta_{i j},
$$

because of the orthonormality of the eigenmodels $\mathbf{m}_{i}$, that is, $\mathbf{m}_{j}^{T} \mathbf{m}_{i}=\delta_{i j}$. Equation (6.48) means that also the synthetic data vectors $\mathbf{d}_{i}$ are orthonormal. At this point, we assume that the number of observations $N$ is less than the number of model parameters, $n$, that is, $N \leq n$. In case that $r<N$, we extend the set of the $r$ vectors $\mathbf{d}_{i}$ into a complete orthonormal basis of $\mathbb{R}^{N}$. It then follows that

$$
\mathbf{d}_{i}^{T} \mathbf{G m}_{j}=\sigma_{j} \mathbf{d}_{i}^{T} \mathbf{d}_{j}= \begin{cases}\sigma_{j} \delta_{i j}, & i=1, \ldots, \min (r, N), \\ 0, & i>\min (r, N) .\end{cases}
$$

Now collecting the $N$ vectors $\mathbf{d}_{i}$ as columns into the matrix

$$
\mathbf{D}=\left[\mathbf{d}_{1}, \ldots, \mathbf{d}_{N}\right] \in \mathbb{R}^{N \times N},
$$

and the $n$ vectors $\mathbf{m}_{i}$ as columns into the matrix

$$
\mathbf{M}=\left[\mathbf{m}_{1}, \ldots, \mathbf{m}_{n}\right] \in \mathbb{R}^{n \times n},
$$


we obtain

$$
\mathbf{D}^{T} \mathbf{G M}=\Lambda,
$$

where $\Lambda \in \mathbb{R}^{N \times n}$ is a diagonal matrix containing the $\sigma_{j}$ values. Written explicitly,

$$
\Lambda=\left[\begin{array}{ccccccccc}
\sigma_{1}>0 & 0 & \ldots & \ldots & \ldots & \ldots & 0 & \ldots & 0 \\
0 & \sigma_{2}>0 & \ldots & \ldots & \ldots & \ldots & 0 & \ldots & 0 \\
\vdots & \ddots & \ddots & \ddots & \ddots & \ddots & \vdots & \ddots & \vdots \\
\vdots & \ldots & \ldots & \sigma_{r}>0 & 0 & \ldots & 0 & \ldots & 0 \\
\vdots & \ldots & \ldots & 0 & \sigma_{r+1}=0 & \ldots & 0 & \ldots & 0 \\
\vdots & \ddots & \ddots & \ddots & \ddots & \ddots & \vdots & \ddots & \vdots \\
0 & 0 & \ldots & \ldots & \ldots & \ldots & \sigma_{N}=0 & \ldots & 0
\end{array}\right]
$$

Left-multiplying equation (6.51) with $\mathbf{D}$, and right-multiplying it with $\mathbf{M}^{T}$, we finally obtain

$$
\mathbf{G}=\mathbf{D} \Lambda \mathbf{M}^{T} \text {. }
$$

Equation (6.53) is referred to as the singular-value decomposition or SVD of the matrix $\mathbf{G}$. The entries of $\Lambda$ are the singular values of $\mathbf{G}$, and the columns of $\mathbf{D}$ and $\mathbf{M}$ are its left- and right-singular vectors, respectively. For the case $N>n$, equation (6.53) can be derived with the matrix $\mathbf{G G}^{T}$ as starting point, which leads to an explicit $\Lambda$ taking the form

$$
\Lambda=\left[\begin{array}{ccccccc}
\sigma_{1}>0 & 0 & \ldots & \ldots & \ldots & \ldots & 0 \\
0 & \sigma_{2}>0 & \ldots & \ldots & \ldots & \ldots & 0 \\
\vdots & \ddots & \ddots & \ddots & \ddots & \ddots & \vdots \\
\vdots & \ldots & \ldots & \sigma_{r}>0 & 0 & \ldots & 0 \\
\vdots & \ldots & \ldots & 0 & \sigma_{r+1}=0 & \ldots & 0 \\
\vdots & \ddots & \ddots & \ddots & \ddots & \ddots & \vdots \\
\vdots & \ddots & \ddots & \ddots & \ddots & \ddots & \sigma_{n}=0 \\
\vdots & \ddots & \ddots & \ddots & \ddots & \ddots & \vdots \\
0 & 0 & \ldots & \ldots & \ldots & \ldots & 0
\end{array}\right]
$$

As one might expect, the singular-value decomposition is closely related to the Moore-Penrose inverse, $\left(\mathbf{G}^{T} \mathbf{G}\right)^{-1} \mathbf{G}^{T}$, if it exists. In fact, for some arbitrary $\mathbf{m} \in \mathbb{R}^{n}$, we have $\mathbf{G m}=\mathbf{d}$ and $\mathbf{G}^{T} \mathbf{G m}=\mathbf{G}^{T} \mathbf{d}$. Assuming that $\mathbf{G}^{T} \mathbf{G}$ is invertible, we find $\mathbf{m}=\left(\mathbf{G}^{T} \mathbf{G}\right)^{-1} \mathbf{G}^{T} \mathbf{d}$. Similarly, using equation (6.53), the forward problem can be expressed as

$$
\mathbf{D} \Lambda \mathbf{M}^{T} \mathbf{m}=\mathbf{d} .
$$

Using the orthonormality of $\mathbf{D}$ and $\mathbf{M}$, yields

$$
\mathbf{m}=\mathbf{M} \Lambda^{-1} \mathbf{D}^{T} \mathbf{d},
$$

where $\Lambda^{-1} \in \mathbb{R}^{n \times N}$ is defined as the diagonal matrix with entries $1 / \sigma_{i}$ for the $r$ positive $\sigma_{i}$, and 0 otherwise, that is, 


$$
\Lambda^{-1}=\left[\begin{array}{ccccccc}
\sigma_{1}^{-1} & 0 & \ldots & \ldots & \ldots & \ldots & 0 \\
0 & \sigma_{2}^{-1} & \ldots & \ldots & \ldots & \ldots & 0 \\
\vdots & \ddots & \ddots & \ddots & \ddots & \ddots & \vdots \\
\vdots & \ldots & \ldots & \sigma_{r}^{-1} & 0 & \ldots & 0 \\
\vdots & \ldots & \ldots & 0 & 0 & \ldots & 0 \\
\vdots & \ddots & \ddots & \ddots & \ddots & \ddots & \vdots \\
0 & 0 & \ldots & \ldots & \ldots & \ldots & 0
\end{array}\right]
$$

It follows that the Moore-Penrose inverse has the alternative representation

$$
\left(\mathbf{G}^{T} \mathbf{G}\right)^{-1} \mathbf{G}^{T}=\mathbf{M} \Lambda^{-1} \mathbf{D}^{T}
$$

Thus, using equation (6.58), the least-squares problem may also be solved via a singular-value decomposition, instead of by direct inversion of $\mathbf{G}^{T} \mathbf{G}$. However, the actual benefit of the singular-value decomposition is its ability to provide an explicit representation of those parts of the model space $\mathbb{M}$ that can or cannot be discovered by the data, as we shall see in the following paragraphs.

\subsubsection{The nullspace *}

Being a set of $n$ orthonormal vectors, the eigenvectors $\mathbf{m}_{i}$ of $\mathbf{G}^{T} \mathbf{G}$ form a basis of the model space $\mathbb{M}$, meaning that any model $\mathbf{m} \in \mathbb{M}$ can be represented as a linear combination

$$
\mathbf{m}=\sum_{i=1}^{n} \mu_{i} \mathbf{m}_{i}
$$

with some real numbers $\mu_{i}$. Now assume that our model coincides precisely with the $k^{\text {th }}$ eigenvector, meaning that $\mathbf{m}=\mathbf{m}_{k}$. Inserting this choice of a model into equation (6.55), we find

$$
\mathbf{d}=\mathbf{G m}_{k}=\mathbf{D} \Lambda \mathbf{M}^{T} \mathbf{m}_{k}=\mathbf{D} \Lambda \mathbf{e}_{k}=\sigma_{k} \mathbf{D} \mathbf{e}_{k}=\sigma_{k} \mathbf{d}_{k}
$$

where $\mathbf{e}_{k}$ is the unit vector with components $\left(\mathbf{e}_{k}\right)_{i}=\delta_{k i}$. If the $k^{\text {th }}$ singular value happens to be 0 , or when $k>\min (n, N)$, the right-hand side of equation (6.60) vanishes. We are then left with

$$
\mathbf{d}=\mathbf{0}
$$

Equation (6.61) has profound implications, as it suggests that certain models cannot be seen by the data. The collection of all of these invisible models, that is, the subspace of $\mathbb{M}$ spanned by eigenvectors with vanishing singular values, is referred to as the nullspace or kernel of the forward modelling operator $\mathbf{G}$. It is symbolised by $\operatorname{ker} \mathbf{G}$.

Using, for instance, the least-squares method, we may find an estimated model, $\mathbf{m}^{\text {est }}$ that produces estimated synthetic data, $\mathbf{d}^{\text {est }}$. The presence of a nullspace implies that the estimated model is nonunique, in the sense that we can add any model $\mathbf{m}_{0}$ from inside the nullspace, and the estimated data do not change:

$$
\mathbf{G}\left(\mathbf{m}^{\mathrm{est}}+\mathbf{m}_{0}\right)=\mathbf{G} \mathbf{m}^{\mathrm{est}}+\mathbf{G} \mathbf{m}_{0}=\mathbf{G} \mathbf{m}^{\mathrm{est}}=\mathbf{d}^{\mathrm{est}}, \quad \text { if } \quad \mathbf{m}_{0} \in \operatorname{ker} \mathbf{G} .
$$

In other words, $\mathbf{m}^{\text {est }}$ and $\mathbf{m}^{\text {est }}+\mathbf{m}_{0}$ are indistinguishable from the data perspective, because both produce exactly identical synthetic data $\mathbf{d}^{\text {est }}$.

Conversely, it follows that only those eigenvectors $\mathbf{m}_{i}$ with nonzero singular values $\sigma_{i}$ make any contribution to the data. Contrasting the nullspace, these $r \leq n$ eigenvectors are collected into the activated model space. Via 
the forward modelling matrix $\mathbf{G}$, the activated model space is mapped into the activated data space, spanned by the vectors $\mathbf{d}_{1}, \ldots, \mathbf{d}_{r}$, according to equation (6.60). The nonactivated parts of the model space, that is, the nullspace, have no impact on the synthetic data. The nonactivated parts of the data space correspond to synthetic data that no possible model can produce. The relation between the activated data and model spaces is illustrated in figure 6.13.

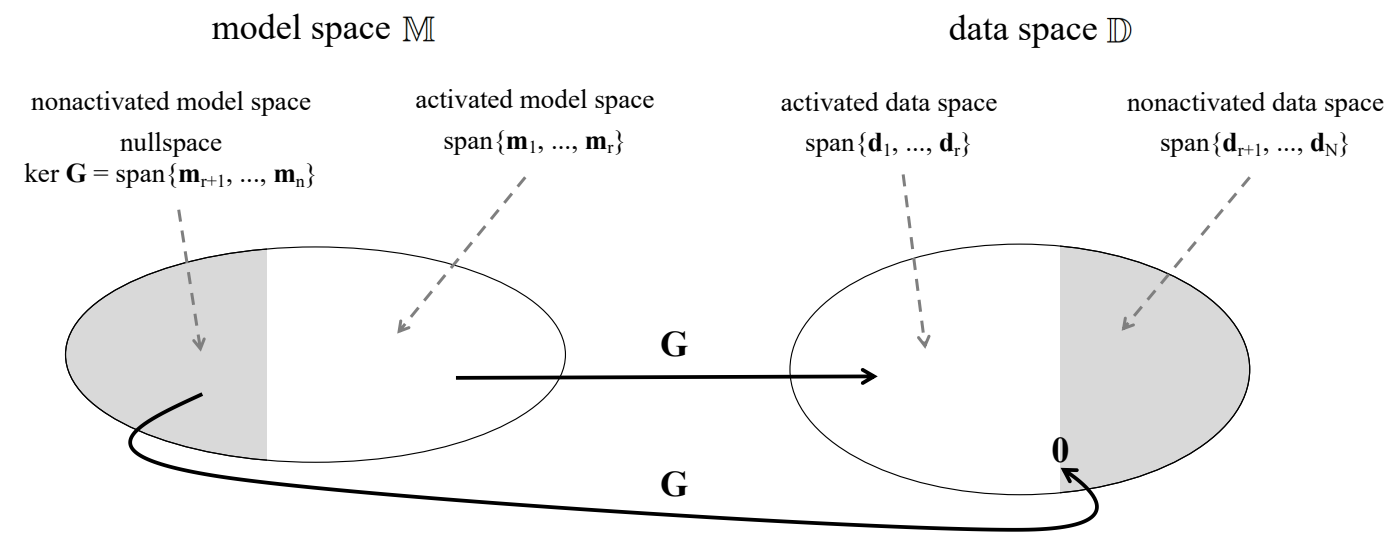

Fig. 6.13: Schematic illustration of the relation between the activated and nonactivated model and data spaces, assuming that the first $r$ singular values are nonzero. The activated model space is spanned by the first $r$ rightsingular vectors of $\mathbf{G}$ (eigenvalues of $\mathbf{G}^{T} \mathbf{G}$ ). The forward modelling matrix $\mathbf{G}$ maps the activated model space $\operatorname{span}\left\{\mathbf{m}_{1}, \ldots, \mathbf{m}_{r}\right\}$ to the activated data space, spanned by the first $r$ left-singular vectors $\mathbf{d}_{i}, \ldots, \mathbf{d}_{r}$. The complete nullspace, $\operatorname{ker} \mathbf{G}$, spanned by the right-singular vectors $\mathbf{m}_{r+1}, \ldots, \mathbf{m}_{n}$, is mapped by $\mathbf{G}$ to a single data vector, namely $\mathbf{0}$, which is the only vector that resides within both the activated and nonactivated data space. (However, $\mathbf{d}=\mathbf{0}$ may not be a physically meaningful data vector.) The nonactivated data space, spanned by the remaining vectors $\mathbf{d}_{r+1}, \ldots, \mathbf{d}_{N}$ is not accessible from any of the vectors in model space $\mathbb{M}$.

Returning again to our model problem from section 6.2, we can explicitly compute members of the activated and nonactivated model space, using singular-value decomposition, as outlined above. Since $N<n$, all rightsingular vectors $\mathbf{m}_{i}$ with index $i>N=231$ are from the outset within the nullspace (nonactivated model space). The first three members of the activated model space, and the last three members of the nullspace are displayed in figure 6.14. Loosely speaking, members of the activated model space contain large-scale structure visible by the data. Members of the nullspace tend to contain small-scale structure that may be added to another model without having any effect on the synthetic data.

The observation that the nullspace mostly contains small-scale structure, is intuitively reasonable. An observed traveltime represents an average over slowness variations along the ray that connects source and receiver. Thus, small-scale variations that are roughly as often positive as they are negative, may average out. However, it has been noted by Lévêque et al. (1993) that this intuition may be misleading. There are, in fact, counterexamples where large-scale structures are contained in the nullspace.

\subsection{Most-squares and extremal bounds analysis}

The least-squares method, covered in the previous sections, yields an estimated model $\mathbf{m}^{\text {est }}=\tilde{\mathbf{m}}$ that minimises the quadratic misfit function (6.2) and allows us to quantify its quality in terms of the posterior model covariance $\tilde{\mathbf{C}}_{M}$ and the model resolution $\mathbf{R}_{M}$. While $\tilde{\mathbf{C}}_{M}$ determines the bounds within which model parameters may 

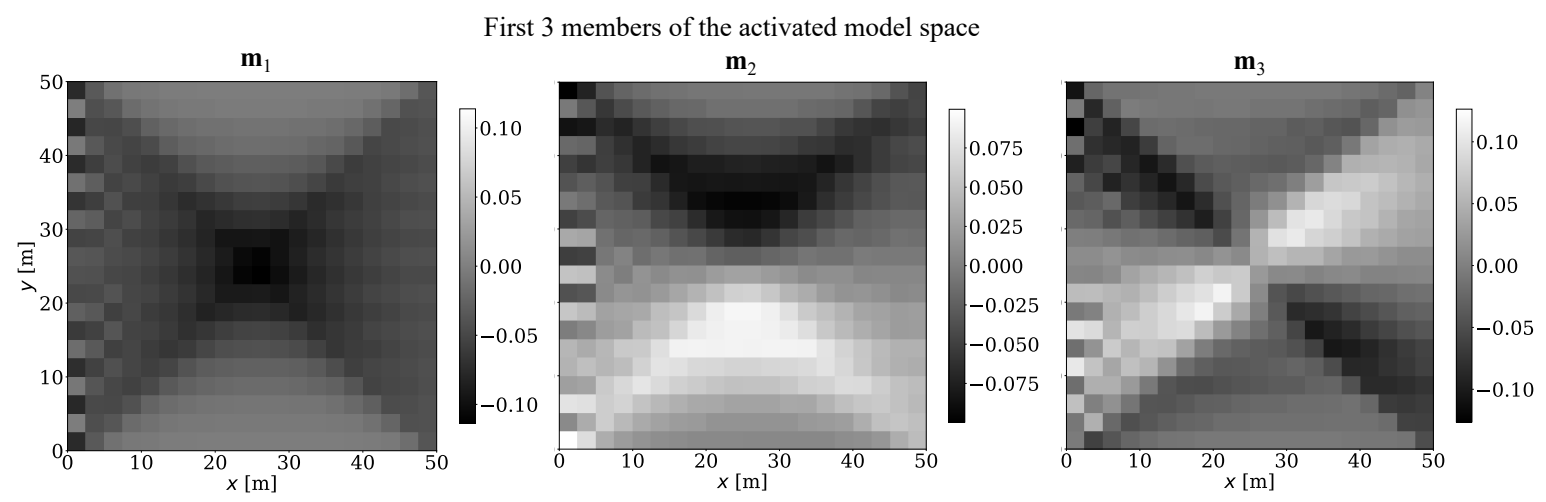

Last 3 members of the nonactivated model space (nullspace)
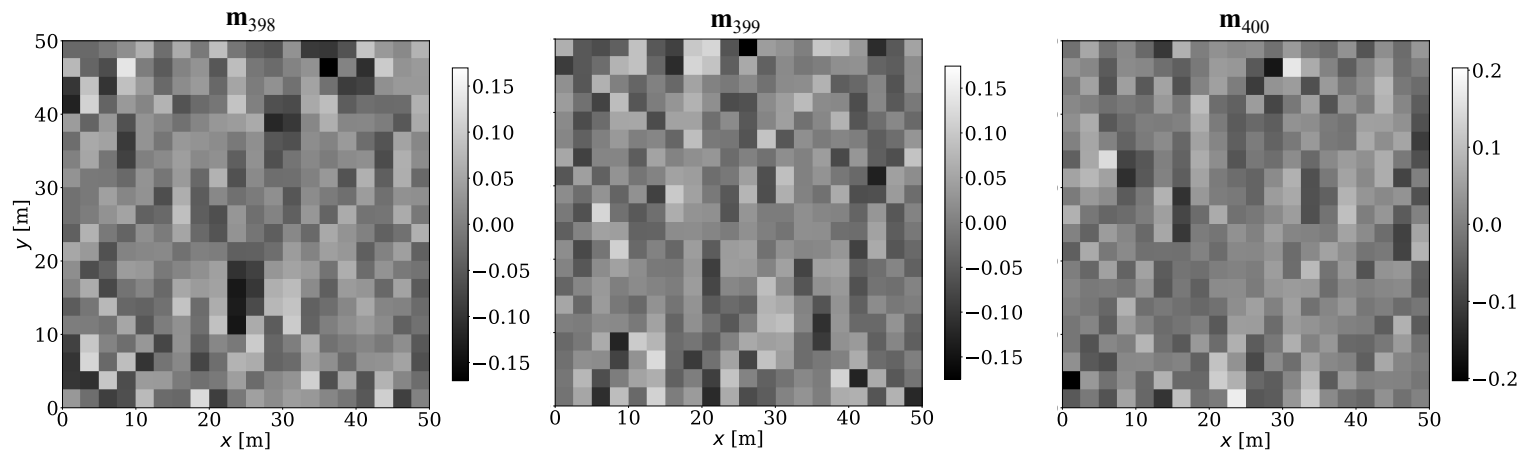

Fig. 6.14: Selected members of the activated and nonactivated model space. Top: The first three members of the activated model space represent large-scale slowness patterns that are visible by the (synthetic) data. Bottom: The last three members of the nullspace contain small-scale structure that can be added to another model without having any effect on the synthetic data.

plausibly vary, the least-squares method itself does not provide a mechanism to actually construct alternative models that still produce an acceptable misfit.

A computationally efficient approach to the construction of alternative models, that does not require Monte Carlo sampling, is the most-squares method, proposed by Jackson (1976). The most-squares method allows us to find models that are as extreme as possible, while remaining within the bounds of misfits that we find acceptable. It is therefore also known as extremal bounds analysis.

To develop the method, we first consider an extremal misfit $\chi^{\text {ext }}$ that is just on the edge of being acceptable. Typically, $\chi^{\text {ext }}$ will be defined as a function of the observational errors. Our goal is to extremise the quantity $\mathbf{b}^{T} \mathbf{m}$, subject to the constraint $\chi(\mathbf{m})=\chi^{\text {ext }}$. The vector $\mathbf{b}$ can be chosen freely in order to extract a specific aspect of the model. Choosing, for example, $b_{i}=\delta_{i k}$, provides the extremal bounds of the individual model parameter $m_{k}$. Alternatively, $b_{i}=1 / n$, yields the extremal variations of the model average. We can solve this constrained optimisation problem using the Lagrange multiplier approach (see appendix 15.1), with the Lagrange function $L(\mathbf{m})$ defined as

$$
L(\mathbf{m})=\mathbf{b}^{T} \mathbf{m}+\mu\left[\chi(\mathbf{m})-\chi^{\mathrm{ext}}\right]=\mathbf{b}^{T} \mathbf{m}+\mu\left[\frac{1}{2}(\mathbf{m}-\tilde{\mathbf{m}})^{T} \tilde{\mathbf{C}}_{M}^{-1}(\mathbf{m}-\tilde{\mathbf{m}})+\chi^{\mathrm{min}}-\chi^{\mathrm{ext}}\right],
$$

where we expressed $\chi(\mathbf{m})$ in terms of $\tilde{\mathbf{m}}$ and $\tilde{\mathbf{C}}_{M}$, as in equation (6.3). The scalar $\mu$ is the Lagrange multiplier, which remains to be determined. Forcing the gradient of $L(\mathbf{m})$ to zero, yields an explicit expression for the extremal model $\mathbf{m}^{\text {ext }}$, 


$$
\mathbf{m}^{\mathrm{ext}}=\tilde{\mathbf{m}}-\frac{1}{\mu} \tilde{\mathbf{C}}_{M} \mathbf{b} .
$$

Realising that the posterior model covariance $\tilde{\mathbf{C}}_{M}$ tends to act as a smoothing operator, equation (6.64) reveals that the extremal model $\mathbf{m}^{\text {ext }}$ equals the mean model $\tilde{\mathbf{m}}$, perturbed by a smooth version of the vector $\mathbf{b}$.

To determine the Lagrange multiplier $\mu$, we note that $L\left(\mathbf{m}^{\text {ext }}\right)=\mathbf{b}^{T} \mathbf{m}^{\text {ext }}$, and therefore,

$$
\mathbf{0}=\frac{1}{2}\left(\mathbf{m}^{\mathrm{ext}}-\tilde{\mathbf{m}}\right)^{T} \tilde{\mathbf{C}}_{M}^{-1}\left(\mathbf{m}^{\mathrm{ext}}-\tilde{\mathbf{m}}\right)+\chi^{\mathrm{min}}-\chi^{\mathrm{ext}}
$$

Substituting the expression for $\mathbf{m}^{\text {ext }}$ from (6.64), we find

$$
\mu= \pm \sqrt{\frac{\mathbf{b}^{T} \tilde{\mathbf{C}}_{M} \mathbf{b}}{\chi^{\mathrm{ext}}-\chi^{\min }}}
$$

Equation (6.67) reveals that there are two most extremal perturbations of the mean model $\tilde{\mathbf{m}}$, which are proportional to $\sqrt{\chi^{\mathrm{ext}}-\chi^{\mathrm{min}}}$. Combining equations 6.64) and 6.67), we also see that the allowable perturbations are proportional to the posterior standard deviation, as intuitively expected.

With these developments, we return one last time to our model problem from section 6.2. Figure 6.15a shows the extremal variations with respect to the mean model $\tilde{\mathbf{m}}$ when $\chi^{\text {ext }}=\chi^{\min }+1$ and $b_{i}=\delta_{i k}$. This means that we allow the misfit to increase from around 1.2 , as in figure 6.5 , to 2.2 by making the individual model parameter $m_{k}$ as large or small as possible. To achieve this goal, the extremal model perturbation $\mathbf{m}^{\text {ext }}-\tilde{\mathbf{m}}$ attains large values in the vicinity of $m_{k}$ itself, but also has structure of opposite sign at larger distance from $m_{k}$, which ensures that traveltime increases (or decreases) caused by large variations of $m_{k}$ are compensated.

An alternative scenario is presented in figure $6.15 \mathrm{~b}$, where $b_{i}=1 / n$, meaning that we try to extremise the average of the model parameters

$$
\mathbf{b}^{T} \mathbf{m}=\frac{1}{n} \sum_{i=1}^{n} m_{n} .
$$

The corresponding extremal variations $\mathbf{m}^{\mathrm{ext}}-\tilde{\mathbf{m}}$ tend to be largest in regions where coverage is poor. In contrast, admissible slowness variations are small in the well-covered parts of the domain, where even small slowness variations would lead to significant increases of the traveltime misfit.

a) Extremal local perturbation, $b_{i}=\delta_{i k}$

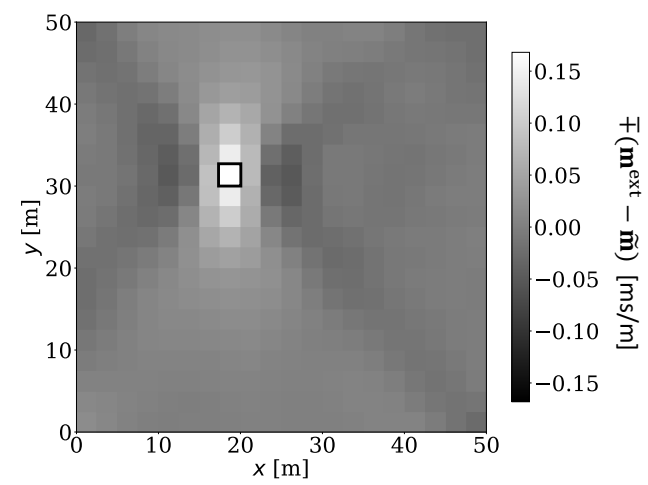

b) Extremal average model, $b_{i}=1 / n$

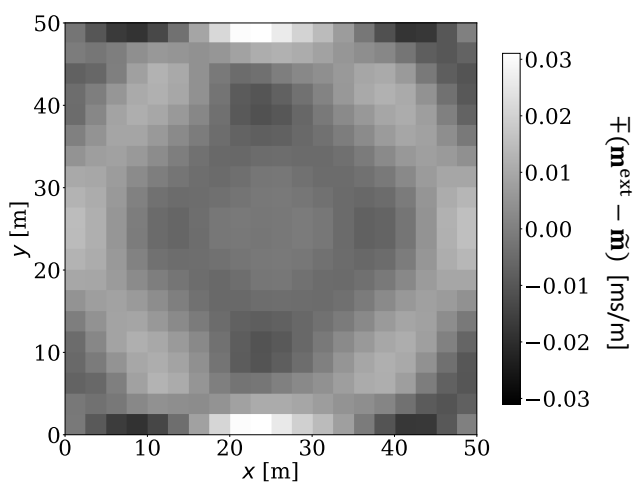

Fig. 6.15: Model variations $\pm\left(\mathbf{m}^{\text {ext }}-\tilde{\mathbf{m}}\right)$ that minimise or maximise (a) an individual model parameter $m_{k}$, marked by a black square, and (b) the average of the model parameters. 
Somewhat paradoxically, the most-squares method, as presented in the previous paragraphs, ultimately rests on the least-squares solution of an inverse problem with Gaussian priors and a linear forward problem. An extension to nonlinear forward problems that employs an iterative scheme to estimate approximate extremal bounds can be found in Meju (2009).

\subsection{Stochastic methods and matrix probing}

For linear inverse problems, the resolution and posterior covariance matrices contain most of the information needed to assess the quality of the maximum likelihood model $\tilde{\mathbf{m}}$. However, when the model space dimension $n$ is large, these matrices may be impossible to compute or store explicitly. Though it has been argued that growing computational resources may come to the rescue (e.g. Soldati et al., 2006), resolution and posterior covariance matrices are still hardly being computed. To some extent, this may be the result of an interesting psychological effect: We seem to be driven towards the construction of models that are as big (and detailed) as allowed by our currently available resources. As a consequence, the even larger resources needed to assess if these details are actually resolved, are frequently not available. ${ }^{4}$

The problem of having to compute properties of a matrix that is too big to compute or store, is the central theme of matrix probing methods. While the family of matrix probing methods has grown rapidly in recent years, they all share the common concept of estimating specific matrix properties, for instance, the trace or large eigenvalues, through the multiplication of random vectors with the matrix. In many cases, the matrixvector product can be computed efficiently, without having to compute the matrix explicitly. An extensive summary of matrix probing methods can be found in Halko et al. (2011). Applications to resolution analysis in inverse problems have been presented, for example, by An (2012), Trampert \& Fichtner (2013) and Fichtner \& van Leeuwen (2015).

To illustrate the concept, our focus will be on a specific radom probing method, known as Hutchinson's method (Hutchinson, 1990), which can be used to estimate the trace of a matrix. Since the trace of the model resolution matrix $\mathbf{R}_{M}$ has the concrete interpretation as the number of resolved model parameters, we take $\mathbf{R}_{M}$ as working example.

As first step, we generate a set of $N$ Rademacher vectors $\mathbf{a}^{n}(n=1, \ldots, N)$, that is, vectors where the entries are randomly either 1 or -1 , and uncorrelated in the sense

$$
\lim _{N \rightarrow \infty} \frac{1}{N} \sum_{n=1}^{N} a_{i}^{n} a_{j}^{n}=0 \quad \text { for } i \neq j
$$

The multiplication of $\mathbf{a}^{n}$ with $\mathbf{R}_{M}$ from the left and the right, gives

$$
\begin{aligned}
\left(\mathbf{a}^{n}\right)^{T} \mathbf{R}_{M} \mathbf{a}^{n} & =\sum_{i=1}^{N} R_{M, i i}\left(a_{i}^{n}\right)^{2}+\sum_{i=1}^{N} \sum_{j=1, j \neq i}^{N} a_{i} R_{M, i j} a_{j} \\
& =\sum_{i=1}^{N} R_{M, i i}+\sum_{\substack { i=1 \\
\begin{subarray}{c}{j=1 \\
j \neq i{ i = 1 \\
\begin{subarray} { c } { j = 1 \\
j \neq i } }\end{subarray}}^{N} a_{i} R_{M, i j} a_{j},
\end{aligned}
$$

where we used $\left(a_{i}^{n}\right)^{2}=1$ for any $i$ and $n$. Taking the average over all realisations of random vectors, we obtain

\footnotetext{
${ }^{4}$ A corolloary of this effect is the fact that our supercomputers will never be large enough to compute the resolution matrices that we are really interested in. Also, while supercomputers are often said to grow fast, they actually grow pretty slowly compared to the scaling properties of our inverse problems.
} 


$$
\frac{1}{N} \sum_{n=1}^{N}\left(\mathbf{a}^{n}\right)^{T} \mathbf{R}_{M} \mathbf{a}^{n}=\sum_{i=1}^{N} R_{M, i i}+\sum_{i=1}^{N} \sum_{\substack{j=1 \\ j \neq i}}^{N} R_{M, i j}\left[\frac{1}{N} \sum_{n=1}^{N} a_{i}^{n} a_{j}^{n}\right] .
$$

Considering equation (6.68), this condenses to

$$
\frac{1}{N} \sum_{n=1}^{N}\left(\mathbf{a}^{n}\right)^{T} \mathbf{R}_{M} \mathbf{a}^{n} \approx \sum_{i=1}^{N} R_{M, i i}=\operatorname{tr} \mathbf{R}_{M}
$$

where the $\approx$ sign results from considering only a finite number of random realisations $N$. Equation (6.71) provides a simple recipe for the estimation of $\operatorname{tr} \mathbf{R}_{M}$. We multiply $\mathbf{R}_{M}$ from both sides with a realisation of a random Rademacher vector and then average over several realisations. What makes (6.71) efficient and practical is that the computation of $\mathbf{R}_{M} \mathbf{a}^{n}$ actually does not require an explicit version of $\mathbf{R}_{M}$. In fact, we have

$$
\mathbf{R}_{M} \mathbf{a}^{n}=\mathbf{G}^{-g} \mathbf{G a}^{n}=\mathbf{G}^{-g} \mathbf{d}_{a},
$$

where $\mathbf{d}_{a}$ are the data corresponding to the random vector $\mathbf{a}^{n}$, which is here interpreted as a model with random entries of 1 or -1 . According to (6.72), the computation of $\mathbf{R}_{M} \mathbf{a}^{n}$ is equal to the solution of the linear inverse problem with synthetic data $\mathbf{d}_{a}$, which does not require explicit matrices.

The method represented by equation (6.71) can be modified to estimate the trace of a submatrix of $\mathbf{R}_{M}$. For instance, multiplying $\mathbf{R}_{M}$ from the right with random realisations of

$$
\mathbf{a}=(\underbrace{0, \ldots, 0}_{k}, a_{1}, \ldots, a_{s}, \underbrace{0, \ldots, 0}_{n-k-s})^{T},
$$

and from the left with

$$
\mathbf{a}=(\underbrace{0, \ldots, 0}_{n-k-s}, a_{1}, \ldots, a_{s}, \underbrace{0, \ldots, 0}_{k})^{T},
$$

gives an approximation of the trace of the $s \times s$ submatrix located between columns $k$ and $k+s$, and between rows $n-k-s$ and $n-k$.

The quality of an approxmation (6.71) depends on the number of random realisations $N$; and the number of realisations needed to obtain a useful approximation generally depends on properties of $\mathbf{R}_{M}$. When $\mathbf{R}_{M}$ is diagonally dominant, the cross terms on the right-hand side of (6.70) tend to vanish more quickly than in cases where $\mathbf{R}$ is a dense matrix with entries of nearly equal size.

In many tomographic inverse problems, the structure of $\mathbf{R}_{M}$ is indeed favourable. Often, the Earth model is parameterised in terms of $n_{v}$ vertical and $n_{h}$ horizontal basis functions, so that $n=n_{v} n_{h}$. The principal diagonal indicates how well the amplitude of a given parameter can be recovered. After $n_{h}$ horizontal entries along a row, the next diagonal indicates how much a parameter at a given horizontal position is correlated with the one at the same horizontal position but at the following depth index, and so on.

An example for this parameterisation is the global seismic shear velocity model S40RTS by Ritsema et al. (2011), built laterally from spherical harmonics up to degree 40 (1681 coefficients) and vertically from 21 cubic splines. As illustrated in figure $6.16 \mathrm{~b}$, the resolution matrix of S40RTS has dominant entries along the main diagonal, showing a progressive decay with increasing spherical harmonic degree. For any specific row of the resolution matrix, the off-diagonal terms are comparatively small, except for the same horizontal parameter at different depth indices. Figure $6.16 \mathrm{~b}$ shows trace estimates of the main diagonal and all the minor diagonals (the diagonals shifted by a certain index from the main diagonal) for $N=1$, that is, the extreme case where only a single realisation of random vectors was used. For index 1 (main diagonal), the random probing estimate of the trace is 8009 . The exact value, which we can compute because $\mathbf{R}_{M}$ is actually small enough to be computed explicitly, is 8007 . This illustrates that one random vector may be sufficient the matrix is strongly diagonally dominant. The correlation at position 2 corresponds to the trace of the next diagonal, etc. The next significant diagonal is at position 1682, meaning that there is a correlation with the following depth layer. Eventually, 
the modulo-1681 diagonals disappear in the background noise, which may be taken as a rough estimate of the accuracy of the method.

a) Shear wavespeed variations in S40RTS
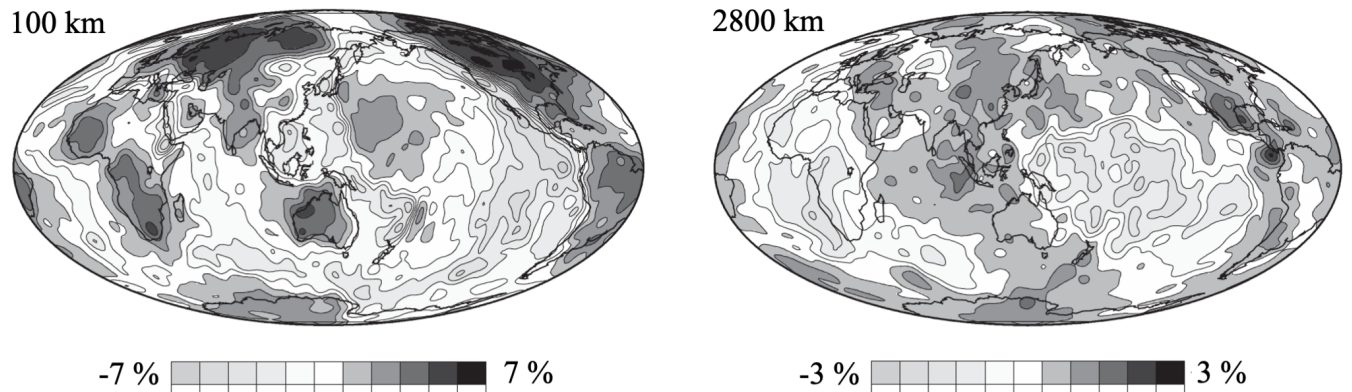

b) Diagonal ( $\rightarrow$ and row 1683 ( $)$ of $\mathbf{R}_{M}$

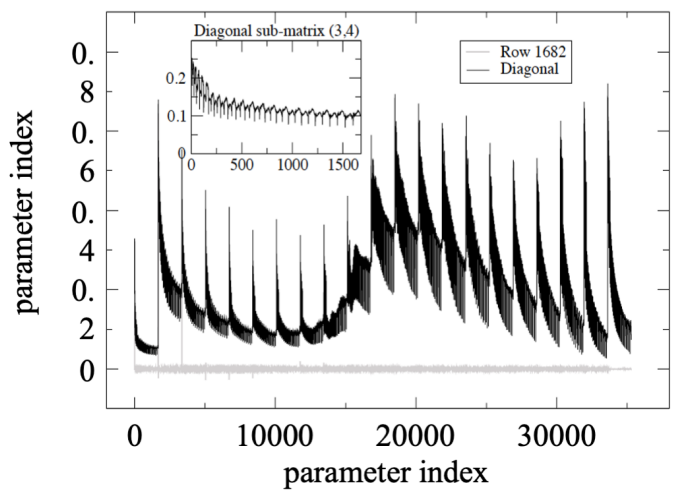

c) Estimated traces of submatrices

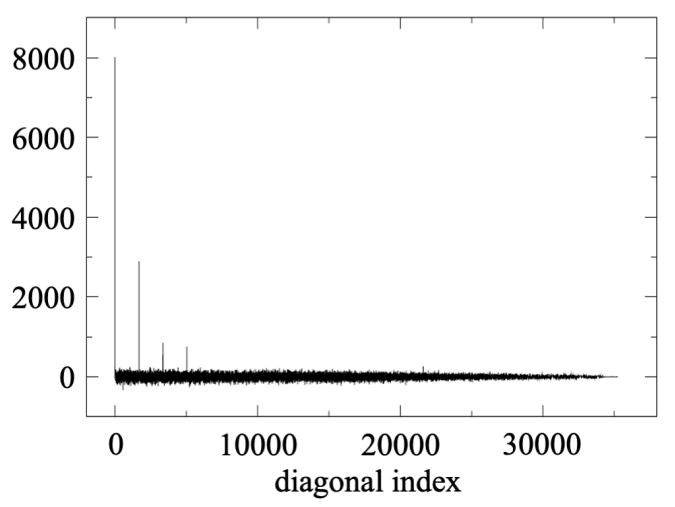

Fig. 6.16: Random probing of the resolution matrix of the global shear velocity model S40RTS (Ritsema et al., 2011). (a) Relative lateral shear velocity variations at $100 \mathrm{~km}$ and at $2800 \mathrm{~km}$ depth. (b) Diagonal (black) and row number 1682 of the resolution matrix $\mathbf{R}_{M}$. (c) Random probing estimates of the traces of the main diagonal and all minor diagonals of $\mathbf{R}_{M}$.

\subsection{The geometry of least-squares}

A very different but also very insightful perspective of the linear least-squares method was proposed by Tarantola \& Valette (1982) who considered a joint data-model space, $\mathbb{X}=\mathbb{D} \times \mathbb{M}$, consisting of $(N+n)$-dimensional vectors

$$
\mathbf{x}=\left[\begin{array}{c}
\mathbf{d} \\
\mathbf{m}
\end{array}\right] \text {. }
$$

Rewriting the linear forward problem as $\mathbf{d}-\mathbf{G m}=\mathbf{0}$, the forward problem in the joint data-model space can be expressed as

$$
\mathbf{F x}=\left[\begin{array}{ll}
\mathbf{I} & -\mathbf{G}
\end{array}\right]\left[\begin{array}{c}
\mathbf{d} \\
\mathbf{m}
\end{array}\right]=\mathbf{0},
$$

where $\mathbf{F}=\left[\begin{array}{ll}\mathbf{I} & -\mathbf{G}\end{array}\right]$ is a matrix of dimension $N \times(N+n)$. Using this notation, and defining 


$$
\mathbf{x}_{0}=\left[\begin{array}{c}
\mathbf{d}^{\text {obs }} \\
\mathbf{m}^{\text {prior }}
\end{array}\right], \quad \mathbf{C}_{0}=\left[\begin{array}{cc}
\mathbf{C}_{D} & \mathbf{0} \\
\mathbf{0} & \mathbf{C}_{M}
\end{array}\right],
$$

the least-squares misfit of equation (6.2) takes the form

$$
\chi(\mathbf{x})=\frac{1}{2}\left(\mathbf{x}-\mathbf{x}_{0}\right)^{T} \mathbf{C}_{0}^{-1}\left(\mathbf{x}-\mathbf{x}_{0}\right), \quad \text { subject to } \quad \mathbf{F x}=\mathbf{0} .
$$

We can find the maximum-likelihood solution $\tilde{\mathbf{x}}$ of (6.78) using the Lagrange multiplier method (see appendix 15.1). For this, we minimise the auxiliary misfit function

$$
\chi_{\lambda}(\mathbf{x})=\frac{1}{2}\left(\mathbf{x}-\mathbf{x}_{0}\right)^{T} \mathbf{C}_{0}^{-1}\left(\mathbf{x}-\mathbf{x}_{0}\right)-\lambda^{T} \mathbf{F} \mathbf{x}
$$

where $\lambda$ is a vector-valued Lagrange multiplier. Forcing the gradient of $\chi_{\lambda}$ to zero, we obtain

$$
\mathbf{0}=\frac{1}{2}\left(\tilde{\mathbf{x}}-\mathbf{x}_{0}\right)^{T} \mathbf{C}_{0}^{-1}-\lambda^{T} \mathbf{F}
$$

Transposing and rearranging equation (6.80), gives

$$
\mathbf{C}_{0} \mathbf{F}^{T} \lambda=\tilde{\mathbf{x}}-\mathbf{x}_{0}
$$

Left-multiplying equation (6.81) by $\mathbf{F}$ and assuming that $\mathbf{F C}_{0} \mathbf{F}^{T}$ is invertible, then yields an explicit expression for the Lagrange multiplier,

$$
\lambda=\left(\mathbf{F} \mathbf{C}_{0} \mathbf{F}^{T}\right)^{-1} \mathbf{F}\left(\tilde{\mathbf{x}}-\mathbf{x}_{0}\right) .
$$

Realising that $\mathbf{F} \tilde{\mathbf{x}}=\mathbf{0}$ and resubstituting $\lambda$ back into equation (6.81), finally gives an explicit solution for the maximum-likelihood vector $\tilde{\mathbf{x}}$,

$$
\tilde{\mathbf{x}}=\mathbf{x}_{0}-\mathbf{C}_{0} \mathbf{F}^{T}\left(\mathbf{F C}_{0} \mathbf{F}^{T}\right)^{-1} \mathbf{F x}_{0}
$$

Equation (6.83) yields, of course, exactly the same maximum-likelihood model $\tilde{\mathbf{m}}$ as equation (6.13). The maximum-likelihood data are $\tilde{\mathbf{d}}=\mathbf{G} \tilde{\mathbf{m}}$, as one may easily show with the help of equation (6.17).

\subsubsection{Projections}

Before we discover the real beauty of equation (6.83) and its consequences, we make a small detour. For notational convenience, we define two operators,

$$
\mathbf{Q}=\mathbf{C}_{0} \mathbf{F}^{T}\left(\mathbf{F C}_{0} \mathbf{F}^{T}\right)^{-1} \mathbf{F}
$$

and

$$
\mathbf{P}=\mathbf{I}-\mathbf{Q}
$$

The definition of $\mathbf{Q}$ directly implies

$$
\mathbf{Q Q}=\mathbf{C}_{0} \mathbf{F}^{T}\left(\mathbf{F C}_{0} \mathbf{F}^{T}\right)^{-1}\left(\mathbf{F C}_{0} \mathbf{F}^{T}\right)\left(\mathbf{F C}_{0} \mathbf{F}^{T}\right)^{-1} \mathbf{F}=\mathbf{Q}
$$

Equation (6.86) implies that the matrix $\mathbf{Q}$ represents a projection ${ }^{5}$, meaning that any subsequent applications of $\mathbf{Q}$ to some $\mathbf{Q x}$ have no effect; $\mathbf{Q}^{n} \mathbf{Q x}=\mathbf{Q x}$. Using (6.86), we find

$$
\mathbf{P P}=(\mathbf{I}-\mathbf{Q})(\mathbf{I}-\mathbf{Q})=\mathbf{I}-2 \mathbf{Q}+\mathbf{Q Q}=\mathbf{I}-\mathbf{Q}=\mathbf{P} .
$$

\footnotetext{
${ }^{5}$ In fact, any matrix $\mathbf{A}$ that satisfies $\mathbf{A A}=\mathbf{A}$ is by definition a projection.
} 
Thus, $\mathbf{P}$ is a projection as well. Furthermore, we see directly from equation (6.84) that

$$
\mathbf{Q C}_{0}=\mathbf{C}_{0} \mathbf{F}^{T}\left(\mathbf{F C}_{0} \mathbf{F}^{T}\right)^{-1} \mathbf{F C}_{0}=\mathbf{C}_{0}\left[\mathbf{C}_{0} \mathbf{F}^{T}\left(\mathbf{F C}_{0} \mathbf{F}^{T}\right)^{-1} \mathbf{F}\right]^{T}=\mathbf{C}_{0} \mathbf{Q}^{T},
$$

which then implies

$$
\mathbf{P C}_{0}=\mathbf{C}_{0}-\mathbf{Q C}_{0}=\mathbf{C}_{0}-\mathbf{C}_{0} \mathbf{Q}^{T}=\mathbf{C}_{0} \mathbf{P}^{T}
$$

With these beautifully symmetric identities in mind, we return to our original problem of understanding the deeper meaning of equation (6.83).

\subsubsection{Posterior $=$ projection $($ prior $)$}

From the definition of the projector $\mathbf{P}$ in equation (6.85), it follows immediately that the least-squares solution from (6.83) can be written as

$$
\tilde{\mathbf{x}}=\mathbf{P} \mathbf{x}_{0} \text {. }
$$

The posterior is the projection of the prior onto a typically lower-dimensional subspace of the joint data-model space $\mathbb{X}$. This relation between prior and posterior is schematically illustrated in figure 6.17

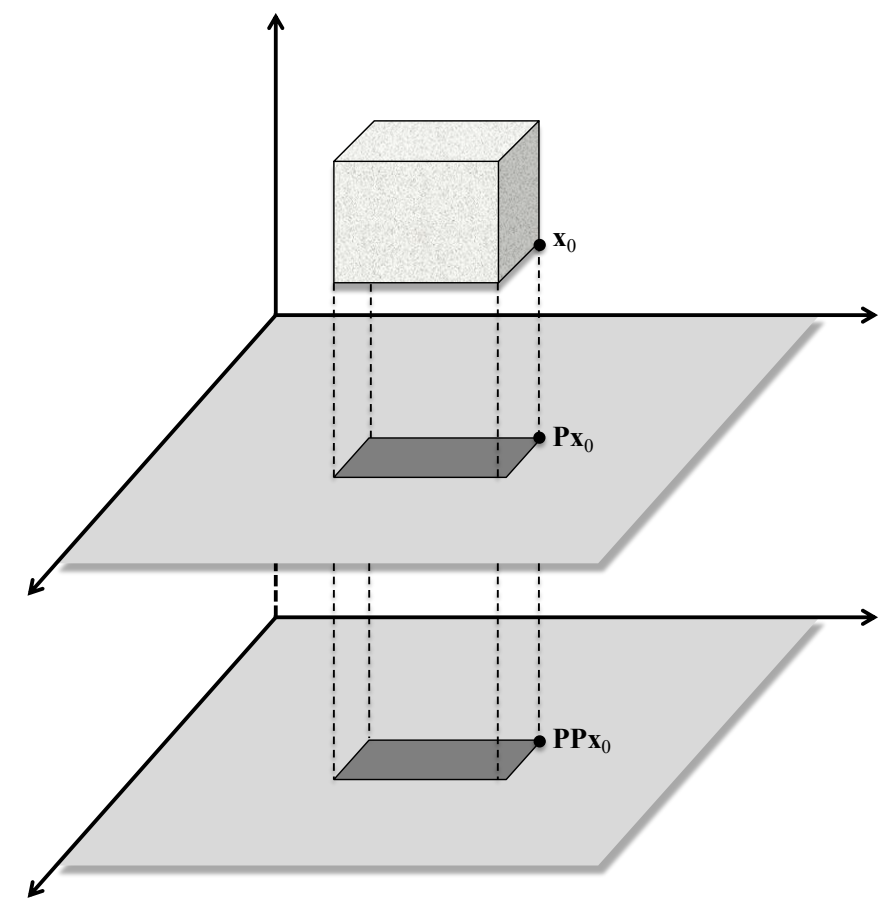

Fig. 6.17: The posterior $\tilde{\mathbf{x}}$ is the projection $\mathbf{P} \mathbf{x}_{0}$ of the prior $\mathbf{x}_{0}$. In optical terms, the posterior is a lowerdimensional shadow of the prior in the joint data-model space.

One of the implications of (6.90) is that the use of any image of $\mathbf{P}$ as target model in a synthetic inversion is a trivial task. In other words, if we were trying to reconstruct some $\tilde{\mathbf{x}}$ to demonstrate the performance of our least-squares inversion, we would of course obtain a perfect result, because $\mathbf{P} \tilde{\mathbf{x}}=\tilde{\mathbf{x}}$. This illustrates the limited meaning of synthetic inversions as an indicator of resolution. Willingly or accidentally choosing an image of $\mathbf{P}$ 
always produces perfect results.

Using equation (6.90), we also find that the difference between $\mathbf{x}_{0}$ and $\tilde{\mathbf{x}}$ is in the imaging nullspace:

$$
\mathbf{P} \Delta \mathbf{x}=\mathbf{P}\left(\tilde{\mathbf{x}}-\mathbf{x}_{0}\right)=\mathbf{P P} \mathbf{x}_{0}-\mathbf{P} \mathbf{x}_{0}=\mathbf{0} .
$$

In other words, $\Delta \mathbf{x}$ is exactly non-reconstructable. Finally, we use equation (6.90) to derive an expression for the posterior covariance $\tilde{\mathbf{C}}$. Expressing $\tilde{\mathbf{C}}$ in terms of expectations $\mu$, we find

$$
\begin{aligned}
\tilde{\mathbf{C}} & =\mu\left([\tilde{\mathbf{x}}-\mu(\tilde{\mathbf{x}})][\tilde{\mathbf{x}}-\mu(\tilde{\mathbf{x}})]^{T}\right) \\
& =\mu\left(\left[\mathbf{P} \mathbf{x}_{0}-\mu\left(\mathbf{P} \mathbf{x}_{0}\right)\right]\left[\mathbf{P} \mathbf{x}_{0}-\mu\left(\mathbf{P} \mathbf{x}_{0}\right)\right]^{T}\right) \\
& =\mathbf{P} \mu\left(\left[\mathbf{x}_{0}-\mu\left(\mathbf{x}_{0}\right)\right]\left[\mathbf{x}_{0}-\mu\left(\mathbf{x}_{0}\right)\right]^{T} \mathbf{P}^{T}\right) \\
& =\mathbf{P C}_{0} \mathbf{P}^{T}
\end{aligned}
$$

Using equation (6.89) and the fact that $\mathbf{P}$ is a projection, finally gives the complementary equation to (6.90),

$$
\tilde{\mathbf{C}}=\mathbf{P C}_{0}
$$

An interesting implication of equation (6.93) is that using $\tilde{\mathbf{C}}$ as new prior to potentially improve the solution (reduce its uncertainty) has actually no effect. In other words, there is no point in iterating a linear least-squares problem unless new independent data are added. 


\section{Chapter 7 \\ Backus-Gilbert theory}

An Earth model is a mathematical abstraction which suffices for some geophysical discussion.

It follows that the inverse problem for a given finite collection of [data] consists in describing the infinite-dimensional manifold of all acceptable Earth models.

George E. Backus and J. Freeman Gilbert, The resolving power of gross Earth data, 1968.

Throughout the previous chapters, and also in most of the following ones, the model space $\mathbb{M}$ is finitedimensional. In some cases, the number of parameters that we wish to infer may indeed be finite, for example, when we try to constrain the position of some object in 3-D space or the timing of some event. Quite often, however, we enforce the finite dimensionality of $\mathbb{M}$ artificially via the discretisation of a model parameter $\mathfrak{m}(\mathbf{x})$ that is actually a continuous function of position $\mathbf{x}$. As illustrated by equation (1.1), $\mathfrak{m}(\mathbf{x})$ may be discretised through an approximate expansion in terms of a finite number of basis functions. This comes with the distinct advantage of being able to work with vectors and matrices, instead of having to unfold the somewhat cumbersome machinery of functional analysis. The price to pay is that our inferences are flawed, or at least affected, by the entirely subjective choice of basis functions.

Despite the fact that the vast majority of inverse problems that involve distributed model parameters is solved by discretisation, the study of continuous inverse problems can provide deep insight into the nature of scientific inference that is independent of some subjective discretisation. One of the most profound contributions in this context was made by George Edward Backus and James Freeman Gilbert in a series of monumental papers that are often considered the foundation of geophysical inverse theory (Backus \& Gilbert, 1967, 1968, 1970). From today's perspective, the relevance of their work goes far beyond geophysics, and so we will present its most important elements in the following sections.

\subsection{The resolving power of perfect data}

Backus-Gilbert theory rests on the notion of linear data functionals that link a synthetic datum $d_{i}$ to the continuously distributed model $\mathfrak{m}(\mathbf{x})$ via a data kernel $G_{i}(\mathbf{x})$, that would in today's language mostly be called a sensitivity kernel,

$$
d_{i}=\int_{V} G_{i}(\mathbf{x}) \mathfrak{m}(\mathbf{x}) d \mathbf{x} .
$$


The integral is taken over the volume $V$ where $\mathfrak{m}(\mathbf{x})$ is defined. Backus and Gilbert, working in a geophysical context, referred to (7.1) as a gross Earth functional. Though (7.1) may initially seem abstract and unmotivated, it covers a broad range of applications. Newton's law of gravitation, for example, states that the gravitational acceleration at some point $\mathbf{x}^{\prime}$ in space is proportional to an integral over a mass density distribution $\rho(\mathbf{x})$ times $\left|\mathbf{x}-\mathbf{x}^{\prime}\right|^{-2}$. The same mathematical form, though with symbols of different physical meaning, can be found in Coulomb's law describing the forces between charged particles, and in the Biot-Savart equation that relates an electrical current density to the resulting magnetic field. Also the forward problem in the linear traveltime tomography, considered in section 6.2, is a special case of (7.1), where the transit time of a wave is given by an integral over slowness along the ray path. Again, mathematically identical forms describe many other physical phenomena, for instance, the attenuation of light or X-ray beams, captured by the Beer-Lambert law.

To begin simple, we limit ourselves to the one-dimensional case, and we consider observed data $d_{i}^{\text {obs }}$ that are perfect in the sense of being error-free and perfectly explained by some model $\hat{\mathfrak{m}}$, that is,

$$
d_{i}^{\mathrm{obs}}=\int_{V} G_{i}(z) \hat{\mathfrak{m}}(z) d z
$$

Based on (7.2), the probably most straightforward and also most profound realisation of Backus and Gilbert was that we observe spatial averages of the model parameters $\hat{\mathfrak{m}}(z)$, and therefore, only average properties can be inferred from observations. A generic average over $\hat{\mathfrak{m}}(z)$ may be written in the form of an integral,

$$
\langle A, \hat{\mathfrak{m}}\rangle=\int_{V} A(z) \hat{\mathfrak{m}}(z) d z
$$

where $A(z)$ is an averaging kernel. To ensure that the averages are intuitively interpretable, we require $A(z)$ to be normalised, so that

$$
1=\int_{V} A(z) d z
$$

Ideally, we would like the averaging kernel to be as concentrated as possible. In fact, if we could construct $A(z)$ to equal to the $\delta$-function $\delta\left(z-z_{0}\right)$, the average in (7.3) would simplify to $\langle A, \hat{\mathfrak{m}}\rangle=\mathfrak{m}\left(z_{0}\right)$, meaning that we could infer $\mathfrak{m}$ at a specific point without averaging-induced blurring. In practice, this ideal scenario is unlikely to be realised, but we may still hope to construct an averaging kernel that is somewhat close to the $\delta$-function $\delta\left(z-z_{0}\right)$.

To obtain a constructive method, we try to find $A(z)$ in the form of a linear combination of the data kernels,

$$
A(z)=\sum_{i=1}^{N} a_{i} G_{i}(z) .
$$

Inserting (7.5) into (7.3) shows that the average of some acceptable model $\hat{\mathfrak{m}}$ can be computed easily from the observed data,

$$
\langle A, \hat{\mathfrak{m}}\rangle=\sum_{i=1}^{N} a_{i} d_{i}^{\mathrm{obs}}=\mathbf{a}^{T} \mathbf{d}^{\mathrm{obs}} .
$$

The seemingly harmless equation (7.6) has an important implication: While the left-hand side depends on one of potentially many acceptable model $\hat{\mathfrak{m}}$, the right-hand side does not. Hence, for some chosen coefficient vector $\mathbf{a}=\left(a_{1}, \ldots, a_{N}\right)^{T}$, the average on the right-hand side is actually unique. Hence, it does not matter which of the acceptable models we choose to average, the result is always the same. Though there is not a single acceptable model, the spatial average over acceptable Earth models is actually unique.

It remains to determine coefficients $a_{i}$ such that the averaging kernel $A(z)$ is close to $\delta\left(z-z_{0}\right)$ for a fixed position $z_{0}$. This requires a measure of deltaness, or the extent to which $A(z)$ is localised around $z_{0}$. While numerous deltaness criteria $s\left(A, z_{0}\right)$ may be found, we adopt the pragmatic choice of Backus \& Gilbert (1970), 


$$
s\left(A, z_{0}\right)=12 \int_{V}\left(z-z_{0}\right)^{2} A(z)^{2} d z .
$$

The quantity $s\left(A, z_{0}\right)$ has the physical unit metres, and indeed measures the width of $A(z)$ around $z_{0}$. The more $A(z)$ is concentrated around $z_{0}$, the smaller $s\left(A, z_{0}\right)$ will be. Conversely, when $A(z)$ is widely dispersed, $s\left(A, z_{0}\right)$ will take large values. The factor 12 in (7.7) is motivated by the special case $A(z)=\ell^{-1}$ within the interval $\left[z_{0}-\ell / 2, z_{0}+\ell / 2\right]$, which leads to $s\left(A, z_{0}\right)=\ell$. Hence, in this sense, $s\left(A, z_{0}\right)$ as defined in (7.7) can be interpreted as the averaging or resolution length.

To minimise $s\left(A, z_{0}\right)$ subject to the normalisation constraint (7.4), we insert (7.5) into (7.7) and force all partial derivatives $\partial / \partial a_{i}$ of the Lagrange function (appendix 15.1)

$$
L(\mathbf{a}, \lambda)=12 \int_{V}\left(z-z_{0}\right)^{2}\left[\sum_{i=1}^{N} a_{i} G_{i}(z)\right]^{2} d z-\lambda\left[1-\sum_{i=1}^{N} a_{i} \int_{V} G_{i}(z) d z\right]
$$

to zero. Defining, in the interest of a simplified notation, the vector $\mathbf{u}$ with components

$$
u_{i}=\int_{V} G_{i}(z) d z
$$

and the symmetric matrix $\mathbf{S}$ with components

$$
S_{i k}=24 \int_{V}\left(z-z_{0}\right)^{2} G_{i}(z) G_{k}(z) d z,
$$

gives the coefficient vector a as the solution of a linear system of equations

$$
\mathbf{S a}=\lambda \mathbf{u}, \quad \lambda=-\mathbf{a}^{T} \mathbf{S a}
$$

The system (7.11) can be solved by first inverting the vector-matrix equation for $\mathbf{a}^{\prime}=\mathbf{a} / \lambda$ and then determining the Lagrange multiplier $\lambda$ from the scaling relation $\lambda=\mathbf{a}^{T} \mathbf{S a}$.

\subsection{Model problem: Love waves in layered media}

To fill these abstract developments with life, we consider the specific case of Love ${ }^{1}$ waves propagating in an isotropic elastic medium. Schematically illustrated in figure 7.1, Love waves are a special kind of surface waves, meaning that they propagate along the surface of a solid medium, with an amplitude that decays rapidly with increasing depth. The polarisation direction of Love waves is horizontal and perpendicular to the propagation direction. Hence, they are transverse waves, just as electromagnetic waves. A detailed mathematical treatment of Love waves can be found in Appendix 16.2.

In the interest of simplicity, we consider Love waves propagating through a medium that is composed of a homogeneous layer in the depth interval $H \geq z \geq 0$, that is located above a half-space at $z \leq 0$. The layer and the half-space are characterised by their mass density $\rho$ and shear modulus $\mu$. The latter controls the deformation of the medium when it is subjected to shear forces.

The phase velocity $c(f)$, that is, the propagation speed of a monochromatic Love wave, depends on the oscillation frequency $f$. Furthermore, for a given frequency, more than one Love wave solution may exist. The

${ }^{1}$ Augustus Edward Hough Love (1863 - 1940) was an English mathematician and physicist, best known for his work on elasticity and wave propagation. For the mathematical analysis of the surface waves, named after him, he received the Adams Prize in 1911. 


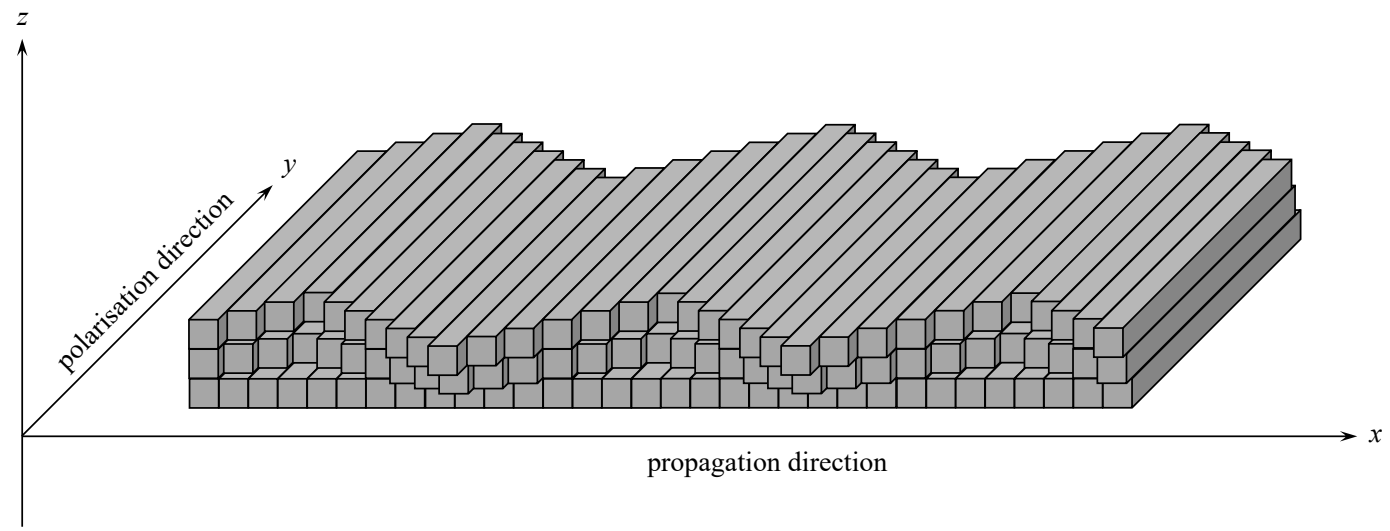

Fig. 7.1: Schematic illustration of a Love wave with polarisation in $y$-direction and propagation in $x$-direction. Being a surface wave, the amplitude of a Love wave decays rapidly with increasing depth, that is, with decreasing $z$.

solution with the lowest phase velocity, $c_{0}(f)$, is referred to as the fundamental mode, whereas all other solutions with phase velocities $c_{i>0}(f)>c_{0}(f)$ are termed overtones or higher modes. While the fundamental mode always exists, the higher modes only appear from a certain threshold frequency upwards.

Figure 7.2 illustrates the frequency dependence of Love wave modes, known as dispersion curve, for the concrete case of a layer with a thickness of $H=10^{\prime} 000 \mathrm{~m}$, that may, for example, simulate the crust of the Earth. Its mass density and shear modulus are $\rho=2700 \mathrm{~kg} / \mathrm{m}^{3}$ and $\mu=10.8 \cdot 10^{9} \mathrm{~N} / \mathrm{m}^{2}$. The underlying half-space is characterised by $\rho=3100 \mathrm{~kg} / \mathrm{m}^{3}$ and $\mu=27.9 \cdot 10^{9} \mathrm{~N} / \mathrm{m}^{2}$.

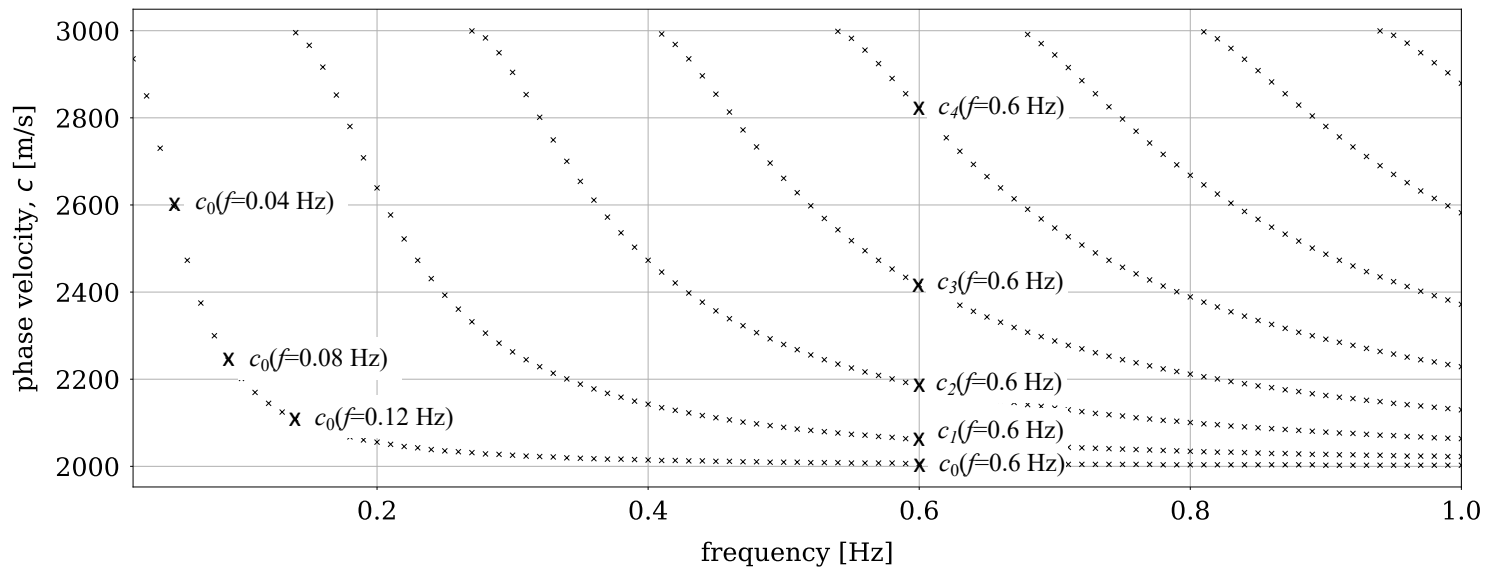

Fig. 7.2: Love wave phase velocity dispersion curves for a homogeneous layer $\left(H=10^{\prime} 000 \mathrm{~m}, \rho=2700 \mathrm{~kg} / \mathrm{m}^{3}\right.$ and $\left.\mu=10.8 \cdot 10^{9} \mathrm{~N} / \mathrm{m}^{2}\right)$ located above a homogeneous half space $\left(\rho=3100 \mathrm{~kg} / \mathrm{m}^{3}\right.$ and $\left.\mu=27.9 \cdot 10^{9} \mathrm{~N} / \mathrm{m}^{2}\right)$. Each of the little crosses marks the phase velocity $c$ of a Love wave mode at a given frequency $f$. The larger crosses indicate the modes for which data kernels are displayed in figure 7.3. [Backus-Gilbert]

The phase velocities of actual Love wave modes, $c_{i}^{\text {obs }}(f)$, can be measured from their traveltime between two sensors, and they can be compared to phase velocities $c_{i}(f)$ computed for a medium with density $\rho(z)$ and 
shear modulus $\mu(z)$. The relative phase velocity differences,

$$
\frac{\delta c_{i}^{\mathrm{obs}}(f)}{c_{i}^{\mathrm{obs}}(f)}=\frac{c_{i}^{\mathrm{obs}}(f)-c_{i}(f)}{c_{i}^{\mathrm{obs}}(f)},
$$

are the components of the observed data vector $\mathbf{d}^{\text {obs }}$. In most realistic scenarios, $\mathbf{d}^{\text {obs }}$ will not be equal to $\mathbf{0}$, meaning that the elastic medium with properties $\rho(z)$ and $\mu(z)$ cannot reproduce the observed phase velocities exactly. For this, $\rho(z)$ and $\mu(z)$ need to be perturbed by some $\delta \rho(z)$ and $\delta \mu(z)$, respectively. Hence, the question arises how well these necessary perturbations can be resolved, given the data $\mathbf{d}^{\mathrm{obs}}$.

As demonstrated in appendix 16.2 , a datum $d_{j}^{\text {obs }}$ is related to small shear modulus perturbations $\delta \mu(z)$ via the linear equation

$$
d_{j}^{\mathrm{obs}}=\int_{-\infty}^{H} K_{j}(z) \delta \mu(z) d z,
$$

with a sensitivity kernel $K_{j}(z)$ that can be computed from the depth-dependent amplitude of the Love wave mode with label $j$. This label represents a specific frequency $f$ and mode number $i \geq 0$. A similar relation can be derived for density variations $\delta \rho(z)$, which we will, however, disregard for the moment. Figure 7.3 shows the sensitivity kernels for the Love wave modes marked by large crosses in figure 7.2.

In the context of Backus-Gilbert theory, the sensitivity kernels play the role of data kernels, as introduced in equation (7.2). Hence, they can be used to construct optimally $\delta$-like averaging kernels via the solution of the linear system (7.11). A small collection of averaging kernels involving the fundamental and first three higher modes at frequencies $f=0.1,0.2, \ldots, 1.0 \mathrm{~Hz}$ is presented in figure 7.4a. It shows that the averaging length scales $s$ are on the order of a few kilometres, the exception being the much narrower averaging kernel that is localised near the interface between the upper layer and the underlying half space. The importance of including higher-mode surface wave observations is highlighted in figure 7.4b, where the higher modes are left out. As a consequence, the averaging kernels broaden significantly, especially within the layer, where their displacement amplitudes are largest.

A comparison between the leftmost panels in figures figure 7.4a,b also sheds some light onto the functioning of the deltaness criterion (7.7). Though the kernel around $z_{0}=2500 \mathrm{~m}$ visually appears more concentrated when higher-mode observations are included, the exclusion of higher modes seems to actually reduce the averaging length from $s=3470 \mathrm{~m}$ to $s=2729 \mathrm{~m}$. The reason for this discrepancy between visual perception and plain numbers is the term $\left(z-z_{0}\right)^{2}$ in the deltaness criterion (7.7), which tends to give significant importance to small side lobes even when these are at large distance from $z_{0}$. As discussed in section 7.4, many other deltaness criteria may be designed. However, assigning less importance to distant side lobes will unavoidably produce more distant side lobes in the averaging kernels.

\subsection{Inferences from inaccurate data}

In any realistic application, data $d_{i}^{\text {obs }}$ are not perfect but contaminated by some error $\Delta d_{i}^{k}$, which can be interpreted as the $k^{\text {th }}$ realisation of a random variable. Given some averaging kernel $A(z)$, represented by a coefficient vector $\mathbf{a}$, these observational errors translate into an error of the average, given by

$$
\Delta\langle A, \hat{\mathfrak{m}}\rangle^{k}=\sum_{i=1}^{N} a_{i} \Delta d_{i}^{k}
$$

Though we do not know the individual errors $\Delta d_{i}^{k}$, we may have an estimate of their statistical properties from a series of $N_{s}$ repeated experiments. Assuming that the errors have zero mean, when averaged over the $N_{s}$ 

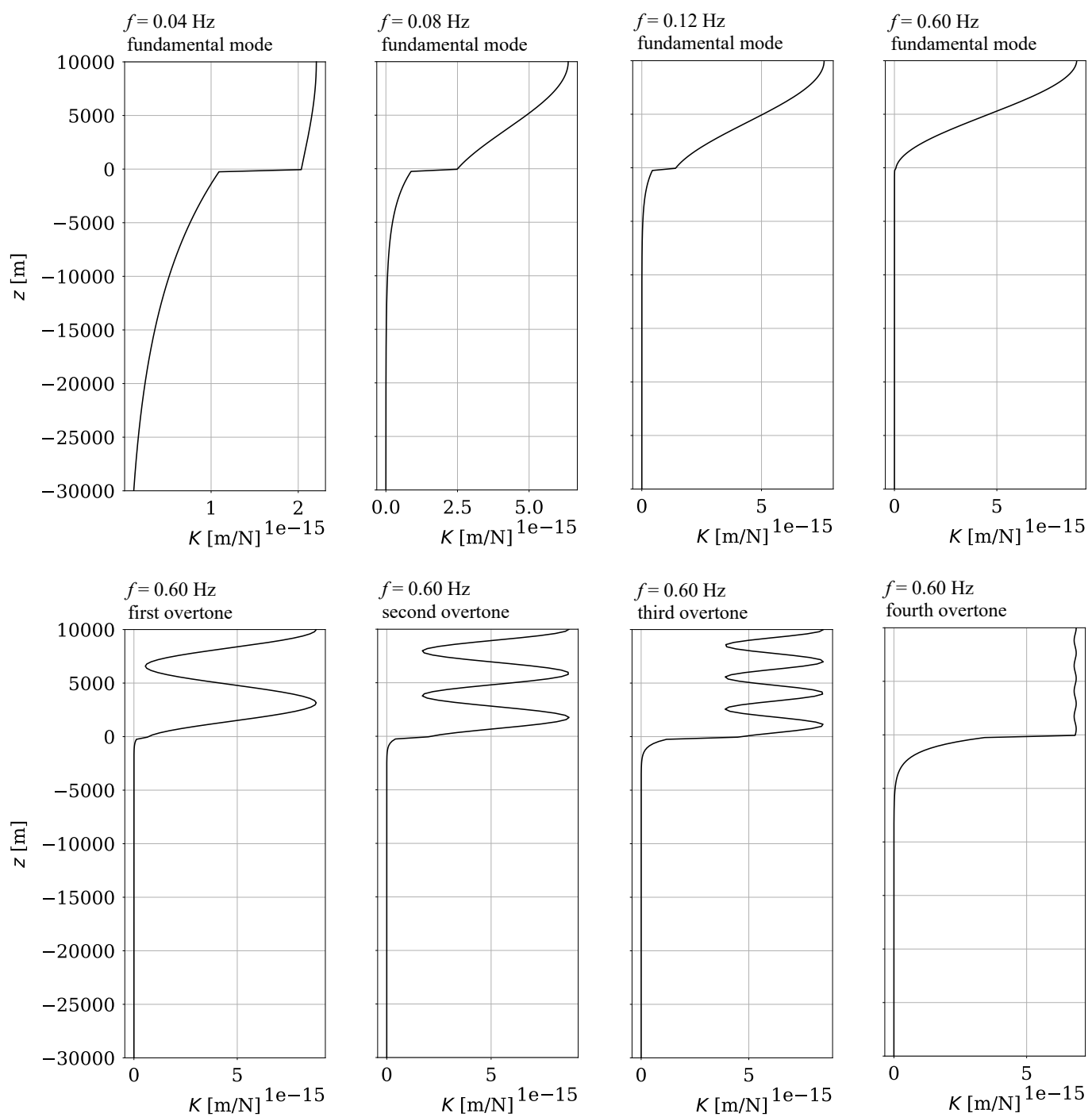

Fig. 7.3: Sensivitiy kernels $K_{j}(z)$ for the selection of Love wave modes marked by large crosses in figure 7.2. [Backus-Gilbert]

realisations, we may for example estimate the covariance matrix of the underlying distribution using equation (5.30),

$$
\left(\mathbf{C}_{D}\right)_{i j} \approx \frac{1}{N_{s}} \sum_{k=1}^{N_{s}} \Delta d_{i}^{k} \Delta d_{j}^{k} .
$$

In the interest of a simpler notation, we will replace $\approx$ by $=$ in the following paragraphs, keeping in mind that this is an approximation. On the basis of (7.15), we can derive the variance $\sigma^{2}\langle A, \hat{\mathfrak{m}}\rangle$ of the averaging error $\Delta\langle A, \hat{\mathfrak{m}}\rangle^{k}$, 
a) Averaging kernels for fundamental- and higher-mode Love waves, $f=0.1,0.2, \ldots, 1.0 \mathrm{~Hz}$
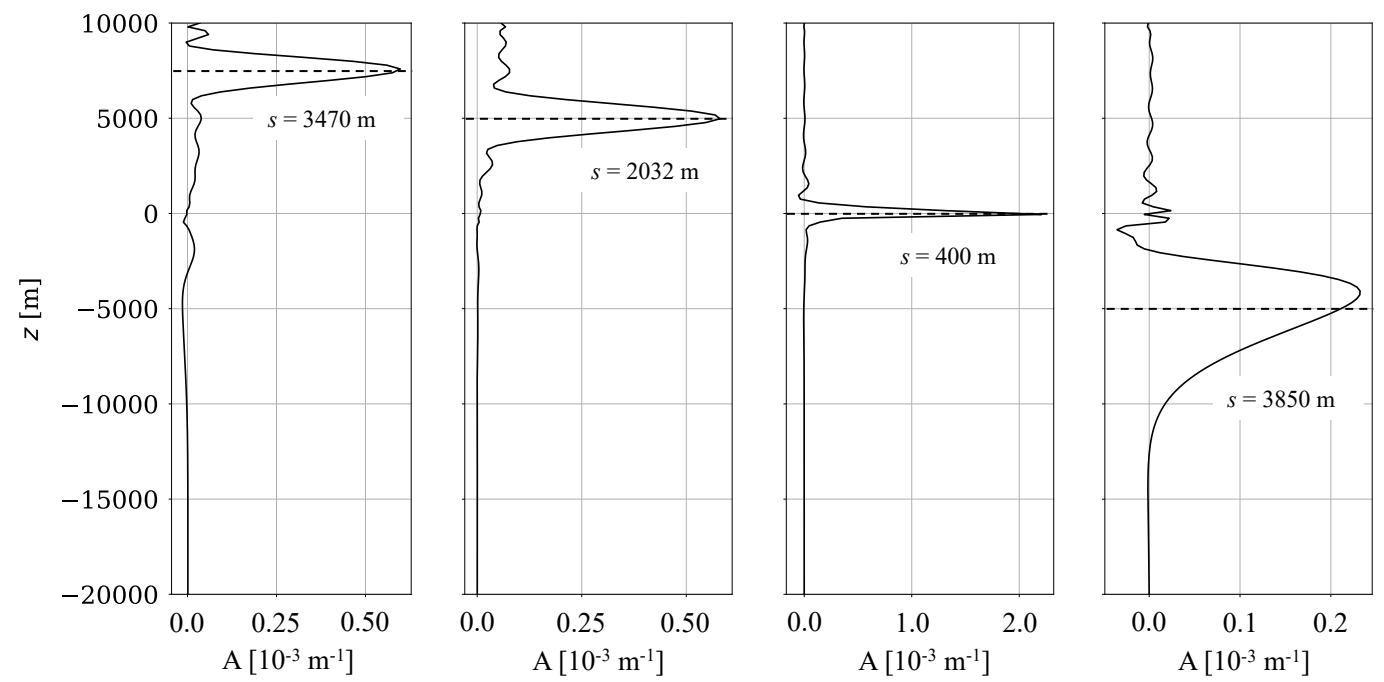

b) Averaging kernels for only fundamental-mode Love waves, $f=0.1,0.2, \ldots, 1.0 \mathrm{~Hz}$
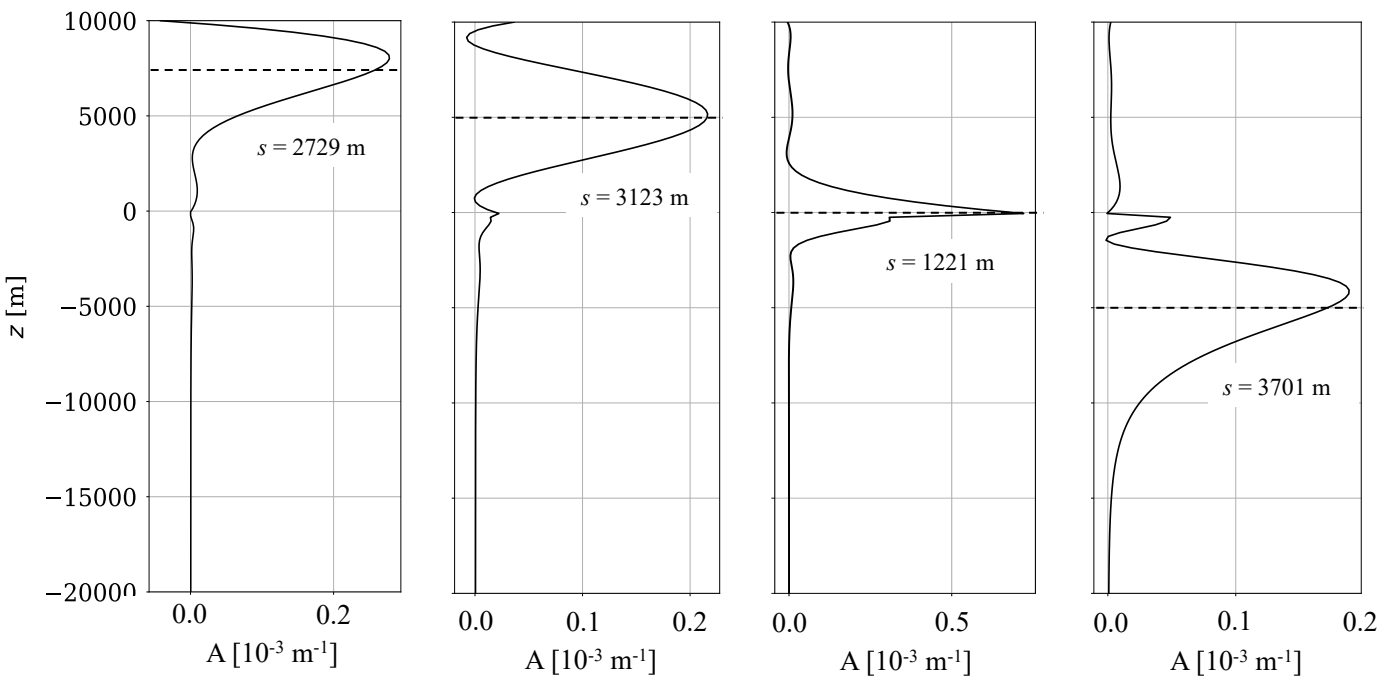

Fig. 7.4: Love wave averaging kernels for phase velocity measurements at frequencies $f=0.1,0.2, \ldots, 1.0 \mathrm{~Hz}$. The depths $z_{0}$ are marked by horizontal dashed lines. Averaging length scales $s$ are indicated in the subfigures. (a) Including fundamental modes and the first three overtones. (b) Including only the fundamental modes. [Backus-Gilbert] 


$$
\begin{gathered}
\sigma^{2}\langle A, \hat{\mathfrak{m}}\rangle=\frac{1}{N_{s}} \sum_{k=1}^{N_{s}}\left[\Delta\langle A, \hat{\mathfrak{m}}\rangle^{k}\right]^{2}=\frac{1}{N_{s}} \sum_{k=1}^{N_{s}}\left[\sum_{i, j=1}^{N} a_{i} a_{j} \Delta d_{i}^{k} \Delta d_{j}^{k}\right]=\sum_{i, j=1}^{N} a_{i} a_{j} \frac{1}{N_{s}} \sum_{k=1}^{N_{s}} \Delta d_{i}^{k} \Delta d_{j}^{k} \\
\sum_{i, j=1}^{N} a_{i}\left(\mathbf{C}_{D}\right)_{i j} a_{j}=\mathbf{a}^{T} \mathbf{C}_{D} \mathbf{a} .
\end{gathered}
$$

Its square root, the standard deviation $\sigma\langle A, \hat{\mathfrak{m}}\rangle=\sqrt{\sigma^{2}\langle A, \hat{\mathfrak{m}}\rangle}$, serves as an uncertainty measure of the averages inferred from the data. The standard deviation $\sigma\langle A, \hat{\mathfrak{m}}\rangle$ and the averaging length scale $s\left(A, z_{0}\right)$ have an antagonistic relationship. Ideally, we would like to minimise both simultaneously. However, it is intuitively clear that increasing the averaging length scale will reduce the standard deviation of the averages, and vice versa. This trade-off becomes more explicit when we try to jointly minimise the weighted sum

$$
s\left(A, z_{0}\right)+\frac{\gamma}{2} \sigma^{2}\langle A, \hat{\mathfrak{m}}\rangle=\frac{1}{2} \mathbf{a}^{T} \mathbf{S a}+\frac{\gamma}{2} \mathbf{a}^{T} \mathbf{C}_{D} \mathbf{a} .
$$

Imposing the constraint (7.4) leads to a modified version of the linear system (7.11),

$$
\left(\mathbf{S}+\gamma \mathbf{C}_{D}\right) \mathbf{a}=\lambda \mathbf{u}, \quad \lambda=-\mathbf{a}^{T} \mathbf{S a} .
$$

The scalar $\gamma$ balances the extent to which preference is given to one or the other term. If the standard deviation and the averaging length scale were independent quantities, the result of the Backus-Gilbert optimisation would be independent of the choice of $\gamma$, at least to within machine precision.

Returning to the Love wave example from section 7.2, we can visualise the trade-off between the standard deviation of the inferred average and the averaging length scale. Choosing the specific case where we want to localise the average around $z_{0}=-5000 \mathrm{~m}$, and somewhat optimistically assuming that the standard deviations of all Love wave mode observations are independent and equal to $1 \mathrm{~m} / \mathrm{s}$, that is $\mathbf{C}_{D}=\mathbf{I}$, we obtain the relation between $\sigma\langle A, \hat{\mathfrak{m}}\rangle$ and $s\left(A, z_{0}\right)$ shown in figure 7.5a. As expected, increasing averaging length scales result in decreasing standard deviations of the averages. The trade-off graph has the characteristic shape of an $L$-curve, that we previously encountered in section 6.3 on regularisation in least-squares inversion. It allows us to pick a specific value for $\gamma$ that produces a desired level of certainty. Figure $7.5 \mathrm{~b}$ displays two of the broadened averaging kernels. By construction, they are less focused around the target depth, and their peaks are shifted towards the bottom of the layer at $z=0 \mathrm{~m}$, where averages can be inferred with less uncertainty.

\subsection{Concluding remarks}

In addition to providing invaluable physical insight, the Backus-Gilbert method has the outstanding advantage of producing a unique inference in the form of a parameter average for a specified standard deviation. The beauty and elegance of Backus' and Gilbert's approach continues to inspire research (e.g. Zaroli, 2019; Zaroli et al., 2017).

The undeniable advantages of the method come a the price of having to construct and solve the linear system (7.18) for each value of $z_{0}$ that is of interest, and potentially for a range of $\gamma$ values. For the large datasets that are available today and for 3-D problems, this can rapidly become computationally prohibitive. Possibly, this is one of the reasons why the Backus-Gilbert approach is not applied very often in practice.

A conceptually more profound difficulty is related to the construction of models that can be used to make predictions. The Backus-Gilbert approach delivers a collection of unique local averages around selected positions $z_{0}$ that may be spaced arbitrarily closely. It is, therefore, tempting to construct an approximation $\tilde{m}(z)$ by setting $\tilde{\mathfrak{m}}\left(z_{0}\right)$ equal to the local average around $z_{0}$. However, it is not guaranteed that this collection of averages actually explains the ensemble of all data to within their uncertainties. It is important to understand that this potential failure to explain existing or predict future data is not the failure of the Backus-Gilbert method itself, 
a) L-curve

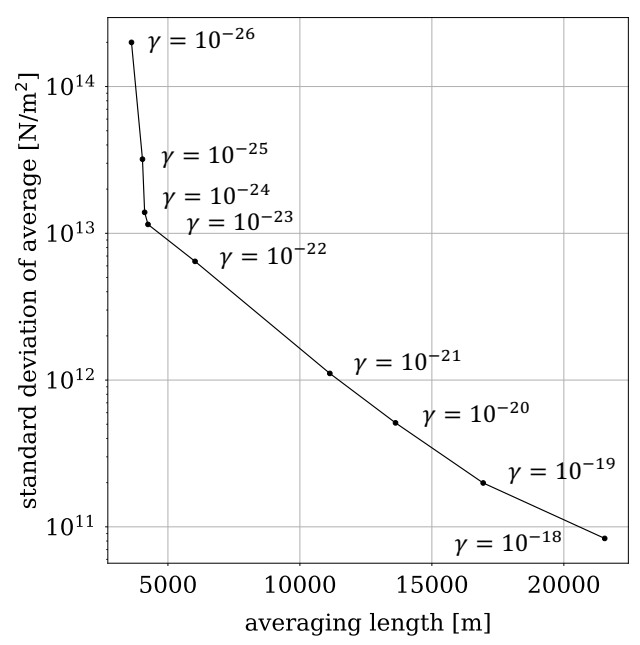

b) averaging kernels

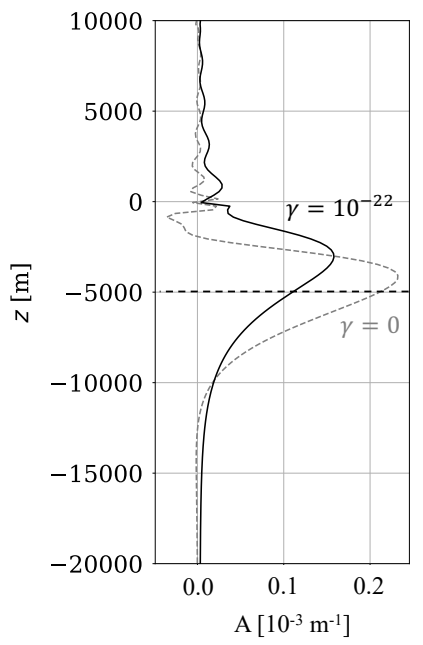

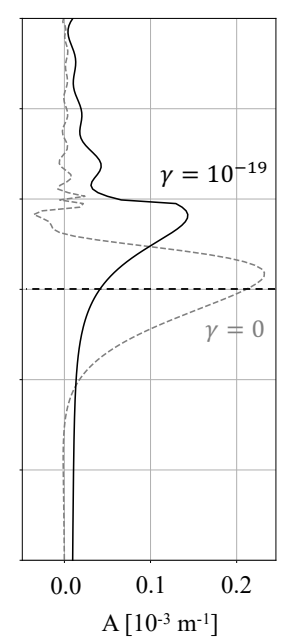

Fig. 7.5: Standard deviation vs. averaging. (a) The L-curve showing the standard deviation of the average $\sigma\langle A, \hat{\mathfrak{m}}\rangle$ as a function of the averaging length scale $s\left(A, z_{0}\right)$ for a range of weights $\gamma$. (b) Averaging kernels for $z_{0}=-5000 \mathrm{~m}$ and $\gamma$ set to $10^{-22}$ and $10^{-19}$, respectively. The averaging kernel for $\gamma=0$, which is identical to the kernel around $z_{0}=-5000 \mathrm{~m}$ in figure 7.4a, is shown as grey dashed curve. [Backus-Gilbert]

because it results from using a method for a purpose for which it has never been designed.

The deltaness criterion in equation (7.7) is meaningful and convenient. However, it is by no means the only possible option. In fact, one may define some function $J\left(z, z_{0}\right)$ that vanishes for $z=z_{0}$ and increases monotonically with increasing distance of $z$ from $z_{0}$, in order to define a generalised deltaness criterion,

$$
s_{J}\left(A, z_{0}\right)=\int_{V} J\left(z, z_{0}\right) A(z)^{2} d z .
$$

Finally it remains to repeat the obvious: The Backus-Gilbert approach is strictly speaking limited to linear forward problems. For mildly nonlinear problems, including, for instance, the Love wave example in section 7.2, linearisation around a meaningful prior model can provide useful results. An extension to arbitrary nonlinear problems, however, has not been proposed so far. 



\section{Chapter 8 \\ Weakly nonlinear problems and optimisation *}

A view of nature as dense and nonlinear is at the core of our contemporary

science. Process and order emerge subtly.

Gregory Benford, 1995.

We have so far considered two methodological extremes: In chapters 2 to 5 we introduced the very general and holistic framework of Bayesian inference and its practical implementation using Monte Carlo methods. Apart from computational difficulties related to the potentially large number of samples required to approximate the posterior, Bayesian inference has no inherent limitations. This was contrasted in chapter 6 by the leastsquares method for linear forward problems. Often, linearity is merely a convenient approximation, and the explicit solution of least-squares problems may require the injection of artificial prior knowledge, referred to as regularisation.

In the following sections we will consider approaches that are located inbetween the extremes, and that do not require strict linearity of the forward problem,

$$
\mathbf{d}=\mathbf{G}(\mathbf{m}) \text {. }
$$

Instead, weak forms of nonlinearity will be allowed. The price to pay for more flexibility is a reduction of scope. Instead of trying to characterise the complete posterior distribution, we will mostly limit ourselves to finding an approximation of the posterior maximum-likelihood model $\hat{\mathbf{m}}$ which minimises some misfit functional, for instance, the least-squares misfit

$$
\chi(\mathbf{m})=\frac{1}{2}\left(\mathbf{m}-\mathbf{m}^{\text {prior }}\right)^{T} \mathbf{C}_{M}^{-1}\left(\mathbf{m}-\mathbf{m}^{\text {prior }}\right)+\frac{1}{2}\left[\mathbf{G}(\mathbf{m})-\mathbf{d}^{\mathrm{obs}}\right]^{T} \mathbf{C}_{D}^{-1}\left[\mathbf{G}(\mathbf{m})-\mathbf{d}^{\mathrm{obs}}\right] .
$$

Additionally, within a neighbourhood of the maximum-likelihood model, we may find an approximation of the posterior covariance.

\subsection{Iterative linearisation *}

Probably the most straightforward approach to the least-squares solution of an inverse problem with nonlinear foward modelling equations (8.1), is brute-force linearisation. In fact, a first-order Taylor expansion of equation (8.1) around an initial model $\mathbf{m}_{0}$, yields

$$
\mathbf{G}(\mathbf{m}) \doteq \mathbf{G}\left(\mathbf{m}_{0}\right)+\mathbf{J}_{0}\left(\mathbf{m}-\mathbf{m}_{0}\right),
$$


where $\doteq$ denotes a first-order approximation, and $\mathbf{J}_{0}$ is the Jacobian of $\mathbf{G}$ evaluated at $\mathbf{m}_{0}$. The components of $\mathbf{J}_{0}$ are given by

$$
J_{0, i j}=\left.\frac{\partial G_{i}}{\partial m_{j}}\right|_{\mathbf{m}=\mathbf{m}_{0}} .
$$

Substituting the approximation (8.4) into the least-squares misfit (8.2), yields

$$
\chi(\mathbf{m}) \doteq \frac{1}{2}\left(\mathbf{m}-\mathbf{m}^{\text {prior }}\right)^{T} \mathbf{C}_{M}^{-1}\left(\mathbf{m}-\mathbf{m}^{\text {prior }}\right)+\frac{1}{2}\left(\mathbf{J}_{0} \mathbf{m}-\mathbf{d}_{0}^{\text {obs }}\right)^{T} \mathbf{C}_{D}^{-1}\left(\mathbf{J}_{0} \mathbf{m}-\mathbf{d}_{0}^{\text {obs }}\right),
$$

where we defined

$$
\mathbf{d}_{0}^{\mathrm{obs}}=\mathbf{d}^{\mathrm{obs}}+\mathbf{J}_{0} \mathbf{m}_{0}-\mathbf{G}\left(\mathbf{m}_{0}\right) .
$$

Structurally, equation (8.5) is identical to the least-squares misfit for linear forward problems, given in equation (6.2). The only differences are that the forward modelling matrix $\mathbf{G}$ is replaced by the Jacobian matrix $\mathbf{J}_{0}$, and that $\mathbf{d}^{\text {obs }}$ is replaced by the modified observations $\mathbf{d}_{0}^{\text {obs }}$. Thus, we may employ the machinery derived for linear forward problems in chapter 6 in order to compute a least-squares solution. In particular, using the third of the three equivalent expressions in equation (6.13), we find

$$
\mathbf{m}_{1}=\mathbf{m}^{\text {prior }}+\mathbf{C}_{M} \mathbf{J}_{0}^{T}\left(\mathbf{C}_{D}+\mathbf{J}_{0} \mathbf{C}_{M} \mathbf{J}_{0}^{T}\right)^{-1}\left(\mathbf{d}_{0}^{\text {obs }}-\mathbf{J}_{0} \mathbf{m}_{0}\right) .
$$

Depending on how close $\mathbf{G}$ is to being linear, $\mathbf{m}_{1}$ may or may not be a good approximation of the maximumlikelihood model $\hat{\mathbf{m}}$. Improvements may be obtained by iteratively updating the reference model used in the linear approximation (8.3). Replacing $\mathbf{m}_{0}$ in the approximation by $\mathbf{m}_{1}$, computed via equation (8.7), we obtain a simple iterative scheme:

$$
\begin{aligned}
\mathbf{m}_{2} & =\mathbf{m}^{\text {prior }}+\mathbf{C}_{M} \mathbf{J}_{1}^{T}\left(\mathbf{C}_{D}+\mathbf{J}_{1} \mathbf{C}_{M} \mathbf{J}_{1}^{T}\right)^{-1}\left(\mathbf{d}_{1}^{\text {obs }}-\mathbf{J}_{1} \mathbf{m}_{1}\right), \\
\ldots & =\ldots \\
\mathbf{m}_{i+1} & =\mathbf{m}^{\text {prior }}+\mathbf{C}_{M} \mathbf{J}_{i}^{T}\left(\mathbf{C}_{D}+\mathbf{J}_{i} \mathbf{C}_{M} \mathbf{J}_{i}^{T}\right)^{-1}\left(\mathbf{d}_{i}^{\text {obs }}-\mathbf{J}_{i} \mathbf{m}_{i}\right) .
\end{aligned}
$$

Convergence of the scheme will depend critically on the properties of the forward problem, but also on the choice of the initial model $\mathbf{m}_{0}$, as we will see in the following examples.

Finding the frequency of a sinusoid: In the interest of a simple visual illustration, we consider a synthetic, one-dimensional problem where the forward modelling equations are given by

$$
d_{i}=\sin \left(m t_{i}\right),
$$

with time $t$ and circular frequency $m$. Setting the prior covariances to $\mathbf{C}_{M}=\mathbf{I}$ and $\mathbf{C}_{D}=\mathbf{I}$, also for simplicity, defines the least-squares misfit function

$$
\chi(m)=\frac{1}{2} \sum_{i=1}^{N}\left[d_{i}^{\mathrm{obs}}-\sin \left(m t_{i}\right)\right]^{2}+\frac{1}{2}\left(m-m^{\text {prior }}\right)^{2} .
$$

As target model parameter, we choose the circular frequency $m^{\text {target }}=2 \mathrm{~s}^{-1}$, and we place observations in intervals of $0.1 \mathrm{~s}$ between $t=0 \mathrm{~s}$ and $t=4 \pi \mathrm{s}$. The artificial observations and the synthetic data for the prior model $m^{\text {prior }}=1.8 \mathrm{~s}^{-1}$ are shown in figure 8.1a. Using the linearisation approach in the form of equation (8.7), produces a first guess, $m_{1}=1.899 \mathrm{~s}^{-1}$, which still deviates from $m^{\text {target }}=2 \mathrm{~s}^{-1}$. This is because the forward modelling equation is nonlinear, implying that the approximated misfit function, displayed in figure $8.1 \mathrm{~b}$, does not exactly match the correct misfit function. Note, in particular, that the correct misfit function has multiple local minima. This cannot be reproduced by a quadratic approximation that always has at most one global minimum. 
a) observed $(\bullet)$ and synthetic $(\bullet-\bullet)$ observations

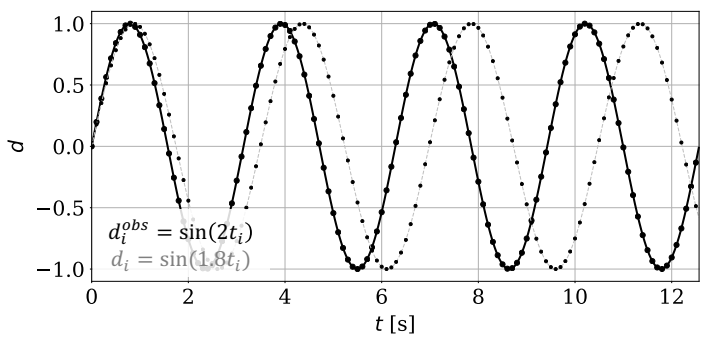

b) misfit $(\bullet \bullet)$ and linearised misfit $(\bullet-\bullet)$

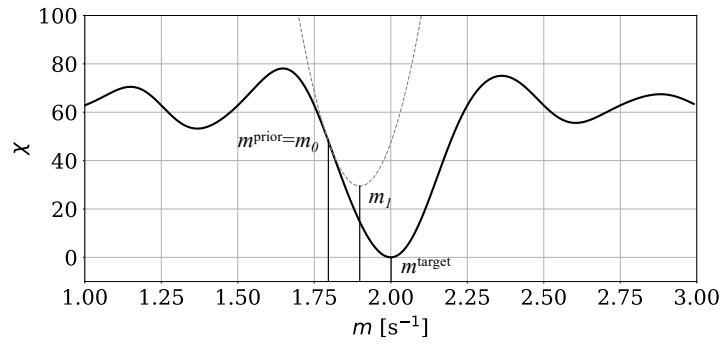

Fig. 8.1: Data and misfit function for the nonlinear forward problem of equation (8.10). (a): Artificial observations with target circular frequency $m^{\text {target }}=2 \mathrm{~s}^{-1}$ are plotted in black, and synthetic observations with prior circular frequency $m^{\text {prior }}=1.8 \mathrm{~s}^{-1}$ in grey. Actual observation points are shown as black and grey circles. (b): The linearisation of the forward modelling equation leads to a quadratic approximation (grey) of the original, non-quadratic misfit function (black). [Iterative linearisation]

Iterative improvement: The solution $m_{1}$ of the previous example can be improved by iteration, using equation (8.8). The iterative procedure for 2 iterations is shown in figure 8.2. It can be repeated arbitrarily often in order to obtain increasingly better approximations of the maximum-likelihood model.

a) observed $(\bullet)$ and updated synthetic $(\bullet-\bullet)$ observations

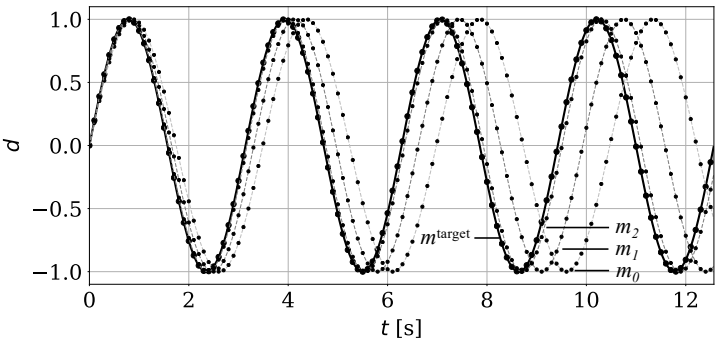

b) misfit $(\bullet)$ and updated linearised misfit $(\bullet-\bullet)$

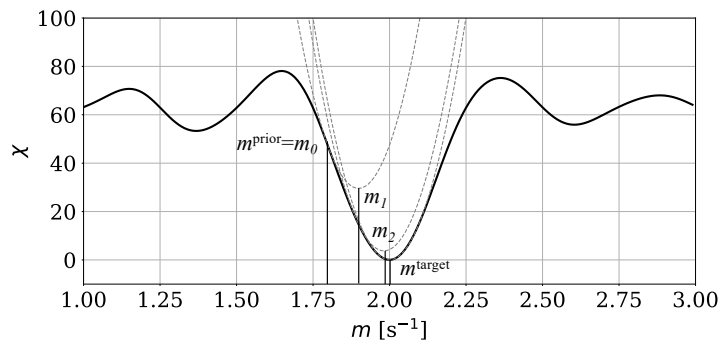

Fig. 8.2: Iteratively updated synthetic data and linearised misfit function. (a): Each iteration further improves the match between the artificial observations and the synthetic data. (b): In the course of the iteration, quadratic approximations of the misfit function are recomputed around the successively updated solutions. [Iterative linearisation]

Importance of the initial model: The choice of the initial model in the previous two examples was ad hoc. As shown in figure 8.3, other choices may lead to very different results that do not converge towards the global optimum.

Figures 8.2 and 8.3 illustrate important properties and limitations of the linearisation approach. Most importantly, the iteration is local, meaning that all iterative solutions are within the same misfit valley as the initial model. In other words, the iteration cannot walk uphill, only downhill. As a consequence, the iteration may get stuck in a local minimum, without any possibility to escape from the local valley into the neighbouring valley where the global minimum is located. This property is in contrast to Monte Carlo methods such as Simulated Annealing (section 5.6), which perform global optimisation, at least for suitably chosen tuning parameters.

The strong dependence of the iteration on the choice of the initial model also gives more precise meaning to the term weakly nonlinear. In fact, weak is also relative to the initial model. A strongly nonlinear and oscillatory 
a) observed $(\bullet)$ ) and updated synthetic $(\bullet-\bullet)$ observations

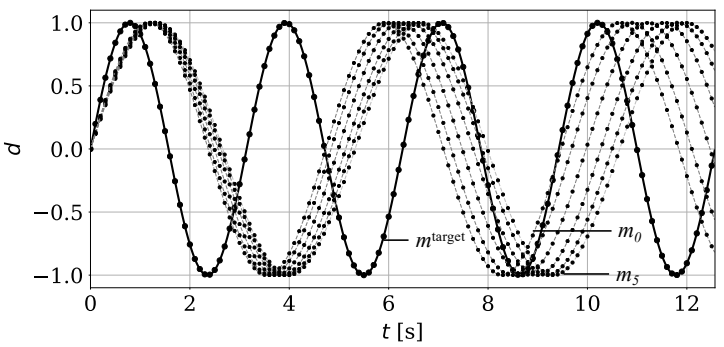

b) misfit $(\bullet)$ and updated linearised misfit $(\bullet-\bullet)$

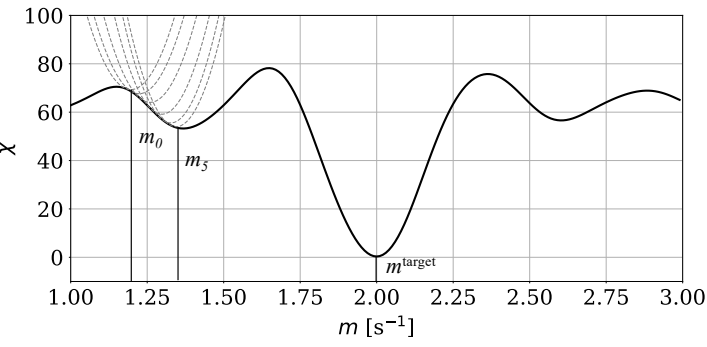

Fig. 8.3: The same as in figure 8.2, but for a different initial model $m_{0}=1.18 \mathrm{~s}^{-1}$. The iteration converges towards the nearest local minimum but not to the global minimum of the misfit function. [Iterative linearisation]

misfit function may still be tractable with iterative linearisation, provided that the initial model is sufficiently close to the global minimum. Finding a suitable initial model is therefore of outstanding importance for many nonlinear inverse problems.

The presence of local minima is closely related to the concept of convexity. A misfit function $\chi(\mathbf{m})$ is said to be convex when its graph between $\mathbf{m}$ and any other $\mathbf{m}^{\prime}$ generally lies above the tangent plane at $\mathbf{m}$, that is, when

$$
\chi\left(\mathbf{m}^{\prime}\right) \geq \chi(\mathbf{m})+\nabla \chi(\mathbf{m})^{T}\left(\mathbf{m}^{\prime}-\mathbf{m}\right)
$$

A convex function has no local minima, meaning that the iteration will eventually always converge towards the unique global mimimum, regardless of the initial model. In practice, it is of course difficult to know a priori if a misfit function is convex. However, convexity is often a useful mathematical tool in the analysis of optimisation algorithms.

Many methods exist for the approximation of the maximum-likelihood model or various other aspects of an inverse problem solution. Frequently, one method is claimed to be superior to another one. While this may seem intuitively obvious, the opposite can be shown rigorously in the form of the No-Free-Lunch theorem, demonstrated in chapter 12. At least statistically, all sampling or optimisation methods are equally inefficient. Efficiency only arises in the context of specific problems for which we have prior knowledge about its properties. This prior knowledge guides us in the choice of a suitable algorithm that may then indeed be more efficient than another one.

The iterative linearisation of the least-squares solution, outlined in the previous paragraphs can provide quality estimates of the solution in the form of a covariance or resolution matrix, which is meaningful in the vicinity of the global optimum. This advantage, however, comes at the price of having to store and invert a potentially large matrix, and it motivates the development of matrix-free optimisation or descent methods. Before the presentation of specific methods, we will first take a brief look at some basic properties of such algorithms.

\subsection{General descent methods *}

First, in the interest of generality, we leave the framework of the least-squares method in order to allow for other types of misfit functions. These may be derived, for instance, from non-Gaussian observational errors or non-Gaussian prior knowledge in model space. 


\subsubsection{Descent directions *}

Our general goal is to improve the initial model $\mathbf{m}_{0}$ by adding to it an update $\gamma_{0} \mathbf{h}_{0}$, where $\mathbf{h}_{0}$ is the descent direction and $\gamma_{0}>0$ is the step length:

$$
\mathbf{m}_{1}=\mathbf{m}_{0}+\gamma_{0} \mathbf{h}_{0} .
$$

For the construction of a suitable descent direction, we require that the misfit is actually reduced during the step, meaning that

$$
\chi\left(\mathbf{m}_{1}\right)=\chi\left(\mathbf{m}_{0}+\gamma_{0} \mathbf{h}_{0}\right)<\chi\left(\mathbf{m}_{0}\right)
$$

for all positive step lengths $\gamma_{0}$ that are smaller than a maximum step length $\gamma_{\max }$. Rearranging (8.14) and taking the limit $\gamma_{0} \rightarrow 0$, yields

$$
\mathbf{h}_{0}^{T} \nabla \chi\left(\mathbf{m}_{0}\right)=\lim _{\gamma_{0} \rightarrow 0} \frac{1}{\gamma_{0}}\left[\chi\left(\mathbf{m}_{0}+\gamma_{0} \mathbf{h}_{0}\right)-\chi\left(\mathbf{m}_{0}\right)\right]<0,
$$

where $\mathbf{h}_{0}^{T} \nabla \chi\left(\mathbf{m}_{0}\right)$ is the directional derivative of the misfit $\chi$ in the direction of $\mathbf{h}_{0}$. The condition $\mathbf{h}_{0}^{T} \nabla \chi\left(\mathbf{m}_{0}\right)<$ 0 can be considered as the definition of a local descent direction, that is, a direction indicating where we need to go from $\mathbf{m}_{0}$ in order to reduce the misfit. It follows immediately that a descent direction always exists as long as $\nabla \chi\left(\mathbf{m}_{0}\right) \neq 0$. To see this, we choose

$$
\mathbf{h}_{0}=-\nabla \chi\left(\mathbf{m}_{0}\right)
$$

and therefore

$$
\mathbf{h}_{0}^{T} \nabla \chi\left(\mathbf{m}_{0}\right)=-\left[\nabla \chi\left(\mathbf{m}_{0}\right)\right]^{2}<0 .
$$

This means that choosing $\mathbf{h}_{0}$ from (8.16) to be the negative gradient of the misfit, satisfies condition (8.15). Many other descent directions are possible. In fact, when $\mathbf{A}$ is a positive definite matrix, the vector

$$
\mathbf{h}_{0}=-\mathbf{A} \nabla \chi\left(\mathbf{m}_{0}\right)
$$

is also a descent direction because

$$
\mathbf{h}_{0}^{T} \nabla \chi\left(\mathbf{m}_{0}\right)=-\nabla \chi\left(\mathbf{m}_{0}\right)^{T} \mathbf{A}^{T} \nabla \chi\left(\mathbf{m}_{0}\right)<0 .
$$

In an iterative minimisation, we successively update models by going from the current model $\mathbf{m}_{i}$ along a descent direction $\mathbf{h}_{i}=-\mathbf{A}_{i} \nabla \chi\left(\mathbf{m}_{i}\right)$ towards the improved model $\mathbf{m}_{i+1}$. The general descent algorithm can be summarised as follows:

\section{General descent method}

1. Choose an initial model, $\mathbf{m}_{0}$, and set $i=0$.

2. Compute the descent direction

$$
\mathbf{h}_{i}=-\mathbf{A}_{i} \nabla \chi\left(\mathbf{m}_{i}\right)
$$

3. Update $\mathbf{m}_{i}$ according to

$$
\mathbf{m}_{i+1}=\mathbf{m}_{i}+\gamma_{i} \mathbf{h}_{i}
$$

with a suitable step length $\gamma_{i}$ that ensures $\chi\left(\mathbf{m}_{i+1}\right)<\chi\left(\mathbf{m}_{i}\right)$.

4. Set $i \rightarrow i+1$ and go back to (2).

This is repeated until the misfit is as small as permitted by the errors in the data.

A critical element of the above algorithm is the gradient $\nabla \chi$, which may be expensive to compute. The choice of the positive definite matrices $\mathbf{A}_{i}$ characterises different descent methods, and it controls the speed of conver- 
gence towards the optimum $\hat{\mathbf{m}}$.

Given the availability of sufficient computational resources, as well as a suitable initial model, the minimum of $\chi$ can be approximated arbitrarily closely with the help of descent methods. However, the approximation of $\hat{\mathbf{m}}$ may explain the observed data so well that the remaining residuals are smaller than the uncertainty in the data. In the case of a pure least-squares problem, such over-fitting could be prevented by adjusting the prior data and model covariance matrices. When iterative methods are used to approximate the maximum-likelihood model, the iteration may alternatively be terminated before the root-mean-square misfit drops significantly below 1 . In this sense, the early termination of the iteration acts as implicit regularisation. Especially in cases where iterative updating is computationally expensive, this approach may be preferred over repeated iterations with different prior covariance matrices.

\subsubsection{Optimal step length*}

To complete the description of the general descent method, it remains to construct an efficient step length for each iteration. Ideally, we would like to find an optimal $\gamma_{i}^{\mathrm{opt}}$ such that $\chi\left(\mathbf{m}_{i+1}\right)=\chi\left(\mathbf{m}_{i}+\gamma_{i}^{\mathrm{opt}} \mathbf{h}_{i}\right)$ is minimal. A necessary condition for the optimality of $\gamma_{i}$ is then

$$
\left.\frac{d}{d \gamma_{i}} \chi\left(\mathbf{m}_{i}+\gamma_{i} \mathbf{h}_{i}\right)\right|_{\gamma_{i}=\gamma_{i}^{\mathrm{opt}}}=0
$$

Evaluating the derivative in (8.22), gives

$$
\left.\mathbf{h}_{i}^{T} \nabla \chi\left(\mathbf{m}_{i}+\gamma_{i} \mathbf{h}_{i}\right)\right|_{\gamma_{i}=\gamma_{i}^{\mathrm{opt}}}=0
$$

Equation (8.23) defines the optimal $\gamma_{i}$ implicitly. To obtain an explicit approximation, we employ a first-order approximation of $\nabla \chi\left(\mathbf{m}_{i}+\gamma_{i}^{\mathrm{opt}} \mathbf{h}_{i}\right)$,

$$
\nabla \chi\left(\mathbf{m}_{i}+\gamma_{i}^{\mathrm{opt}} \mathbf{h}_{i}\right) \doteq \nabla \chi\left(\mathbf{m}_{i}\right)+\gamma_{i}^{\mathrm{opt}} \mathbf{H}\left(\mathbf{m}_{i}\right) \mathbf{h}_{i}
$$

where the Hessian matrix contains the second derivatives of this misfit function $\chi$,

$$
H_{j k}\left(\mathbf{m}_{i}\right)=\left.\frac{\partial^{2} \chi}{\partial m_{j} \partial m_{k}}\right|_{\mathbf{m}=\mathbf{m}_{i}} .
$$

Substituting equation (8.24) into equation (8.23), yields

$$
0=\mathbf{h}_{i}^{T} \nabla \chi\left(\mathbf{m}_{i}\right)+\gamma_{i}^{\mathrm{opt}} \mathbf{h}_{i}^{T} \mathbf{H}\left(\mathbf{m}_{i}\right) \mathbf{h}_{i} .
$$

Solving for $\gamma_{i}^{\text {opt }}$ then gives

$$
\gamma_{i}^{\mathrm{opt}}=-\frac{\mathbf{h}_{i}^{T} \nabla \chi\left(\mathbf{m}_{i}\right)}{\mathbf{h}_{i}^{T} \mathbf{H}\left(\mathbf{m}_{i}\right) \mathbf{h}_{i}} .
$$

A commonly used alternative to equation (8.27) that allows us to circumvent the computation of the Hessian times vector, $\mathbf{H}_{\chi}\left(\mathbf{m}_{i}\right) \mathbf{h}_{i}$, is a line search: For this we choose a small number of trial step lengths, $\gamma_{i}^{(k)}(k=$ $1, \ldots, p)$, and evaluate the corresponding misfit functionals $\chi\left(\mathbf{m}_{i}+\gamma_{i}^{(k)} \mathbf{h}_{i}\right)$. These are then used to approximate $\chi\left(\mathbf{m}_{i}+\gamma_{i} \mathbf{h}_{i}\right)$ by an interpolating polynomial of degree $p-1$. The minimum of the polynomial is then an estimate of the optimal step length. Depending on the complexity of the misfit function, $\chi\left(\mathbf{m}_{i}+\gamma_{i} \mathbf{h}_{i}\right)$ may be sufficiently quadratic with respect to $\gamma_{i}$ so that a second-order polynomial yields a good approximation.

The choice of the trial step lengths is crucial for the success of a line search. The only obvious trial step length is $\gamma_{i}^{(0)}=0$, because the corresponding misfit, $\chi\left(\mathbf{m}_{i}\right)$, is already known. Any other $\gamma_{i}^{(k)}$ can be found most 
efficiently with the help of intuition that usually provides us with a range of step lengths that lead to physically plausible models.

\subsubsection{Test functions *}

The performance of various optimisation methods depends not only on the method itself but also on the function to which it is applied. Since it is often difficult to obtain general but still practically useful convergence results for specific methods, it is customary to test them on more or less pathological functions. We introduce some of them here, for later use in 2-D numerical examples.

The simple quadratic function

$$
\chi(\mathbf{m})=m_{1}^{2}+m_{2}^{2}+m_{1} m_{2},
$$

has a single optimum at $\left(m_{1}, m_{2}\right)=(0,0)$ and is perfectly convex. The Rosenbrock function (Rosenbrock, 1960)

$$
\chi(\mathbf{m})=\left(1-m_{1}^{2}\right)+100\left(m_{2}-m_{1}^{2}\right)^{2},
$$

also has a global optimum, located at $\left(m_{1}, m_{2}\right)=(1,1)$. It is characterised by an elongated, curved valley, along which the first derivative of $\chi$ takes very small values. The Himmelblau function (Himmelblau, 1972)

$$
\chi(\mathbf{m})=\left(m_{1}^{2}+m_{2}-11\right)^{2}+\left(x_{1}+x_{2}^{2}-7\right)^{2},
$$

is a fourth-order polynomial with 4 global minima where the function is equal to 0 . The Himmelblau function also has 4 saddle points and a local maximum. Finally, the Bazaraa-Shetty function (Bazaraa \& Shetty, 1976)

$$
\chi(\mathbf{m})=\left(m_{1}-2\right)^{4}+\left(m_{1}-2 m_{2}\right)^{2},
$$

is also a fourth-order polynomial. It has a global minimum at $\left(m_{1}, m_{2}\right)=(2,1)$.

\subsection{The method of steepest descent *}

To improve $\mathbf{m}_{0}$ as much as possible in the first iteration already, we try to find the descent direction, $\mathbf{h}_{0}$, that leads to the maximum decrease of $\chi$ for a small fixed step length, $\gamma_{0}$. For this we determine $\mathbf{h}_{0}$ with $\left|\mathbf{h}_{0}\right|=1$ such that

$$
\chi\left(\mathbf{m}_{1}\right)-\chi\left(\mathbf{m}_{0}\right)=\chi\left(\mathbf{m}_{0}+\gamma_{0} \mathbf{h}_{0}\right)-\chi\left(\mathbf{m}_{0}\right) \approx \gamma_{0} \mathbf{h}_{0}^{T} \nabla \chi\left(\mathbf{m}_{0}\right)
$$

is minimal. Assuming that $\mathbf{h}_{0}$ is a descent direction in the sense of equation (8.15), yields the inequality

$$
\gamma_{0} \mathbf{h}_{0}^{T} \nabla \chi\left(\mathbf{m}_{0}\right) \geq-\gamma_{0}\left|\nabla \chi\left(\mathbf{m}_{0}\right)\right|\left|\mathbf{h}_{0}\right|=-\gamma_{0}\left|\nabla \chi\left(\mathbf{m}_{0}\right)\right|,
$$

meaning that the quantity that we seek to minimise, $\gamma_{0} \mathbf{h}_{0}^{T} \nabla \chi\left(\mathbf{m}_{0}\right)$, is always larger than or equal to $-\gamma_{0}\left|\nabla \chi\left(\mathbf{m}_{0}\right)\right|$. The minimum of $\gamma_{0} \mathbf{h}^{T} \nabla \chi\left(\mathbf{m}_{0}\right)$ corresponds to the direction $\mathbf{h}_{0}$ for which the equal sign holds in (8.33). This is the case for

$$
\mathbf{h}_{0}=-\frac{\nabla \chi\left(\mathbf{m}_{0}\right)}{\left|\nabla \chi\left(\mathbf{m}_{0}\right)\right|}
$$

which is the direction of steepest descent. Our finding that $\mathbf{h}_{0}$ as defined in (8.34) leads to the most rapid descent for a given small step length $\gamma_{0}$, suggests to repeat this procedure by iteratively moving from a current model $\mathbf{m}_{i}$ along the local descent direction $-\nabla \chi\left(\mathbf{m}_{i}\right)$ towards the updated model $\mathbf{m}_{i+1}$. This is the concept of the steepest descent method: 


\section{Method of steepest descent}

1. Choose an initial model, $\mathbf{m}_{0}$, and set $i=0$.

2. Compute the gradient for the current model, $\nabla \chi\left(\mathbf{m}_{i}\right)$.

3. Update $\mathbf{m}_{i}$ according to

$$
\mathbf{m}_{i+1}=\mathbf{m}_{i}-\gamma_{i} \nabla \chi\left(\mathbf{m}_{i}\right),
$$

with a suitable step length $\gamma_{i}$ that ensures $\chi\left(\mathbf{m}_{i+1}\right)<\chi\left(\mathbf{m}_{i}\right)$.

4. Set $i \rightarrow i+1$, go back to (2) and repeat until the data are explained sufficiently well.

While being conceptually simple and attractive, the steepest descent method is rarely used in practice because it tends to converge rather slowly towards an acceptable model. This is because a succession of descent directions that are locally optimal may not necessarily be optimal from a global perspective.

Steepest-descent optimisation in two dimensions: Figure 8.4 visualises the steepest-descent optimisation of the quadratic function (8.28) (left) and the Rosenbrock function (8.29) (right). For the quadratic function, the steepest-descent algorithm closely approaches the minimum at $\mathbf{m}=(0,0)$ within few iterations. However, for the Rosenbrock function, steepest descent shows a characteristic zig-zagging from the initial model through the very flat valley and towards the minimum at $\mathbf{m}=(1,1)$, which it has not yet reached after the 1000 iterations shown in the figure.

a) quadratic function

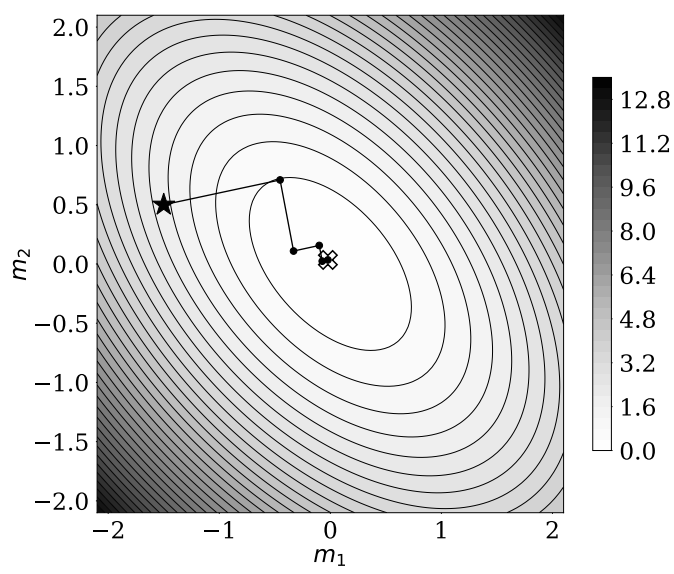

b) Rosenbrock function

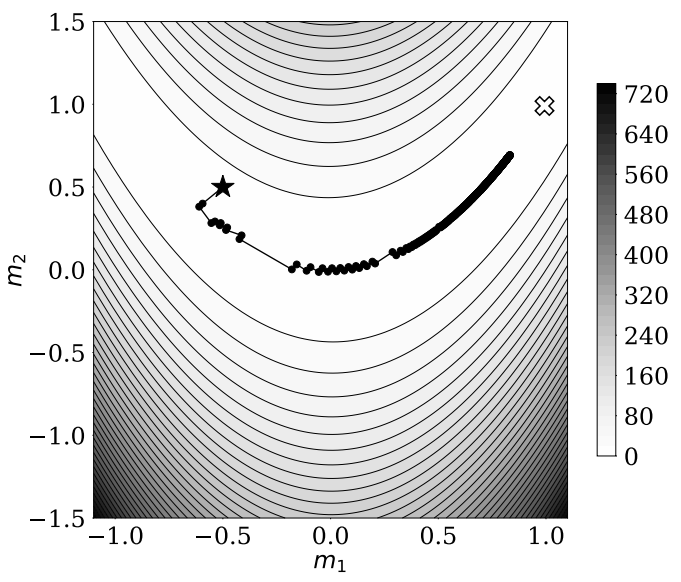

Fig. 8.4: Steepest-descent optimisation of the quadratic function (8.28) (a, 5 iterations) and of the Rosenbrock function (8.29) (b, 1000 iterations). Shown are the misfit functions in the form of grey scale values and contours, and the iteration path. The star marks the initial model, and the cross is at the position of the global minimum. Each black dot represents one iteration. The Rosenbrock function features a very flat, U-shaped valley that provokes zig-zagging and very slow convergence. [Nonlinear optimisation in 2D] 


\subsection{Newton's method and its variants *}

In the steepest descent algorithm from section $8.3, \mathbf{h}_{i}$ is determined only from first-derivative information at the current iterate $\mathbf{m}_{i}$. Newton's method modifies the steepest descent direction with the help of secondderivative information, which may lead to faster convergence. The algorithm exploits the equivalence of finding an extremum of the misfit, $\chi$, and finding a zero of its gradient, $\nabla \chi$. Upon using the necessary condition that the gradient vanishes at the optimum, $\nabla \chi(\hat{\mathbf{m}})=\mathbf{0}$, the linear approximation of $\nabla \chi(\hat{\mathbf{m}})$ around a nearby model $\mathbf{m}$ reads

$$
\mathbf{0}=\nabla \chi(\hat{\mathbf{m}}) \approx \nabla \chi(\mathbf{m})+\mathbf{H}(\mathbf{m})(\hat{\mathbf{m}}-\mathbf{m})
$$

Formally solving for $\hat{\mathbf{m}}$, gives

$$
\hat{\mathbf{m}} \approx \mathbf{m}-\mathbf{H}^{-1}(\mathbf{m}) \nabla \chi(\mathbf{m}) .
$$

Equation (8.37) suggests the following scheme:

\section{Newton's method}

1. Choose an initial model, $\mathbf{m}_{0}$, and set $i=0$.

2. Compute the gradient for the current model, $\nabla \chi\left(\mathbf{m}_{i}\right)$.

3. Determine the descent direction, $\mathbf{h}_{i}$, as the solution of

$$
\mathbf{H}\left(\mathbf{m}_{i}\right) \mathbf{h}_{i}=-\nabla \chi\left(\mathbf{m}_{i}\right)
$$

4. Update $\mathbf{m}_{i}$ according to

$$
\mathbf{m}_{i+1}=\mathbf{m}_{i}+\mathbf{h}_{i} .
$$

5. Set $i \rightarrow i+1$, go back to (2) and repeat as often as needed.

In each iteration, Newton's method requires the computation of the Hessian times a vector, and the solution of the linear system (8.38), which is known as the Newton equation. Its solution, the descent direction $\mathbf{h}_{i}$, can be found, for instance, with the help of iterative matrix solvers (e.g. Quarteroni et al., 2000). Note that Newton's method corresponds to choosing the matrix $\mathbf{A}_{i}$ from equation (8.20) as

$$
\mathbf{A}_{i}=\mathbf{H}^{-1}\left(\mathbf{m}_{i}\right)
$$

Provided that $\mathbf{H}\left(\mathbf{m}_{i}\right)$ is positive definite, $-\mathbf{H}^{-1}\left(\mathbf{m}_{i}\right) \nabla \chi\left(\mathbf{m}_{i}\right)$ is a descent direction. The beauty of Newton's method becomes apparent when it is applied to a misfit function that is actually quadratic, so that the approximation in equation (8.36) is exact. In fact, using the least-squares misfit for the linear forward problem from equation (6.2), we find

$$
\nabla \chi\left(\mathbf{m}_{0}\right)=\mathbf{C}_{M}^{-1}\left(\mathbf{m}_{0}-\mathbf{m}^{\text {prior }}\right)+\mathbf{G}^{T} \mathbf{C}_{D}^{-1}\left(\mathbf{G m}_{0}-\mathbf{d}^{\mathrm{obs}}\right)
$$

and

$$
\mathbf{H}\left(\mathbf{m}_{0}\right)=\mathbf{C}_{M}^{-1}+\mathbf{G}^{T} \mathbf{C}_{D}^{-1} \mathbf{G} .
$$

Using equation (8.38), the descent direction $\mathbf{h}_{0}$ is then given by

$$
\begin{aligned}
& \mathbf{h}_{0}=-\left(\mathbf{C}_{M}^{-1}+\mathbf{G}^{T} \mathbf{C}_{D}^{-1} \mathbf{G}\right)^{-1}\left[\mathbf{C}_{M}^{-1}\left(\mathbf{m}_{0}-\mathbf{m}^{\text {prior }}\right)+\mathbf{G}^{T} \mathbf{C}_{D}^{-1}\left(\mathbf{G} \mathbf{m}_{0}-\mathbf{d}^{\text {obs }}\right)\right] \\
& =-\left(\mathbf{C}_{M}^{-1}+\mathbf{G}^{T} \mathbf{C}_{D}^{-1} \mathbf{G}\right)^{-1}\left[\left(\mathbf{C}_{M}^{-1}+\mathbf{G}^{T} \mathbf{C}_{D}^{-1} \mathbf{G}\right) \mathbf{m}_{0}-\mathbf{C}_{M}^{-1} \mathbf{m}^{\text {prior }}-\mathbf{G}^{T} \mathbf{C}_{D}^{-1} \mathbf{d}^{\text {obs }}\right] \\
& =-\mathbf{m}_{0}+\left(\mathbf{C}_{M}^{-1}+\mathbf{G}^{T} \mathbf{C}_{D}^{-1} \mathbf{G}\right)^{-1}\left(\mathbf{C}_{M}^{-1} \mathbf{m}^{\text {prior }}+\mathbf{G}^{T} \mathbf{C}_{D}^{-1} \mathbf{d}^{\text {obs }}\right) .
\end{aligned}
$$


According to equation (8.39), the first update, $\mathbf{m}_{1}$, is now given by

$$
\mathbf{m}_{1}=\mathbf{m}_{0}+\mathbf{h}_{0}=\left(\mathbf{C}_{M}^{-1}+\mathbf{G}^{T} \mathbf{C}_{D}^{-1} \mathbf{G}\right)^{-1}\left(\mathbf{C}_{M}^{-1} \mathbf{m}^{\text {prior }}+\mathbf{G}^{T} \mathbf{C}_{D}^{-1} \mathbf{d}^{\mathrm{obs}}\right) .
$$

Comparing with equation (6.8), we see that already the first update is indeed equal to the minimum of the least-squares misfit function for a linear forward problem, that is

$$
\mathbf{m}_{1}=\hat{\mathbf{m}} .
$$

Though equation (8.45) is strictly valid only in the case of a linear forward problem, it still suggests fast convergence in cases where the misfit function is nearly quadratic.

Newton optimisation in two dimensions: We consider Newton's method applied to various (pathological) misfit functions $\chi$ in two dimensions. For the purely quadratic function, Newton's method converges in only one iteration to the global minimum, by construction. This is illustrated in figure 8.5a for the quadratic function (8.28). For the Rosenbrock function (8.29), shown in figure 8.5b, around 5 iterations are needed to approximate the minimum very closely. Compare this result to figure $8.4 \mathrm{~b}$, where more than 1000 steepest-descent iterations were not sufficient to reach the minimum. However, we also observe that misfit actually increases in the second iteration. This undesirable phenomenon, known as over-shooting, occurs when the Hessian is not positive definite, or at least close to having zero eigenvalues. In this example, we are simply lucky that the over-shooting can be compensated in subsequent iterations. The Himmelblau function (8.30) (Himmelblau, 1972), shown in figure $8.5 \mathrm{c}$, is a fourth-order polynomial with 4 global minima. Around 5 iterations lead to a close approximation of one of them. Finally, the Bazaraa-Shetty function (8.31), in figure $8.5 \mathrm{~d}$, is also a fourth-order polynomial. It has a global minimum at $\left(m_{1}, m_{2}\right)=(2,1)$ where the Hessian becomes singular. Thus, the iteration becomes numerically unstable just before the minimum is reached.

Newton optimisation of the Himmelblau function: Having multiple minima, maxima and saddle points, the iterative optimisation of the Himmelblau function (8.30) depends critically on the initial model. Figure 8.6 shows two examples for initial models that are different from the one shown in figure $8.5 \mathrm{c}$. In the left panel, the iteration reaches the local maximum. In the right panel, the iteration crosses a ridge in the first step, meaning that it locally ascends. This is because the Hessian is negative definite at the position of the initial model.

Before we consider variants of the pure Newton method, we note that the examples shown above are of course to some extent pathologic. In practice, few misfit functions will be perfectly quadratic or have a set of four global minima. These examples are intended to illustrate strengths and weaknesses of different methods in a range of scenarios. Another, more profound, reason for working with (toy) examples is indeed the No-Free-Lunch theorem, shown in Appendix 12. It states that we cannot prove the universal superiority of any optimisation method for all possible misfit functions. As a consequence, we can merely study exemplary misfit functions that have some of the properties that we may expect in real-world applications or in the worst-case scenario.

\subsubsection{Damped and regularised Newton methods}

Newton's method relies critically on the appropriateness of the local approximation from equation (8.36). When the current iterate, $\mathbf{m}_{i}$, is far from the optimum, the Hessian, $\mathbf{H}\left(\mathbf{m}_{i}\right)$, may have negative eigenvalues and be illconditioned or even singular. This can result in very slow convergence, movement in non-descent directions and heavy oscillations that diverge from the solution ${ }^{1}$.

Being potentially unsuitable in its raw form, Newton's method has been modified in many different ways. In

\footnotetext{
${ }^{1}$ In fact, one may easily show that Newton's method may actually ascend towards a (local) maximum in cases where the misfit function $\chi$ is not convex but concave, that is, when $\mathbf{H}$ is negative definite.
} 
a) quadratic function

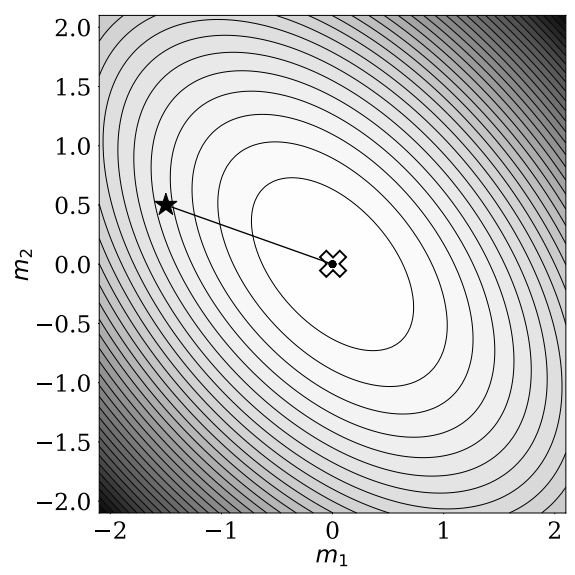

c) Himmelblau function

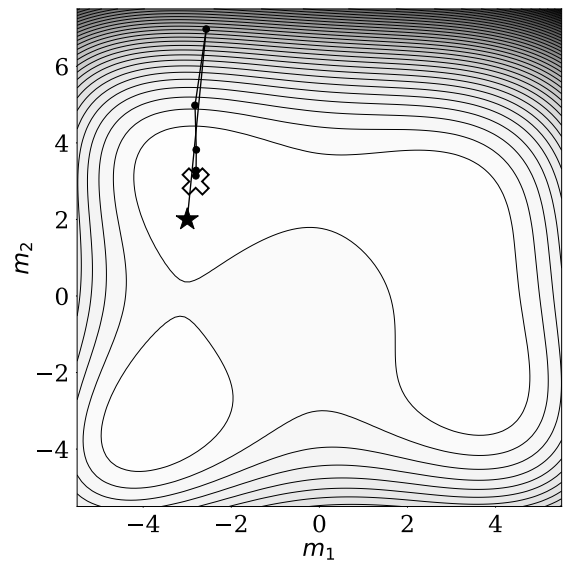

b) Rosenbrock function

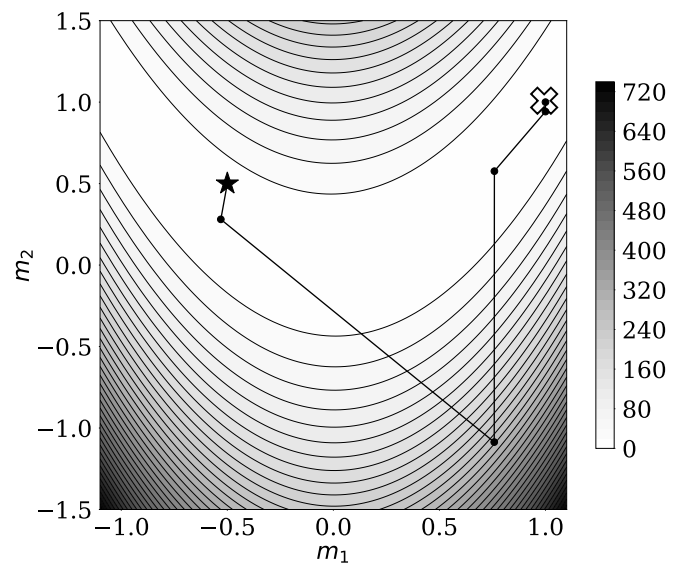

d) Bazaraa-Shetty function

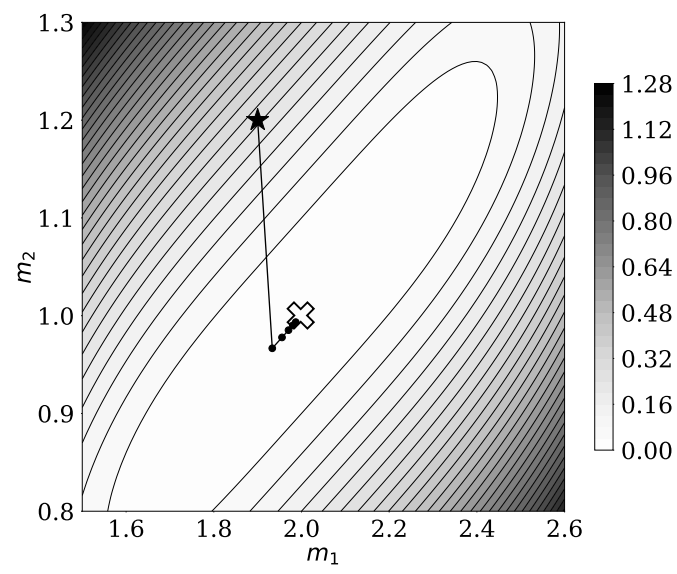

Fig. 8.5: Newton optimisation of various test misfit functions. (a) Perfect convergence in one iteration for the quadratic function (8.28). (b) Optimisation of the Rosenbrock function (8.29), including over-shooting after the first iteration. (c) Convergence toward one of the global minima of the Himmelblau function (8.30). (d) Optimisation of the Bazaraa-Shetty function (8.31), terminated when the Hessian becomes nearly singular. Global minima are shown as crosses, and initial models as stars. [Nonlinear optimisation in 2D]

the damped version of the algorithm, the update $\mathbf{h}_{i}=-\mathbf{H}^{-1}\left(\mathbf{m}_{i}\right) \nabla \chi\left(\mathbf{m}_{i}\right)$ is multiplied by a scalar parameter $\alpha \in(0,1]$ :

$$
\mathbf{m}_{i+1}=\mathbf{m}_{i}-\alpha \mathbf{H}^{-1}\left(\mathbf{m}_{i}\right) \nabla \chi\left(\mathbf{m}_{i}\right)
$$

Using a conventional line search, $\alpha$ is chosen such that $\mathbf{m}_{i+1}$ reduces $\chi$ as much as possible. Since $\mathbf{H}\left(\mathbf{m}_{i}\right) \operatorname{could}$ be singular, its inversion may require regularisation. The regularised Newton method therefore adds a scalar multiple of the unit matrix, $\mathbf{I}$, to $\mathbf{H}\left(\mathbf{m}_{i}\right)$ :

$$
\mathbf{m}_{i+1}=\mathbf{m}_{i}-\left[\mathbf{H}\left(\mathbf{m}_{i}\right)+\beta \mathbf{I}\right]^{-1} \nabla \chi\left(\mathbf{m}_{i}\right)
$$

Just as $\alpha$, the parameter $\beta$ may be found with the help of a line search. Note that the regularised Newton method approaches the steepest descent method for large values of $\beta$. 
Newton optimisation of the Himmelblau function
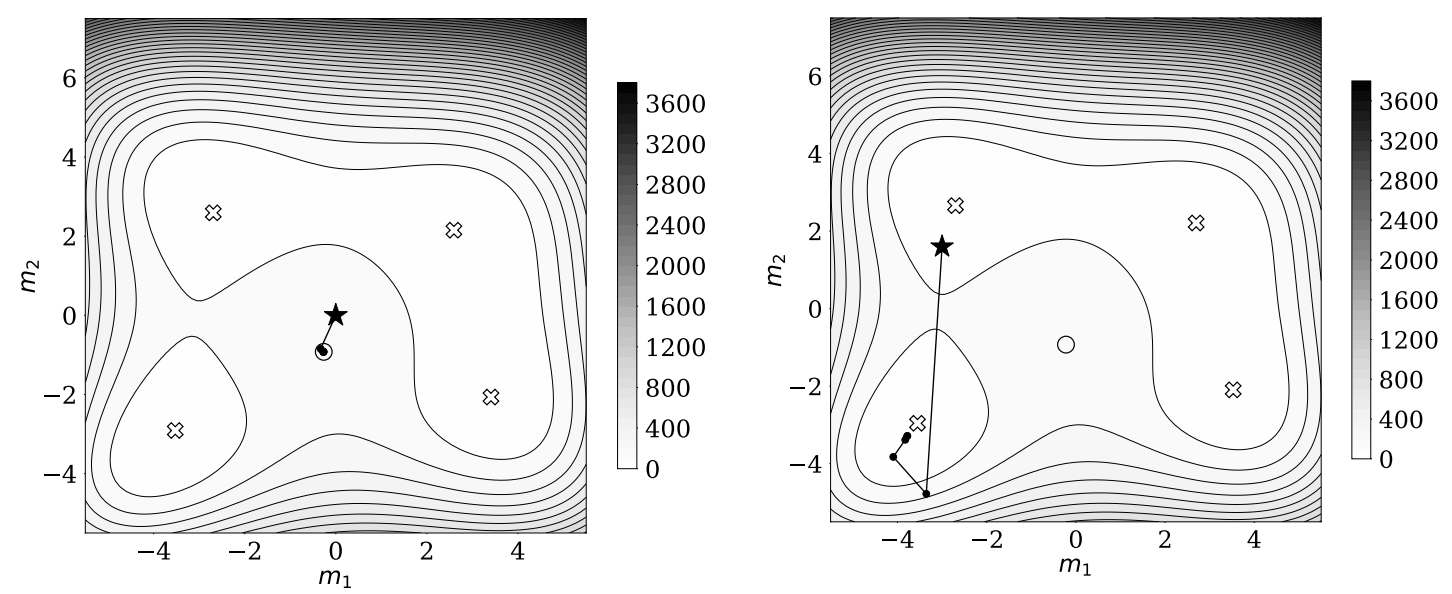

Fig. 8.6: Newton optimisation of the Himmelblau function (8.30) for different initial models. Global minima are shown as crosses, and the local maximum as circle. [Nonlinear optimisation in 2D]

\subsubsection{Gauss-Newton, Levenberg and Levenberg-Marquardt methods}

A whole class of methods aims to increase computational efficiency by avoiding the potentially expensive calculation of second derivatives in the Hessian. Ignoring, for convencience, any explicit dependence of the misfit $\chi$ on $\mathbf{m}$, we note that $\chi$ is related to $\mathbf{m}$ via the forward modelling operator $\mathbf{G}$, that is

$$
\chi=\chi[\mathbf{G}(\mathbf{m})]
$$

Differentiating $\chi$ twice with respect to $\mathbf{m}$ and making repeated use of the chain rule, we find, in index notation,

$$
H_{i j}=\frac{\partial^{2} \chi}{\partial m_{i} \partial m_{j}}=\frac{\partial^{2} \chi}{\partial G_{k} \partial G_{l}} \frac{\partial G_{l}}{\partial m_{j}} \frac{\partial G_{k}}{\partial m_{i}}+\frac{\partial \chi}{\partial G_{k}} \frac{\partial^{2} G_{k}}{\partial m_{i} \partial m_{j}} .
$$

The first term on the right-hand side of equation (8.49) only contains the often easily computable second derivative of $\chi$ with respect to $\mathbf{G}$, as well as the first derivative $\frac{\partial G_{l}}{\partial m_{j}}$, which is equal to the Jacobian $J_{l j}$. The difficult part, that is, the second derivatives of the forward modelling operator, are contained in the second term on the right-hand side of equation (8.48). Thus, a natural simplification is to replace the complete Hessian in Newton's method by the approximate Hessian

$$
\bar{H}_{i j}=\frac{\partial^{2} \chi}{\partial G_{k} \partial G_{l}} \frac{\partial G_{l}}{\partial m_{j}} \frac{\partial G_{k}}{\partial m_{i}}
$$

The Newton-like method based on the approximate Hessian,

$$
\mathbf{m}_{i+1}=\mathbf{m}_{i}-\overline{\mathbf{H}}^{-1}\left(\mathbf{m}_{i}\right) \nabla \chi\left(\mathbf{m}_{i}\right)
$$

is known as the Gauss-Newton method. Its regularised version

$$
\mathbf{m}_{i+1}=\mathbf{m}_{i}-\left[\overline{\mathbf{H}}\left(\mathbf{m}_{i}\right)+\beta \mathbf{I}\right]^{-1} \nabla \chi\left(\mathbf{m}_{i}\right),
$$


is the Levenberg method (Levenberg, 1944). Marquardt (1963) suggested to replace the identity matrix, I, in equation (8.52) by the diagonal of the approximate Hessian, $\overline{\mathbf{H}}\left(\mathbf{m}_{i}\right)$, in order to overcome slow convergence in the directions where the gradient is small. The resulting algorithm

$$
\mathbf{m}_{i+1}=\mathbf{m}_{i}-\left[\overline{\mathbf{H}}\left(\mathbf{m}_{i}\right)+\beta \operatorname{diag} \overline{\mathbf{H}}\left(\mathbf{m}_{i}\right)\right]^{-1} \nabla \chi\left(\mathbf{m}_{i}\right),
$$

is referred to as the Levenberg-Marquardt method.

\subsection{The conjugate-gradient method}

We have seen, so far, that Newton's method and its variants often converge faster than the steepest-descent method, though at the expense of having to compute the inverse of the (approximate) Hessian applied to a vector. While this is no problem for the two-dimensional toy examples considered in figures 8.5 and 8.6, the computational costs may become prohibitive in higher-dimensional problems. The steepest-descent method is hardly an alternative, because it tends to converge slowly. As illustrated in figure 8.4, this slow convergence partly results from the algorithm walking repeatedly in directions in which it has walked before.

This deficiency of steepest descent is the main motivation behind the conjugate-gradient method. Instead of reusing descent directions that have been used in previous iterations, the conjugate-gradient method constructs a sequence of descent directions that are mutually orthogonal. In the following, we will first derive the method for purely quadratic misfit functions, then investigate more rigorously the benefit of using conjugate directions, and finally propose several generalisations for arbitrary misfit functions.

\subsubsection{Derivation of the method for quadratic misfit functions}

The conjugate-gradient method for quadratic misfit functions can be understood as a method for the direct inversion of the vector-matrix equation $\mathbf{G m}=\mathbf{d}$. Left-multiplication with $\mathbf{G}^{T}$, gives

$$
\mathbf{H m}=\mathbf{b}
$$

with the symmetric matrix $\mathbf{H}=\mathbf{G}^{T} \mathbf{G}$, and the constant vector $\mathbf{b}=\mathbf{G}^{T} \mathbf{d}$. Though this may not be the case in practice, we assume for the moment that $\mathbf{H}$ is positive definite. First, we note that the solution of equation (8.54) is equivalent to the minimisation of the quadratic misfit function

$$
\chi(\mathbf{m})=\frac{1}{2} \mathbf{m}^{T} \mathbf{H} \mathbf{m}-\mathbf{m}^{T} \mathbf{b},
$$

where $\mathbf{H}$ is the Hessian of $\chi$. Indeed, forcing the gradient of $\chi$ to $\mathbf{0}$, returns equation (8.54), which has a unique solution as a consequence of the assumed positive definiteness. To minimise $\chi$ using a descent method, we update the current model $\mathbf{m}_{i}$ along a descent direction $\mathbf{h}_{i}$ with some step length $\gamma$. This modifies the misfit from $\chi\left(\mathbf{m}_{i}\right)$ to $\chi\left(\mathbf{m}_{i}+\gamma \mathbf{h}_{i}\right)$. Differentiating $\chi\left(\mathbf{m}_{i}+\gamma \mathbf{h}_{i}\right)$ with respect to $\gamma$ and setting the derivative to 0 , yields the optimal step length

$$
\gamma_{i}=\frac{\mathbf{h}_{i}^{T} \mathbf{r}_{i}}{\mathbf{h}_{i}^{T} \mathbf{H} \mathbf{h}_{i}}
$$

where we define the residual $\mathbf{r}_{i}$ as

$$
\mathbf{r}_{i}=\mathbf{b}-\mathbf{H m}_{i} .
$$

In fact, equation (8.56) is a special case of the optimal step length equation (8.27) for non-quadratic misfit functions. We arrived at $\mathbf{m}_{i}$ through a sequence of updates in some directions $\mathbf{h}_{j}$, with $j=0, \ldots, i-1$. To avoid 
convergence problems as in the steepest descent method, we would like to ensure that $\mathbf{m}_{i}$ cannot be updated in any of the previous directions without actually increasing the misfit. Thus, in symbols, we require that

$$
\chi\left(\mathbf{m}_{i}\right) \leq \chi\left(\mathbf{m}_{i}+\gamma \mathbf{h}_{j}\right), \quad \text { for any } \gamma \text { and } j=0, \ldots, i-1 .
$$

An iterate $\mathbf{m}_{i}$ that satisfies equation (8.58) is said to be optimal with respect to the directions $\mathbf{h}_{j}$. Clearly, equation (8.58) implies that $\chi\left(\mathbf{m}_{i}+\gamma \mathbf{h}_{j}\right)$ has a minimum at $\gamma=0$. Thus, its derivative must vanish at $\gamma=0$, which is equivalent to

$$
\mathbf{h}_{j}^{T} \mathbf{r}_{i}=0, \quad j=0, \ldots, i-1 .
$$

Equation (8.59) means that model $\mathbf{m}_{i}$ is optimal with respect to all the previous descent directions provided that they are orthogonal to the current residual $\mathbf{r}_{i}=\mathbf{b}-\mathbf{H m}_{i}$.

Interestingly, we have already computed an optimal model unconsciously during the first iteration of the steepest-descent method. In fact, the first steepest-descent update $\mathbf{m}_{1}$ is optimal with respect to the initial descent direction $-\nabla \chi\left(\mathbf{m}_{0}\right)=\mathbf{h}_{0}=\mathbf{r}_{0}$. To see this, we simply compute

$$
\mathbf{h}_{0}^{T} \mathbf{r}_{1}=\mathbf{r}_{0}^{T}\left(\mathbf{b}-\mathbf{H} \mathbf{m}_{0}-\gamma_{0} \mathbf{H} \mathbf{h}_{0}\right)=\mathbf{r}_{0}^{T} \mathbf{r}_{0}-\gamma_{0} \mathbf{r}_{0}^{T} \mathbf{H} \mathbf{h}_{0}=0,
$$

where the last identity follows from the subsitution of $\gamma_{0}$ by equation (8.56) for $k=0$. Thus, in a steepestdescent method, the first update is, by design, optimal to the previous descent direction.

The question then is, how to automatically construct a whole sequence of descent directions such that the current iterate is always optimal with respect to them, in the sense of equation (8.59). For this, we consider a descent direction $\mathbf{h}_{i}$ that updates $\mathbf{m}_{i}$ to $\mathbf{m}_{i+1}$, that is

$$
\mathbf{m}_{i+1}=\mathbf{m}_{i}+\mathbf{h}_{i} .
$$

Using equation (8.59), we find a necessary condition for $\mathbf{h}_{i}$,

$$
\mathbf{h}_{j}^{T} \mathbf{r}_{i+1}=\mathbf{h}_{j}^{T}\left(\mathbf{b}-\mathbf{H} \mathbf{m}_{i}-\mathbf{H h} \mathbf{h}_{i}\right)=\mathbf{h}_{j}^{T}\left(\mathbf{r}_{i}-\mathbf{H h}{ }_{i}\right) .
$$

Assuming that (8.59) is satisfied for all $j=0, \ldots, i-1$, we then find that $\mathbf{r}_{i+1}$ is also optimal to the previous descent directions, when

$$
\mathbf{h}_{j}^{T} \mathbf{H h} \mathbf{h}_{i}=0, \quad j=0, \ldots, i-1 .
$$

Vectors $\mathbf{h}_{j}$ and $\mathbf{h}_{i}$ that satisfy (8.63) are said to be H-orthogonal. Equation (8.63) thus implies that descent directions must be mutually $\mathrm{H}$-orthogonal in order to ensure that successive iterates remain optimal with respect to the earlier descent directions.

Equipped with a criterion that the descent directions need to meet, we proceed with their actual construction. For simplicity, we try an ansatz where the next descent direction $\mathbf{h}_{i+1}$ is equal to the direction of steepest descent, $-\nabla \chi\left(\mathbf{m}_{i+1}\right)=\mathbf{r}_{i+1}$ plus a scaled version of the previous descent direction $\mathbf{h}_{i}$, that is

$$
\mathbf{h}_{i+1}=\mathbf{r}_{i+1}-\beta_{i} \mathbf{h}_{i} .
$$

Requiring condition (8.63) to hold for $\mathbf{h}_{i}$ and $\mathbf{h}_{i+1}$, gives an expression for the scaling factor $\beta_{i}$,

$$
\beta_{i}=\frac{\mathbf{h}_{i}^{T} \mathbf{H r}_{i+1}}{\mathbf{h}_{i}^{T} \mathbf{H h}_{i}} .
$$

It remains to be shown that

$$
\mathbf{h}_{j}^{T} \mathbf{H h}_{i+1}=0
$$


also holds for $j \neq i$, that is, for all $j=0, \ldots, i-1$. We try to do so by induction with respect to the iteration index $i$. First, for $i=0$, we have

$$
\mathbf{h}_{0}^{T} \mathbf{H} \mathbf{h}_{1}=\mathbf{r}_{0}^{T} \mathbf{H}\left(\mathbf{r}_{1}-\beta_{0} \mathbf{r}_{0}\right)=0,
$$

where we directly used the definition of $\beta_{0}$. Thus, we have established the induction anchor. To proceed, we assume that $\mathbf{m}_{i}$ is optimal with respect to the descent directions $\mathbf{h}_{j}$ for $j=0, \ldots, i-1$, and that they are mutually H-orthogonal. We then find

$$
\mathbf{h}_{j}^{T} \mathbf{r}_{i+1}=\mathbf{h}_{j}^{T}\left[\mathbf{b}-\mathbf{H}\left(\mathbf{m}_{i}+\gamma_{i} \mathbf{h}_{i}\right)\right]=\mathbf{h}_{j}^{T} \mathbf{r}_{i}-\gamma_{i} \mathbf{h}_{j}^{T} \mathbf{H} \mathbf{h}_{i}=0, \quad \text { for } j=0, \ldots, i .
$$

For $j<i$, the term $\mathbf{h}_{j}^{T} \mathbf{r}_{i}$ vanishes because $\mathbf{m}_{i}$ is optimal with respect to the directions $\mathbf{h}_{j}$, and the second term, $\mathbf{h}_{j}^{T} \mathbf{H h} \mathbf{h}_{i}$, vanishes because of the assumed H-orthogonality. For $j=i$, equation (8.68) holds because of the definition of $\gamma_{i}$ from (8.56). Thus, we already know that $\mathbf{m}_{i+1}$ is optimal with respect to the descent directions $\mathbf{h}_{j \leq i}$.

To finally show (8.66), we note that equation (8.68) implies orthogonality of $\mathbf{r}_{i+1}$ with respect to all previous descent directions $\mathbf{h}_{j}$ with $j=0, \ldots, i$, and thus orthogonality to the space $H_{i}=\operatorname{span}\left\{\mathbf{h}_{0}, \ldots, \mathbf{h}_{i}\right\}$. Furthermore, equation (8.64) suggests that the residual $\mathbf{r}_{i}$ is a linear combination of the descent directions $\mathbf{h}_{i}$ and $\mathbf{h}_{i-1}$, which implies that they span the same space, $H_{i}=\operatorname{span}\left\{\mathbf{r}_{0}, \ldots, \mathbf{r}_{i}\right\}$. In symbols:

$$
\mathbf{r}_{i+1} \perp H_{i}=\operatorname{span}\left\{\mathbf{h}_{0}, \ldots, \mathbf{h}_{i}\right\}=\operatorname{span}\left\{\mathbf{r}_{0}, \ldots, \mathbf{r}_{i}\right\} .
$$

Furthermore, we have

$$
\mathbf{H h}_{j}=\frac{1}{\gamma_{j}}\left(\mathbf{r}_{j}-\mathbf{r}_{j+1}\right)
$$

which implies that

$$
\mathbf{H h}_{j} \in \operatorname{span}\left\{\mathbf{r}_{0}, \ldots, \mathbf{r}_{j+1}\right\} \subset \operatorname{span}\left\{\mathbf{h}_{0}, \ldots, \mathbf{h}_{i}\right\}=H_{i}, \quad \text { for } j=0, \ldots, i-1 .
$$

As a consequence of (8.71), each $\mathbf{H} \mathbf{h}_{j}$ with $j=0, \ldots, i-1$ can be expressed as a linear combination of vectors $\mathbf{h}_{0}$ to $\mathbf{h}_{i}$. Then, using (8.64) and the assumed H-orthogonality of $\mathbf{h}_{j<i}$ and $\mathbf{h}_{i}$, we have

$$
\mathbf{h}_{j}^{T} \mathbf{H} \mathbf{h}_{i+1}=\left(\mathbf{H} \mathbf{h}_{j}\right)^{T} \mathbf{h}_{i+1}=\left(\mathbf{H} \mathbf{h}_{j}\right)^{T} \mathbf{r}_{i+1}=0, \quad \text { for } j=0, \ldots, i-1 .
$$

The last identity follows from (8.68) and $\mathbf{H h} \mathbf{h}_{j} \in H_{i}$. For $j=i$, (8.66) follows from the construction of $\beta_{i}$. This concludes the inductive proof that the orthogonality relations (8.59) and (8.63) for $i$ and $j=0, \ldots, i-1$ imply the same orthogonality relations for $i+1$ and $j=0, \ldots, i$.

It remains to collect the previous equations into an algorithm: 


\section{Conjugate-gradient method for quadratic misfit functions}

1. Choose an initial model, $\mathbf{m}_{0}$. Set $i=0$ and $\mathbf{h}_{0}=\mathbf{r}_{0}$.

2. Compute the optimal step length $\gamma_{i}$ as

$$
\gamma_{i}=\frac{\mathbf{h}_{i}^{T} \mathbf{r}_{i}}{\mathbf{h}_{i}^{T} \mathbf{H} \mathbf{h}_{i}}
$$

3. Update $\mathbf{m}_{i}$ via

$$
\mathbf{m}_{i+1}=\mathbf{m}_{i}+\gamma_{i} \mathbf{h}_{i}
$$

4. Compute the residual (gradient) for the next iterate, $\mathbf{r}_{i+1}$.

5. Compute the descent direction for the next iteration, $\mathbf{h}_{i+1}$, according to

$$
\begin{gathered}
\beta_{i}=\frac{\mathbf{h}_{i}^{T} \mathbf{H r}_{i+1}}{\mathbf{h}_{i}^{T} \mathbf{H h}_{i}}, \\
\mathbf{h}_{i+1}=\mathbf{r}_{i+1}-\beta_{i} \mathbf{h}_{i} .
\end{gathered}
$$

6. Set $i \rightarrow i+1$, go back to (2) and repeat as often as needed.

An interesting corollary of the orthogonality relations (8.59) and (8.63) is that also the residuals are automatically mutually orthogonal. In fact, using the definition of the optimal step length $\gamma_{i}$ and $\mathbf{r}_{i+1}=\mathbf{r}_{i}-\gamma_{i} \mathbf{H} \mathbf{h}_{i}$, we find

$$
\mathbf{r}_{i}^{T} \mathbf{r}_{i+1}=\mathbf{r}_{i}^{T} \mathbf{r}_{i}-\frac{\mathbf{h}_{i}^{T} \mathbf{r}_{i}}{\mathbf{h}_{i}^{T} \mathbf{H} \mathbf{h}_{i}} \mathbf{r}_{i}^{T} \mathbf{H} \mathbf{h}_{i} .
$$

Furthermore, the mutual H-orthogonality of the descent directions implies

$$
\mathbf{r}_{i}^{T} \mathbf{H} \mathbf{h}_{i}=\left(\mathbf{h}_{i}+\beta_{i} \mathbf{h}_{i-1}\right)^{T} \mathbf{H} \mathbf{h}_{i}=\mathbf{h}_{i}^{T} \mathbf{H} \mathbf{h}_{i}
$$

and from the orthogonality of descent directions and residuals, we have

$$
\mathbf{h}_{i}^{T} \mathbf{r}_{i}=\left(\mathbf{r}_{i}-\beta_{i-1} \mathbf{h}_{i-1}\right)^{T} \mathbf{r}_{i}=\mathbf{r}_{i}^{T} \mathbf{r}_{i}
$$

Substituting equations (8.78) and (8.79) back into equation (8.77), we find

$$
\mathbf{r}_{i}^{T} \mathbf{r}_{i+1}=0
$$

Which is the mutual orthogonality of the residuals. Equation (8.80) allows us to derive various alternatives to equation (8.75) for the computation of $\beta_{i}$, which will be useful in the derivation of conjugate-gradient algorithms for non-quadratic misfit function that we will cover in section 8.5.3. In fact, using (8.80), yields

$$
\mathbf{h}_{i}^{T} \mathbf{H} \mathbf{r}_{i+1}=\frac{1}{\gamma_{i}}\left(\mathbf{r}_{i}-\mathbf{r}_{i+1}\right)^{T} \mathbf{r}_{i+1}=-\frac{1}{\gamma_{i}}\left|\mathbf{r}_{i+1}\right|^{2},
$$

and

$$
\mathbf{h}_{i}^{T} \mathbf{H} \mathbf{h}_{i}=\frac{1}{\gamma_{i}}\left(\mathbf{r}_{i}-\mathbf{r}_{i+1}\right)^{T} \mathbf{h}_{i}=\frac{1}{\gamma_{i}} \mathbf{r}_{i}^{T} \mathbf{h}_{i}=\frac{1}{\gamma_{i}}\left|\mathbf{r}_{i}\right|^{2} .
$$

With the help of equations (8.81) and (8.82), $\beta_{i}$ can now be computed without the explicit vector-matrix product:

$$
\beta_{i}=-\frac{\left|\mathbf{r}_{i+1}\right|^{2}}{\left|\mathbf{r}_{i}\right|^{2}} .
$$

Alternatively, we have, again as a result of orthogonality, 


$$
\beta_{i}=-\frac{\mathbf{r}_{i+1}^{T}\left(\mathbf{r}_{i+1}-\mathbf{r}_{i}\right)}{\left|\mathbf{r}_{i}\right|^{2}}
$$

and

$$
\beta_{i}=-\frac{\mathbf{r}_{i+1}^{T}\left(\mathbf{r}_{i+1}-\mathbf{r}_{i}\right)}{\mathbf{h}_{i}^{T}\left(\mathbf{r}_{i}-\mathbf{r}_{i+1}\right)}
$$

\subsubsection{The benefit of using conjugate descent directions}

To reveal the benefit of conjugate directions in the case of a quadratic misfit function with an $n \times n$-matrix $\mathbf{H}$, we compute the $n^{\text {th }}$ iterate $\mathbf{m}_{n}$. Starting from some model $\mathbf{m}_{j+1}$, the repeated application of the general iterative scheme, $\mathbf{m}_{i+1}=\mathbf{m}_{i}+\gamma_{i} \mathbf{h}_{i}$, yields

$$
\mathbf{m}_{n}=\mathbf{m}_{j+1}+\sum_{i=j+1}^{n-1} \gamma_{i} \mathbf{h}_{i},
$$

for $j$ between 0 and $n-1$. Subtracting $\hat{\mathbf{m}}$ from both sides of equation (8.86) and multiplying by $\mathbf{H}$, gives

$$
-\mathbf{r}_{n}=-\mathbf{r}_{j+1}+\sum_{i=j+1}^{n-1} \gamma_{i} \mathbf{H} \mathbf{h}_{i}
$$

With the help of the first orthogonality condition from equation (8.59), we find

$$
-\mathbf{h}_{j}^{T} \mathbf{r}_{n}=\sum_{i=j+1}^{n-1} \gamma_{i} \mathbf{h}_{j}^{T} \mathbf{H} \mathbf{h}_{i} .
$$

Since the descent directions are H-orthogonal in the sense of equation (8.63), the right-hand side vanishes,

$$
\mathbf{h}_{j}^{T} \mathbf{r}_{n}=0
$$

for any $j$ between 0 and $n-1$. Since the mutually orthogonal descent directions, $\mathbf{h}_{0}, \mathbf{h}_{1}, \ldots, \mathbf{h}_{n-1}$, form a basis of the $n$-dimensional model space $\mathbb{M}$, it follows from equation (8.89) that $\mathbf{r}_{n}=\mathbf{0}$. This means that the $n^{\text {th }}$ iterate $\mathbf{m}_{n}$ is equal to the minimum $\hat{\mathbf{m}}$ of the quadratic misfit functional $\chi$, that is, equal to the solution of the linear equation $\mathbf{H x}=\mathbf{b}$. Clearly, the advantage of using an algorithm that satisfies the orthogonality conditions (8.59) and (8.63) is that the solution is reached after at most $n$ iterations, provided that numerical errors are negligible. This is in contrast to the steepest-descent method where the minimum may not be reached within any finite number of iterations.

\subsubsection{The conjugate-gradient method for non-quadratic misfit functions}

The conjugate-gradient method for general, non-quadratic misfit functions is essentially a leap of faith from the quadratic version considered above. It rests on the simple realisation that the residual is equal to the negative gradient of the misfit, that is,

$$
\mathbf{r}_{i}=-\nabla \chi\left(\mathbf{m}_{i}\right)
$$

Thus, replacing residuals by negative gradients in the original algorithm, we obtain the conjugate-gradient method for non-quadratic misfit functions: 


\section{Conjugate-gradient method for general misfit functions}

1. Choose an initial model, $\mathbf{m}_{0}$. Set $i=0$ and $\mathbf{h}_{0}=-\nabla \chi\left(\mathbf{m}_{0}\right)$.

2. Compute the optimal step length $\gamma_{i}$, either according to (8.27) or using a line search.

3. Update $\mathbf{m}_{i}$ via

$$
\mathbf{m}_{i+1}=\mathbf{m}_{i}+\gamma_{i} \mathbf{h}_{i}
$$

4. Compute the gradient for the next iterate, $\nabla \chi\left(\mathbf{m}_{i+1}\right)$.

5. Compute the descent direction for the next iteration, $\mathbf{h}_{i+1}$, according to

$$
\begin{gathered}
\beta_{i}=-\frac{\left|\nabla \chi\left(\mathbf{m}_{i+1}\right)\right|_{2}^{2}}{\left|\nabla \chi\left(\mathbf{m}_{i}\right)\right|_{2}^{2}}, \\
\mathbf{h}_{i+1}=-\nabla \chi\left(\mathbf{m}_{i+1}\right)-\beta_{i} \mathbf{h}_{i} .
\end{gathered}
$$

6. Set $i \rightarrow i+1$, go back to (2) and repeat as often as needed.

Of course, for a non-quadratic misfit, the descent directions are not generally orthogonal, and the algorithm will not generally converge to the minimum in at most $n$ iterations. The conjugate-gradient algorithm as outlined above, is commonly referred to as the Fletcher-Reeves method (Fletcher \& Reeves, 1964). Popular variants follow directly from the alternative equations (8.84) and (8.85) for the computation of $\beta_{i}$. The Polak-Ribière method (Polak \& Ribière, 1969) defines $\beta_{i}$ as

$$
\beta_{i}=-\frac{\nabla \chi\left(\mathbf{m}_{i+1}\right)^{T}\left[\nabla \chi\left(\mathbf{m}_{i+1}\right)-\nabla \chi\left(\mathbf{m}_{i}\right)\right]}{\nabla \chi\left(\mathbf{m}_{i}\right)^{T} \nabla \chi\left(\mathbf{m}_{i}\right)},
$$

and the Hestenes-Stiefel method (Hestenes \& Stiefel, 1952) uses

$$
\beta_{i}=-\frac{\nabla \chi\left(\mathbf{m}_{i+1}\right)^{T}\left[\nabla \chi\left(\mathbf{m}_{i+1}\right)-\nabla \chi\left(\mathbf{m}_{i}\right)\right]}{\mathbf{h}_{i}^{T}\left[\nabla \chi\left(\mathbf{m}_{i+1}\right)-\nabla \chi\left(\mathbf{m}_{i}\right)\right]}
$$

All definitions of $\beta_{i}$ are equivalent for quadratic $\chi$. In the more general case, the preferred formula appears to be a matter of personal preference, and again, the No-Free-Lunch theorem precludes any statements concerning general universality of any of the variants.

Nonlinear conjugate gradients: Figure 8.7 shows examples of nonlinear optimisation using the FletcherReeves variant of the conjugate-gradient method. For the quadratic function (8.28), the method converges to the global minimum in two iterations, as predicted theoretically for a two-dimensional model space. In the case of the Rosenbrock function (8.29), more iterations are needed to approach the minimum because it is not quadratic.

A comparison to the other two conjugate-gradient variants, Polak-Ribière and Hestenes-Stiefel, is shown in figure 8.8. In this particular example, differences between the two methods are of little practical relevance.

\subsection{Quasi-Newton methods}

The Gauss-Newton, Levenberg and Levenberg-Marquardt methods, introduced in section 8.4.2, implement strategies to circumvent the often computationally expensive calculation of the full Hessian, $\mathbf{H}$. Their common theme is the approximation of second derivatives by first derivatives. 
a) quadratic function

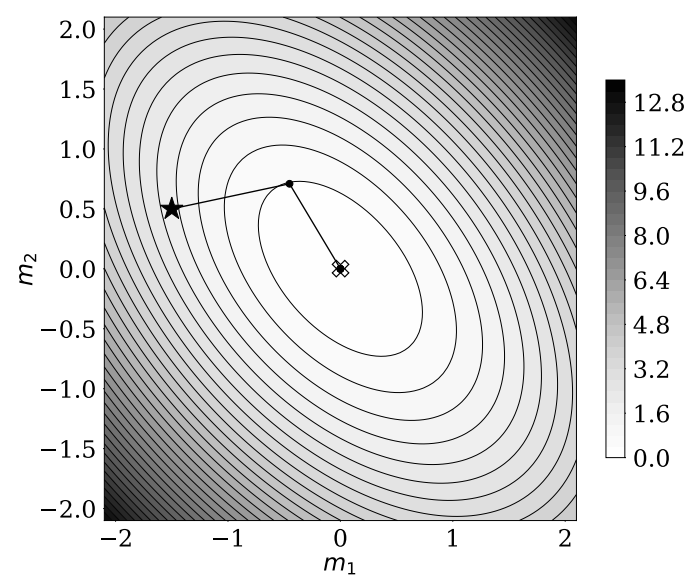

b) Rosenbrock function

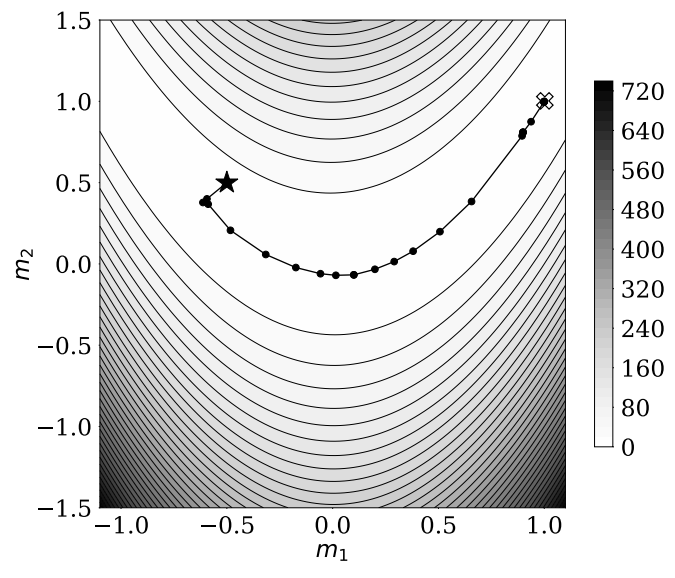

Fig. 8.7: Nonlinear optimisation of an exactly quadratic function (8.28) (a) and the Rosenbrock function (8.29) (b) using the Fletcher-Reeves variant of conjugate gradients. As predicted theoretically, the conjugate-gradient method converges in $n=2$ iterations to the global minimum of the quadratic function. Around 25 iterations are needed to approach the global minimum of the Rosenbrock function. [Nonlinear optimisation in 2D]

a) Polak-Ribière

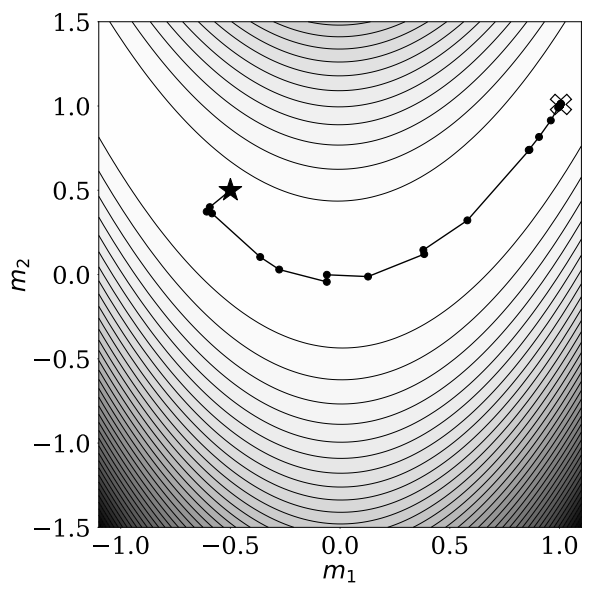

b) Hestenes-Stiefel

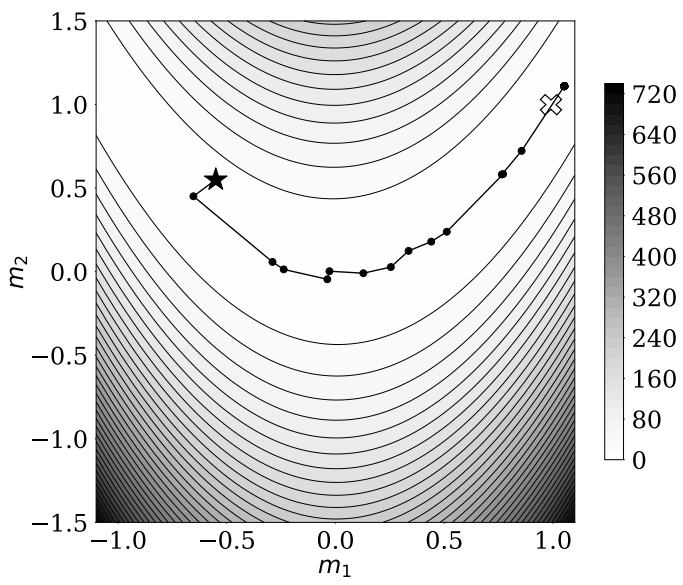

Fig. 8.8: Comparison of the Polak-Ribière (a) and the Hestenes-Stiefel (b) variants of the conjugate-gradient algorithm for the Rosenbrock function (8.29). Though the Hestenes-Stiefel algorithm misses the global optimum slightly due to getting stuck in a region of very small gradients, there are few practically significant differences between the two methods in this specific scenario. [Nonlinear optimisation in 2D] 
Quasi-Newton methods follow a different approach by trying to construct the matrices $\mathbf{A}_{i}$ in the general descent scheme (8.20) such that they approximate the inverse Hessian, $\mathbf{H}^{-1}$. Starting from some initial guess $\mathbf{A}_{0}$, the matrix is updated in each iteration, to yield matrices $\mathbf{A}_{1}, \mathbf{A}_{2}, \ldots$, each being hopefully a better approximation of $\mathbf{H}^{-1}$ than its predecessor. The descent directions are then chosen as $\mathbf{h}_{i}=-\mathbf{A}_{i} \nabla \chi\left(\mathbf{m}_{i}\right)$. To illustrate the basic idea of how to obtain the sequence of matrices $\mathbf{A}_{i}$, we begin with the simplifying assumption that the misfit $\chi$ is perfectly quadratic in $\mathbf{m}$,

$$
\chi(\mathbf{m})=\frac{1}{2} \mathbf{m}^{T} \mathbf{H m}+\mathbf{b}^{T} \mathbf{m} .
$$

Taking the first derivative of equation (8.96), implies the following equation,

$$
\mathbf{H}\left(\mathbf{m}_{i+1}-\mathbf{m}_{i}\right)=\nabla \chi\left(\mathbf{m}_{i+1}\right)-\nabla \chi\left(\mathbf{m}_{i}\right) .
$$

This suggests that, for $\mathbf{A}_{i+1}$ to be a reasonable approximation of $\mathbf{H}^{-1}$, we may want to impose the condition

$$
\mathbf{A}_{i+1}^{-1}\left(\mathbf{m}_{i+1}-\mathbf{m}_{i}\right)=\nabla \chi\left(\mathbf{m}_{i+1}\right)-\nabla \chi\left(\mathbf{m}_{i}\right) \text {. }
$$

Equation (8.98), known as the quasi-Newton equation or secant equation, does not uniquely determine the matrix $\mathbf{A}_{i+1}$ or its inverse. Numerous options have been proposed, some of which will be explained in the following sections. Before doing so, we lighten the notation by assigning new symbols to the model and gradient differences in the quasi-Newton equation,

$$
\mathbf{s}_{i}=\mathbf{m}_{i+1}-\mathbf{m}_{i}, \quad \mathbf{y}_{i}=\nabla \chi\left(\mathbf{m}_{i+1}\right)-\nabla \chi\left(\mathbf{m}_{i}\right),
$$

so that it simplifies to

$$
\mathbf{A}_{i+1}^{-1} \mathbf{s}_{i}=\mathbf{y}_{i} .
$$

\subsubsection{The SR1 method}

The SR1 method derives its name from a symmetric, rank-1 update of $\mathbf{A}_{i}^{-1}$ that takes the general form

$$
\mathbf{A}_{i+1}^{-1}=\mathbf{A}_{i}^{-1}+\sigma \mathbf{v v}^{T},
$$

with $\sigma= \pm 1$ and a vector $\mathbf{v}$ that remains to be determined. Substituting (8.101) into (8.100), gives

$$
\mathbf{y}_{i}-\mathbf{A}_{i}^{-1} \mathbf{s}_{i}=\left(\sigma \mathbf{v}^{T} \mathbf{s}_{i}\right) \mathbf{v} .
$$

Since the term in brackets is a scalar, we infer that the vector $\mathbf{v}$ must be parallel to the vector $\mathbf{y}_{i}-\mathbf{A}_{i}^{-1} \mathbf{s}_{i}$, meaning that it can be written as

$$
\mathbf{v}=\gamma\left(\mathbf{y}_{i}-\mathbf{A}_{i}^{-1} \mathbf{s}_{i}\right)
$$

Upon inserting (8.103) back into (8.102), we find

$$
\mathbf{y}_{i}-\mathbf{A}_{i}^{-1} \mathbf{s}_{i}=\sigma \gamma^{2}\left[\mathbf{s}_{i}^{T}\left(\mathbf{y}_{i}-\mathbf{A}_{i}^{-1} \mathbf{s}_{i}\right)\right]\left(\mathbf{y}_{i}-\mathbf{A}_{i}^{-1} \mathbf{s}_{i}\right) .
$$

Realising that the term in square brackets must be equal to 1 , we can uniquely determine $\sigma$ and $\gamma$,

$$
\sigma=\operatorname{sign}\left[\mathbf{s}_{i}^{T}\left(\mathbf{y}_{i}-\mathbf{A}_{i}^{-1} \mathbf{s}_{i}\right)\right], \quad \gamma=\frac{1}{\sqrt{\left|\mathbf{s}_{i}^{T}\left(\mathbf{y}_{i}-\mathbf{A}_{i}^{-1} \mathbf{s}_{i}\right)\right|}} .
$$

Finally combining (8.105) with (8.101), we obtain the complete update, 


$$
\mathbf{A}_{i+1}^{-1}=\mathbf{A}_{i}^{-1}+\frac{\left(\mathbf{y}_{i}-\mathbf{A}_{i}^{-1} \mathbf{s}_{i}\right)\left(\mathbf{y}_{i}-\mathbf{A}_{i}^{-1} \mathbf{s}_{i}\right)^{T}}{\mathbf{s}_{i}^{T}\left(\mathbf{y}_{i}-\mathbf{A}_{i}^{-1} \mathbf{s}_{i}\right)}
$$

Invoking the Sherman-Morrison formula, given in the grey box below, yields the corresponding update equation for $\mathbf{A}$,

$$
\mathbf{A}_{i+1}=\mathbf{A}_{i}+\frac{\left(\mathbf{s}_{i}-\mathbf{A}_{i} \mathbf{y}_{i}\right)\left(\mathbf{s}_{i}-\mathbf{A}_{i} \mathbf{y}_{i}\right)^{T}}{\left(\mathbf{s}_{i}-\mathbf{A}_{i} \mathbf{y}_{i}\right)^{T} \mathbf{y}_{i}}
$$

The Sherman-Morrison formula: Named after Jack Sherman and Winifred J. Morrison (Sherman \& Morrison, 1950), the equation provides the inverse of a rank-one update of a square and invertible matrix A,

$$
\overline{\mathbf{A}}=\mathbf{A}+\mathbf{a b}^{T},
$$

with arbitrary vectors $\mathbf{a}$ and $\mathbf{b}$. This inverse is given by

$$
\overline{\mathbf{A}}^{-1}=\mathbf{A}^{-1}-\frac{\mathbf{A}^{-1} \mathbf{a b}^{T} \mathbf{A}^{-1}}{1+\mathbf{b}^{T} \mathbf{A}^{-1} \mathbf{a}},
$$

as can be easily verified by multiplying (8.108) and (8.109). In our context, the Sherman-Morrison formula is a particularly useful tool for the derivation of quasi-Newton updates and their inverses.

Equation (8.107) together with the general gradient descent scheme (8.20) forms the SR1 method:

\section{SR1 method}

1. Choose an initial model, $\mathbf{m}_{0}$, and an initial, positive definite matrix $\mathbf{A}_{0}$. Set $i=0$.

2. Compute the descent direction $\mathbf{h}_{i}=-\mathbf{A}_{i} \nabla \chi\left(\mathbf{m}_{i}\right)$.

3. Compute the optimal step length, $\gamma_{i}$, using a line search.

4. Update $\mathbf{m}_{i}$ via

$$
\mathbf{m}_{i+1}=\mathbf{m}_{i}+\gamma_{i} \mathbf{h}_{i}
$$

5. Compute the new gradient, $\nabla \chi\left(\mathbf{m}_{i+1}\right)$, and the differences

$$
\mathbf{s}_{i}=\mathbf{m}_{i+1}-\mathbf{m}_{i}, \quad \mathbf{y}_{i}=\nabla \chi\left(\mathbf{m}_{i+1}\right)-\nabla \chi\left(\mathbf{m}_{i}\right) .
$$

6. Update the matrix,

$$
\mathbf{A}_{i+1}=\mathbf{A}_{i}+\frac{\left(\mathbf{s}_{i}-\mathbf{A}_{i} \mathbf{y}_{i}\right)\left(\mathbf{s}_{i}-\mathbf{A}_{i} \mathbf{y}_{i}\right)^{T}}{\left(\mathbf{s}_{i}-\mathbf{A}_{i} \mathbf{y}_{i}\right)^{T} \mathbf{y}_{i}} .
$$

7. Set $i \rightarrow i+1$, go back to (2) and repeat as often as needed.

Though the SR1 method is beautifully simple, it does not come without problems. In fact, the denominator in (8.106) and (8.107) may become small and negative, meaning that the update is not guaranteed to be positive definite. As a consequence, the line search for an optimal step length will fail. Furthermore, a small denominator may lead to numerical instabilities. In practice, this may be avoided by simply skipping updates, where the absolute value of the denominator is below some user-defined threshold, which then is a tuning parameter of the method.

A more profound limitation of SR1 relates to the special case $\mathbf{s}_{i}^{T}\left(\mathbf{y}_{i}-\mathbf{A}_{i}^{-1} \mathbf{s}_{i}\right)=0$ and $\mathbf{y}_{i}-\mathbf{A}_{i}^{-1} \mathbf{s}_{i} \neq \mathbf{0}$, which contradicts equation (8.104). Hence, a symmetric, rank-1 update actually does not exist. This suggests that updates of at least rank 2 are required in order to ensure that updates exist and are positive definite. A popular option is the BFGS method, treated in the following section. 


\subsubsection{The BFGS method}

Simultaneously studied by Charles George Broyden, Roger Fletcher, Donald Goldfarb and David Shanno (Broyden, 1970; Fletcher, 1970; Goldfarb, 1970; Shanno, 1970), the method named after them applies specific modifications to the current matrix $\mathbf{A}_{i}$ to obtain an updated matrix $\mathbf{A}_{i+1}$ that remains positive definite. To derive the updating equations, we first compute an auxiliary matrix,

$$
\tilde{\mathbf{A}}_{i}^{-1}=\mathbf{A}_{i}^{-1}-\frac{\left(\mathbf{A}_{i}^{-1} \mathbf{s}_{i}\right)\left(\mathbf{A}_{i}^{-1} \mathbf{s}_{i}\right)^{T}}{\mathbf{s}_{i}^{T} \mathbf{A}_{i}^{-1} \mathbf{s}_{i}}
$$

Equation (8.113) constitutes a rank-1 modification of $\mathbf{A}_{i}^{-1}$ because the update matrix $\left(\mathbf{A}_{i}^{-1} \mathbf{s}_{i}\right)\left(\mathbf{A}_{i}^{-1} \mathbf{s}_{i}\right)^{T}$ has only one linearly independent row. Multiplying $\tilde{\mathbf{A}}_{i}^{-1}$ with $\mathbf{s}_{i}$ from the right, we find $\tilde{\mathbf{A}}_{i}^{-1} \mathbf{s}_{i}=\mathbf{0}$. To satisfy the quasi-Newton equation, we add another rank-1 modification,

$$
\mathbf{A}_{i+1}^{-1}=\tilde{\mathbf{A}}_{i}^{-1}+\frac{\mathbf{y}_{i} \mathbf{y}_{i}^{T}}{\mathbf{y}_{i}^{T} \mathbf{s}_{i}}
$$

Combining (8.113) and (8.114), we find the complete BFGS update formula

$$
\mathbf{A}_{i+1}^{-1}=\mathbf{A}_{i}^{-1}-\frac{\left(\mathbf{A}_{i}^{-1} \mathbf{s}_{i}\right)\left(\mathbf{A}_{i}^{-1} \mathbf{s}_{i}\right)^{T}}{\mathbf{s}_{i}^{T} \mathbf{A}_{i}^{-1} \mathbf{s}_{i}}+\frac{\mathbf{y}_{i} \mathbf{y}_{i}^{T}}{\mathbf{y}_{i}^{T} \mathbf{s}_{i}}
$$

As in the SR1 method, the matrices $\mathbf{A}_{i}^{-1}$ serve as approximations to the Hessian $\mathbf{H}$ in the sense of the quasiNewton equation, and they can be used to compute descent directions $\mathbf{h}_{i}=-\mathbf{A}_{i} \nabla \chi\left(\mathbf{m}_{i}\right)$. To avoid the potentially expensive inversion of $\mathbf{A}_{i}^{-1}$, updates of $\mathbf{A}_{i}$ may again be computed directly with the help of the ShermanMorrison formula (8.109), leading to

$$
\mathbf{A}_{i+1}=\left(\mathbf{I}-\rho_{i} \mathbf{s}_{i} \mathbf{y}_{i}^{T}\right) \mathbf{A}_{i}\left(\mathbf{I}-\rho_{i} \mathbf{y}_{i} \mathbf{s}_{i}^{T}\right)+\rho_{i} \mathbf{s}_{i} \mathbf{s}_{i}^{T}
$$

with

$$
\rho_{i}=\frac{1}{\mathbf{y}_{i}^{T} \mathbf{s}_{i}} .
$$

Already equation (8.117) indicates that the BFGS method requires $\mathbf{y}_{i}^{T} \mathbf{s}_{i}$ to be non-zero. Additional constraints on $\mathbf{y}_{i}^{T} \mathbf{s}_{i}$ arise from the desideratum that updates remain positive definite. In fact, left- and right-multiplying (8.116) with some arbitrary vector $\mathbf{x}$, gives

$$
\mathbf{x}^{T} \mathbf{A}_{i+1} \mathbf{x}=\mathbf{z}^{T} \mathbf{A}_{i} \mathbf{z}+\rho_{i}\left(\mathbf{x}^{T} \mathbf{s}_{i}\right)^{2},
$$

where we defined $\mathbf{z}=\left(\mathbf{I}-\rho_{i} \mathbf{y}_{i} \mathbf{s}_{i}^{T}\right) \mathbf{x}$. It follows from (8.118) that positive definiteness of $\mathbf{A}_{i+1}$, given the positive definiteness of $\mathbf{A}_{i}$, can only be guaranteed if

$$
\rho_{i}^{-1}=\mathbf{y}_{i}^{T} \mathbf{s}_{i}>0 .
$$

Relation (8.119) is commonly referred to as the curvature condition. It is generally met for convex misfit functions. If convexity cannot be ensured a priori, which is usually the case in practice, BFGS updates of $\mathbf{A}_{i}$ need to be skipped it iterations where the curvature condition is not met. We may now formulate the BFGS algorithm: 


\section{BFGS method}

1. Choose an initial model, $\mathbf{m}_{0}$, and an initial, positive definite matrix $\mathbf{A}_{0}$. Set $i=0$.

2. Compute the descent direction $\mathbf{h}_{i}=-\mathbf{A}_{i} \nabla \chi\left(\mathbf{m}_{i}\right)$.

3. Compute the optimal step length, $\gamma_{i}$, using a line search.

4. Update $\mathbf{m}_{i}$ via

$$
\mathbf{m}_{i+1}=\mathbf{m}_{i}+\gamma_{i} \mathbf{h}_{i} .
$$

5. Compute the new gradient, $\nabla \chi\left(\mathbf{m}_{i+1}\right)$, and the differences

$$
\mathbf{s}_{i}=\mathbf{m}_{i+1}-\mathbf{m}_{i}, \quad \mathbf{y}_{i}=\nabla \chi\left(\mathbf{m}_{i+1}\right)-\nabla \chi\left(\mathbf{m}_{i}\right) .
$$

6. Update the matrix,

$$
\mathbf{A}_{i+1}=\left(\mathbf{I}-\rho_{i} \mathbf{s}_{i} \mathbf{y}_{i}^{T}\right) \mathbf{A}_{i}\left(\mathbf{I}-\rho_{i} \mathbf{y}_{i} \mathbf{s}_{i}^{T}\right)+\rho_{i} \mathbf{s}_{i} \mathbf{s}_{i}^{T},
$$

with $\rho_{i}=\frac{1}{\mathbf{y}_{i}^{T} \mathbf{s}_{i}}$.

7. Set $i \rightarrow i+1$, go back to (2) and repeat as often as needed.

The update formulas (8.115) and (8.116) may seem somewhat complicated for just satisfying positive definiteness and the quasi-Newton equation. In fact, for quadratic misfit functions, it can be shown that these choices have additional benefits (e.g. Alt, 2002; Nocedal \& Wright, 1999). Most importantly, the BFGS iteration for quadratic misfit functions produces descent directions that are mutually H-orthogonal in the sense of equation (8.63). This implies, as shown in section 8.5.2, that the iteration terminates after at most $n$ iterations with the exact optimal model, $\hat{\mathbf{m}}=\mathbf{m}_{n}$; assuming, of course, that rounding errors can be neglected. Furthermore, the matrices $\mathbf{A}_{i}$ converge towards the inverse Hessian, and after $n$ iterations we have $\mathbf{A}_{n}=\mathbf{H}^{-1}$.

In the vicinity of an extremum where a general misfit $\chi$ can be approximated by a quadratic function, BFGS may indeed compute a good approximation of the local Hessian that helps to accelerate convergence. Yet, convergence within a finite number of iterations, as for quadratic misfits, cannot be guaranteed.

\subsubsection{The L-BFGS method}

A drawback of the BFGS method is the need to explicitly compute and store the inverse Hessian approximations $\mathbf{A}_{i}$. The dimension of $\mathbf{A}_{i}$ is $n \times n$, where $n$ is the dimension of the model space $\mathbb{M}$. It follows that the computational and storage requirements of BFGS quickly become prohibitive as the model space dimension increases. This deficiency motivates an approximate version of BFGS, known as limited-memory BFGS or $L$ BFGS method (e.g. Liu \& Nocedal, 1989; Nocedal, 1980).

Before explaining the details of L-BFGS, we demonstrate that the BFGS descent directions can in fact be computed through a sequence of vector-vector multiplications, without any need to explicitly compute or store a matrix. For this, we write the BFGS update of the matrix $\mathbf{A}_{i}$ in equation (8.116) in the more condensed form

$$
\mathbf{A}_{i+1}=\mathbf{V}_{i}^{T} \mathbf{A}_{i} \mathbf{V}_{i}+\rho_{i} \mathbf{s}_{i} \mathbf{s}_{i}^{T}
$$

with the matrix $\mathbf{V}_{i}$ defined as

$$
\mathbf{V}_{i}=\mathbf{I}-\rho_{i} \mathbf{y}_{i} \mathbf{s}_{i}^{T} .
$$

Starting from the initial matrix $\mathbf{A}_{0}$, the repeated application of equation (8.123) yields 


$$
\begin{aligned}
\mathbf{A}_{i} & =\left(\mathbf{V}_{i-1}^{T} \cdots \mathbf{V}_{0}^{T}\right) \mathbf{A}_{0}\left(\mathbf{V}_{0} \cdots \mathbf{V}_{i-1}\right) \\
& +\rho_{0}\left(\mathbf{V}_{i-1}^{T} \cdots \mathbf{V}_{1}^{T}\right) \mathbf{s}_{0} \mathbf{s}_{0}^{T}\left(\mathbf{V}_{1} \cdots \mathbf{V}_{i-1}\right) \\
& +\rho_{1}\left(\mathbf{V}_{i-1}^{T} \cdots \mathbf{V}_{2}^{T}\right) \mathbf{s}_{1} \mathbf{s}_{1}^{T}\left(\mathbf{V}_{2} \cdots \mathbf{V}_{i-1}\right) \\
& +\ldots \\
& +\rho_{i-1} \mathbf{s}_{i-1} \mathbf{s}_{i-1}^{T} .
\end{aligned}
$$

To see how we can obtain the descent direction $\mathbf{h}_{i}=-\mathbf{A}_{i} \nabla \chi\left(\mathbf{m}_{i}\right)$ via equation (8.125) without explicitly building a matrix, we start with the term $\mathbf{A}_{0}\left(\mathbf{V}_{i-1} \cdots \mathbf{V}_{0}^{T}\right) \nabla \chi\left(\mathbf{m}_{i}\right)$, which can be computed with following iteration:

\section{Iteration 1}

1. Set the vector $\mathbf{q}=\nabla \chi\left(\mathbf{m}_{i}\right)$, and the iteration index $n=i-1$.

2. Compute the scalar

$$
\alpha_{n}=\rho_{n} \mathbf{s}_{n}^{T} \mathbf{q}
$$

with $\rho_{n}$ defined in equation (8.117).

3. Set

$$
\mathbf{q} \rightarrow \mathbf{q}-\alpha_{n} \mathbf{y}_{n} .
$$

4. Decrease the iteration index by 1 , that is, $n \rightarrow n-1$. If $n \geq 0$ go back to 2 . Otherwise go to 5 .

5. Compute the vector

$$
\mathbf{r}=\mathbf{A}_{0} \mathbf{q}
$$

As one can see by writing the algorithm explicitly for $n=i-1, \ldots, 0$, that it generates the following sequence of $\mathbf{q}$ vectors:

$$
\left\{\nabla \chi\left(\mathbf{m}_{i}\right), \quad \mathbf{V}_{i-1} \nabla \chi\left(\mathbf{m}_{i}\right), \quad \mathbf{V}_{i-2} \mathbf{V}_{i-1} \nabla \chi\left(\mathbf{m}_{i}\right), \quad \ldots \quad, \mathbf{V}_{0} \cdots \mathbf{V}_{i-1} \nabla \chi\left(\mathbf{m}_{i}\right)\right\} .
$$

The final multiplication with $\mathbf{A}_{0}$ then yields $\mathbf{r}=\mathbf{A}_{0}\left(\mathbf{V}_{0} \cdots \mathbf{V}_{i-1}\right) \nabla \chi\left(\mathbf{m}_{i}\right)$, as desired. Since $\mathbf{q}$ from the individual iterations is subsequently not needed, its values are overwritten, which saves storage. Along the way, Iteration 1 also produces a series of scalars $\alpha_{n}$,

$$
\begin{aligned}
\alpha_{i-1} & =\rho_{i-1} \mathbf{s}_{i-1}^{T} \nabla \chi\left(\mathbf{m}_{i}\right) \\
\alpha_{i-2} & =\rho_{i-2} \mathbf{s}_{i-2}^{T} \mathbf{V}_{i-1} \nabla \chi\left(\mathbf{m}_{i}\right), \\
\alpha_{i-3} & =\rho_{i-3} \mathbf{s}_{i-3}^{T} \mathbf{V}_{i-2} \mathbf{V}_{i-1} \nabla \chi\left(\mathbf{m}_{i}\right), \\
\ldots & =\ldots \\
\alpha_{0} & =\rho_{0} \mathbf{s}_{0}^{T} \mathbf{V}_{1} \cdots \mathbf{V}_{i-1} \nabla \chi\left(\mathbf{m}_{i}\right) .
\end{aligned}
$$

The comparison of this sequence with equation (8.125) suggests that the values of $\alpha_{n}$ may be reused to complete the computation of $\mathbf{A}_{i} \nabla \chi\left(\mathbf{m}_{i}\right)$. Indeed, this can be done using a second iteration:

\section{Iteration 2}

1. Set the iteration index $n=0$.

2. Compute the scalar

$$
\beta=\rho_{n} \mathbf{y}_{n}^{T} \mathbf{r}
$$

3. Set

$$
\mathbf{r} \rightarrow \mathbf{r}+\mathbf{s}_{n}\left(\alpha_{n}-\beta\right) .
$$

4. Increase the iteration index by 1 , that is, $n \rightarrow n+1$. If $n \geq i-1$ go back to 2 . Otherwise stop. 
In the first iteration, for $n=0$, this algorithm transforms the initial $\mathbf{r}=\mathbf{A}_{0}\left(\mathbf{V}_{0} \cdots \mathbf{V}_{i-1}\right) \nabla \chi\left(\mathbf{m}_{i}\right)$ from Iteration 1 into the new

$$
\mathbf{r}=\mathbf{V}_{0}^{T} \mathbf{A}_{0}\left(\mathbf{V}_{0} \cdots \mathbf{V}_{i-1}\right) \nabla \chi\left(\mathbf{m}_{i}\right)+\rho_{0} \mathbf{s}_{0} \mathbf{s}_{0}^{T}\left(\mathbf{V}_{1} \cdots \mathbf{V}_{i-1}\right) \nabla \chi\left(\mathbf{m}_{i}\right)
$$

In other words, the initial $\mathbf{r}$ is left-multiplied by $\mathbf{V}_{0}^{T}$ and the term $\rho_{0} \mathbf{s}_{0} \mathbf{s}_{0}^{T}\left(\mathbf{V}_{1} \cdots \mathbf{V}_{i-1}\right) \nabla \chi\left(\mathbf{m}_{i}\right)$ is added. In the next iteration, the algorithm then left-multiplies by $\mathbf{V}_{1}^{T}$ and adds $\rho_{1} \mathbf{s}_{1} \mathbf{s}_{1}^{T}\left(\mathbf{V}_{2} \cdots \mathbf{V}_{i-1}\right) \nabla \chi\left(\mathbf{m}_{i}\right)$, and so on and so forth. The final $\mathbf{r}$ is then the negative descent direction in the $i^{\text {th }}$ BFGS iteration,

$$
\mathbf{r}=\mathbf{A}_{i} \nabla \chi\left(\mathbf{m}_{i}\right)=-\mathbf{h}_{i}
$$

The only matrix-vector operation contained in Iterations 1 and 2 is the multiplication of $\mathbf{A}_{0}$ with $\mathbf{q}$ in equation (8.128). However, choosing the initial matrix $\mathbf{A}_{0}$ to be diagonal, the computational and storage requirements of $\mathbf{A}_{0} \mathbf{q}$ are the same as for a vector-vector multiplication. Thus, we have already shown that the BFGS algorithm can be used without explicitly computing and storing a large, and usually dense, matrix.

The problem that remains to be solved is the increasing number of vectors $\mathbf{s}_{i}$ and $\mathbf{y}_{i}$ that we need to store. In each BFGS iteration, a new pair of vectors is added. In the ideal case of a quadratic misfit function, where the BFGS iteration converges in $n$ steps, we would need to store $2 n$ vectors of dimension $n$, that is, $2 n^{2}$ numbers. We are therefore back to our original problem of having to store a quantity of numbers that grows quadratically with model space dimension.

At this point, the basic idea of L-BFGS is very simple: Instead of using all vector pairs $\left(\mathbf{s}_{0}, \mathbf{y}_{0}\right), \ldots,\left(\mathbf{s}_{i-1}, \mathbf{y}_{i-1}\right)$ in Iterations 1 and 2, we only use the last $k$ vector pairs $\left(\mathbf{s}_{i-k}, \mathbf{y}_{i-k}\right), \ldots,\left(\mathbf{s}_{i-1}, \mathbf{y}_{i-1}\right)$, where $k$ is typically a small number between 3 and 20. After each completed model update, we delete the vector pair with the lowest index, and we store the next vector pair. Only in the first $k$ iterations, all vector pairs are used, as in the original BFGS algorithm. Limiting the number of vector pairs to $k$, reduces the storage requirements to $2 \mathrm{kn}$. The price to pay is an incorrect computation of the descent direction. The L-BFGS algorithm can be summarised as follows:

\section{L-BFGS method}

1. Choose an initial model, $\mathbf{m}_{0}$, a maximum number of vector pairs, $k$, and set the iteration index $i=0$.

2. If $i=0$, compute the descent direction $\mathbf{h}_{0}=-\nabla \chi\left(\mathbf{m}_{0}\right)$. Otherwise, compute the descent direction $\mathbf{h}_{i}$ by running Iterations 1 and 2 with the $k$ most recent vector pairs $\left(\mathbf{s}_{i-k}, \mathbf{y}_{i-k}\right), \ldots,\left(\mathbf{s}_{i-1}, \mathbf{y}_{i-1}\right)$ and an initial, positive definite matrix $\mathbf{A}_{0, i}$.

3. Compute the optimal step length, $\gamma_{i}$, using a line search.

4. Update $\mathbf{m}_{i}$ via

$$
\mathbf{m}_{i+1}=\mathbf{m}_{i}+\gamma_{i} \mathbf{h}_{i}
$$

5. Compute the new gradient, $\nabla \chi\left(\mathbf{m}_{i+1}\right)$, and the differences

$$
\mathbf{s}_{i}=\mathbf{m}_{i+1}-\mathbf{m}_{i}, \quad \mathbf{y}_{i}=\nabla \chi\left(\mathbf{m}_{i+1}\right)-\nabla \chi\left(\mathbf{m}_{i}\right)
$$

6. Update the set of vector pairs by deleting the one with the lowest index, and storing the new one, computed in the previous step.

7. Set $i \rightarrow i+1$, go back to (2) and repeat as often as needed.

An interesting aspect of the L-BFGS method is that the initial matrix $\mathbf{A}_{0, i}$ may be chosen differently in each iteration. The only requirements are that $\mathbf{A}_{0, i}$ be positive definite and nearly diagonal, so that $\mathbf{A}_{0, i} \mathbf{q}$ from equation (8.128) can be computed easily.

A comparison of BFGS and L-BFGS for the Rosenbrock function is shown in figure 8.9. It highlights that the convergence of L-BFGS may approach that of BFGS already for small values of $k$, that is, when only a small number of model and gradient evaluations are used for the inverse Hessian approximation. In fact, in this specific case, the two methods are nearly indistinguishable for $k>2$.

The number $k$ of stored models and gradients is a tuning parameter of the L-BFGS method. Suitable values 
are typically found by trial and error, and mostly do not exceed 10, even for problems of considerably higher dimension than in our toy example. Ignoring the storage requirements, one might think that $k$ should be rather larger in order to provide good approximations of the inverse Hessian. However, large values of $k$ also imply a long memory and, therefore, a reduced ability of the method to adapt to changing curvature (second derivatives) along the iteration path.

a) misfit as a function of iteration

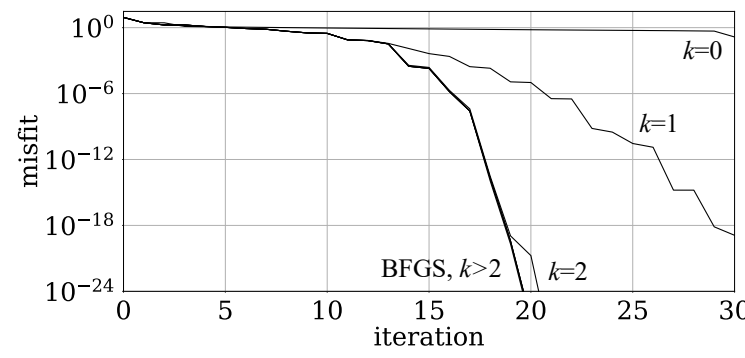

b) L-BFGS trajectory $(k=3)$, Rosenbrock function

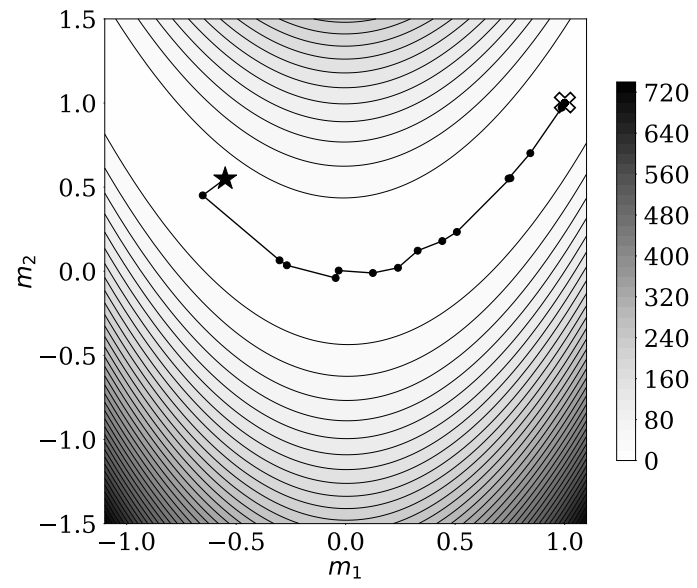

Fig. 8.9: BFGS and L-BFGS optimisation for the Rosenbrock function. (a) Misfit $\chi$ as a function of iteration number for BFGS and L-BFGS with $k=0,1,2,3,4,5$. The case $k=0$ corresponds to the method of steepest descent. For $k>0$, the misfit evolution of L-BFGS is nearly indistinguishable from BFGS. (b) Iteration trajectory for L-BFGS with $k=5$. The star marks the initial model, and the cross the global minimum. [Nonlinear optimisation in 2D]

\subsection{The best method}

Figure 8.10 shows a comparison of the optimisation methods covered in the previous sections in terms of their convergence towards zero misfit and the true optimum. Similar to figure $8.5 \mathrm{~b}$, the Roenbrock function (8.29) is used as test function. The figure suggests that the steepest-descent algorithm converges slowly, to the extent that it hardly makes progress after the first 2 iterations. In contrast, Newton's method converges faster than any of the other methods. This comes at the expense of having to compute second derivatives and the inverse of the Hessian. Therefore, despite its fast convergence, Newton's method is no option in many realistic, large-scale inverse problems. Conjugate-gradient and quasi-Newton methods (BFGS and L-BFGS) can be regarded as a compromise. They do not use second derivatives, which slows down convergence. Yet, approximate Hessians and conjugate directions lead to significant improvements compared to steepest descent.

While being specific to the Rosenbrock function (8.29), the curves shown in figure 8.10 have some general validity. In most real-world applications, the steepest-descent method is uselessly slow, and Newton's method is fast but unaffordable. As a consequence, most large-scale, nonlinear optimisation problems are currently solved with some conjugate-gradient or quasi-Newton variant.

Nevertheless, it is important to again remember the No-Free-Lunch theorem, shown in chapter 12 . The effi- 
a) misfit evolution

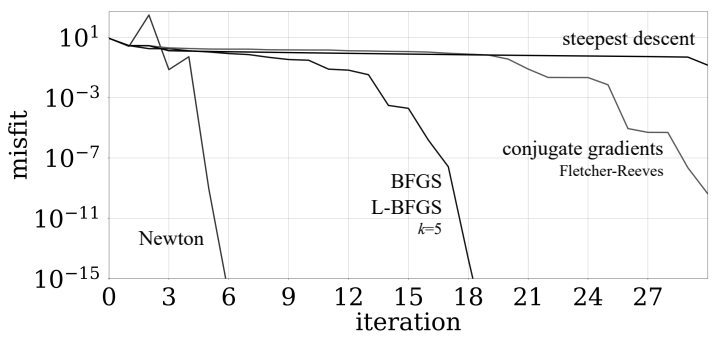

b) distance to optimal model

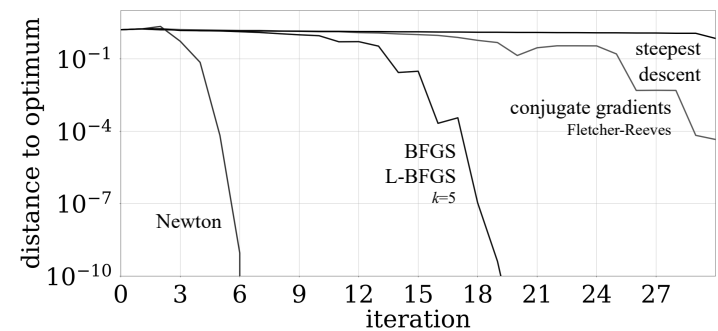

Fig. 8.10: Convergence comparison of steepest descent, the Fletcher-Reeves variant of conjugate gradients, Newton's method, BFGS and L-BFGS. The test function is the Rosenbrock function (8.29), as in figure 8.5b. (a) Misfit as a function of iteration. (b) Distance $\left|\mathbf{m}_{i}-\hat{\mathbf{m}}\right|$ between the current model $\mathbf{m}_{i}$ and the optimal model m. [Nonlinear optimisation in 2D]

ciency of any method is dependent on the problem to which it is applied. It is, for instance, trivial to construct a misfit function for which the steepest-descent method converges in exactly 1 iteration, therefore outperforming any other method that is computationally or algorithmically more complex. In this sense, the best method does not exist.

A distinct benefit of iterative linearisation, introduced in section 8.1, is the possibility to compute the posterior covariance, the resolution matrix, or other related quality measures, in the vicinity of the optimum. Furthermore, when the misfit function is nearly quadratic, iterative minimisation often converges more quickly than steepest-descent and conjugate-gradient methods. This comes at the price of having to compute the Jacobian $\mathbf{J}$ explicitly, which may be impossible unless the forward operator $\mathbf{G}$ can be differentiated (semi-)analytically. As we will see in chapter 9 , the direct computation of the misfit gradient $\nabla \chi$ with the help of adjoint methods is usually more efficient.

The absence of an explicit matrix inverse in the steepest-descent, conjugate-gradient, and quasi-Newton methods has occasionally been misinterpreted as the absence of regularisation, typically needed to make some (nearly) singular matrix invertible. However, the necessarily finite number of iterations in non-linear optimisation methods acts as implicit regularisation; the occurrence of instabilities is prevented by stopping after a sufficiently small number of iterations. In contrast to explicit regularisation, the effect of implicit regularisation on resolution is typically much more difficult to quantify.

Quasi-Newton methods, and L-BFGS in particular, have recently gained popularity in the context of multiparameter inversion. Frequently, the sensitivity of a misfit to different classes of physical parameters differs substantially. A simple steepest-descent method would therefore preferentially update one but not the other parameter class. A beneficial effect of applying an approximate inverse Hessian to $\nabla \chi$ is the automatic scaling of different sensitivities, which leads to a more balanced convergence of different parameter classes.

In many cases, quasi-Newton methods converge faster than steepest-decent and conjugate-gradient methods. As a consequence, the resulting models often tend to show more detail after a smaller number of iterations. Frequently, this is misinterpreted as an ability of quasi-Newton methods to provide higher resolution. In fact, however, resolution is entirely controlled by the misfit $\chi$, which depends on the data $\mathbf{d}^{\text {obs }}$ and the forward model G. Resolution is a property of the misfit and not a function of the method that we use to explore model space or to find an optimum. Eventually, also the most inefficient method gets to the optimum. 



\section{Chapter 9 Adjoint methods}

Don't drink and derive!

Unknown but obviously experienced author.

In many of the previous chapters, derivatives of some suitably chosen misfit function $\chi(\mathbf{m})$ play a central role. For example, in the Metropolis-adjusted Langevin algorithm (MALA) (section 5.5.3) and in Hamiltonian Monte Carlo (HMC) (section 5.7), the misfit is the negative logarithm of the posterior probability density, and its derivative is used to compute a quasi-random test model. In a linearised version of the Backus-Gilbert method, derivatives of some observables serve as data kernels, which are used to construct linear combinations of the data that minimise the extent of spatial averaging. Furthermore, all of the descent methods presented in chapter 8 rest on the availability of first or even second derivatives of $\chi$. This naturally raises the question how these derivatives can be computed efficiently.

The task is complicated by the fact that the dependence of $\chi$ on $\mathbf{m}$ is usually not direct. Instead, the model parameters $\mathbf{m}$ are mostly used to compute some physical field $\mathbf{u}$, for instance, the gravity field around the Earth, the electromagnetic field caused by solar winds, acoustic or elastic wavefields used to probe the interior of inaccessible bodies, the temperature field in the atmosphere, or flow fields (currents) in the oceans. Specific aspects of these fields, often in the form of finite number of point-wise measurements, are then extracted as synthetic data $\mathbf{d}=\mathbf{G}(\mathbf{m})$ and compared to observations $\mathbf{d}^{\text {obs }}$. A useful measure of the discrepancy between $\mathbf{d}^{\text {obs }}$ and $\mathbf{d}$ finally defines the misfit $\chi$. Hence, the computation of the single number $\chi$ entails a whole sequence of operations, often including the solution of a differential equation that links $\mathbf{u}$ to $\mathbf{m}$.

A naïve brute-force approach to the calculation of partial derivatives $\partial \chi / \partial m_{i}$ is the second-order finitedifference approximation

$$
\left.\frac{\partial \chi}{\partial m_{i}} \approx \frac{\partial \chi}{\partial m_{i}}\right|_{F D}=\frac{\chi\left[\mathbf{u}\left(m_{1}, \ldots, m_{i}+\Delta m_{i}, \ldots, m_{n}\right)\right]-\chi\left[\mathbf{u}\left(m_{1}, \ldots, m_{i}-\Delta m_{i}, \ldots, m_{n}\right)\right]}{2 \Delta m_{i}}
$$

with some small increment $\Delta m_{i}$ of model parameter $m_{i}$. While being conceptually simple, the approximation (9.1) is often computationally prohibitive. In fact, accumulating the complete gradient $\nabla \chi$ requires $2 n$ computations of the field $\mathbf{u}$, that is, $2 n$ solutions of the forward modelling equations. This may be reduced to $n+1$ when the second-order approximation is replaced by a less accurate first-order variant. Nevertheless, considering that modern inverse problems may have millions or more model parameters, and that most methods to approximate inverse problem solutions require large numbers of gradient evaluations, finite-differencing of any order is clearly not an option in many cases.

The most widely used approach to compute the derivatives of $\chi$ efficiently is the adjoint method; so called because it involves the adjoint or transpose of the forward modelling operator that connects $\mathbf{u}$ and $\mathbf{m}$. In the following sections, we will provide detailed derivations of the adjoint method, complemented by numerous 
examples that should help to train physical intuition. In section 9.1, we begin with the discrete adjoint method, which starts under the assumption that the forward modelling equations are either inherently discrete or suitably discretised versions of some continuous equations. In this context, the discrete adjoint method is closely related to the concept of first discretise then optimise, where originally continuous equations are first discretised before deriving expressions for the misfit derivative and using it in a gradient-based optimisation scheme. This is in contrast to the first optimise then discretise approach, where derivatives with respect to continuously defined model parameters are derived first, before being discretised to yield partial derivatives with respect to individual elements of a discrete model vector. The first optimise then discretise paradigm goes hand in hand with the continuous adjoint method, covered in section 9.2.

\subsection{Discrete adjoint method}

\subsubsection{Derivation of the adjoint equations}

We begin our development of the discrete adjoint method with the definition of a misfit $\chi$. For this, we consider the discretised version of some physical field $\mathbf{u}$. Originally, that is, before discretisation, it may have been a continuously defined field in space and time. In the interest of greater generality, we will allow $\mathbf{u}$ to be complexvalued. A matrix $\mathbf{D}$ extracts some components from $\mathbf{u}$ in order to produce the synthetic data,

$$
\mathbf{d}=\mathbf{D u} .
$$

For example, when $\mathbf{u}$ is given at a finite number of spatial grid points, used to compute a finite-difference approximation of a differential equation, then $\mathbf{D}$ may select those grid points where some measurement devices are located. Similarly, we define observed data as $\mathbf{d}^{\mathrm{obs}}=\mathbf{D u}^{\mathrm{obs}}$. To quantify the difference between computed and observed data, we may define the quadratic misfit

$$
\chi=\frac{1}{2}\left(\mathbf{d}-\mathbf{d}^{\mathrm{obs}}\right)^{H} \mathbf{C}_{D}^{-1}\left(\mathbf{d}-\mathbf{d}^{\mathrm{obs}}\right)=\frac{1}{2}\left(\mathbf{u}-\mathbf{u}^{\mathrm{obs}}\right)^{H} \mathbf{C}^{-1}\left(\mathbf{u}-\mathbf{u}^{\mathrm{obs}}\right),
$$

where ${ }^{H}$ denotes the complex-conjugate or Hermitian transpose, and $\mathbf{C}^{-1}=\mathbf{D}^{H} \mathbf{C}_{D}^{-1} \mathbf{D}$. As we will see in section 9.2.5, other misfit definitions are possible. However, the simplistic choice in equation (9.3) is the most useful starting point. The field $\mathbf{u}$ is often not given explicitly, but only implicitly as the solution of some forward modelling equation

$$
\mathbf{L}[\mathbf{u}(\mathbf{m}), \mathbf{m}]=\mathbf{f},
$$

where $\mathbf{L}$ is some operator, and $\mathbf{f}$ is typically a forcing term. Hence, evaluating the misfit (9.3) first requires the solution of (9.4), which may already be a formidable numerical challenge.

Having set the stage, we now turn to the computation of the partial derivatives of $\chi$ with respect to the individual model parameters $m_{i}$, which is given by

$$
\frac{\partial \chi}{\partial m_{i}}=\frac{1}{2} \frac{\partial \mathbf{u}^{H}}{\partial m_{i}} \mathbf{C}^{-1}\left(\mathbf{u}-\mathbf{u}^{\mathrm{obs}}\right)+\frac{1}{2}\left(\mathbf{u}-\mathbf{u}^{\mathrm{obs}}\right)^{H} \mathbf{C}^{-1} \frac{\partial \mathbf{u}}{\partial m_{i}} .
$$

The difficulty in equation (9.5) lies in the presence of $\partial \mathbf{u} / \partial m_{i}$, which we cannot compute easily. To eliminate $\partial \mathbf{u} / \partial m_{i}$, from (9.5), we make use of the derivative of the forward modelling equation (9.4),

$$
\frac{\partial}{\partial m_{i}} \mathbf{L}[\mathbf{u}(\mathbf{m}), \mathbf{m}]+\mathbf{J} \frac{\partial \mathbf{u}}{\partial m_{i}}=\mathbf{0},
$$

where we defined the Jacobian $\mathbf{J}$ with components 


$$
J_{i j}=\frac{\partial L_{i}}{\partial u_{j}}
$$

In the next step, we multiply (9.6) with an arbitrary vector $\mathbf{v} / 2$,

$$
\frac{1}{2} \mathbf{v}^{H} \frac{\partial}{\partial m_{i}} \mathbf{L}[\mathbf{u}(\mathbf{m}), \mathbf{m}]+\frac{1}{2} \mathbf{v}^{H} \mathbf{J} \frac{\partial \mathbf{u}}{\partial m_{i}}=0 .
$$

Adding (9.8) plus its complex conjugate to (9.5), we obtain

$$
\frac{\partial \chi}{\partial m_{i}}=\operatorname{Re}\left[\left(\left(\mathbf{u}-\mathbf{u}^{\mathrm{obs}}\right)^{H} \mathbf{C}^{-1}+\mathbf{v}^{H} \mathbf{J}\right) \frac{\partial \mathbf{u}}{\partial m_{i}}+\mathbf{v}^{H} \frac{\partial}{\partial m_{i}} \mathbf{L}[\mathbf{u}(\mathbf{m}), \mathbf{m}]\right] .
$$

We may now eliminate $\partial \mathbf{u} / \partial m_{i}$ from (9.9) by defining the so far arbitrary vector $\mathbf{v}$ as the solution of the vector-matrix equation

$$
\mathbf{J}^{H} \mathbf{v}=-\mathbf{C}^{-H}\left(\mathbf{u}-\mathbf{u}^{\text {obs }}\right)
$$

Equation (9.10) is referred to as the discrete adjoint equation. Its right-hand side is the adjoint source. It produces the solution, $\mathbf{v}$, called the adjoint field. Specifying $\mathbf{v}$ via (9.10), condenses the partial derivative (9.9) to

$$
\frac{\partial \chi}{\partial m_{i}}=\operatorname{Re}^{H} \frac{\partial}{\partial m_{i}} \mathbf{L}[\mathbf{u}(\mathbf{m}), \mathbf{m}]
$$

Equations (9.10) and (9.11) deserve some discussion. Most importantly, we see that, by design, the calculation of $\frac{\partial \chi}{\partial m_{i}}$ does not actually require the partial derivatives of $\mathbf{u}$. Instead, the difficulty is transferred to the more manageable task deriving the Jacobian $\mathbf{J}$ and solving the vector-matrix adjoint equation (9.10) for the adjoint field $\mathbf{v}$. Once the adjoint field $\mathbf{v}$ is computed, it is simply multiplied with $\partial \mathbf{L} / \partial m_{i}$. Interestingly, the adjoint field is completely determined by the residuals $\mathbf{C}^{-H}\left(\mathbf{u}-\mathbf{u}^{\text {obs }}\right)$, and it does not depend on the component $i$ of the partial derivative. Hence, the adjoint field needs to be computed only once in order to obtain all $n$ partial derivatives of $\chi$. This is in stark contrast to the $n+1$ solutions of the forward modelling equations, needed to approximate the partial derivatives of $\chi$ by finite-differencing, as in equation (9.1).

Some special cases are of particular interest. When the forward modelling equations are linear in the discrete field $\mathbf{u}$, we may rewrite $(9.4)$ as

$$
\mathbf{L}[\mathbf{u}(\mathbf{m}), \mathbf{m}]=\mathbf{L}(\mathbf{m}) \mathbf{u}=\mathbf{f},
$$

with a matrix $\mathbf{L}$. The Jacobian $\mathbf{J}$ is then equal to $\mathbf{L}$ itself, and equations (9.10) and (9.11) simplify to

$$
\mathbf{L}^{H} \mathbf{v}=-\mathbf{C}^{-H}\left(\mathbf{u}-\mathbf{u}^{\text {obs }}\right)
$$

and

$$
\frac{\partial \chi}{\partial m_{i}}=\operatorname{Rev}^{H} \frac{\partial \mathbf{L}}{\partial m_{i}} \mathbf{u}
$$

Should $\mathbf{L}$ be Hermitian, equation (9.13) can be further simplified by replacing $\mathbf{L}^{H}$ by $\mathbf{L}$.

\subsubsection{Model problem: The frequency-domain wave equation with attenuation in 1-D}

To illustrate the developments in the previous section, we consider the simple but instructive example of a 1-D wave equation in the frequency domain,

$$
\omega^{2} u(\omega, x)+c^{2}(x) \frac{\partial^{2}}{\partial x^{2}} u(\omega, x)=-f(\omega, x) .
$$


Equation (9.15) describes the propagation of a 1-D wave $u$ with circular frequency $\omega$ that has been excited by the external force $f$. The wave propagates along the $x$-axis with a speed given by the real part $c^{\mathrm{re}}(x)$ of the complex-valued parameter $c(x)$. The imaginary part, $c^{\mathrm{im}}(x)=\operatorname{Im} c(x)$ controls the attenuation of the wave, that is, the conversion of kinetic and potential energy of the wave into heat. Attenuation increases with increasing $c^{\mathrm{im}}(x)$. Despite being simple, equation (9.15) captures a broad range of wave propagation phenomena, ranging from the vibration of a bar or guitar string to the propagation of laser pulses through an optical fibre.

For arbitrary distributions of $c(x)$, equation (9.15) does not have an analytical solution, and so we resort to a numerical approximation. Though more sophisticated methods for the numerical solution of the wave equation exist (e.g. Igel, 2016), we implement a second-order finite-difference approximation of the spatial derivative term in (9.15),

$$
c^{2}(x) \frac{\partial^{2}}{\partial x^{2}} u(\omega, x) \approx \frac{c_{i}^{2}}{\Delta x^{2}}\left(u_{i+1}-2 u_{i}+u_{i-1}\right), \quad \text { for } i=1, \ldots, N_{g}-1 .
$$

The subscripts $i-1, i$ and $i+1$ denote evaluation of a position-dependent quantity at $x=x_{i}-\Delta x, x_{i}$ and $x_{i}+\Delta x$, respectively. At the domain edges, we set $u_{0}=u_{N_{g}}=0$, which effectively implements reflecting boundaries. To obtain useful numerical solutions, the grid point spacing $\Delta x$ should be around an order of magnitude smaller than the shortest wavelength $\min 2 \pi c^{\mathrm{re}} / \omega$. The values of the real and imaginary parts of $c(x)$ sampled at the grid points, that $c_{i}^{\text {re }}$ and $c_{i}^{\mathrm{im}}$, constitute the components of the discrete model parameter vector $\mathbf{m}$. Combining (9.15) and (9.16), we find the discrete forward modelling equations in index notation,

$$
\sum_{k=1}^{n} L_{i k} u_{k}=\omega^{2} u_{i}+\frac{c_{i}^{2}}{\Delta x^{2}}\left(u_{i+1}-2 u_{i}+u_{i-1}\right)=-f_{i}
$$

The forward modelling operator in (9.17) is linear and fully represented by the matrix non-Hermitian matrix $\mathbf{L}$ with components $L_{i k}$.

As a concrete example, we mimic the propagation of seismic waves excited by earthquakes. In the absence of observed data, and in order to have a more controlled numerical experiment, we compute artificial observations using the complex-valued velocity distribution shown in figure 9.1a as solid black line. The velocity distribution used to compute synthetic observations, shown as grey dashed curve, is a smoother version of this distribution. This reflects the common situation that only a blurred image of Earth structure is known a priori.

We discretise the interval between $x=0 \mathrm{~km}$ and $x=1000 \mathrm{~km}$ using 1001 grid points, meaning that the grid spacing in $\Delta x=1.0 \mathrm{~km}$. Two example wavefields for this medium are displayed in figure $9.1 \mathrm{~b}$ for frequencies of $f=0.25 \mathrm{~Hz}$ and $f=0.15$. As expected, the wavefield amplitudes decay with increasing distance from the source due to attenuation.

The data vectors $\mathbf{d}^{\mathrm{obs}}$ and $\mathbf{d}$ for this example consist of the wavefields $u^{\mathrm{obs}}$ and $u$ for both frequencies $(f=0.25$ $\mathrm{Hz}$ and $f=0.15$ ) sampled at the 10 receiver positions, which marked by triangles in figure $9.1 \mathrm{~b}$. Setting $\mathbf{C}_{D}=0.01 \mathrm{I} \mathrm{mm} \mathrm{s}$, for simplicity, yields a misfit value of $\chi=767.78$.

The discrepancy between $u^{\mathrm{obs}}$ and $u$ at the measurement locations defines the adjoint source, that is, the righthand side of the adjoint equation (9.13). Inserting the definition of the forward modelling matrix $\mathbf{L}$ from (9.17) into the generic equation for partial misfit derivatives (9.14), yields the derivatives of $\chi$ with respect to the real and imaginary parts of velocity sampled at the grid points,

$$
\frac{\partial \chi}{\partial c_{i}^{\mathrm{re}}}=4 \operatorname{Re}\left(c_{i} v_{i}^{*} e_{i}\right)
$$

and

$$
\frac{\partial \chi}{\partial c_{i}^{\mathrm{im}}}=-4 \operatorname{Im}\left(c_{i} v_{i}^{*} e_{i}\right),
$$

with the discrete second derivative of the wavefield, 
a) Distributions of real and imaginary velocities
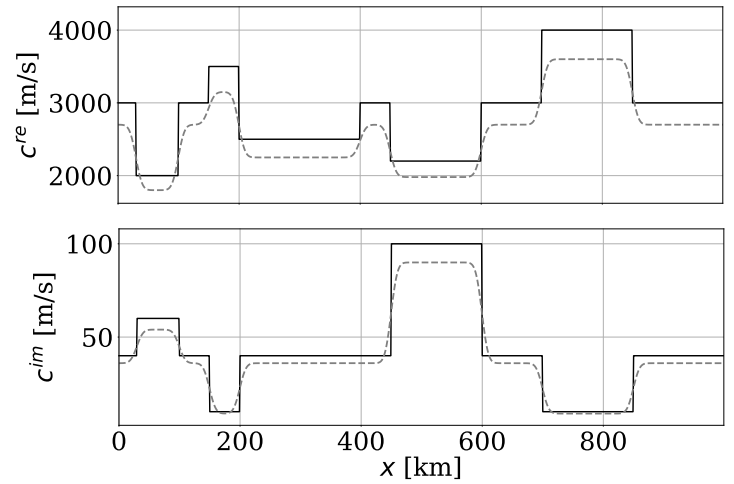

used for $u^{\text {obs }}$ b) Wavefields
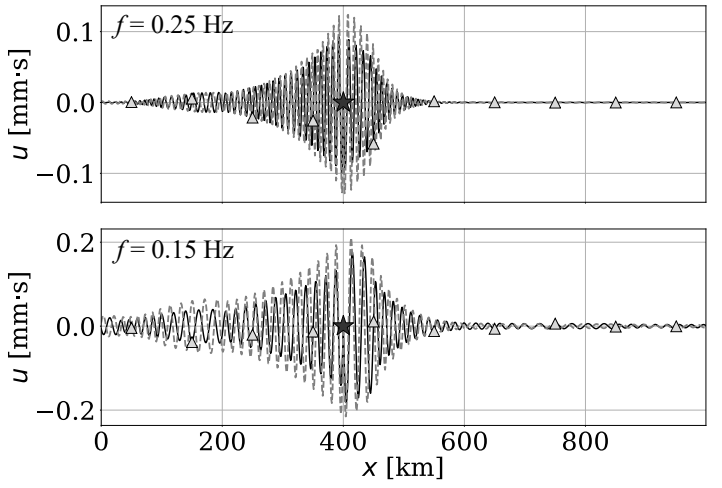

$u^{\text {obs }} \quad \cdots \quad u \quad \downarrow$ source $\Delta$ receivers

Fig. 9.1: Models and wavefields for 1-D frequency-domain wave propagation. (a) Real part $c^{\mathrm{re}}(x)$ (top) and imaginary part $c^{\mathrm{im}}(x)$ of the complex-valued velocity $c(x)$ (bottom). The distribution plotted as solid black curve is used to compute artificial observations $u^{\mathrm{obs}}(\omega, x)$. The smoother grey dashed distribution serves to compute the synthetic wavefield $u(\omega, x)$. (b) Examples of frequency-domain observed (black solid) and synthetic (grey dashed) wavefields for frequencies of $f=0.25 \mathrm{~Hz}$ (top) and $f=0.15 \mathrm{~Hz}$ (bottom). The source position is indicated by a star, and the receiver positions by triangles. [FD1D visco-elastic]

$$
e_{i}=\frac{1}{\Delta x^{2}}\left(u_{i+1}-2 u_{i}+u_{i+1}\right)
$$

Applying these equations to our model problem from figure 9.1, yields the partial derivatives shown in figure 9.2.

a) Derivatives with respect to real part of velocity

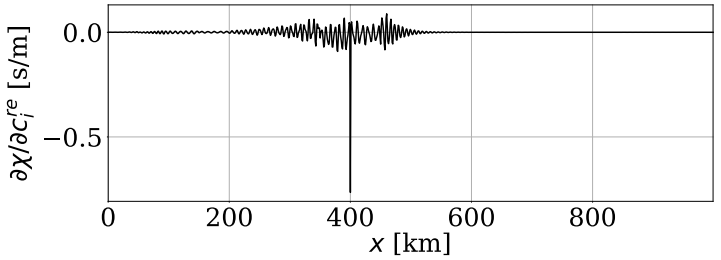

b) Derivatives with respect to imaginary part of velocity

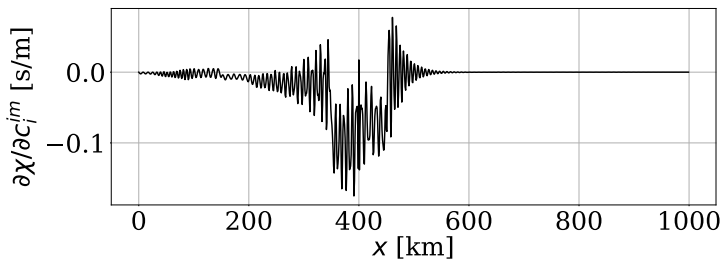

Fig. 9.2: Partial derivatives of the misfit $\chi$ with respect to the real part $c_{i}^{\mathrm{re}}(\mathbf{a})$ and the imaginary $\operatorname{part} c_{i}^{\mathrm{im}}(\mathbf{b})$ of the sampled complex-valued velocity $c(x)$. [FD1D visco-elastic]

In total, figure 9.2 displays 2002 partial derivatives, two at each of the 1001 grid points, one of them for the real part, the other for the imaginary part of the complex-valued velocity $c_{i}$. All that was required to obtain the complete misfit gradient, was one solution of the forward problem (9.17), followed by one solution of the adjoint problem (9.13). Since the forward modelling equations are linear in $\mathbf{u}$, the adjoint problem is also a matrix-vector equation, involving $\mathbf{L}^{H}$ instead of $\mathbf{L}$. The complex conjugation $\mathbf{L}^{H}$ means that the imaginary part of the velocity, $c_{i}^{\mathrm{im}}$, is replaced by $-c_{i}^{\mathrm{im}}$. Therefore, the adjoint wavefield experiences an energy increase during its propagation, which may be interpreted as anti-attenuation.

Two-dimensional variants of the discrete forward and adjoint problems covered in the previous paragraphs have 
been studied extensively in the classic work of Pratt et al. (1998) and Pratt (1999), and applied, for example, to geophysical and medical imaging problems, broadly known as full-waveform inversion (e.g. Afanasiev et al., 2014; Bleibinhaus et al., 2007; Pratt et al., 2007; Smithyman et al., 2009).

\subsubsection{Gradient testing}

Especially in the case of more complicated forward problems, the derivation and implementation of the adjoint equations can be a challenging task, and even experienced programmers may struggle to write correct code. While approximate gradients may be useful for some applications, most of the gradient descent methods presented in chapter 8 require accurate gradients in order to ensure reasonably fast convergence. Hence, it is useful to perform simple tests that make a correct implementation at least more plausible.

Arguably the simplest of these tests involves the finite-difference approximation in equation (9.1), or higherorder variants of it. For many practical problems, brute-force finite-differencing for all components of the gradient vector is no viable option. However, a few partial derivatives may be tested without excessive computational effort.

In the deliberately simplistic case of our 1-D model problem, the complete gradient vector can be approximated by finite differences and compared to the adjoint-based result. This is illustrated in figure $9.3 \mathrm{a}$ for the derivatives with respect to the real part of velocity and for a velocity increment of $\Delta c_{i}^{\mathrm{re}}=0.01 \mathrm{~m} / \mathrm{s}$. The approximation error in figure $9.3 \mathrm{~b}$, that is, the difference between finite-difference approximation and adjoint-based derivatives, is around 9 orders of magnitude smaller than the partial derivatives themselves. This suggests that the implementation is indeed correct.

a) Finite-difference approximation

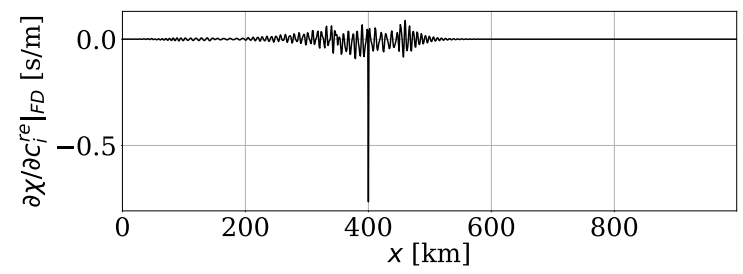

b) Approximation error

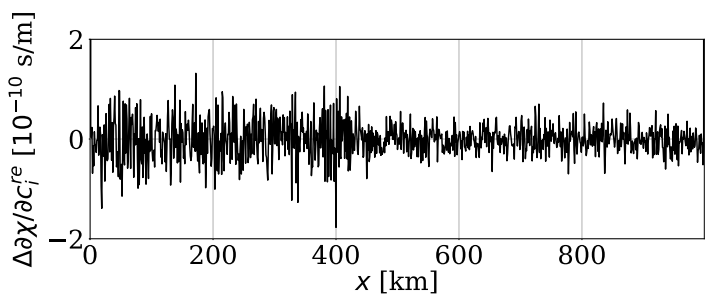

Fig. 9.3: Comparison of partial derivatives obtained by the finite-difference approximation (9.1) and the discrete adjoint method. (a) Second-order finite-difference approximations of the partial derivatives of $\chi$ with respect to the real part of velocity. The velocity increment used in the finite-difference calculations is $\Delta c_{i}^{\mathrm{re}}=0.01 \mathrm{~m} /$. Visually, the result is identical to the adjoint-based partial derivatives in figure 9.2a. (b) Difference between the adjoint-based partial derivatives and their finite-difference approximation. [FD1D visco-elastic]

We can obtain a different and very instructive perspective on the difference between finite-difference and adjoint-based derivatives by considering only one specific model parameter $m_{i}$, and varying the finite-difference increment $\Delta m_{i}$. For large increments, the difference will be controlled by the error of the finite-difference approximation. As the increment becomes smaller, the difference between finite-difference approximation and adjoint-based derivative decreases, but only to the point where floating-point errors start to dominate. Further decreasing the increment, increases the difference because the division of a very small number by another very small number in (9.1) cannot be represented with sufficient accuracy in a digital computer with limited precision. This behaviour results in characteristic error curves that are commonly referred to as hockey stick plots. Their appearance is a useful indicator, but no guarantee, for a correct adjoint method implementation. 
a) Real part of velocity

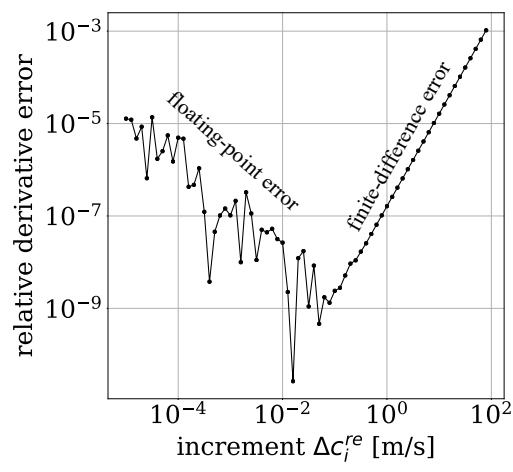

b) Imaginary part of velocity

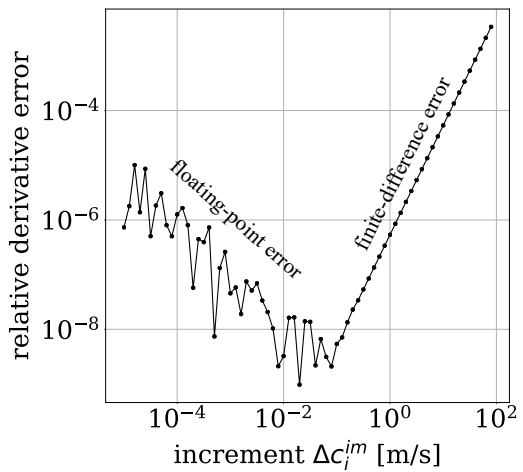

c) Hockey stick (not logarithmic)

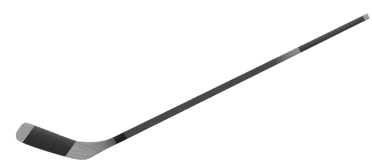

Fig. 9.4: Hockey stick plots. Relative absolute difference between partial derivatives approximated by finite differences and computed with the adjoint method, as a function of the finite-difference increment. The handle of the hockey stick is related to increasing finite-difference errors. The head is a consequence of floating-point errors that start to dominate when the finite-difference increment becomes very small. Panels (a) and (b) show hockey stick plots for the real and imaginary parts of velocity at position $x_{i}=50 \mathrm{~km}$. (c) A real hockey stick for comparison. [FD1D visco-elastic]

\subsection{Continuous adjoint method}

The discrete adjoint method, covered in the previous section, allows us to efficiently compute the partial derivatives of a misfit function, provided that the model parameters are either inherently discrete or suitably discretised. Hence, from a pragmatic point of view, the problem may be considered solved at this point.

In the case of inherently continuous model parameters, $\mathfrak{m}(\mathbf{x})$, however, the discrete adjoint method suffers from the disadvantage that we need to choose some discretisation a priori. While being useful in numerical optimisation, the discretisation-specific partial derivatives may hide discretisation-independent physics. In fact, much of the physical intuition needed to solve ill-posed inverse problems in a meaningful fashion, derives from the (visual) analysis of discretisation-independent first derivatives. Furthermore, some methods, including the Backus-Gilbert method from chapter 7, require a continuous version of derivatives in the form of data kernels.

In analogy to the discrete case, the forward modelling equation,

$$
\mathbf{L}[\mathbf{u}(\mathfrak{m}), \mathfrak{m}]=\mathbf{f}
$$

serves as our starting point. Though (9.21) looks very similar to its discrete counterpart (9.4), it is usually very different in nature. In many cases, $\mathbf{L}$ is a differential operator that links the field $\mathbf{u}$ to the continuous distribution of model parameters $\mathfrak{m}(\mathbf{x})$ and the forcing term $\mathbf{f}$.

In the case of gravity, for example, equation (9.21) would be Gauss' law of gravitation, $\nabla \cdot \mathbf{g}(\mathbf{x})=-4 \pi G \rho(\mathbf{x})$, where the differential operator $L=\nabla$. relates the gravitational acceleration $\mathbf{u}(\mathbf{x})=\mathbf{g}(\mathbf{x})$ to the forcing term $\mathbf{f}(\mathbf{x})=-4 \pi G \rho(\mathbf{x})$. The latter depends on the distribution of mass density $\mathfrak{m}(\mathbf{x})=\rho(\mathbf{x})$ within some volume and on the gravitational constant $G=6.674 \cdot 10^{-11} \mathrm{~m}^{3} \mathrm{~kg}^{-1} \mathrm{~s}^{-2}$.

The solution $\mathbf{u}$ of (9.21) is the field $\mathbf{u}$, which may - depending on the specifics of $\mathbf{L}$ - be a continuously defined function of space $\mathbf{x}$, time $t$, or both. The comparison of the computed field $\mathbf{u}$ to the observable field $\mathbf{u}^{\text {obs }}$, typically at a discrete number of measurement points, defines a misfit

$$
\chi=\chi[\mathbf{u}(\mathfrak{m})] .
$$


Contrary to the discrete case, $\chi$ does not depend on a vector with a finite number of components, but on the continuously defined functions $\mathbf{u}$ and $\mathfrak{m}$. Such scalar-valued functions of functions are commonly referred to as functionals.

Our interest is to compute the small variations $\delta \chi$ of the misfit functional, induced by small variations $\delta \mathbf{u}$, which are the result of arbitrary small model parameter perturbations $\delta \mathfrak{m}$. Doing so efficiently, without having to re-solve the forward modelling equations for any imaginable $\delta \mathfrak{m}$, is the purpose of the continuous adjoint method, which we will develop in the following paragraphs. Though the terminology and symbolisms will be slightly different, we will rediscover many of the concepts and approaches that we already encountered in the discrete case.

Before delving into the derivation, we will take a closer look at what a continuous derivative is supposed to mean.

\subsubsection{Fréchet derivatives and Fréchet kernels}

We begin with some perturbation $\delta \mathbf{u}$ of the field $\mathbf{u}$. This perturbation results in a relative change of the misfit, $\delta \chi$, which we can express in terms of the derivative

$$
\delta \chi(\mathbf{u} ; \delta \mathbf{u})=\lim _{\varepsilon \rightarrow 0} \frac{1}{\varepsilon}[\chi(\mathbf{u}+\varepsilon \delta \mathbf{u})-\chi(\mathbf{u})] .
$$

Provided that the limit exists, $\delta \chi(\mathbf{u} ; \delta \mathbf{u})$ is called the functional derivative or Fréchet ${ }^{1}$ derivative of $\chi$ at $\mathbf{u}$ in the direction $\delta \mathbf{u}$.In the special case where $\mathbf{u}$ is just a constant vector and not a vector-valued function, the definition (9.23) is equivalent to the directional derivative $\delta \mathbf{u}^{T} \nabla \chi(\mathbf{u})$.

It is not difficult to see that $\delta \chi(\mathbf{u} ; \delta \mathbf{u})$ is linear in the direction $\delta \mathbf{u}$. In fact, for two different perturbations $\delta \mathbf{u}_{1}$ and $\delta \mathbf{u}_{2}$, we find

$$
\begin{aligned}
\delta \chi\left(\mathbf{u} ; \delta \mathbf{u}_{1}+\delta \mathbf{u}_{2}\right) & =\lim _{\varepsilon \rightarrow 0} \frac{1}{\varepsilon}\left[\chi\left(\mathbf{u}+\varepsilon \delta \mathbf{u}_{1}+\varepsilon \delta \mathbf{u}_{2}\right)-\chi(\mathbf{u})\right] \\
& =\lim _{\varepsilon \rightarrow 0} \frac{1}{\varepsilon}\left[\chi\left(\mathbf{u}+\varepsilon \delta \mathbf{u}_{1}+\varepsilon \delta \mathbf{u}_{2}\right)-\chi\left(\mathbf{u}+\varepsilon \delta \mathbf{u}_{2}\right)\right]+\lim _{\varepsilon \rightarrow 0} \frac{1}{\varepsilon}\left[\chi\left(\mathbf{u}+\varepsilon \delta \mathbf{u}_{2}\right)-\chi(\mathbf{u})\right] \\
& =\delta \chi\left(\mathbf{u} ; \delta \mathbf{u}_{1}\right)+\delta \chi\left(\mathbf{u} ; \delta \mathbf{u}_{2}\right) .
\end{aligned}
$$

The linearity of $\delta \chi$ with respect to $\delta \mathbf{u}$ has an important practical implication that results from Riesz ${ }^{2}$ representation theorem. Demonstrated in appendix 15.2, the representation theorem states that $\delta \chi(\mathbf{u} ; \delta \mathbf{u})$ can generally be written in the form of a scalar product

$$
\delta \chi(\mathbf{u} ; \delta \mathbf{u})=-\left(\mathbf{f}^{\dagger}(\mathbf{u}), \delta \mathbf{u}\right)
$$

with some uniquely defined Fréchet kernel. The minus sign in (9.25) is a matter of convention, the usefulness of which will become evident a little later.

\footnotetext{
${ }^{1}$ Maurice René Fréchet (1878 - 1973) was a French mathematician, whose potential was recognised early on by the then already famous Jacques Hadamard, who supported him throughout his early career. His scientific trajectory was disrupted by the turbulences of his time, and by World War I in particular. Remarkably, while serving close to the front line for two and a half years, he produced a number of elaborate mathematical papers. He later taught mathematics at various institutions in France.

${ }^{2}$ Frigyes Riesz (1880 - 1956) was a Hungarian mathematician, known for his fundamental contributions to functional and spectral analysis. In addition to his scientific work, often published under the name Frédéric Riesz, also some anecdotes about his somewhat unconventional lectures have been transmitted. Apparently, he used to enter the lecture hall with two assistants. One would read Riesz' lecture notes, while the other one copied the relevant equations onto the blackboard. Riesz is reported to have nodded occasionally.
} 
Scalar products: From geometry in two or three dimensions, we are most familiar with the scalar product

$$
(\mathbf{a}, \mathbf{b})=\mathbf{a}^{H} \mathbf{b}=\sum_{i} a_{i}^{*} b_{i},
$$

between two vectors, $\mathbf{a}$ and $\mathbf{b}$, which we assume to be complex-valued for greater generality. The superscript ${ }^{H}$ in (9.26) denotes the complex conjugate of the vector transpose, and the index $i$ runs from 1 to the number of vector dimensions. For any choice of vectors, the scalar product can be shown to have the following properties: (1) Linearity in the first argument,

$$
\left(\mathbf{a}_{1}+\mathbf{a}_{2}, \mathbf{b}\right)=\left(\mathbf{a}_{1}, \mathbf{b}\right)+\left(\mathbf{a}_{2}, \mathbf{b}\right),
$$

(2) conjugate symmetry,

$$
(\mathbf{a}, \mathbf{b})=(\mathbf{b}, \mathbf{a})^{*}
$$

and (3) positive definiteness,

$$
(\mathbf{a}, \mathbf{a})>0, \quad \text { for } \mathbf{a} \neq \mathbf{0} .
$$

The positive definiteness (9.29) ensures that the scalar product can be used to define a norm or length $\|\mathbf{a}\|$ of the vector a via

$$
\|\mathbf{a}\|^{2}=(\mathbf{a}, \mathbf{a}) .
$$

Relations (9.27) to (9.28), satisfied by the vector scalar product, also serve as defining properties for scalar products between other mathematical objects. Considering, for example, two complex-valued functions $a(\mathbf{x})$ and $b(\mathbf{x})$, defined in some volume $V$, a scalar product may be defined as

$$
(a, b)=\int_{V} a^{*}(\mathbf{x}) b(\mathbf{x}) d \mathbf{x}
$$

Similarly, for complex-valued vectorial functions $\mathbf{a}(\mathbf{x})$ and $\mathbf{b}(\mathbf{x})$ we may define

$$
(\mathbf{a}, \mathbf{b})=\int_{V} \mathbf{a}^{H}(\mathbf{x}) \mathbf{b}(\mathbf{x}) d \mathbf{x} .
$$

Generally, there is more than one possible definition of a scalar product, and a particular choice may depend on practical and application-specific considerations. For example,

$$
(\mathbf{a}, \mathbf{b})=\int_{V} \mathbf{a}^{H}(\mathbf{x}) \mathbf{A} \mathbf{b}(\mathbf{x}) d \mathbf{x},
$$

is also a valid scalar product for any positive definite matrix $\mathbf{A}$.

As an illustration of (9.25), we consider some time- and space-dependent observed field $\mathbf{u}^{\mathrm{obs}}\left(\mathbf{x}^{r}, t\right)$, and the corresponding computed field $\mathbf{u}\left(\mathbf{x}^{r}, t\right)$, sampled at some measurement point $\mathbf{x}^{r}$. The field may, for instance, be temperature in the atmosphere, or an ultrasonic wavefield emitted into a human body. A potentially useful measure of the discrepancy between $\mathbf{u}^{\text {obs }}\left(\mathbf{x}^{r}, t\right)$ and $\mathbf{u}\left(\mathbf{x}^{r}, t\right)$ is the quadratic misfit integrated over the measurement time interval $T=\left[t_{0}, t_{1}\right]$

$$
\chi=\frac{1}{2} \int_{t_{0}}^{t_{1}}\left[\mathbf{u}\left(\mathbf{x}^{r}, t\right)-\mathbf{u}^{\mathrm{obs}}\left(\mathbf{x}^{r}, t\right)\right]^{2} d t=\frac{1}{2} \int_{t_{0}}^{t_{1}} \int_{V}\left[\mathbf{u}(\mathbf{x}, t)-\mathbf{u}^{\mathrm{obs}}(\mathbf{x}, t)\right]^{2} \delta\left(\mathbf{x}-\mathbf{x}^{r}\right) d t d \mathbf{x} .
$$


The Fréchet derivative of (9.34) is

$$
\delta \chi(\mathbf{u} ; \delta \mathbf{u})=\int_{t_{0}}^{t_{1}} \int_{V}\left[\mathbf{u}\left(\mathbf{x}^{r}, t\right)-\mathbf{u}^{\mathrm{obs}}\left(\mathbf{x}^{r}, t\right)\right] \delta\left(\mathbf{x}-\mathbf{x}^{r}\right) \delta \mathbf{u}(\mathbf{x}, t) d t d \mathbf{x} .
$$

On comparison with (9.25), we find that the appropriate scalar product is defined by the integral $\int_{t_{0}}^{t_{1}}() d t. d \mathbf{x}$, and that the Fréchet kernel is given by

$$
\mathbf{f}^{\dagger}(\mathbf{x}, t)=-\left[\mathbf{u}\left(\mathbf{x}^{r}, t\right)-\mathbf{u}^{\mathrm{obs}}\left(\mathbf{x}^{r}, t\right)\right] \delta\left(\mathbf{x}-\mathbf{x}^{r}\right) .
$$

The function $\mathbf{f}^{\dagger}$ is localised at the receiver position $\mathbf{x}^{r}$, and its time evolution is controlled by the linear field residuals, that is, by the difference between observed and computed field values, $\mathbf{u}\left(\mathbf{x}^{r}, t\right)-\mathbf{u}^{\mathrm{obs}}\left(\mathbf{x}^{r}, t\right)$. We will see later that $\mathbf{f}^{\dagger}$ will contain some form of residuals, irrespective of the precise definition of the misfit functional.

\subsubsection{Derivation of the continuous adjoint equations}

While the expression (9.25) for the Fréchet derivative of the misfit functional $\chi$ is formally correct, it is of little practical use because it does not allow us to compute $\delta \chi$ directly for some arbitrary model perturbation $\delta \mathfrak{m}$. Instead, it forces us to first compute the field perturbation $\delta \mathbf{u}$ induced by $\delta \mathfrak{m}$, for instance, via the solution of another forward problem with $\mathfrak{m}+\delta \mathfrak{m}$ as model parameter. This indirect computation of $\delta \chi$ can be prohibitively expensive when the number of desired model perturbations is large. Therefore, we wish to eliminate the explicit occurrence of $\delta \mathbf{u}$ from (9.25). As in the discrete case, we can achieve this goal by making use of the forward modelling equation (9.21).

Making use of the chain rule, we can compute the Fréchet derivative of (9.21) with respect to $\mathfrak{m}$, taking into account that the right-hand side does not depend on $\mathfrak{m}$,

$$
\mathbf{J}[\mathbf{u}(\mathfrak{m}), \mathfrak{m} ; \delta \mathbf{u}]+\delta \mathbf{L}[\mathbf{u}(\mathfrak{m}), \mathfrak{m} ; \delta \mathfrak{m}]=\mathbf{0} .
$$

In equation (9.37), $\mathbf{J}$ denotes the Jacobian $\nabla \mathbf{L}$ of the forward modelling operator $\mathbf{L}$, defined as the Fréchet derivative with respect to $\mathbf{u}$,

$$
\mathbf{J}[\mathbf{u}(\mathfrak{m}), \mathfrak{m} ; \delta \mathbf{u}]=\lim _{\varepsilon \rightarrow 0} \frac{1}{\varepsilon}[\mathbf{L}[\mathbf{u}(\mathfrak{m})+\varepsilon \delta \mathbf{u}(\mathfrak{m}), \mathfrak{m}]-\mathbf{L}[\mathbf{u}(\mathfrak{m}), \mathfrak{m}]] .
$$

The second term in (9.37) is to be understood as the Fréchet derivative of $\mathbf{L}$ with respect to the explicit dependence on $\mathfrak{m}$, that is, the second argument in $\mathbf{L}[\mathbf{u}(\mathfrak{m}), \mathfrak{m}]$. Forming the scalar product of (9.37) with some arbitrary vector $\mathbf{v}$ and adding the result to the Fréchet derivative of the misfit functional $\chi$ in equation (9.25), we obtain

$$
\delta \chi=-\left(\mathbf{f}^{\dagger}(\mathbf{u}), \delta \mathbf{u}\right)+(\mathbf{J}(\delta \mathbf{u}), \mathbf{v})+(\delta \mathbf{L}(\delta \mathfrak{m}), \mathbf{v}),
$$

where we omitted the dependencies on $\mathbf{u}$ and $\mathfrak{m}$ in the interest of notational clarity. To eliminate $\delta \mathbf{u}$ from (9.39), the term $(\mathbf{J}(\delta \mathbf{u}), \mathbf{v})$ needs to be rearranged such that $\delta \mathbf{u}$ is isolated. This can be achieved by invoking the adjoint operator $\mathbf{J}^{\dagger}$ of $\mathbf{J}$, defined through the relation

$$
(\mathbf{J}(\delta \mathbf{u}), \mathbf{v})=\left(\mathbf{J}^{\dagger}(\mathbf{v}), \delta \mathbf{u}\right)
$$

which must hold for any $\delta u$ and $v$. Similar to the transpose of a matrix, the adjoint of $\mathbf{J}$ allows us to swap the positions of $\mathbf{v}$ and $\delta \mathbf{u}$ under a scalar product. At this point, we silently assume that the adjoint $\mathbf{J}^{\dagger}$ actually exists. As we will see later, its actual derivation can be a quite formidable task. Replacing $(\mathbf{J}(\delta \mathbf{u}, \mathbf{v}))$ in (9.39) by the right-hand side of (9.40), and sorting terms, we find 


$$
\delta \chi=\left(\mathbf{J}^{\dagger}(\mathbf{v})-\mathbf{f}^{\dagger}, \delta \mathbf{u}\right)+(\delta \mathbf{L}(\delta \mathfrak{m}), \mathbf{v}) .
$$

It is now possible to eliminate $\delta \mathbf{u}$ by specifying the so far arbitrary $\mathbf{v}$ as the solution of the adjoint equation

$$
\mathbf{J}^{\dagger}(\mathbf{v})=\mathbf{f}^{\dagger}
$$

which is the continuous analogue of the discrete adjoint equation (9.10). Upon imposing (9.42), the Fréchet derivative of the misfit functional condenses to

$$
\delta \chi=(\delta \mathbf{L}(\delta \mathfrak{m}), \mathbf{v}) .
$$

The discrete version of (9.43) is equation (9.11). Equations (9.42) and (9.43) suggest a conceptually simple recipe for the calculation of the Fréchet derivative $\delta \chi$ :

1) Solve the forward problem (9.21) to obtain the field $\mathbf{u}$.

2) Compare the observed field $\mathbf{u}^{\text {obs }}$ and the computed field $\mathbf{u}$ using a suitable misfit $\chi$, which then determines the adjoint source $\mathbf{f}^{\dagger}$.

3) Using $\mathbf{f}^{\dagger}$ on the right-hand side, solve the adjoint equation (9.42) for the adjoint field $\mathbf{v}$.

4) Insert $\mathbf{v}$ into (9.43) to compute the Fréchet derivative $\delta \chi$.

By design, the forward field $\mathbf{u}$ and the adjoint field $\mathbf{v}$ need to be computed only once in order to obtain $\delta \chi$ for any arbitrary $\delta \mathfrak{m}$. An important special cases arises when the forward modelling operator $\mathbf{L}$ is linear in $\mathbf{u}$, so that $\mathbf{J}[\mathbf{u}(\mathfrak{m}), \mathfrak{m} ; \delta \mathbf{u}]=\mathbf{L}[\delta \mathbf{u}(\mathfrak{m}), \mathfrak{m}]$. Invoking the adjoint operator $\mathbf{L}^{\dagger}$ of $\mathbf{L}$, the adjoint equation (9.42) may then be rewritten as

$$
\mathbf{L}^{\dagger}(\mathbf{v})=\mathbf{f}^{\dagger} \text {. }
$$

As a final step in our derivation of the continuous adjoint method, we take a closer look at the misfit functional derivative (9.43). The scalar product $(\delta \mathbf{L}(\delta \mathfrak{m}), \mathbf{v})$ involves the Fréchet derivative of $\mathbf{L}$. As we have seen in section 9.2.1, this derivative is linear in $\delta \mathfrak{m}$. Consequently, the complete scalar product is a linear functional in $\delta \mathfrak{m}$. It then follows again from Riesz' representation theorem, that $\delta \chi$ can be expressed in terms of a scalar product between $\delta \mathfrak{m}$ and some Fréchet kernel $K$,

$$
\delta \chi=(K, \delta \mathfrak{m}) .
$$

Since $K$ refers to the derivative of the misfit, that is, to the sensitivity of the misfit with respect to model perturbations, it is frequently referred to as sensitivity kernel.

\subsubsection{Kernels and gradients - Linking to the discrete case}

In the previous paragraphs, we have seen that the discrete and continuous adjoint equations 'look' similar, and that their respective derivations follow nearly identical arguments. By investigating the relation between continuous sensitivity kernels and discrete finite-dimensional gradients, the link can be made even more explicit.

In fact, a sensitivity kernel represents the Fréchet derivative with respect to a continuous distributions of model parameters $\mathfrak{m}(\mathbf{x})$. However, the numerical optimisation schemes described in chapter 8 operate with gradients. To make the transition from kernels to gradients, we recall the representation of a continuous model parameter $\mathfrak{m}(\mathbf{x})$ in terms of a finite number of basis functions $b_{i}(\mathbf{x})$ from equation (1.1) in chapter 1, 


$$
\mathfrak{m}(\mathbf{x})=\sum_{i=1}^{n} m_{i} b_{i}(\mathbf{x})
$$

The expansion coefficients $m_{i}$ constitute the components of the discrete, finite-dimensional model vector $\mathbf{m}=$ $\left(m_{1}, \ldots, m_{n}\right)^{T}$. Our interest is in the partial derivatives of the misfit, $\partial \chi / \partial m_{i}$, defined as

$$
\frac{\partial \chi}{\partial m_{i}}=\lim _{\varepsilon \rightarrow 0} \frac{1}{\varepsilon}\left[\chi\left(\ldots\left(m_{i}+\varepsilon\right) b_{i} \ldots\right)-\chi\left(\ldots m_{i} b_{i} \ldots\right)\right] .
$$

From equation (9.47) we see that the partial derivative $\partial \chi / \partial m_{i}$ equals the Fréchet derivative $\delta \chi$ for a model perturbation $\delta m(\mathbf{x})=b_{i}(\mathbf{x})$. Inserting this form of the derivative into the representation of $\delta \chi$ in terms of sensitivity kernels (9.45), yields

$$
\frac{\partial \chi}{\partial m_{i}}=\int_{V} K(\mathbf{x}) b_{i}(\mathbf{x}) d \mathbf{x}
$$

Thus, the partial derivative of $\chi$ with respect to a coefficient $m_{i}$ is the projection of the kernel $K(\mathbf{x})$ onto the corresponding basis function $b_{i}(\mathbf{x})$.

\subsubsection{Model problem: The scalar wave equation}

In order to bring these rather abstract developments to life, we consider the concrete example of the scalar wave equation

$$
L[u(c), c]=\frac{1}{c^{2}(\mathbf{x})} \frac{\partial^{2}}{\partial t^{2}} u(\mathbf{x}, t)-\Delta u(\mathbf{x}, t)=f(\mathbf{x}, t),
$$

where the field $u(\mathbf{x}, t)$ is a wave propagating with the position-dependent phase velocity $c(\mathbf{x})=\mathfrak{m}(\mathbf{x})$, and $\Delta$ is the Laplace operator. The right-hand side $f(\mathbf{x}, t)$ is an external forcing term that excites the wave. Equations similar to (9.49) and their adjoints have been studied early-on in the context of seismic imaging to reveal the internal structure of the Earth (e.g. Bamberger et al., 1982; Lailly, 1983; Tarantola, 1984), and are now used in a wide range of wave-based imaging applications, often collectively referred to as full-waveform inversion. To ensure the existence of a unique solution, the wave equation (9.49) must be complemented by initial and boundary conditions. For simplicity, we set all wave motion at the initial time $t=t_{0}$ to 0 ,

$$
\left.u(\mathbf{x}, t)\right|_{t=t_{0}}=\left.\frac{\partial}{\partial t} u(\mathbf{x}, t)\right|_{t=t_{0}}=0,
$$

and choose the homogeneous Dirichlet boundary condition

$$
\left.u(\mathbf{x}, t)\right|_{\mathbf{x} \in \partial V}=0,
$$

where $\partial V$ denotes the boundary of the domain of interest $V$. Our first task is to derive the adjoint of the forward modelling operator $L$ in equation (9.49). Since $L$ is linear in $u$, this requires us to find an operator $L^{\dagger}$ that satisfies the slightly simplified version of the defining relation of the adjoint (9.40),

$$
(L(\delta u), v)=\left(L^{\dagger}(v), \delta u\right),
$$

for any $\delta u$ and $v$, and some scalar product $(.,$.$) . Motivated by the example in section 9.2.1, we choose the$ scalar product defined by the integral $\int_{V} \int_{T} d t d \mathbf{x}$, with a time interval $T=\left[t_{0}, t_{1}\right]$ during which we make our observations. We begin by writing out the left-hand side of (9.52) explicitly, omitting all time- and space dependencies in the notation, 


$$
(L(\delta u), v)=\int_{V} \int_{T}\left(\frac{1}{c^{2}} \frac{\partial^{2} \delta u}{\partial t^{2}}-\Delta \delta u\right) v d t d \mathbf{x} .
$$

In the interest of a transparent development, we analyse the temporal and spatial derivative terms in (9.53) separately. First, repeated integration by parts allows us to transform the second time derivative of the wavefield perturbation $\delta u$ into a second time derivative of the adjoint field $v$,

$$
\int_{V} \int_{T} v \frac{1}{c^{2}} \frac{\partial^{2} \delta u}{\partial t^{2}} d t d \mathbf{x}=\left.\int_{V} v \frac{1}{c^{2}} \frac{\partial \delta u}{\partial t} d \mathbf{x}\right|_{t=t_{0}} ^{t=t_{1}}-\left.\int_{V} \delta u \frac{1}{c^{2}} \frac{\partial v}{\partial t} d \mathbf{x}\right|_{t=t_{0}} ^{t=t_{1}}+\int_{V} \int_{T} \delta u \frac{1}{c^{2}} \frac{\partial^{2} v}{\partial t^{2}} d t d \mathbf{x} .
$$

While the third term on the right-hand side already has the desired form of a scalar product, the first two terms do not. Their lower integral bounds at $t=t_{0}$ vanish as a consequence of the zero initial conditions (9.50) that we imposed upon any wavefield $u$, and therefore also upon any wavefield perturbation $\delta u$. The contributions from the upper integral bounds at $t=t_{1}$ remain, unless we impose the terminal conditions

$$
\left.v(\mathbf{x}, t)\right|_{t=t_{1}}=\left.\frac{\partial}{\partial t} v(\mathbf{x}, t)\right|_{t=t_{1}}=0
$$

upon the adjoint field $v$. This leaves us with

$$
\int_{V} \int_{T} v \frac{1}{c^{2}} \frac{\partial^{2} \delta u}{\partial t^{2}} \delta u d t d \mathbf{x}=\int_{V} \int_{T} \delta u \frac{1}{c^{2}} \frac{\partial^{2} v}{\partial t^{2}} d t d \mathbf{x}
$$

We now turn to the spatial derivative term in (9.53) that involves the Laplacian. Making use of the relation

$$
\nabla \cdot(u \nabla v)-u \Delta v=\nabla \cdot(v \nabla u)-v \Delta u
$$

we obtain

$$
\int_{V} \int_{T} v \Delta \delta u d t d \mathbf{x}=\int_{V} \int_{T} \nabla \cdot(\delta u \nabla v) d t d \mathbf{x}-\int_{V} \int_{T} \nabla \cdot(v \nabla \delta u) d t d \mathbf{x}+\int_{V} \int_{T} u \Delta v d t d \mathbf{x} .
$$

Invoking Gauss' integral theorem, we can transform the first two terms on the right-hand side into integrals over the surface $\partial V$ of the domain,

$$
\int_{V} \int_{T} v \Delta \delta u d t d \mathbf{x}=\int_{\partial V} \int_{T} \mathbf{n} \cdot(\delta u \nabla v) d t d \mathbf{x}-\int_{\partial V} \int_{T} \mathbf{n} \cdot(v \nabla \delta u) d t d \mathbf{x}+\int_{V} \int_{T} u \Delta v d t d \mathbf{x},
$$

where $\mathbf{n}$ denotes the outward-pointing normal vector. The first term on the right-hand side vanishes because the boundary condition (9.51) states that any wavefield $u$, including the perturbation $\delta u$, is zero along the boundary $\partial V$. To ensure that the complete right-hand side takes the desired form of a scalar product, we furthermore impose a Dirichlet boundary condition for the adjoint field

$$
\left.v(\mathbf{x}, t)\right|_{\mathbf{x} \in \partial V}=0
$$

Finally collecting the temporal and spatial derivative parts, we find the transformed version of equation (9.53),

$$
(L(\delta u), v)=\int_{V} \int_{T}\left(\frac{1}{c^{2}} \frac{\partial^{2} \delta u}{\partial t^{2}}-\Delta \delta u\right) v d t d \mathbf{x}=\int_{V} \int_{T}\left(\frac{1}{c^{2}} \frac{\partial^{2} v}{\partial t^{2}}-\Delta v\right) \delta u d t d \mathbf{x}=\left(L^{\dagger}(v), \delta u\right) .
$$

From (9.62) we infer that the adjoint operator $L^{\dagger}$ is given by 


$$
L^{\dagger}[v(c), c]=\frac{1}{c^{2}(\mathbf{x})} \frac{\partial^{2}}{\partial t^{2}} v(\mathbf{x}, t)-\Delta v(\mathbf{x}, t)
$$

The results found so far merit some discussion. Most importantly, it is a pleasant surprise that the adjoint operator (9.62) is identical to the forward modelling operator (9.49), that is, $L=L^{\dagger}$. In such cases, we say that the operator $L$ is self-adjoint. Typically, self-adjointess goes hand in hand with energy conservation in systems governed by linear equations. It comes with the useful side effect that the numerical code used to solve the forward modelling equations can be used to solve the adjoint equations, as well. Even the boundary conditions are identical.

The major difference between the forward and the adjoint wavefields lies in the terminal conditions, which the latter has to satisfy. This means that the adjoint wavefield, after having propagated through the medium for some time, somehow needs to vanish. While this is easy to write down in an equation, the practical implementation is not trivial. In the vast majority of applications, the adjoint equations are simply solved backward in time, thereby turning the terminal conditions of $v(\mathbf{x}, t)$ into initial conditions of its time-reversed version $v(\mathbf{x},-t)$.

It remains to find an explicit expression of the misfit functional derivative, symbolically given as $\delta \chi=$ $(\delta \mathbf{L}(\delta \mathfrak{m}), \mathbf{v})$ in equation (9.43). For the Fréchet derivative of the forward modelling operator $L$ with respect to the model parameter $\mathfrak{m}=c$, we find

$$
\delta L[u(c), c ; \delta c]=-\frac{\delta c}{c^{3}} \frac{\partial^{2} u}{\partial t^{2}}
$$

Inserting (9.63) into (9.43), we obtain

$$
\delta \chi=-\int_{V} \int_{T}\left(\frac{1}{c^{3}} v \frac{\partial^{2} u}{\partial t^{2}}\right) \delta c d t d \mathbf{x}=\int_{V} \int_{T}\left(\frac{1}{c^{3}} \frac{\partial v}{\partial t} \frac{\partial u}{\partial t}\right) \delta c d t d \mathbf{x}
$$

Equation (9.64) states that we can compute the Fréchet derivative of the misfit functional $\chi$ in any direction (for any perturbation) $\delta c$ by solving the forward and adjoint equations just once, for $u$ and $v$, respectively, and then integrating the product of their time derivatives over time and space. Comparing the defining equation (9.48) of the sensitivity kernel $K$, to equation (9.65), we infer

$$
K(\mathbf{x}, t)=\frac{1}{c^{3}} \frac{\partial v}{\partial t} \frac{\partial u}{\partial t}
$$

Since the phase velocity $c$ is time-independent, it is customary to further integrate $K(\mathbf{x}, t)$ over time and to analyse the time-independent sensitivity

$$
K(\mathbf{x})=\int_{T} K(\mathbf{x}, t) d t=\int_{T} \frac{1}{c^{3}} \frac{\partial v}{\partial t} \frac{\partial u}{\partial t} d t
$$

If needed for numerical optimisation, this kernel may then be projected onto a finite number of basis functions in order to obtain the finite-dimensional gradient vector, as suggested by equation (9.48).

Calculating the units of sensitivity kernels: Equation (9.66) suggests that the physical unit of sensitivity kernels may be obtained from the units of the forward and adjoint wavefields. While the unit of the forward wavefield is trivially known, the unit of the adjoint wavefield is less straightforward. Focusing on equation (9.66), a much simpler way of obtaining the unit of $K$ is often overlooked: From equation (9.45) we know that

$$
\delta \chi=\int_{V} K(\mathbf{x}) \delta \mathfrak{m}(\mathbf{x}) d \mathbf{x}
$$


Knowing the unit of the misfit $\chi$ and the model parameters $\mathfrak{m}$, it immediately follows that the unit of $K$ is given by

$$
[K]=\frac{[\chi]}{[\mathfrak{m}] \mathrm{m}^{q}},
$$

where the integer $q$ is the dimension of the volume $V$, that is, $q=1$ in one dimension, $q=2$ in two dimensions, ....

Figure 9.5 illustrates the geometry behind the adjoint method for the scalar or similar wave equations. The source emits a wave that propagates through the medium towards a receiver. The adjoint source, located at the receiver position, emits the adjoint wavefield, which can be imagined as travelling in reverse time. Regions where the forward and the adjoint fields overlap define an influence zone, where sensitivity is non-zero. Depending on the properties of the medium, this influence zone is roughly cigar shaped.

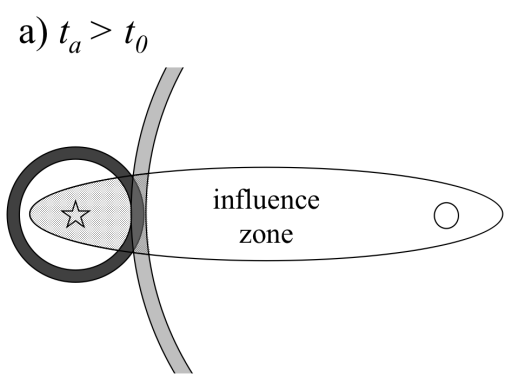

c) $t_{c}>t_{b}$

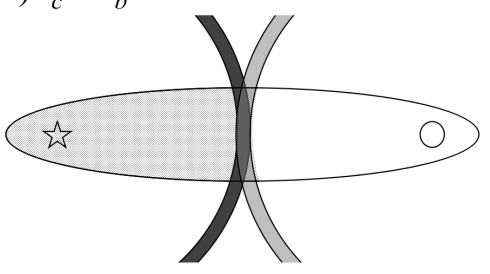

b) $t_{b}>t_{a}$

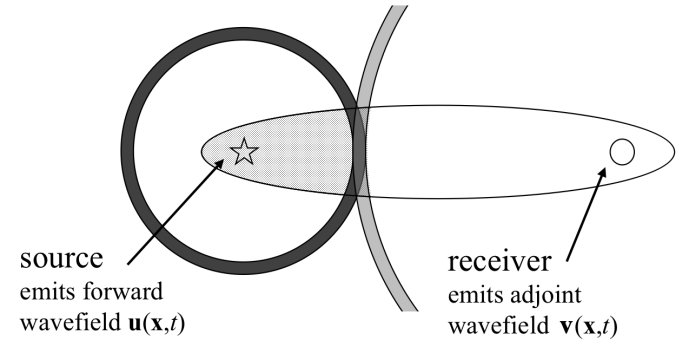

d) $t_{d}>t_{c}$

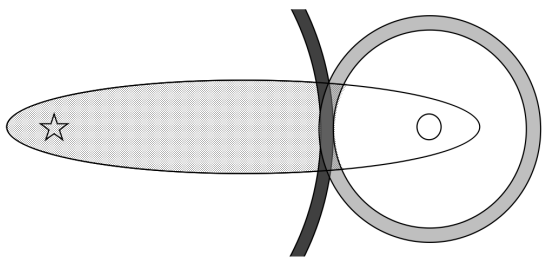

Fig. 9.5: Schematic summary of the geometry behind the adjoint method for the scalar or similar wave equations. (a) After some time $t_{a}>t_{0}$, the forward wavefield, shown as black circle, has propagated some distance away from the source, marked by the star. It overlaps with the adjoint wavefield, represented by the grey circle segment, within a small volume, which contributes to the influence zone. (b) - (d) As time progresses, the forward wavefield propagates towards the receiver, marked by an open circle. Seen in reverse time, that is, from (d) - (a), the adjoint field propagates outwards from the receiver position, into the medium. The resulting influence zone marks the region of non-zero sensitivity.

To further illustrate the adjoint equations and their application to the scalar wave equation (9.49), we consider the concrete example of the 2-D domain shown in figure 9.6a. Extending $100 \mathrm{~m}$ in $x$ - and $y$-direction, the phase velocity is constant at $600 \mathrm{~m} / \mathrm{s}$, with the exception of a small inclusion where the phase velocity is reduced to $500 \mathrm{~m} / \mathrm{s}$. We use this medium to compute artificial observations $u^{\text {obs }}\left(\mathbf{x}^{r}, t\right)$, which we use in lieu of actual observations in order to make this example more intuitive. Since the wave equation cannot be solved analytically for an arbitrarily heterogeneous medium, we resort to a numerical approximation, explained in detail in section 16.3. The reference medium, $c(\mathbf{x})=600 \mathrm{~m} / \mathrm{s}$ is perfectly homogeneous and produces the wavefield $u(\mathbf{x}, t)$, visualised in figure $9.6 \mathrm{~b}$. 
a) velocity distribution [perturbed medium]

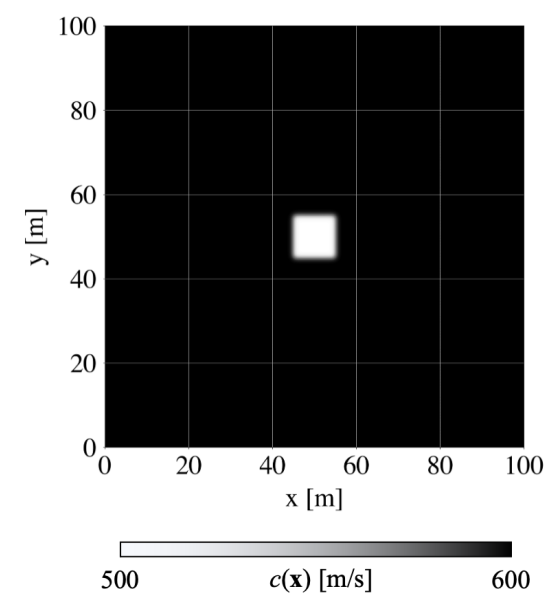

b) wavefield at $0.12 \mathrm{~s}$ [homog. medium]

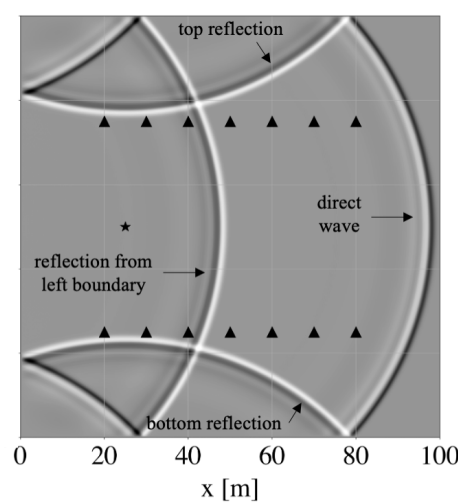

c) wavefield at $0.12 \mathrm{~s}$ [pert. medium]

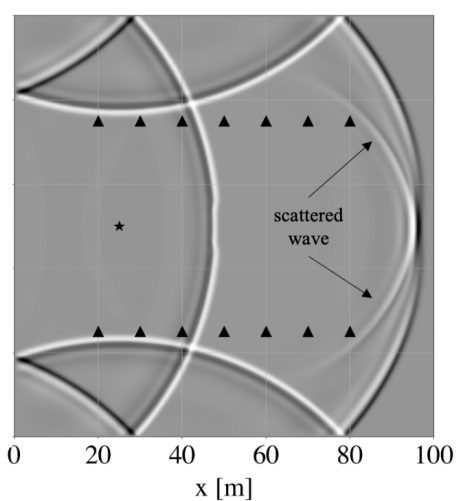

Fig. 9.6: Summary of the medium and wavefields used as examples for the sensitivity kernel calculation. (a) Phase velocity distribution used to compute artificial observations $u^{\text {obs }}\left(\mathbf{x}^{r}, t\right)$. (b) Wavefield snapshot at $t=0.12$ $\mathrm{s}$ for the reference medium with phase velocity $c(\mathbf{x})=600 \mathrm{~m} / \mathrm{s}$. The source is marked by a black star, and receiver positions $\mathbf{x}^{r}$ by black triangles. The direct wave, as well as reflections from the top, bottom and left boundaries are visible. (c) The wavefield for the perturbed medium in panel (a) contains an additional scattered wave, caused by the low-velocity inclusion. In a real-world application, this wavefield is only known at the receiver locations and not throughout the whole domain. [FD2Dpy]

The subtle differences between artificial observations $\mathbf{u}^{\text {obs }}\left(\mathbf{x}^{r}, t\right)$ and the forward wavefield $\mathbf{u}\left(\mathbf{x}^{r}, t\right)$ at the receiver locations $\mathbf{x}^{r}$, for $r=1, \ldots, N_{r}$ are shown in figure 9.7a. They define the waveform misfit

$$
\chi=\frac{1}{2} \sum_{r=1}^{N_{r}} \int_{t_{0}}^{t_{1}}\left[\mathbf{u}\left(\mathbf{x}^{r}, t\right)-\mathbf{u}^{\mathrm{obs}}\left(\mathbf{x}^{r}, t\right)\right]^{2} d t=\frac{1}{2} \int_{t_{0}}^{t_{1}} \int_{V}\left[\mathbf{u}(\mathbf{x}, t)-\mathbf{u}^{\mathrm{obs}}(\mathbf{x}, t)\right]^{2} \delta\left(\mathbf{x}-\mathbf{x}^{r}\right) d t d \mathbf{x},
$$

which is a slight generalisation of (9.34) for multiple receivers. The corresponding adjoint source is the superposition of individual adjoint sources for each receiver,

$$
\mathbf{f}^{\dagger}(\mathbf{x}, t)=-\sum_{r=1}^{N_{r}}\left[\mathbf{u}\left(\mathbf{x}^{r}, t\right)-\mathbf{u}^{\mathrm{obs}}\left(\mathbf{x}^{r}, t\right)\right] \delta\left(\mathbf{x}-\mathbf{x}^{r}\right)
$$

Injecting the sum of point sources into the adjoint equations, yields the sensitivity kernel displayed in figure 9.7b. The kernel is non-zero in regions where perturbations of the phase velocity $c(\mathbf{x})$ have an effect on the misfit. This includes the small area where the low-velocity perturbation is located, but also other parts of the medium.

\subsubsection{Misfit functionals and adjoint sources}

Having seen an example derivation of an adjoint operator in the previous section, we turn our attention to the right-hand side of the adjoint equation, that is, the adjoint source $\mathbf{f}^{\dagger}$. As we have shown in section (9.2.1), the adjoint source is uniquely defined by our - to some degree subjective - choice of a suitable misfit functional $\chi$, and it can be understood as the Fréchet kernel of $\chi$ with respect to field perturbations $\delta \mathbf{u}$. 
a) wavefield recordings

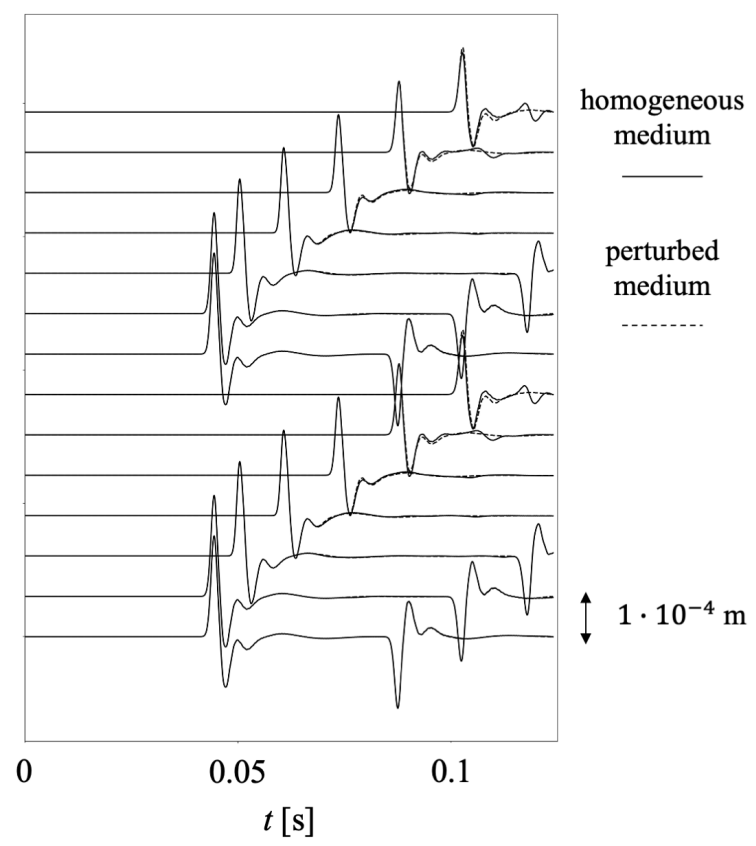

b) sensitivity kernel

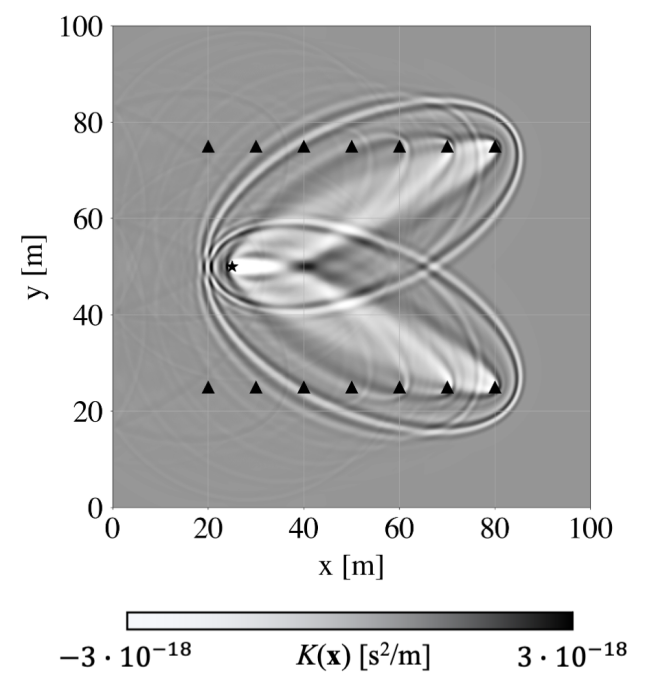

Fig. 9.7: Wavefield recordings and the resulting sensitivity kernel. (a) Wavefields for the homogeneous medium (solid) and the perturbed medium (dashed), sampled at the receiver positions $\mathbf{x}^{r}$. The subtle differences between the two define the misfit (9.69) and the corresponding adjoint source (9.70). (b) The sensitivity kernel outlines those parts of the medium where a phase velocity perturbation $\delta c(\mathbf{x})$ affects the misfit $\chi$. It can be interpreted as the negative direction of steepest descent. [FD2Dpy]

The list of misfit functionals that have been used for different purposes only in the field of wave-based imaging, is practically endless (e.g. Bozdağ et al., 2011; Brossier et al., 2010; Fichtner et al., 2008; Luo \& Schuster, 1991; van Leeuwen \& Mulder, 2010). We therefore limit ourselves to two examples that are primarily intended to outline the general procedure to obtain $\mathbf{f}^{\dagger}$ for a specific choice of $\chi$, however motivated.

\subsubsection{1 $L_{2}$ time series misfit}

As a starting point, we return to the $L_{2}$ time misfit already introduced in (9.34). To be more realistic, we now consider the $i$-component of a vector-valued, windowed and filtered time series recording,

$$
\chi=\frac{1}{2} \int_{T}\left[w(t) F(t) *\left(u_{i}\left(\mathbf{x}^{r}, t\right)-u_{i}^{\mathrm{obs}}\left(\mathbf{x}^{r}, t\right)\right)\right]^{2} d t .
$$

The convolutional filter $F(t)$, and the time windowing function $w(t)$ may serve, for instance, to isolate a specific parts of the recorded time series within a defined frequency band. To obtain the adjoint source corresponding to this choice of misfit, the variation $\delta \chi$ with respect to an infinitesimal field perturbation $\delta \mathbf{u}$ must be brought into the generic form (9.25). Using the identity

$$
\int a(t)[F(t) * b(t)] d t=\int b(t)[F(-t) * a(t)] d t,
$$


which holds for any functions $a(t)$ and $b(t)$, we find that

$$
\delta \chi=\int_{T} \int_{V} w^{2}(t)\left[F(-t) * F(t) *\left(u_{i}\left(\mathbf{x}^{r}, t\right)-u_{i}^{\mathrm{obs}}\left(\mathbf{x}^{r}, t\right)\right)\right] \delta\left(\mathbf{x}^{r}-\mathbf{x}\right) \mathbf{e}_{i}^{T} \delta \mathbf{u}(\mathbf{x}, t) d t d \mathbf{x},
$$

where $\mathbf{e}_{i}$ is the unit vector pointing in the $i$-direction. Comparing (9.113) with the generic form for adjoint sources (9.25), we infer that the adjoint source is

$$
\mathbf{f}^{\dagger}(\mathbf{x}, t)=-w^{2}(t)\left[F(-t) * F(t) *\left(u_{i}\left(\mathbf{x}^{r}, t\right)-u_{i}^{\mathrm{obs}}\left(\mathbf{x}_{r}, t\right)\right)\right] \delta\left(\mathbf{x}^{r}-\mathbf{x}\right) \mathbf{e}_{i}^{T}
$$

This adjoint source has several interesting properties. It is point-localised at the receiver point $\mathbf{x}^{r}$, and is a vector force acting in the direction parallel to the field component used to measure the misfit, that is, the $i$-component. The adjoint source-time function is a filtered version of the windowed residuals $u_{i}\left(\mathbf{x}_{r}, t\right)-u_{i}^{\text {obs }}\left(\mathbf{x}^{r}, t\right)$. The time window $w(t)$ is applied twice to the residual, and so appears squared. Further, the filter $F$ is also applied twice, once forward in time and once in reverse time. Thus, the complete effective filter $F(-t) * F(t)$ has zero phase. This property indirectly ensures that the adjoint field is properly aligned in time so that it interacts correctly with the forward field in the calculation of sensitivity kernels.

\subsubsection{Cross-correlation time shifts}

The cross-correlation time shift $\mathscr{T}$ is defined as the time where the cross-correlation function between observed and computed time series at some receiver position $\mathbf{x}^{r}$,

$$
C_{\left\{u_{i}^{\mathrm{obs}}, u_{i}\right\}}(\tau)=\int_{T} u_{i}^{\mathrm{obs}}\left(\mathbf{x}^{r}, t\right) u_{i}\left(\mathbf{x}^{r}, t+\tau\right) d t
$$

attains its global maximum. This definition requires $\mathscr{T}>0$ when the observed field arrives earlier than the simulated one, and $\mathscr{T}<0$ when the observations arrive later. The misfit functional that we wish to minimise, is now defined as

$$
\chi=\frac{1}{2} \mathscr{T}^{2} .
$$

Though (9.77) does not provide an explicit expression for $\mathscr{T}$, we can derive an explicit expression for its Fréchet derivative. For this, we note that $C_{\left\{u_{i}^{\mathrm{obs}}, u_{i}\right\}}(\tau)$ attains a maximum for $\tau=\mathscr{T}$, meaning that

$$
0=\frac{d}{d \tau} C_{\left\{u_{i}^{\mathrm{obs}}, u_{i}\right\}}(\mathscr{T})=-\int_{T} \frac{\partial}{\partial t} u_{i}^{\mathrm{obs}}\left(\mathbf{x}^{r}, t-\mathscr{T}\right) u_{i}\left(\mathbf{x}_{r}, t\right) d t .
$$

Using implicit function differentiation, yields the Fréchet derivative of $\chi$ :

$$
\delta \chi=\mathscr{T} \delta \mathscr{T}=\frac{\mathscr{T} \int_{T} \frac{\partial}{\partial t} u_{i}^{\mathrm{obs}}\left(\mathbf{x}^{r}, t-\mathscr{T}\right) \delta u_{i}\left(\mathbf{x}^{r}, t\right) d t}{\int_{T} \frac{\partial^{2}}{\partial t^{2}} u_{i}^{\mathrm{obs}}\left(\mathbf{x}^{r}, t-\mathscr{T}\right) u_{i}\left(\mathbf{x}^{r}, t\right) d t} .
$$

Making the simplifying assumption that the observed field $u_{i}^{\text {obs }}$ and the computed field $u_{i}$ are identical but shifted in time by $\mathscr{T}$, we find that $u_{i}^{\text {obs }}\left(\mathbf{x}^{r}, t-\mathscr{T}\right)=u_{i}\left(\mathbf{x}^{r}, t\right)$, and (9.78) condenses to

$$
\delta \chi=-\frac{\mathscr{T}}{E} \int_{T} \frac{\partial}{\partial t} u_{i}\left(\mathbf{x}^{r}, t\right) \delta u_{i}\left(\mathbf{x}^{r}, t\right) d t
$$


where the signal energy $E$ is defined as

$$
E=\int_{T}\left[\frac{\partial}{\partial t} u_{i}\left(\mathbf{x}^{r}, t\right)\right]^{2} d t
$$

A slight modification now allows us to bring (9.79) into the generic form (9.25),

$$
\delta \chi=-\frac{\mathscr{T}}{E} \int_{T} \int_{V} \frac{\partial}{\partial t} u_{i}\left(\mathbf{x}^{r}, t\right) \delta\left(\mathbf{x}-\mathbf{x}^{r}\right) \mathbf{e}_{i} \cdot \delta \mathbf{u}(\mathbf{x}, t) d t d \mathbf{x}
$$

Comparing (9.81) and (9.25), we infer that the adjoint source for the cross-correlation time shift misfit is given by

$$
\mathbf{f}^{\dagger}(\mathbf{x}, t)=\frac{\mathscr{T}}{E} \frac{\partial}{\partial t} u_{i}\left(\mathbf{x}^{r}, t\right) \delta\left(\mathbf{x}-\mathbf{x}^{r}\right) \mathbf{e}_{i}
$$

As a result of the point-wise measurement, the adjoint source is again localised at the receiver point. The adjoint source time function is determined by the time derivative of the computed field $\partial u_{i}\left(\mathbf{x}^{r}, t\right) / \partial t$, and the normalisation factor $E$ ensures that traveltime sensitivity does not depend on the amplitude of the forward field. The adjoint source, and therefore the sensitivity kernels, are quasi-independent of the data, except for the constant scaling factor $\mathscr{T}$. This is a result of our simplifying assumption that the observed and computed waveforms are sufficiently similar to allow for the replacement of $u_{i}^{\text {obs }}\left(\mathbf{x}^{r}, t-\mathscr{T}\right)$ by $u_{i}\left(\mathbf{x}^{r}, t\right)$.

Back-of-the-envelope checking sensitivity kernel values: Though the adjoint method is elegant and simple on paper, its correct numerical implementation is often not straightforward. Therefore, it is useful to have some simple tools to roughly check the accuracy of the results before running a more extensive gradient test, as described in section 9.1.3.

The cross-correlation time shift $\mathscr{T}$ is particularly useful in this context. In fact, consider a medium with a homogeneous velocity distribution $c(\mathbf{x})=$ const. Perturbing $c(\mathbf{x})$ by some small and constant $\delta c$ changes the traveltime of a wave propagating from a source at position $\mathbf{x}^{s}$ to some receiver at $\mathbf{x}^{r}$ by

$$
\mathscr{T}_{\delta c}=\frac{\left|\mathbf{x}^{r}-\mathbf{x}^{s}\right|}{c(\mathbf{x})+\delta c}-\frac{\left|\mathbf{x}^{r}-\mathbf{x}^{s}\right|}{c(\mathbf{x})} .
$$

The value of $\mathscr{T}_{\delta c}$, obtained from simple physical intuition, should be approximately equal to the crosscorrelation traveltime shift predicted by the adjoint-based sensitivity kernel, that is,

$$
\mathscr{T}_{\delta c} \approx \frac{\delta c}{\mathscr{T}} \int_{V} K(\mathbf{x}) d \mathbf{x}
$$

where $K(\mathbf{x})$ is the sensitivity kernel for the misfit $\chi=\frac{1}{2} \mathscr{T}^{2}$, as defined before in equation (9.76). Equations (9.83) and (9.84) provide a simple means of checking not the small details but the average amplitude of the sensitivity kernel $K(\mathbf{x})$. It is one, and possibly the simplest, of many examples for testing basic physics against a rather complex numerical adjoint machinery.

Equipped with equation (9.82), we return to the model problem in section 9.2.4, and continue using the scalar wave equation as an illustrative example, though with some small adaptations.

Taking a closer look at equation (9.78), we realise that the variation of the time shift, $\delta \mathscr{T}$, can be expressed as 


$$
\delta \mathscr{T}=\frac{\int_{T} \frac{\partial}{\partial t} u^{\mathrm{obs}}\left(\mathbf{x}^{r}, t-\mathscr{T}\right) \delta u\left(\mathbf{x}^{r}, t\right) d t}{\int_{T} \frac{\partial^{2}}{\partial t^{2}} u^{\mathrm{obs}}\left(\mathbf{x}^{r}, t-\mathscr{T}\right) u\left(\mathbf{x}^{r}, t\right) d t},
$$

where we omitted the subscript $i$, to account for the fact the the wavefield in this example is scalar. Following the same logic as before, we find the corresponding adjoint source,

$$
f^{\dagger}(\mathbf{x}, t)=\frac{1}{E} \frac{\partial}{\partial t} u\left(\mathbf{x}^{r}, t\right) \delta\left(\mathbf{x}-\mathbf{x}^{r}\right),
$$

with the scalar version of $E$ from (9.80). Equation (9.86) suggests that we can compute sensitivity kernels for time shift variations $\delta \mathscr{T}$ without actually having measured a time shift $\mathscr{T}$. This allows us to study the sensitivity of different wave arrivals purely on the basis of forward problem solutions.

Figure 9.8 illustrates how (9.86) may be used to understand the nature of various wave arrivals. With the identical computational setup as in figures 9.6 and 9.7, we approximately solve the scalar wave equation (9.49) with the finite-difference method described in section 16.3. For a receiver at $50 \mathrm{~m}$ distance from the source, this produces the wavefield recording $u\left(\mathbf{x}^{r}, t\right)$ in figure $9.8 \mathrm{a}$. In addition to the direct wave from source to receiver, the time series contains various other arrivals, corresponding to single or multiple reflections off the domain boundaries. Multiplying $u\left(\mathbf{x}^{r}, t\right)$ by a time window $w(t)$ that isolates individual arrivals, we can analyse the influence zoneinfluence zone or the wave path of each of them.

Centring the time window $w(t)$ on the direct arrival around $0.09 \mathrm{~s}$, produces the traveltime sensitivity kernel displayed in figure $9.8 \mathrm{~b}$. As intuitively expected, the kernel $K(\mathbf{x})$ connects source and receiver, and surrounds the ray path. It marks the area where velocity perturbations $\delta c(\mathbf{x})$ have a non-zero effect on the time shift $\mathscr{T}$. In this sense, $K(\mathbf{x})$ may be interpreted as a generalised Fresnel zone, the width of which is proportional to

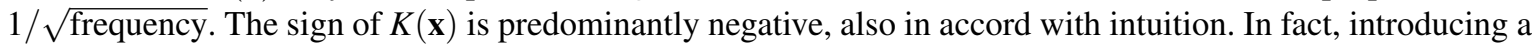
positive velocity perturbation $\delta c(\mathbf{x})$ causes the wave to travel faster and to arrive earlier. Hence, the time shift $\mathscr{T}$ should become smaller (possibly negative), meaning that $\delta \mathscr{T}=\int_{V} K(\mathbf{x}) \delta c(\mathbf{x}) d \mathbf{x}$ should be negative.

The traveltime sensitivity kernel in figure $9.8 \mathrm{~b}$ also has several apparently paradoxical features. Within a thin fringe, sensitivity is slightly positive. Therefore, a positive velocity perturbation $\delta c(\mathbf{x})$ that is localised inside this fringe will cause the wave to arrive a little later, and vice versa. Furthermore, sensitivity attains a local minimum along the ray path. This is in contrast to ray theory where all traveltime sensitivity is concentrated on the infinitely thin ray and zero anywhere else. Alluding to the curved and hollow shape of traveltime sensitivity kernels in the Earth, $K(\mathbf{x})$ is often somewhat jokingly referred to as a banana-doughnut kernel, and the counterintuitive vanishing of sensitivity along the ray is known as the banana-doughnut paradox (e.g. Dahlen et al., 2000; Marquering et al., 1999; Tong et al., 1998).

Just as regions of positive sensitivity, the banana-doughnut paradox is not a paradox but the result of comparing apples to oranges. The traveltime in ray theory is the onset time of an impulsive wavefront with infinitely broad frequency content. The traveltime shift defined by cross-correlation does not measure any onsets. It translates distortions of waveforms with finite frequency content, induced by velocity variations, into a measure of arrival time difference. As may be verified by numerical experiments (e.g. Tong et al., 1998), these distortions are indeed small when $\delta c(\mathbf{x})$ is located near the ray path, and they may be such that positive correlation shift results from a positive $\delta c(\mathbf{x})$. In summary, the ray theoretical and the cross-correlation time shift are different physical quantities, and this difference becomes apparent in the form of various finite-frequency effects, which are to some extent counter-intuitive but not paradoxical.

Placing $w(t)$ around the second arrival in 9.8a produces the sensitivity kernel in figure 9.8c. It reveals that the time window contains the reflections off the left and right boundaries of the domain, which happen to arrive at $\mathbf{x}^{r}$ at the same time. A thin elliptical feature surrounds the broad sensitivity along the reflected wave path. What may initially be mistaken as a numerical artefact is in fact the region where a small velocity perturbation $\delta c(\mathbf{x})$ produces a scattered wave that arrives just within the time window $w(t)$ around the second arrival. This means that the combined traveltime from the source to some point along the scattering ellipse and from there to the 
a) displacement field $u(\mathbf{x}, t)[\mathrm{m}]$ at receiver $\mathbf{x}^{r}$

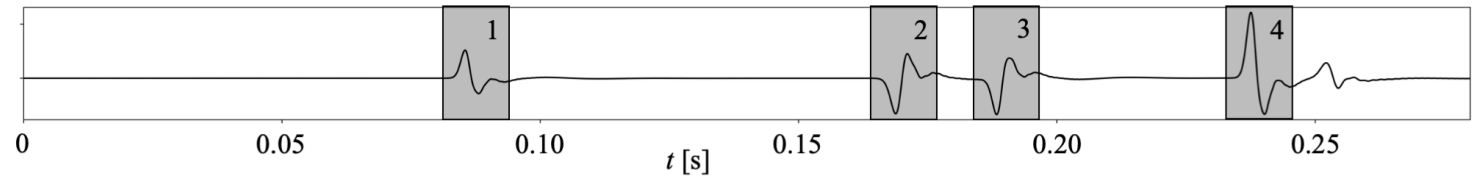

b) sensitivity kernel for time window 1

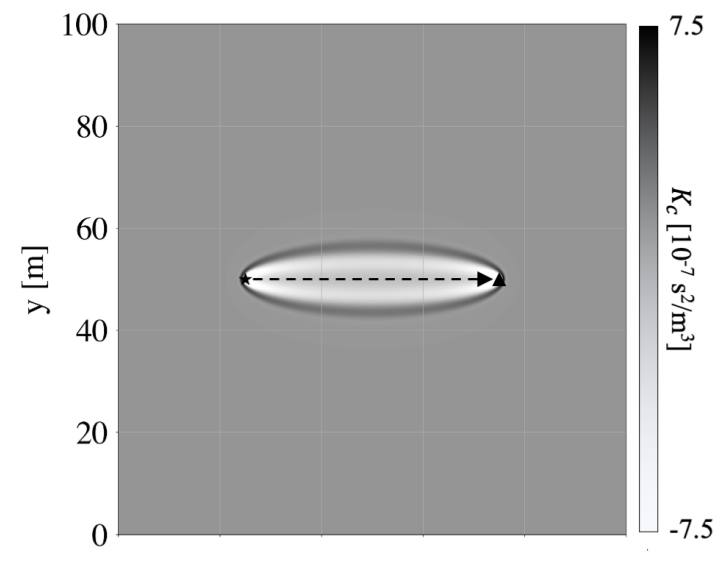

d) sensitivity kernel for time window 3

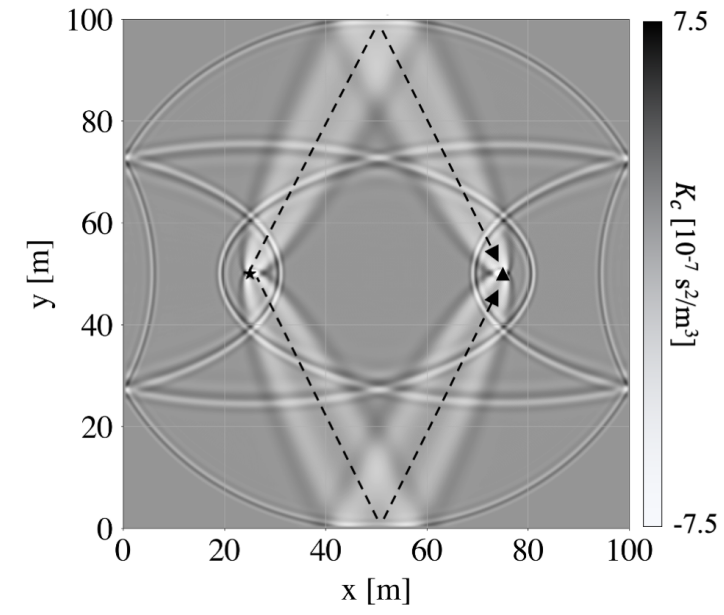

c) sensitivity kernel for time window 2

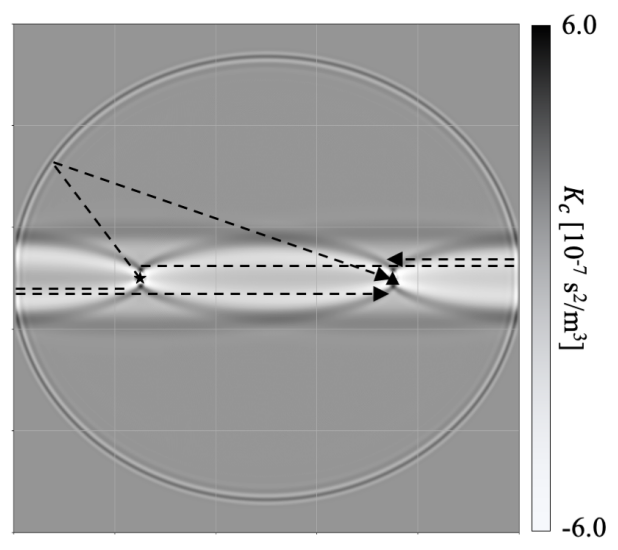

e) sensitivity kernel for time window 4

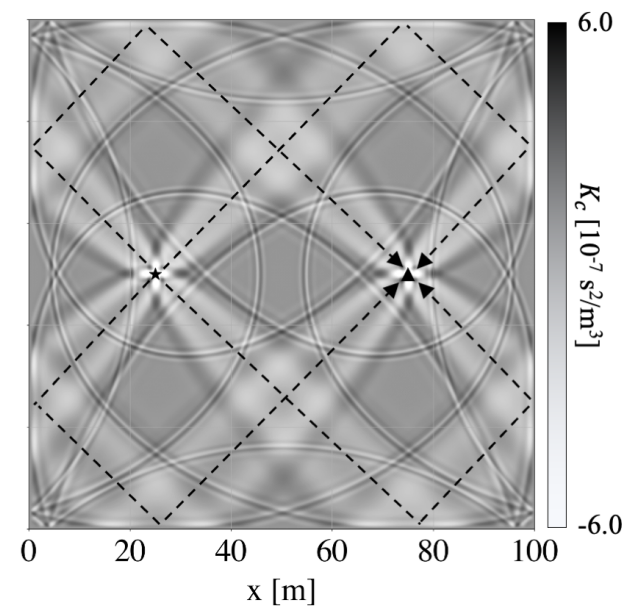

Fig. 9.8: Wavefield recordings and corresponding traveltime sensitivity kernels using the model problem setup from section 9.2.4 and figures 9.6 and 9.7. (a) Displacement field $u(\mathbf{x}, t)$ at the receiver position $\mathbf{x}=\mathbf{x}^{r}$. Different arrivals correspond to the direct wave and to various reflections off the boundary. Traveltime sensitivity kernels for the grey-shaded time windows are shown below. (b) Traveltime sensitivity kernel for the direct wave travelling from source (star) to receiver (triangle). The ray path, corresponding to a wave with infinitely high frequency, is indicated by a dashed arrow. (c) - (e) Traveltime sensitivity kernels for time windows 2, 3 and 4, containing (multiple) reflections off the domain boundary. [FD2Dpy] 
receiver is approximately identical to the traveltimes of the boundary-reflected waves.

Shifting the time window further onto other arrivals, produces sensitivity kernels with increasing complexity, such as those shown in figures $9.8 \mathrm{~d}$ and $9.8 \mathrm{e}$. They outline the influence zones of waves reflecting once off the top and bottom boundaries, or multiple times off a combination of boundaries. Also more complicated versions of the scattering ellipse from figure $9.8 \mathrm{c}$ can be distinguished, though their precise nature is more difficult to disentangle.

Very generally speaking, a sensitivity analysis as presented above can help us to understand how different aspects of the data react to model perturbations in different parts of the domain. This knowledge is invaluable for the design of the experiment, which often aims to maximise resolution while minimising computational and experimental costs.

\subsubsection{Model problem: The visco-elastic wave equation}

Building on the scalar example in section 9.2.4, we can also understand more complicated vectorial forward modelling equations and their adjoints. For this, we consider the special case where the operator $\mathbf{L}$ represents the visco-elastic wave equation, which governs the propagation of mechanical waves through continuous media, for instance, the propagation of ultrasound waves through an engineering part in non-destructive testing, or the propagation of seismic waves excited by earthquakes. Derivations similar to the one presented below, including more detail, may be found in Tarantola (1988), Tromp et al. (2005) or Fichtner et al. (2006a,b).

The elastic wave equation is equivalent to Newton's second law, which balances inertial forces against internal stresses and an external excitation (e.g. Aki \& Richards, 2002; Dahlen \& Tromp, 1998; Kennett, 2001). It is given by

$$
\mathbf{L}[\mathbf{u}(\mathfrak{m}), \mathfrak{m}]=\rho(\mathbf{x}) \frac{\partial^{2}}{\partial t^{2}} \mathbf{u}(\mathbf{x}, t)-\nabla \cdot \boldsymbol{\sigma}(\mathbf{x}, t)=\mathbf{f}(\mathbf{x}, t),
$$

where $\mathbf{u}$ is the elastic displacement field, $\rho$ the distribution of mass density, $\boldsymbol{\sigma}$ the stress tensor, and $\mathbf{f}$ an external force density. Assuming a linear visco-elastic rheology, the stress tensor $\sigma$ is related to the displacement gradient $\nabla \mathbf{u}$ via the constitutive relation

$$
\boldsymbol{\sigma}(\mathbf{x}, t)=\int_{\tau=t_{0}}^{\infty} \dot{\mathbf{c}}(\mathbf{x}, t-\tau): \nabla \mathbf{u}(\mathbf{x}, \tau) d \tau .
$$

The constitutive relation, which is a form of Hooke's law involves the time derivative $\dot{\mathbf{c}}$ of the fourth-order elastic tensor $\mathbf{c}$, which satisfies the symmetry relations $c_{i j k l}=c_{j i k l}=c_{k l i j}$. The symbol : in equation (9.88) denotes the tensor contraction, written in index notation as $(\dot{\mathbf{c}}: \nabla \mathbf{u})_{i j}=\sum_{k, l=1}^{3} \dot{c}_{i j k l} \partial u_{l} / \partial x_{k}$. The time dependence of the elastic tensor is responsible for visco-elastic attenuation, that is, the irreversible transformation of potential and kinetic energy of the wave into heat. In the specific context of the elastic wave equation, the continuous parameter vector $\mathfrak{m}$ is

$$
\mathfrak{m}(\mathbf{x})=[\rho(\mathbf{x}), \mathbf{c}(\mathbf{x}, t)] .
$$

The operator $\mathbf{L}$ is complemented by the initial and boundary conditions

$$
\left.\mathbf{u}\right|_{t \leq t_{0}}=\mathbf{0},\left.\quad \frac{\partial \mathbf{u}}{\partial t}\right|_{t \leq t_{0}}=\mathbf{0},\left.\quad \mathbf{n} \cdot \boldsymbol{\sigma}\right|_{\mathbf{x} \in \partial V}=\mathbf{0},
$$

with $\mathbf{n}$ defined as the outward-pointing normal vector on the boundary $\partial V$ of the medium. The conditions in (9.90) ensure that the medium is initially at rest, and that stresses normal to the boundary vanish.

As for the scalar wave equation, the operator $\mathbf{L}$ is linear in the wavefield $\mathbf{u}$. Hence, to compute the Fréchet derivative $\delta \chi$, we require the adjoint $\mathbf{L}^{\dagger}$ of $\mathbf{L}$. To find $\mathbf{L}^{\dagger}$, we first write an explicit form for the left-hand side 
of (9.40),

$$
\begin{aligned}
(\mathbf{L}(\delta \mathbf{u}), \mathbf{v}) & =\int_{T} \int_{V} \mathbf{v} \cdot \mathbf{L}(\delta \mathbf{u}) d t d \mathbf{x} \\
& =\int_{T} \int_{V} \rho \mathbf{v} \cdot \delta \frac{\partial^{2} \mathbf{u}}{\partial t^{2}} d t d \mathbf{x}-\int_{T} \int_{V} \mathbf{v} \cdot\left[\nabla \cdot \int_{\tau=t_{0}}^{t} \dot{\mathbf{c}}(t-\tau): \nabla \delta \mathbf{u}(\tau) d \tau\right] d t d \mathbf{x},
\end{aligned}
$$

where we omitted all explicit dependencies on $\mathbf{x}$ and $t$ for notational clarity. To isolate $\delta \mathbf{u}$, we start with the first term on the right-hand side of (9.91),

$$
\left(\mathbf{v}, \rho \frac{\partial^{2} \delta \mathbf{u}}{\partial t^{2}}\right)=\int_{T} \int_{V} \rho \mathbf{v} \cdot \frac{\partial^{2} \delta \mathbf{u}}{\partial t^{2}} d t d \mathbf{x}
$$

Double integration by parts yields

$$
\int_{T} \int_{V} \rho \mathbf{v} \cdot \frac{\delta^{2} \delta \mathbf{u}}{\partial t^{2}}=\int_{T} \int_{V} \rho \delta \mathbf{u} \cdot \frac{\partial^{2} \mathbf{v}}{\partial t^{2}}+\left.\int_{V} \rho \mathbf{v} \cdot \frac{\partial \delta \mathbf{u}}{\partial t} d \mathbf{x}\right|_{t=t_{1}}-\left.\int_{V} \rho \delta \mathbf{u} \cdot \frac{\partial \mathbf{v}}{\partial t} d \mathbf{x}\right|_{t=t_{1}}
$$

where we already used the initial conditions in (9.90). To bring (9.93) into the required form of a scalar product, we impose terminal conditions upon the adjoint field $\mathbf{v}$,

$$
\left.\mathbf{v}\right|_{t \geq t_{1}}=\mathbf{0},\left.\quad \frac{\partial \mathbf{v}}{\partial t}\right|_{t \geq t_{1}}=\mathbf{0}
$$

We are then left with the first contribution to the adjoint operator,

$$
\left(\rho \frac{\partial^{2} \delta \mathbf{u}}{\partial t^{2}}, \mathbf{v}\right)=\left(\rho \frac{\partial^{2} \mathbf{v}}{\partial t^{2}}, \delta \mathbf{u}\right)
$$

For the contribution that involves the spatial derivatives of $\delta \mathbf{u}$, we need to rearrange the expression

$$
\Gamma=(\nabla \cdot \boldsymbol{\sigma}, \mathbf{v})=\int_{V} \int_{T} \mathbf{v} \cdot\left[\nabla \cdot \int_{\tau=t_{0}}^{t} \dot{\mathbf{c}}(t-\tau): \nabla \delta \mathbf{u}(\tau) d \tau\right] d \mathbf{x} d t
$$

Recalling the symmetries of the elastic tensor $\mathbf{c}$, we find the relation

$$
\mathbf{v} \cdot[\nabla \cdot(\dot{\mathbf{c}}: \nabla \delta \mathbf{u})]=\nabla \cdot(\mathbf{v} \cdot \dot{\mathbf{c}}: \nabla \delta \mathbf{u})-\nabla \cdot(\delta \mathbf{u} \cdot \dot{\mathbf{c}}: \nabla \mathbf{v})+\delta \mathbf{u} \cdot[\nabla \cdot(\dot{\mathbf{c}}: \nabla \mathbf{v})]
$$

Using equation (9.97), we can rewrite (9.96) as

$$
\begin{aligned}
\Gamma & =\int_{V} \int_{t=t_{0}}^{t_{1}} \int_{\tau=t_{0}}^{t} \nabla \cdot[\mathbf{v}(t) \cdot \dot{\mathbf{c}}(t-\tau): \nabla \delta \mathbf{u}(\tau)] d \mathbf{x} d t d \tau-\int_{V} \int_{t=t_{0}}^{t_{1}} \int_{\tau=t_{0}}^{t} \nabla \cdot[\delta \mathbf{u}(\tau) \cdot \dot{\mathbf{c}}(t-\tau): \nabla \mathbf{v}(t)] d \mathbf{x} d t d \tau \\
& +\int_{V} \int_{t=t_{0}}^{t_{1}} \int_{\tau=t_{0}}^{t} \delta \mathbf{u}(\tau) \cdot[\nabla \cdot(\dot{\mathbf{c}}(t-\tau): \nabla \mathbf{v}(\tau))] d \mathbf{x} d t d \tau
\end{aligned}
$$

Invoking Gauss' theorem in combination with the integral identity 


$$
\int_{\tau=t_{0}}^{t} \int_{t=t_{0}}^{t_{1}} d \tau d t=\int_{t=\tau}^{t_{1}} \int_{\tau=t_{0}}^{t_{1}} d t d \tau
$$

we can write $\Gamma$ such that two of the contributing integrands can be eliminated,

$$
\begin{aligned}
\Gamma & =\int_{\partial V} \int_{t=t_{0}}^{t_{1}} \mathbf{v}(t) \cdot\left[\int_{\tau=t_{0}}^{t} \dot{\mathbf{c}}(t-\tau): \nabla \delta \mathbf{u}(\tau) d \tau\right] \cdot \mathbf{n} d \mathbf{x} d t-\int_{\partial V} \int_{\tau=t_{0}}^{t_{1}} \delta \mathbf{u}(\tau) \cdot\left[\int_{t=\tau}^{t_{1}} \dot{\mathbf{c}}(t-\tau): \nabla \mathbf{v}(t) d t\right] \cdot \mathbf{n} \\
& +\int_{V} \int_{\tau=t_{0}}^{t_{1}} \delta \mathbf{u}(\tau) \cdot\left[\nabla \cdot \int_{t=\tau}^{t_{1}} \dot{\mathbf{c}}(t-\tau): \nabla \mathbf{v}(t) d t\right] d \mathbf{x} d \tau .
\end{aligned}
$$

The first term in (9.100) vanishes because the expression in square brackets is the stress tensor $\boldsymbol{\sigma}$, and $\boldsymbol{\sigma} \cdot \mathbf{n}=\mathbf{0}$ on the boundary $\partial V$ as a consequence of the boundary condition (9.90). Since the adjoint field $\mathbf{v}$ is so far only constrained by the terminal conditions (9.94), we can impose a boundary condition that forces the second term to zero,

$$
\left.\mathbf{n} \cdot \boldsymbol{\sigma}^{\dagger}\right|_{\mathbf{x} \in \partial V}=\mathbf{0}
$$

where we define the adjoint stress tensor $\boldsymbol{\sigma}^{\dagger}$ as

$$
\boldsymbol{\sigma}^{\dagger}(t)=\int_{\tau=t}^{t_{1}} \dot{\mathbf{c}}(\tau-t): \nabla \mathbf{v}(\tau) d \tau
$$

The third integrand in (9.100) is already in the form that we require. Thus, it only remains to assemble the complete adjoint operator $\mathbf{L}^{\dagger}$ by combining equations (9.95) and (9.100). We thereby obtain

$$
\left(\mathbf{L}^{\dagger}(\mathbf{v}), \delta \mathbf{u}\right)=\left(\rho \frac{\partial^{2} \mathbf{v}}{\partial t^{2}}, \delta \mathbf{u}\right)-\Gamma=\left(\rho \frac{\partial^{2} \mathbf{v}}{\partial t^{2}}-\nabla \cdot \boldsymbol{\sigma}^{\dagger}, \delta \mathbf{u},\right)
$$

The result is that the adjoint operator $\mathbf{L}^{\dagger}$ is given by

$$
\mathbf{L}^{\dagger}[\mathbf{v}(\mathfrak{m}), \mathfrak{m}]=\rho \frac{\partial^{2} \mathbf{v}}{\partial t^{2}}-\nabla \cdot \boldsymbol{\sigma}^{\dagger}
$$

Hence, the complete adjoint equation is

$$
\rho \frac{\partial^{2} \mathbf{v}}{\partial t^{2}}-\nabla \cdot \boldsymbol{\sigma}^{\dagger}=\mathbf{f}^{\dagger}
$$

subject to the terminal and boundary conditions

$$
\left.\mathbf{v}\right|_{t \geq t_{1}}=\mathbf{0},\left.\quad \frac{\partial \mathbf{v}}{\partial t}\right|_{t \geq t_{1}}=\mathbf{0},\left.\quad \mathbf{n} \cdot \boldsymbol{\sigma}^{\dagger}\right|_{\mathbf{x} \in \partial V}=\mathbf{0} .
$$

In the definition of the adjoint stress tensor (9.102) we note that the time dependence is reversed relative to the time dependence of the regular stress tensor in (9.88). While regular stresses at time $t$ depend on the history of deformation prior to $t$, adjoint stresses at $t$ depend on future deformation at later times. This peculiarity leads to anti-attenuation, that we previously encountered in the 1-D discrete wave equation, studies in section 9.1.2. Both anti-attenuation and terminal conditions can be handled numerically by solving the adjoint equations in reverse time. In non-dissipative media, where the time dependence of the elastic tensor is given by $\dot{\mathbf{c}}(t)=\mathbf{c} \delta(t)$, the operator $\mathbf{L}$ is self-adjoint, meaning that $\mathbf{L}=\mathbf{L}^{\dagger}$.

As a final step, we assemble the Fréchet derivative of the misfit functional, for which we require the Fréchet 
derivative of the forward modelling operator $\mathbf{L}$ with respect to the continously defined model parameters, $\rho$ and c. In the interest of simplicity, we consider a non-dissipative medium, where

$$
\delta \mathbf{L}[\mathbf{u}(\mathfrak{m}), \mathfrak{m} ; \delta \mathfrak{m}]=\delta \rho(\mathbf{x}) \frac{\partial^{2}}{\partial t^{2}} \mathbf{u}(\mathbf{x}, t)-\nabla \cdot[\delta \mathbf{c}(\mathbf{x}): \nabla \mathbf{u}(\mathbf{x}, t)] .
$$

It now follows from (9.43) that $\delta \chi$ is given by

$$
\delta \chi=-\int_{T} \int_{V} \delta \rho(\mathbf{x}) \frac{\partial}{\partial t} \mathbf{v}(\mathbf{x}, t) \cdot \frac{\partial}{\partial t} \mathbf{u}(\mathbf{x}, t) d t d \mathbf{x}+\int_{T} \int_{V} \nabla \mathbf{v}(\mathbf{x}, t): \delta \mathbf{c}(\mathbf{x}): \nabla \mathbf{u}(\mathbf{x}, t) d t d \mathbf{x} .
$$

Equation (9.109) can be written more succinctly in terms of time-independent sensitivity kernels for density and the elastic tensor

$$
\delta \chi=\int_{V} K_{\rho}(\mathbf{x}) \delta \rho(\mathbf{x}) d \mathbf{x}+\int_{V} \mathbf{K}_{\mathbf{c}}(\mathbf{x}):: \delta \mathbf{c}(\mathbf{x}) d \mathbf{x}
$$

where :: denotes the tensor contraction $\mathbf{K}:: \delta \mathbf{c}=\sum_{i, j, k, l=1}^{3} K_{i j k l} \delta c_{i j k l}$. Comparing (9.108) and (9.109), we see that the kernels $K_{\rho}$ and $\mathbf{K}_{\mathbf{c}}$ are defined as

$$
K_{\rho}(\mathbf{x})=-\int_{T} \frac{\partial}{\partial t} \mathbf{v}(\mathbf{x}, t) \cdot \frac{\partial}{\partial t} \mathbf{u}(\mathbf{x}, t) d t, \quad \mathbf{K}_{\mathbf{c}}(\mathbf{x})=\int_{T} \nabla \mathbf{v}(\mathbf{x}, t) \otimes \nabla \mathbf{u}(\mathbf{x}, t) d t .
$$

The symbol $\otimes$ in (9.110) indicates the outer product $A_{i j} B_{k l}$ of two matrices $\mathbf{A}$ and $\mathbf{B}$. On the basis of (9.110), one may derive sensitivity kernels for other quantities that can be computed from the distributions of density and elastic parameters, such as wave speeds, attenuation parameters, or model parameters describing anisotropy (e.g. Fichtner, 2010; Fichtner \& van Driel, 2014; Sieminski et al., 2007a,b; Tromp et al., 2005).

The adjoint equations for the visco-elastic wave equation are a central building block of full-waveform inversion methods that combine numerical wavefield simulations and nonlinear optimisation schemes, such as those described in chapter 8. An essential benefit of full-waveform inversion is, as the name suggests, the ability to exploit complete waveform recordings with the goal to improve tomographic resolution. Despite being computationally more expensive than methods based on ray theory, this has led to numerous applications, especially in medical and seismic imaging (e.g. Bozdağ et al., 2016; Chen et al., 2007; Fichtner et al., 2009; Marty et al., 2021; Rickers et al., 2013; Tape et al., 2010).

Being a vector-valued equation with tensor-valued model parameters, the visco-elastic wave equation is not well suited for short computational examples based on a few lines of programme code. In order to still obtain some physical intuition, and to illustrate the range of possible applications, the following paragraphs contain some numerical examples computed with a finite-element solver of the visco-elastic wave equation (Afanasiev et al., 2019).

Sensitivity kernels for alternative sets of model parameters (change of variables): Visco-elastic wave propagation is controlled by the spatial distributions of density $\rho$ and the elastic parameters $c_{i j k l}$, sensitivity kernels for which can be computed via equation (9.110). In many applications, however, wave propagation velocities computed from $\rho$ and $c_{i j k l}$ are of greater interest. This raises the general question how sensitivity kernels $K_{i}(\mathbf{x})$ for some physical model parameters $\mathfrak{m}_{1}(\mathbf{x}), \ldots, \mathfrak{m}_{p}(\mathbf{x})$ can be transformed into sensitivity kernels $K_{i}^{*}(\mathbf{x})$ for the derived parameters $\mathfrak{m}_{1}^{*}(\mathbf{x}), \ldots, \mathfrak{m}_{p}^{*}(\mathbf{x})$, defined through some transformation equations

$$
\mathfrak{m}_{i}^{*}(\mathbf{x})=f_{i}\left[\mathfrak{m}_{1}(\mathbf{x}), \ldots, \mathfrak{m}_{p}(\mathbf{x})\right] .
$$

To solve this problem, we write the misfit variation $\delta \chi$ in terms of both $K_{i}(\mathbf{x})$ and $K_{i}^{*}(\mathbf{x})$, 


$$
\delta \chi=\sum_{i=1}^{p} \int_{V} K_{i}(\mathbf{x}) \delta \mathfrak{m}_{i}(\mathbf{x}) d \mathbf{x}=\sum_{i=1}^{p} \int_{V} K_{i}^{*}(\mathbf{x}) \delta \mathfrak{m}_{i}^{*}(\mathbf{x}) d \mathbf{x}
$$

Equation (9.112) simply states the requirement that a small change in misfit $\delta \chi$ must be the same, no matter if it originates from variations $\delta \mathfrak{m}_{i}$ or the corresponding variations $\delta \mathfrak{m}_{i}^{*}$. From the differentiation of (9.111) we obtain a relation between the different model parameter variations,

$$
\delta \mathfrak{m}_{i}^{*}=\sum_{j=1}^{p} J_{i j}(\mathbf{x}) \delta \mathfrak{m}_{j}(\mathbf{x}), \quad \text { for } i=1, \ldots, p,
$$

with the Jacobian of the transformation,

$$
J_{i j}(\mathbf{x})=\frac{\partial f_{i}}{\partial \mathfrak{m}_{j}}(\mathbf{x}) .
$$

Inserting (9.113) into (9.112), we obtain

$$
\sum_{i=1}^{p} \int_{V} K_{i}(\mathbf{x}) \delta \mathfrak{m}_{i}(\mathbf{x}) d \mathbf{x}=\sum_{i=1}^{p} \int_{V}\left[\sum_{j=1}^{p} K_{j}^{*}(\mathbf{x}) J_{j i}(\mathbf{x})\right] \delta \mathfrak{m}_{i}(\mathbf{x}) d \mathbf{x} .
$$

Since equation (9.115) must hold for any set of perturbations $\delta \mathfrak{m}_{i}$, the kernels $K_{i}(\mathbf{x})$ on the left-hand side must equal the expression in square brackets on the right-hand side, that is,

$$
K_{i}(\mathbf{x})=\sum_{j=1}^{p} K_{j}^{*}(\mathbf{x}) J_{j i}(\mathbf{x})
$$

Inverting the linear system of $p$ equations in (9.116), yields the transformed sensitivity kernels,

$$
K_{i}^{*}(\mathbf{x})=\sum_{j=1}^{p} K_{j}(\mathbf{x}), J_{i j}^{-1},
$$

where $J_{i j}^{-1}$ are the components of the inverse Jacobian. In addition to providing sensitivity kernels for parameters that are of interest in a particular application, transformations of the form (9.117) can be used in experimental design, with the goal to find a parameterisation where sensitivity for as many parameters as possible is negligible (e.g. Bernauer et al., 2014; Sieminski et al., 2009).

\subsubsection{Kernel examples: Global seismic wave propagation}

The propagation of seismic waves through the interior of the Earth is among the many phenomena described by the visco-elastic wave equation. Excited by a wide range of possible sources, including earthquakes, explosions, ocean waves, and anthropogenic activity, various types of seismic waves are present in the Earth. Surface waves, as their name suggests, are largely confined to the surface of the Earth, and have amplitudes that decay approximately exponentially with increasing depth. Body waves, in contrast, propagate to greater depth, thereby probing the deeper parts of our planet. They can be classified into shear waves and compressional waves, depending on their sense of particle motion. Along their path, body waves may reflect off and diffract along several internal discontinuities, defined, for instance, by the boundaries between crust, mantle and core of the Earth. 
The traveltimes of seismic waves carry information on the 3-D internal structure of the Earth that can be analysed quantitatively in terms of sensitivity kernels with respect to various material parameters, including the compressional wave velocity $\alpha$ and the shear wave velocity $\beta$, both of which can be derived from the distributions of density and the elastic tensor (e.g. Aki \& Richards, 2002).

Figure 9.9 displays a small collection of sensitivity kernels with respect to $\alpha$ and $\beta$ for cross-correlation traveltime measurements $\mathscr{T}$, as defined in section 9.2.6. The model $\mathfrak{m}$, used to compute synthetic seismograms, is a spherically symmetric approximation of Earth structure that is frequently used as a reference for 3-D structural variations inferred by seismic tomography (Kennett et al., 1995). The simplest case is that of a P-wave, which is a compressional wave travelling from some source near the surface, through the crust and mantle, and finally towards some receiver where it can be recorded. Being a compressional wave, the P-wave is primarily sensitive to the compressional wave velocity $\alpha$, as shown in figure $9.9 \mathrm{a}$. In contrast, its sensitivity to the shear wave velocity $\beta$, shown in figure $9.9 \mathrm{~b}$, is negligibly small. The PP-wave, in figure $9.9 \mathrm{c}$, is somewhat more complicated. Emitted from the source, it propagates downwards into the Earth, returns upwards, reflects at the surface to propagate downwards again, and after returning upwards once more, it is recorded at the receiver position. This complex trajectory, which can be summarised in the form of a thin ray path, translates into a more complicated distribution of sensitivity. Clearly, a PP-wave 'sees' very different parts of the Earth than a simple P-wave. The final example in figure $9.9 \mathrm{~d}$ is the sensitivity distribution with respect to $\alpha$ of a $\mathrm{P}_{\text {diff }}$ wave that diffracts along the boundary between the solid mantle and the fluid outer core. A wider range of examples may be found in Dahlen \& Baig (2002), Tromp et al. (2005), Liu \& Tromp (2008) or Fichtner (2010).

\subsubsection{Kernel examples: Transcranial ultrasound imaging}

At spatial and temporal scales that are around 50 million times smaller than those used in whole-Earth imaging, the visco-elastic wave equation can be used to simulate the propagation of ultrasound waves through the human brain. While ultrasound imaging of soft tissues, such as the human breast, is well-established (e.g. Boehm \& Fichtner, 2018; Pratt et al., 2007), ultrasound imaging of the brain remains a challenge due to the complex interactions between the wavefield and the skull (e.g. Guasch et al., 2020; Marty et al., 2021). The strong material contrast at the tissue-bone interface produces strong reflections, thereby limiting the transmission of ultrasound energy into the brain. Furthermore, conversions at the interface of compressional waves into shear waves and vice versa, complicate the wavefield and its analysis.

Transcranial imaging with ultrasound requires profound understanding of the wavefield generated by transducer arrays because different types of waves may be sensitive to different parts of the brain. Much of this understanding derives from the analysis of sensitivity kernels, a collection of which is presented in figure 9.10. The underlying structure, known as MIDA model (Iacono et al., 2015), includes, among other anatomical features, the outer skin layer, the scull, and different parts of the brain. To obtain a measure of wave amplitude as a function of brain structure, the misfit (measurement) functional is chosen as $\chi=\int p^{2}\left(\mathbf{x}^{r}, t\right) d t$, where $p^{2}\left(\mathbf{x}^{r}, t\right)$ is the pressure field recorded at transducer position $\mathbf{x}^{r}$.

Depending on the configuration of the source and receiver transducer, the wavefield senses different parts of the brain. This insight can be used to design imaging methods that harness the wavefield complexities in order to successively recover more and more detailed information about the structure of the brain (Marty et al., 2021). 
a) P-wave traveltime sensitivity

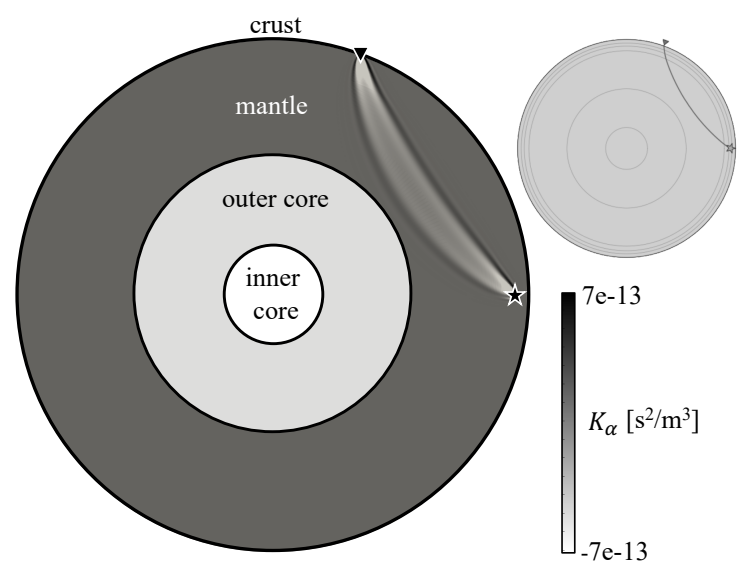

c) PP-wave traveltime sensitivity

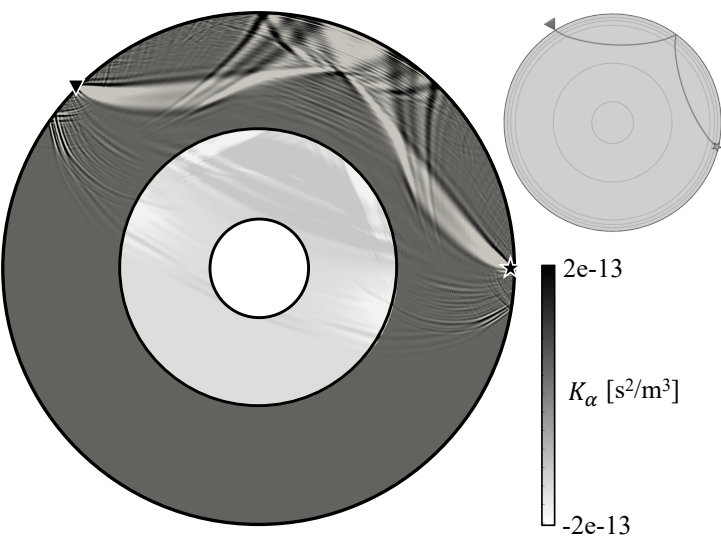

b) P-wave traveltime sensitivity

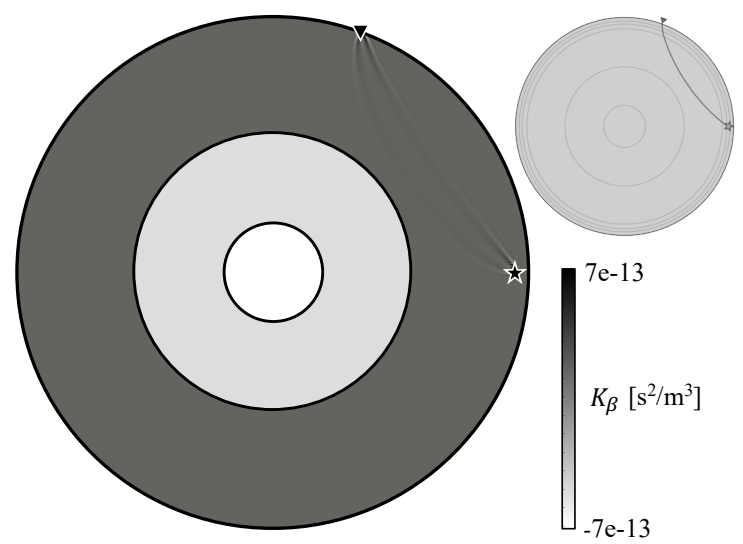

d) $P_{\text {diff-wave traveltime sensitivity }}$

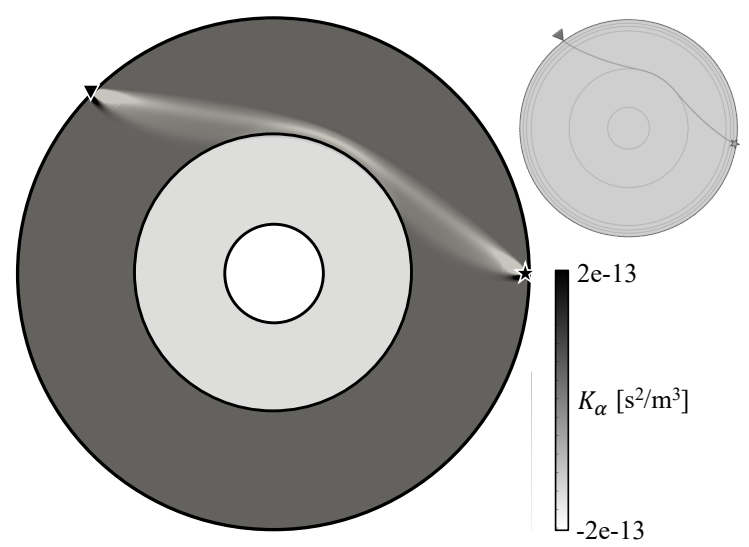

Fig. 9.9: Sensitivity kernels of various compressional waves with respect to the compressional wave velocity, $K_{\alpha}$, and the shear wave velocity, $K_{\beta}$. Simplified ray paths for the different types of waves are shown as small insets. The source position is indicated by a star, and the receiver position by a triangle. (a) The P-wave is the simplest kind of compressional wave, which propagates through the Earth's crust and mantle, directly from source to receiver. (b) In contrast to the sensitivity with respect to $\alpha$, shown in (a), sensitivity of the P-wave with respect to $\beta$ is very small. (c) The PP-wave is a compressional wave that experiences a surface reflection along its path from source to receiver. $(\mathbf{d})$ The $\mathrm{P}_{\text {diff }}$ wave diffracts along the boundary between the Earth mantle and outer core. 
a) MIDA model of the human head

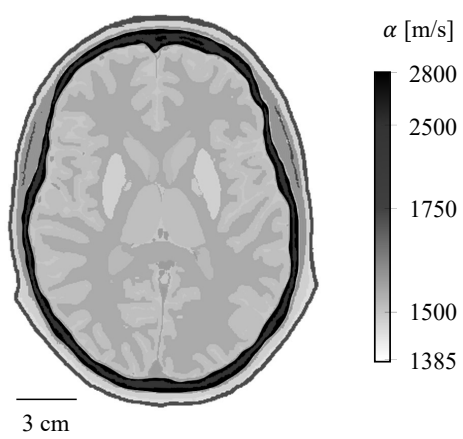

b) skull diffraction

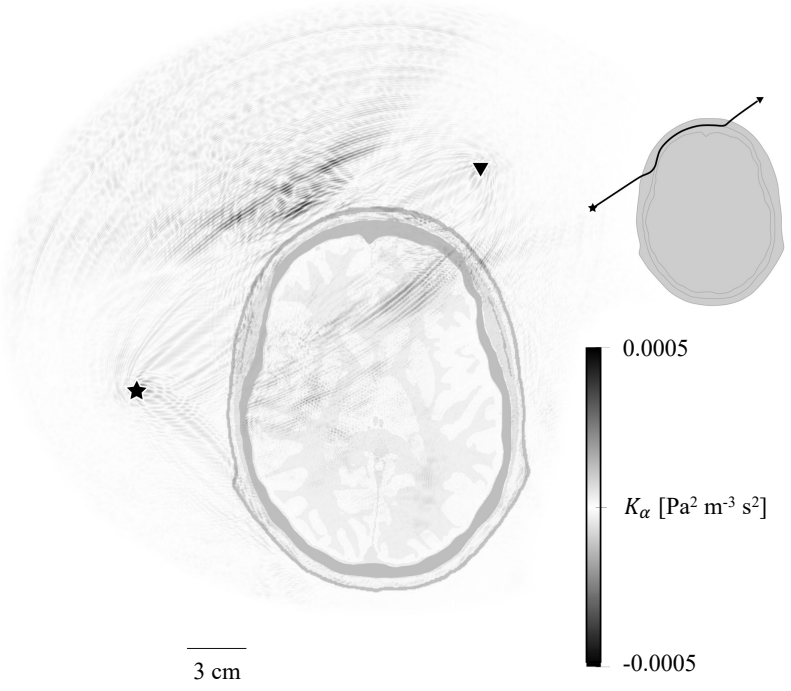

c) skin diffraction

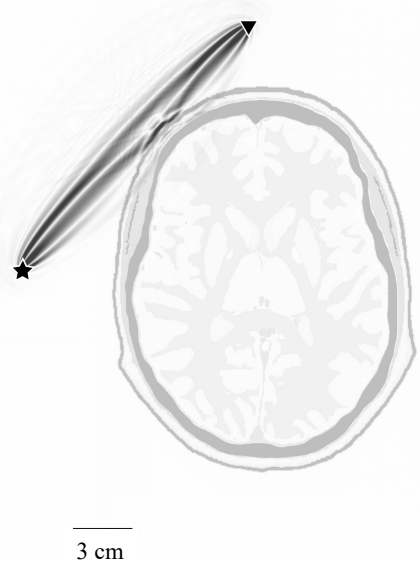

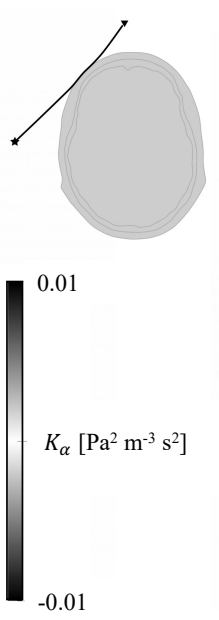

d) transmission and reflection

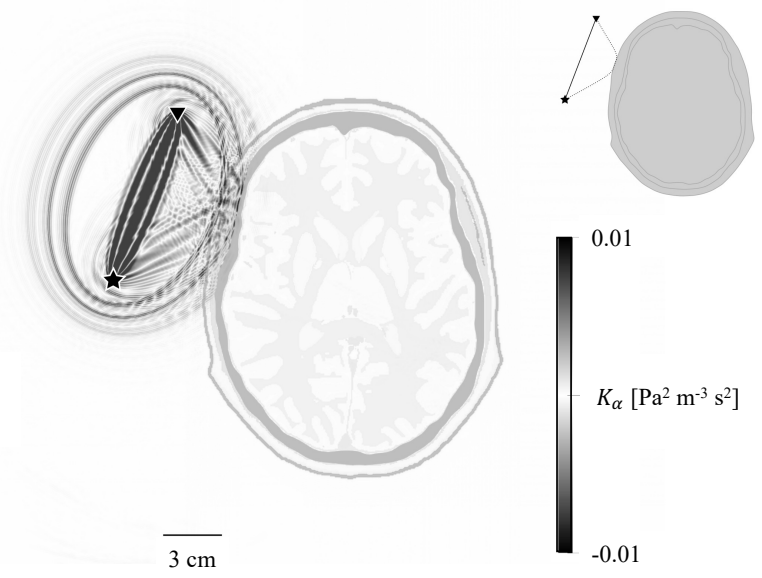

Fig. 9.10: Sensitivity kernels for transcranial ultrasound imaging. (a) Compressional wave velocity $\alpha$ in the MIDA model of the human head (Iacono et al., 2015). The contrast between the high velocities of the skull and the low velocities of the brain are clearly visible. (b) - (d) Sensitivity kernels with respect to $\alpha$ for different parts of an ultrasound recording, comprising transmitted, reflected and diffracted waves. The source location is marked by a black star, and the receiver location by a black triangle. The dominant frequency of the wavefield is $500 \mathrm{kHz}$. (Figure produced by Patrick Marty.) 

Part III

Analytically Solvable Inverse Problems 



\section{Chapter 10 \\ Inverse scattering in 1-D and the Marchenko equation}

Less computing! More thinking!

Brian L. N. Kennett, 4th QUEST Workshop, Benodet, 2013.

Waves propagating through a medium are scattered at heterogeneities. The scattered waves transport information about these heterogeneities towards receivers where the waves are recorded. The reconstruction of the heterogeneities as a function of space using recordings of scattered waves is known as the inverse scattering problem. In one dimension, the inverse scattering problem can be solved even when only one receiver is used. This requires the solution of an integral equation, known as the Marchenko equation, which we will derive in the following paragraphs.

\subsection{Introduction and problem statement}

We consider the one-dimensional wave equation

$$
\rho(x) \frac{\partial^{2}}{\partial t^{2}} u(x, t)-\frac{\partial^{2}}{\partial x^{2}}\left[\rho(x) c^{2}(x) u(x, t)\right]=0
$$

where $u$ is the particle displacement as a function of space $x$ and time $t, \rho$ is mass density, and $c$ is the wave speed. Our goal is to recover the medium properties $\rho(x)$ and $c(x)$ based on observations of the wave at some position $x_{0}$.

The geometric setup is illustrated in figure 10.1. We assume that a wave arrives from the left, where it first propagates through a homogeneous medium with constant density and velocity. At $x=0$, the wave reaches the heterogeneous part of the medium where density and velocity are spatially variable. The heterogeneities produce a scattered wave that propagates back into the homogeneous part, where it is recorded at $x=x_{0}$.

To reconstruct $\rho$ and $c$ in the heterogeneous part of the medium based on observations of the scattered waves, we will proceed as follows: In section 10.2, we first transform the wave equation (10.1) into a different partial differential equation, called the Schrödinger equation. This will reveal that $\rho$ and $c$ can actually not be constrained independently. Instead, we can only recover a function of $\rho$ times $c$, known as the scattering potential. In section 10.3, we will see that solutions to the Schrödinger equation are propagating pulses, and that the amplitude of these pulses is related to the scattering potential. Finally, in sections 10.4 and 10.5, we will demonstrate how the recording of the scattered wave at only one position can be used to construct a new wavefield from which the scattering potential as a function of space can be reconstructed directly. 


\section{homogeneous heterogeneous}

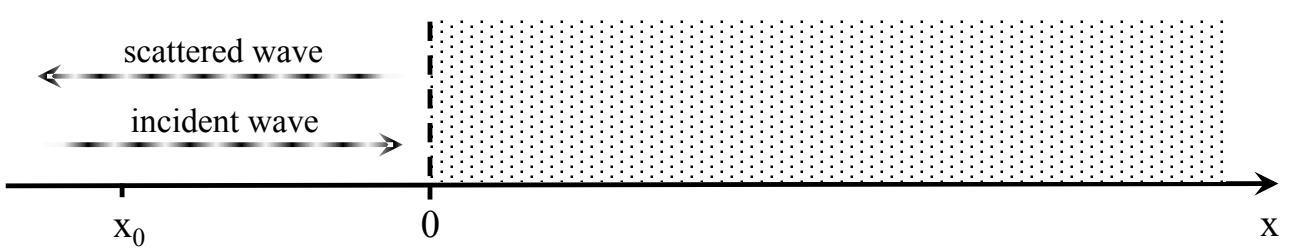

Fig. 10.1: Setup of the scattering experiment. After propagating through a homogeneous part of the medium at $x<0$, the incident wave reaches the heterogeneous part of the medium at $x>0$. The heterogeneities produce a back-scattered wave that is recorded at the observation point $x=x_{0}$.

In our developments, we will mostly follow the classic work of Burridge (1980). The analytical examples are taken from Ware \& Aki (1969). Additional numerical analyses can be found in Symes (1980), Santosa (1982), Bube \& Burridge (1983) and Ahmad \& Razzaghi (1998).

The Marchenko method, as presented here, is strictly valid only in 1-D, and an exact extension to higher dimensions has so far not been found. However, conceptually similar, approximate approaches in 2-D and 3-D have been proposed (e.g. Wapenaar et al., 2013, 2014).

\subsection{Transformation of the wave equation}

Inverse scattering problems similar to the one that we just formulated have been studied extensively in quantum mechanics. To take advantage of the developments in this field, we transform the wave equation (10.1) into a Schrödinger ${ }^{1}$ equation, originally invented to describe the state of a quantum-mechanical system. As a first step, we replace the space coordinate $x$ by the one-way traveltime $\tau$ that the wave requires to reach that position. Each small increment in position, $d x$, corresponds to a small increment in the traveltime, $d \tau$, given by

$$
d \tau=\frac{d x}{c(x)} .
$$

Integrating equation (10.2) gives the traveltime of the wave as a function of position,

$$
\tau(x)=\int_{0}^{x} \frac{d x^{\prime}}{c\left(x^{\prime}\right)} .
$$

The choice of the lower integral bound in equation (10.3) determines the location of zero traveltime. In fact, $\tau=0$ just when the wave reaches the heterogeneous part of the medium, which starts at $x=0$. While still being in the homogeneous part at $x<0$, traveltime $\tau$ is negative. Within $x>0$, traveltime is positive. Using equation (10.2), we may now replace the space derivative in (10.1) by a time derivative. In fact, we have

$$
\frac{\partial}{\partial x}\left(\rho c^{2} \frac{\partial u}{\partial x}\right)=\frac{d \tau}{d x} \frac{\partial}{\partial \tau}\left(\rho c^{2} \frac{d \tau}{d x} \frac{\partial u}{\partial \tau}\right)=c^{-1} \frac{\partial}{\partial \tau}\left(\rho c \frac{\partial u}{\partial \tau}\right)
$$

Multiplying (10.4) by $c$ and reinserting the result into (10.1), then gives the wave equation in the traveltime domain,

\footnotetext{
${ }^{1}$ Erwin Schrödinger (1887 - 1961) was an Austrian physicist. For his contributions to the development of quantum mechanics, he received the Nobel Prize in 1933, together with Paul Dirac. He discovered the equation named after him in 1926, and used it successfully to explain the energy spectrum of the hydrogen atom.
} 


$$
\rho c \frac{\partial^{2} u}{\partial t^{2}}-\frac{\partial}{\partial \tau}\left(\rho c \frac{\partial u}{\partial \tau}\right)=0 .
$$

Looking at equation (10.5), it becomes clear that the one-dimensional wave equation only has one effective medium parameter, namely the product of density and velocity, $\rho c$, known as impedance. Therefore, we replace $\rho c$ by the new parameter

$$
\eta=\sqrt{\rho c}
$$

which gives the wave equation

$$
\eta^{2} \frac{\partial^{2} u}{\partial t^{2}}-\frac{\partial}{\partial \tau}\left(\eta^{2} \frac{\partial u}{\partial \tau}\right)=0
$$

Introducing the scaled wavefield

$$
\psi=\eta u
$$

further transforms the wave equation to

$$
\frac{\partial^{2} \psi}{\partial t^{2}}-\frac{\partial^{2} \psi}{\partial \tau^{2}}+\frac{1}{\eta} \frac{d^{2} \eta}{d \tau^{2}} \psi=0
$$

Finally, for convenience, we define the scattering potential

$$
q=\frac{1}{\eta} \frac{d^{2} \eta}{d \tau^{2}}
$$

which leads to the Schrödinger equation

$$
\frac{\partial^{2} \psi}{\partial t^{2}}-\frac{\partial^{2} \psi}{\partial \tau^{2}}+q \psi=0
$$

The scattering potential $q$, defined in equation (10.10), depends on the second derivative of the square root of the impedance, $\eta=\sqrt{\rho c}$. This implies that $q$ is small when the curvature of the medium properties is small. In fact, when $\eta$ increases linearly as a function of one-way traveltime $\tau$, the second derivative vanishes, and $q=0$. Consequently, the Schrödinger equation (10.12) simplifies to a wave equation with wave speed 1 . This already suggests that solutions to the Schrödinger equation are wave-like.

An analytical solution: For arbitrary distributions of $\rho$ and $c$, the Schrödinger equation needs to be solved numerically. There are, however, few cases where an analytical solution is possible. One of these is given by

$$
\rho(x)=1+[\sqrt{1+2 x}-1] H(x)
$$

and

$$
c(x)=1+[\sqrt{1+2 x}-1] H(x),
$$

where $H$ is the Heaviside function. The traveltime $\tau$ follows from its definition in equation (10.3). For $x>0$, we have

$$
\tau(x)=\int_{0}^{x} \frac{d x^{\prime}}{c\left(x^{\prime}\right)}=\int_{0}^{x} \frac{d x^{\prime}}{\sqrt{1+2 x^{\prime}}}=\sqrt{1+2 x}-1
$$

and for $x<0$,

$$
\tau(x)=x
$$

It follows that the impedance is given by

$$
\rho(\tau) c(\tau)=\eta^{2}(\tau)=\left\{\begin{array}{lll}
1 & \text { for } & \tau<0, \\
(1+\tau)^{2} & \text { for } & \tau>0 .
\end{array}\right.
$$


The distribution of $c$ in the space domain and of $\eta$ in the one-way traveltime domain are shown in figure 10.2. Computing the derivatives
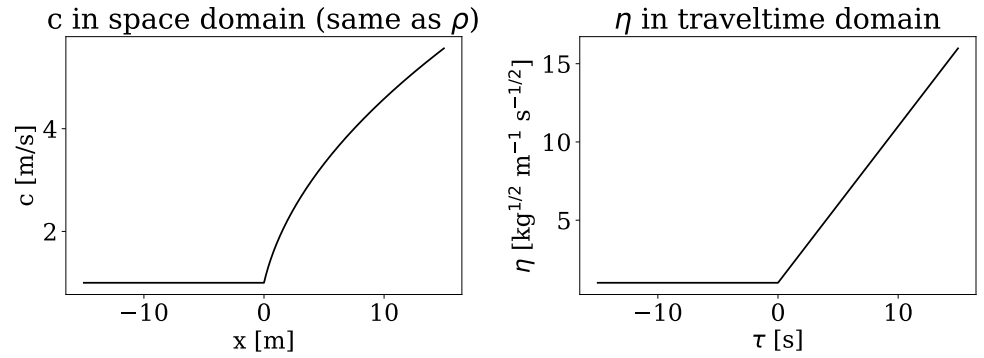

Fig. 10.2: Wave speed $c(x)$ in the space domain (left) and the square root of impedance $\eta(\tau)$ in the traveltime domain (right).

$$
\frac{d \eta}{d \tau}=H(\tau), \quad \text { and } \quad \frac{d^{2} \eta}{d \tau^{2}}=\delta(\tau)
$$

we then find the scattering potential

$$
q(\tau)=\delta(\tau) .
$$

An analytical solution of the Schrödinger equation (10.12) with scattering potential (10.18) is

$$
\psi_{f}(\tau, t)=\left\{\begin{array}{lll}
\delta(\tau-t)-\frac{1}{2} e^{-\frac{1}{2}(\tau+t)} H(\tau+t) & \text { for } \quad \tau<0 \\
\delta(\tau-t)-\frac{1}{2} e^{-\frac{1}{2}(\tau-t)} H(\tau-t) & \text { for } \quad \tau>0
\end{array}\right.
$$

For negative traveltimes, $\tau<0$, the wave consists of a $\delta$-pulse travelling to the right, which corresponds to the incident wave sketched in figure 10.1. Superimposed is a damped Heaviside function that propagates to the left. This is the scattered wave in figure 10.1, which results from the interaction of the incident wave with the non-zero scattering potential $q$. For $\tau>0$, the wave is a superposition of a $\delta$-pulse that continues travelling to the right, and a damped Heaviside function that travels to the right as well. These two constitute the transmitted wave. The complete wavefield $\psi$ is shown in figure 10.3.

Equation (10.19) constitutes a special solution of the Schrödinger equation. It is special not only for the type of medium for which it is valid (see equations 10.12 and 10.13), but also for the initial state, that is, the single $\delta$-pulse arriving from the left. In the following, we call such solutions with an incoming $\delta$-pulse a fundamental solution. For later convenience we will write the fundamental solution in the form

$$
\psi_{f}(\tau, t)=\delta(\tau-t)+R(\tau, t),
$$

where the non- $\delta$ component $R$ is the scattered wavefield. For $\tau<0$, it purely consists of the leftward travelling reflected wave set off by the nonzero scattering potential at $\tau \geq 0$. Thus, for $\tau<0$ we may write the fundamental solution as

$$
\psi_{f}(\tau, t)=\delta(\tau-t)+R(t+\tau) .
$$

The observations from which we try to recover the scattering potential are the reflection data recorded at the one-way traveltime position $\tau_{0}$, that is, $R\left(t+\tau_{0}\right)$. 

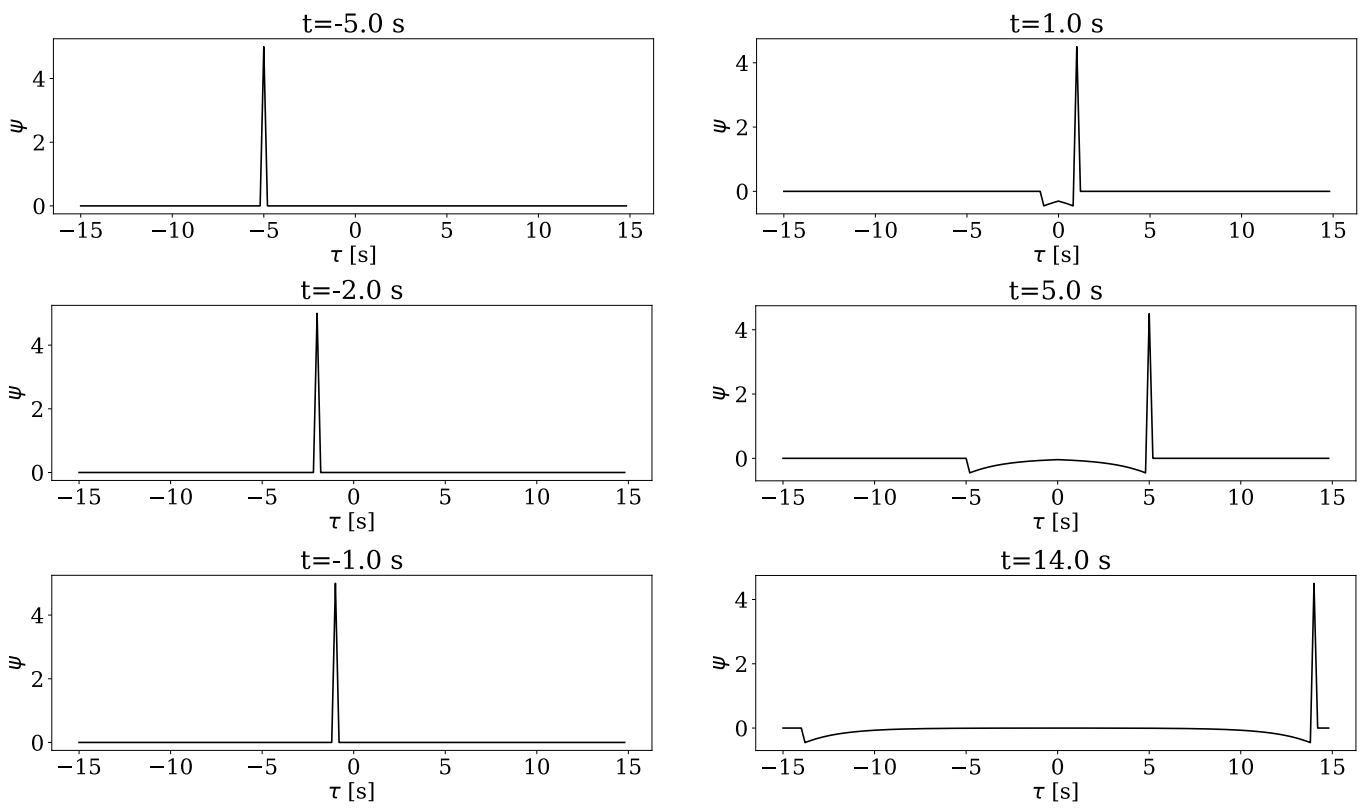

Fig. 10.3: The wavefield $\psi_{f}(\tau, t)$ from equation (10.19) for the medium shown in figure 10.2. Wavefields produced by an incoming $\delta$-pulse are called fundamental solutions.

\subsection{Propagating pulses}

In the analytical example we saw that the solution takes the form of pulses that propagate with velocity \pm 1 along the one-way traveltime axis. For a more general medium with a more complicated distribution of the scattering potential $q(\tau)$, a similarly simple analytical solution may not be available. Instead, we try to find an approximate solution by making a qualified guess or ansatz of what a solution may look like. Using our intuition that $\psi(\tau, t)$ should roughly take the form of pulses propagating with some velocity along the $\tau$ axis, we try

$$
\psi(\tau, t)=\sum_{n=0}^{\infty} a_{n}(\tau) f_{n}[t-\phi(\tau)] .
$$

Equation (10.22) is a superposition of wave shapes or wavelets $f_{n}$ with amplitude $a_{n}$ dependent on $\tau$. As time $t$ progresses, each wavelet is shifted by a phase $\phi$, which depends on $\tau$ as well. Our goal is to determine $a_{n}$, $f_{n}$ and $\phi$ by substituting the ansatz (10.22) into the Schrödinger equation (10.12). To reduce the number of unknowns, we impose an additional relation between the wavelets with successive indices,

$$
\frac{d f_{n}(z)}{d z}=f_{n-1}(z)
$$

In more than one dimension, that is, for a two- or three-dimensional $\tau$, the solution ansatz described by equations (10.22) and (10.23) is known as the ray expansion. It leads to the description of waves in terms of rays, well-known from geometric optics.

Equation (10.23) is a recursion relation. Knowing the wavelet $f_{n}$ for a specific $n$, we can compute the other wavelets either by differentiation (to get lower indices) or integration (to get higher indices). Again looking at the analytical solution from section 10.2 , we make the guess that

$$
f_{0}(z)=\delta(z),
$$


may be a good starting point. Using the recursion in (10.23) we then find

$$
f_{1}(z)=H(z), \quad f_{2}(z)=z H(z), \quad f_{3}(z)=\frac{1}{2} z^{2} H(z), \quad f_{4}(z)=\frac{1}{6} z^{3} H(z), \quad \ldots,
$$

or more generally

$$
f_{n}(z)=\frac{1}{(n-1) !} z^{n-1} H(z), \quad \text { for } n>0 .
$$

Equations (10.25) and (10.26) reveal that the sequence of wavelets constitutes a Taylor expansion of a more general wave shape for $z=t-\phi(\tau) \geq 0$. The first four wavelets are illustrated in figure 10.4. For a specific position in the medium, expressed by the one-way traveltime $\tau$, each wavelet $f_{n}(t-\phi(\tau))$ arrives at time $t=\phi(\tau)$. The initial amplitude of the complete wave is determined by the coefficients $a_{0}$ and $a_{1}$ of the first two wavelets $f_{0}(t-\phi(\tau))$ and $f_{1}(t-\phi(\tau))$. This is because the other wavelets $f_{n>1}(t-\phi(\tau))$ are zero at time $t=\phi(\tau)$.
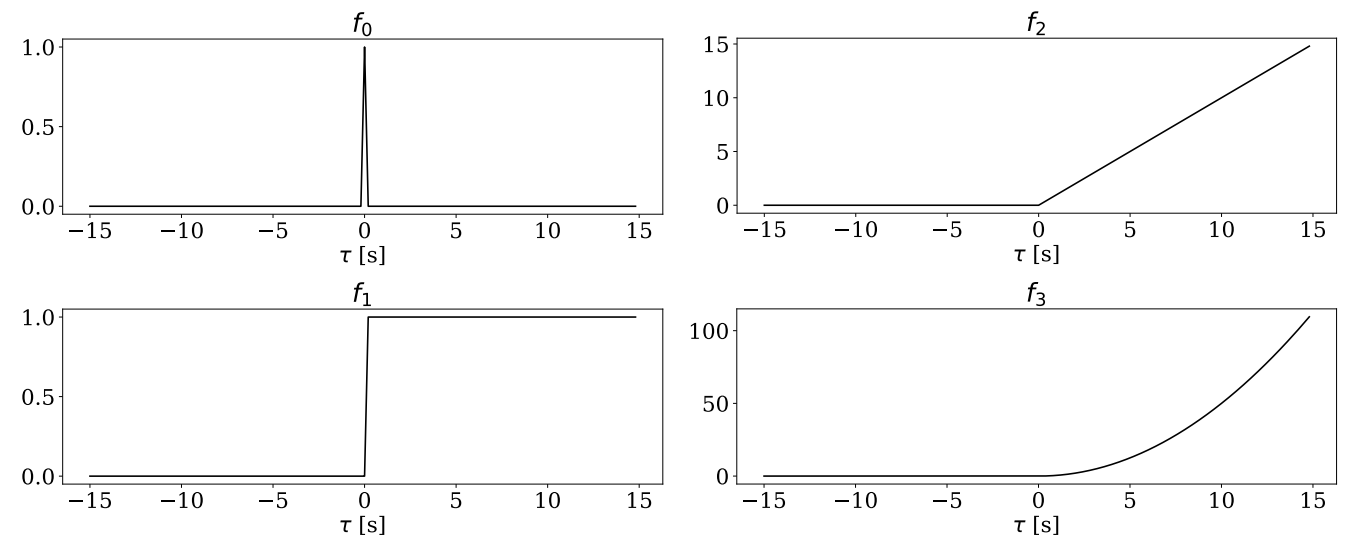

Fig. 10.4: The first four wavelets, $f_{0}$ to $f_{3}$, entering the expansion in equation (10.25).

Inserting the expansion (10.22) into the Schrödinger equation (10.12), yields

$$
0=\sum_{n=0}^{\infty}\left[\left(1-\left(\frac{d \phi}{d \tau}\right)^{2}\right) a_{n}+2 \frac{d a_{n-1}}{d \tau} \frac{d \phi}{d \tau}+q a_{n-2}-\frac{d^{2} a_{n-2}}{d \tau^{2}}+a_{n-1} \frac{d^{2} \phi}{d \tau^{2}}\right] f_{n-2}(t-\phi(\tau)),
$$

where, for convencience, we defined $a_{-2}=a_{-1}=0$, and used the recursion relation (10.23). Since the sum in equation (10.27) must vanish for all times $t$, the terms in square brackets must vanish for each individual $n>0$. Considering each $n$ separately, allows us to determine the amplitude coefficients $a_{n}$ and the phase function $\phi$. Starting with $n=0$, we get

$$
\left[1-\left(\frac{d \phi}{d \tau}\right)^{2}\right] a_{0}=0
$$

It follows that

$$
\left|\frac{d \phi}{d \tau}\right|=1
$$


Equation (10.28) is known as the eikonal equation ${ }^{2}$. It describes the evolution of the phase function with position, expressed by the one-way traveltime $\tau$. The eikonal equation has two solutions. Taking $d \phi / d \tau=1$, and requiring that $\phi=0$ for $\tau=0$, gives

$$
\phi=\tau
$$

This corresponds to a wave that travels from left to right with speed 1 . Alternatively, taking $d \phi / d \tau=-1$, yields

$$
\phi=-\tau,
$$

which corresponds to a wave travelling from right to left with speed 1. The results from equations (10.30) and (10.31) are in accord with the transformation of the wave equation to the Schrödinger equation in section 10.2. In fact, we replaced the space variable $x$ by the one-way traveltime $\tau$. We therefore expect that the time needed to reach a certain position in $\tau$-space is actually the traveltime of the wave. Equations (10.30) and (10.31) also imply that all waves propagate with speed 1 , either in positive or in negative $\tau$-direction.

We continue with $n=1$. Realising that the eikonal equation implies $d^{2} \phi / d \tau^{2}=0$, we obtain

$$
0=\frac{d a_{0}}{d \tau} \frac{d \phi}{d \tau}= \pm \frac{d a_{0}}{d \tau}
$$

If follows that the amplitude coefficient $a_{0}$ is constant. The actual value will depend on the strength of the source that sets off the wave in the first place. Here, for convenience, we set

$$
a_{0}=1 \text {. }
$$

Equation (10.33) implies that the $\delta$-pulse travels with constant amplitude. No matter what the distribution of the scattering potential $q(\tau)$ is, the $\delta$-component of the wave does not change its amplitude.

Finally, we consider $n=2$. Taking into account that $d^{2} a_{0} / d \tau^{2}=0$ and $d^{2} \phi / d \tau^{2}=0$, we find

$$
2 \frac{d a_{1}}{d \tau}=-q \frac{d \phi}{d \tau}
$$

where $d \phi / d \tau$ is either 1 for a wave travelling to the right, or -1 for a wave travelling to the left. Equation (10.34) is a special form of a transport equation. It describes how amplitude is transported through the medium. When the scattering potential is positive, $q>0$, the amplitude $a_{1}$ of wavelet $f_{1}$ decreases as it propagates from left to right. In contrast, a negative scattering potential, $q<0$, increases the amplitude of a rightward travelling wavelet.

\subsection{The annihilator wavefield}

Equation (10.34) has other interesting implications for the inverse scattering problem. In fact, we already noted that the initial amplitude of the complete wave at $t=\phi(\tau)$ is controlled only by the amplitudes of the first two wavelets, $f_{0}$ and $f_{1}$. This is because all other wavelets, $f_{n>1}$ start with zero amplitude, as shown in figure 10.4. From equation (10.33) we know that the amplitude $a_{0}$ of the $\delta$-component $f_{0}=\delta$ is constant. It therefore follows that information on the scattering potential $q$ may be extracted by measuring the amplitude of the wave right at time $t=\tau$. In fact, assume that the wavefield has the form

$$
\psi_{a}(\tau, t)=\delta(t-\tau)+A(\tau, t) .
$$

\footnotetext{
${ }^{2}$ The eikonal, from the Greek word eikon, meaning image, is the path of a light ray. The word is also used for the term $t-\phi(\tau)$ in the ray expansion.
} 
Looking at the ray expansion in equation (10.22), this is the wavefield with $a_{0}=1$ and $f_{0}=\delta$. The non- $\delta$ component $A(\tau, t)$ is given by

$$
A(\tau, t)=a_{1} H(t-\tau)+\sum_{n=2}^{\infty} a_{n}(\tau) f_{n}(t-\tau) .
$$

Observing the wavefield $\psi_{a}$ at position $\tau$ at time $t=\tau$, gives

$$
A(\tau, \tau)=a_{1},
$$

and making use of equation (10.34), we may indeed recover the scattering potential,

$$
2 \frac{d A(\tau, \tau)}{d \tau}=2 \frac{d a_{1}(\tau)}{d \tau}=-q(\tau) \frac{d \phi}{d \tau} .
$$

While equation (10.38) formally provides a recipe for the reconstruction of $q(\tau)$ based on observations of the non- $\delta$ component of the wavefield $\psi_{a}$, there are still two complications that prevent it from being immediately useful for the solution of the inverse scattering problem:

1) Equation (10.38) requires that the wavefield $\psi_{a}$ actually be known at any position, that is, for any $\tau$. However, we only have an observation of the fundamental solution $\psi_{f}$ at a specific $\tau_{0}=x_{0}>0$.

2) Equations (10.36) to (10.38) assume that there is only one Heaviside function propagating to the right, towards larger $\tau$. However, as illustrated in figures 10.1 and 10.3, the incoming $\delta$-pulse also generates a scattered wavefield that travels to the left. The presence of more than one wavefront would make the evaluation at $t=\tau$ ambiguous.

To overcome the first complication, we need to find a way to compute the wavefield $\psi_{a}$ for all $\tau$ from a single observation of the fundamental solution $\psi_{f}$ at $\tau_{0}$. For the second, we need to construct a wavefield $\psi_{a}$ without a scattered wave. The complete suppression of a scattered wave may be achieved by injecting another wavelet coming from the right. If properly constructed, this wavelet perfectly annihilates the scattered wave caused by the incoming pulse $\delta(t-\tau)$. For $t \ll 0$, that is, before entering the heterogeneity from the right, this annihilator wavefield is equal to $A(\tau, t)$. This concept is illustrated in figure 10.5 .

Before trying to construct $A(\tau, t)$, we first consider its support, that is, the domain where it is nonzero. For negative times, $t<0$, the annihilator comes propagating from the right, with speed 1 . Therefore, it is nonzero only for one-way traveltimes $\tau>t$. At time $t=0$, the annihilator must have reached $\tau=0$ in order to compensate the scattered wave set off by the incoming $\delta$-pulse at this position. Subsequently, for positive times, $t>0$, the annihilator must propagate towards the right, along with the $\delta$-pulse, in order to ensure that there is only a single wavefront for which the transport equation (10.38) can be evaluated unambiguously. In summary, it follows that the annihilator $A(\tau, t)$ is nonzero only within the domain

$$
\tau \geq|t|
$$

\subsection{Construction of the annihilator wavefield}

To construct the annihilator, we first note that the wave equation and the Schrödinger equation are time-reversal symmetric, meaning that the sign of the time variable $t$ in a solution can be flipped, and it is still a solution. Thus, the time-reverse version of equation (10.35),

$$
\psi_{a}(\tau,-t)=\delta(\tau+t)+A(\tau,-t)
$$




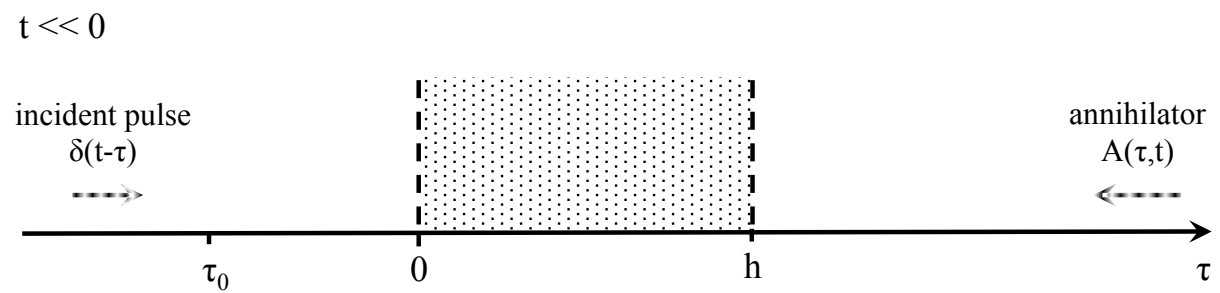

$\mathrm{t}>0$

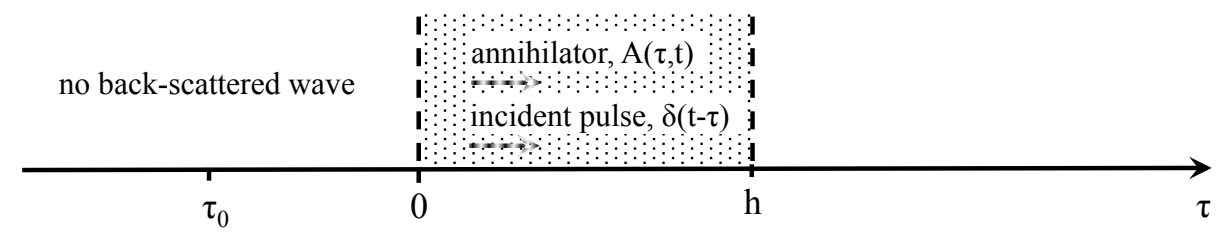

$t>0$

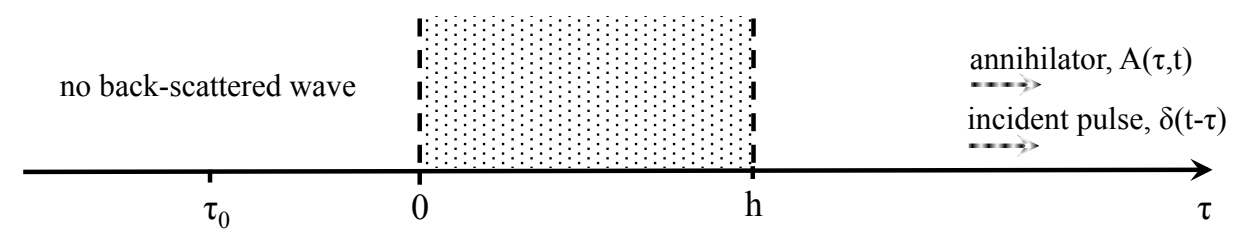

Fig. 10.5: Illustration of the annihilator concept. For large negative times, $t \ll 0$, the incident pulse $\delta(t-\tau)$ arrives from the left, and the annihilator, $A(\tau, t)$, arrives from the right. The scattering potential $q(\tau)$ is zero outside the one-way traveltime interval from $\tau=0$ to $\tau=h$. For small positive times, $t>0$, the annihilator propagates to the right, compensating perfectly for the back-scattered wave set off by the rightward travelling pulse. Finally, for large positive times, $t \gg 0$, both the pulse and the annihilator propagate to the right within the part of the medium where the scattering potential vanishes.

is also a solution of the Schrödinger equation. Shifting in time by some amount $-s$, that is, replacing $t$ by $t-s$, produces yet another solution

$$
\psi_{a}(\tau, s-t)=\delta(\tau+t-s)+A(\tau, s-t) .
$$

In the next step, we multiply (10.41) by some arbitrary function $B(s)$ and integrate over $s$ from $-\infty$ to $\infty$,

$$
\int_{-\infty}^{\infty} \psi_{a}(\tau, s-t) B(s) d s=B(t+\tau)+\int_{-\infty}^{\infty} B(s) A(\tau, s-t) d s .
$$

Since the multiplication by $B(s)$ and the integration over $s$ are independent of $t$ and $\tau$, it follows that (10.42) is a solution of the Schrödinger equation as well. We can tighten the integral bounds on the right-hand side of (10.42) by considering the support of $A$, specified in relation (10.39). In fact, requiring $|s-t| \leq \tau$ for $A(\tau, s-t)$ to be nonzero, translates to $s \leq t+\tau$ for $s>t$, and to $s \geq t-\tau$ for $s<t$. Thus, the solution may be rewritten as

$$
\int_{-\infty}^{\infty} \psi_{a}(\tau, s-t) B(s) d s=B(t+\tau)+\int_{t-\tau}^{t+\tau} B(s) A(\tau, s-t) d s .
$$

Substituting $s$ by $s+t$, transforms the right-hand side of equation (10.43) to 


$$
\int_{-\infty}^{\infty} \psi_{a}(\tau, s-t) B(s) d s=B(t+\tau)+\int_{-\tau}^{\tau} B(s+t) A(\tau, s) d s .
$$

Finally, we add $\psi_{a}$ from equation (10.35) to (10.44) to obtain the following solution to the Schrödinger equation

$$
\begin{aligned}
\psi(\tau, t) & =\psi_{a}(\tau, t)+\int_{-\infty}^{\infty} \psi_{a}(\tau, s-t) B(s) d s \\
& =\delta(t-\tau)+B(t+\tau)+A(\tau, t)+\int_{-\tau}^{\tau} B(s+t) A(\tau, s) d s
\end{aligned}
$$

Though the solution in equation (10.45) may seem somewhat arbitrary, it has properties that will turn out to be useful for our purpose. From (10.39) we know that the annihilator $A$ vanishes for $\tau<0$, which implies

$$
\left.\psi(\tau, t)\right|_{\tau<0}=\delta(t-\tau)+B(t+\tau)
$$

Equation (10.46) holds for any function $B$. In particular, it holds when $B$ is chosen to equal the reflection data $R$, which specialises $\psi$ to

$$
\left.\psi(\tau, t)\right|_{\tau<0}=\delta(t-\tau)+R(t+\tau) .
$$

A comparison with equation (10.21) reveals that $\psi(\tau, t)$ equals the fundamental solution $\psi_{f}(\tau, t)$ for $\tau<0$ and for all times $t$. This implies that $\psi(\tau, t)$ must equal $\psi_{f}(\tau, t)$ also for all $\tau$, because any differences between $\psi(\tau, t)$ and $\psi_{f}(\tau, t)$ in $\tau>0$ would ultimately propagate to $\tau<0$. Thus, in summary, we have

$$
\psi(\tau, t)=\psi_{f}(\tau, t) .
$$

Furthermore, we know that the fundamental solution vanishes for $\tau>t$, that is, ahead of the leftward propagating wavefront. Combining these considerations with the explicit representation of $\psi$ in (10.45), it follows that

$$
0=R(t+\tau)+A(\tau, t)+\int_{-\tau}^{\tau} R(s+t) A(\tau, s) d s, \quad \text { for } \tau>t
$$

Equation (10.49) is the celebrated Marchenko ${ }^{3}$ equation (Agranovich \& Marchenko, 1963). It is an implicit integral equation that includes the known reflection data $R$ and the unknown annihilator $A$. Solving the integral equation means to find $A(\tau, t)$ such that (10.49) is satisfied for all $\tau$ and $t$. Once the annihilator is found, the scattering potential $q$ can be recovered via a simple differentiation, as shown in equation (10.38). Thus, solving (10.49) effectively corresponds to the solution of the inverse scattering problem in the one-way traveltime domain.

Noticing that the reflection response $R(s+t)$ vanishes for $s+t<0$, the lower intergal bound in (10.49) may be shifted to $-t$, which leads to the slightly different equation

$$
0=R(t+\tau)+A(\tau, t)+\int_{-t}^{\tau} R(s+t) A(\tau, s) d s, \quad \text { for } \tau>t
$$

An analytical inverse scattering solution: To illustrate this concept, we return to the analytical forward problem solution in section 10.2. From equation (10.19) we know that the reflection data for this simple case are given by

\footnotetext{
${ }^{3}$ Vladimir Alexandrovich Marchenko, born in 1922, is a Ukrainian mathematical physicist. He is best known for his contributions to the study of partial differential equations.
} 


$$
R(t)=-\frac{1}{2} e^{-\frac{1}{2} t} H(t)
$$

We show that, given these reflection data, the solution to the Marchenko equation (10.49) is given by

$$
A(\tau, t)=\frac{1}{2} H(\tau+t), \quad \text { for } \tau>t
$$

and zero otherwise, that is, for $\tau<t$. In fact, inserting (10.51) and (10.52) into the integral of (10.49), gives

$$
\int_{-\tau}^{\tau} R(s+t) A(\tau, s) d s=-\frac{1}{4} \int_{-t}^{\tau} e^{-\frac{1}{2}(s+t)} d s
$$

For $-t \geq \tau$, the integral vanishes. For $-t<\tau$, we have

$$
\int_{-\tau}^{\tau} R(s+t) A(\tau, s) d s=-\frac{1}{2}+\frac{1}{2} e^{-\frac{1}{2}(\tau+t)}
$$

Thus, the general solution of the integral is

$$
\int_{-\tau}^{\tau} R(s+t) A(\tau, s) d s=-\frac{1}{2} H(\tau+t)+\frac{1}{2} e^{-\frac{1}{2}(\tau+t)} H(\tau+t)=-A(\tau, t)-R(t+\tau),
$$

which implies that $A$ from equation (10.52) indeed solves the Marchenko equation. Evaluating $A$ at $t=\tau$ and differentiating with respect to $\tau$, yields the scattering potential, according to equation (10.38),

$$
q(\tau)=2 \frac{d A(\tau, \tau)}{d \tau}=\frac{d H(\tau)}{d \tau}=\delta(\tau)
$$

Here we used the fact that the annihilator propagates to the left for $t<\tau$, so that $d \phi / d \tau=-1$. This solution agrees with the original scattering potential from equation (10.18), confirming that the inverse scattering problem has indeed been solved. For illustration, the wavefield $\psi_{a}(\tau, t)$ is shown in figure 10.6.

\subsection{Numerical solution of the Marchenko equation}

In our examples, we so far considered a simple case where both the forward problem (Schrödinger's equation) and the Marchenko equation (10.49) can be solved analytically. For a general distribution of the scattering potential, solutions to these equations must be found numerically. For the Schrödinger equation, this may be achieved, for instance, using well-established finite-difference methods (e.g., Igel, 2016). The Marchenko equation may be solved by a simple fix-point iteration. For this, we write equation (10.49) in fix point form,

$$
A(\tau, t)=-R(t+\tau)-\int_{-\tau}^{\tau} R(s+t) A(\tau, s) d s
$$

Starting from an initial guess of $A(\tau, t)$, denoted $A_{0}(\tau, t)$, we may then compute an improved update

$$
A_{1}(\tau, t)=-R(t+\tau)-\int_{-\tau}^{\tau} R(s+t) A_{0}(\tau, s) d s
$$

Iterating this procedure, produces a sequence of updates 

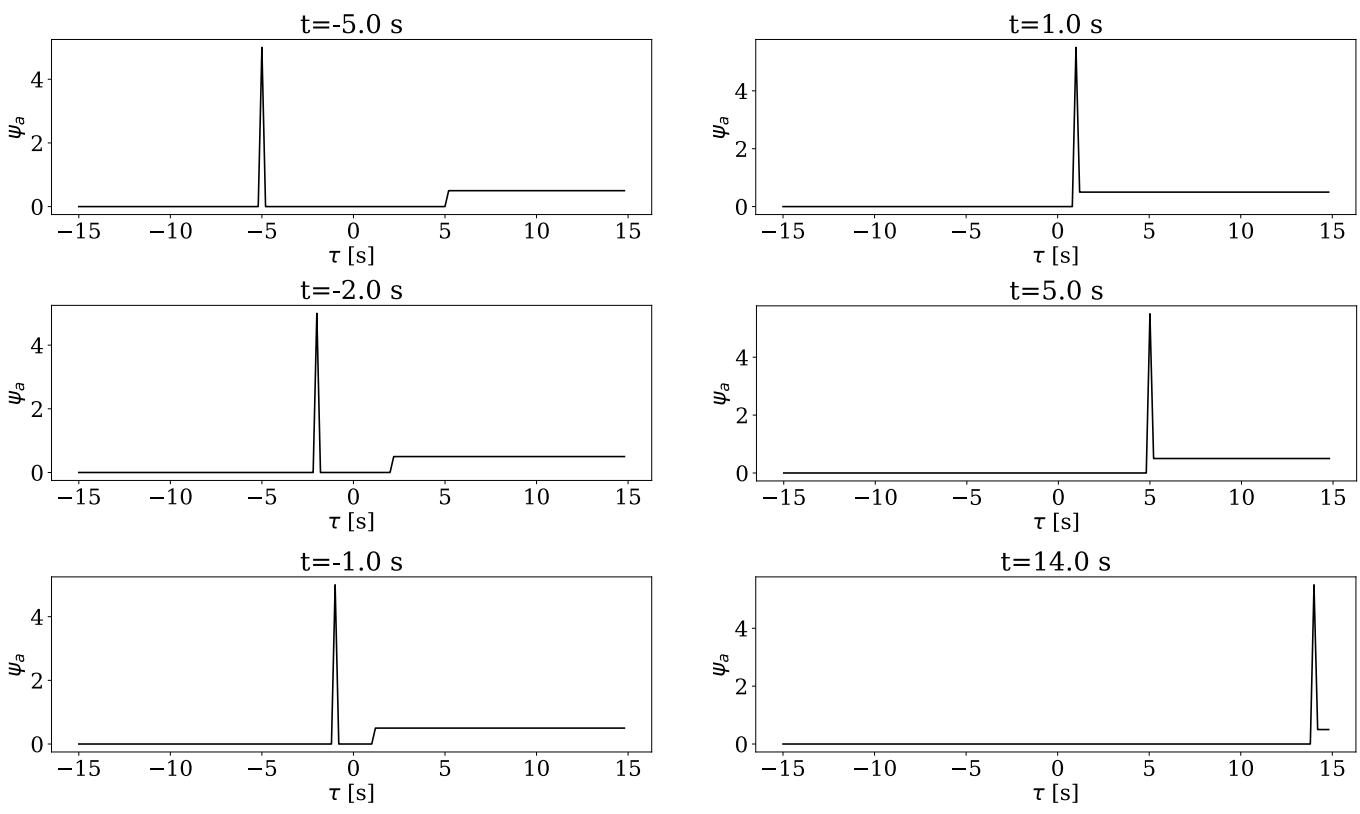

Fig. 10.6: Snapshots of the complete wavefield $\psi_{a}(\tau, t)$. Note the absence of a back-scattered wave for $\tau<0$.

$$
A_{i+1}(\tau, t)=-R(t+\tau)-\int_{-\tau}^{\tau} R(s+t) A_{i}(\tau, s) d s,
$$

which typically converges quickly towards an acceptable solution of the Marchenko equation.

Numerical solution of the Marchenko equation: As an example, we consider the velocity distribution, $c(x)$ shown in the left panel of figure 10.7. The density distribution is constant, $\rho(x)=1$. The corresponding distribution of the scattering potential in the one-way traveltime domain, $q(\tau)$, is shown to the right.

Propagating a pulse through the scattering potential using a finite-difference method, produces the fundamental solution, $\psi_{a}$, shown at time $t=18 \mathrm{~s}$ in figure 10.8. The part of $\psi_{a}$ in $\tau<0$ defines the scattering data $R$ that enter the Marchenko equation. Performing a fix-point iteration, as described in equation (10.59), produces an approximation of $A(\tau, t)$, which is the wave right of the dashed line in figure 10.8. Propagating the annihilator, $\psi_{a}$, forward in time, again using a finite-difference method, produces the wavefield shown in the right panel of figure 10.8 for $t=18 \mathrm{~s}$.

Indeed, the prominent back-scattered wave present in the fundamental solution has largely disappeared, as desired. However, a very small wave in the left part of the domain remains as a consequence of limited numerical accuracy. 

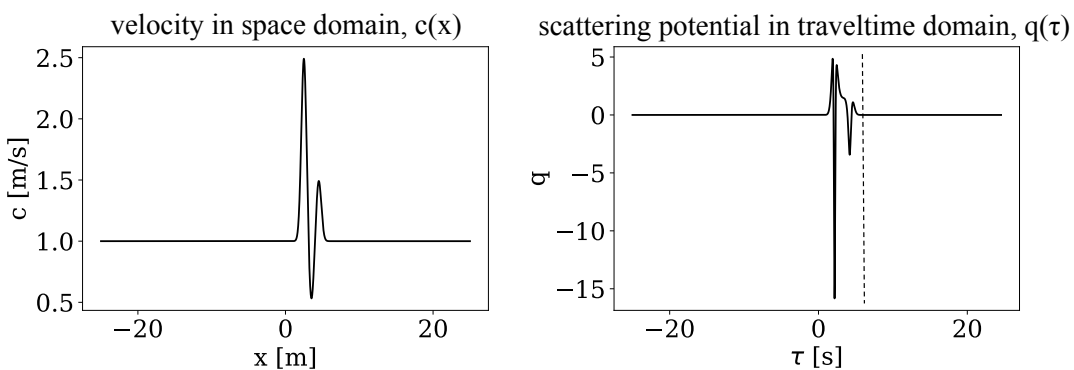

Fig. 10.7: Heterogeneous distribution of velocity in the space domain (left) and of the scattering potential in the one-way traveltime domain (right). The dashed line marks the right boundary of the non-zero scattering potential.
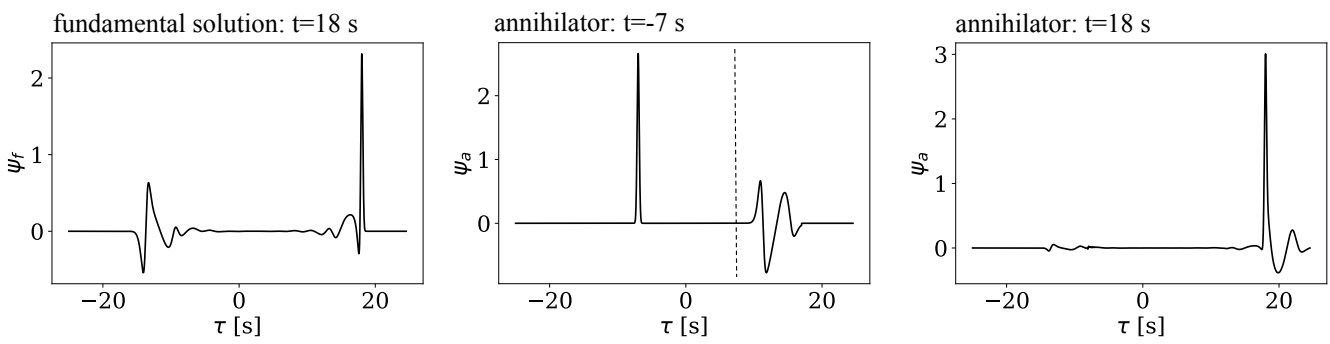

Fig. 10.8: Fundamental solution $\psi_{f}$ at $t=18 \mathrm{~s}$ (left), and the annihilator $\psi_{a}$ at $t=-7 \mathrm{~s}$ (centre) and $t=18$ (right). The solution to the Marchenko equation, $A(\tau, t)$ is the wave shape in the annihilator at $t=-7 \mathrm{~s}$ for $\tau>0$. The medium is homogeneous with zero scattering potential to the right of the dashed line. 



\section{Chapter 11 \\ Tomography and the central slice theorem}

There was virtually no response. The most interesting request for a reprint came from the Swiss Centre for Avalanche Research. The method would work for deposits of snow on mountains if one could get either the detector or the source into the mountain under the snow!

Allan McLeod Cormack, Nobel Prize lecture, 1979.

First demonstrated by the Austrian mathematician Johann Radon ${ }^{1}$ (Radon, 1917), the central slice theorem, also known as projection slice theorem or Fourier slice theorem, forms the basis of radiological tomography. Its potential for medical applications was first realised by Allan McLeod Cormack (Cormack, 1963) and Sir Godfrey Newbold Hounsfield (Hounsfield, 1973), who received the 1979 Nobel Prize in Physiology or Medicine for their work on X-ray computed tomography.

Given this historical background, we will initially consider the special case of X-ray tomography. Later we will see that the central slice theorem is useful for other types of tomography as well. Our interest is in the reconstruction of an object that cannot be accessed directly but probed by X-rays. The object, schematically illustrated in figure 11.1a, is characterised by the distribution of the absorption coefficient, $g(\mathbf{x})$, where $\mathbf{x}=(x, y)^{T}$ is the position vector in 2D. When an X-ray penetrates the object in $y$-direction, its initial intensity $I_{0}$ decays as a consequence of energy absorption. The receiver located on the other side of the object records the intensity

$$
I(x)=I_{0} e^{-\int g(\mathbf{x}) d y},
$$

where the is along the path of an X-ray. Equation (11.1) is known as the Beer-Lambert law. Dividing by $I_{0}$ and taking the natural logarithm of equation (11.1), yields the projection $P(x)$ of the object,

$$
P(x)=\ln \left(\frac{I_{0}}{I(x)}\right)=\int_{-\infty}^{\infty} g(\mathbf{x}) d y .
$$

In equation (11.2) we assumed that the absorption coefficient $g(\mathbf{x})$ is zero outside some finite domain, so that we can take the integral from $-\infty$ to $\infty$. It is intuitively clear that the object cannot be reconstructed from the projection $P(x)$ because infinitely many objects may produce the same projection.

To overcome this inherent non-uniqueness, we rotate the object by some angle $\varphi$, defined to be positive in counter-clockwise direction. After rotation, the absorption coefficient at position $(x, y)^{T}$ is equal to the absorp-

${ }^{1}$ Johann Radon (1887-1956) was an Austrian mathematician who specialised in functional analysis and geometry. Though is myopia saved him from a direct participation in World War I, the political instabilities of his days severly perturbed his scientific career, and so he studied and taught at 9 different universities. In addition to his mathematical talent, he was known for his friendly and noble personality, and for his love for classical literature and music. 
tion coefficient at the original position

$$
\left[\begin{array}{l}
x^{\prime} \\
y^{\prime}
\end{array}\right]=\left[\begin{array}{cc}
\cos \varphi & \sin \varphi \\
-\sin \varphi & \cos \varphi
\end{array}\right]\left[\begin{array}{l}
x \\
y
\end{array}\right]=\mathbf{R}_{\varphi} \mathbf{x}
$$

where $\mathbf{R}$ is a rotation matrix (meaning it is orthogonal, $\mathbf{R}^{T}=\mathbf{R}$ ). It follows that the X-rays now probe the rotated function

$$
g(\varphi, \mathbf{x})=g\left(\mathbf{R}_{\varphi} \mathbf{x}\right)
$$

thereby producing the projection

$$
P(\varphi, x)=\int_{-\infty}^{\infty} g(\varphi, \mathbf{x}) d y .
$$

Equation (11.5) is known as the Radon transform of $g$. More commonly, it is written in a form where the sources and receivers are rotated, instead of rotating the object. The two approaches are equivalent.

a) Projection of the object

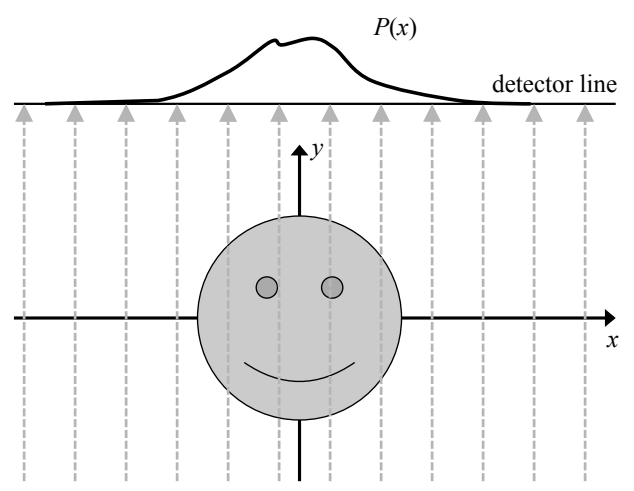

b) Projection of the rotated object

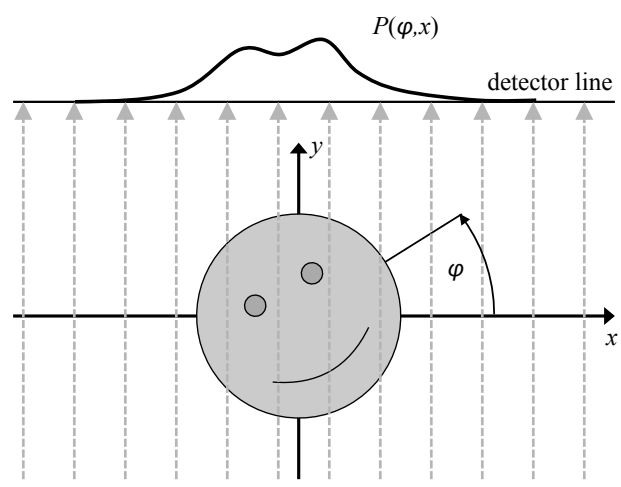

Fig. 11.1: Schematic illustration of the experimental setup for X-ray tomography using the central slice theorem. (a) X-ray penetrate an object (smiley) parallel to the $y$-direction. This produces the projection $P(x)$ on the detector line. (b) When the objected is rotated by some angle $\varphi$, the X-rays produce a different projection, $P(\varphi, x)$. The object can be reconstructed when the projections for all angles $\varphi$ between 0 and $\pi$ have been measured.

In the next step, we compute the spatial Fourier transform of $P(\varphi, x)$ with respect to $x$,

$$
\hat{P}\left(\varphi, k_{x}\right)=\int_{-\infty}^{\infty} P(\varphi, x) e^{-i k_{x} x} d x=\int_{-\infty}^{\infty} \int_{-\infty}^{\infty} g(\varphi, \mathbf{x}) e^{-i k_{x} x} d x d y=\left.\int_{-\infty}^{\infty} g(\varphi, \mathbf{x}) e^{-i \mathbf{k}^{T} \mathbf{x}} d x d y\right|_{k_{y}=0},
$$

where $\mathbf{k}=\left(k_{x}, k_{y}\right)^{T}$ is the $2 \mathrm{D}$ wave number vector. Using the definition of the rotated absorption coefficient in equation (11.4), we can transform equation (11.6) into

$$
\hat{P}\left(\varphi, k_{x}\right)=\left.\int_{-\infty}^{\infty} \int_{-\infty}^{\infty} g(\mathbf{x}) e^{-i\left(\mathbf{R}_{\varphi} \mathbf{k}\right)^{T} \mathbf{x}} d x d y\right|_{k_{y}=0} .
$$


Substituting the explicit expression of the rotation matrix $\mathbf{R}_{\varphi}$ from equation (11.3), gives

$$
\hat{P}\left(\varphi, k_{x}\right)=\int_{-\infty}^{\infty} \int_{-\infty}^{\infty} g(\mathbf{x}) e^{-i\left(x k_{x} \cos \varphi-y k_{x} \sin \varphi\right)} d x d y .
$$

Equation (11.8) means that the Fourier transformed projections of the rotated object, $\hat{P}\left(\varphi, k_{x}\right)$, are equal to the 2D spatial Fourier transform of the unrotated object, $g(\mathbf{x})$, for a limited range of wave number vectors

$$
\left[\begin{array}{c}
k_{x} \cos \varphi \\
-k_{x} \sin \varphi
\end{array}\right]
$$

that is

$$
\hat{P}\left(\varphi, k_{x}\right)=\hat{g}\left(k_{x} \cos \varphi,-k_{x} \sin \varphi\right)
$$

For instance, when $\varphi=0$, the Fourier transformed projection only contains the wave numbers $\left(k_{x}, 0\right)^{T}$ of $g(\mathbf{x})$. When $\varphi=\pi / 2$, only the wave numbers $\left(0,-k_{x}\right)^{T}$ are recorded. Ultimately, when projections for all angles $\varphi$ between 0 and $\pi$ have been recorded, they can be combined into the complete 2D spatial Fourier transform of the object $\hat{g}(\mathbf{k})$, which may then be inverted to yield the object itself,

$$
g(\mathbf{x})=\frac{1}{4 \pi^{2}} \int_{-\infty}^{\infty} \int_{-\infty}^{\infty} \hat{g}(\mathbf{k}) e^{i \mathbf{k}^{T} \mathbf{x}} d k_{x} d k_{y} .
$$

In summary, we have the following procedure: The object with absorption coefficient $g(\mathbf{x})$ is probed by X-rays travelling parallel to the $y$-direction. This produces a projection of the object that depends on the $x$-coordinate. Rotating the object gives a sequence of projections, each of which 'sees' the object from a different angle. The 1D Fourier transforms of all projections can be combined in the complete 2D Fourier transform of the object. Applying the 2D inverse Fourier transform then reconstructs the object.

As noted above, the central slice theorem can also be useful for other types of tomography. Instead of measuring intensities of X-rays, we may measure the arrival times $T(x)$ of acoustic or elastic waves that propagate through the object. In fact, denoting by $v(\mathbf{x})$ the wave propagation velocity in the object, the traveltimes recorded as a function of the $x$-coordinate are given by

$$
T(x)=\int_{-\infty}^{\infty} \frac{1}{v(\mathbf{x})} d y .
$$

Replacing velocity by slowness, $s=v^{-1}$, yields a forward modelling equation that is identical to the BeerLambert law (11.2),

$$
T(x)=\int_{-\infty}^{\infty} s(\mathbf{x}) d y .
$$

This suggests that the central slice theorem may equally be used to infer the 2D distribution of slowness using traveltime measurements under all possible angles.

The central slice theorem demonstrates that medium properties may be perfectly reconstructed under ideal conditions, meaning that there is no inherent non-uniqueness in the inverse problem. The ideal conditions include the absence of measurement errors, infinitely dense data coverage, and the propagation of waves (electromagnetic, acoustic, elastic, ...) along infinitely thin and perfectly straight rays. The latter is to some extent a contradiction in itself because rays are generally bent away from straight lines in the presence of heterogeneities (non-zero absorption, non-zero slowness variations, ...). 

Part IV

Advanced and Integrated Topics 



\section{Chapter 12 \\ The No-Free-Lunch theorem}

Alice: Would you tell me, please, which way I ought to go from here?

The Cheshire Cat: That depends a good deal on where you want to get to.

Alice: I don't much care where.

The Cheshire Cat: Then it doesn't much matter which way you go.

Alice: ... So long as I get somewhere.

The Cheshire Cat: Oh, you're sure to do that, if only you walk long enough.

Lewis Caroll, Alice's Adventures in Wonderland, 1865.

Charles Lutwidge Dodgson (1832-1898), better known as Lewis Caroll, was in fact a talented mathematician, educated at Oxford University. In addition to his famous writings Alice's Adventures in Wonderland and Through the Looking-Glass, he wrote numerous books on geometry, linear algebra and logic. Did he foresee the No-Free-Lunch theorem?

So far, we have encountered a wide variety of algorithms to sample probability densities or to estimate maximum-likelihood models. We are now interested in seeing if any of them can be genuinely better than another. For this, we consider our model space in a slightly different way than before. In fact, $\mathbb{M}$ is discretised such that the components $m_{i}$ of each $N_{M}$-dimensional model $\mathbf{m} \in \mathbb{M}$ can only take a finite number of values, for instance, those numbers that can be represented on a computer. In this sense, the model space consists of all possible combinations of $N_{M}$ values. We denote the total number of these combinations by $|\mathbb{M}|$.

For a simple illustration, we consider the case shown in figure 12.2, where $N_{M}=2$. Each of the two model parameters, $m_{1}$ and $m_{2}$ can only take the values 0 or 1 . It follows that the total number of different models is $|\mathbb{M}|=2^{2}=4$.

Then there is a set $\mathbb{S}$ of misfit values $s$. Since $\mathbb{M}$ is discrete, the attainable misfit values must be discrete as well. We denote the number of all possible distinct misfit values by $|\mathbb{S}|$. The discrete models and the misfit values are related by misfit functions $\chi$, the ensemble of which forms the set $\mathbb{X}$. These misfit functions are in fact made of two components, namely the forward problem that relates a model to some synthetic data, and an actual measurement that compares observed and synthetic data to produce a misfit.

With both $\mathbb{M}$ and $\mathbb{S}$ being of finite dimension, it follows that the number of distinct misfit functions $\chi$ that map $\mathbb{M}$ to $\mathbb{S}$ has finite dimension, too. In the example shown in figure 12.2, the misfit values $s$ have a binary representation with 3 bits, meaning that there are $|\mathbb{S}|=8$ different ones. The first misfit function $\chi_{1}$ maps any of the four possible models to the misfit value $s=(0,0,0)$. The second misfit function $\chi_{2}$ maps the first model to the misfit value $s=(0,0,1)$ and all the other models to $s=(0,0,0)$, and so on. In total, there are $|\mathbb{X}|=8^{4}=4096$ distinct misfit functions. More generally, we find

$$
|\mathbb{X}|=|\mathbb{S}|^{|\mathbb{M}|}
$$




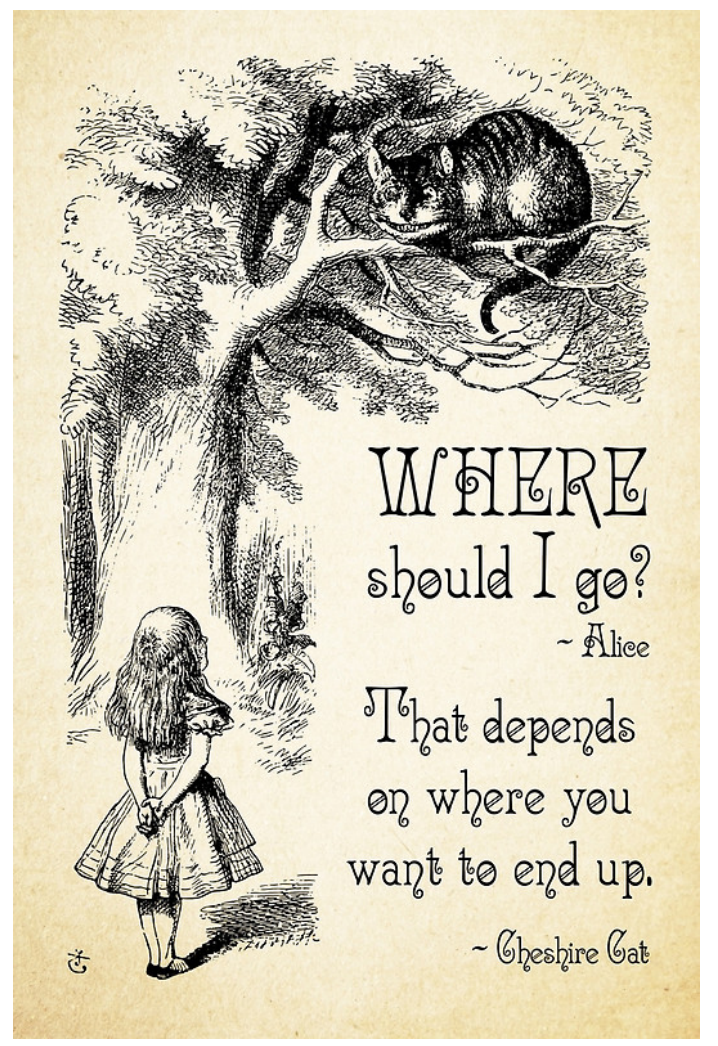

Fig. 12.1: Original illustration by John Tenniel (1820-1914) of the Cheshire cat, responding to Alice's question about the best algorithm for her nonlinear optimisation problem.

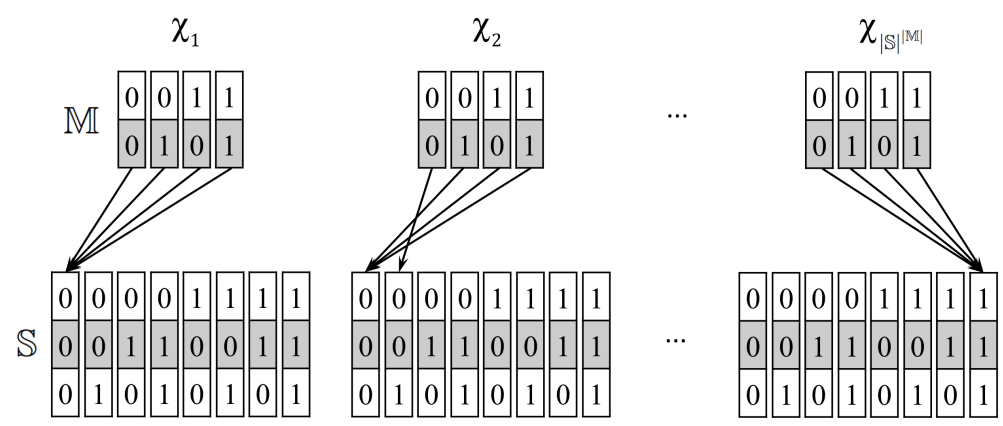

Fig. 12.2: Illustration of the mapping between the finite-dimensional model space $\mathbb{M}$ to the finite-dimensional space of attainable misfit values $|\mathbb{S}|$. In the case shown here, models are represented by two bits, meaning that there are 4 different models. The attainable misfit values are represented by 3 bits, which allows for 8 different values. In total, there are $|\mathbb{X}|=8^{4}=4096$ distinct misfit functions connecting models and misfit values.

Now assume there is an algorithm $a(\chi)$ that works with a specific misfit function $\chi \in \mathbb{X}$ in order to generate a specific sequence of $m<|\mathbb{M}|$ models $M=\left\{\mathbf{m}_{1}, \ldots, \mathbf{m}_{m}\right\}$, corresponding to a specific sequence of misfit values $\left\{s_{1}, \ldots, s_{m}\right\}$. Depending on the algorithm, this sequence may be generated for different purposes, for instance, sampling a probability density or finding an optimal model that minimises the misfit.

Since $m$ is smaller than the total number of possible models, there must be other misfit functions $\chi^{\prime}$ that produce 
the exact same sequence of misfit values $\left\{s_{1}, \ldots, s_{m}\right\}$ for the model sequence $M=\left\{\mathbf{m}_{1}, \ldots, \mathbf{m}_{m}\right\}$, while being distinct from $\chi$. In fact, all misfit functions $\chi^{\prime}$ that assign $\left\{s_{1}, \ldots, s_{m}\right\}$ to $\left\{\mathbf{m}_{1}, \ldots, \mathbf{m}_{m}\right\}$, and some other values $\left\{s_{m+1}, s_{m+2} \ldots\right\}$ to models $\left\{\mathbf{m}_{m+1}, \mathbf{m}_{m+2} \ldots\right\}$ that have not been drawn, are different from $\chi$. In total, there are

$$
\left|\mathbb{X}_{M}\right|=|\mathbb{S}|^{|\mathbb{M}|-m}
$$

such misfit functions that also relate the specific sequence $M=\left\{\mathbf{m}_{1}, \ldots, \mathbf{m}_{m}\right\}$ to the same misfit values $\left\{s_{1}, \ldots, s_{m}\right\}$. So, in this sense, there $\left|\mathbb{X}_{M}\right|=|\mathbb{S}|^{|\mathbb{M}|-m}$ misfit functions that are equivalent for this specific algorithm.

Now we close our eyes and blindly apply the algorithm $a$ to randomly chosen misfit functions, assuming that each of the $|\mathbb{X}|$ misfit functions is drawn with equal probability. Then it follows that the probability of encountering the sequence $\left\{s_{1}, \ldots, s_{m}\right\}$, given the algorithm $a$ and the number $m$, is given by

$$
P\left(s_{1}, \ldots, s_{m} \mid a, m\right)=\frac{\left|\mathbb{X}_{M}\right|}{|\mathbb{X}|}=|\mathbb{S}|^{-m} .
$$

An interesting aspect of equation (12.4) is that the right-hand side is independent of the algorithm used to produce $M=\left\{\mathbf{m}_{1}, \ldots, \mathbf{m}_{m}\right\}$. Thus, we could have used another algorithm $a^{\prime}$ to produce some $M^{\prime}=\left\{\mathbf{m}_{1}^{\prime}, \ldots, \mathbf{m}_{m}^{\prime}\right\}$, and the probability of finding the exact same sequence $\left\{s_{1}, \ldots, s_{m}\right\}$ would still be

$$
P\left(s_{1}, \ldots, s_{m} \mid a^{\prime}, m\right)=\frac{\left|\mathbb{X}_{M}\right|}{|\mathbb{X}|}=|\mathbb{S}|^{-m} .
$$

This result has profound implications: If we choose an algorithm and apply it randomly to whatever problem, characterised by its misfit function, then the probability of encountering a specific sequence of misfit values is independent of the actual algorithm.

We now turn our attention to measuring the performance of an algorithm. Typically, algorithm performance is assessed on the basis of the sequence of misfit values $\left\{s_{1}, \ldots, s_{m}\right\}$ that they produce. Denote the performance measure by $\Phi$, we symbolically have

$$
\Phi=\Phi\left(s_{1}, \ldots, s_{m}\right) .
$$

For example, if the algorithm is intended to minimise misfit as fast as possible, then $\Phi$ would measure how quickly the numbers in the sequence decrease. Since we know already that the probability of actually finding the sequence $\left\{s_{1}, \ldots, s_{m}\right\}$ is independent of the algorithm, it follows that the probability of finding a certain performance is independent of the algorithm as well. In other words, assuming that we take some algorithm and randomly apply it to all kinds of problems (misfit functions) then the probability of achieving a certain performance is the same as if we had chosen another algorithm.

What we just expressed, is the essential content of the No-Free-Lunch theorem, of which different variants can be found in the literature (e.g. Mosegaard, 2012; Wolpert \& Macready, 1997). Statistically, all algorithms are equally good or bad. The beauty of the theorem is its counter-intuitive nature, because we seem to know that certain algorithms perform better than others.

The solution to the apparent paradox lies in the blindness in the choice of misfit functions. In fact, (12.4) critically relies on the assumption that each of all the misfit functions is drawn with equal probability. However, in practice this is not the case. What we do instead is to apply an algorithm only to well-chosen misfit functions (inverse problems) for which we empirically, analytically or intuitively know that it works rather well. In this regard, we inject prior knowledge into the problem. It follows that no algorithm is particularly efficient by itself. What makes it efficient is its application to well-chosen problems!

An everyday-life illustration of the No-Free-Lunch theorem is shown in figure 12.3, where we consider the problem of getting from A to B using different vehicles (algorithms). The performance measure is speed. Though certain vehicles are often considered superior, their actual usefulness depends critically on the details of the problem. A race car applied to the problem of traversing Amsterdam during rush certainly performs 
worse than a bicycle. (In fact, you do not even need a road bike. Any good old Dutch bicyle is faster than any race car in Amsterdam!)

a) Traversing Amsterdam during rush hour

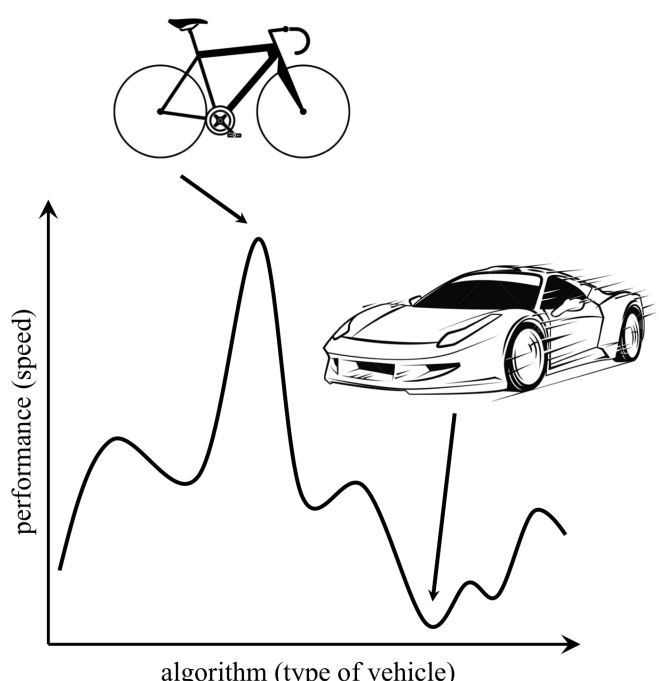

b) Traversing a sandy desert

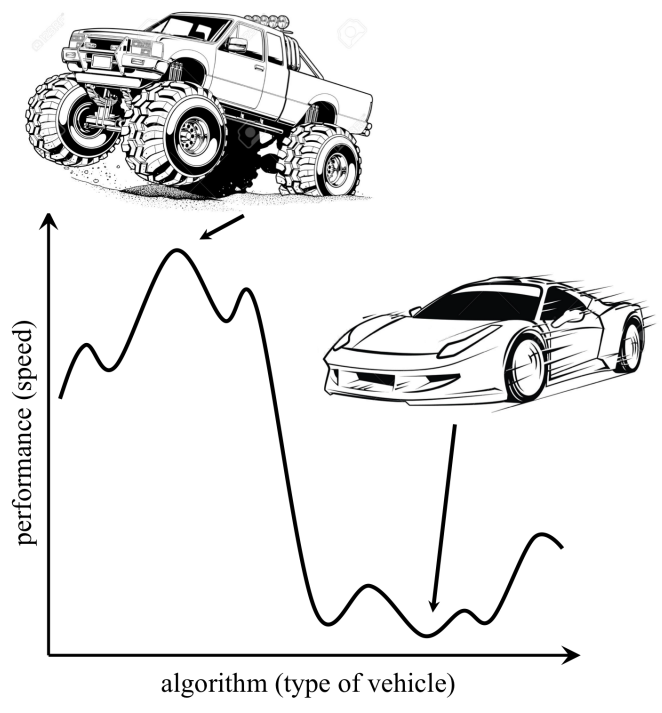

Fig. 12.3: Practical illustration of the No-Free-Lunch theorem. (Idea borrowed from Klaus Mosegaard.) Though a race car may intuitively be seen as superior, its performance in fact depends critically on the problem to which it is applied. 


\section{Chapter 13 Nullspace shuttles}

Ground Control to Major Tom, take your protein pills and put your helmet on

Ground Control to Major Tom, commencing countdown, engines on

Check ignition and may God's love be with you

This is Ground Control to Major Tom, you've really made the grade

And the papers want to know whose shirts you wear

Now it's time to leave the capsule if you dare

This is Major Tom to Ground Contol, I'm stepping through the door

And I'm floating in a most peculiar way, and the stars look different today

For here am I sitting in a tin can far above the world

Planet Earth is blue, and there's nothing I can do

David Bowie, Space Oddity, 1969.

After asking Ground Control to tell his wife he loves her, our hero Major Tom indeed leaves his tin can to explore space, loses radio contact, and finally closes by repeating the words "And there is nothing I can do." Space exploration has always been a somewhat dangerous business, maybe too dangerous for the average reader. And so we will content ourselves with the development of methods, collectively known as nullspace shuttles, that allow us to explore the nullspace of inverse problems without Monte Carlo sampling. These methods may be used to find families of solutions that are particularly interesting or in agreement with knowledge that we did not initially impose, or they may be used for targeted hypothesis testing: Can a certain feature be added to a model without changing the misfit significantly? We will start in section 13.1 with a nullspace shuttle for linear inverse problems, introduced in a classic paper by Deal \& Nolet (1996). Subsequently, in section 13.2, we will extend this concept to general nonlinear problems by borrowing concepts of the Hamiltonian Monte Carlo method (section 5.7, Fichtner \& Zunino (2019)).

\subsection{Linear nullspace shuttles}

Already in section 6.5.1, we rigorously defined the nullspace $\operatorname{ker} \mathbf{G}$ of a linear forward modelling operator $\mathbf{G}$ as the subspace of model space $\mathbf{M}$ that has exactly zero effect on the synthetic data. Choosing a model $\mathbf{m}_{0} \in \operatorname{ker} \mathbf{G}$ and adding this to some estimated model $\mathbf{m}^{\text {est }}$ produces the estimated data

$$
\mathbf{G}\left(\mathbf{m}^{\text {est }}+\mathbf{m}_{0}\right)=\mathbf{G} \mathbf{m}^{\text {est }}+\mathbf{G} \mathbf{m}_{0}=\mathbf{G m}^{\mathrm{est}}=\mathbf{d}^{\mathrm{est}}, \quad \text { if } \quad \mathbf{m}_{0} \in \operatorname{ker} \mathbf{G} .
$$

Thus, adding an element of the nullspace to an estimated model does not affect the difference $\left|\mathbf{d}^{\text {obs }}-\mathbf{d}^{\text {est }}\right|$. Simplistically assuming a least-squares misfit with infinite prior model covariance, 


$$
\chi(\mathbf{m})=\frac{1}{2}\left(\mathbf{d}^{\mathrm{obs}}-\mathbf{G m}\right)^{T} \mathbf{C}_{D}^{-1}\left(\mathbf{d}^{\mathrm{obs}}-\mathbf{G m}\right),
$$

we may use nullspace elements constructively in order to modify $\mathbf{m}^{\text {est }}$ a posteriori without increasing this misfit $\chi\left(\mathbf{m}^{\text {est }}\right)$ at all. Such a posteriori modifications may be desirable when new independent information becomes available after solving the inverse problem. Alternatively, nullspace elements may be added in order to display a family of alternative models that are still consistent with the observations.

In practice, we may want to go beyond nullspace exploration sensu stricto. Since observations are generally contaminated by errors, we may add models $\Delta \mathbf{m}$ that change the observations up to a (small) tolerance $\varepsilon$, that is,

$$
\left|\Delta \mathbf{d}^{\text {est }}\right|=|\mathbf{G} \Delta \mathbf{m}|<\varepsilon .
$$

Recalling the singular-value decomposition of $\mathbf{G}$, introduced in equation (6.55), we can use (13.3) to relate the tolerance $\varepsilon$ to the singular values $\sigma_{i}$ of $\mathbf{G}$. In fact, assuming that $\Delta \mathbf{m}$ is a normalised eigenvector $\mathbf{m}_{i}$ of $\mathbf{G}^{T} \mathbf{G}$, or right-singular vector, we find

$$
\left|\mathbf{G m}_{i}\right|=\left|\mathbf{D} \Lambda \mathbf{M}^{T} \mathbf{m}_{i}\right|=\left|\mathbf{D} \Lambda \mathbf{e}_{i}\right|=\left|\sigma_{i} \mathbf{d}_{i}\right|=\sigma_{i},
$$

where $\mathbf{D}$ and $\mathbf{M}$ are the matrices of left- and right-singular vectors. Comparing (13.3) and (13.4), it follows that the tolerance $\varepsilon$ directly determines the cutoff singular value $\sigma_{\varepsilon}$. Adding to $\mathbf{m}^{\text {est }}$ a right-singular vector $\mathbf{m}_{i}$ (or eigenvector of $\mathbf{G}^{T} \mathbf{G}$ ) corresponding to some $\sigma_{i}<\sigma_{\varepsilon}=\varepsilon$ will not change the estimated data $\mathbf{d}^{\text {est }}$ by more than $\varepsilon$. Since the singular values are commonly ordered from largest to smallest, $\varepsilon$ also defines a cutoff index $k$ such that $\sigma_{i}<\sigma_{k}$ for all $k>i$. The space spanned by the right-singular vector $\mathbf{m}_{i}$ with $\sigma_{i}<\varepsilon$ is the effective or generalised nullspace, $\operatorname{ker}_{\varepsilon} \mathbf{G}$.

Equipped with the effective nullspace concept, we may now apply some a posteriori modifications to the previously estimated model $\mathbf{m}^{\text {est }}$, thus producing a new, filtered model

$$
\mathbf{m}^{\text {filt }}=\mathbf{F}\left(\mathbf{m}^{\text {est }}\right) .
$$

The function, or filter, $\mathbf{F}$ may, for instance, smooth or roughen $\mathbf{m}^{\text {est }}$, or it may introduce some new features. Since $\mathbf{F}$ is arbitrary, the filtered model $\mathbf{m}^{\text {filt }}$ is not automatically guaranteed to have a misfit that falls within the tolerance. Some additional adjustments of $\mathbf{m}^{\text {filt }}$ may still be needed. For this, we interpret the action of $\mathbf{F}$ as an addition of some differential model $\Delta \mathbf{m}$ to $\mathbf{m}^{\text {est }}$,

$$
\mathbf{m}^{\text {filt }}=\mathbf{m}^{\text {est }}+\Delta \mathbf{m} .
$$

In terms of the $n$ eigenvectors $\mathbf{m}_{i}$ of $\mathbf{G}^{T} \mathbf{G}$, the differential model may be written as

$$
\Delta \mathbf{m}=\sum_{i=1}^{n} \Delta \mu_{i} \mathbf{m}_{i},
$$

with some expansion coefficients $\Delta \mu_{i}$. To ensure that the addition of $\Delta \mathbf{m}$ to $\mathbf{m}^{\text {est }}$ does not change the misfit significantly, we need to eliminate all contributions in (13.7) with $i<k$. This can be achieved by projecting $\Delta \mathbf{m}$ onto the effective nullspace. Defining $\mathbf{M}_{k}$ as the matrix filled with the first $k<n$ eigenvectors of $\mathbf{G}^{T} \mathbf{G}$, that is,

$$
\mathbf{M}_{k}=\left(\mathbf{m}_{1}, \ldots, \mathbf{k}\right),
$$

we find

$$
\mathbf{M}_{k} \mathbf{M}_{k}^{T} \Delta \mathbf{m}=\sum_{i=1}^{k} \Delta \mu_{i} \mathbf{m}_{i} .
$$

Subtracting (13.9) from $\Delta \mathbf{m}$ leaves the components of $\Delta \mathbf{m}$ that lie within the effective nullspace, 


$$
\Delta \mathbf{m}^{\mathrm{c}}=\Delta \mathbf{m}-\mathbf{M}_{k} \mathbf{M}_{k}^{T} \Delta \mathbf{m}=\sum_{i=k+1}^{n} \Delta \mu_{i} \mathbf{m}_{i} .
$$

Equation (13.10) means that $\Delta \mathbf{m}^{\mathrm{c}}$ is the result of applying the projector $\mathbf{I}-\mathbf{M}_{k} \mathbf{M}_{k}^{T}$ to the differential model $\Delta \mathbf{m}$ produced by the filter $\mathbf{F}$. Finally, we obtain a conservatively filtered model by replacing $\Delta \mathbf{m}$ in (13.6) with its filtered version $\Delta \mathbf{m}^{\mathrm{c}}$,

$$
\mathbf{m}^{\mathrm{c}}=\mathbf{m}^{\mathrm{est}}+\Delta \mathbf{m}^{\mathrm{c}} \text {. }
$$

By design, $\mathbf{m}^{\mathrm{c}}$ should leave the misfit nearly unchanged.

While being beautifully simple, the application of the linear nullspace shuttle to large-scale problems is nontrivial because it requires knowledge of the first $k$ right-singular vectors and the associated singular values. An approximate solution suggested by Deal \& Nolet (1996) and applied to large-scale tomographic problems by de Wit et al. (2012), circumvents the need to compute the singular-value decomposition. For this we note that $\Delta \mathbf{m}$ can generally be decomposed into a part $\Delta \mathbf{m}^{\text {null }}$ that lies in the nullspace of $\mathbf{G}$, and a part $\Delta \mathbf{m}^{\text {range }}$ that lies in the range of $\mathbf{G}$,

$$
\Delta \mathbf{m}=\Delta \mathbf{m}^{\text {null }}+\Delta \mathbf{m}^{\text {range }} .
$$

Applying $\mathbf{G}$ to (13.12), gives, by the definitions of nullspace and range,

$$
\mathbf{G} \Delta \mathbf{m}=\mathbf{h}=\mathbf{G} \Delta \mathbf{m}^{\text {range }} \text {. }
$$

Using, for instance, the least-squares approach, we may find an approximate solution $\Delta \tilde{\mathbf{m}}^{\text {range }}$ of (13.13). Subtracting the approximate solution from $\Delta \mathbf{m}$ yields an approximate nullspace component,

$$
\Delta \tilde{\mathbf{m}}^{\text {null }} \approx \Delta \mathbf{m}-\Delta \tilde{\mathbf{m}}^{\text {range }}
$$

The advantage of the approximate approach is, by construction, the absence of an explicit singular-value decomposition, which is not computationally feasible for large-scale problems. However, this comes at the price of having to solve the linear system (13.13) each time a new $\Delta \mathbf{m}$ is to be tested and projected onto the effective nullspace.

The nullspace shuttle presented in the previous paragraphs is only applicable when the forward problem is linear. Furthermore, it ignores prior information in model space, for example, in the form of a finite posterior covariance matrix. Hence, some of the conservatively filtered models may actually be in conflict with a nontrivial prior. Both issues nonlinearity and prior knowledge can be addressed by the nonlinear (Hamiltonian) nullspace shuttle.

\subsection{Nonlinear Hamiltonian nullspace shuttles}

The nonlinear nullspace shuttle Fichtner \& Zunino (2019) drops the two key limitations of the linear nullspace shuttle introduced in section 13.1, namely that the forward problem is linear and that the misfit $\chi$ has the form of the simplified least-squares misfit (13.2). To initiate the nullspace exploration, we assume that we have estimated some plausible model $\mathbf{m}^{\text {est }}$ that approximately minimises the misfit $\chi$. This may have been achieved, for example, using one of the nonlinear optimisation methods covered in chapter 8. Owing to the presence of observational uncertainties, alternative models $\mathbf{m}^{\text {est }}+\Delta \mathbf{m}$ may still be plausible if their associated misfit remains below a given tolerance $\varepsilon^{2}$,

$$
\chi\left(\mathbf{m}^{\mathrm{est}}+\Delta \mathbf{m}\right) \leq \chi\left(\mathbf{m}^{\mathrm{est}}\right)+\varepsilon^{2}
$$

In analogy to the linear case, we refer to the space spanned by those $\Delta \mathbf{m}$ that satisfy (13.15) as the effective or generalised nullspace.

The nonlinear nullspace shuttle rests on the interpretation of the model $\mathbf{m}$ in terms of an $n$-dimensional position 
vector that marks the location of an imaginary particle in $n$-dimensional space. The location is a function of an artificially introduced time variable $t$, and so each time corresponds to some new model $\mathbf{m}(t)$. To guide the movement of the particle through model space, we construct artificial equations of motion, borrowing the basic concept from Hamilton's equations in classical mechanics. For this, we first define the potential energy $U$ of the particle to equal the misfit,

$$
U(\mathbf{m})=\chi(\mathbf{m}) .
$$

The potential energy induces an artificial force proportional to $-\nabla U[\mathbf{m}(t)]$, which effectively pulls the model $\mathbf{m}(t)$ in the direction of steepest descent, towards a model $\mathbf{m}(t+\delta t)$ with lower misfit. The straight movement in the direction of $-\nabla U[\mathbf{m}(t)]$ is complemented by the presence of an artificial, $n$-dimensional momentum $\mathbf{p}(t)$. Together with an equally artificial, symmetric and positive definite $n \times n$ mass matrix, $\mathbf{M}$, the momentum defines a kinetic energy

$$
K(\mathbf{p})=\frac{1}{2} \mathbf{p}^{T} \mathbf{M}^{-1} \mathbf{p} .
$$

The sum of potential and kinetic energies defines the artificial Hamiltonian,

$$
H(\mathbf{m}, \mathbf{p})=U(\mathbf{m})+K(\mathbf{p})
$$

of the system. Equipped with the artificial energies $U, K$ and $H=U+K$, the movement of the particle is fully described by Hamilton's equations (e.g. Landau \& Lifshitz, 1976; Symon, 1971),

$$
\frac{d m_{i}}{d t}=\frac{\partial H}{\partial p_{i}}, \quad \frac{d p_{i}}{d t}=-\frac{\partial H}{\partial m_{i}}, \quad i=1, \ldots, n .
$$

As demonstrated in section 5.7.1, the Hamiltonian is preserved along a trajectory. Hence, starting at some approximate minimum $\mathbf{m}^{\text {est }}$ of $\chi$, the solution of Hamilton's equations (13.18) produces a continuous sequence of models $\mathbf{m}(t)$ and associated momenta $\mathbf{p}(t)$ that satisfy

$$
H[\mathbf{m}(t), \mathbf{p}(t)]=\chi[\mathbf{m}(t)]+\frac{1}{2} \mathbf{p}(t)^{T} \mathbf{M}^{-1} \mathbf{p}(t)=H\left(\mathbf{m}^{\text {est }}, \mathbf{p}^{\text {init }}\right)=\chi\left(\mathbf{m}^{\text {est }}\right)+\frac{1}{2}\left(\mathbf{p}^{\text {init }}\right)^{T} \mathbf{M}^{-1} \mathbf{p}^{\text {init }} .
$$

When the initial momentum $\mathbf{p}^{\text {init }}$ is chosen such that

$$
K\left(\mathbf{p}^{\text {init }}\right)=\frac{1}{2}\left(\mathbf{p}^{\text {init }}\right)^{T} \mathbf{M}^{-1} \mathbf{p}^{\text {init }}=\varepsilon^{2},
$$

equation (13.20) implies

$$
\chi[\mathbf{m}(t)] \leq \chi\left(\mathbf{m}^{\mathrm{est}}\right)+\varepsilon^{2},
$$

because the positive definiteness of the mass matrix $\mathbf{M}$ ensures $\mathbf{p}(t)^{T} \mathbf{M}^{-1} \mathbf{p}(t)$ for all momenta $\mathbf{p}(t)$. It follows that all models $\mathbf{m}(t)$ along the Hamiltonian trajectory are within the effective nullspace.

In the vast majority of interesting cases, Hamilton's equations (13.19) must be solved numerically, using, for example, the leapfrog scheme with time step $\Delta t$ :

$$
\begin{aligned}
p_{i}(t+\Delta t / 2) & =p_{i}(t)-\left.\frac{1}{2} \Delta t \frac{\partial U}{\partial m_{i}}\right|_{t}, \\
m_{i}(t+\Delta t) & =m_{i}(t)+\left.\Delta t \frac{\partial K}{\partial p_{i}}\right|_{t+\Delta t / 2}, \\
p_{i}(t+\Delta t) & =p_{i}(t+\Delta t / 2)-\left.\frac{1}{2} \Delta t \frac{\partial U}{\partial m_{i}}\right|_{t+\Delta t} .
\end{aligned}
$$

Though other numerical integrators may as well be used, the leap-frog scheme allows us to easily illustrate how the specific choices of initial momentum and mass matrix control the orbital properies of the trajectories. 
In the extreme case where a misfit increase above $\chi\left(\mathbf{m}^{\text {est }}\right.$ cannot be tolerated, we set $\varepsilon=0$. Equation (13.15) then implies vanishing initial momentum, $\mathbf{p}^{\text {init }}=\mathbf{0}$, and the first iteration of the leapfrog scheme from $t=0$ to $t=\Delta t$ becomes

$$
m_{i}(\Delta t)=m_{i}^{\text {est }}-\frac{1}{2} \Delta t^{2} \sum_{k=1}^{n} M_{i k}^{-1} \frac{\partial \chi\left(\mathbf{m}^{\text {est }}\right)}{\partial m_{k}} .
$$

Equation (13.26) reveals an interesting relation between effective nullspace exploration and the gradient descent methods from chapter 8 . In fact, the special case of choosing a mass matrix equal to the identity matrix, $\mathbf{M}=\mathbf{I}$, transforms (13.26) into a steepest-descent step with steplength $\Delta t^{2} / 2$. Similarly, choosing $\mathbf{M}$ to equals the Hessian $\mathbf{H}$ of $\chi$, we retrieve a variant of Newton's method with steplength $\Delta t^{2} / 2$. Since the mass matrix is positive definite, the first step of the zero-tolerance scenario is generally in a descent direction, as discussed in section 8.2.

When the estimated model $\mathbf{m}^{\text {est }}$ is sufficiently close to the minimum of $\chi$ to ignore the first derivative $\partial \chi\left(\mathbf{m}^{\text {est }}\right) / \partial m_{i}=\partial U\left(\mathbf{m}^{\text {est }}\right)$, the first step of the leapfrog scheme specialises to

$$
m_{i}(\Delta t)=m_{i}^{\mathrm{est}}+\sum_{k=1}^{n} \Delta t M_{i k}^{-1} p_{k}^{\mathrm{init}}
$$

Equation (13.27) provides the opportunity to add specific model perturbation $\Delta \mathbf{m}$ to the estimated model $\mathbf{m}^{\text {est }}$. In fact, choosing the initial momentum

$$
\mathbf{p}^{\text {init }}=\gamma \mathbf{M} \Delta \mathbf{m},
$$

with the factor $\gamma$ set to satisfy $K\left(\mathbf{p}^{\text {init }}\right)=\varepsilon^{2}$, the first leapfrog iteration adds a scaled version of $\Delta \mathbf{m}$ to $\mathbf{m}^{\text {est }}$. Subsequent iterations will add more of $\Delta \mathbf{m}$ until an increasing misfit derivative starts to dominate equation, thereby deflecting the trajectory towards a different direction.

A slight rearrangement of equation (13.24) shows that the mass matrix generally acts to modify the direction of the trajectory,

$$
\mathbf{m}(t+\Delta t)=\mathbf{m}(t)+\Delta t \mathbf{M}^{-1} \mathbf{p}(t+\Delta t / 2) .
$$

Choosing $\mathbf{M}^{-1}$ to be a smoothing operator, for example, will lead to a preferential exploration of smoother models in the effective nullspace. A roughening operator, in contrast, will produce a sequence of rougher alternative models.

Analytic one-dimensional nullspace shuttle: The concept of the nonlinear Hamiltonian nullspace shuttle can be illustrated most easily with a one-dimensional model space and an analytically defined misfit function $\chi(m)$. An example is shown in figure 13.1 for the misfit function

$$
\chi(m)=U(m)=1-\cos \left(0.2 m^{2}\right)+0.025 m^{2} .
$$

By design, the Hamiltonian trajectories are curves of constant total energy $H$. For a comparatively low tolerance, as in figure 13.1a, the trajectory remains entirely within the basin of attraction where the estimated model is located. Increasing the tolerance, as shown in figure $13.1 \mathrm{~b}$, still produces constant-energy trajectories in the combined model-momentum space (phase space), but the projection of the trajectory in model space is able to transition between different basins of attraction.

Nonlinear 2-D traveltime tomography: A more realistic example in the form of a synthetic 2-D nonlinear traveltime tomography is shown in figure 13.2 (Fichtner \& Zunino, 2019). The misfit $\chi=U$ is defined as the squared sum of traveltime residuals for all source-receiver pairs, and the forward problem is the eikonal equation, which describes the geometry of ray in a (heterogeneous) medium (Cerveny, 2001). For a given tolerance, the Hamiltonian trajectory consists of a sequence of P-wave velocity models that are, by construction, all consistent with the observed traveltimes because their misfit (potential energy) is bounded above by the constant Hamiltonian $H$. 
a) low tolerance, $\varepsilon^{2}=0.12$

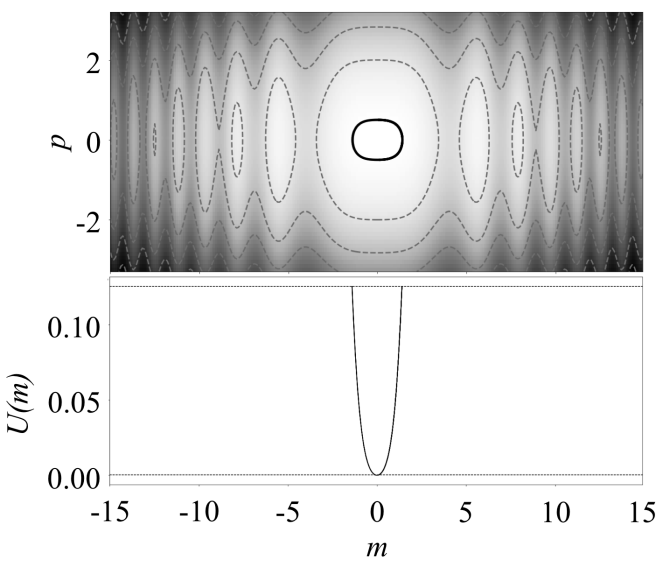

b) high tolerance, $\varepsilon^{2}=3.0$

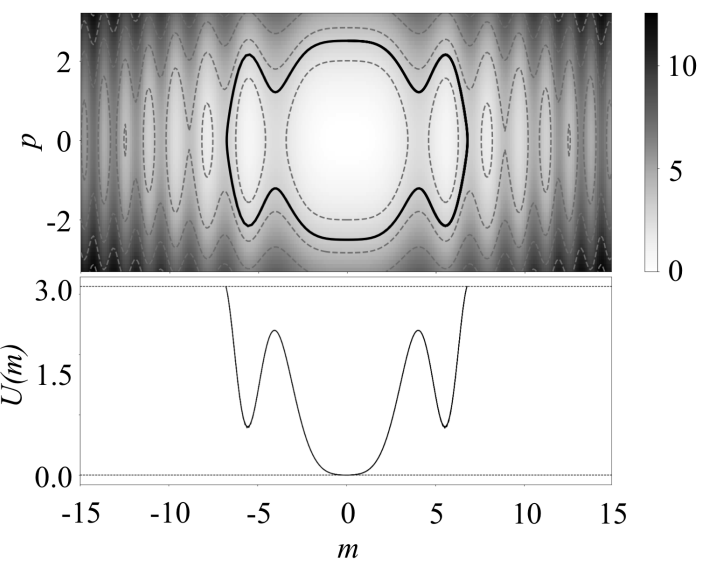

Fig. 13.1: Hamiltonian trajectories in model-momentum space (top) and their projection in model space (below) The total energy $H$ is shown in grey scale, with dashed curves marking levels of contant energy. The trajectory is shown a solid black curve. For a low tolerance (a), the trajectory remains close to the global optimum, whereas it may sample neighbouring basins of attraction in model space when the tolerance is higher (b). [Hamiltonian Nullspace Shuttle - 2D analytic]

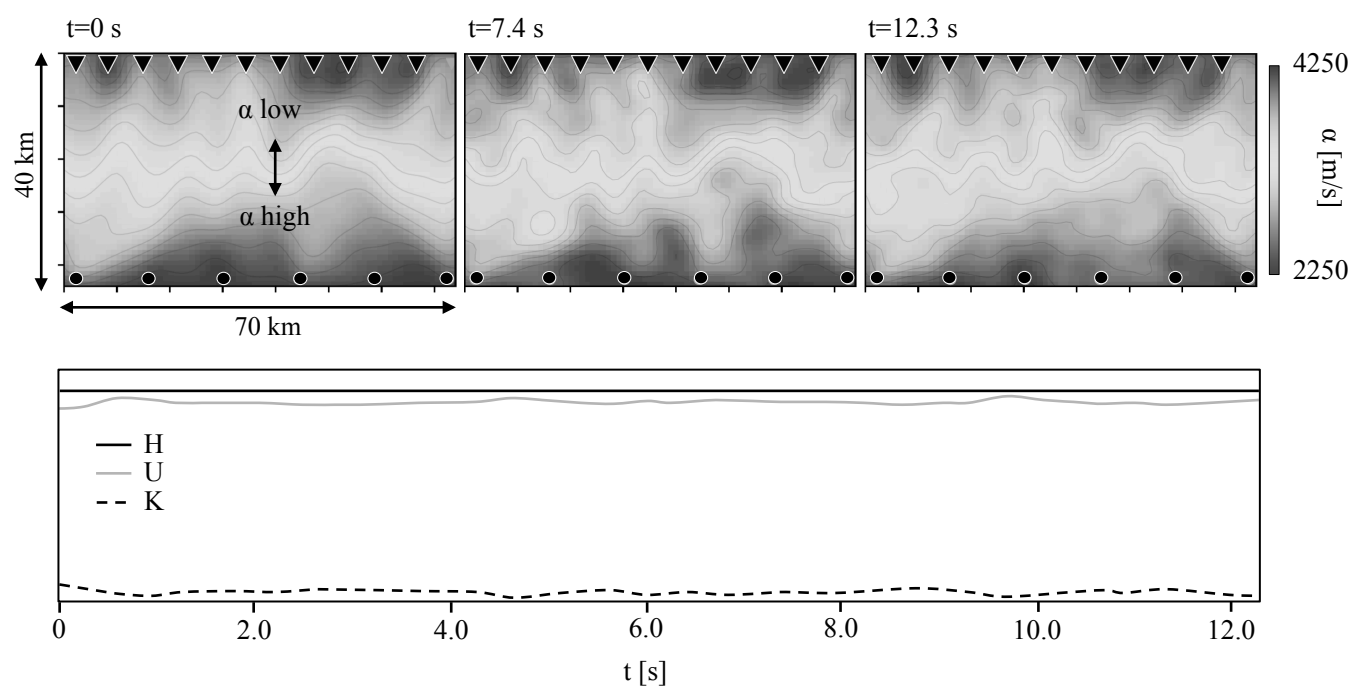

Fig. 13.2: Snapshots along the nullspace trajectory of a 2-D nonlinear traveltime tomography (top). Sources near the bottom of the domain are marked by black circles, receivers near the surface are shown as black triangles. All models along the trajectors explain traveltimes of waves from sources to receivers within a given tolerance. The evolution of the total energy $H$, the potential energy (misfit) $U$, and the kinetic energy $K$ is shown in the lower panel. Note that the potential energy $U$ is, by design, always smaller than $H$. 
In most cases, the tolerance $\varepsilon$ is not a hard bound but in itself uncertain, suggesting that it should be treated as a random variable. Equation (13.21) implies that normally distributed tolerances with zero mean and standard deviations $\varepsilon$ correspond to random momentum vectors $\mathbf{p}$ drawn from an $n$-dimensional Gaussian distribution with covariance matrix $2 \varepsilon \mathbf{M}$.

Allowing the tolerance $\varepsilon$ to exceed the hard bound with some probability gives rise to a randomised version of the nonlinear Hamiltonian nullspace shuttle. After some simulation time, the trajectory is stopped, and a new initial momentum is drawn randomly from the Gaussian with covariance matrix $2 \varepsilon \mathbf{M}$. The subsequent trajectory will be on a somewhat lower or higher energy level, thus giving rise to a broader nullspace exploration. The randomised nullspace shuttle is nearly identical to the Hamiltonian Monte Carlo algorithm, covered in section 5.7. The only difference is the absence of a Metropolis rule that decides about the acceptance or rejection of a move.

Randomised analytic one-dimensional nullspace shuttle: We return to the analytic example, with the misfit given by equation (13.30), and consider a randomised version of the algorithm. The tolerance is now interpreted as a random, normally distributed variable with zero mean and standard deviation $\varepsilon$. The corresponding trajectories are shown in figure 13.3. For a low tolerance (standard deviation), the nullspace mostly explores the vicinity of the maximum-likelihood model. As the tolerance increases, the shuttle becomes more exploratory and ventures into neighbouring basins of attraction.

a) low tolerance, $\varepsilon^{2}=0.12$

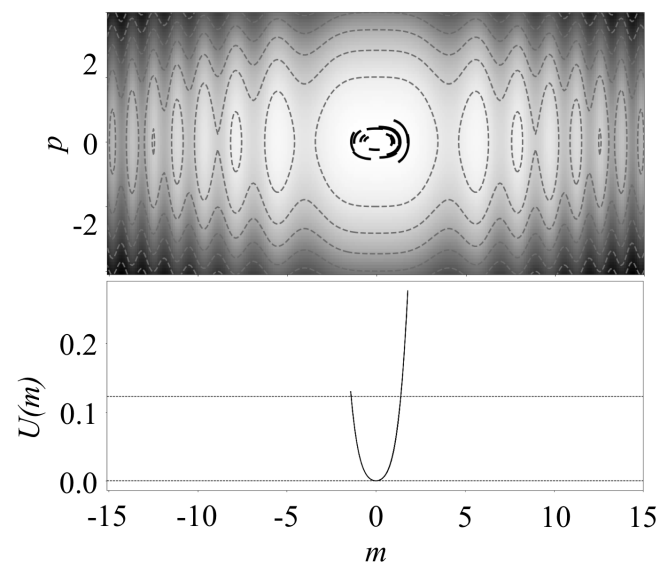

b) high tolerance, $\varepsilon^{2}=2.1$

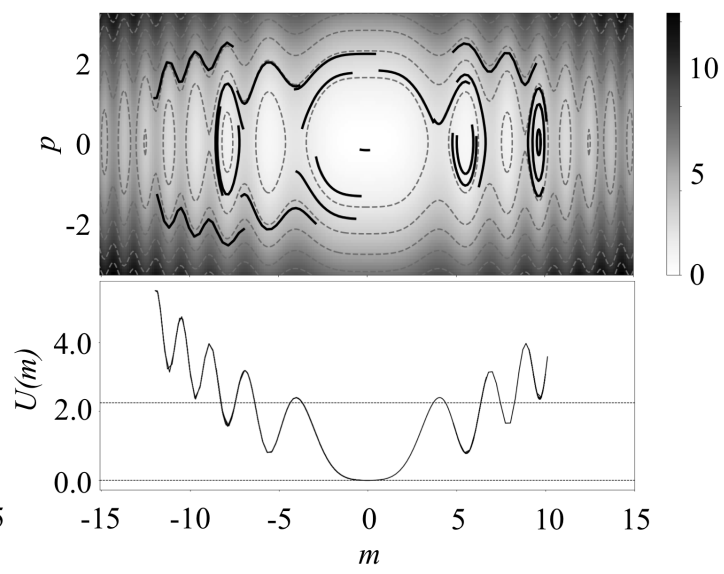

Fig. 13.3: Randomised version of the nonlinear Hamiltonian nullspace shuttle. Trajectories are shown in the top panels, superimposed on the total energy $H$ in phase space. The projections in model space are shown below. For sufficiently high tolerances, the shuttle is able to explore the nullspace more exhaustively. [Hamiltonian Nullspace Shuttle - 2D analytic] 



\section{Chapter 14 Autotuning Monte Carlo}

It may be mentioned in this connection that the maximum displacement $\alpha$ must be chosen with some care; if too large, most moves will be forbidden, and if too small, the configuration will not change enough. In either case it will then take longer to come to equilibrium.

Nicholas Metropolis, Arianna W. Rosenbluth, Marshall N. Rosenbluth, Ausgusta H. Teller and Edward Teller, Equation of State Calculations by Fast Computing Machines, 1953.

Already in the early days when computers started to be powerful enough to make statistical computing practically feasible, it was clear that Monte Carlo algorithms require careful tuning in order to be useful. The variable $\alpha$, mentioned by Metropolis and co-workers in their influential paper, is the tuning parameter of a Monte Carlo sampler, used to explore the phase space of a thermodynamical system, and it corresponds to the search radius $\sigma$ in the local Metropolis-Hastings algorithm in section 5.5.2. When $\sigma$ is too small, almost all moves of the random walker are accepted, but model space exploration may be uselessly slow. Conversely, a value of $\sigma$ that is too large leads to the frequent rejection of a proposed move, meaning that the random walker is stuck at the same position for a long time. Tuning Monte Carlo algorithms by hand can be a tedious task, especially for algorithms where the number of tuning parameters is large, such as Hamiltonian Monte Carlo. It is therefore desirable to develop autotuning strategies that find a nearly optimal set of tuning parameters on the fly, that is, while sampling the distribution that we are interested in. The following chapter describes such an autotuning strategy using the example of a Hamiltonian Monte Carlo algorithm. It closely follows the method presented in Fichtner et al. (2021).

\subsection{Motivation and context}

Metropolis et al.'s tuning parameter $\alpha$ determines the width of the Gaussian proposal distribution in a local Metropolis-Hastings sampler, and it thereby controls convergence speed. In a more general context, the Metropolis rule (5.57) suggests that the perfect proposal distribution is the target distribution itself. All moves would be accepted, and the random walker would sample the target distribution right from the start. However, if we were able to draw samples directly from the target distribution, we would defeat the purpose of actually running a Monte Carlo algorithm in the first place. Nevertheless, this idealistic thought experiment suggests that at least some approximation of the target distribution may be a useful proposal distribution that speeds up convergence.

The Metropolis-adjusted Langevin algorithm (MALA), introduced in section 5.5.3, can be interpreted as a first step in this direction. Instead of making proposals that are blind to the properties of the target distribution, it 
exploits local gradient information in order to make proposals that are on average more plausible. Continuing this line of thought, Hessian-aware Monte Carlo sampling takes advantage of second derivatives, that is, of the local curvature of the target distribution. Early examples of Hessian-aware Monte Carlo methods took the form of modified Metropolis-Hastings algorithms, where the proposal density is a function of the explicitly computed Hessian (Chib et al., 1998; Geweke \& Tanizaki, 1999). Conceptually closely related is the work of Qi \& Minka (2002), who proposed a variant of the Metropolis-Hastings algorithm where the target distribution is approximated locally by a Gaussian, the covariance of which is determined by the local Hessian. For their method, the Hessian and its Cholesky decomposition must be known explicitly, thereby imposing a limitation to rather small problems. Along similar lines, Martin et al. (2012), employed second-order adjoint techniques to compute local Hessian-vector products (Fichtner \& Trampert, 2011; Santosa \& Symes, 1988), which then provide a low-rank approximation of the Hessian. The latter enables the application of their method to higherdimensional problems. An efficient alternative to second-order adjoints, suggested by Hanson \& Cunningham (1998), is the use of the BFGS method from section 8.6.2 to approximate the Hessian-vector products needed for sampling.

In Hamiltonian Monte Carlo, the mass matrix $\mathbf{M}$ effectively controls the properties of the proposal distribution. As we have already seen in section 5.7.7, the mass matrix affects the geometry of Hamiltonian trajectories, and thereby the convergence speed of the algorithm. This is once more illustrated in figures 14.1 and 14.2, where we consider the example of a 1000-dimensional Gaussian,

$$
\rho\left(\mathbf{m} \mid \mathbf{d}^{\mathrm{obs}}\right) \propto e^{-\frac{1}{2} \mathbf{m}^{T} \mathbf{C}^{-1} \mathbf{m}},
$$

where the covariance matrix $\mathbf{C}$ is diagonal with entries ranging linearly from $C_{1,1}=0.01$ to $C_{1000,1000}=1.0$. Figure 14.1 summarises the result after drawing 2000 samples using the trivial choice $\mathbf{M}=\mathbf{I}$. While the approximated 1-D marginal of parameter $m_{1}$ in figure 14.1a resembles the desired Gaussian with standard deviation 0.1 , the 1-D marginal of $m_{1000}$ appears bimodal instead of Gaussian, indicating that the number of samples is insufficient. This apparently insufficient convergence towards the target marginal can be explained by the effective sample size $N_{\text {eff }}$, previously introduced in equation (5.71), or its normalised version $N_{\text {eff }} / N_{s}$, known as the effective sample fraction. For $m_{1}$, we find $N_{\text {eff }} / N_{s}=0.3286$, meaning that about 3 samples are needed in order to draw an independent one. In contrast, $N_{\text {eff }} / N_{s}=0.0047$ for $m_{1000}$, which suggests that more than 200 samples are needed in $m_{1000}$ direction in order to achieve sample independence. Figure $14.1 \mathrm{~d}$ explains this observation at the level of Hamiltonian trajectories. In $m_{1}$-direction, the particle makes rapid progress, thereby producing independent samples quickly. Progress in $m_{1000}$-direction, however, is comparatively slow, resulting in strong correlations of subsequent samples.

Changing the mass matrix from $\mathbf{M}=\mathbf{I}$ to $\mathbf{M}=\mathbf{C}^{-1}$ has a profound impact on the sampler, as shown in figure 14.2. The marginals for both $m_{1}$ and $m_{1000}$ are close to being Gaussian, and even though the effective sample fraction of $m_{1}$ decreased slightly, it increased by a factor of $>30$ for $m_{1000}$. Hence, overall, $\sim 30$ times less samples are needed to achieve a similar level of convergence.

Though knowing $\mathbf{C}^{-1}$ defeats the purpose of sampling, the Gaussian example suggests that the local Hessian of the potential energy $U(\mathbf{m})=\log \rho\left(\mathbf{m} \mid \mathbf{d}^{\text {obs }}\right)$ may be a useful mass matrix. Explicitly computing or storing the Hessian is prohibitive in higher-dimensional applications, and so we need to find computationally efficient approximations. In the following sections, we introduce an autotuning approach that employs a variant of the L-BFGS method, derived in section 8.6.3, in order to find nearly optimal mass matrices on the fly, that is, while sampling the target distribution. 
a) 1-D marginal of $m_{l}$

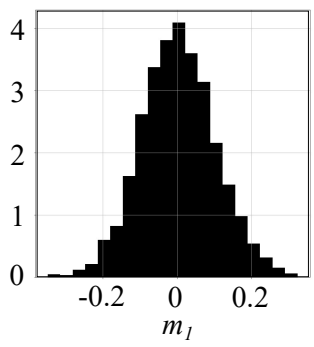

b) 1-D marginal of $m_{1000}$

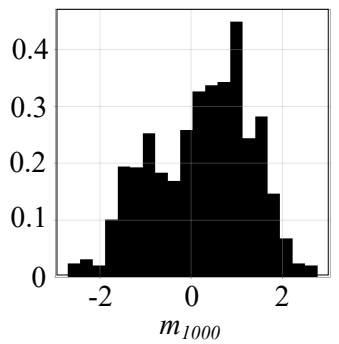

c) auto-correlations $\left(m_{1}, m_{1000}\right)$

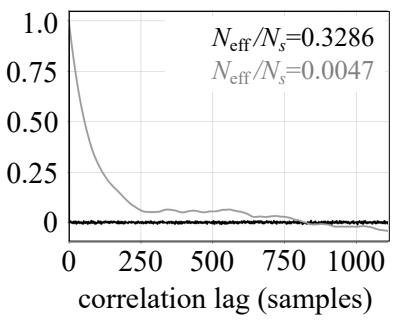

d) representative trajectory

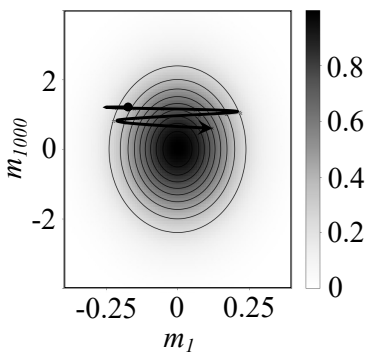

Fig. 14.1: Hamiltonian Monte Carlo sampling of the 1000-dimensional Gaussian in equation (14.1). The model covariance matrix $\mathbf{C}$ is diagonal, with elements ranging linearly from $C_{1,1}=0.01$ to $C_{1000,1000}=1.0$. The mass matrix $\mathbf{M}$ is equal to the unit matrix I. Of the 3000 samples used, 1000 are ignored as burn-in. (a,b) Sampleapproximated one-dimensional marginals for parameters $m_{1}$ and $m_{1000}$. (c) Auto-correlations averaged over 100 Hamiltonian Monte Carlo runs of the sample chains for $m_{1}$ and $m_{1000}$, with corresponding effective sample fractions. (d) Two-dimensional projection of a representative Hamiltonian trajectory, with the target Gaussian shown in the brackground.

a) 1-D marginal of $m_{1}$

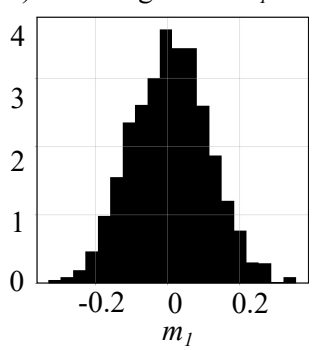

b) 1-D marginal of $m_{1000}$

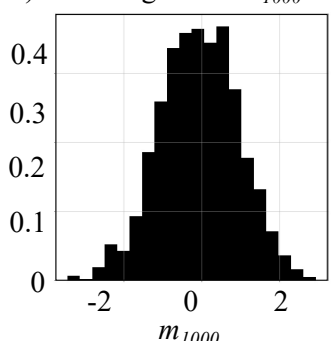

c) auto-correlations $\left(m_{l}, m_{1000}\right)$

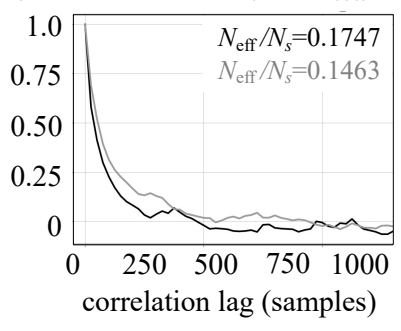

d) representative trajectory

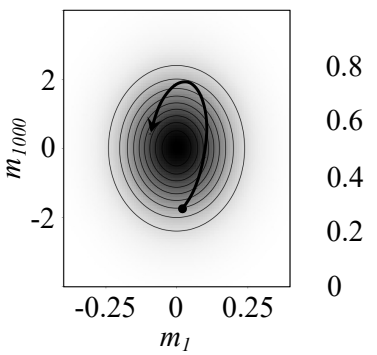

Fig. 14.2: Hamiltonian Monte Carlo sampling with a setup identical to the one in figure 14.1, except for choosing the mass matrix equal to the inverse covariance, that is, $\mathbf{M}=\mathbf{C}^{-1}$. (a,b) One-dimensional marginals for parameters $m_{1}$ and $m_{1000}$. (c) Auto-correlations averaged over 100 Hamiltonian Monte Carlo runs for $m_{1}$ and $m_{1000}$, with corresponding effective sample fractions. (d) Two-dimensional projection of a representative Hamiltonian trajectory, with the target Gaussian shown in greyscale in the brackground.

\subsection{Autotuning by limited-memory quasi-Newton updating}

To make the methodological developments more digestible, we begin with a brief recapitulation of the BFGS method, covered before in section 8.6.2. This will be followed by its factorised version, as well as several strategies to improve convergence.

\subsubsection{BFGS updating of the HMC mass matrix}

In nonlinear optimisation, the BFGS method (Broyden, 1970; Fletcher, 1970; Goldfarb, 1970; Nocedal \& Wright, 1999; Shanno, 1970) is used for the iterative approximation of the local inverse Hessian $\mathbf{A}=\mathbf{H}^{-1}$, which then serves as a computationally less expensive substitute of the exact Hessian in a quasi-Newton iteration, introduced earlier in section 8.6. To summarise BFGS, we introduce the auxiliary vectors

$$
\mathbf{s}_{k}=\mathbf{m}_{k+1}-\mathbf{m}_{k}, \quad \mathbf{y}_{k}=\nabla U\left(\mathbf{m}_{k+1}\right)-\nabla U\left(\mathbf{m}_{k}\right),
$$


where the potential energy $U$ equals the misfit $\chi$, and the $k^{\text {th }}$ iterate $\mathbf{m}_{k}$ in the BFGS sequence is taken to be the $k^{\text {th }}$ sample $\mathbf{m}^{k}$ of the Hamiltonian Monte Carlo sample chain. Starting from a positive definite initial guess $\mathbf{A}_{0}$ of the inverse Hessian of $U$, the BFGS iteration computes successive approximations of $\mathbf{A}$ as

$$
\mathbf{A}_{k+1}=\left(\mathbf{I}-\rho_{k} \mathbf{s}_{k} \mathbf{y}_{k}^{T}\right) \mathbf{A}_{k}\left(\mathbf{I}-\rho_{k} \mathbf{y}_{k} \mathbf{s}_{k}^{T}\right)+\rho_{k} \mathbf{s}_{k} \mathbf{s}_{k}^{T},
$$

with the scaling factor

$$
\rho_{k}=\frac{1}{\mathbf{y}_{k}^{T} \mathbf{s}_{k}} .
$$

Equations (14.2) to (14.4) are identical to equations (8.99), (8.116) and (8.117). The quantity $\rho_{k}$ must be strictly positive to ensure that successive updates are positive definite. The corresponding approximation of the Hessian, $\mathbf{A}_{k+1}^{-1}=\mathbf{H}_{k+1}$ can be derived from the application of the Sherman-Morrison formula (8.109) (Bartlett, 1951; Nocedal \& Wright, 1999) to equation (14.3). Assuming that $\mathbf{A}_{k+1}^{-1}$ approximates the local Hessian $\mathbf{H}\left(\mathbf{m}_{k+1}\right)$, we may use $\mathbf{H}_{k+1}$ as mass matrix to draw the subsequent sample $\mathbf{m}^{k+1}=\mathbf{m}_{k+1}$.

\subsubsection{Factorised BFGS method}

As shown in section 5.4, generating random momenta $\mathbf{p}$ from a Gaussian distribution with covariance matrix $\mathbf{M}=\mathbf{A}_{k}^{-1}$ for Hamiltonian Monte Carlo sampling, requires a factorisation of the mass matrix, $\mathbf{M}=\mathbf{S S}^{T}$. This may, for instance, be the Cholesky decomposition (3.40). However, the number of operations needed to compute $\mathbf{S}$ via a Cholesky decomposition is of order $n^{3}$, meaning that it is not affordable for many relevant applications.

To design a scalable algorithm, we try to compute $\mathbf{S}$ directly through a modified version of the BFGS equations, referred to as factorised BFGS (F-BFGS). Closely following the approach of Broodlie et al. (1973), we first write the BFGS update from (14.3) in a factorised form,

$$
\mathbf{A}_{k+1}=\left(\mathbf{I}+\mathbf{u}_{k} \mathbf{v}_{k}^{T}\right) \mathbf{A}_{k}\left(\mathbf{I}+\mathbf{u}_{k} \mathbf{v}_{k}^{T}\right)^{T},
$$

where $\mathbf{u}_{k}$ and $\mathbf{v}_{k}$ are two vectors for which we still need to find explicit expressions. For this, we first notice that (14.5) may be expanded into

$$
\mathbf{A}_{k+1}=\mathbf{A}_{k}+\mathbf{a}_{k} \mathbf{a}_{k}^{T}-\mathbf{b}_{k} \mathbf{a}_{k}^{T}-\mathbf{a}_{k} \mathbf{b}_{k}^{T},
$$

where we defined the auxiliary variables

$$
\begin{aligned}
\gamma_{k}^{2} & =\rho_{k}^{2} \mathbf{y}_{k}^{T} \mathbf{A}_{k} \mathbf{y}_{k}+\rho_{k}, \\
\mathbf{a}_{k} & =\gamma_{k} \mathbf{s}_{k}, \\
\mathbf{b}_{k} & =\frac{\rho_{k}}{\gamma_{k}} \mathbf{A}_{k} \mathbf{y}_{k} .
\end{aligned}
$$

Expanding (14.5) and comparing to (14.7), suggests the following ansatz for the vectors $\mathbf{u}_{k}$ and $\mathbf{v}_{k}$,

$$
\begin{aligned}
& \mathbf{u}_{k}=\mathbf{a}_{k}, \\
& \mathbf{v}_{k}=-\mathbf{A}_{k}^{-1}\left(\mathbf{b}_{k}+\theta \mathbf{a}_{k}\right),
\end{aligned}
$$

with some scalar $\theta$. To determine $\theta$, we substitute equations (14.8)a,b into (14.5), which yields

$$
\mathbf{A}_{k+1}=\mathbf{A}_{k}+\mathbf{a}_{k} \mathbf{a}_{k}^{T}\left[\left(\mathbf{b}_{k}+\theta \mathbf{a}_{k}\right)^{T} \mathbf{A}_{k}^{-1}\left(\mathbf{b}_{k}+\theta \mathbf{a}_{k}\right)-2 \theta\right]-\mathbf{b}_{k} \mathbf{a}_{k}^{T}-\mathbf{a}_{k} \mathbf{b}_{k}^{T} .
$$

Comparing (14.9) to Eq. (14.6) shows that $\theta$ must satisfy the quadratic equation 
14.2 Autotuning by limited-memory quasi-Newton updating

$$
\left(\mathbf{b}_{k}+\theta \mathbf{a}_{k}\right)^{T} \mathbf{A}_{k}^{-1}\left(\mathbf{b}_{k}+\theta \mathbf{a}_{k}\right)-2 \theta=1 .
$$

Slightly reorganising (14.10), gives

$$
\left(\mathbf{a}_{k}^{T} \mathbf{A}_{k}^{-1} \mathbf{a}_{k}\right) \theta^{2}+2\left(\mathbf{a}_{k}^{T} \mathbf{A}_{k}^{-1} \mathbf{b}_{k}-1\right) \theta+\left(\mathbf{b}_{k}^{T} \mathbf{A}_{k}^{-1} \mathbf{b}_{k}-1\right)=0 .
$$

To express the polynomial coefficients in (14.11) in terms of $\mathbf{s}_{k}$ and $\mathbf{y}_{k}$, we re-substitute equations (14.7)b,c, which leads to

$$
\left(\gamma_{k}^{2} \mathbf{s}_{k}^{T} \mathbf{A}_{k}^{-1} \mathbf{s}_{k}\right) \theta^{2}=\frac{\rho_{k}}{\gamma_{k}^{2}}
$$

The quadratic equation (14.12) has two real-valued solutions for $\theta$ provided that $\rho_{k}>0$. This condition is identical to the requirement for positive-definite BFGS updates (e.g. Nocedal \& Wright, 1999).

This set of equations yields a recipe for the factorised BFGS (F-BFGS) updating of $\mathbf{A}_{k}$ based on the computation of the vectors $\mathbf{u}_{k}$ and $\mathbf{v}_{k}$ through equations (14.7) and (14.8). A factorised update of $\mathbf{A}_{k}^{-1}$ follows from the inversion of (14.5),

$$
\mathbf{H}_{k+1}=\left(\mathbf{I}+\mathbf{v}_{k} \mathbf{u}_{k}^{T}\right)^{-1} \mathbf{H}_{k}\left(\mathbf{I}+\mathbf{u}_{k} \mathbf{v}_{k}^{T}\right)^{-1},
$$

combined with the Shermann-Morrison formula (8.109),

$$
\left(\mathbf{I}+\mathbf{v}_{k} \mathbf{u}_{k}^{T}\right)^{-1}=\mathbf{I}-\frac{\mathbf{v}_{k} \mathbf{u}_{k}^{T}}{1+\mathbf{v}_{k}^{T} \mathbf{u}_{k}} .
$$

Assuming that a factorisation $\mathbf{A}_{k}^{-1}=\mathbf{S}_{k} \mathbf{S}_{k}^{T}$ is available from previous F-BFGS iterations, equations (14.13) and (14.14) imply that the updated matrix factor $\mathbf{S}_{k+1}$ and its inverse $\mathbf{S}_{k+1}^{-1}$ are given by

$$
\mathbf{S}_{k+1}=\left(\mathbf{I}-\frac{\mathbf{v}_{k} \mathbf{u}_{k}^{T}}{1+\mathbf{v}_{k}^{T} \mathbf{u}_{k}}\right) \mathbf{S}_{k}, \quad \mathbf{S}_{k+1}^{-1}=\mathbf{S}_{k}^{-1}\left(\mathbf{I}+\mathbf{v}_{k} \mathbf{u}_{k}^{T}\right)
$$

Knowing the matrix factors $\mathbf{S}_{k+1}$ and $\mathbf{S}_{k+1}^{-1}$, allows us to compute all (inverse) Hessian-vector products and to generate random momenta from a Gaussian with covariance $\mathbf{M}=\mathbf{H}_{k}$.

\subsubsection{Limited-memory factorised BFGS method (LF-BFGS)}

The factorised updating equations (14.15) enable a limited-memory approach, similar to the standard limitedmemory BFGS concept of Nocedal (1980) and Liu \& Nocedal (1989), that we introduced in section 8.6.3. For this, we let $\mathbf{h}$ be a arbitrary vector and write

$$
\mathbf{S}_{k+1} \mathbf{h}=\left(\mathbf{I}-\frac{\mathbf{v}_{k} \mathbf{u}_{k}^{T}}{1+\mathbf{v}_{k}^{T} \mathbf{u}_{k}}\right)\left(\mathbf{I}-\frac{\mathbf{v}_{k-1} \mathbf{u}_{k-1}^{T}}{1+\mathbf{v}_{k-1}^{T} \mathbf{u}_{k-1}}\right) \ldots\left(\mathbf{I}-\frac{\mathbf{v}_{0} \mathbf{u}_{0}^{T}}{1+\mathbf{v}_{0}^{T} \mathbf{u}_{0}}\right) \mathbf{S}_{0} \mathbf{h} .
$$

Typically, the initial matrix factor $\mathbf{S}_{0}$ is equal the unit matrix $\mathbf{I}$. Defining the vector $\mathbf{h}_{0}=\mathbf{S}_{0} \mathbf{h}$, equation (14.16) takes the form of a sequential update,

$$
\mathbf{h}_{i+1}=\left(\mathbf{I}-\frac{\mathbf{v}_{i} \mathbf{u}_{i}^{T}}{1+\mathbf{v}_{i}^{T} \mathbf{u}_{i}}\right) \mathbf{h}_{i}=\mathbf{h}_{i}-\mathbf{v}_{i} \frac{\mathbf{u}_{i}^{T} \mathbf{h}_{i}}{1+\mathbf{v}_{i}^{T} \mathbf{u}_{i}}, \quad i=0, \ldots, k
$$

which eventually gives

$$
\mathbf{S}_{k+1} \mathbf{h}=\mathbf{h}_{k+1} .
$$

Equation (14.18) has the important benefit of only containing vector-vector products, thereby eliminating the need to explicitly compute and store complete matrices. Furthermore, the sequence can be limited to the last 
$\ell+1$ vector pairs $\left(\mathbf{u}_{k}, \mathbf{v}_{k}\right), \ldots,\left(\mathbf{u}_{k-\ell}, \mathbf{v}_{k-\ell}\right)$, which further reduces storage requirements at the expense of a more incorrect but hopefully still acceptable Hessian approximation (Nocedal \& Wright, 1999). Following this approach, similar equations can be found for products of $\mathbf{h}$ with the inverse and transpose of $\mathbf{S}_{k+1}$.

\subsubsection{Improving convergence and stability}

\subsubsection{Iterative updating of the initial matrix}

In order to accelerate the convergence of the LF-BFGS iteration, the initial matrix factor $\mathbf{S}_{0}$ should be updated. A constant $\mathbf{S}_{0}$ implies that the LF-BFGS algorithm has a memory of $\ell$ samples, which may be small compared to the model space dimension $n$. Updating $\mathbf{S}_{0}$ may increase the length of the memory, meaning that more than $\ell$ samples effectively contribute to the Hessian approximation.

Most straightforwardly, $\mathbf{S}_{0}$ is replaced in regular intervals, typically every $\ell$ samples, by the square root of the diagonal elements of the current Hessian approximation, that is,

$$
\sqrt{\operatorname{diag} \mathbf{A}_{k}^{-1}} \rightarrow \mathbf{S}_{0}
$$

Importantly, any updating of $\mathbf{S}_{0}$ requires a recalculation of the vector sequences $\mathbf{u}_{0}, \mathbf{u}_{1}, \ldots$ and $\mathbf{v}_{0}, \mathbf{v}_{1}, \ldots$, as they depend on $\mathbf{S}_{0}$.

\subsubsection{Integration length}

The choice of a suitable integration length $\tau$ is a balancing act between a large $\tau$ that ensures rapid model space exploration and a small $\tau$ to limit computational cost. Using the LF-BFGS Hessian $\mathbf{H}_{k}$ as mass matrix provides some useful guidance. As $\mathbf{A}_{k}^{-1}$, and therefore $\mathbf{M}$, approach the true Hessian $\mathbf{H}$, the Hamiltonian trajectories converge towards segments of $n$-dimensional circles with circular frequency $2 \pi$, as demonstrated in section 5.7.7. Therefore, in the case of a roughly constant Hessian, we observe approximate Poincaré recurrence for $\tau=2 \pi$, meaning that the trajectory nearly reaches its starting point. After only half the time, about half the trajectory has been traversed.

When the Hessian is not approximately constant, the above argument looses precision, of course. Nevertheless, setting $\tau \approx \pi$ with some random variations to avoid cyclic behaviour of the sampler (Mackenzie, 1989) is an empirically useful choice.

\subsubsection{Initial and adaptive time stepping}

As in chapter 5.7, we solve Hamilton's equations numerically, using the leap-frog algorithm. The leap-frog integration time step $h$ is controlled by the need to maintain reasonably high acceptance rates and ensure numerical stability. As demonstrated in section 15.3, numerical stability requires $h \leq 2 / \sqrt{\lambda_{\max }\left(\mathbf{M}^{-1} \mathbf{H}\right)}$, where $\lambda_{\max }\left(\mathbf{M}^{-1} \mathbf{H}\right)$ is the maximum eigenvalue of the matrix product $\mathbf{M}^{-1} \mathbf{H}$.

A conservative initial estimate of $h$ may be obtained by trial and error. Alternatively, one may have estimates of the maximum eigenvalue of the Hessian near the initial model, $\lambda_{\max }\left[\mathbf{H}\left(\mathbf{m}_{0}\right)\right]$, for instance, from physical arguments or the application of second-order adjoint methods (e.g. Fichtner \& Trampert, 2011; Pratt et al., 1998; Santosa \& Symes, 1988). Setting the initial mass matrix to $\lambda_{\max }\left[\mathbf{H}\left(\mathbf{m}_{0}\right)\right] \mathbf{I}$ then causes the maximum allowable $h$ to be around 2 , meaning that $h \approx 1$ is likely to be a useful starting point.

Successive updating of the mass matrix with the LF-BFGS Hessian approximation affects numerical stability because the maximum eigenvalue of $\mathbf{M}^{-1} \mathbf{H}$ changes. Since repeated eigenvalue estimations are computation- 
ally prohibitive, an alternative approach for the adjustment of $h$ is required. For this, we may exploit the otherwise undesirable fact that energy conservation of the leap-frog scheme is only correct to first order in $h$, as shown in section 15.3. The deterioration of energy conservation may therefore be used as a proxy for upcoming numerical instability.

In practice, the adaptation of $h$ is most easily implemented by monitoring the acceptance rate $R$ averaged over roughly $\ell$ samples. The decrease of $R$ below some threshold $R_{\min }$ relates directly to decreasing energy conservation, suggesting that $h$ should be reduced to a smaller value $\gamma h$ with $\gamma<1$. Conversely, when $R$ is above some threshold $R_{\max }$, the time step may be increased to $h / \gamma$ to reduce computational costs. Empirically, $R_{\min }=0.65$, $R_{\max }=0.85$ and $\gamma=0.80$ are useful values.

\subsubsection{Loss of the Markov property and variations of the theme}

The sequence of samples produced by the LF-BFGS approximation of the mass matrix algorithm is not an exact Markov chain where the next model only depends on the current one. In fact, the next model depends on the current mass matrix, which is controlled by $\ell>1$ previous misfit and gradient evaluations. Hence, the random sampling process is not memoryless, as required by the detailed balance proof for Hamiltonian Monte Carlo in section 5.7.4. Similar to other approximate Markov chain methods (e.g. Bardenet et al., 2014; Fox \& Nicholls, 1997; Korattikara et al., 2014; Scott et al., 2016), the autotuning algorithm may effectively sample a different distribution, thereby introducing bias.

In realistic applications, where the target distribution $\rho\left(\mathbf{m} \mid \mathbf{d}^{\text {obs }}\right)$ is unknown from the outset, the bias may be difficult to estimate. Nevertheless, the Hessian-aware algorithm may still produce independent samples more efficiently than a Hamiltonian sampler with unit mass matrix.

In the following paragraphs, we introduce two variations of the theme that preserve the Markov property, however, possibly at the expense of reduced efficiency (depending on the specifics of an application).

\subsubsection{Diagonal freezing}

In cases where the Hessian is a priori known to be roughly diagonal and roughly invariant, sampling may be accelerated by estimating the diagonal of the Hessian with a few short sample chains starting from different initial models. The resulting approximation of the Hessian diagonal is then used as a constant mass matrix in a sample chain that is sufficiently long to ensure convergence. The freezing of the diagonal after its initial estimation has the advantage of avoiding both the computational cost of on-the-fly autotuning and the potential bias introduced by an otherwise inexact Markov chain.

\subsubsection{Macroscopic autotuning}

When the generalised nullspace has fine-scale structure, for instance, in the form of numerous local minima, superimposed on some broad-scale background that is roughly Gaussian, we may borrow basic ideas from tempering (e.g. Geyer \& Thompson, 1995; Kirkpatrick et al., 1983; Marinari \& Parisi, 1992; Sambridge, 2014), introduced earlier in section 5.6 on Simulated Annealing. Instead of considering the original canonical distribution

$$
\rho(\mathbf{m}, \mathbf{p})=e^{-H(\mathbf{m}, \mathbf{p})}=e^{-U(\mathbf{m})} e^{-\frac{1}{2} \mathbf{p}^{T} \mathbf{M}^{-1} \mathbf{p}},
$$

we design a tempered version,

$$
\rho^{1 / T}(\mathbf{m}, \mathbf{p})=e^{-H(\mathbf{m}, \mathbf{p}) / T}=e^{-U(\mathbf{m}) / T} e^{-\frac{1}{2} \mathbf{p}^{T}\left(\mathbf{M}_{T}^{-1}\right) \mathbf{p}},
$$


with a temperature $T>1$ and a tempered or macroscopic mass matrix

$$
\mathbf{M}_{T}^{-1}=\frac{1}{T} \mathbf{M}^{-1}
$$

By construction, tempering suppresses detail while enhancing and broadening macroscopic features of the distribution, as illustrated in Fig. 14.3. The macroscopic shape of the posterior distribution may thus be captured using an LF-BFGS approximation of the macroscopic Hessian, again using a small number of short sampling chains, possibly starting from different initial models. Subsequently, the macroscopic Hessian in LF-BFGS representation can be scaled back to a hopefully useful and constant mass matrix of the actual problem using equation (14.22).

a) original probability density (non-Gaussian)

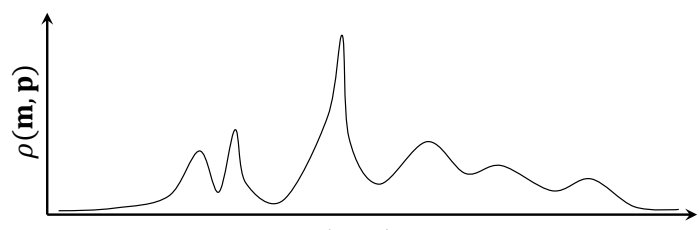

$(\mathbf{m}, \mathbf{p})$ b) tempered probability density ('more' Gaussian)

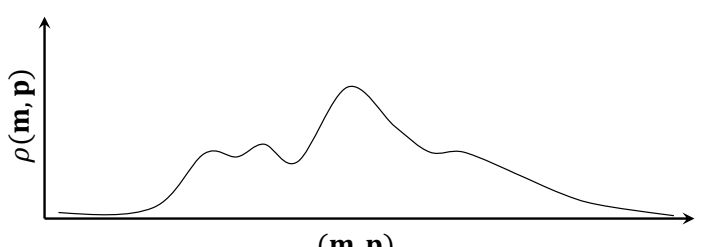

$(\mathbf{m}, \mathbf{p})$

Fig. 14.3: Schematic illustration of the effect of tempering on the canonical distribution $\rho(\mathbf{m}, \mathbf{p})$. Tempering transforms the multi-modal distribution in (a) into the smoother, more Gaussian-like distribution in (b), thereby enhancing macroscopic features at the expense of small-scale detail..

\subsection{Performance analysis using analytical test functions}

In the absence of a universally valid quantifier of Markov chain convergence (e.g. Cowles \& Carlin, 1996; Roy, 2019), we assess the performance of the autotuning strategy using analytical test functions. Unavoidably, this approach is limited by the small number of test functions that we can consider, but it still provides useful indications about the circumstances under which the algorithms are useful.

\subsubsection{Return to the high-dimensional Gaussian}

Starting with the simplest possible case, we return to the sampling of the 1000-dimensional Gaussian, previously presented as motivating example in section 14.1. Updating the initial mass matrix $\mathbf{M}=\mathbf{I}$ with the autotuning procedure, reproduces figure 14.2 almost exactly. We achieve effective sample sizes as if we had used $\mathbf{M}=\mathbf{H}=\mathbf{C}^{-1}$ from the outset. The time-step adaptivity guided by the average acceptance rate ensures that the leap-frog integration remains numerically stable. This is summarised in figure 14.4. Since the target distribution is Gaussian, the LF-BFGS approximation to the Hessian eventually becomes stationary in this example, meaning that the initially approximate Markov chain converges towards an exact Markov chain. 
a) average acceptance rate

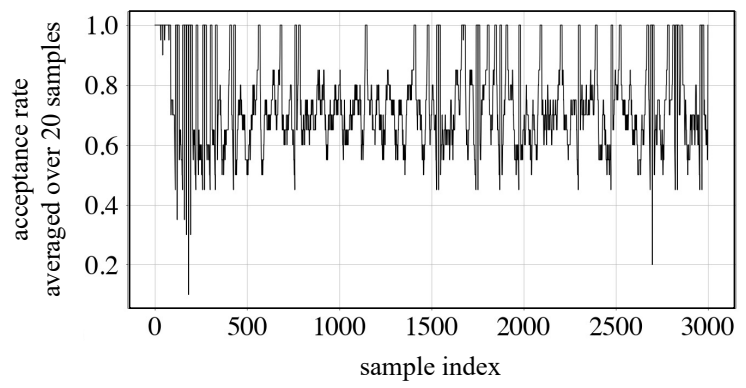

b) leap-frog integration time step

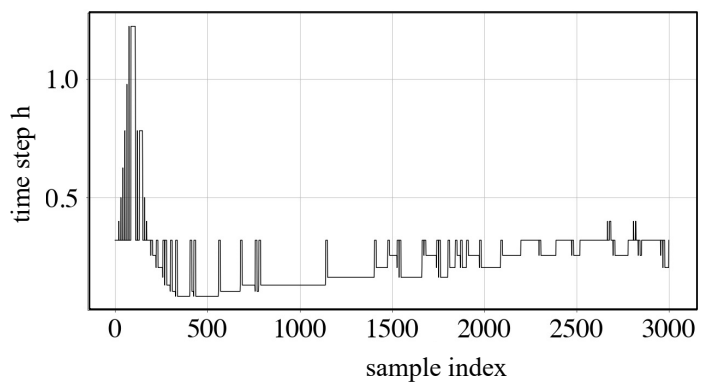

Fig. 14.4: Time-step adaptivity during autotuning of the Hamiltonian Monte Carlo sampler. (a) Acceptance rate $R$ averaged over 20 samples. (b) Variable integration time step $h$ that aims to keep $R$ between the threshold values $R_{\max }=0.85$ and $R_{\min }=0.5$.

\subsubsection{Modified Styblinski-Tang function}

Originally developed for the benchmarking of global optimisation algorithms in the context of neural network design, the $n$-dimensional Styblinski-Tang function (Styblinski \& Tang, 1990) is non-convex and multi-modal, with $2^{n}$ local minima. Since the Styblinski-Tang function can take negative values, we use a modified version, described in detail in section 15.4.1, in order to define a valid target probability density. Furthermore, we introduce inter-parameter trade-offs, and we again choose the model space dimension $n=1000$.

As a reference, we consider a chain of 1 million samples computed with constant unit mass matrix, $\mathbf{M}=\mathbf{I}$, and constant time step, $h$. Somewhat laborious trial and error yields values for $h$ and the integration length $\tau$ such that the effective sample fraction of the least constrained parameter, $m_{1000}$ in this case, is maximised. Specifically, we found $h=0.35$ and $\tau=2.45$ to produce a maximum effective sample fraction of $1.1 \cdot 10^{-4}$. Small changes of $h$ and $\tau$ may increase the effective sample fraction slightly, but order-of-magnitude improvements are unlikely. The small value of the effective sample fraction reflects the number of samples required to switch between different modes of the modified Styblinski-Tang probability density. This is in contrast to the Gaussian example, where the effective sample fraction describes the (in-)dependence of samples within the only existing mode.

Using the diagonal freezing variant of autotuning from section 14.2.5.1, we compute a constant diagonal mass matrix by averaging the diagonals of LF-BFGS Hessian approximations obtained from 10 sample chains. Each of these chains starts from a different, randomly selected initial model and only contains 200 samples. The resulting effective sample fraction is $5.5 \cdot 10^{-4}$, that is, 5 times larger than in the most optimal case with unit mass matrix. This suggests, that the sampler can switch into a different mode of the distribution about every 2000 samples, instead of every 10000 samples.

The differences in effective sample fractions translate to differences in convergence, which we can express in terms of the discrepancy between the exact marginal $\rho\left(m_{1}, m_{1000}\right)$ and the sample-approximated marginal $\tilde{\rho}\left(m_{1}, m_{1000}\right)$, quantified by the Kullback-Leibler divergence (3.25),

$$
D_{K L}=\int \tilde{\rho}\left(m_{1}, m_{1000}\right) \log _{10} \frac{\tilde{\rho}\left(m_{1}, m_{1000}\right)}{\rho\left(m_{1}, m_{1000}\right)} d m_{1} d m_{1000}
$$

In this context, $D_{K L}$ can be interpreted as a loss of information (in digits) that results from an inaccurate approximation of the exact distribution. As illustrated in figure 14.5, the autotuning variant of the Hamiltonian Monte Carlo sampler approximates the exact marginal with order of magnitude 10000 samples, assuming $D_{K L}=0.1$ as a reasonable threshold. Around 5 times more samples are needed without autotuning. 
a) Kullback-Leibler divergence

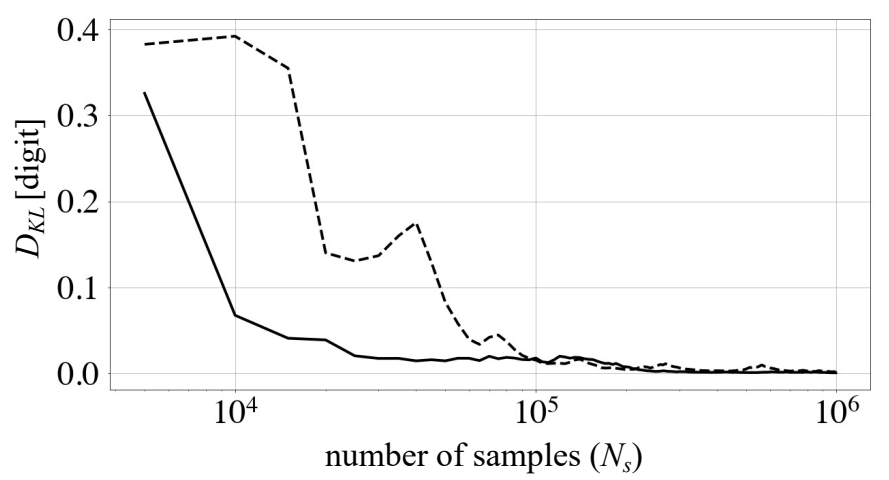

with autotuning

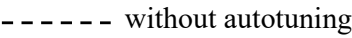

b) 2-D posterior without autotuning
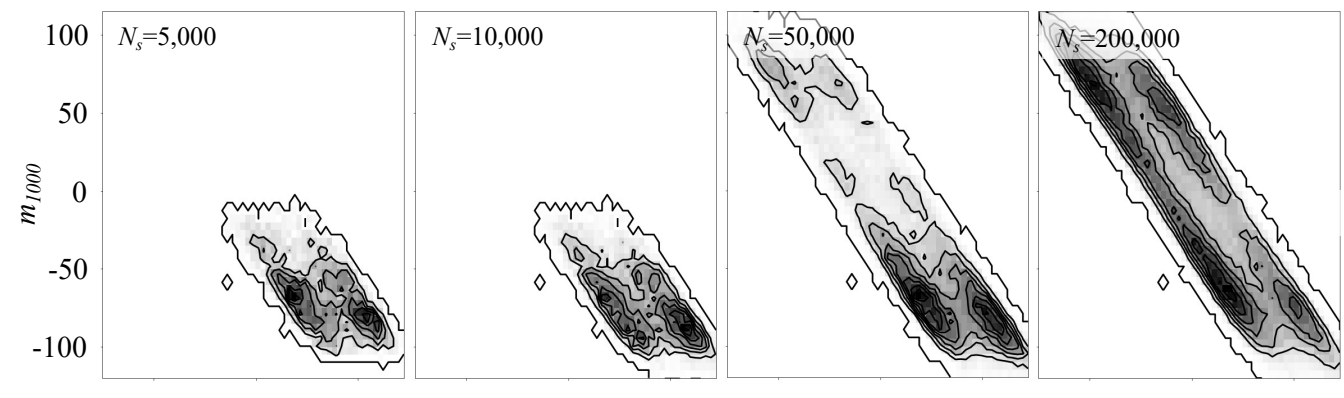

c) 2-D posterior with autotuning

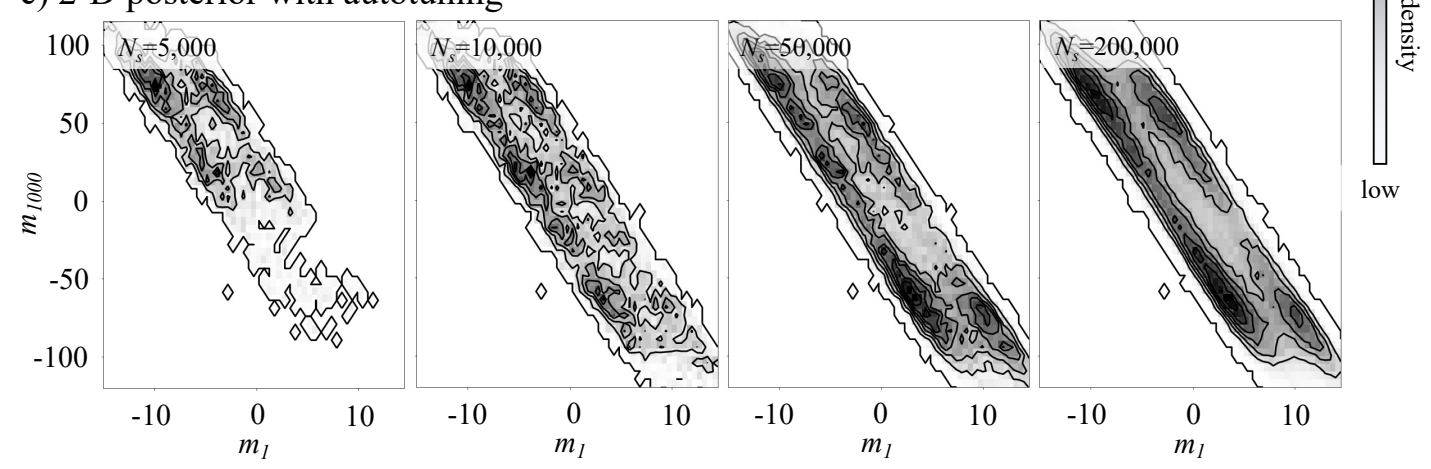

Fig. 14.5: Convergence summary of the 2-D marginal for the parameter pair $\left(m_{1}, m_{1000}\right)$ for the Styblinski-Tang probability density. (a) Kullback-Leibler divergence, $D_{K L}$, of the 2-D distribution, without autotuning (dashed curve) and with autotuning (solid curve). (b) Selection of 2-D posterior histograms as a function of the sample size $N_{s}$. The exact analytical posterior is shown in figure 15.1. 


\subsubsection{Modified Rastrigin function}

Similar to the Styblinski-Tang function, the 2-D version of the Rastrigin function was initially proposed as a performance test function for optimisation algorithms (Rastrigin, 1974). Its higher-dimensional generalisation, proposed by Rudolph (1990), is given by equation (15.52) in section 15.4.2. Being highly oscillatory, the Rastrigin function is non-convex and equipped with an infinite number of local maxima. Since the Rastrigin function is positive semi-definite, it can be used directly as a misfit function. Yet, to mimic realistic inverse problems more closely, we introduce inter-parameter correlations and variable parameter sensitivities, as we previously did for the Styblinski-Tang function. The resulting modified Rastrigin function is defined through equation (15.53), and some illustrations of the function itself and its associated probability density are shown in figure 15.2. For the model space dimension, we again choose $n=1000$.

To establish a reference, we disable auto-tuning and repeat the trial-and-error search over the integration time step, $h$, and the integration length, $\tau$, with the aim to maximise the effective sample fraction of the least constrained parameter, $m_{1000}$. Nearly optimal values for chains with 1 million samples are $h=0.02$ and $\tau=1.4$, leading to low effective sample fractions of $7.0 \cdot 10^{-6}$ for $m_{1}$ and $7.1 \cdot 10^{-6}$ for $m_{1000}$. The corresponding autocorrelation graphs are shown in figure 14.6a.

To improve convergence, we employ the macroscopic auto-tuning approach presented in section 14.2.5.2, using the temperature $T=100$ and only 500 samples. The resulting LF-BFGS representation of the mass matrix $\mathbf{M}_{T}$ is then rescaled and kept constant during the subsequent sampling of the modified Rastrigin probability density. The resulting auto-correlation graphs are shown in figure $14.6 \mathrm{~b}$. Relative to the previous chain without autotuning, effective sample fractions increase by a factor of around 50 , to $3.6 \cdot 10^{-4}$ for $m_{1}$, and $3.0 \cdot 10^{-4}$ for $m_{1000}$.

a) auto-correlations without auto-tuning $\left(m_{1}, m_{1000}\right)$

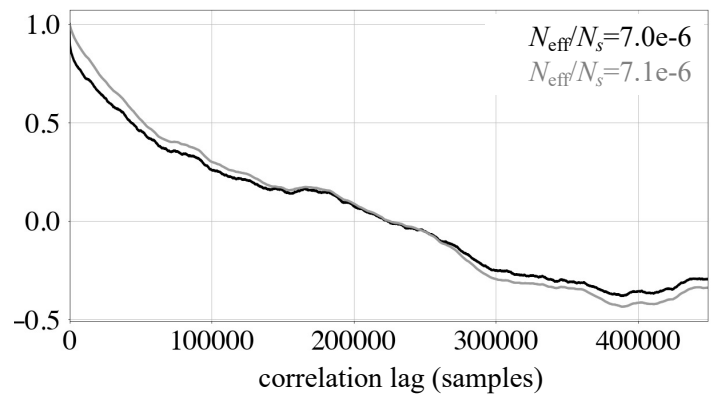

b) auto-correlations with auto-tuning $\left(m_{1}, m_{1000}\right)$

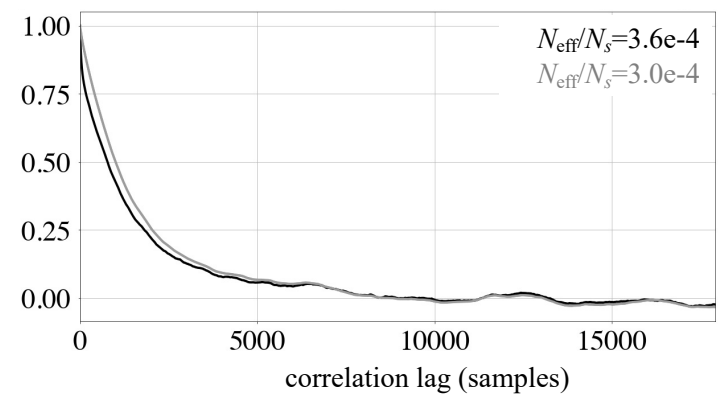

Fig. 14.6: Auto-correlations of the most constrained parameter, $m_{1}$, and the least constrained parameter, $m_{1000}$, of the modified Rastrigin function, averaged over 10 realisations of sample chains with 1 million samples each. (a) Without auto-tuning, auto-correlation lengths are on the order of 200000, meaning that around 200000 samples are needed to switch between modes of the modified Rastrigin function. The corresponding effective sample fractions, $N_{\text {eff }} / N_{s}$, are around $7 \cdot 10^{-6}$. (b) Auto-correlations and effective sample fractions when macroscopic auto-tuning is used. The effective sample fractions increased by a factor of about 50.

\subsection{Still no free lunch!}

The autotuning approach for the mass matrix and the time step has the potential to greatly increase the effective sample size and thereby accelerate the convergence of Monte Carlo integration. Its apparent capability of 
adapting optimally to any problem is, however, deceiving. In fact, the No-Free-Lunch theorem (e.g. Mosegaard, 2012; Wolpert \& Macready, 1997), a variant of which we derived in chapter 12, establishes that the efficiency of any method is not a universal property. Instead, efficiency derives from careful tuning of an algorithm towards a specific class of problems. Despite their ability to autotune, the family of algorithms proposed in this chapter of course does not beat the theorem.

As discussed, for instance, by Rosenthal (2011), efficiency itself is a diffuse term. It may refer to distributional convergence, asymptotic variance, mixing or other properties of the sample chain. Though to some extent related, the choice of these measures of efficiency is somewhat subjective. In our numerical examples, we considered the Kullback-Leibler divergence, auto-correlations and effective sample sizes (which are also not uniquely defined). These choices are pragmatic but also guided by our explicit goal to efficiently generate independent samples. It cannot be excluded that other efficiency measures would lead to different results.

Finally we note that the autotuning method is itself tuned towards problems with specific properties, that may, admittedly, not be well-known or clearly defined beforehand. These include the ability to approximate the not generally positive definite Hessian by a positive definite version of it, and in terms of a small number $\ell$ (typically below 100) of parameter-gradient pairs. Furthermore, the scaling analysis presented by Neal (2011) suggests that Hamiltonian approaches become increasingly efficient with increasing model space dimension, compared to standard Metropolis-Hastings. Hence, for low-dimensional problems (where 'low' is not clearly defined), the latter may in fact be more suitable. 
Part V

Appendix 



\section{Chapter 15 Mathematical tools}

This chapter contains a selection of mathematical tools and results that are either frequently used or too detailed for the main text.

\subsection{Lagrange multiplier method}

The method of Lagrange multipliers is a particularly useful tool for the optimisation of a function $f(\mathbf{x})$ under the equality contraint $g(\mathbf{x})=0$. To derive the method, we start with the two-dimensional case where $\mathbf{x}=(x, y)$, and we assume that a constrained extremum occurs at some point $\left(x_{0}, y_{0}\right)$.

The constraint $g(x, y)=0$ defines a curve in the $x$-y-plane that we parameterise in terms of an arc length parameter $t$,

$$
x=x(t), \quad y=y(t),
$$

where $t$ is chosen such that the extremum of $f[x(t), y(t)]$ occurs at $t_{0}$,

$$
f\left(x_{0}, y_{0}\right)=f\left[x\left(t_{0}\right), y\left(t_{0}\right)\right]
$$

Differentiating $f[x(t), y(t)]$ with respect to $t$ and evaluating the derivative at the extremum $t_{0}$, gives

$$
\frac{\partial f}{\partial x} \frac{d x}{d t}+\left.\frac{\partial f}{\partial y} \frac{d y}{d t}\right|_{t=t_{0}}=\left.\mathbf{T}^{T} \nabla f\right|_{t=t_{0}}=0 .
$$

Equation (15.3) states that the gradient $\nabla f$ is either equal to $\mathbf{0}$ or perpendicular to the tangent $\mathbf{T}\left(t_{0}\right)=$ $\left.(d x / d t, d y / d t)^{T}\right|_{t=t_{0}}$ of the constraint curve.

Now the constraint curve itself is a level curve of the function $g(x, y)$. Therefore, $\nabla g\left(x_{0}, y_{0}\right)$ must also be perpendicular to the tangent $\left.(d x / d t, d y / d t)^{T}\right|_{t=t_{0}}$, unless it happens to be $\mathbf{0}$. It follows that $\nabla f$ and $\nabla g$ are parallel when they are unequal to $\mathbf{0}$, and hence there must be some number $\lambda$, called the Lagrange multiplier, such that

$$
\nabla f\left(x_{0}, y_{0}\right)=\lambda \nabla g\left(x_{0}, y_{0}\right)
$$

Equation (15.4) can be turned into a constructive method. First, we define the Lagrange function

$$
L(x, y, \lambda)=f(x, y)-\lambda g(x, y)
$$

Subsequently, we find the $x$ and $y$ where the partial derivatives of $L$ vanish, which gives an extremum as a function of the Langrange multiplier $\lambda$. It finally remains to determine $\lambda$ from the constraints.

The previous argument can be extended to functions $f(\mathbf{x})$ where $\mathbf{x}$ has arbitrary dimension $n \geq 2$. Analogous 
to (15.3), $\nabla f$ is perpendicular to the $(n-1)$-dimensional tangent plane in the constrained extremum point $\mathbf{x}_{0}$. Also, $\nabla g$ is perpendicular to any $(n-1)$-dimensional level surface, including the constraint surface. Hence, $\nabla f$ and $\nabla g$ are parallel in $\mathbf{x}_{0}$, and so we find a generalised version of (15.4),

$$
\nabla f\left(\mathbf{x}_{0}\right)=\lambda \nabla g\left(\mathbf{x}_{0}\right)
$$

It follows that we can find the constrained extremum by setting the partial derivatives of the Lagrange function,

$$
L(\mathbf{x}, \lambda)=f(\mathbf{x})-\lambda g(\mathbf{x})
$$

to $\mathbf{0}$. Equation (15.7) states that the constrained optimisation problem can be solved through the unconstrained optimisation of the Lagrange function, and it suggests a strategy for the incorporation of additional constraints. In fact, for two constraints $g_{1}(\mathbf{x})=0$ and $g_{2}(\mathbf{x})=0$, one may first use the argument for a single constraint with $g_{1}$. This will lead to the unconstrained optimisation problem of $f(\mathbf{x})-\lambda_{1} g_{1}(\mathbf{x})$. Then simply iterating the argument with the added constraint $g_{2}$, yields the Lagrange function

$$
L(\mathbf{x}, \lambda)=f(\mathbf{x})-\lambda_{1} g_{1}(\mathbf{x})-\lambda_{2} g_{2}(\mathbf{x}) .
$$

To ensure that the resulting system of equations has a solution, the number of constraints must not exceed $n-1$.

\subsection{Riesz' representation theorem}

Riesz' representation theorem is one of the major results of the theory of Hilbert spaces, which are general vector spaces with a scalar product. It was proven independently by Frigyes Riesz and Maurice René Fréchet, and is therefore also sometimes referred to as the Riesz-Fréchet representation theorem. We use the theorem in section 9.2, where it serves as one of the cornerstones of the continuous adjoint method. To demonstrate Riesz' representation theorem, we consider an arbitrary vector space $\mathbb{V}$ with elements $\mathbf{u}$, and a linear functional $F(\mathbf{u})$. The elements may be actual finite-dimensional vectors or functions, for which some scalar product $(\mathbf{u}, \mathbf{v})$ is defined. Our goal is to show that $F(\mathbf{u})$ can generally be written in terms of the scalar product as

$$
F(\mathbf{u})=(\mathbf{f}, \mathbf{u})
$$

with a unique vector space element $\mathbf{f}$. For the proof, we proceed in three steps: First, we show that the activated vector space of $F$, that is, the orthogonal complement of its nullspace or kernel $\operatorname{ker} F$ has either dimension 0 or 1 . Based on this result, we then construct a possible $\mathbf{f}$ explicitly. Finally, we demonstrate that $\mathbf{f}$ is indeed uniquely defined.

So we begin with the definition of the nullspace, which we encountered previously in section 6.5 .1 and in the specific context of a linear forward modelling operator $\mathbf{G}$ that has the form of a matrix. For our more general linear operator $F$, the nullspace is defined as the subspace of vectors that are mapped to 0 , that is,

$$
\operatorname{ker} F=\{\mathbf{u} \in \mathbb{V} \mid F(\mathbf{u})=0\}
$$

The activated vector space act $F$, analogous to the activated model space in section 6.5.1, is the orthogonal complement of $\operatorname{ker} F$, and its members are mapped to non-zero values. First, we assume that the dimension of act $F$ is greater than 1 . This means that there are at least two vectors, $\mathbf{u}_{1}$ and $\mathbf{u}_{2}$ that span a subspace of act $F$ via the linear combination

$$
\mathbf{u}=a_{1} \mathbf{u}_{1}+a_{2} \mathbf{u}_{2}
$$

Applying $F$ to (15.11), gives

$$
F(\mathbf{u})=F\left(a_{1} \mathbf{u}_{1}+a_{2} \mathbf{u}_{2}\right)=a_{1} F\left(\mathbf{u}_{1}\right)+a_{2} F\left(\mathbf{u}_{2}\right) .
$$


Since $\mathbf{u}_{1}$ and $\mathbf{u}_{2}$ are elements of act $F$, we have $F\left(\mathbf{u}_{1}\right) \neq 0$ and $F\left(\mathbf{u}_{2}\right) \neq 0$. Now consider the linear homogeneous equation

$$
F(\mathbf{u})=0 .
$$

We can find at least one non-zero solution, $\left(a_{1}^{\prime}, a_{2}^{\prime}\right) \neq(0,0)$, for instance by choosing an arbitrary $a_{2}^{\prime} \neq 0$ and then computing $a_{1}^{\prime}$ as

$$
a_{1}^{\prime}=-a_{2}^{\prime} \frac{F\left(\mathbf{u}_{2}\right)}{F\left(\mathbf{u}_{1}\right)} .
$$

With $a_{1}^{\prime}$ and $a_{2}^{\prime}$ we can form the vector

$$
\mathbf{u}^{\prime}=a_{1}^{\prime} \mathbf{u}_{1}+a_{2}^{\prime} \mathbf{u}_{2} .
$$

At this point, we encounter a contradiction. On the one hand, we know that the non-zero vector $\mathbf{u}^{\prime}$ is an element of the activated model space act $F$ because it is a linear combination of $\mathbf{u}_{1}$ and $\mathbf{u}_{2}$. On the other hand, we found non-zero coefficients $a_{1}^{\prime}$ and $a_{2}^{\prime}$ such that $F\left(\mathbf{u}^{\prime}\right)=0$, meaning that $\mathbf{u}^{\prime}$ is in the nullspace $\operatorname{ker} F$. Consequently, our starting assumption that the dimension of act $F$ is greater than 1 must be incorrect.

Let us consider the remaining two cases. When the dimension of act $F$ equals 0 , it only contains one element, namely $\mathbf{0}$. Therefore, all non-zero vectors $\mathbf{u}$ are within the nullspace, meaning that

$$
F(\mathbf{u})=0=(\mathbf{0}, \mathbf{u}) .
$$

Hence, the choice $\mathbf{f}=\mathbf{0}$ produces the desired form (15.9). Finally, when the dimension of act $F$ is 1 , all elements of act $F$ can be written as $a \mathbf{e}$, with a unit vector $\mathbf{e}$ and an arbitrary scalar $a$. Furthermore, all elements of $\mathbb{V}$ take the form

$$
\mathbf{u}=\mathbf{v}+a \mathbf{e},
$$

with some element $\mathbf{v}$ of the nullspace. The application of $F$ to (15.17) gives

$$
F(\mathbf{u})=F(\mathbf{v}+a \mathbf{e})=F(\mathbf{v})+F(a \mathbf{e})=a F(\mathbf{e}) .
$$

Since $\mathbf{e}$ is, by construction, a unit vector with $(\mathbf{e}, \mathbf{e})=1$, we may alternatively write $F(\mathbf{u})$ as

$$
F(\mathbf{u})=a F(\mathbf{e})=a F(\mathbf{e})(\mathbf{e}, \mathbf{e})=\left(a \mathbf{e}, F(\mathbf{e})^{*} \mathbf{e}\right) .
$$

Exploiting the fact that $\mathbf{v}$ is orthogonal to $\mathbf{e}$, that is, $(\mathbf{v}, \mathbf{e})=0$, we may sneak $\mathbf{v}$ into the right-hand side of (15.19),

$$
F(\mathbf{u})=\left(\mathbf{v}+a \mathbf{e}, F(\mathbf{e})^{*} \mathbf{e}\right)=\left(\mathbf{u}, F(\mathbf{e})^{*} \mathbf{e}\right) .
$$

Choosing

$$
\mathbf{f}=F(\mathbf{e})^{*} \mathbf{e},
$$

we again arrive at (15.9). It follows that we can indeed always find some vector $\mathbf{f}$ such that $F(\mathbf{u})$ can be expressed in terms of a scalar product between $\mathbf{f}$ and $\mathbf{u}$.

To demonstrate that $\mathbf{f}$ is uniquely defined, we assume that there are two vectors, $\mathbf{f}_{1}$ and $\mathbf{f}_{2}$ with

$$
F(\mathbf{u})=\left(\mathbf{f}_{1}, \mathbf{u}\right)=\left(\mathbf{f}_{2}, \mathbf{u}\right) .
$$

It follows from (15.22) that

$$
\left(\mathbf{u}, \mathbf{f}_{1}-\mathbf{f}_{2}\right)=0,
$$

for any arbitrary vector $\mathbf{u}$. Thus, for the specific choice $\mathbf{u}=\mathbf{f}_{1}-\mathbf{f}_{2}$ we find

$$
\left(\mathbf{f}_{1}-\mathbf{f}_{2}, \mathbf{f}_{1}-\mathbf{f}_{2}\right)=\left\|\mathbf{f}_{1}-\mathbf{f}_{2}\right\|^{2}=0 .
$$

By the defining properties of scalar products, (9.27) to (9.28), equation (15.24) can hold only when $\mathbf{f}_{1}=\mathbf{f}_{2}$. Therefore, the vector $\mathbf{f}$ is indeed unique. 


\subsection{Analysis of numerical time integration}

Most differential equations that describe the time evolution of some system cannot be integrated analytically. Therefore, numerical time integrators are needed. In most cases, these numerical integrators are not unconditionally stable, and they may not preserve the total energy of the system. In the following paragraphs, we show how stability and energy dissipation of numerical integrators may be analysed, using the example of the leap-frog scheme, which plays an important role in Hamiltonian Monte Carlo sampling. Before we begin, we briefly recall the leap-frog scheme equations,

$$
\begin{aligned}
\mathbf{p}(t+h / 2) & =\mathbf{p}(t)-\frac{1}{2} h \frac{\partial U(t)}{\partial \mathbf{m}} \\
\mathbf{m}(t+h) & =\mathbf{m}(t)+h \mathbf{M}^{-1} \mathbf{p}(t+h / 2) \\
\mathbf{p}(t+h) & =\mathbf{p}(t+h / 2)-\frac{1}{2} h \frac{\partial U(t+h)}{\partial \mathbf{m}},
\end{aligned}
$$

where $h$ is the time increment.

\subsubsection{Numerical stability}

To analyse the stability of the leap-frog scheme as a function of $h$, we first substitute (15.25) into (15.26), which yields

$$
\mathbf{m}(t+h)=\mathbf{m}(t)+h \mathbf{M}^{-1}\left[\mathbf{p}(t)-\frac{1}{2} h \frac{\partial U(t)}{\partial \mathbf{m}}\right] .
$$

Combining (15.28) with the corresponding equation for $\mathbf{m}(t-h)$, gives an iterative scheme for the models $\mathbf{m}$ without the momenta $\mathbf{p}$,

$$
\mathbf{m}(t+h)=2 \mathbf{m}(t)-\mathbf{m}(t-h)-h^{2} \mathbf{M}^{-1} \frac{\partial U(t)}{\partial \mathbf{m}} .
$$

Equation (15.29) is a central finite-difference equation, which implies that the scheme is of second order in $h$. To analyse stability, we assume that $\mathbf{m}(t-h)$ has been perturbed by some error $\delta \mathbf{m}(t-h)$, for example due to limited floating-point precision. Our interest is in the propagation of this error through the integration scheme. Including the errors at time steps $t$ and $t+h$ in the scheme, gives

$$
\mathbf{m}(t+h)+\delta \mathbf{m}(t+h)=2 \mathbf{m}(t)+2 \delta \mathbf{m}(t)-\mathbf{m}(t-h)-\delta \mathbf{m}(t-h)-h^{2} \mathbf{M}^{-1} \frac{\partial U(t)}{\partial \mathbf{m}}-h^{2} \mathbf{M}^{-1} \mathbf{H}(t) \delta \mathbf{m}(t),
$$

where we used the first-order approximation

$$
\frac{\partial U[\mathbf{m}(t)+\delta \mathbf{m}(t)]}{\partial \mathbf{m}} \approx \mathbf{H}(t) \delta \mathbf{m}(t)
$$

with the Hessian H. Eliminating a copy of (15.29) from (15.30), we can express the temporal evolution of the error terms,

$$
\delta \mathbf{m}(t+h)=2 \delta \mathbf{m}(t)-\delta \mathbf{m}(t-h)-h^{2} \mathbf{M}^{-1} \mathbf{H}(t) \delta \mathbf{m}(t) .
$$

Since $\mathbf{M}^{-1} \mathbf{H}(t)$ is symmetric, there exists an orthogonal matrix $\mathbf{Q}$ such that

$$
\delta \mathbf{q}(t+h)=2 \delta \mathbf{q}(t)-\delta \mathbf{q}(t-h)-\Delta t^{2} \Lambda(t) \delta \mathbf{q}(t),
$$

where $\delta \mathbf{q}=\mathbf{Q}^{T} \delta \mathbf{m}$ and $\Lambda$ the diagonal matrix filled with the eigenvalues $\lambda_{i}$ of $\mathbf{M}^{-1} \mathbf{H}$. Equation (15.33) allows us to consider the decoupled scalar equations, 
15.3 Analysis of numerical time integration

$$
\delta q_{i}(t+h)=2 \delta q_{i}(t)-\delta q_{i}(t-h)-h^{2} \lambda_{i}(t) \delta q_{i}(t)
$$

Assuming that $\lambda_{i}$ is approximately constant along the trajectory, we can analyse (15.34) with the ansatz

$$
\delta q_{i}(t+h)=g_{i} \delta q_{i}(t), \quad \delta q_{i}(t)=g_{i} \delta q_{i}(t-h),
$$

which leads to the quadratic equation

$$
g_{i}^{2}-\left(2-h^{2} \lambda_{i}\right) g_{i}+1=0
$$

Solutions are

$$
g_{i}=\frac{1}{2}\left(2-h^{2} \lambda_{i}\right) \pm \sqrt{\frac{1}{4}\left(2-h^{2} \lambda_{i}\right)^{2}-1} .
$$

For long-term stability of the integration scheme, errors must not amplify, meaning that $\left|g_{i}\right| \leq 1$ is required. Upon inspection of (15.38), this implies

$$
h \leq \frac{2}{\sqrt{\lambda_{i}}}, \quad \lambda_{i} \geq 0
$$

Hence, the integration is long-term unstable when any of the eigenvalues of $\mathbf{M}^{-1} \mathbf{H}(t)$ is smaller than 0 . When all $\lambda_{i} \geq 0$, the maximum integration time step $h$ is controlled by the largest eigenvalue of $\mathbf{M}^{-1} \mathbf{H}(t)$.

\subsubsection{Energy dissipation}

To analyse the extent to which the leapfrog scheme dissipates the total energy $H$ of a Hamiltonian system, we first restate Hamilton's equations, encountered before in section 5.7.1,

$$
\frac{d m_{i}}{d t}=\frac{\partial H}{\partial p_{i}}, \quad \frac{d p_{i}}{d t}=-\frac{\partial H}{\partial m_{i}} .
$$

Our goal is to find an equation for the time derivative of $H$ under the leap-frog approximation. For this, we substitute

$$
\frac{\partial U(t)}{\partial \mathbf{m}}=\frac{\partial H(t)}{\partial \mathbf{m}}, \quad \text { and } \quad \mathbf{M}^{-1} \mathbf{p}(t)=\frac{\partial H(t)}{\partial \mathbf{p}}
$$

into equations (15.25) to (15.27). This leads to

$$
\frac{\partial H(t)}{\partial \mathbf{m}}=\frac{2}{h}[\mathbf{p}(t)-\mathbf{p}(t+h / 2)]
$$

and

$$
\frac{\partial H(t)}{\partial \mathbf{p}}=\frac{1}{h}[\mathbf{m}(t+h / 2)-\mathbf{m}(t-h / 2)] .
$$

Using (15.41) and (15.42), it follows that

$$
[\mathbf{m}(t+h / 2)-\mathbf{m}(t-h / 2)]^{T} \frac{\partial H(t)}{\partial \mathbf{m}}+2[\mathbf{p}(t+h / 2)-\mathbf{p}(t)]^{T} \frac{\partial H(t)}{\partial \mathbf{p}}=0 .
$$

The terms that appear in square brackets in equation (15.43) can be written as approximate time derivatives of $\mathbf{m}$ and $\mathbf{p}$, using a Taylor expansion:

$$
\mathbf{m}(t+h / 2)-\mathbf{m}(t-h / 2)=h \frac{d \mathbf{m}(t)}{d t}+\mathscr{O}\left(h^{3}\right),
$$

and 


$$
\mathbf{p}(t+h / 2)-\mathbf{p}(t)=\frac{1}{2} h \frac{d \mathbf{p}(t)}{d t}+\mathscr{O}\left(h^{2}\right) .
$$

Finally combining equations (15.43) to (15.45), we obtain

$$
0=\frac{\partial H(t)}{\partial \mathbf{m}} \frac{d \mathbf{m}(t)}{d t}+\frac{\partial H(t)}{\partial \mathbf{p}} \frac{d \mathbf{p}(t)}{d t}+\mathscr{O}(h)=\frac{d H(t)}{d t}+\mathscr{O}(h)
$$

It follows that the leapfrog scheme preserves the total energy $H$ only to first order in $h$. This is in contrast to the integration of $\mathbf{m}$ and $\mathbf{p}$, which is of second order in $h$.

\subsection{High-dimensional analytical test functions}

The convergence and performance, however measured, of many sampling and optimisation algorithms is often difficult to analyse. It has therefore become common practice to study such algorithms when applied to analytical test functions with features that we think are either representative of real-world scenarios or particularly challenging. In section 8.2.3 we already encountered a collection of 2-D test functions, primarily designed to visualise the behaviour of nonlinear optimisation algorithms. In the following paragraphs, we complement this collection with high-dimensional, multi-modal test functions that are particularly useful in the analysis of Monte Carlo sampling algorithms, including those presented in chapters 5 and 14.

\subsubsection{Modified Styblinski-Tang function}

The Styblinski-Tang function, originally proposed as analytical, non-convex, multi-modal benchmark function for optimisation algorithms (Styblinski \& Tang, 1990), is defined as

$$
f(\mathbf{m})=\frac{1}{2} \sum_{i=1}^{n}\left(m_{i}^{4}-16 m_{i}^{2}+5 m_{i}\right) \text {. }
$$

The function reaches its global minimum $f_{\min }=-39.16599 n$ at $\left(m_{1}, \ldots, m_{n}\right)=(-2.903534, \ldots,-2.903534)$, and has additional $2^{n}-1$ additional local minima. The 1-D Styblinski-Tang function is illustrated in figure 15.1a. To be a more useful and interesting high-dimensional test function, we modify equation (15.47) as follows,

$$
\chi(\mathbf{m})=\frac{n}{f_{\min }}\left[\frac{1}{2} \sum_{i=1}^{n}\left(x_{i}^{4}-16 x_{i}^{2}+5 x_{i}\right)+f_{\min }\right], \quad \text { with } \quad \mathbf{x}=\mathbf{A m} .
$$

The addition of $f_{\min }$ ensures that misfits are generally non-negative. The symmetric $n \times n$ matrix $\mathbf{A}$ serves to introduce variable sensitivities of model parameters, as well as inter-parameter trade-offs. Based on the analytical misfit definition in (15.48), we can define a probability density

$$
\rho(\mathbf{m})=k e^{-\chi(\mathbf{m}},
$$

which may be used to test sampling algorithms. The normalisation constant $k$ could be computed with some effort but is of little interested in most applications. For our experiments in section 14.3, we specifically set the diagonal elements to

$$
A_{i, i}=e^{-3 i / n}
$$

which mimics the exponential decay of sensitivity, often found in practice (e.g. Curtis, 1999a,b). For the offdiagonal elements, we choose

$$
A_{1, n}=A_{n, 1}=0.1, \quad A_{2, n-1}=A_{n-1,2}=0.1, \quad A_{3, n-2}=A_{n-2,3}=0.1, \quad \ldots,
$$


in order to introduce inter-parameter trade-offs, while still being able to compute marginals and moments semianalytically. The probability density corresponding to the 1-D, modified Styblinski-Tang function, as well as a 2 -D slice through its 1000 -D version are shown in figures $15.1 \mathrm{~b}$ and $15.1 \mathrm{c}$, respectively.

a) 1-D Styblinski-Tang function $f(m)$

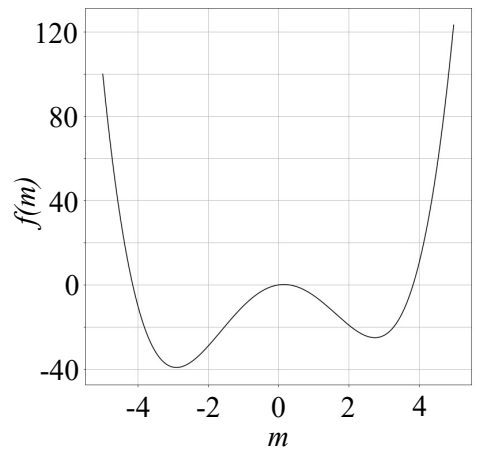

b) 1-D prob. density $\rho(m)=e^{-\chi(m)}$

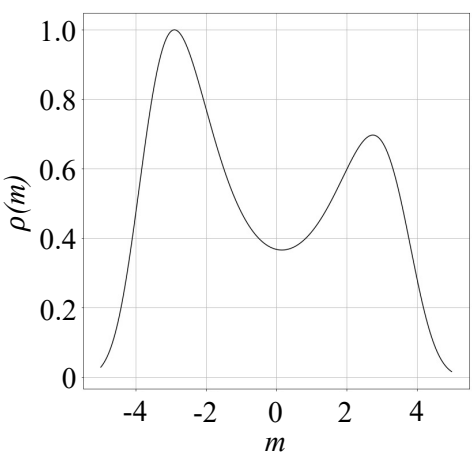

c) 1000-D probability density

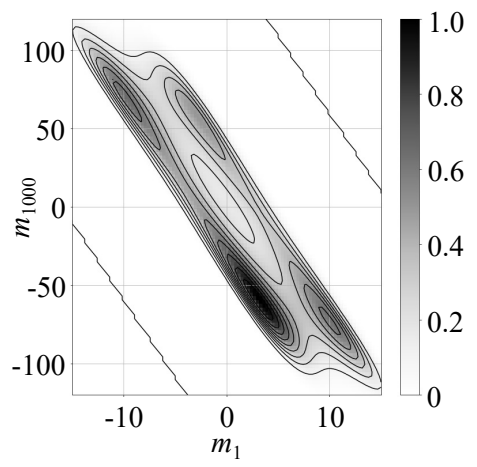

Fig. 15.1: Styblinski-Tang test functions. (a) Original one-dimensional Styblinski-Tang function $f(m)$, as defined in equation (15.47). (b) Non-normalised probability density $\rho(m)=e^{-\chi(m)}$, corresponding to the 1-D modified Styblinski-Tang function $\chi(m)$, defined in equation (15.48). (c) Normalised 2-D slice through the 1000-D modified Styblinski-Tang probability density $\rho(m)=e^{-\chi(m)}$ based on equations (15.48) $-(15.51)$ at $\left(m_{2}, \ldots, m_{999}\right)=(0, \ldots, 0)$.

\subsubsection{Modified Rastrigin function}

Similar to the Styblinski-Tang function, the 2-D Rastrigin function was originally proposed as a test function for the performance analysis of optimisation algorithms (Rastrigin, 1974). Its higher-dimensional version, introduced by Rudolph (1990), is defined as

$$
f(\mathbf{m})=10 n+\sum_{i=1}^{n}\left[m_{i}^{2}-10 \cos \left(2 \pi m_{i}\right)\right] .
$$

The Rastrigin function is non-convex and equipped with an infinite number of local minima. To make $f(\mathbf{m})$ a more suitable mimic of misfit functions encountered in realistic inverse problems, we introduce parameter scalings and correlations in analogy to equations (15.50) and (15.51) for the Styblinski-Tang function. This leads to the modified Rastrigin function

$$
\chi(\mathbf{m})=10 n+\sum_{i=1}^{n}\left[x_{i}^{2}-10 \cos \left(2 \pi x_{i}\right)\right], \quad \text { with } \quad \mathbf{x}=\mathbf{A m} .
$$

Images of the (modified) Rastrigin function and of the corresponding probability density are shown in figure 15.2 . 
a) 1-D Rastrigin function $f(m)$

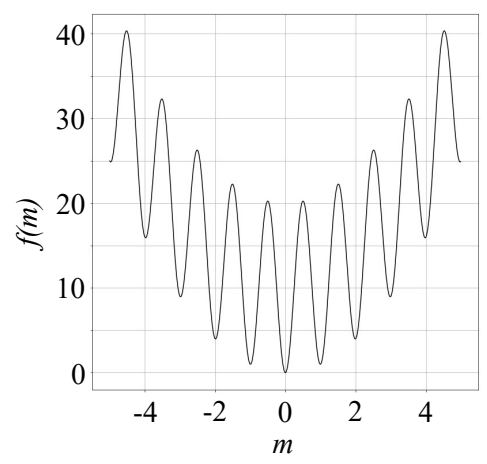

b) 1-D prob. density $\rho(m)=e^{-\chi(m)}$

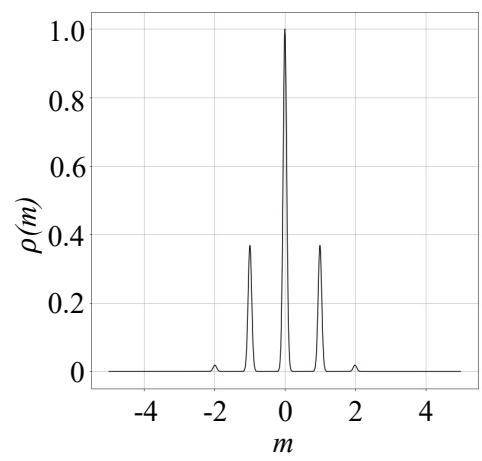

c) $1000-\mathrm{D}$ probability density

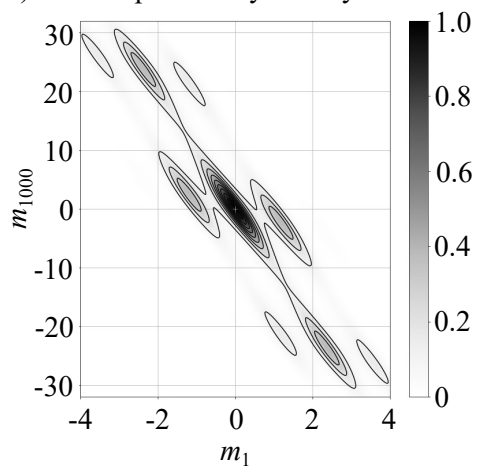

Fig. 15.2: Rastrigin test functions. (a) One-dimensional Rastrigin function $f(m)$, as defined in equation (15.52). (b) Non-normalised probability density $\rho(m)=e^{-\chi(m)}$, corresponding to the 1-D Rastrigin function $\chi(m)$. (c) Normalised 2-D slice through the 1000-D modified Rastrigin probability density $\rho(m)=e^{-\chi(m)}$ defined by equations (15.53), (15.50) and (15.51) at $\left(m_{2}, \ldots, m_{999}\right)=(0, \ldots, 0)$. 


\section{Chapter 16 \\ Physical and numerical models}

In the following chapter, we provide a collection of physical and numerical models used throughout the text to illustrate various inverse problems and solution methods. Unavoidably, this can only be a superficial treatment that cannot replace specialised textbooks. However, it should provide the necessary mathematical context needed to reproduce the examples.

\subsection{Straight-ray tomography}

Our goal is to estimate the variations in slowness (inverse of velocity) within a bounded medium using travel time information of the first wave arrivals from sources at positions $\mathbf{x}_{i}$ to receivers at positions $\mathbf{x}_{j}$. Adopting the simplified concept that waves can be represented as rays, which, in fact, is a high-frequency approximation (e.g. Cerveny, 2001), the traveltime $t_{i}$ of the $i^{\text {th }}$ wave from the source at $\mathbf{x}_{i}$ can be computed as an integral along a ray,

$$
t_{i}=\int_{\text {ray }_{i}} \frac{d l}{v(\mathbf{x})}=\int_{\text {ray }_{i}} s(\mathbf{x}) d l
$$

where $v(\mathbf{x})$ is the spatial velocity distribution of the medium, and $s(\mathbf{x})=1 / v(\mathbf{x})$ is the corresponding slowness. Equation (16.1) reveals that the parameterisation in terms of slowness is indeed to be preferred over velocity, as it leads to a linear relation between model parameters and data. As a first approximation, we represent the continuous medium using discrete blocks of constant slowness, as shown in figure 16.1. This transforms the integral in equation (16.1) into a finite sum,

$$
t_{i}=\sum_{j=1}^{n} l_{i j} s_{j}
$$

where $l_{i j}$ represents the length of the ray path with index $i$ in the cell $j, s_{j}$ is the value of the slowness in the cell $j$, and $n$ is the total number of cells in the medium. Note that $l_{i j}=0$ for those blocks that the ray actually does not cross.

As a consequence of Snell's law, rays bend away from a straight line when the slowness distribution is heterogeneous. Therefore, ray paths depend on the slowness structure of the medium itself, leading to nonlinearity in equation (16.2). For simplicity, however, we consider the simplest, linearised, ray tracing approach where Snell's law at all cell boundaries is ignored, so that waves propagating from sources to receivers can be approximated by straight lines. Therefore, considering an experiment with a total number of $N$ rays, the forward problem described in equation (16.2) can be expressed as a linear system of equations,

$$
\mathbf{d}=\mathbf{G m},
$$

with 


$$
\mathbf{d}=\left[\begin{array}{c}
t_{1} \\
\vdots \\
t_{i} \\
\vdots \\
t_{N}
\end{array}\right], \quad \mathbf{A}=\left[\begin{array}{ccc}
l_{11} & \ldots & l_{1 n} \\
\vdots & & \vdots \\
l_{i 1} & \ddots & l_{i n} \\
\vdots & & \vdots \\
l_{N 1} & \ldots & l_{N n}
\end{array}\right] \text {, and } \quad \mathbf{m}=\left[\begin{array}{c}
s_{1} \\
\vdots \\
s_{j} \\
\vdots \\
s_{n}
\end{array}\right]
$$

The number of rows $N$ in the forward modelling matrix $\mathbf{G}$ in equation (16.4) is equal to the total number of rays, whereas the number of columns $n$ is equal to the number of cells used to describe the medium. The physical interpretation of $\mathbf{G}$ is illustrated in figure 16.1a.

a) forward modelling matrix

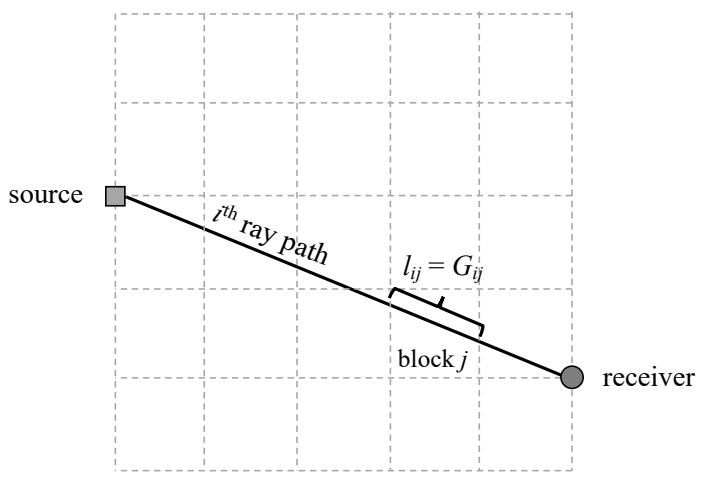

b) ray path parameterisation

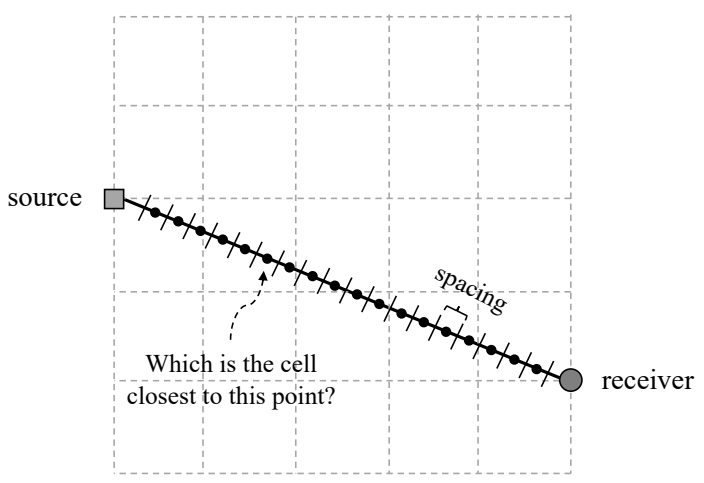

Fig. 16.1: Ray path geometry. (a) Schematic representation of the $i^{\text {th }}$ ray traveling from source to receiver in a discretised 2-D medium. The illustration shows the physical meaning of the element $G_{i j}$ of the forward modelling matrix $\mathbf{G}$ in equation (16.3). (b) Illustration showing a possible parameterisation of the ray path.

Given the locations of sources, receivers, and grid points, used to discretise the medium, the elements of $\mathbf{G}$ can easily be computed if the ray path is parameterised. Starting from the source position, one may take small steps in the receiver direction and check in which cell the ray is located at each step. For this purpose, we consider the closest cell to each point, as shown in figure 16.1b. In case the ray path is just at the boundary between cells, the ray length is equally distributed among all involved cells.

\subsection{Love waves in layered media}

In this section, we are concerned with the propagation of waves, represented by the displacement field $\mathbf{u}(\mathbf{x}, t)$ with unit $\mathrm{m}$, through an elastic medium with mass density distribution $\rho(\mathbf{x})$. When external forces are absent, the deformation of such a medium is governed by Newton's second law, which balances the inertial force density $\rho \frac{\partial^{2}}{\partial t^{2}} \mathbf{u}(\mathbf{x}, t)$ against the divergence of internal stresses $\nabla \cdot \boldsymbol{\sigma}(\mathbf{x}, t)$, caused by the deformation (e.g. Aki \& Richards, 2002; Dahlen \& Tromp, 1998; Kennett, 2001)

$$
\rho \frac{\partial^{2}}{\partial t^{2}} \mathbf{u}(\mathbf{x}, t)=\nabla \cdot \sigma(\mathbf{x}, t)
$$


The stress tensor $\boldsymbol{\sigma}(\mathbf{x}, t)$, with unit $\mathrm{N} / \mathrm{m}^{2}$, is a symmetric, second-order tensor. When the deformation of the medium is small, it can be related to spatial variations in the displacement $\mathbf{u}(\mathbf{x}, t)$ using Hooke's law,

$$
\sigma_{i j}(\mathbf{x}, t)=\frac{1}{2} \sum_{k, l=1}^{3} c_{i j k l}(\mathbf{x})\left(\frac{\partial u_{k}(\mathbf{x}, t)}{\partial x_{l}}+\frac{\partial u_{l}(\mathbf{x}, t)}{\partial x_{k}}\right) .
$$

The elastic properties of the medium are collected in the components $c_{i j k l}(\mathbf{x})$ of the elastic tensor, which satisfies the symmetry relations $c_{i j k l}(\mathbf{x})=c_{j i k l}(\mathbf{x})=c_{k l i j}(\mathbf{x})$. The unit of $c_{i j k l}$ is $\mathrm{N} / \mathrm{m}^{2}$. In total, there are 21 independent elastic tensor components, describing a general anisotropic medium. When the medium is isotropic, the number of independent components is reduced to 2 , and $c_{i j k l}(\mathbf{x})$ takes the form

$$
c_{i j k l}(\mathbf{x})=\left(\kappa(\mathbf{x})-\frac{2}{3} \mu(\mathbf{x})\right) \delta_{i j} \delta_{k l}+\mu(\mathbf{x}) \delta_{i k} \delta_{j l}+\mu(\mathbf{x}) \delta_{i l} \delta_{j k},
$$

with the bulk modulus $\kappa(\mathbf{x})$ that describes the behaviour of the medium under compression, and the shear modulus $\mu(\mathbf{x})$ that controls the deformation of the medium under the influence of shear stresses. Together, equations (16.5) and (16.6) constitute the elastic wave equation. It must be complemented by initial conditions, and the boundary condition that normal stresses vanish along the medium boundary,

$$
\boldsymbol{\sigma} \cdot \mathbf{n}=\mathbf{0}
$$

where $\mathbf{n}$ is the outward-pointing surface normal.

For arbitrary heterogeneous and anisotropic media, it cannot be solved analytically, and numerical methods must be employed to obtain approximate solutions (e.g. Igel, 2016). However, special cases exist, for which simple and insightful solutions can be obtained analytically. A classical example are Love waves, which are horizontally polarised waves that travel along the surface of the medium.

\subsubsection{Displacement and stress functions of Love waves}

Love waves are special solutions of the elastic wave equation that are bound to the surface of the medium, meaning that they are surface waves with amplitude decaying exponentially with depth. For a mathematical description of Love waves, we will loosely follow the classic by Takeuchi \& Saito (1972).

In order to obtain a closed-form solution, we consider the special case of an isotropic medium composed of a homogeneous layer in the depth interval $0 \leq z \leq H$, above a homogeneous half space at $z \leq 0$. We assume that the wave travels in $x$-direction and is polarised in $y$-direction. Hence, for some circular frequency $\omega$ and wave number $k$, we may choose the ansatz

$$
u_{x}(\mathbf{x}, t)=0, \quad u_{y}(\mathbf{x}, t)=y_{1}(z, \omega, k) e^{i(\omega t-k x)}, \quad u_{z}(\mathbf{x}, t)=0,
$$

with the displacement function $y_{1}$. Inserting (16.9) into the equations of motion (16.5) to (16.7) yields a system of two ordinary differential equations,

$$
\begin{aligned}
& \frac{d y_{1}}{d z}=\frac{1}{\mu} y_{2}, \\
& \frac{d y_{2}}{d z}=\left(k^{2} \mu-\omega^{2} \rho\right) y_{1} .
\end{aligned}
$$

Both the stress function $y_{2}$ and the displacement function $y_{1}$ are required to be continuous and to vanish with increasing depth, that is, for $z \rightarrow-\infty$. Furthermore, to satisfy the surface boundary condition (16.8), we require 


$$
\left.y_{2}(z, \omega, k)\right|_{z=H}=0 .
$$

In the upper layer, we find the solution

$$
\begin{aligned}
& y_{1}(z)=A \cos \left(v_{1} z\right)+\frac{\mu_{2} v_{2}}{\mu_{2} v_{1}} A \sin \left(v_{1} z\right), \\
& y_{2}(z)=-A \mu_{1} v_{1} \sin \left(v_{1} z\right)+A \mu_{2} v_{2} \cos \left(v_{1} z\right),
\end{aligned}
$$

with some arbitrary constant $A$ that determines the amplitude of the wave. In the underlying half-space, we find

$$
\begin{aligned}
& y_{1}(z)=A e^{v_{2} z} \\
& y_{2}(z)=\mu_{2} v_{2} A e^{v_{2} z} .
\end{aligned}
$$

The positive scalars $v_{1}$ and $v_{2}$ are defined as

$$
\begin{aligned}
& v_{1}^{2}=\frac{\rho_{1} \omega^{2}}{\mu_{1}}-k^{2}>0, \\
& v_{2}^{2}=k^{2}-\frac{\rho_{2} \omega^{2}}{\mu_{2}}>0 .
\end{aligned}
$$

It follows immediately from the last two relations that a Love wave can only exist when

$$
\frac{\mu_{1}}{\rho_{1}}<\frac{\omega^{2}}{k^{2}}<\frac{\mu_{2}}{\rho_{2}} .
$$

Noticing that $\beta_{1,2}=\sqrt{\mu_{1,2} / \rho_{1,2}}$ is the propagation velocity of a wave in a homogeneous full space with density $\rho_{1,2}$ and elastic modulus $\mu_{1,2},(16.19)$ corresponds to the requirement of a low-velocity layer located over a halfspace of higher velocity.

So far, $\omega$ and $k$ are unconstrained and can be varied independently. However, the boundary condition (16.12) imposes a dispersion relation between $\omega$ and $k$. In fact, inserting (16.14) into (16.12), we find the characteristic equation for Love waves,

$$
\mu_{2} v_{2} \cos \left(v_{1} H\right)=\mu_{1} v_{1} \sin \left(v_{1} H\right) .
$$

To illustrate the previous developments we consider the concrete case of a layer with a thickness of $H=10^{\prime} 000$ $\mathrm{m}$, which may represent the crust of the Earth. Its material properties are $\beta_{1}=2000 \mathrm{~m} / \mathrm{s}$ and $\rho_{1}=2700 \mathrm{~kg} / \mathrm{m}^{3}$. The underlying half-space is characterised by $\beta_{2}=3000 \mathrm{~m} / \mathrm{s}$ and $\rho_{2}=3100 \mathrm{~kg} / \mathrm{m}^{3}$. Figure $16.2 \mathrm{a}$ displays the left- and right-hand sides of the characteristic function (16.20) as a function of the phase velocity

$$
c=\frac{\omega}{k},
$$

for a fixed circular frequency $\omega=2 \pi \mathrm{Hz}$. The phase velocity is the propagation velocity of individual peaks and troughs of a monochromatic Love wave, as in equation (16.9). Solutions to the characteristic equation exist for a discrete number of phase velocities, marked by the intersection of the curves. The solution with the lowest phase velocity is referred to as the fundamental mode, whereas all other solutions at higher phase velocities are known as higher modes or overtones. While the fundamental mode generally exists, the higher modes only appear from a certain threshold frequency upwards. A collection of Love wave modes for a range of frequencies $f=\frac{\omega}{2 \pi}$ is displayed in figure $16.2 \mathrm{~b}$.

Figure 16.3 picks out the displacement functions for a fixed frequency of $f=0.5 \mathrm{~Hz}$ and an arbitrarily chosen amplitude factor $A=1$. Shown are the fundamental mode and the first three overtones. Due to the exponential decay in the lower half-space, Love wave displacement is largely localised inside the low-velocity layer. The phase velocity of the fundamental mode is close to the velocity of the layer, $\beta_{1}$. With increasing frequency, phase velocity approaches the velocity $\beta_{2}$ of the underlying half-space. 
a) characteristic function

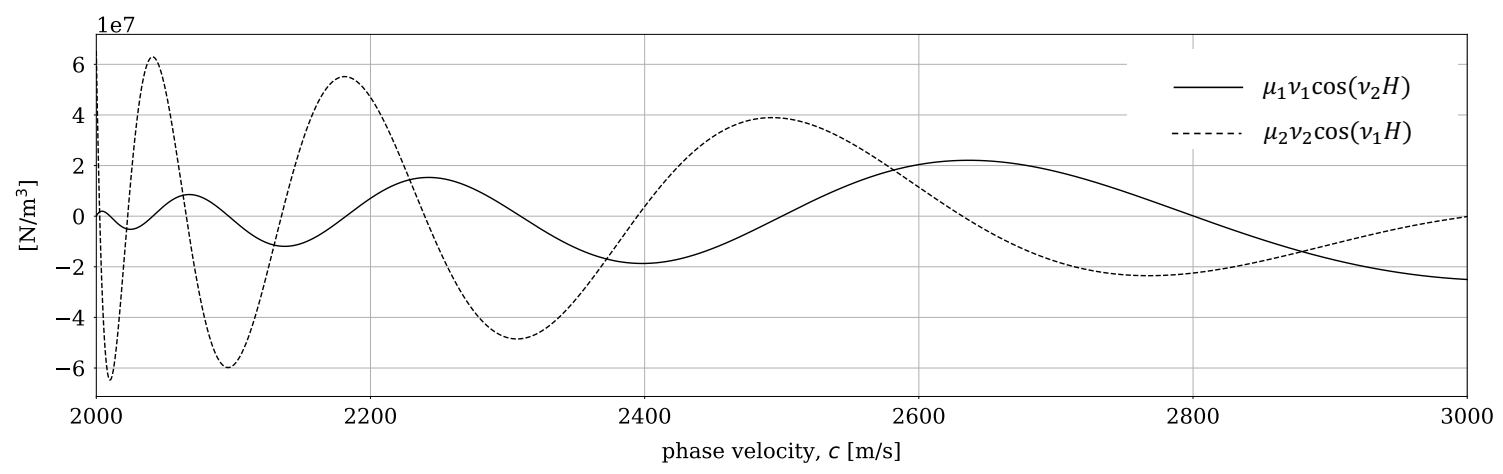

b) phase velocity dispersion curve

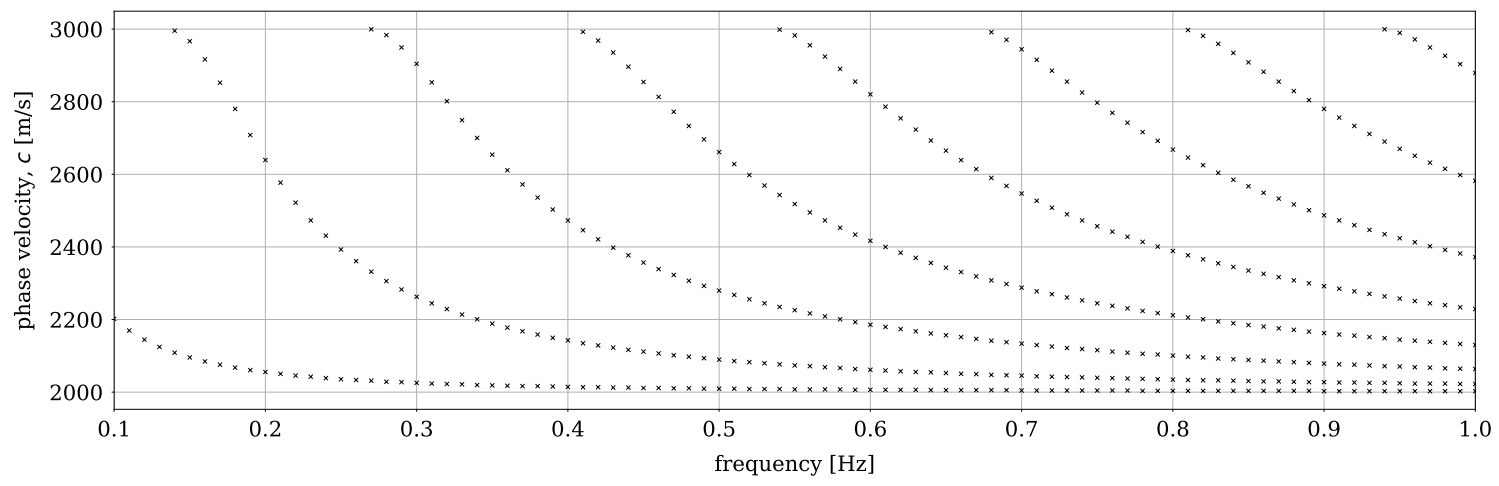

Fig. 16.2: Characteristic function and dispersion of Love waves. (a) Illustration of the left- and right-hand sides of the characteristic function (16.20) for a fixed circular frequency $\omega=2 \pi \mathrm{Hz}$. Solutions exist for phase velocities $c=\omega / k$ (or wave numbers $k$ ) where the two curves intersect. (b) Phase velocities $c$ as a function of frequency $f=\frac{\omega}{2 \pi}$ that solve the characteristic equation. The lowest phase velocity corresponds to the fundamental mode. Higher phase velocities are referred to as higher modes or overtones. [Backus-Gilbert]

\subsubsection{Energy equation and sensitivity kernels}

In order to solve inverse problems involving Love wave observations, we often face the difficulty of having to modify the material parameters such that the phase velocity approaches our observations. Hence, we are interested in variations $\delta c$, induced by variations $\delta \mu$ and $\delta \rho$. In principle, we would obtain $\delta c$ with high accuracy with the help of a finite-difference approximation. This, however, would require repeated solutions of the forward problem. We shall derive a more efficient and physically insightful solution. For this, we first reconsider equation (16.11), which we multiply by another Love wave displacement function $y_{1}^{\prime}$, obtained, for instance, for a different combination of $\omega$ and $k$. Integrating from $-\infty$ to $H$ then yields

$$
\omega^{2} \int_{-\infty}^{H} \rho y_{1} y_{1}^{\prime} d z=k^{2} \int_{-\infty}^{H} \mu y_{1} y_{1}^{\prime} d z-\int_{-\infty}^{H} \frac{d}{d z} y_{2} y_{1}^{\prime} d z,
$$

where we omitted all dependencies on $\omega, k$ and $\mathbf{x}$ in the interest of notational simplicity. Recalling that $y_{2}$ vanishes at $z=H$, and that $y_{1}$ tends to 0 for $z \rightarrow-\infty$, the last term in (16.22) can be integrated by parts. Then making use of (16.10), we obtain 
Love wave displacement functions, $f=0.5 \mathrm{~Hz}$
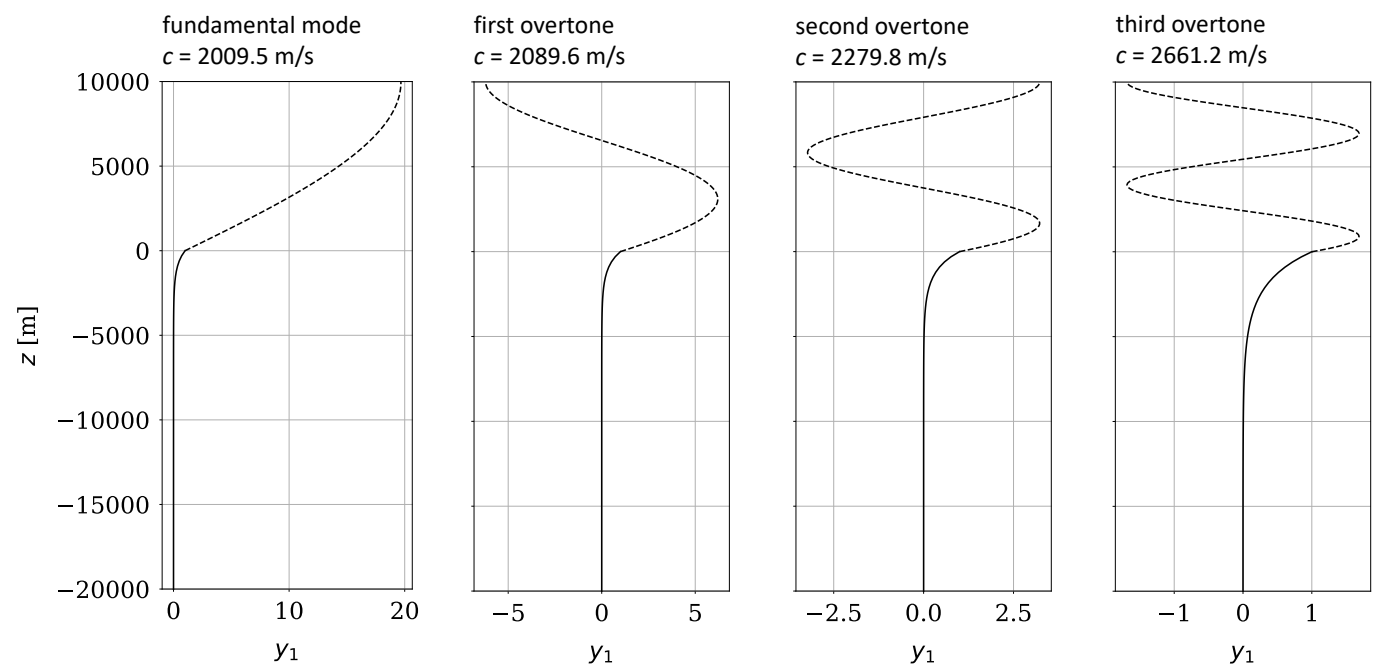

Fig. 16.3: Love wave displacement functions $y_{1}$ for a fixed frequency of $f=0.5 \mathrm{~Hz}$. Shown are the fundamental mode and the first three overtones as a function of depth, together with their respective phase velocities. The displacement in the low-velocity layer is shown as dashed curve. [Backus-Gilbert]

$$
\omega^{2} \int_{-\infty}^{H} \rho y_{1} y_{1}^{\prime} d z=k^{2} \int_{-\infty}^{H} \mu y_{1} y_{1}^{\prime} d z+\int_{-\infty}^{H} \mu \frac{d y_{1}}{d z} \frac{d y_{1}^{\prime}}{d z} d z .
$$

Equation (16.23) establishes a relation between two Love wave solutions $\left(y_{1}, y_{2}\right)$ and $\left(y_{1}^{\prime}, y_{2}^{\prime}\right)$. A special case occurs when both solutions are identical, so that (16.23) simplifies to

$$
\omega^{2} \int_{-\infty}^{H} \rho y_{1}^{2} d z=k^{2} \int_{-\infty}^{H} \mu y_{1}^{2} d z+\int_{-\infty}^{H} \mu\left(\frac{d y_{1}}{d z}\right)^{2} d z
$$

which is known as the energy equation. The energy equation represents the balance between kinetic energy on the left-hand side and potential elastic energy on the right-hand side, and it serves as our starting point for the derivation of an explicit relation between phase velocity and material parameter variations.

For simplicity, we only consider perturbations of the shear modulus $\mu$, neglecting variations of the mass density $\rho$. For some fixed circular frequency $\omega$, we assume that the shear modulus changes from $\mu$ to $\mu+\delta \mu$, thereby inducing a change in wave number from $k$ to $k+\delta k$, a change displacement from $y_{1}$ to $y_{1}+\delta y_{1}$, and a change in stress from $y_{2}$ to $y_{2}+\delta y_{2}$. Inserting the perturbed quantities into (16.24) gives the energy equation for the perturbed Love wave,

$$
\omega^{2} \int_{-\infty}^{H} \rho\left(y_{1}+\delta y_{1}\right)^{2} d z=(k+\delta k)^{2} \int_{-\infty}^{H}(\mu+\delta \mu)\left(y_{1}+\delta y_{1}\right)^{2} d z+\int_{-\infty}^{H}(\mu+\delta \mu)\left(\frac{d\left(y_{1}+\delta y_{1}\right)}{d z}\right)^{2} d z .
$$

Equation (16.25) can be simplified substantially by neglecting second-order terms, subtracting the energy equation (16.24). Furthermore, realising that the displacement perturbation itself satisfies the equations of motion (16.10) and (16.10), allows us to eliminate a copy of (16.23) with $y_{1}^{\prime}=\delta y_{1}$. Finally, we are left with 


$$
0=\int_{-\infty}^{H}\left[k^{2} y_{1}^{2}+\left(\frac{d y_{1}}{d z}\right)^{2}\right] \delta \mu d z+2 k \delta k \int_{-\infty}^{H} \mu y_{1}^{2} d z .
$$

Rearranging (16.26) and making use of the relation $\delta c / c=-\delta k / k$, we finally obtain an explicit expression for the phase velocity variation induced by a shear modulus variation,

$$
\frac{\delta c}{c}=\frac{\int_{-\infty}^{H}\left[k^{2} y_{1}^{2}+\left(\frac{d y_{1}}{d z}\right)^{2}\right] \delta \mu d z}{2 k^{2} \int_{-\infty}^{H} \mu y_{1}^{2} d z} .
$$

Equation (16.27) can be written in the more convenient and succinct form

$$
\frac{\delta c}{c}=\int_{-\infty}^{H} K(z) \delta \mu(z) d z
$$

with the sensitivity kernel

$$
K(z)=\frac{k^{2} y_{1}(z)^{2}+\frac{1}{\mu^{2}} y_{2}(z)^{2}}{2 k^{2} \int_{-\infty}^{H} \mu(z) y_{1}(z)^{2} d z},
$$

where we used (16.10) and reintroduced the dependence on $z$ for clarity. The depth-dependent sensitivity kernel $K(z)$ represents the first-order dependence of phase velocity $c$ on the distribution of the shear modulus $\mu(z)$. Following the same procedure as above, as sensitivity kernel for variations in density, $\delta \rho(z)$, can be derived.

The sensitivity kernels for the Love wave modes considered in the example of figure 16.3 are displayed in figure 16.4. While the fundamental mode is only sensitive to shear velocity variations close to the surface, the higher modes also sense slightly deeper structure.

Love wave sensitivity kernels for shear modulus variations, $f=0.5 \mathrm{~Hz}$
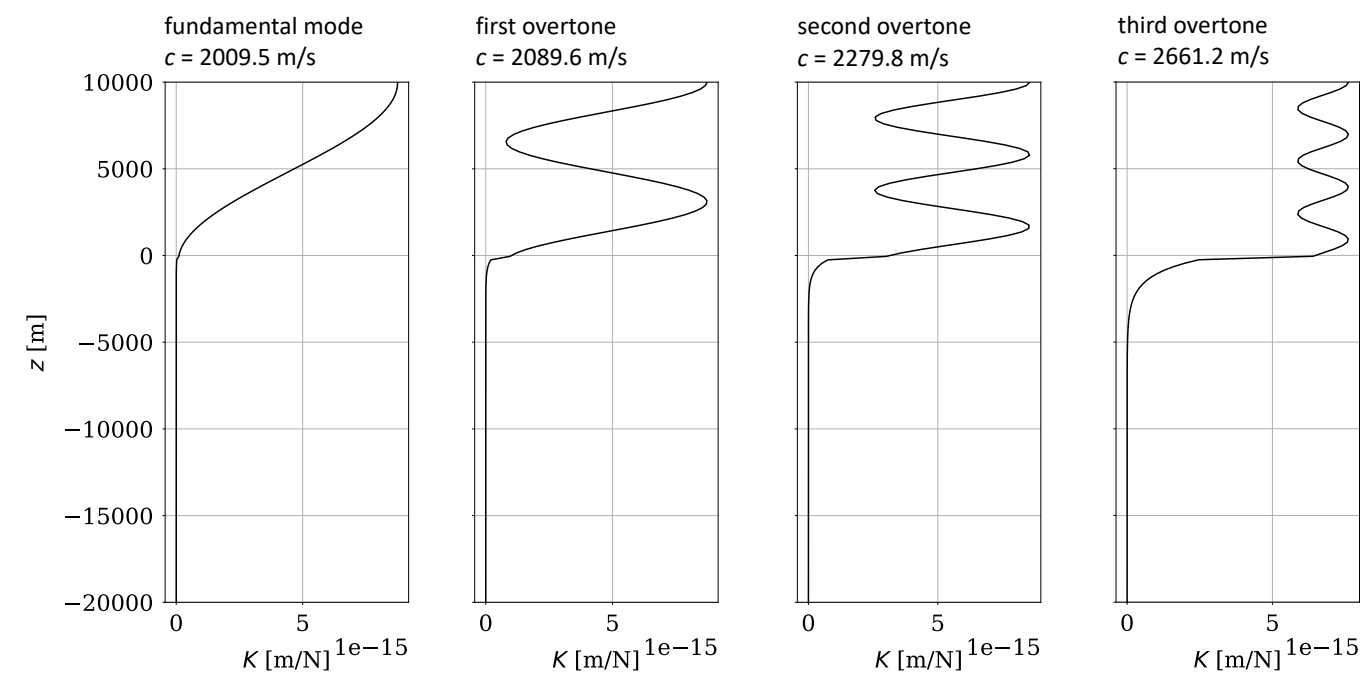

Fig. 16.4: Love wave sensitivity kernels $K(z)$ for shear velocity variations $\delta \mu(z)$. The kernels correspond exactly to the Love wave modes shown in figure 16.3. [Backus-Gilbert] 


\subsection{Finite-difference solution of the 2-D scalar wave equation}

Wave motion is among the most widespread physical phenomena. It occurs in fields as diverse as continuum mechanics, electromagnetics, relativity, and quantum mechanics. Though details may vary between applications, the propagation of waves can be described mathematically by a wave equation, which balances the acceleration of the wavefield against some internal resisting forces. With the exception of few special cases, including layered and homogeneous media, the wave equation cannot be solved analytically, that is, in closed form. Therefore, we need to resort to numerical methods, which at least provide good approximations of the exact solution in more complicated media. One of these numerical approximations, based on a finite-difference approximation in time and space, is the topic of this section.

As an easily tractable special case, we consider the 2-D scalar wave equation

$$
\frac{1}{c^{2}(x, y)} \frac{\partial^{2}}{\partial t^{2}} u(x, y, t)-\Delta u(x, y, t)=f(x, y, t),
$$

which determines the wavefield $u$ at position $(x, y)$ and time $t$, as a function of the wave speed distribution $c(x, y)$ and the external force $f(x, y, t)$. Among the many methods that have been proposed for the numerical solution of wave equations (e.g. Igel, 2016), we employ the staggered-grid finite-difference method, introduced by Virieux (1984). This choice is primarily motivated by its relative simplicity combined with favourable numerical properties.

The method rests on a reformulation of the second-order differential equation (16.30) in terms of a system of first-order differential equations,

$$
\begin{aligned}
& \frac{1}{c^{2}(x, y, t)} \frac{\partial}{\partial t} \dot{u}(x, y, t)-\frac{\partial}{\partial x} \sigma_{x}(x, y, t)-\frac{\partial}{\partial y} \sigma_{y}(x, y, t)=f(x, y, t) \\
& \frac{\partial}{\partial t} \sigma_{x}(x, y, t)=\frac{\partial}{\partial x} \dot{u}(x, y, t) \\
& \frac{\partial}{\partial t} \sigma_{y}(x, y, t)=\frac{\partial}{\partial y} \dot{u}(x, y, t)
\end{aligned}
$$

with the time derivative $\dot{u}=\partial u / \partial t$. A finite-difference method discretises the continuous equations by considering their values only at a finite set of spatial grid points with spacing $\Delta x$ in $x$-direction and $\Delta y$ in $y$-direction. Spatial derivatives are then replaced by finite-difference approximations.

In a regular finite-difference grid, as shown in figure $16.5 \mathrm{a}$, the discretised versions $\dot{u}_{i, j}, \sigma_{x, i, j}$ and $\sigma_{y, i, j}$ of the field variables, $\dot{u}, \sigma_{x}$ and $\sigma_{y}$ are defined at identical grid points, namely at $x=i \Delta x$ and $y=j \Delta y$, for integervalued $i$ and $j$.

A staggered-grid, in contrast, defines different discrete field variables on different grids that are offset by $\Delta x / 2$ and $\Delta y / 2$, as illustrated in figure $16.5 \mathrm{~b}$. While a staggered grid is conceptually more complicated than a regular grid, it comes with the benefit of reducing the grid spacing by a factor of 2 without increasing the number of grid points. Using a central finite-difference approximation of second order, we find the following space-discrete version of equations (16.31) to (16.33),

$$
\begin{aligned}
\frac{\partial}{\partial t} \dot{u}_{i, j}(t) & =a_{i, j}(t), \\
\frac{\partial}{\partial t} \sigma_{x, i, j}(t) & =\frac{1}{\Delta x}\left[\dot{u}_{i+1, j}(t)-\dot{u}_{i, j}(t)\right], \\
\frac{\partial}{\partial t} \sigma_{y, i, j}(t) & =\frac{1}{\Delta y}\left[\dot{u}_{i, j+1}(t)-\dot{u}_{i, j}(t)\right],
\end{aligned}
$$

with the discrete acceleration 
16.3 Finite-difference solution of the 2-D scalar wave equation

$$
a_{i, j}(t)=c_{i, j}^{2} f_{i, j}(t)+\frac{c_{i, j}^{2}}{\Delta x}\left[\sigma_{x, i, j}(t)-\sigma_{x, i-1, j}(t)\right]+\frac{c_{i, j}^{2}}{\Delta y}\left[\sigma_{y, i, j}(t)-\sigma_{y, i, j-1}(t)\right] .
$$

Both the discrete velocity $c_{i, j}$ and the discrete force $f_{i, j}(t)$ are defined at positions $x=i \Delta x$ and $y=j \Delta y$. Equations (16.34) to (16.36) are a set of ordinary differential equations, where the right-hand sides can be evaluated using the values of the field variables at the discrete collection of grid points.
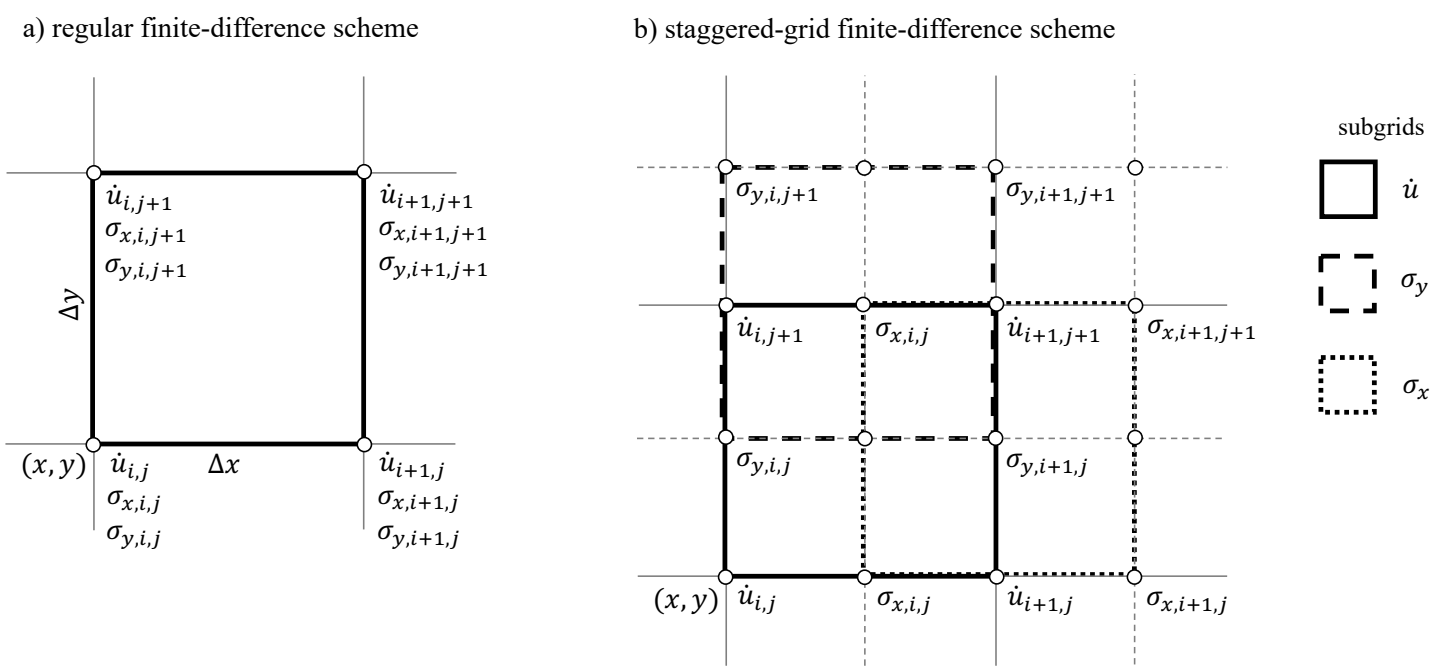

Fig. 16.5: Regular and staggered-grid finite-difference schemes. (a) In a regular finite-difference scheme, all discretised field variables are defined at the same grid points. (b) In a staggered grid, different field variables are defined at different grids that are offset by $\Delta x / 2$ and $\Delta y / 2$ with respect to each other.

To arrive at a fully discrete scheme, it remains to replace the time derivatives with a suitable finite-difference approximation. For this, we introduce the time increment $\Delta t$ and advance the field variables from time $t$ to $t+\Delta t$ using the second-order scheme

$$
\begin{aligned}
\dot{u}_{i, j}(t+\Delta t) & =\dot{u}_{i, j}(t)+\Delta t a_{i, j}(t+\Delta t / 2), \\
\sigma_{x, i, j}(t+\Delta t / 2) & =\sigma_{x, i, j}(t-\Delta t / 2)+\frac{\Delta t}{\Delta x}\left[\dot{u}_{i+1, j}(t)-\dot{u}_{i, j}(t)\right], \\
\sigma_{y, i, j}(t+\Delta t / 2) & =\sigma_{y, i, j}(t-\Delta t / 2)+\frac{\Delta t}{\Delta y}\left[\dot{u}_{i, j+1}(t)-\dot{u}_{i, j}(t)\right] .
\end{aligned}
$$

Equations (16.38) to (16.40) implement a staggered-grid approach in time, and they provide a computational recipe for the iterative, approximate solution of the scalar wave equation (16.30): Starting with $\sigma_{x, i, j}(t-\Delta t / 2)$, $\sigma_{y, i, j}(t-\Delta t / 2)$ and $\dot{u}_{i, j}(t)$, we use equations (16.39) and (16.40) to obtain the updates $\sigma_{x, i, j}(t+\Delta t / 2)$ and $\sigma_{y, i, j}(t+\Delta t / 2)$. Inserting them into (16.37), we can compute the discrete acceleration $a_{i, j}(t+\Delta t / 2)$. Finally, this is used to update the wavefield from time $t$ to $t+\Delta t$ via equation (16.38). This procedure is then repeated until the wavefield has been propagated through the medium for a sufficiently long time.

To implement the Dirichlet condition $\left.u(\mathbf{x}, t)\right|_{\mathbf{x} \in \partial V}=0$ (see (9.51)), we set all velocity values at or behind the domain boundary $\partial V$ to 0 . For the quiescent initial conditions $\left.u\right|_{t=0}=0$ and $\left.\dot{u}\right|_{t=0}=0$ it suffices to initialise all fields with 0 for time steps $t=0$ and $t=-\Delta t / 2$.

Figure 16.6 illustrates the finite-difference solution for the specific case where the wave speed distribution is constant at $c(x, y)=600 \mathrm{~m} / \mathrm{s}$. The grid point spacing is $0.1 \mathrm{~m}$ in both coordinate directions, which extent 100 
$\mathrm{m}$. The time increment is $\Delta t=8.0 \cdot 10^{-5} \mathrm{~s}$. Recordings at a selection of receiver positions are shown in figure 16.7.
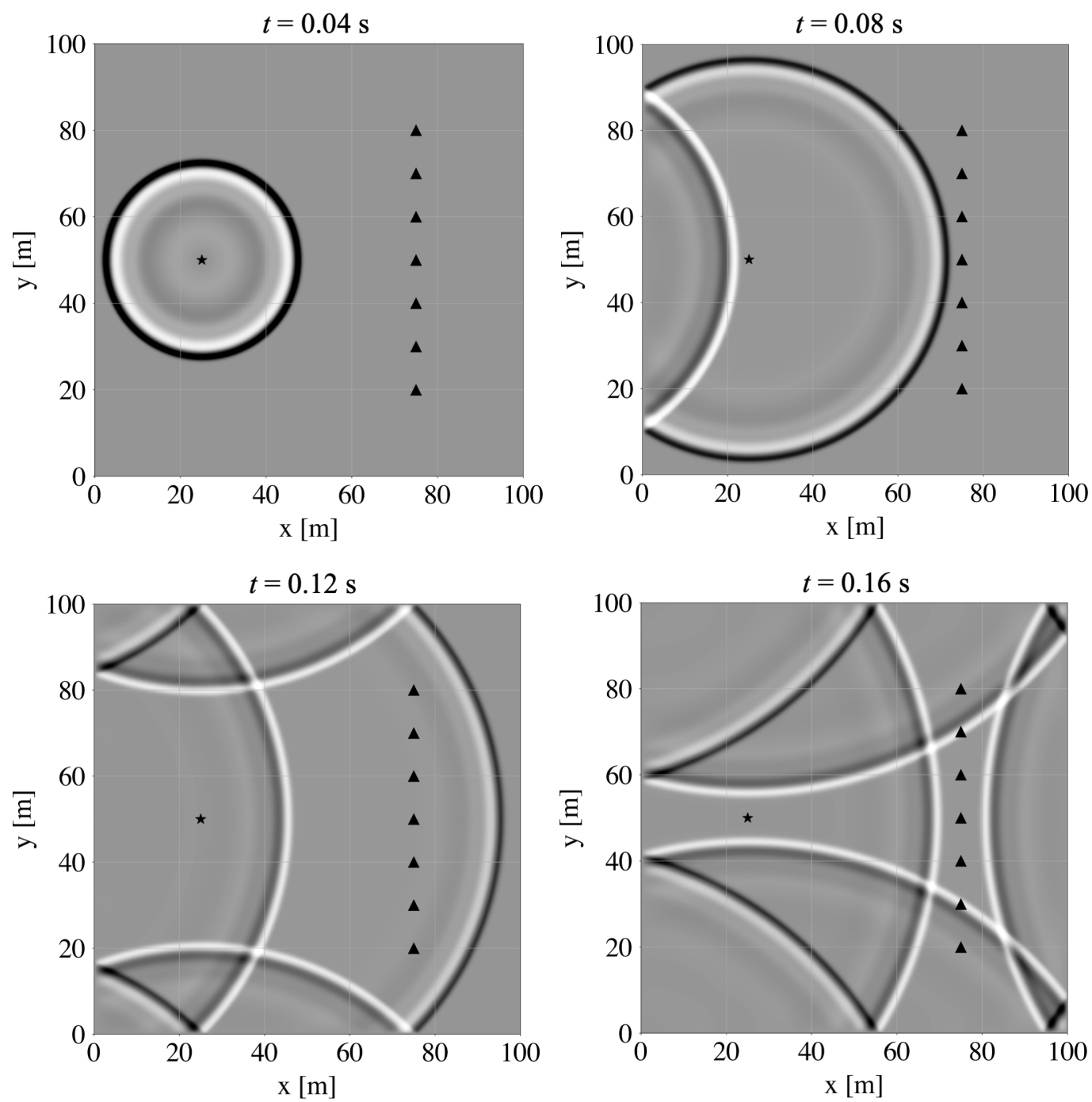

Fig. 16.6: Wavefield snapshots of $u(x, y, t)$ for different times $t$, computed with the second-order, staggeredgrid, finite-difference method. The source location is marked by a black star, and receiver locations, for which recordings are shown in figure 16.7, are indicated by black triangles. The grid point spacing is $\Delta x=\Delta y=0.1$ $\mathrm{m}$, and the time increment is $\Delta t=8.0 \cdot 10^{-5} \mathrm{~s}$. Throughout the domain, the medium is homogeneous, with wave speed $c(x, y)=600 \mathrm{~m} / \mathrm{s}$. [Run in binder. 0X/FD2Dpy]

Some care must be taken in the choice of the time increment $\Delta t$, as too large values will result in numerical instability. The maximum possible value for $\Delta t$ is determined by the Courant-Friedrichs-Lewy or CFL condition (e.g. Igel, 2016; Quarteroni et al., 2000),

$$
\Delta t_{\max }=C \frac{\max c(x, y)}{\min (\Delta x, \Delta y)},
$$




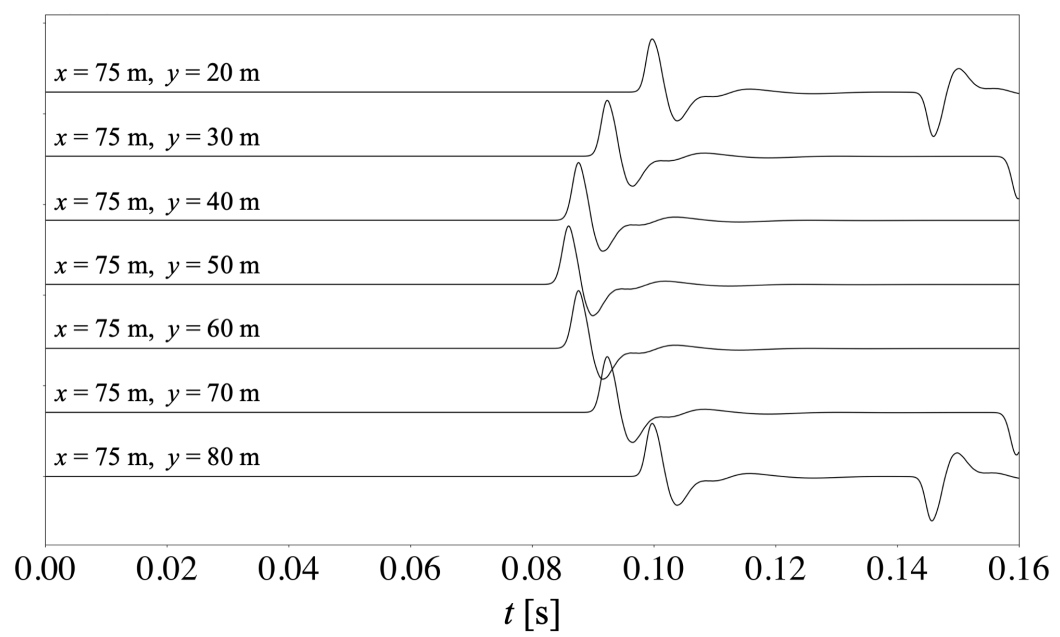

Fig. 16.7: Wavefield recordings of $u(x, y, t)$ at the receiver positions indicated in figure 16.6. [Run in binder. 0X/FD2Dpy]

where $\max c(x, y)$ is the maximum wave speed in the domain, and $\min (\Delta x, \Delta y)$ is the minimum grid spacing. The constant $C$ is the $C F L$ number. It depends on the specific numerical method, and is equal to 0.5 for the second-order, staggered-grid scheme, described above. An example of an unstable solution is shown in figure $16.8 \mathrm{a}$.

The numerical accuracy, that is, the difference between the exact solution and its numerical approximation, is largely controlled by the number of finite-difference grid points per wavelength. For the method presented here, are rule of thumb is that around 20 grid points per wavelength should be used. Figure $16.8 \mathrm{~b}$ provides an example of numerical inaccuracies that result from a grid point spacing that is too large. 
a) Numerical instability

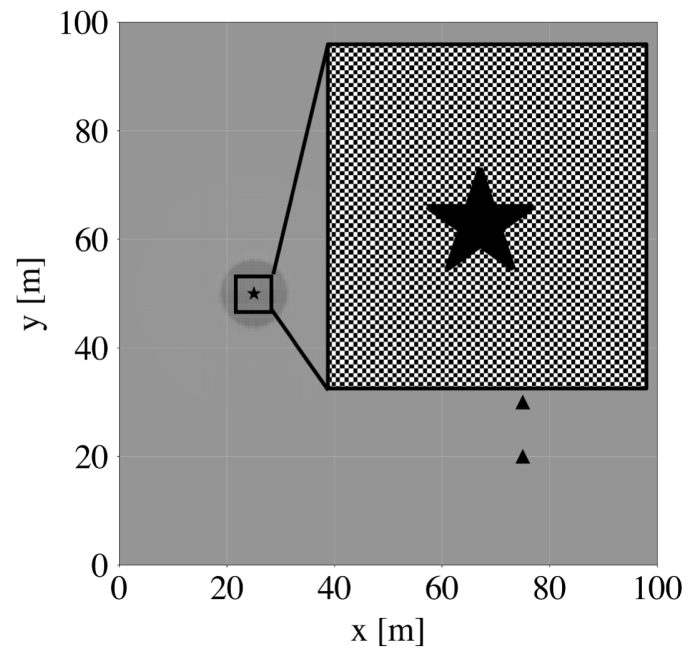

b) Inaccurate solution

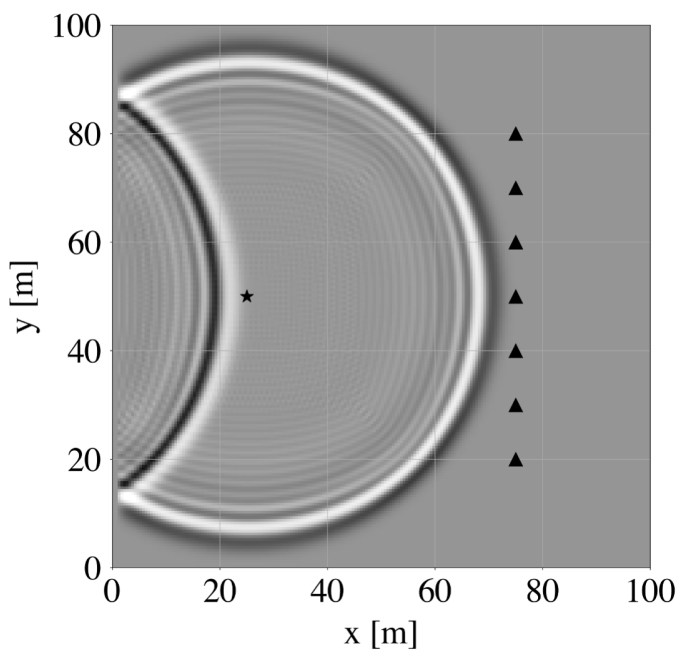

Fig. 16.8: Wavefield snapshots of $u(x, y, t)$ similar to those shown in figure 16.6, but for numerical parameters that produce inaccurate solutions. (a) When the time increment $\Delta t$ is too large, the solution becomes unstable, meaning that wavefield values become excessively large or numerically infinite, and exhibit unphysical patterns, as shown in the zoom around the source location. In this example, the time increment is $\Delta t=12.0 \cdot 10^{-5}$ $\mathrm{s}$, which is significantly above the limit $\Delta t_{\max }=8.3 \cdot 10^{-5} \mathrm{~s}$, imposed by the CFL condition. (b) Increasing the grid point spacing to an extent that the wave shape cannot be represented anymore, leads to numerical accuracies. In this example, the grid point spacing was increased from $0.1 \mathrm{~m}$ to $0.5 \mathrm{~m}$. The resulting inaccuracies, known as numerical dispersion, are visible as long-lasting ringing in the tail of the wavefront. [Run in binder. 0X/FD2Dpy] 


\section{Literature}





\section{Bibliography}

Afanasiev, M. V., Boehm, C., van Driel, M., Krischer, L., Rietmann, M., May, D. A., Knepley, M. G. \& Fichtner, A., 2019. Modular and flexible spectral-element waveform modelling in two and three dimensions. Geophys. J. Int., 216, doi: 10.1093/gji/ggy469.

Afanasiev, M. V., Pratt, R. G., Kamei, R. \& McDowell, G., 2014. Waveform-based simulated annealing of crosshole transmission data: A semi-global method for estimating seismic anisotropy. Geophys. J. Int., 199, 1586-1607.

Agranovich, Z. S. \& Marchenko, V. A., 1963. The inverse problem of scattering theory. Gordon and Breach, New York.

Ahmad, F. \& Razzaghi, M., 1998. A numerical solution to the Gel'Fand-Levitan-Marchenko equation. Appl. Math. Comp., 89, 31-39.

Aki, K. \& Richards, P., 2002. Quantitative Seismology. University Science Books.

Alt, W., 2002. Nichtlineare Optimierung. Vieweg und Sohn, Braunschweig/Wiesbaden.

An, M., 2012. A simple method for determining the spatial resolution of a general inverse problem. Geophys. J. Int., 191, 849-864.

Backus, G. E. \& Gilbert, F., 1967. Numerical application of a formalism for geophysical inverse problems. Geophys. J. Roy. Astr. Soc., 13, 247-276.

Backus, G. E. \& Gilbert, F., 1968. The resolving power of gross Earth data. Geophys. J. Roy. Astr. Soc., 16, 169-205.

Backus, G. E. \& Gilbert, F., 1970. Uniqueness in the inversion of inaccurate gross Earth data. Phil. Trans. R. Soc. London, A, 266, 123-192.

Bamberger, A., Chavent, G., Hemons, C. \& Lailly, P., 1982. Inversion of normal incidence seismograms. Geophysics, 47, 757-770.

Bardenet, R., Doucet, A. \& Holmes, C., 2014. Towards scaling up Markov chain Monte Carlo: an adaptive subsampling approach. In Proc. 31st Int. Conf. Machine Learning, pp. 405-413.

Bartlett, M. S., 1951. An inverse matrix adjustement arising in discriminant analysis. Ann. Math. Statist., 22, $107-111$.

Bayes, T. \& Price, R., 1763. An essay toward solving a problem in the doctrine of chances. Phil. Trans. R. Soc. London, 53, 370-418.

Bazaraa, M. S. \& Shetty, C. M., 1976. Foundations of optimization. Springer, Berlin, Heidelberg, New York.

Bernauer, M., Fichtner, A. \& Igel, H., 2014. Optimal observables for multi-parameter seismic tomography. Geophys. J. Int., 198, 1241-1254.

Betancourt, M., 2017. A conceptual introduction to Hamiltonian Monte Carlo. arXiv:1701.02434 [stat.ME].

Bishop, C. M., 2006. Pattern recognition and machine learning. Springer, New York.

Biswas, R. \& Sen, M., 2017. 2D full-waveform inversion and uncertainty estimation using the reversible jump Hamiltonian Monte Carlo. SEG Expanded Abstracts, 1280-1285.

Blanes, S., Casas, F. \& Sanz-Serna, J. M., 2014. Numerical integrators for the Hybrid Monte Carlo method. SIAM J. Sci. Comp., 36, A1556-A1580.

Bleibinhaus, F., Hole, J. A. \& Ryberg, T., 2007. Structure of the California Coast Ranges and San Andreas Fault at SAFOD from seismic waveform inversion and reflection imaging. J. Geophys. Res., 112, doi:10.1029/2006JB004611.

Boehm, C. \& Fichtner, A., 2018. Lazy wave propagation. Geophys. J. Int., 216, doi: 10.1093/gji/ggy295.

Bozdağ, E., Peter, D., Lefebvre, M., Komatitsch, D., Tromp, J., Hill, J., Podhorszki, N. \& Pugmire, D., 2016. Global adjoint tomography: First-generation model. Geophys. J. Int., 207, 1739-1766.

Bozdağ, E., Trampert, J. \& Tromp, J., 2011. Misfit functions for full waveform inversion based on instantaneous phase and envelope measurements. Geophys. J. Int., 185, 845-870.

Broodlie, K. W., Gourlay, A. R. \& Greenstadt, J., 1973. Rank-one and rank-two corrections to positive definite matrices expressed in product form. J. Inst. Maths. Applics., 11, 73-82.

Brossier, R., Operto, S. \& Virieux, J., 2010. Which data residual norm for robust elastic frequency-domain full waveform inversion? Geophysics, 75, R37-R46. 
Broyden, C. G., 1970. The convergence of a class of double-rank minimization algorithms. J. Inst. Math. Appl., 6, 76-90.

Bube, K. P. \& Burridge, R., 1983. The one-dimensional inverse problem of reflection seismology. SIAM Review, 25, 497-559.

Burridge, R., 1980. The Gelfand-Levitan, the Marchenko, and the Gopinath-Sondhi integral equations of inverse scattering theory, regarded in the context of inverse impulse-response problems. Wave Motion, $\mathbf{2}$, 305-323.

Cerveny, V., 2001. Seismic ray theory. Cambridge University Press.

Chen, P., Zhao, L. \& Jordan, T. H., 2007. Full 3D tomography for the crustal structure of the Los Angeles region. Bull. Seismol. Soc. Am., 97, 1094-1120.

Chib, S., Greenberg, E. \& Winkelmann, R., 1998. Posterior simulation and Bayes factors in panel count data models. J. Economet., 86, 33-54.

Cormack, A. M., 1963. Representation of a function by its line integrals, with some radiological applications. J. Appl. Phys., 34, 2722-2727.

Cowles, M. K. \& Carlin, B. P., 1996. Markov chain monte carlo convergence diagnostics: A comparative review. J. Am. Stat. Ass., 91, 883-904.

Crank, J. \& Nicolson, P., 1947. A practical method for numerical evaluation of solutions of partial differential equations of the heat conduction type. Proc. Camb. Phil. Soc., 43, 50-67.

Curtis, A., 1999a. Optimal design of focused experiments and surveys. Geophys. J. Int., 139, 205-215.

Curtis, A., 1999b. Optimal experimental design: cross-borehole tomographic examples. Geophys. J. Int., 136, 637-650.

Dahlen, F., Hung, S.-H. \& Nolet, G., 2000. Fréchet kernels for finite-frequency traveltimes - I. Theory. Geophys. J. Int., 141, 157-174.

Dahlen, F. A. \& Baig, F. A., 2002. Fréchet kernels for body wave amplitudes. Geophys. J. Int., 150, 440-466.

Dahlen, F. A. \& Tromp, J., 1998. Theoretical Global Seismology. Princeton University Press. NJ.

de Vogelaere, R., 1956. Methods of integration which preserve the contact transformation property of Hamiltonian equations. Technical Report Report No. 4, Department of Mathematics, University of Notre Dame, Notre Dame, Ind., USA.

de Wit, R. W. L., Trampert, J. \& van der Hilst, R. D., 2012. Toward quantifying uncertainty in travel time tomography using the null-space shuttle. J. Geophys. Res., 117, doi:10.1029/2011JB008754.

Deal, M. M. \& Nolet, G., 1996. Nullspace shuttles. Geophys. J. Int., 124, 372-380.

Duane, S., Kennedy, A. D., Pendleton, B. J. \& Roweth, D., 1987. Hybrid Monte Carlo. Phys. Lett. B, 195, 216-222.

Dubbledam, D., Calero, S., Ellis, D. E. \& Snurr, R. Q., 2016. RASPA: molecular simulation software for adsorption and diffusion in flexible nanoporous materials. Mol. Sim., 42, 81-101.

Elhatisari, S., Lee, D., Rupak, G., Epelbaum, E., Krebs, H., Lahde, T. A., Luu, T. \& ner, U.-G. M., 2015. Ab initio alpha-alpha scattering. Nature, 258, 111-113.

Fichtner, A., 2010. Full Seismic Waveform Modelling and Inversion. Springer, Heidelberg.

Fichtner, A., Bunge, H.-P. \& Igel, H., 2006a. The adjoint method in seismology - I. Theory. Phys. Earth Planet. Inter., 157, 86-104.

Fichtner, A., Bunge, H.-P. \& Igel, H., 2006b. The adjoint method in seismology - II. Applications: traveltimes and sensitivity functionals. Phys. Earth Planet. Inter, 157, 105-123.

Fichtner, A., Kennett, B. L. N., Igel, H. \& Bunge, H.-P., 2008. Theoretical background for continental- and global-scale full-waveform inversion in the time-frequency domain. Geophys. J. Int., 175, 665-685.

Fichtner, A., Kennett, B. L. N., Igel, H. \& Bunge, H.-P., 2009. Full seismic waveform tomography for uppermantle structure in the Australasian region using adjoint methods. Geophys. J. Int., 179, 1703-1725.

Fichtner, A. \& Simute, S., 2018. Hamiltonian Monte Carlo inversion of seismic sources in complex media. J. Geophys. Res., 123, doi:10.1002/2017JB015249.

Fichtner, A. \& Trampert, J., 2011. Hessian kernels of seismic data functionals based upon adjoint techniques. Geophys. J. Int., 185, 775-798.

Fichtner, A. \& van Driel, M., 2014. Models and Fréchet kernels for frequency-(in)dependent Q. Geophys. J. Int., 198, 1878-1889. 
Fichtner, A. \& van Leeuwen, T., 2015. Resolution analysis by random probing. J. Geophys. Res., 120, doi:10.1002/2015JB012106.

Fichtner, A. \& Zunino, A., 2019. Hamiltonian nullspace shuttles. Geophys. Res. Lett., 46, doi:10.1029/2018GL080931.

Fichtner, A., Zunino, A. \& Gebraad, L., 2019. Hamiltonian Monte Carlo solution of tomographic inverse problems. Geophys. J. Int., 216, doi:10.1093/gji/ggy496.

Fichtner, A., Zunino, A., Gebraad, L. \& Boehm, C., 2021. Autotuning Hamiltonian Monte Carlo for efficient generalised nullspace exploration. Geophys. J. Int., in press.

Fletcher, R., 1970. A new approach to variable metric algorithms. Comp. J., 13, 317-322.

Fletcher, R. \& Reeves, C. M., 1964. Function minimization by conjugate gradients. Comp. J., 7, $149-154$.

Fox, C. \& Nicholls, G., 1997. Sampling conductivity images via mcmc. In K. V. Mardia, C. A. Gill, \& R. G. Aykroyd (Eds.), The Art and Science of Bayesian Image Analysis, pp. 91-100. Leeds University Press, Leeds, U.K.

Gauss, C. F., 1809. Theoria motus corporum coelestium in sectionibus conicis solem ambientium. Frid. Perthes et I. H. Besser.

Gebraad, L., Boehm, C. \& Fichtner, A., 2020. Bayesian elastic full-waveform inversion using Hamiltonian Monte Carlo. J. Geophys. Res., 125.

Gelman, A., Carlin, J. B., Stern, H. S., Dunson, D. B., Vehtari, A. \& Rubin, D. B., 2013. Bayesian Data Analysis (3 ed.). CRC Press, Boca Raton, FL.

Geweke, J. \& Tanizaki, T., 1999. On Markov-chain Monte Carlo methods for nonlinear and non-Gaussian state-space models. Comm. Stat. Sim. Comp., 28, 867-894.

Geyer, C. J. \& Thompson, E. A., 1995. Annealing Markov Chain Monte Carlo with applications to ancestral inference. J. Am. Stat. Assoc., 90, 909-920.

Goldfarb, D., 1970. A Family of Variable Metric Updates Derived by Variational Means. Math. Comp., 24, 23-26.

Green, P. J., 1995. Reversible jump Markov Chain Monte Carlo computation and Bayesian model determination. Biometrika, 82, 711-732.

Green, P. J. \& Hastie, D. I., 2009. Reversible jump MCMC.

Guasch, L., Calderón Agudo, O., Nachev, P. \& Warner, M., 2020. Full-waveform inversion imaging of the human brain. NPJ Digital Medicine, 28, doi:10.1038/s41746-020-0240-8.

Gull, S. F., 1988. Bayesian inductive inference and maximum entropy. In Maximum-entropy and Bayesian methods in science and engineering, pp. 53-74.

Gutmann, M. U. \& Hyvärinen, A., 2012. Noise-contrastive estimation of unnormalized statistical models with applications to natural image statistics. J. Mach. Learning. Res., 13, 307-361.

Halko, N., Martinsson, P. G. \& Tropp, J. A., 2011. Finding structure with randomness: Probabilistic algorithms for constructing approximate matrix decompositions. SIAM Review, 53, 217-288.

Hanson, K. M. \& Cunningham, G. S., 1998. Posterior sampling with improved efficiency. Proc. SPIE Medical Imaging, 3338, 371-382.

Hestenes, M. R. \& Stiefel, E. L., 1952. Methods of conjugate gradients for solving linear systems. J. Res. Natl. Bur. Stand., 49, 409-436.

Himmelblau, D. M., 1972. Applied nonlinear programming. Mc Graw Hill.

Hoffmann, M. D. \& Gelman, A., 2014. The No-U-Turn sampler. J. Mach. Learn. Res., 15, 1593-1623.

Honkela, A., Peltonen, J., Topa, H., Charapitsa, I., Matarese, F., Grote, K., Stunnenberg, H. G., Reid, G., Lawrence, N. D. \& Rattray, M., 2015. Genome-wide modeling of transcription kinetics reveals patterns of RNA production delays. Proc. Nat. Acad. Sci., 112, 13115-13120.

Hounsfield, G. N., 1973. Computerized transverse axial scanning (tomography): Part i. description of system. British J. Radiology, 46, 1016-1022.

Hutchinson, M. F., 1990. A stochastic estimator of the trace of the influence matrix for Laplacian smoothing splines. Comm. Stat. Sim., 19, 433-450.

Iacono, M. I., Neufeld, E., Akinnagabe, E., Bower, K., Wolf, J., Vogiatzis, O., Sharma, D., Lloyd, B., Wilm, B. J., Wyss, M., Pruessmann, K. P., Jakab, A., Makris, N., Cohen, E. D., Kuster, N., Kainz, W. \& Angelone, 
L. M., 2015. A multimodal imaging-based detailed anatomical model of the human head and neck. PLOS ONE, 10, e0124126.

Igel, H., 2016. Computational seismology: A practical introduction. Oxford University Press.

Ingber, L., 1989. Very fast simulated reannealing. Math. Comp. Modeling, 12, 967-993.

Jackson, D. D., 1976. Most squares inversion. J. Geophys. Res., 81, 1027 - 1030.

Jaynes, E. T., 2003. Probability Theory - The Logic of Science. Cambridge University Press, Cambridge, UK. Jeffreys, H., 1939. Theory of Probability. Oxford University Press, Oxford, UK.

Kennett, B. L. N., 2001. The seismic wavefield I. - Introduction and theoretical development. Cambridge University Press.

Kennett, B. L. N., Engdahl, E. R. \& Buland, R., 1995. Constraints on seismic velocities in the Earth from traveltimes. Geophys. J. Int., 122, 108-124.

Kirkpatrick, S., Gelatt, C. D. \& Vecchi, M. P., 1983. Optimization by Simulated Annealing. Science, 220, 671-680.

Kolmogorov, A. N., 1950. Foundations of the theory of probability. Chelsea Pub. Co., New York.

Korattikara, A., Chen, Y. \& Welling, M., 2014. Asuterity in McMC land: Cutting the Metropolis-Hastings budget. arXiv, 1304.5299, 1-23.

Lailly, P., 1983. The seismic inverse problem as a sequence of before stack migrations. In J. Bednar, R. Redner, E. Robinson, \& A. Weglein (Eds.), Conference on Inverse Scattering: Theory and Application. Soc. Industr. appl. Math., Philadelphia, PA.

Landau, L. D. \& Lifshitz, E. M., 1976. Course of Theoretical Physics, Volume 1, Mechanics, 3rd edition. Elsevier Butterworth Heinemann, Amsterdam.

Leimkuhler, B. \& Reich, S., 1994. Simulating Hamiltonian systems. Cambirdge University Press, Cambridge, UK.

Levenberg, K., 1944. A method for the solution of certain non-linear problems in least-squares. Q. Appl. Math., 2, 164-168.

Lévêque, J. J., Rivera, L. \& Wittlinger, G., 1993. On the use of the checkerboard test to assess the resolution of tomographic inversions. Geophys. J. Int., 115, 313-318.

Liu, D. C. \& Nocedal, J., 1989. On the limited-memory BFGS method for large-scale optimisation. Mathematical Programming, 45, 503-528.

Liu, Q. \& Tromp, J., 2008. Finite-frequency sensitivity kernels for global seismic wave propagation based upon adjoint methods. Geophys. J. Int., 174, 265-286.

Luo, Y. \& Schuster, G. T., 1991. Wave-equation traveltime inversion. Geophysics, 56, 645-653.

Mackenzie, P. B., 1989. An improved hybrid Monte Carlo method. Phys. Lett. B., 226, 369-371.

Marinari, E. \& Parisi, G., 1992. Simulated tempering: a new Monte Carlo scheme. Europhys. Lett., 19, 451458.

Marquardt, D., 1963. An algorithm for least-squares estimation of nonlinear parameters. SIAM J. Appl. Math., 11, 431-441.

Marquering, H., Dahlen, F. A. \& Nolet, G., 1999. Three-dimensional sensitivity kernels for finite-frequency traveltimes: the banana-doughnut paradox. Geophys. J. Int., 137, 805-815.

Martin, J., Wilcox, L. C., Burstedde, C. \& Ghattas, O., 2012. A stochastic Newton MCMC method for largescale statistical inverse problems with application to seismic inversion. SIAM J. Sci. Comp., 34, A1460A1487.

Marty, P., Boehm, C. \& Fichtner, A., 2021. Acoustoelastic full-waveform inversion for transcranial ultrasound computed tomography. Proc. SPIE 11602, Medical Imaging 2021, doi:10.1117/12.2581029.

Meju, M. A., 2009. Regularised extremal bounds analysis (REBA): An approach to quantifying uncertainty in nonlinear geophysical inverse problems. Geophys. Res. Lett., 36, doi:10:1029/2008GL036407.

Menke, W., 2012. Geophysical data analysis: Discrete inverse theory. Academic Press.

Metropolis, N., 1987. The beginning of the Monte Carlo method. Los Alamos Science, Special Issue, 125-130.

Metropolis, N., Rosenbluth, A. W., Rosenbluth, M. N., Teller, A. H. \& Teller, E., 1953. Equations of state calculations by fast computing machines. J. Chem. Phys., 21, 1087-1092.

Mosegaard, K., 2012. Limits to nonlinear inversion, pp. 11-21. Springer, Berlin, Heidelberg. 
Muir, J. B. \& Tkalčić, H., 2015. Probabilistic joint inversion of lowermost mantle P-wave velocities and core mantle boundary topography using differential travel times and hierarchical Hamiltonian Monte-Carlo sampling. In AGU 2015 Fall meeting, pp. S14A-03. AGU.

Muir, J. B. \& Tkalčić, H., 2020. Probabalistic lowermost mantle P-Wave tomography from hierarchical Hamiltonian Monte Carlo and model parametrization cross-validation. Geophys. J. Int., under review.

Nakata, N., Gualtieri, L. \& Fichtner, A., 2019. Seismic Ambient Noise. Cambridge University Press.

Neal, R. M., 1996. Bayesian learning for neural networks. Springer, New York.

Neal, R. M., 2011. MCMC using Hamiltonian dynamics. In Handbook of Markov Chain Monte Carlo, pp. Chapter 5.

Nocedal, J., 1980. Updating quasi-Newton matrices with limited storage. Math. Comp., 35, 773-782.

Nocedal, J. \& Wright, S. J., 1999. Numerical Optimization. Springer, New York.

Polak, E. \& Ribière, G., 1969. Note sur la convergence de la méthode de directions conjuguées. Revue Fr. Inf. Rech. Oper., 16-R1, 35-43.

Popper, K., 1935. Logik der Forschung. Zur Erkenntnistheorie der modernen Naturwissenschaft. Springer.

Pratt, R., Shin, C. \& Hicks, G., 1998. Gauss-Newton and full Newton methods in frequency domain seismic waveform inversion. Geophys. J. Int., 133, 341-362.

Pratt, R. G., 1999. Seismic waveform inversion in the frequency domain, part 1: Theory and verification in a physical scale model. Geophysics, 64, 888-901.

Pratt, R. G., Huang, L., Duric, N. \& Littrup, P., 2007. Sound-speed and attenuation imaging of breast tissue using waveform tomography of transmission ultrasound data. SPIE Medical Imaging 2007, 1523 - 1534.

Qi, Y. \& Minka, T. P., 2002. Hessian-based Markov chain Monte Carlo algorithms. In First Cape Cod Workshop on Monte Carlo Methods.

Quarteroni, A., Sacco, R. \& Saleri, F., 2000. Numerical Mathematics. Springer, New York.

Quispel, G. R. W. \& McLaren, D. I., 2008. A new class of energy-preserving numerical integration methods. J. Phys. A, 41, doi:10.1088/1751-8113/41/4/045206.

Radon, J., 1917. Über die bestimmung von funktionen durch ihre integralwerte längs gewisser mannigfaltigkeiten. Berichte über die Verhandlungen der Königlich-Sächsischen Akademie der Wissenschaften zu Leipzig, Mathematisch-Physische Klasse, 69, 262-277.

Rastrigin, L. A., 1974. Systems of extremal control. Nauka, Moskow.

Rickers, F., Fichtner, A. \& Trampert, J., 2013. The Iceland - Jan Mayen plume system and its impact on mantle dynamics in the North Atlantic region: Evidence from full-waveform inversion. Earth Planet. Sci. Lett., 367, 39-51.

Ripley, B. D., 1987. Statistical Simulation. Wiley, New York.

Ritsema, J., Deuss, A., van Heijst, H. J. \& Woodhouse, J. H., 2011. S40RTS: a degree-40 shear-velocity model for the mantle from new Rayleigh wave dispersion, teleseismic traveltime and normal-mode splitting function measurements. Geophys. J. Int., 184, 1223-1236.

Rosenbrock, H. H., 1960. An automatic method for finding the greatest or least value of a function. Comp. J., 3, 175-184.

Rosenthal, J. S., 2011. Optimal proposal distributions and adpative MCMC. In Handbook of Markov Chain Monte Carlo, pp. Chapter 4.

Roy, V., 2019. Convergence diagnostics for Markov chain Monte Carlo. arXiv, [stat.CO], 1909.11827v2.

Rudolph, G., 1990. Globale Optimierung mit parallelen Evolutionsstrategien. Diploma thesis, Universität Dortmund.

Ruth, R., 1983. A canonical integration technique. IEEE Trans. Nucl. Sci., 30, 2669-2671.

Sambridge, M. S., 2014. A parallel tempering algorithm for probabilistic sampling and multi-modal optimization. Geophys. J. Int., 196, 357-374.

Sambridge, M. S., Gallagher, K., Jackson, A. \& Rickwood, P., 2006. Trans-dimensional inverse problems, model comparison, and the evidence. Geophys. J. Int., 167, 528-542.

Santosa, F., 1982. Numerical scheme for the inversion of acoustical impedance profile based on the gelfandlevitan method. Geophys. J. R. astr. Soc., 70, 229-243.

Santosa, F. \& Symes, W. W., 1988. Computation of the Hessian for least-squares solutions of inverse problems of reflection seismology. Inverse Problems, 4, 211-233. 
Sanz-Serna, J. M. \& Calvo, M. P., 1994. Numerical Hamiltonian problems. Chapman and Hall, London, UK.

Scott, S. L., Blocker, A. W., Bonassi, F. V., Chipman, H. A., George, E. I. \& McCulloch, R. E., 2016. Bayes and Big Data: The consensus Monte Carlo algorithm. Int. J. Manage. Sci. Eng. Manage., 11, 78-88.

Seah, Y.-L., Shang, J., Ng, H. K., Nott, D. J. \& Englert, B.-G., 2015. Monte Carlo sampling from the quantum state space. II. New J. Phys., 17, doi:10.1088/1367-2630/17/4/043018.

Sen, M. K. \& Biswas, R., 2017. Tansdimensional seismic inversion using the reversible jump Hamiltonian Monte Carlo algorithm. Geophysics, 82, R119-R134.

Sen, M. K. \& Stoffa, P. L., 2013. Global optimization methods in geophysical inversion. Cambridge, UK: Cambridge University Press.

Shanno, D. F., 1970. Conditioning of quasi-Newton methods for function minimization. Math. Comp., 24, 647-656.

Sherman, J. \& Morrison, W. J., 1950. Adjustment of an inverse matrix corresponding to a change in one element of a given matrix. Ann. Math. Stat., 21, 124-127.

Sieminski, A., Liu, Q., Trampert, J. \& Tromp, J., 2007a. Finite-frequency sensitivity of body waves to anisotropy based upon adjoint methods. Geophys. J. Int., 171, 368-389.

Sieminski, A., Liu, Q., Trampert, J. \& Tromp, J., 2007b. Finite-frequency sensitivity of surface waves to anisotropy based upon adjoint methods. Geophys. J. Int., 168, 1153-1174.

Sieminski, A., Trampert, J. \& Tromp, J., 2009. Principal component analysis of anisotropic finite-frequency kernels. Geophys. J. Int., 179, 1186-1198.

Simo, J. C., Tarnow, N. \& Wong, K. K., 1992. Exactly energy-momentum conserving algorithms and symplectic schemes for nonlinear dynamics. Comp. Meth. Appl. Mech. Eng., 100, 63-116.

Smithyman, B., Pratt, R. G., Hayles, J. \& Wittebolle, R., 2009. Detecting near-surface objects with seismic waveform tomography. Geophysics, 74, WCC119-WCC127.

Snieder, R. \& Trampert, J., 1999. Inverse problems in geophysics. In A. Wirgin (Ed.), Wavefield Inversion, pp. 119-190.

Soldati, G., Boschi, L. \& Piersanti, A., 2006. Global seismic tomography and modern parallel computers. Ann. Geophys., 49, 977-986.

Styblinski, M. A. \& Tang, T.-S., 1990. Experiments in nonconvex optimization: Stochastic approximation with function smoothing and simulated annealing. Neural Networks, 3, 467-483.

Symes, W. W., 1980. Numerical stability in an inverse scattering problem. SIAM J. Num. Analysis, 17, 707-732.

Symon, K. R., 1971. Mechanics. Addison-Wesley, Reading, Massachusetts.

Szu, H. \& Hartley, R., 1987. Fast simulated annealing. Phys. Lett. A, 122, 157-162.

Takeuchi, H. \& Saito, M., 1972. Seismic surface waves. in: Methods in Computational Physics, editor B. A. Bolt, 11, 217-295.

Tape, C., Liu, Q., Maggi, A. \& Tromp, J., 2010. Seismic tomography of the southern California crust based upon spectral-element and adjoint methods. Geophys. J. Int., 180, 433-462.

Tarantola, A., 1984. Inversion of seismic reflection data in the acoustic approximation. Geophysics, 49, 12591266.

Tarantola, A., 1988. Theoretical background for the inversion of seismic waveforms, including elasticity and attenuation. Pure Appl. Geophys., 128, 365-399.

Tarantola, A., 2005. Inverse problem theory and methods for model parameter estimation, 2 nd edition. Society for Industrial and Applied Mathematics, Philadephia.

Tarantola, A. \& Valette, B., 1982. Generalized nonlinear inverse problems solved using the least squares criterion. Rev. Geophys., 20, 219-232.

Tong, J., Dahlen, F. A., Nolet, G. \& Marquering, H., 1998. Diffraction effects upon finite-frequency travel times: A simple 2-D example. Geophys. Res. Lett., 25, 1983-1986.

Trampert, J. \& Fichtner, A., 2013. Resolution tests revisited: The power of random numbers. Geophys. J. Int., 192, 676-680.

Tromp, J., Tape, C. \& Liu, Q., 2005. Seismic tomography, adjoint methods, time reversal and banana-doughnut kernels. Geophys. J. Int., 160, 195-216.

Unwin, S. D., 2004. The probability of God. Crown Publishing Group. 
van Leeuwen, T. \& Mulder, W. A., 2010. A correlation-based misfit criterion for wave-equation traveltime tomography. Geophys. J. Int., 182, 1383-1394.

Virieux, J., 1984. SH wave propagation in heterogeneous media: velocity-stress finite difference method. Geophysics, 49, 1933-1942.

Wapenaar, K., Broggini, F., Slob, E. \& Snieder, R., 2013. Three-dimensional single-sided Marchenko inverse scattering, data-driven focusing, Green's function retrieval, and their mutual relations. Phys. Rev. Lett., 110, doi:10.1103/PhysRevLett.110.084301.

Wapenaar, K., Thorbecke, J., van der Neut, J., Broggini, F., Slob, E. \& Snieder, R., 2014. Marchenko imaging. Geophysics, 79, 1WA39-WA57.

Ware, J. A. \& Aki, K., 1969. Continuous and discrete inverse-scattering problems in a stratified elastic medium. i. plane waves at normal incidence. J. Acoust. Soc. Am., 45, 911-921.

Wei, Q., Dobigeon, N. \& Tourneret, J.-Y., 2015. Bayesian fusion of multi-band images. IEEE J. Select. Topics Signal Proc., 9, doi:10.1109/JSTSP.2015.2407855.

Wolpert, D. H. \& Macready, W. G., 1997. No Free Lunch Theorems for optimization. IEEE Trans. Evolutionary Comp., 1, 67-82.

Zaroli, C., 2019. Seismic tomography using parameter-free Backus-Gilbert inversion. Geophys. J. Int., 218, $619-630$.

Zaroli, C., Koelemeijer, P. \& Lambotte, S., 2017. Towards seeing the Earth's interior through unbiased tomographic lenses. Geophys. Res. Lett., 44, doi:10.1002/2017GL074996. 



\section{Index}

$\delta$-distribution, 40

absolute information content, 44

absorption coefficient, 237

acceptance criterion, 87

acceptance probability, 87

acceptance rate, 90

activated data space, 144

activated model space, 143, 270

activated vector space, 270

adaptive time step, 260, 262

adjoint equation, 193, 201

adjoint field, 193, 201

adjoint method, 191

continuous, 192, 197

discrete, 192

second order, 256

adjoint methods, 94

adjoint operator, 200

adjoint source, 193, 201, 206, 207, 209

adjoint stress tensor, 214

ambient seismic noise, 53

annealing, 97

annihilator wavefield, 230

anti-attenuation, 195, 214

apple for breakfast, 25

autocorrelation of samples, 91

autotuning Hamiltonian Monte Carlo, 255

autotuning Monte Carlo sampling, 255

average model, 82

averaging kernel, 135, 154

averaging length, 155

Backus-Gilbert method, 153, 191, 197

banana-doughnut kernel, 210

banana-doughnut paradox, 210

basis functions, 9, 201

Bayes' theorem, 27, 31, 56, 57

transdimensional, 110

Bayesian interpretation, 43

Bazaraa-Shetty function, 169, 172

Beer-Lambert law, 237

Bessel function, 68

BFGS method, 184, 256, 257

factorised, 258 limited memory (L-BFGS), 185

binomial coefficient, 28

binomial distribution, 28

birth-death algorithm, 119

blunder, 8

body wave, 216

Box-Muller transform, 85

Brownian motion, 95

bulk modulus, 279

burn-in phase, 80,257

canonical distribution, 102, 261

Cauchy distribution, 51, 60

Central Limit Theorem, 66

central slice theorem, 237

CFL number, 287

characteristic equation (Love wave), 280

Cholesky decomposition, 46, 47, 85, 258

circular mean, 69

circular normal distribution (von Mises distribution), 60, 69

comparing apples and oranges, 210

condition number, 129

conditional probability, 27

conjugate-gradient method, 175

Fletcher-Reeves, 180, 181

Hestenes-Stiefel, 180, 181

Polak-Ribière, 180, 181

conjunction of events, 26

conjunction of probability densities, 48

conservatively filtered model, 249

consistency problem, 17,19

constitutive relation, 212

contrary events, 26

control parameters, 8

convexity, 166, 184

convolution integral, 50

cooling schedule, 98

correlation

cross-correlation, 208

time shift, 208

correlation length, 130

Courant-Friedrichs-Lewy (CFL) condition, 286

covariance matrix, 41

data, 19, 55

model, 56 
cross-hole tomography, 126

cumulative density function, 84

curse of dimensionality, 90, 97, 99, 119

curvature condition, 184

damping, 132

data covariance matrix, 19, 55

data importance, 138

data kernel, 153, 191

data kernels, 197

data resolution matrix, 138

data space, 8

David Bowie, 247

de Moivre-Laplace approximation, 29

deltaness measure, 154

dependent parameters, 41, 42, 46

descent direction, 167

descent method, 166, 191

detailed balance, 78, 103, 111, 113, 115

continuous, 80

determinedness, 13

deterministic inversion, 21, 63

diagonal freezing, 261

diffeomorphism, 116

diffusion equation, 66

direction of steepest ascent, 94

direction of steepest descent, 169, 250

Dirichlet boundary condition, 202, 285

disjunction of events, 26

dispersion curve (Love wave), 156

dispersion relation (Love wave), 280

displacement function (Love wave), 279

effective sample fraction, 256

effective sample size, 91, 256

eikonal equation, 229

elastic tensor, 212

elastic wave equation, 279

energy conservation, 101

energy equation (Love wave), 282

ENIAC, 73

entropy, 44

equileverage design, 138

equilibrium random walk, 78, 81

equipotential surface, 11

error propagation, 135

even-determined problem, 13

event, 25

evidence, 57,58

conditional, 111

total, 111

expectation, 26, 82

extended data, 124

extended forward modelling operator, 124

extremal bounds analysis, 145

f-divergence, 44

Fermat's principle, 126

filtering, 207

finite-frequency effects, 210

first discretise then optimise, 192

first optimise then discretise, 192
Fisher distribution, 69

fix-point iteration, 233

formal resolution, 9

forward modelling operator, 9

Fourier slice theorem, 237

Fréchet derivative, 198

Fréchet kernel, 198, 201

frequentist interpretation, 43

Fresnel zone, generalised, 210

full-waveform inversion, 202

functional, 198

functional derivative (Fréchet derivative), 198

fundamental mode (Love wave), 156, 280

fundamental solution, 226

gamma function, 75

Gauss elimination, 47

Gauss' law of gravitation, 11, 197

Gauss-Newton method, 174

Gaussian distribution, 29, 39, 64

generalised inverse, 135

generalised momentum, 100, 250

generalised position, 100, 250

global optimisation, 165

global optimiser, 98

Goldilocks principle, 91

gradient tests, 196

gravitating phenomenon (Gaussian distribution), 65

gravity potential, 11

gross Earth functional, 154

hairy caterpillar, 91

Hamilton's equations, 100, 250

Hamiltonian, 100, 250

Hamiltonian Monte Carlo, 94, 99, 191, 253, 255, 256, 272

Hamiltonian Monte Carlo algorithm, 94

Hessian, 168

approximate, 174

Hessian-aware Monte Carlo sampling, 255, 256

higher modes (Love wave), 156, 280

Himmelblau function, 169, 172

hockey stick plot, 196

homogeneous distribution, 42, 56

Hooke's law, 212, 279

Hutchinson's method, 147

hypothesis testing, 247

iid (independent and identically distributed), 60

ill-conditioned problem, 129

imaging nullspace, 152

impedance, 225

implicit regularisation, 168

importance sampling, 78

impulse response, 136

inconsistent events, 26

independent events, 26

independent parameters, 41, 42, 46

independent random variables, 83

influence zone, 205

information content, 44

invariance principle, 70

inverse crime, 129, 136 
inverse probability integral transform, 85 inverse scattering problem, 223

inverse transform sampling, 85

inversion

direct, 2

indirect, 2

Jacobian, 164, 174, 192, 216

Jeffreys parameters, 70

kernel, 143, 270

kinetic energy, 100, 250

Kolmogorov axioms, 34

Kullback-Leibler divergence, 44, 263

kurtosis, 63

L-BFGS method, 185, 256

factorised, 259

L-curve, 132, 160

Lagrange function, 269

Lagrange multiplier method, 269

Landon derivation, 65

Langevin diffusion, 95

Langevin equation, 95

leap-frog scheme, 104, 250, 260, 272

energy dissipation, 260, 273

numerical stability, 260, 272

least-squares problem, 17, 64, 123, 247

left-singular vector, 142, 248

Levenberg method, 175

Levenberg-Marquardt method, 175

Lighthouse problem, 59

likelihood function, 57

line fitting as inverse problem, 14

line search, 168, 173

linear data functional, 153

linear inverse problem, 14

linearisation, 163

local minimum, 165

local optimisation, 165

logarithmic parameters, 70

logical and (conjunction), 26

logical or (disjunction), 26

logical product, 26

logical sum (disjunction), 26

logistic rule, 87,88

Love wave, 155, 279

macroscopic autotuning, 261

Major Tom, 247

MAP model, 63

Marchenko equation, 232

marginal probability, 32

marginal probability density, 49

Markov chain, 89, 261

Markov chain Monte Carlo (MCMC), 89

bias, 261

mixing, 91

mass matrix, 108, 250, 256, 257

tempered, 262

matrix probing, 147

matrix-free optimisation, 166 maximum a posteriori (MAP), 63

maximum-entropy principle, 66

mean model, 62

mean of a Gaussian, 29, 39

meateorite impact in the backyard, 43

medical ultrasound tomography, 126

Metropolis rule, 87, 88, 103, 116, 253

Metropolis-adjusted Langevin algorithm (MALA), 94, 191, 255

Metropolis-Hastings algorithm, 119, 255

global, 88, 99, 112, 114

local, 90, 99

misfit function, 64, 123, 191

misfit functional, 208

mixed-determined problem, 13

model covariance, 56

model resolution matrix, 135

model space, 8

moments of a distribution, 62

Monte Carlo integration, 81

Monte Carlo methods, 78

Moore-Penrose inverse, 17, 127, 139, 142

most-squares method, 145

movie strategie, 61

Newton equation, 171

Newton's method, 171, 251

damped, 172

Gauss-Newton, 174

Levenberg, 175

Levenberg-Marquardt, 175

regularised, 172

Newton's second law, 212, 278

No-Free-Lunch theorem, 166, 172, 188, 243

non-uniqueness, 10

coverage, 11

gravity, 11

inherent, 11

norm of a vector or function, 199

normal (Gaussian) distribution, 39

normalisable probability density, 39

nullspace, 143, 247, 270

effective, 248

generalised, 248

nullspace shuttle, 247

Hamiltonian, 249

linear, 247

nonlinear, 249

nonlinear, randomised, 253

orbital properties, 250

number of effectively resolved parameters, 135, 137

numerical dispersion, 288

Occam's razor, 110, 134

one-way traveltime, 224

optimal design, 8

outlier, 8

over-damping, 132

over-determined problem, 13

over-fitting, 20, 132, 168

over-shooting, 172

over-smoothing, 132 
overtones (Love wave), 156, 280

parsimony, 134

natural, 110

phase space, 101, 251

phase velocity, 155, 202, 280

Poincaré recurrence, 260

point-spread function, 135

Poisson equation, 11

posterior covariance, 62

posterior in model space, 56

posterior maximum-likelihood model, 63

posterior model covariance, 123

posterior model mean, 123

posterior probability, 31

potential energy, 100, 250

prior in data space, 55,64

prior in model space, 55, 56, 64

prior knowledge, 31

prior probability, 30

probabilistic inversion, 21, 63

probability density, 32,38

product, 50

quotient, 50

sum, 50

probability distribution, 28

product rule, 34

projection slice theorem, 237

pseudoinverse of a matrix, 17

quasi-Newton equation, 182

quasi-Newton method, 180, 257

Rademacher vector, 147

Radon transform, 238

Radon-Nikodym theorem, 38

random errors, 8

random walk, 78

random-walk Metropolis-Hastings (RWMH), 94

rank-1 modification, 184

Rastrigin function, 275

ray density plot, 126

ray expansion, 227

reflection data, 226

regularisation, 9, 132, 136

explicit, 189

implicit, 189

relative information content, 44

residual, 175

resolution length, 155

resolution matrix, 135

reversible-jump algorithm, 114

detailed balance, 115

Metropolis rule, 116

Riesz' representation theorem, 198, 201, 270

right-singular vector, 142, 248

robustness, 21

root-mean-square misfit, 20, 130, 132, 168

Rosenbrock function, 169, 170, 172, 180, 181

sample covariance, 82

sample mean, 82 samples of a distribution, 78

sampling, 72

sampling a distribution, 78

scalar product, 199

scalar wave equation, 202

scattering ellipse, 210

scattering potential, 225

Schrödinger equation, 224, 225

search radius, 91

secant equation, 182

second-order adjoint method, 256

seismic wave, 216

self-adjoint operator, 204, 214

sensitivity analysis, 212

sensitivity kernel, 153, 157, 201, 204, 215, 283

change of variables, 215

shear modulus, 279

Sherman-Morrison formula, 183

signal energy, 209

Simulated Annealing, 97, 261

singular value, 142

singular vector, 142

singular-value decomposition, 142, 248

skewness, 63

smoothing, 132, 251

Snell's law, 277

sparse matrix, 127

spatial resolution, 9

SR1 method, 182

staggered-grid finite-difference method, 284

standard deviation, 83

standard deviation of a Gaussian, 29, 39

steepest descent method, 94, 169, 173, 251

step length, 167

optimal, 168

stress function (Love wave), 279

Styblinski-Tang function, 274

sum rule, 34

support of a function, 230

surface wave, 216

symplectic integrator, 104

synthetic inversion, 129

systematic errors, 8

tempering, 97, 261

terminal conditions, 203, 213

test functions, 169

high-dimensional, 274

time reversability, 101

time reversal, 204

tomographic telescope, 136

trace plots, 91

transcranial ultrasound, 217

transdimensional inverse problems, 9, 58, 110

transdimensional sampling, 80

naive, 111

reversible-jump algorithm, 114

transformation rule for probability densities, 45

transition probability, 78

Gaussian, 90

transition probability density, 80

transport equation, 229 
traveltime sensitivity kernel, 210

traveltime tomography, 126, 277

triangular matrix

lower, 47

upper, 47

truth table, 34

typical set, 75, 80

unconditional probability, 27

under-damping, 132

under-determined problem, 13, 126

under-fitting, 20, 130, 132

under-smoothing, 132 uniform distribution, 40

variance, 82

Venn diagram, 57

vertical seismic profiling, 15

visco-elastic attenuation, 212

visco-elastic wave equation, 212

von Mises distribution, 60, 68

wave equation, 193

wave path, 210

weight matrix, 124

well-conditioned problem, 129 